Cochrane Database of Systematic Reviews

\title{
Multifactorial and multiple component interventions for preventing falls in older people living in the community (Review)
}

Hopewell S, Adedire O, Copsey BJ, Boniface GJ, Sherrington C, Clemson L, Close JCT, Lamb SE 
TABLE OF CONTENTS

HEADER . . . . . . . . . . . . . . . . . . . . . . . . . . . . . . . . . . . . . . . 1

ABSTRACT . . . . . . . . . . . . . . . . . . . . . . . . . . . . . . . . . . . . . . . . . . . . . . .

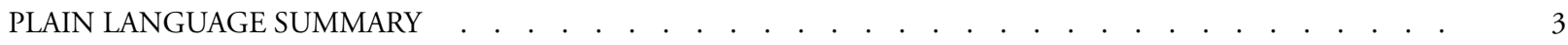

SUMMARY OF FINDINGS FOR THE MAIN COMPARISON . . . . . . . . . . . . . . . . . . . . . . . . . . . . 5

BACKGROUND . . . . . . . . . . . . . . . . . . . . . . . . . . . . . . . . . . . . . 9

OBJECTIVES . . . . . . . . . . . . . . . . . . . . . . . . . . . . . . . . . . . . . . . . . . . . . . . . . .

METHODS . . . . . . . . . . . . . . . . . . . . . . . . . . . . . . . . . . . . . . . . . .

RESUlTS . . . . . . . . . . . . . . . . . . . . . . . . . . . . . . . . . . . . . . . . . . 15

Figure 1. . . . . . . . . . . . . . . . . . . . . . . . . . . . . . . . . . . . . . . 16

Figure 2. . . . . . . . . . . . . . . . . . . . . . . . . . . . . . . . . . . . . . 20

Figure 3. . . . . . . . . . . . . . . . . . . . . . . . . . . . . . . . . . . . . . . 21

Figure 4. . . . . . . . . . . . . . . . . . . . . . . . . . . . . . . . . . . . . . 23

Figure 5. . . . . . . . . . . . . . . . . . . . . . . . . . . . . . . . . . . . . .

Figure 6. . . . . . . . . . . . . . . . . . . . . . . . . . . . . . . . . . . . . . .

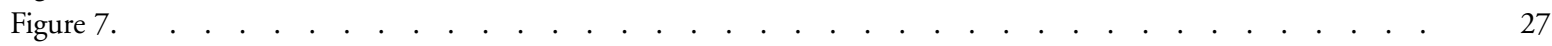

ADDITIONAL SUMMARY OF FINDINGS . . . . . . . . . . . . . . . . . . . . . . . . . . . . . . . . .

DISCUSSION . . . . . . . . . . . . . . . . . . . . . . . . . . . . . . . . . . . . . . . . . 42

AUTHORS' CONCLUSIONS . . . . . . . . . . . . . . . . . . . . . . . . . . . . . . . . . . . . 45

ACKNOWLEDGEMENTS . . . . . . . . . . . . . . . . . . . . . . . . . . . . . . . . . . . . . 46

REFERENCES . . . . . . . . . . . . . . . . . . . . . . . . . . . . . . . . . . . . . . . . 46

CHARACTERISTICS OF STUDIES . . . . . . . . . . . . . . . . . . . . . . . . . . . . . . . . . . . . . . . . . . .

DATA AND ANALYSES . . . . . . . . . . . . . . . . . . . . . . . . . . . . . . . . . . . . . . . . . . . . . . . 192

Analysis 1.1. Comparison 1 Multifactorial intervention vs usual care or attention control, Outcome 1 Rate of falls (falls per person years). . . . . . . . . . . . . . . . . . . . . . . . . . . . . . . . . . . . . . . . . . . 200

Analysis 1.2. Comparison 1 Multifactorial intervention vs usual care or attention control, Outcome 2 Number of people sustaining one or more falls. . . . . . . . . . . . . . . . . . . . . . . . . . . . . . . . . . . . . . . . . . . $\quad . \quad 20$

Analysis 1.3. Comparison 1 Multifactorial intervention vs usual care or attention control, Outcome 3 Number of people sustaining recurrent falls (defined as two or more falls in a specified time period).

Analysis 1.4. Comparison 1 Multifactorial intervention vs usual care or attention control, Outcome 4 Number of people sustaining one or more fall-related fractures.

Analysis 1.5. Comparison 1 Multifactorial intervention vs usual care or attention control, Outcome 5 Number of people who experience a fall that required hospital admission. . . . . . . . . . . . . . . . . . . . . . . . .

Analysis 1.6. Comparison 1 Multifactorial intervention vs usual care or attention control, Outcome 6 Number of people who experience a fall that require medical attention. $\quad . \quad$. . . . . . . . . . . . . . . . . . . . . . . 206

Analysis 1.7. Comparison 1 Multifactorial intervention vs usual care or attention control, Outcome 7 Health-related quality of life: endpoint score. . . . . . . . . . . . . . . . . . . . . . . . . . . . . . . . . . . . . . . . . . . . . 207

Analysis 1.8. Comparison 1 Multifactorial intervention vs usual care or attention control, Outcome 8 Health-related quality of life (mental): endpoint score. . . . . . . . . . . . . . . . . . . . . . . . . . . . . . . . . . 208

Analysis 1.9. Comparison 1 Multifactorial intervention vs usual care or attention control, Outcome 9 Health-related quality of life (physical): endpoint score. . . . . . . . . . . . . . . . . . . . . . . . . . . . . . . . . . . . . . . 209

Analysis 2.1. Comparison 2 Multifactorial intervention vs exercise, Outcome 1 Rate of falls (falls per person years). . 209

Analysis 2.2. Comparison 2 Multifactorial intervention vs exercise, Outcome 2 Number of people sustaining one or more falls. . . . . . . . . . . . . . . . . . . . . . . . . . . . . . . . . . . . . . 210

Analysis 3.1. Comparison 3 Multiple intervention vs usual care or attention control, Outcome 1 Rate of falls (falls per person years).

Analysis 3.2. Comparison 3 Multiple intervention vs usual care or attention control, Outcome 2 Number of people sustaining one or more falls. 201 208 210 nalysis 3.3. Comparison 3 Multiple intervention vs usual care or attention control, Outcome 3 Number of people sustaining recurrent falls (defined as two or more falls in a specified time period).

Analysis 3.4. Comparison 3 Multiple intervention vs usual care or attention control, Outcome 4 Number of people sustaining one or more fall-related fractures. 
Analysis 3.5. Comparison 3 Multiple intervention vs usual care or attention control, Outcome 5 Number of people who experience a fall that required hospital admission. $\quad . \quad$. . . . . . . . . . . . . . . . . . . . . .

Analysis 3.6. Comparison 3 Multiple intervention vs usual care or attention control, Outcome 6 Number of people who experience a fall that required medical attention. . . . . . . . . . . . . . . . . . . . . . . . . . . . .

Analysis 3.7. Comparison 3 Multiple intervention vs usual care or attention control, Outcome 7 Health-related quality of life: endpoint score. . . . . . . . . . . . . . . . . . . . . . . . . . . . . . . . . . . . . . . . 217

Analysis 3.8. Comparison 3 Multiple intervention vs usual care or attention control, Outcome 8 Health-related quality of life (mental): endpoint score. . . . . . . . . . . . . . . . . . . . . . . . . . . . . . . . . . . . .

Analysis 3.9. Comparison 3 Multiple intervention vs usual care or attention control, Outcome 9 Health-related quality of life (physical): endpoint score. . . . . . . . . . . . . . . . . . . . . . . . . . . . . . . .

Analysis 4.1. Comparison 4 Multiple intervention vs exercise, Outcome 1 Rate of falls (falls per person years). . . .

Analysis 4.2. Comparison 4 Multiple intervention vs exercise, Outcome 2 Number of people sustaining one or more falls.

Analysis 4.3. Comparison 4 Multiple intervention vs exercise, Outcome 3 Number of people who experience a fall that required hospital admission. . . . . . . . . . . . . . . . . . . . . . . . . . . . . . . . . . . .

Analysis 5.1. Comparison 5 Multifactorial intervention vs control: subgroup analysis by intensity of intervention, Outcome 1 Rate of falls (falls per person years). . . . . . . . . . . . . . . . . . . . . . . . . . . . . . . . . . . . $\quad 222$

Analysis 5.2. Comparison 5 Multifactorial intervention vs control: subgroup analysis by intensity of intervention, Outcome 2 Number of people sustaining one or more falls. $\quad . \quad$. . . . . . . . . . . . . . . . . . . . . . . . . . . . $\quad 224$

Analysis 5.3. Comparison 5 Multifactorial intervention vs control: subgroup analysis by intensity of intervention, Outcome 3 Number of people sustaining recurrent falls (defined as two or more falls in a specified time period). . . . . 226

Analysis 6.1. Comparison 6 Multifactorial intervention vs control: subgroup analysis by falls risk at baseline, Outcome 1 Rate of falls (falls per person years).

Analysis 6.2. Comparison 6 Multifactorial intervention vs control: subgroup analysis by falls risk at baseline, Outcome 2

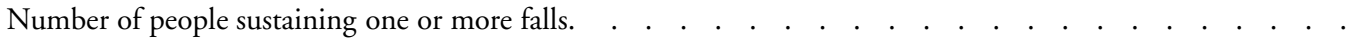

Analysis 6.3. Comparison 6 Multifactorial intervention vs control: subgroup analysis by falls risk at baseline, Outcome 3 Number of people sustaining recurrent falls (defined as two or more falls in a specified time period). . . . . . 224 226

Analysis 7.1. Comparison 7 Multiple intervention vs control: subgroup analysis by falls risk at baseline, Outcome 1 Rate of falls (falls per person years). . . . . . . . . . . . . . . . . . . . . . . . . . . . . . . . . . .

Analysis 7.2. Comparison 7 Multiple intervention vs control: subgroup analysis by falls risk at baseline, Outcome 2 Number

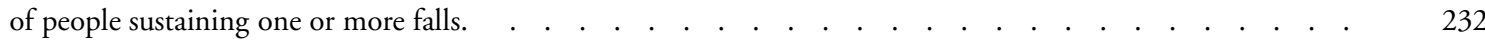

Analysis 7.3. Comparison 7 Multiple intervention vs control: subgroup analysis by falls risk at baseline, Outcome 3 Number of people sustaining recurrent falls (defined as two or more falls in a specified time period). . . . . . . . . . .

Analysis 8.1. Comparison 8 Multifactorial intervention vs control: sensitivity analysis by low risk of selection bias, Outcome 1 Rate of falls (falls per person years). . . . . . . . . . . . . . . . . . . . . . . . . . . . . . . . . . . $\quad 234$

Analysis 8.2. Comparison 8 Multifactorial intervention vs control: sensitivity analysis by low risk of selection bias, Outcome 2 Number of people sustaining one or more falls. $\quad . \quad$. . . . . . . . . . . . . . . . . . . . . . . . . . . .

Analysis 8.3. Comparison 8 Multifactorial intervention vs control: sensitivity analysis by low risk of selection bias, Outcome 3 Number of people sustaining recurrent falls (defined as two or more falls in a specified time period). . . . $\quad 236$

Analysis 9.1. Comparison 9 Multifactorial intervention vs control: sensitivity analysis by low risk of detection bias, Outcome 1 Rate of falls (falls per person years). . . . . . . . . . . . . . . . . . . . . . . . . . . . . 237

Analysis 9.2. Comparison 9 Multifactorial intervention vs control: sensitivity analysis by low risk of detection bias, Outcome 2 Number of people sustaining one or more falls. . . . . . . . . . . . . . . . . . . . . . . . . . . . . . . 238

Analysis 9.3. Comparison 9 Multifactorial intervention vs control: sensitivity analysis by low risk of detection bias, Outcome 3 Number of people sustaining recurrent falls (defined as two or more falls in a specified time period). . . .

Analysis 10.1. Comparison 10 Multifactorial intervention vs control: sensitivity analysis by low risk of attrition bias, Outcome 1 Rate of falls (falls per person years).

Analysis 10.2. Comparison 10 Multifactorial intervention vs control: sensitivity analysis by low risk of attrition bias, Outcome 2 Number of people sustaining one or more falls. . . . . . . . . . . . . . . . . . . . . . . . .

Analysis 10.3. Comparison 10 Multifactorial intervention vs control: sensitivity analysis by low risk of attrition bias, Outcome 3 Number of people sustaining recurrent falls (defined as two or more falls in a specified time period).

Analysis 11.1. Comparison 11 Multifactorial intervention vs control: sensitivity analysis by individual randomisation, Outcome 1 Rate of falls (falls per person years). 
Analysis 11.2. Comparison 11 Multifactorial intervention vs control: sensitivity analysis by individual randomisation,

Outcome 2 Number of people sustaining one or more falls. . . . . . . . . . . . . . . . . . . . . . .

Analysis 11.3. Comparison 11 Multifactorial intervention vs control: sensitivity analysis by individual randomisation,

Outcome 3 Number of people sustaining recurrent falls (defined as two or more falls in a specified time period).

Analysis 12.1. Comparison 12 Multiple intervention vs control: sensitivity analysis by low risk of selection bias, Outcome 1

Rate of falls (falls per person years). . . . . . . . . . . . . . . . . . . . . . . . . . . . . . . . . . . . . 246

Analysis 12.2. Comparison 12 Multiple intervention vs control: sensitivity analysis by low risk of selection bias, Outcome 2

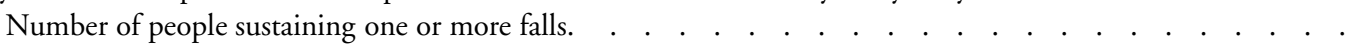

Analysis 12.3. Comparison 12 Multiple intervention vs control: sensitivity analysis by low risk of selection bias, Outcome 3

Number of people sustaining recurrent falls (defined as two or more falls in a specified time period). . . . . . $\quad 248$

Analysis 13.1. Comparison 13 Multiple intervention vs control: sensitivity analysis by low risk of detection bias, Outcome

1 Rate of falls (falls per person years). . . . . . . . . . . . . . . . . . . . . . . . . . . . . . . . . . . . . .

Analysis 13.2. Comparison 13 Multiple intervention vs control: sensitivity analysis by low risk of detection bias, Outcome

2 Number of people sustaining one or more falls. $\quad . \quad$. . . . . . . . . . . . . . . . . . . . . . . . . . . . .

Analysis 13.3. Comparison 13 Multiple intervention vs control: sensitivity analysis by low risk of detection bias, Outcome

3 Number of people sustaining recurrent falls (defined as two or more falls in a specified time period). . . . .

Analysis 14.1. Comparison 14 Multiple intervention vs control: sensitivity analysis by low risk of attrition bias, Outcome 1

Rate of falls (falls per person years). $\quad . \quad$. . . . . . . . . . . . . . . . . . . . . . . . . . . . . . . . . . . $\quad . \quad 252$

Analysis 14.2. Comparison 14 Multiple intervention vs control: sensitivity analysis by low risk of attrition bias, Outcome 2

Number of people sustaining one or more falls. $\quad . \quad$. . . . . . . . . . . . . . . . . . . . . . . . . . . . . . . . $\quad$. 253

Analysis 14.3. Comparison 14 Multiple intervention vs control: sensitivity analysis by low risk of attrition bias, Outcome 3

Number of people sustaining recurrent falls (defined as two or more falls in a specified time period). . . . . .

Analysis 15.1. Comparison 15 Multiple intervention vs control: sensitivity analysis by individual randomisation, Outcome

1 Rate of falls (falls per person years). . . . . . . . . . . . . . . . . . . . . . . . . . . . . . . . . . 255

Analysis 15.2. Comparison 15 Multiple intervention vs control: sensitivity analysis by individual randomisation, Outcome

2 Number of people sustaining one or more falls. . . . . . . . . . . . . . . . . . . . . . . . 256

Analysis 15.3. Comparison 15 Multiple intervention vs control: sensitivity analysis by individual randomisation, Outcome

3 Number of people sustaining recurrent falls (defined as two or more falls in a specified time period). . . . $\quad 257$

ADDITIONAL TABLES . . . . . . . . . . . . . . . . . . . . . . . . . . . . . . . . . . . . 257

APPENDICES . . . . . . . . . . . . . . . . . . . . . . . . . . . . . . . . . . . . . . 283

CONTRIBUTIONS OF AUTHORS . . . . . . . . . . . . . . . . . . . . . . . . . . . . . . . . . . . . . . . . . .

DECLARATIONS OF INTEREST . . . . . . . . . . . . . . . . . . . . . . . . . . . . . . . . . . . . .

SOURCES OF SUPPORT . . . . . . . . . . . . . . . . . . . . . . . . . . . . . . . . . . 305

DIFFERENCES BETWEEN PROTOCOL AND REVIEW . . . . . . . . . . . . . . . . . . . . . . . . . . . . .

NOTES . . . . . . . . . . . . . . . . . . . . . . . . . . . . . . . . . . . . . . 307

Multifactorial and multiple component interventions for preventing falls in older people living in the community (Review) 
[Intervention Review]

\title{
Multifactorial and multiple component interventions for preventing falls in older people living in the community
}

\author{
Sally Hopewell ${ }^{1}$, Olubusola Adedire ${ }^{2}$, Bethan J Copsey ${ }^{1}$, Graham J Boniface ${ }^{3}$, Catherine Sherrington ${ }^{4}$, Lindy Clemson ${ }^{5}$, Jacqueline \\ CT Close ${ }^{6}$, Sarah E Lamb ${ }^{1}$ \\ ${ }^{1}$ Nuffield Department of Orthopaedics, Rheumatology and Musculoskeletal Sciences (NDORMS), University of Oxford, Oxford, UK. \\ ${ }^{2}$ Biomedical Engineering, Oxehealth, Oxford, UK. ${ }^{3}$ Centre for Rehabilitation Research in Oxford (RRIO), Nuffield Department of \\ Orthopaedics, Rheumatology \& Musculoskeletal Sciences (NDORMS), University of Oxford, Oxford, UK. ${ }^{4}$ Musculoskeletal Health \\ Sydney, School of Public Health, The University of Sydney, Sydney, Australia. ${ }^{5}$ Faculty of Health Sciences, The University of Sydney, \\ Lidcombe, Australia. ${ }^{6}$ Falls, Balance and Injury Research Centre, Neuroscience Research Australia, Randwick, Australia
}

Contact address: Sally Hopewell, Nuffield Department of Orthopaedics, Rheumatology and Musculoskeletal Sciences (NDORMS), University of Oxford, Botnar Research Centre, Windmill Road, Oxford, Oxfordshire, OX3 7LD, UK. sally.hopewell@csm.ox.ac.uk.

Editorial group: Cochrane Bone, Joint and Muscle Trauma Group.

Publication status and date: New, published in Issue 7, 2018.

Citation: Hopewell S, Adedire O, Copsey BJ, Boniface GJ, Sherrington C, Clemson L, Close JCT, Lamb SE. Multifactorial and multiple component interventions for preventing falls in older people living in the community. Cochrane Database of Systematic Reviews 2018, Issue 7. Art. No.: CD012221. DOI: 10.1002/14651858.CD012221.pub2.

Copyright @ 2018 The Cochrane Collaboration. Published by John Wiley \& Sons, Ltd.

\begin{abstract}
A B S T R A C T
Background

Falls and fall-related injuries are common, particularly in those aged over 65, with around one-third of older people living in the community falling at least once a year. Falls prevention interventions may comprise single component interventions (e.g. exercise), or involve combinations of two or more different types of intervention (e.g. exercise and medication review). Their delivery can broadly be divided into two main groups: 1) multifactorial interventions where component interventions differ based on individual assessment of risk; or 2) multiple component interventions where the same component interventions are provided to all people.
\end{abstract}

Objectives

To assess the effects (benefits and harms) of multifactorial interventions and multiple component interventions for preventing falls in older people living in the community.

\section{Search methods}

We searched the Cochrane Bone, Joint and Muscle Trauma Group Specialised Register, the Cochrane Central Register of Controlled Trials, MEDLINE, Embase, the Cumulative Index to Nursing and Allied Health Literature, trial registers and reference lists. Date of search: 12 June 2017.

\section{Selection criteria}

Randomised controlled trials, individual or cluster, that evaluated the effects of multifactorial and multiple component interventions on falls in older people living in the community, compared with control (i.e. usual care (no change in usual activities) or attention control (social visits)) or exercise as a single intervention.

Multifactorial and multiple component interventions for preventing falls in older people living in the community (Review)

Copyright () 2018 The Cochrane Collaboration. Published by John Wiley \& Sons, Ltd. 


\section{Data collection and analysis}

Two review authors independently selected studies, assessed risks of bias and extracted data. We calculated the rate ratio (RaR) with 95\% confidence intervals (CIs) for rate of falls. For dichotomous outcomes we used risk ratios (RRs) and 95\% CIs. For continuous outcomes, we used the standardised mean difference (SMD) with $95 \%$ CIs. We pooled data using the random-effects model. We used the GRADE approach to assess the quality of the evidence.

\section{Main results}

We included 62 trials involving 19,935 older people living in the community. The median trial size was 248 participants. Most trials included more women than men. The mean ages in trials ranged from 62 to 85 years (median 77 years). Most trials (43 trials) reported follow-up of 12 months or over. We assessed most trials at unclear or high risk of bias in one or more domains.

Forty-four trials assessed multifactorial interventions and 18 assessed multiple component interventions. $\left(\mathrm{I}^{2}\right.$ not reported if $=0 \%$ ).

\section{Multifactorial interventions versus usual care or attention control}

This comparison was made in 43 trials. Commonly-applied or recommended interventions after assessment of each participant's risk profile were exercise, environment or assistive technologies, medication review and psychological interventions. Multifactorial interventions may reduce the rate of falls compared with control: rate ratio (RaR) 0.77, $95 \%$ CI 0.67 to $0.87 ; 19$ trials; 5853 participants; $\mathrm{I}^{2}=88 \%$; low-quality evidence. Thus if 1000 people were followed over one year, the number of falls may be 1784 (95\% CI 1553 to 2016) after multifactorial intervention versus 2317 after usual care or attention control. There was low-quality evidence of little or no difference in the risks of: falling (i.e. people sustaining one or more fall) (RR 0.96, 95\% CI 0.90 to 1.03; 29 trials; 9637 participants; $\mathrm{I}^{2}=60 \%$ ); recurrent falls (RR 0.87, 95\% CI 0.74 to 1.03; 12 trials; 3368 participants; $\mathrm{I}^{2}=53 \%$ ); fall-related hospital admission (RR 1.00, $95 \%$ CI 0.92 to $1.07 ; 15$ trials; 5227 participants); requiring medical attention (RR 0.91, 95\% CI 0.75 to $1.10 ; 8$ trials; 3078 participants). There is low-quality evidence that multifactorial interventions may reduce the risk of fall-related fractures (RR $0.73,95 \%$ CI 0.53 to $1.01 ; 9$ trials; 2850 participants) and may slightly improve health-related quality of life but not noticeably (SMD $0.19,95 \%$ CI 0.03 to $0.35 ; 9$ trials; 2373 participants; $I^{2}=70 \%$ ). Of three trials reporting on adverse events, one found none, and two reported 12 participants with self-limiting musculoskeletal symptoms in total.

\section{Multifactorial interventions versus exercise}

Very low-quality evidence from one small trial of 51 recently-discharged orthopaedic patients means that we are uncertain of the effects on rate of falls or risk of falling of multifactorial interventions versus exercise alone. Other fall-related outcomes were not assessed.

\section{Multiple component interventions versus usual care or attention control}

The 17 trials that make this comparison usually included exercise and another component, commonly education or home-hazard assessment. There is moderate-quality evidence that multiple interventions probably reduce the rate of falls ( $\mathrm{RaR} 0.74,95 \% \mathrm{CI} 0.60$ to $0.91 ; 6$ trials; 1085 participants; $\mathrm{I}^{2}=45 \%$ ) and risk of falls (RR $0.82,95 \%$ CI 0.74 to $0.90 ; 11$ trials; 1980 participants). There is low-quality evidence that multiple interventions may reduce the risk of recurrent falls, although a small increase cannot be ruled out (RR $0.81,95 \%$ CI 0.63 to 1.05 ; 4 trials; 662 participants). Very low-quality evidence means that we are uncertain of the effects of multiple component interventions on the risk of fall-related fractures ( 2 trials) or fall-related hospital admission ( 1 trial). There is lowquality evidence that multiple interventions may have little or no effect on the risk of requiring medical attention (RR 0.95 , 95\% CI 0.67 to 1.35 ; 1 trial; 291 participants); conversely they may slightly improve health-related quality of life (SMD $0.77,95 \%$ CI 0.16 to 1.39; 4 trials; 391 participants; $\mathrm{I}^{2}=88 \%$ ). Of seven trials reporting on adverse events, five found none, and six minor adverse events were reported in two.

\section{Multiple component interventions versus exercise}

This comparison was tested in five trials. There is low-quality evidence of little or no difference between the two interventions in rate of falls ( 1 trial) and risk of falling (RR $0.93,95 \%$ CI 0.78 to $1.10 ; 3$ trials; 863 participants) and very low-quality evidence, meaning we are uncertain of the effects on hospital admission ( 1 trial). One trial reported two cases of minor joint pain. Other falls outcomes were not reported.

\section{Authors' conclusions}

Multifactorial interventions may reduce the rate of falls compared with usual care or attention control. However, there may be little or no effect on other fall-related outcomes. Multiple component interventions, usually including exercise, may reduce the rate of falls and risk of falling compared with usual care or attention control.

Multifactorial and multiple component interventions for preventing falls in older people living in the community (Review) 


\section{PLAIN LANGUAGE SUMMARY}

Interventions based on individual assessment of falls risk and multiple component interventions for preventing falls in older people in the community

\section{Review question}

To assess whether fall-prevention strategies which target two or more risk factors for falls (multifactorial interventions) or fixed combinations of interventions (multiple component interventions) are effective in preventing falls in older people living in the community.

\section{Background}

As people age they are more likely to fall. Although most fall-related injuries are minor, they can cause significant pain and discomfort, affect a person's confidence and lead to a loss of independence. Some falls can cause serious long-term health problems. A combination of factors increases the risk of falls with ageing, such as weak muscles, stiff joints, hearing problems, changes in sight, side effects of medications, tiredness or confusion. Poor lighting, slippery or uneven surfaces, and issues with poor footwear can also increase the risk of falling.

Different interventions have been developed to help prevent falls in older people. They may involve a single type of intervention, such as exercise to increase muscle strength, or combinations of interventions, such as exercise and adjustment of a person's medication. A combination of two or more components can be delivered as either a multifactorial intervention based on an assessment of a person's risk factors for falling or as a multiple component intervention where the same combination of interventions is provided to all participants.

\section{Search date}

We searched the healthcare literature for reports of randomised controlled trials relevant to this review up to 12 June 2017.

\section{Study characteristics}

We included 62 randomised trials involving 19,935 older participants. Most trials included more women than men; the average ages in the trials ranged from 62 to 85 years. Trials compared the interventions to an inactive control group receiving usual care (no change in usual activities) or a matched level of attention (such as social visits) or to an active control group receiving an exercise programme.

\section{Key results}

We identified 43 trials that compared a multifactorial intervention with an inactive control. Multifactorial interventions led to some reduction in the rate at which people fall compared with the inactive control group, but the quality of evidence was low because of large differences in how studies were conducted. There may be little or no difference in the number of people who experienced one or more falls (fallers), recurrent falls, fall-related fractures, or experienced a fall requiring hospital admission or medical attention. Multifactorial interventions may make little difference to people's health-related quality of life. There was very limited evidence on adverse events related to the intervention; all 12 reported musculoskeletal complaints such as back pain were minor.

We did not find enough evidence to determine the effects of multifactorial interventions compared with exercise as this was only assessed in one small trial.

We identified 18 trials assessing the effects of multiple component interventions. Seventeen compared the intervention with an inactive control group and five compared the intervention with exercise. Seventeen of the trials included exercise in the intervention and another component, often education on falls prevention or home safety assessment. There was limited evidence on adverse events related to the intervention; all six reported events were minor.

Multiple component interventions probably reduce the rate at which people fall and the number of fallers compared with the inactive control group. They may also reduce the number of people who experienced recurrent falls. The evidence was not enough to determine their effects on fall-related fractures or hospital admission. Multiple component interventions may make little or no difference to the risk of a fall requiring medical attention. However, they may slightly improve a person's health-related quality of life.

Trials comparing multiple component interventions with exercise showed there may be little or no difference in the rate at which people fall and the number of fallers, but not enough evidence to determine the effects on hospital admission. Other falls outcomes were not reported.

\section{Quality of the evidence}

Multifactorial and multiple component interventions for preventing falls in older people living in the community (Review)

Copyright ( 2018 The Cochrane Collaboration. Published by John Wiley \& Sons, Ltd. 
We rated the quality of the available evidence as of low or very low quality. This means that we have limited confidence about the results where the evidence is low quality, but are uncertain where the evidence is of very low quality. 


\section{SUMMARY OF FINDINGS FOR THE MAIN COMPARISON [Explanation]}

\section{Multifactorial interventions ${ }^{a}$ compared with usual care or attention control for preventing falls in older people living in the community}

Patient or population: Older people living in the community

Setting: Community (home or places of residence that do not provide residential health-related care)

Intervention: Multifactorial interventions (i.e. where component interventions are based on individual assessment of falls risk) ${ }^{b}$

Comparison: Usual care or attention control

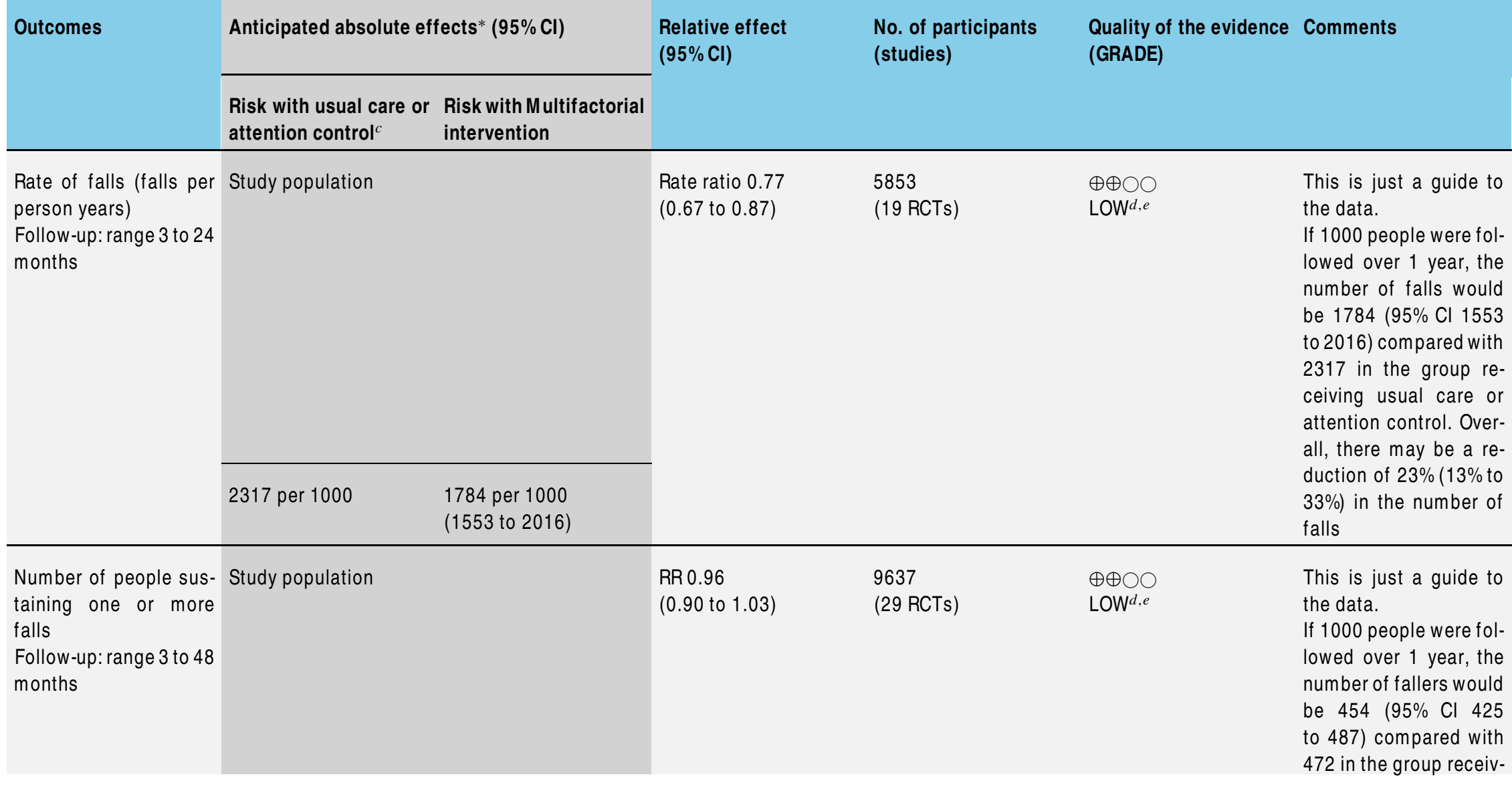




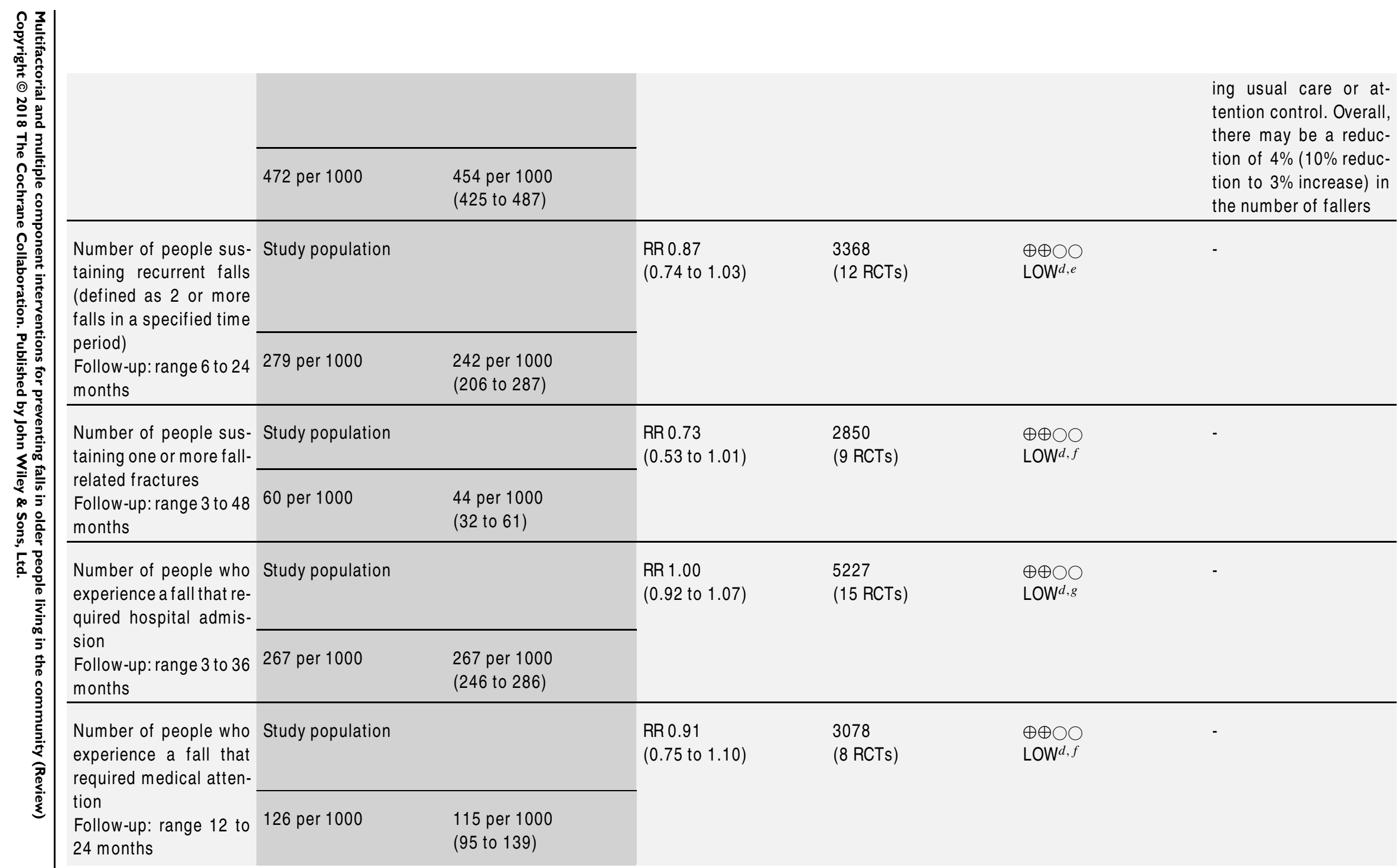




\begin{tabular}{|c|c|c|c|c|c|c|}
\hline $\begin{array}{l}\text { Health-related quality } \\
\text { of life } \\
\text { assessed with: SF- } 36 \\
\text { Scale from: } 0 \text { (worst) to } \\
100 \text { (best) } \\
\text { Follow-up: range } 3 \text { to } 36 \\
\text { months }\end{array}$ & - & $\begin{array}{l}\text { MD } 2.47 \\
(0.39 \text { lower to } 4.55 \\
\text { higher) }\end{array}$ & - & $\begin{array}{l}2373 \\
\text { (9 RCTs) }\end{array}$ & $\begin{array}{l}\oplus \oplus \bigcirc \bigcirc \\
\mathrm{LOW}^{d, e}\end{array}$ & $\begin{array}{l}\text { SMD } 0.19 \text { ( } 95 \% \text { Cl } 0 \text {. } \\
03 \text { to } 0.35) \text { converted } \\
\text { back to MD using SF- } \\
36 \text { scale, based on data } \\
\text { for } 9 \text { trials reporting end } \\
\text { point scores } \\
\text { MID for the SF-36 is typ- } \\
\text { ically } 3 \text { to } 5 \text { (Walters } \\
2003) \\
\text { EQ-5D (0 to } 1 \text {; best } \\
\text { score) changes scores } \\
\text { reported by } 1 \text { other trial } \\
(212 \text { participants) (MD } \\
-0.06,95 \% \mathrm{Cl}-0.10 \text { to } \\
-0.02 \text { ) were also not } \\
\text { important differences }\end{array}$ \\
\hline Adverse effects & See comment & & Not estimable & See comment & - & $\begin{array}{l}\text { Only } 3 \text { trials reported on } \\
\text { adverse events which } \\
\text { may have been related } \\
\text { to the intervention. } 1 \\
\text { trial reported } 2 \text { partic- } \\
\text { ipants with back pain } \\
\text { ( } 2 \% \text { of } 107), 1 \text { trial re- } \\
\text { ported } 10 \text { with mus- } \\
\text { culoskeletal symptoms } \\
\text { ( } 7 \% \text { of } 147 \text { ); the remain- } \\
\text { ing trial found none. All } \\
12 \text { events were self-lim- } \\
\text { iting }\end{array}$ \\
\hline
\end{tabular}

${ }^{*}$ The risk in the intervention group (and its $95 \%$ confidence interval) is based on the assumed risk in the comparison group and the relative effect of the intervention (and its $95 \% \mathrm{Cl})$.

Cl: Confidence interval; MID: Minimal important difference; RR: Risk ratio 


\section{GRADE Working Group grades of evidence}

High quality: We are very confident that the true effect lies close to that of the estimate of the effect

Moderate quality: We are moderately confident in the effect estimate: The true effect is likely to be close to the estimate of the effect, but there is a possibility that it is substantially different

Low quality: Our confidence in the effect estimate is limited: The true effect may be substantially different from the estimate of the effect

Very low quality: We have very little confidence in the effect estimate: The true effect is likely to be substantially different from the estimate of effect

${ }^{a} \mathrm{~A}$ multifactorial intervention is one in which the selection of falls-prevention interventions (such as exercise, home-hazard modification or medication review) prescribed or provided to each individual is matched to their risk-of-falls profile, which is assessed beforehand. This individually-tailored intervention means that after receiving an assessment of known risk factors for falling, individuals are likely to received different combinations of interventions: i.e. one person may receive supervised exercise and home-hazard modification whereas another may receive home-hazard modification and medication review.

${ }^{b}$ Commonly-used component interventions in the 43 trials testing this comparison included exercise, environment/assistive technologies, medication review, and psychological interventions. Given that the selection of component intervention is matched to the individual's risk profile, the clinical heterogeneity within a trial and across trials is to be expected.

'We calculated the risk in the control group based on the number of events and the total number of participants in the control group for each outcome.

${ }^{d}$ Downgraded one level for risk of bias (more than one trial at high or unclear risk of bias).

${ }^{e}$ Downgraded one level for inconsistency (there was moderate to considerable statistical heterogeneity in these outcomes that could not be explained by prespecified sensitivity and subgroup analyses).

${ }^{f}$ Downgraded one level for imprecision (relatively broad overall confidence interval).

${ }^{g}$ Downgraded one level for indirectness (poor reporting meant that it was sometimes unclear how many hospital admissions were falls-related. Therefore, we included outcome data on hospital admissions in general). 


\section{B A C K G R O U N D}

\section{Description of the condition}

Falls and fall-related injuries are common and a serious problem in older people. People over 65 years of age have the highest risk of falling, with an estimated one-third of older people living in the community falling at least once a year (Campbell 1990; NICE 2013). The rate of fall-related injuries also increases with age (Peel 2002). Most fall-related injuries are minor, such as bruising, abrasions, lacerations, strains and sprains, but can still cause significant pain and discomfort. However, some falls can have serious longterm consequences, including fall-related fractures and head injuries (Peel 2002). Around 10\% of falls result in a fracture (Berry 2008; Campbell 1990; Tinetti 1988), and fall-associated fractures in older people are a significant source of morbidity and mortality (Burns 2016; Scuffham 2003).

Despite early attempts to achieve a consensus definition of 'a fall' (Kellogg 1987), many definitions still exist in the literature. It is particularly important to have a clear, simple definition for studies in which older people record their own falls, as their concept of a fall may differ from that of researchers or healthcare professionals ( Zecevic 2006). An international consensus statement defined a fall as "an unexpected event in which the participant comes to rest on the ground, floor or lower level” (Lamb 2005). The recommended wording when asking individuals about falls is "In the past month, have you had any fall including a slip or trip in which you lost your balance and landed on the floor or ground or lower level?” (Lamb 2005).

Epidemiological studies of varying quality have identified a number of risk factors for falling in community-dwelling older people (Deandrea 2010). These risk factors can be broadly categorised as either intrinsic or extrinsic. Intrinsic fall-related risk factors include advanced age, history of previous falls, muscle weakness, gait and balance problems, poor vision, and chronic diseases such as arthritis, diabetes, stroke, Parkinson's, dementia and incontinence. Extrinsic fall-related risk factors include environmental factors such as lack of hand rails, poor lighting, slippery or uneven surfaces, use of walking aids and poor footwear (Todd 2004). It is estimated that around $15 \%$ of falls result from a major external event that would cause most people to fall. A similar percentage of falls result from a single identifiable event such as syncope (fainting). However, most result from multiple interacting factors (e.g. a person has balance problems, poor vision and slips on an uneven surface which results in a fall) (Campbell 2006). Generally, the more risk factors a person has, the greater their chances are of having a fall. Falls can have major psychological consequences, such as a fear of falling and loss of confidence, which can result in self-restricted activity levels and may lead in turn to a reduction in physical function and social interactions (Yardley 2002). There is evidence that exercise interventions in older people living in the community probably reduce fear of falling to a limited extent immediately after the intervention (without increasing the risk or frequency of falls). However, there is insufficient evidence to determine whether this reduces fear beyond the end of the intervention (Kendrick 2014). Falling also puts a strain on the family and is an independent predictor of admission to a nursing home (Laird 2001; Tinetti 1997).

\section{Description of the intervention}

Many interventions and programmes of interventions for preventing falls have been established and evaluated. These are often based on known, modifiable risk factors for falling and some interventions specifically target people at high risk of falling, such as those with a history of falling. Most fall prevention interventions can be classified according to the taxonomy developed by the Prevention of Falls Network Europe (ProFANE) (Lamb 2007; Lamb 2011). Drawing on this, with some modifications that primarily reflect categorisation in Gillespie 2012, the main intervention categories that we use in this review plus examples of individual interventions are shown below.

- Exercises (supervised or unsupervised, or both): including gait, balance and functional training; strength/resistance exercises; flexibility exercises; 3D training (e.g. Tai Chi); general physical activity; endurance training or others.

- Medication (drug target): including vitamin D and calcium supplementation.

- Medication (review): including medication withdrawal, dose reduction or increase, substitution or provision.

- Surgery: including cataract extraction, pacemaker provision, podiatric surgery or others.

- Management of urinary incontinence (e.g. assisted toileting, bladder retraining).

- Fluid or nutrition therapy where the basic objective was to restore the volume and composition of the body fluids to normal with respect to water-electrolyte balance (fluid therapy) or to improve the health status of the individual by adjusting the quantities, qualities and methods of nutrient intake (nutrition therapy).

- Psychological intervention, either individual or in a group: including cognitive (behavioural) interventions.

- Environment/assistive technology: furnishings and adaptations to homes and other premises; aids for personal mobility (e.g. walking aids); aids for communication and signalling (e.g. alarm systems); body-worn aids for personal care and protection (e.g. anti-slip devices for shoes).

- Environment/assistive technology: aids for communication (e.g. eyeglasses, hearing aids). This includes vision assessment.

- Social environment: including staff ratio, staff training, service model change, telephone support, caregiver training, homecare services or others.

- Knowledge/education interventions: including written material, videos and lectures (in addition to the information that 
is given more generally).

Fall prevention interventions may comprise single component interventions from one of the above categories alone (e.g. balance training) or involve combinations of two or more component interventions (e.g. balance training and strength/resistance exercises) from the same category (e.g. exercise); or from different categories (e.g. exercise and medication (drug target)). Delivery of interventions with more than one component intervention from different categories can broadly be divided into the following two main groups.

- Multifactorial interventions, where the component interventions are matched to an individual assessment of risk.

- Multiple component interventions, where the same component interventions are provided to all people (Gillespie 2012; Lamb 2005).

Multifactorial interventions are interventions that involve an assessment of an individual to determine the presence of two or more modifiable risk factors for falling, which is then followed by specific interventions targeting those risk factors (Lamb 2011). Importantly, not all people receive the same combination of interventions. For example, based on an individual's risk profile, one person may receive supervised exercise and home-hazard modification whereas another may receive home-hazard modification and medication modification. The manner in which multifactorial interventions are delivered varies. In some instances, the assessment and linked interventions are by the same provider. In other instances, one provider may undertake the assessment, but linked interventions are provided through referral to other providers or other routes.

Multiple component interventions are those where people receive a fixed combination of two or more fall prevention interventions from the different categories shown above (Lamb 2011). For example, all people at risk of falling will receive the same combination of component interventions, such as supervised exercise, education and home-hazard modification. Provision is regardless of their underlying risk factor profile, which is not usually assessed as part of the intervention (Gillespie 2012). Hence there is no formal tailoring to the exact risk-factor profile of an individual.

\section{How the intervention might work}

Fall prevention interventions aim to minimise known modifiable risk factors for falling, and thereby prevent falls and associated injuries (Todd 2004).

The hypothesis underlying multifactorial interventions is that health providers assess a range of modifiable risk factors for falling and, along with the linked interventions that follow, provide a much more tailored and potentially effective intervention. This assumes a cumulative and reasonably linear association between the number of risk factors and the probability of falling (Tinetti 2003). It assumes all risk factors contribute in a similar way and that increasing the numbers of risk factors assessed reduces the chances of falling, but this assumption may not be true (Gates 2008). Gillespie 2012 found some evidence that multifactorial interventions may reduce the rate of falls (i.e. the total number of falls per unit of person-time that falls were monitored), but not the risk of falling (i.e. the number of people who fell once or more). Of note is the wide variation in the risk factors assessed, and both the type and format of matched interventions described in published interventions. Multifactorial interventions are the recommended approach for falls prevention in the UK (NICE 2013) and recommended as a primary treatment strategy in the guideline for prevention of falls published by the American Geriatrics Society, the British Geriatrics Society and the Australian Commission on Safety and Quality in Healthcare (ACSQH 2009; American Geriatrics Society 2011). Implementation of multifactorial interventions is a challenge because of the time involved, skills demand, sometimes the need for co-ordinated efforts for assessment and intervention delivery (involving multiple health professionals), and associated cost implications (Vieira 2016).

Multiple component interventions also aim to reduce several components of fall risk rather than dealing with single risk factors. However, there is no assessment and individual tailoring of the intervention to risk factors. There is some evidence that multiple component interventions may reduce the rate of falls and risk of falling in older people living in the community. However, additional evidence is needed to determine which are the most effective combinations of component interventions (Gillespie 2012). It might be simpler and cheaper not to undertake complex assessments, but to focus on interventions for the most common risk factors and provide these to all, regardless of exact risk status. The other complication is that it is possible that the populations that receive these interventions may be different.

\section{Why it is important to do this review}

There is some evidence for the effectiveness of multifactorial interventions and multiple component interventions in preventing falls in older people living in the community, based on the findings of a Cochrane Review (Gillespie 2012). An updated review of the effects of these interventions was warranted, given the number of new trials published, the increasing number of older people living in the community and the major long-term consequences associated with falls and fall-related injuries (including disability and reduced quality of life) to both the individual and to society. In the UK, the National Health Service (NHS) is estimated to spend around GBP 2.3 billion each year on fall-related injuries in people over the age of 65 (NICE 2013). Evidence is needed on which interventions are most effective in reducing falls and fallrelated injuries, the results of which will be of major importance to healthcare professionals, policy-makers, consumers, researchers and others with an interest in this topic. Although not a focus of 
our review, having a sufficiently effective intervention is also an integral component of cost effectiveness.

\section{O B J E C T I VES}

To assess the effects (benefits and harms) of multifactorial interventions and multiple component interventions for preventing falls in older people living in the community.

\section{METHODS}

\section{Criteria for considering studies for this review}

\section{Types of studies}

We included randomised controlled trials, either individual or cluster-randomised, that evaluated the effects of multifactorial interventions and multiple component interventions on the incidence of falls in older people living in the community. We excluded trials that explicitly use methods of quasi-randomisation (e.g. allocation to groups by alternation or date of birth).

\section{Types of participants}

We included studies of interventions to prevent falls if they specified an inclusion criterion of participants aged 60 years or over. We also accepted studies that included younger participants if the mean age minus one standard deviation (SD) was more than 60 years. We included studies where most participants recruited were living in the community, either at home or in places of residence that, on the whole, do not provide residential health-related care or rehabilitative services. Studies with mixed populations (community and higher-dependency places of residence) were eligible for inclusion provided separate data were available for those participants living in the community or the numbers in higher-dependency residences were very few and balanced in the comparison groups. We included studies that recruited participants in hospital if most participants were discharged to the community (where most of the intervention is delivered and falls were recorded).

We excluded studies that tested interventions for preventing falls in people after stroke and with Parkinson's disease, as these topic areas are covered by other Cochrane Reviews (Canning 2015; Verheyden 2013).

\section{Types of interventions}

This Cochrane Review focuses on any multifactorial intervention or multiple component intervention designed to reduce falls in older people (i.e. designed to minimise exposure to, or the effect of, any risk factor for falling). We considered these two groups of interventions separately.

We define a multifactorial intervention as one in which interventions from two or more main categories of intervention can be given to participants, but the interventions are linked to each individual's risk profile (usually assessed using a formal process). Importantly, not all participants in a programme receive the same combination of interventions. We distinguished between multifactorial interventions where treatments were actively provided to address identified risk factors and those where the intervention consisted mainly of referral to other services or the provision of information to increase knowledge (e.g. increase the person's awareness about their risk factors to enable them to take decisions). For example:

- Each individual receives an assessment of known risk factors for falling (fall risk assessment) and then receives an intervention to match their risk profile (i.e. one person may receive supervised exercise and home-hazard modification, whereas another may receive home-hazard modification and medication modification).

We define a multiple component intervention as one in which interventions from two or more main categories of intervention are given to all participants of the falls prevention programme. Combinations of interventions and an assessment of relating to another category (e.g. assessment of environment/dwelling units) are also defined as multiple component interventions. For example, all participants of the fall prevention programme receive the following:

- Supervised exercise and medication (vitamin D and calcium supplementation).

- Supervised exercise and environmental assessment of their home.

We have based these definitions on those developed by the Prevention of Falls Network Europe (ProFaNE) (Lamb 2005).

We included studies where the intervention was compared with 'usual care' (i.e. no change in usual activities), an attention control intervention (i.e. an intervention that is not thought to reduce falls, e.g. general health education or social visits) or exercise as a single active falls-prevention intervention. We analysed studies where the control group was usual care or an attention control intervention separately from those with exercise as an 'active' control.

We chose to include exercise as a separate comparator intervention because systematic reviews of fall prevention interventions have consistently shown exercise to be the intervention that has the largest and most consistent evidence base supporting its use (Gillespie 2012; Sherrington 2016b). Impairments of gait and balance are the most commonly-occurring risk factors for falling (Tinetti 1988), and so exercise is the most logical and effective 
intervention. As the evidence base for falls prevention evolves to refine and provide evidence about the best interventions, exercise is the natural active comparator to select.

We did not include comparisons of different multifactorial interventions or different multiple component interventions, comparisons of any multifactorial versus multiple component interventions, or comparisons where the control was a single active intervention, apart from exercise.

\section{Types of outcome measures}

We included studies that reported data related to the rate and number of falls during follow-up (fallers). Prospective daily calendars returned monthly for at least one year from randomisation were the preferred method for recording falls (Lamb 2005). However, we also included studies where falls were recorded retrospectively, or not monitored continuously throughout the trial, as this is still common practice and would have resulted in excluding a number of trials. We included the following outcomes in this review.

\section{Primary outcomes}

- Rate of falls (falls per person-years).

- Number of people who have sustained one or more falls (risk of falling).

- Number of people who have sustained recurrent falls (defined as two or more falls in a specified time period) (risk of recurrent falls).

\section{Secondary outcomes}

- Number of people who have sustained one or more fallrelated fractures.

- Number of people who experienced a fall that required hospital admission.

- Number of people who experienced a fall that required medical attention (e.g. attended hospital emergency department, required general practitioner (GP) consultation).

- Health-related quality of life (measured using validated scale e.g. EQ-5D or similar).

- Adverse effects of the intervention.

\section{Timing of outcome measurement}

For studies with less than 12 months of follow-up, we used the longest duration reported. We planned to make assessments at short-term (less than 12 months) and long-term (12 months or longer) follow-up, but because of the limited number of studies for some outcomes we combined both short- and long-term follow-up and reported duration of follow-up for each study in the Characteristics of included studies.

\section{Other outcomes}

We recorded and reported intervention adherence data, where available, for use in the interpretation of trial and review findings. We noted when trials had performed an economic evaluation, and reported on the key findings.

\section{Search methods for identification of studies}

\section{Electronic searches}

Our search extends that performed up to February 2012 in Gillespie 2012. We searched the Cochrane Bone, Joint and Muscle Trauma Group Specialised Register (February 2012 to 12 June 2017), the Cochrane Central Register of Controlled Trials (CENTRAL) (2012 Issue 3 to 2017 Issue 6), MEDLINE (including Epub Ahead of Print, In-Process \& Other Non-Indexed Citations, MEDLINE Daily and MEDLINE Versions) (January 2012 to 9 June 2017), Embase (January 2012 to 12 June 2017) and the Cumulative Index to Nursing and Allied Health Literature (CINAHL) (January 2012 to 12 June 2017), using tailored search strategies.

In MEDLINE, we combined subject-specific search terms with the sensitivity- and precision-maximising version of the Cochrane Highly Sensitive Search Strategy for identifying randomised trials (Lefebvre 2011). The search strategies for all databases are in Appendix 1.

We also searched the World Health Organization International Clinical Trials Registry Platform (WHO ICTRP) for ongoing and recently-completed trials (14 July 2017). There were no language or publication status restrictions.

\section{Searching other resources}

We checked the references in Gillespie 2012 and other relevant articles. We also identified ongoing and unpublished trials by contacting researchers in the field.

\section{Data collection and analysis}

\section{Selection of studies}

Pairs of review authors (OA, BC, GB, DB) independently screened all titles and abstracts for potentially eligible studies, for which we obtained full-text reports. The same two review authors independently performed study selection. They resolved any disagreements about the inclusion or exclusion of individual studies by discussion or, if necessary, consulted another review author ( $\mathrm{SH}$ or SL). 


\section{Data extraction and management}

Pairs of review authors (OA, BC, GB, SH) independently performed data extraction. We piloted the data extraction form using a representative sample of studies in order to identify any missing items or unclear coding instructions. The pairs of review authors resolved any disagreements by discussion or, if they could not achieve consensus, another review author acted as an arbitrator (SL). The review authors were not blinded to names of authors, institutions, journals or outcomes. We used a standardised data extraction form to record the following items:

- General information: review author's name, date of data extraction, study ID, first author of study, author's contact address (if available), citation of paper and trial objectives.

- Trial details: trial design, location, setting, sample size, inclusion and exclusion criteria, comparability of groups, length of follow-up, stratification, stopping rules and funding source.

- 'Risk of bias' assessment: sequence generation, allocation concealment, blinding (participants, personnel, outcome assessors), incomplete outcome data, selective outcome reporting and other bias (recall bias).

- Characteristics of participants: age, gender, ethnicity, the number randomised, analysed, lost to follow-up and dropouts in each arm (with reasons).

- Interventions: experimental and control interventions, timing of intervention, whether studies assessed adherence (compliance) with interventions and associated data, and additional co-interventions.

- Outcomes measured: rate of falls, number of people sustaining one or more falls, number of people sustaining recurrent falls, number of people sustaining one or more fallrelated fractures, number of people who experience a fall requiring hospital admission, number of people who experience a fall requiring medical attention, health-related quality of life, and adverse effects of the interventions.

- Other details: economic and health-resource information.

We retrieved data from both full-text and abstract reports of studies. Where these sources did not provide sufficient information, we contacted study authors for additional details.

\section{Assessment of risk of bias in included studies}

Two review authors (OA and BC) independently assessed the risks of bias of each included study based on recommendations in the Cochrane Handbook for Systematic Reviews of Interventions (Higgins 2011a). They resolved any disagreements by consensus or, if they could not achieve consensus, a third review author $(\mathrm{SH})$ acted as arbitrator. We assessed the risk of bias for the following domains: sequence generation (selection bias); allocation concealment (selection bias); blinding of participants and personnel (performance bias); blinding of outcome assessment (detection bias); incomplete outcome data (attrition bias); and selective outcome reporting. In our assessment of detection bias, we assessed separately (a) rate of falls and risk of falling; (b) risk of fractures; and (c) requiring hospital admission/medical attention. We also assessed bias in the recall of falls due to less reliable methods of ascertainment (i.e. where falls were recorded retrospectively, or not monitored continuously throughout the trial) (Hannan 2010). Specifically for trials using cluster randomisation, we considered the risk of additional bias relating to recruitment, baseline imbalance, loss of clusters, incorrect analysis and comparability with individually-randomised trials, as described in Chapter 16 of the Cochrane Handbook for Systematic Reviews of Interventions (Higgins 2011).

We rated risk of bias as either low, high or unclear for each domain. We used the criteria for judging risk of bias in fall-prevention trials based on those described by Gillespie 2012 (see Appendix 2).

\section{Measures of treatment effect}

We presented the treatment effect for rate of falls, rate of fall-related fractures and rate of hospital admission as rate ratios (RaRs) with 95\% confidence intervals (CIs). For the number of fallers, number of recurrent fallers, number sustaining fall-related fractures and number sustaining one or more hospital admission, we reported risk ratios (RRs) and 95\% CIs. For continuous outcomes (healthrelated quality of life), we presented the mean difference (MD) with $95 \%$ CIs where the same outcome measure was used, or standardised mean difference (SMD) with 95\% CIs for outcomes measured using different scales. We only used results based on change scores if final values were unavailable.

\section{Primary outcomes}

\section{Rate of falls}

We defined the rate of falls as the total number of falls per unit of person-time that falls were monitored (e.g. falls per person-year). The RaR compares the rate of falls in any two groups during each trial. If appropriate raw data were available, we calculated a RaR (using the total number of falls over the per person-years) and 95\% CI using Stata ${ }^{\circledR}$, and used this in the meta-analysis. We used the reported $\mathrm{RaR}$ and $95 \% \mathrm{CI}$ if appropriate raw data were not available. If included studies reported both adjusted and unadjusted RaRs, we used the unadjusted estimate unless the adjustment was for clustering.

\section{Risk of falling}

We defined the risk of falling separately for the number of people who fell once or more (fallers) and the number of people who sustained recurrent falls (defined as two or more falls). The RR compares the risk of falling in any two groups during each trial. We used the reported estimate of risk (RR) and 95\% CIs if available. If an included study reported both adjusted and unadjusted estimates we used the unadjusted estimate, unless the adjustment was for clustering. If a study reported an odds ratio (or an effect estimate 
and $95 \%$ CI was not reported) and appropriate data were available, we calculated an RR and 95\% CI using Stata 2015.

\section{Secondary outcomes}

Where data were available, we reported RRs and 95\% CIs for the number of participants who sustained one or more fall-related fractures, one or more hospital admissions and one or more adverse events.

\section{Unit of analysis issues}

For studies that were cluster-randomised (e.g. randomised by medical practice), we performed adjustments for clustering according to guidance provided in the Cochrane Handbook for Systematic Reviews of Interventions (Higgins 2011b) if this had not been performed correctly in the original study. We used an intraclass correlation coefficient (ICC) of 0.01, as reported by Smeeth 2002. We did not adjust for the possibility of a clustering effect in studies that randomised by household. We anticipated that trials would be unlikely to report details of clustering by household and that the clustering effect by household would be very small (if any). For studies with multiple intervention groups, we included each pair-wise comparison separately, but with the shared intervention group (typically the control group) divided evenly among the different comparisons. This avoids the loss of valuable information from multiple group studies and avoids problems associated with the same group of participants being included in the analysis twice. We followed guidance provided in the Cochrane Handbook for Systematic Reviews of Interventions (Higgins 2011d) on dealing with multiple groups from one study.

\section{Dealing with missing data}

We attempted to contact study investigators for any key missing or unclear data or information on their trial. To avoid the risk of overly positive answers, we asked open-ended questions (e.g. "Please describe all measures used") followed up by more focused questions if further clarification was required. For all outcomes, we used the number of participants contributing data in each group if this was known; if this was not reported we used the number randomised in each group as long as there was no significant loss to follow-up. We recorded the reasons for missing data across treatment groups. We conducted sensitivity analyses to explore the effects of missing data (defined as those studies at high risk of bias for incomplete outcome data) on the treatment effect. If a study did not report SDs for continuous outcomes, we calculated these from standard errors, CIs or exact probability $(\mathrm{P})$ values where possible. We did not impute missing SDs.

\section{Assessment of heterogeneity}

The decision about whether or not to combine the results of individual studies was dependent on an assessment of clinical and methodological heterogeneity. Where we performed a meta-analysis, we assessed statistical heterogeneity of treatment effects between trials using the $\mathrm{Chi}^{2}$ test with a significance level at $\mathrm{P}<0.1$ and the $\mathrm{I}^{2}$ statistic. We based our interpretation of the $\mathrm{I}^{2}$ statistic results on that suggested by Higgins 2011c: $0 \%$ to $40 \%$ might not be important; $30 \%$ to $60 \%$ may represent moderate heterogeneity; $50 \%$ to $90 \%$ may represent substantial heterogeneity; and $75 \%$ to $100 \%$ may represent very substantial ('considerable') heterogeneity.

\section{Assessment of reporting biases}

If there were more than 10 studies included in the meta-analysis, we explored potential publication bias by generating a funnel plot and tested this statistically using a linear regression test. A P value of less than 0.1 could be an indication of a publication bias or small-study effects.

\section{Data synthesis}

We analysed multifactorial interventions and multiple component interventions separately.

- We analysed multifactorial interventions, whereby participants received different combinations of intervention based on an individual assessment of risk, as one group. We analysed studies where the intervention was compared with 'usual care' (i.e. no change in usual activities) or an attention control intervention (i.e. an intervention that is not thought to reduce falls, e.g. social visits) separately from those that were compared with exercise as a single active falls-prevention intervention.

- We subgrouped multiple component interventions by the combination of interventions (i.e. where the same combination of single categories of intervention are delivered to all participants). Although we planned to analyse and report each combination separately, after finding exercise was a key component in 17 of the 18 studies assessing multiple component interventions, we decided to analyse the different combinations of interventions together in the same analysis and present the pooled results for both analyses (versus usual care and versus exercise).

We used the fall prevention intervention classification system (taxonomy) developed by the Prevention of Falls Network Europe (ProFaNE) (Lamb 2011). These categories include: exercises (supervised/unsupervised), medication (drug target), surgery, management of urinary incontinence, fluid or nutrition therapy, vision assessment, psychological interventions, environment/assistive technology, social environment and interventions to increase knowledge. Full details are available in the ProFaNE Taxonomy Manual.

Where appropriate, we had planned to pool results of comparable studies using both fixed-effect and random-effects models. We decided to use the random-effects model for all analyses, based on a 
careful consideration of the extent of heterogeneity and whether it could be explained, in addition to other factors, such as the number and size of included studies. We used 95\% CIs throughout. We considered not pooling data where there was considerable heterogeneity ( $\mathrm{I}^{2}$ statistic value of greater than $\left.75 \%\right)$ that could not be explained by the diversity of methodological or clinical features among trials. Had we considered it inappropriate to pool data, we would have presented trial data in the analyses or tables for illustrative purposes and reported these in the text.

When we thought it appropriate, we pooled data using the generic inverse variance method in Review Manager 5 (RevMan) (RevMan 2014). This method enables pooling of the adjusted and unadjusted treatment effect estimates (RaRs or RRs) reported in the individual studies or which can be calculated from data presented in the published article (see Measures of treatment effect). The generic inverse variance option in RevMan requires entering the natural logarithm of the RaR or RR and its standard error for each trial; we calculated these using Stata ${ }^{\circledR}$.

\section{Subgroup analysis and investigation of heterogeneity}

Where there was sufficient data for primary outcomes, we explored potential sources of heterogeneity by carrying out the following prespecified subgroup analyses:

- Higher versus lower falls risk at enrolment (i.e. comparing trials with participants selected for inclusion based on history of falling or other specific risk factors for falling, versus unselected).

- For the multifactorial interventions, trials that actively provided treatment to address identified risk factors versus those where the intervention consisted mainly of referral to other services or the provision of information to educate older people and their families about falls and potential risk factors.

Where appropriate, we performed the test for subgroup differences available in RevMan (RevMan 2014). We planned to perform a subgroup analysis for multiple interventions which included a vitamin $\mathrm{D}$ component, comparing trials that recruited participants with lower baseline vitamin $\mathrm{D}$ levels versus those that did not. However, only four (Campbell 2005; Neelemaat 2012; Ng 2015; Uusi-Rasi 2015) of the 15 trials of multiple interventions included a vitamin $\mathrm{D}$ component, and none specified the participants baseline vitamin D level.

\section{Sensitivity analysis}

Where there were sufficient data, we assessed the robustness of our findings by conducting sensitivity analyses. We examined the effects of the following:

- Inclusion of trials at high or unclear risk of selection bias from inadequate concealment of allocation.

- Inclusion of trials at high or unclear risk of detection bias from inadequate blinding of outcome assessors.
- Inclusion of trials at high or unclear risk of attrition bias from incomplete outcome data.

- Cluster versus individual randomised trials.

We did not perform sensitivity analyses based on the choice of statistical model for pooling (fixed-effect versus random-effects). While we visually assessed the effect of time of study publication by sorting the studies in meta-analyses into ascending order by year of publication, we did not identify a suitable cut-off year to select a subgroup of more recent trials; see Differences between protocol and review.

\section{Assessing the quality of the evidence and 'Summary of findings' tables}

We used the GRADE approach to assess the quality of the body of evidence for each primary and secondary outcome listed in the Types of outcome measures section (Schünemann 2011). The quality rating 'high' is reserved for evidence based on randomised controlled trials. We downgraded the quality rating to 'moderate', 'low' or 'very low', depending on the presence and extent of five factors: study limitations, inconsistency of effect, imprecision, indirectness or publication bias. We then prepared a 'Summary of findings' table for each of the main comparisons:

- Multifactorial interventions compared with usual care or attention control

- Multifactorial interventions compared with exercise

- Multiple component interventions compared with usual care or attention control

- Multiple component interventions compared with exercise

\section{RE S U L T S}

\section{Description of studies}

\section{Results of the search}

We found 6080 articles from the following databases: Cochrane Bone, Joint and Muscle Trauma Group Specialised Register (21 records); CENTRAL (1483), MEDLINE (1343), Embase (2170), CINAHL (777), the WHO ICTRP (286). We also identified 41 studies from Gillespie 2012. After removal of duplicates, we screened 3406 records.

The search identified 427 records for potential inclusion, for which we obtained full reports where possible. After further examination, we included 62 studies (in 137 records) (see Characteristics of included studies), we eliminated 271 records of which we kept 42 studies (in 94 records) as excluded studies (see Characteristics of excluded studies). An additional 16 studies (in 19 records) were 
ongoing studies (see Characteristics of ongoing studies). No studies await classification. A flow diagram summarising the study selection process is shown in Figure 1.

Figure I. Study flow diagram

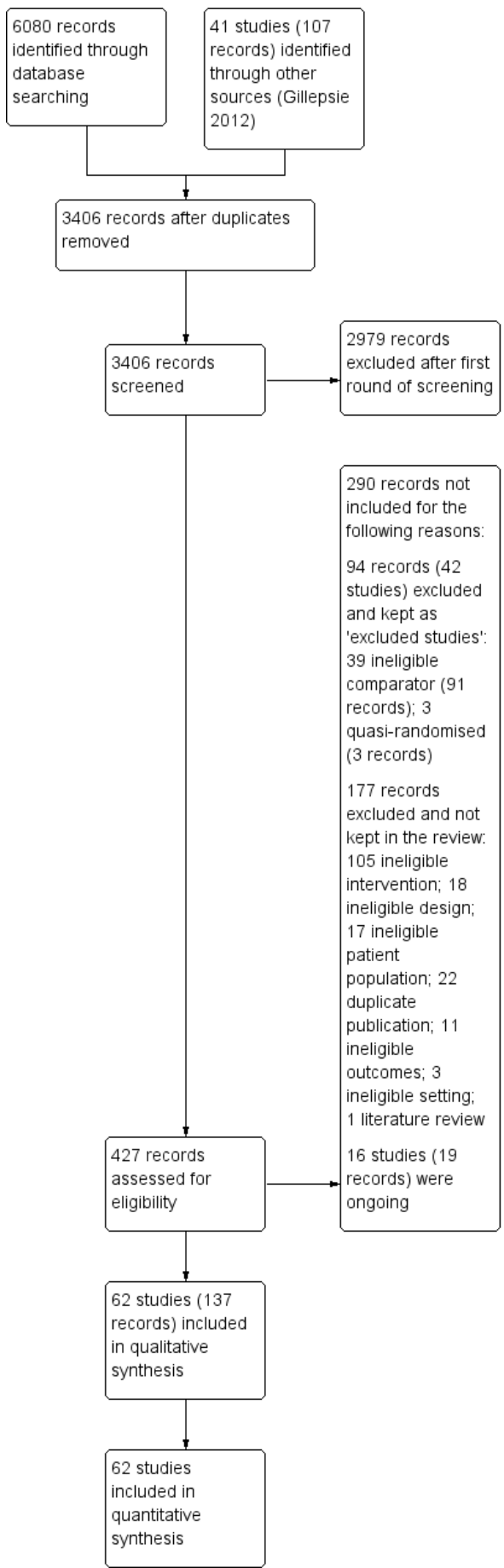

Multifactorial and multiple component interventions for preventing falls in older people living in the community (Review) 


\section{Included studies}

We describe all 62 trials in the Characteristics of included studies and summarise them below:

- 44 trials assessed multifactorial interventions.

- 18 trials assessed multiple component interventions.

\section{Multifactorial interventions}

\section{Trial design}

All 44 trials assessing multifactorial interventions were randomised controlled trials, of which 40 were parallel-group trials and four were cluster-randomised (Coleman 1999; Metzelthin 2013; Spice 2009; Tinetti 1994). Most trials included two arms, four (Carter 1997; Lord 2005; Spice 2009; Wagner 1994) had three arms and one (Markle-Reid 2010) had four arms. Sixteen trials were multicentre trials and 20 were single-centre trials; the number of centres was unclear in the remaining eight trials. The length of follow-up ranged from one month to 48 months. More than half of trials $(\mathrm{n}=$ 23/44) reported 12-month follow-up; 10 trials reported less than 12 months and 11 trials reported more than 12 months followup. SeeTable 1.

\section{Trial setting}

The 44 trials were conducted in 16 different countries, the most common being the UK ( 8 trials), USA (7 trials), the Netherlands (7 trials), Australia (6 trials), and Canada ( 4 trials), with the remainder being conducted in Denmark (1 trial), Finland (1 trial), France (1 trial), Germany (1 trial), Japan (1 trial), New Zealand (1 trial), Spain (1 trial), Sweden (1 trial), Switzerland (1 trial), Taiwan (2 trials), and Thailand (1 trial); see Table 1.

\section{Trial size}

The trials included a total of 15,733 participants. The median number of participants randomised in each trial was 303 (interquartile range (IQR) 156 to 489) with a minimum sample size of 23 participants in Beling 2009 and a maximum of 1559 participants in Wagner 1994. The median number of participants analysed in each trial was 230 (IQR 122 to 367) with a minimum of 19 (Beling 2009) and maximum of 1145 participants (Palvanen 2014). The total number of participants analysed was 11,716; however, this tally does not include the 1559 participants of Wagner 1994, as this trial did not report on the number analysed. Fifteen of the 44 trials reported more than $20 \%$ lost to follow-up. We report full details in the Characteristics of included studies and summarise these details in Table 1.

\section{Participants}

The mean age of participants ranged from 72 (Ciaschini 2009; Kingston 2001; Wagner 1994) to 85 years (Imhof 2012; Luck
2013). Some studies only reported the median age, which ranged from 75 (Lightbody 2002) to 83 years (Logan 2010), the age range which was from 75 to 84 years (Markle-Reid 2010; Van Rossum 1993 ) or the percentage over a certain age range (Carpenter 1990; Carter 1997; Russell 2010; Schrijnemaekers 1995; Vetter 1992). Most trials included more women than men. The median percentage of women included in the trials was 69\% (IQR 65\% to 72\%), and ranged from 2\% in Fabacher 1994 to 100\% in Kingston 2001; two trials (Spice 2009; Vetter 1992) did not report on the percentage of women included. Both trials conducted predominantly in men were carried out by the US Department of Veterans Affairs; 98\% were men in Fabacher 1994 and 97\% in Rubenstein 2007. Thirty-one trials included study participants judged to be at higher risk of falls at enrolment (i.e. participants were selected for inclusion based on a history of falling or other specific risk factors for falling) and 13 trials included participants not judged to be at higher risk of falls (i.e. participants were not selected for inclusion based on history of falling or other specific risk factors for falling). We report full details in the Characteristics of included studies and summarise these details in Table 2.

\section{Interventions}

Of the 44 trials assessing multifactorial interventions, 43 trials (Beling 2009; Carpenter 1990; Carter 1997; Ciaschini 2009; Close 1999; Coleman 1999; Davison 2005; De Vries 2010; Elley 2008; Fabacher 1994; Fairhall 2014; Ferrer 2014; Gallagher 1996; Hendriks 2008; Hogan 2001; Huang 2005; Imhof 2012; Jitapunkul 1998; Kingston 2001; Lightbody 2002; Logan 2010; Lord 2005; Luck 2013; Markle-Reid 2010; Metzelthin 2013; Möller 2014; Newbury 2001; Palvanen 2014; Pardessus 2002; Rubenstein 2007; Russell 2010; Schrijnemaekers 1995; Sheffield 2013; Shyu 2010; Spice 2009; Tinetti 1994; Van Haastregt 2000; Van Rossum 1993; Vetter 1992; Vind 2009; Wagner 1994; Whitehead 2003; Zijlstra 2009) were compared with 'usual care' (i.e. no change in usual activities), or an attention control intervention (i.e. an intervention that is not thought to reduce falls, e.g. general health education or social visits). One trial compared a multifactorial intervention with exercise, a single active falls prevention intervention (Ueda 2017).

Twenty trials actively provided treatment to address identified risk factors as part of the intervention, and in 23 trials the intervention consisted mainly of referral to other services or the provision of information to educate older people and their families about falls and potential risk factors. One trial (Lord 2005) was a threearm trial and included an active intervention, a referral intervention and a control intervention. Twenty-six trials reported assessing adherence (compliance) to the intervention as part of the trial. This was predominantly reported to be assessed by monitoring the 
intervention delivery by attending treatment sessions and phone contact with participants. However, the extent to which participants within the trials complied with the individual treatment components of the intervention was unclear. We report full details in the Characteristics of included studies, and summarise these details in Table 2 .

We summarise details of the key components of each of the multifactorial interventions in Table 3: two or more main categories of intervention could be given to participants, but as the interventions were linked to each individual's risk profile (usually assessed using a formal process), not all participants would have received the same intervention within an individual trial. The most common categories of intervention to be included across individual trials were exercise $(n=37)$ and environment/assistive technologies (e.g. home-hazard assessment and modifications, referral to occupational therapist $)(n=34)$. Medication review $(n=28)$ and psychological interventions (e.g. cognitive behavioural intervention, referral to mental health services) ( $\mathrm{n}=19)$ were also common. Poor reporting for some trials meant that it was not always possible to identify key components of the intervention.

\section{Outcomes}

We report full details of outcomes in the Characteristics of included studies and summarise these details in summary Table 4. Not all trials which assessed an outcome reported results in a way which could be included in a meta-analysis.

- 23 trials assessed the rate of falls

- 35 trials assessed the number of people sustaining one or more falls

- 13 trials assessed the number of people sustaining recurrent falls (defined as two or more falls in a specified time period)

- 9 trials assessed the number of people sustaining one or more fall-related fractures

- 17 trials assessed the number of people who experienced a fall that required hospital admission

- 11 trials assessed the number of people who experienced a fall that required medical attention (e.g. attended a hospital emergency department, required general practitioner (GP) consultation)

- 19 trials assessed health-related quality of life measured using a validated scale; the most commonly-used scale was the SF-36

- 3 trials assessed adverse events that may have been as a result of the intervention.

Economic information was recorded in 13 trials (Close 1999; Coleman 1999; De Vries 2010; Fairhall 2014; Hendriks 2008; Imhof 2012; Lightbody 2002; Logan 2010; Metzelthin 2013; Sheffield 2013; Shyu 2010; Tinetti 1994; Van Rossum 1993). Details are reported in the Characteristics of included studies and summarised in Table 5. All 13 trials provided some information on the cost of delivering the intervention or the cost saving in terms of the total healthcare costs. Only two trials reported information on the cost per fall prevented (De Vries 2010; Hendriks 2008) and two trials on the cost per quality-adjusted life year (QALY) gained (De Vries 2010; Logan 2010).

\section{Multiple component interventions}

\section{Trial design}

All 18 trials assessing multiple component interventions were randomised controlled trials; 13 were parallel-group trials, four used a factorial design and one was cluster-randomised (Huang 2010). Eight trials had two arms, three (Huang 2011; Waterman 2016; Wilder 2001) had three arms and seven (Campbell 2005; Day 2002; Freiberger 2012; Huang 2010; Ng 2015; Sosnoff 2015; Uusi-Rasi 2015) had four or more arms. Nine trials were multicentre trials, and six were single-centre trials; the number of centres was unclear in the other three trials. The length of follow-up ranged from 3 to 24 months, with four trials reporting 12 months follow-up, nine trials reported less than 12 months and five trials reported more than 12 months follow-up. See Table 6.

\section{Trial setting}

The included trials were conducted in 14 different countries, the most common being Australia ( 3 trials), the Netherlands ( 2 trials) and Taiwan ( 2 trials). The remaining were conducted in Canada (1 trial), Finland (1 trial), Germany ( 1 trial), Mexico (1 trial), New Zealand (1 trial), Norway (1 trial), Singapore (1 trial), Slovakia (1 trial), Spain (1 trial), UK (1 trial) and USA (1 trial). See Table 6.

\section{Trial size}

The included trials covered a total of 4202 participants. The median number of participants randomised per trial was 179 (IQR 72 to 310 ), with a minimum sample size of 22 participants (Wesson 2013) and a maximum of 1107 participants (Day 2002). The median number of participants analysed per trial was 157 (IQR 69 to 242) with a minimum of 22 (Wesson 2013) and a maximum of 1090 participants (Day 2002). The total number of participants analysed was 3377 , but this tally does not include the 320 participants of Faes 2011 or the 60 participants of Wilder 2001, because neither trial reported the number of participants in their analyses. Five of the 18 trials reported more than $20 \%$ lost to follow-up. We report full details in the Characteristics of included studies and summarise these details in Table 6.

\section{Participants}

The mean age of participants ranged from 62 (Sosnoff 2015) to 84 years (Campbell 2005). Two trials did not report on the mean age of participants (Huang 2010; Wilder 2001). Most trials included more women than men: the median percentage of women included in the trials was $61 \%$ (IQR $55 \%$ to $71 \%$ ), with a minimum of $41 \%$ in Wesson 2013 and a maximum of $100 \%$ women in Olsen 2014 and Uusi-Rasi 2015. Two trials (Neelemaat 2012; Wilder 2001) did not report on the percentage of women included.

Eleven trials included study participants judged to be at higher risk of falls at enrolment (i.e. participants were selected for inclusion based on a history of falling or other specific risk factors for falling) and seven trials included participants not judged at higher risk 
of falls (i.e. participants were not selected for inclusion based on history of falling or other specific risk factors for falling). We report full details in the Characteristics of included studies and summarise these details in Table 7.

\section{Interventions}

Of the 18 trials assessing multiple component interventions, 17 (Campbell 2005; Clemson 2004; Day 2002; Faes 2011; Freiberger 2012; Hagovska 2016; Huang 2010; Huang 2011; Mendoza-Ruvalcaba 2015; Neelemaat 2012; Ng 2015; Olsen 2014; Serra-Prat 2017; Sosnoff 2015; Waterman 2016; Wesson 2013; Wilder 2001) were compared with 'usual care' (i.e. no change in usual activities), or an attention control intervention (i.e. an intervention that is not thought to reduce falls; e.g. general health education or social visits). Five trials (Day 2002; Huang 2010; Ng 2015; Sosnoff 2015; Uusi-Rasi 2015) compared a multiple component intervention with exercise as a single active fallsprevention intervention.

Seventeen trials included exercise as an intervention in addition to: education ( 4 trials); home safety (3 trials); nutrition ( 2 trials); psychological intervention ( 3 trials); home safety and nutrition (1 trial); home safety and vision assessment (2 trials); or nutrition and psychological intervention ( 2 trials). The remaining trial assessed a nutrition and psychological intervention (Neelemaat 2012). Most of the multiple component interventions included only two components (12 trials) and no trial included an intervention with more than four components. Most multiple component interventions included exercise and another component, commonly education or home-hazard assessment.

Eleven trials reported assessing adherence (compliance) to the intervention as part of the trial. This was predominantly reported as being assessed by monitoring of the intervention delivery by attending treatment sessions and phone contact with participants. However, the extent to which participants complied with the individual treatment components was unclear.

We report full details in the Characteristics of included studies and summarise these details in Table 7.

\section{Outcomes}

We report full details in the Characteristics of included studies and summarise these details in Table 8. Not all trials which assessed an outcome reported results in a way which could be included in a meta-analysis.

- 8 trials assessed the rate of falls

- 14 trials assessed the number of people sustaining one or more falls

- 4 trials assessed the number of people sustaining recurrent falls (defined as two or more falls in a specified time period)

- 2 trials assessed the number of people sustaining one or more fall-related fractures

- 1 trial assessed the number of people who experienced a fall that required hospital admission

- 1 trial assessed the number of people who experienced a fall that required medical attention (e.g. attended a hospital emergency department, required general practitioner (GP) consultation)

- 7 trials assessed health-related quality of life measured using a validated scale; the most commonly-used scale was the SF-36

- 8 trials assessed adverse events which may have been as a result of the intervention

Economic information was recorded in three trials (Campbell 2005; Uusi-Rasi 2015; Waterman 2016).Details are reported in the Characteristics of included studies and summarised in Table 5. All three trials provided some information on the cost of delivering the intervention, or the cost saving in terms of the total healthcare costs, and reported information on the cost per fall prevented; none reported on the cost per QALY gained.

\section{Ongoing studies}

We identified 16 ongoing trials (see Characteristics of ongoing studies). Of these, one study had not yet started recruiting (ACTRN12607000206426), seven are currently open to recruitment (ACTRN12614000827639; ACTRN12615001326583; Close 2014; Hill 2017; Landi 2017; NCT02631330; Sherrington 2016), one is ongoing but no longer recruiting (NCT02374307), and six have recently been completed but the results not yet published (Barker 2015; Blank 2011; ISRCTN21120199; NCT01552551; NCT01713543; Tan 2014). The recruitment status is unknown for one study (NCT01080196).

Ten trials are evaluating multifactorial interventions (ACTRN12607000206426; Barker 2015; Close 2014; ISRCTN21120199; NCT01080196; NCT01552551; NCT01713543; NCT02631330; Sherrington 2016; Tan 2014).

\section{Excluded studies}

We dropped 271 records from the review, for reasons given below. Of these, we retained 42 studies (included in 94 records) as excluded studies. The excluded studies fell into two categories: ineligible comparator and quasi-randomised.

- 39 (in 91 records) studies assessed the effects of multifactorial or multiple component interventions but included an ineligible comparator (see Characteristics of excluded studies).

- 3 (in 3 records) studies assessed the effects of multifactorial or multiple component interventions but were quasi-randomised trials (see Characteristics of excluded studies).

- 105 records assessed an ineligible intervention.

- 17 records included an ineligible participant population.

- 18 were reports of non-randomised studies.

- 22 records were duplicate publications.

- 11 records did not include our outcomes of interest (i.e. relevant outcomes were not assessed or measured).

- 3 records were conducted in an ineligible setting.

- 1 record was a literature review. 


\section{Risk of bias in included studies}

See Figure 2 and Figure 3 for visual representations of the 'Risk of bias' assessments across all included trials and for each individual item in the included trials. See the Characteristics of included studies section 'Risk of bias' table for further information about the bias identified within the individual trial.

Figure 2. Risk of bias graph: review authors' judgements about each risk of bias item presented as percentages across all included studies.

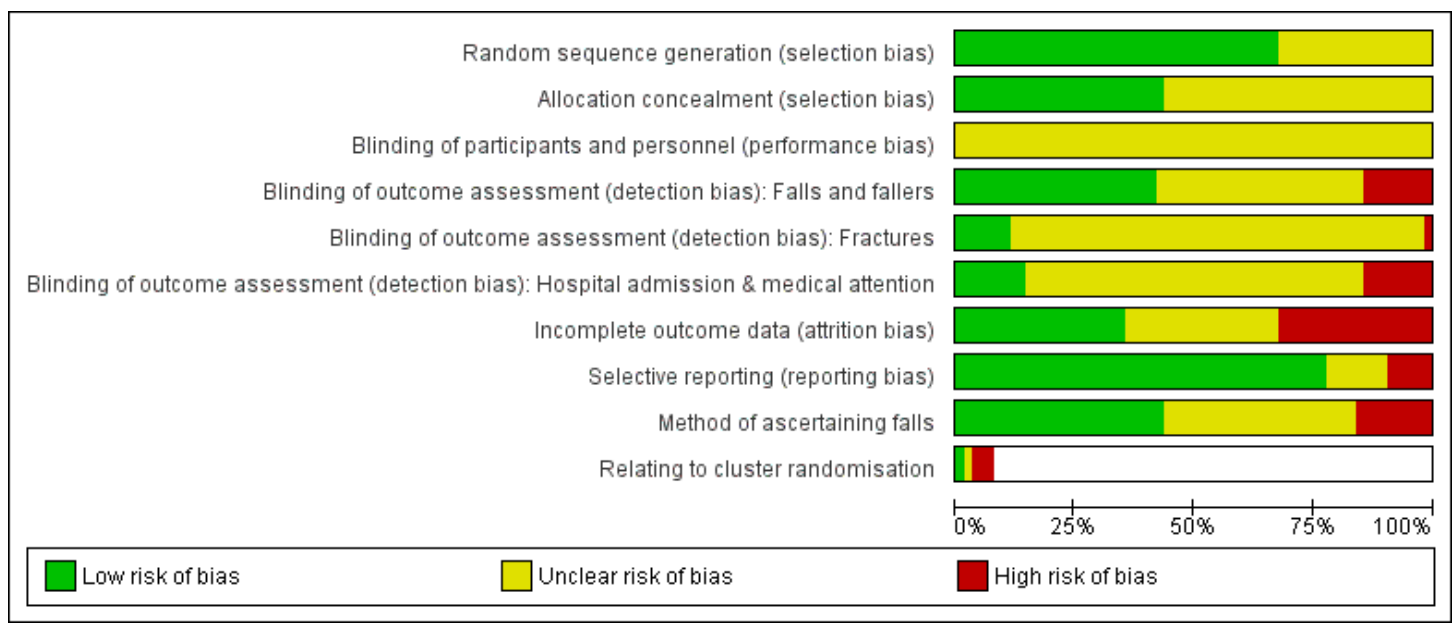


Figure 3. Risk of bias summary: review authors' judgements about each risk of bias item for each included study.

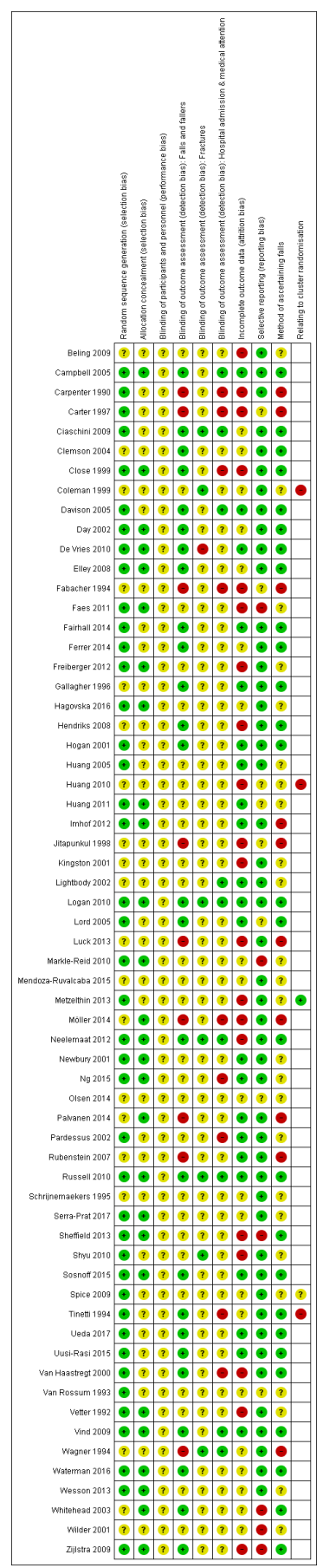

Multifactorial and multiple component interventions for preventing falls in older people living in the community (Review) 


\section{Allocation}

Of the 44 trials assessing multifactorial interventions, we assessed the risk of bias in the generation of allocation sequence as low in $66 \%(n=29 / 44)$ and unclear in the remaining 34\% $(n=15 / 44)$. We judged the methods of concealment of the allocation prior to group assignment as low risk of bias in $34 \%(\mathrm{n}=15 / 44)$ and unclear in the remaining 66\% $(n=29 / 44)$.

Of the 18 trials assessing multiple component interventions, we assessed the risk of bias in the generation of allocation sequence as low in $72 \%(\mathrm{n}=13 / 18)$ and unclear in the remaining $28 \%(\mathrm{n}=$ $5 / 18$ ). We judged methods of concealment of the allocation prior to group assignment as low risk of bias in $67 \%(\mathrm{n}=12 / 18)$ and unclear in the remaining $33 \%(n=6 / 18)$.

\section{Blinding}

\section{Blinding of participants and personnel}

Due to the nature of the interventions, it was not possible to blind the participants and personnel to the allocated group. It was unclear whether awareness of the group allocation would be likely to introduce performance bias, and we therefore assessed the risk of bias for non-blinding as unclear for all trials.

\section{Blinding of outcome assessment}

We assessed the risk of bias for blinding of outcome assessment separately for rate of falls and risk of falling, risk of fractures and requiring hospital admission or medical attention.

\section{Rate of falls and risk of falling}

In trials of multifactorial interventions reporting on the rate or risk of falls, or both, we assessed the risk of detection bias in relation to the methods of ascertainment of the rate or risk of falls to be low in $63 \%(\mathrm{n}=20 / 32)$, unclear in $9 \%(\mathrm{n}=3 / 32)$ and high in the remaining $28 \%(n=9 / 32)$; this was largely due to problematic methods of recording falls (e.g. phone call at six months or verbally at 12-month follow-up visit). In trials of multiple component interventions reporting on the rate or risk of falls, we assessed the risk of detection bias in relation to the methods of ascertainment of the rate or risk of falls to be low in $50 \%(\mathrm{n}=7 / 14)$, and unclear in the remaining $50 \%(\mathrm{n}=7 / 14)$.

\section{Risk of fractures}

In trials of multifactorial interventions reporting on the risk of fracture, we judged the risk of detection bias in relation to the method of ascertainment of fractures as low in $60 \%(n=6 / 10)$, unclear in $30 \%(\mathrm{n}=3 / 10)$ and high in the remaining $10 \%(\mathrm{n}=$ $1 / 10$ ), due to self-report of fractures by participants. In the two trials of multiple component interventions reporting on the risk of fracture, we judged the risk of detection bias in relation to the method of ascertainment of fractures to be low in one trial and unclear in the other.

\section{Requiring hospital admission or medical attention}

In trials of multifactorial interventions reporting on the risk of hospital admission or requiring medical attention, we judged the risk of detection bias in relation to the method of ascertainment of hospital admission or medical attention to be low in 32\% (n $=7 / 22)$, unclear in $32 \%(\mathrm{n}=7 / 22)$ and high in the remaining $36 \%(n=8 / 22)$, due to self-report by participants. In the two trials of multiple component interventions reporting on the risk of hospital admission or requiring medical attention, we judged the risk of detection bias in relation to the method of ascertainment of hospital admission or medical attention to be low in one trial and high in the other.

\section{Incomplete outcome data}

Of the 44 trials assessing multifactorial interventions, we assessed the risk of bias due to attrition bias from incomplete outcome data to be low in $39 \%(\mathrm{n}=17 / 44)$, unclear in $25 \%(\mathrm{n}=11 / 44)$ and high in the remaining $36 \%(16 / 44)$, due to more than $20 \%$ of missing outcome data or with either imbalance in numbers or reasons for missing data across intervention groups. Of the 18 trials assessing multiple component interventions, we assessed risk of attrition bias to be low in $28 \%(\mathrm{n}=5 / 18)$, unclear in $50 \%$ (n $=9 / 18)$ and high in the remaining $22 \%(4 / 18)$.

\section{Selective reporting}

Of the 44 trials assessing multifactorial interventions, we assessed the risk of bias due to selective reporting of outcomes as low in $80 \%(\mathrm{n}=35 / 44)$, unclear in $11 \%(\mathrm{n}=5 / 44)$ and high in the remaining $9 \%(n=4 / 44)$, due to non-reporting of all prespecified outcome or incomplete reporting of study outcomes. Of the 18 trials assessing multiple component interventions, we assessed the risk of bias due to selective reporting of outcomes as low in $72 \%$ $(\mathrm{n}=13 / 18)$, unclear in $17 \%(\mathrm{n}=3 / 18)$ and high in the remaining $11 \%(n=2 / 18)$.

\section{Other potential sources of bias}

Bias in the recall of falls due to less reliable methods of ascertainment 
Of the 44 trials assessing multifactorial interventions, we assessed the risk of bias in the recall of falls (i.e. falls were recorded concurrently using methods such as postcards or monthly fall diaries) to be low risk in $45 \%(n=20 / 44)$. In $23 \%$ of trials $(n=10 / 44)$ there was potential for a high risk of bias in that ascertainment of falling episodes was by participant recall, at intervals during the study or at its conclusion. In $32 \%$ of trials $(n=14 / 44)$ the risk of bias was unclear, as retrospective recall was for a short period only, or details of ascertainment were not described. Of the 18 trials assessing multiple component interventions, we assessed the risk of bias in the recall of falls to be low risk in 39\% $(n=7 / 18)$ and unclear in the remaining $61 \%(\mathrm{n}=11 / 18)$.

\section{Bias specific to cluster-randomised trials}

Of the four cluster-randomised trials that assessed multifactorial interventions, we rated two (Coleman 1999; Tinetti 1994) at high risk of bias because they did not adjust for clustering in their analyses; we rated Spice 2009 at unclear risk of bias because it was unclear how participants were recruited within the clusters of GP practices; and we rated Metzelthin 2013 at low risk of bias. Notably, we assessed all four trials as low risk of bias for baseline imbalance, loss of clusters and comparability with individuallyrandomised trials.

We judged the sole cluster-randomised trial (Huang 2010) assessing multiple interventions to be at high risk of bias, reflecting baseline imbalance between the intervention groups and lack of adjustment for clustering. Furthermore, we rated comparability with individually-randomised trials as unclear, as there was only one trial for the comparison.

\section{Publication bias}

Where there were more than 10 studies included in the metaanalysis, we explored potential publication bias ( $P$ value less than 0.1 ) by generating a funnel plot, and tested this statistically using a linear regression test for the following comparisons and primary outcomes:

Multifactorial interventions versus usual care or attention control:

- Rate of falls: Egger's test bias co-efficient: 1.12; 95\% CI -

1.64 to $3.88 ; \mathrm{P}=0.405$ (funnel plot not shown).

- Number of people who experienced one or more falls: Egger's test bias co-efficient: $0.58,95 \% \mathrm{CI}-0.66$ to $1.82 ; \mathrm{P}=$ 0.350 (Figure 4).

Figure 4. Funnel plot of comparison: Multifactorial intervention vs usual care or attention control: risk of falls

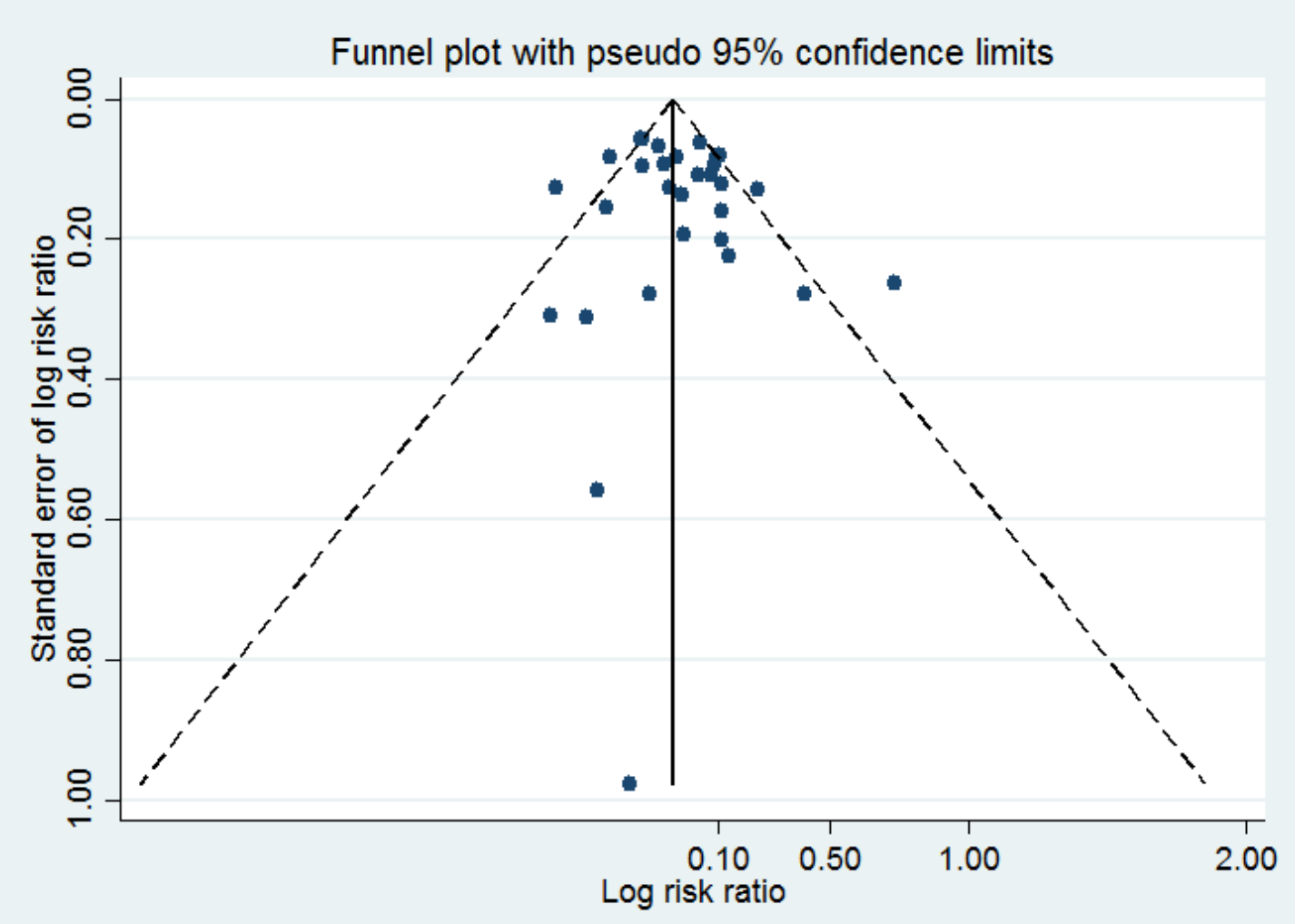

Multifactorial and multiple component interventions for preventing falls in older people living in the community (Review) 
- Number of people who experienced recurrent falls: Egger's test bias co-efficient: $-2.50,95 \% \mathrm{CI}-6.30$ to 1.30 ; $\mathrm{P}=0.174$

(funnel plot not shown).

Multiple component interventions versus usual care or attention control:

- Number of people who experienced one or more falls:

Egger's test bias co-efficient: $-0.42,95 \% \mathrm{CI}-1.40$ to 0.56 ; $\mathrm{P}=$

0.371 (Figure 5).

Figure 5. Funnel plot of comparison: Multiple interventions vs usual care or attention control: rate of falls

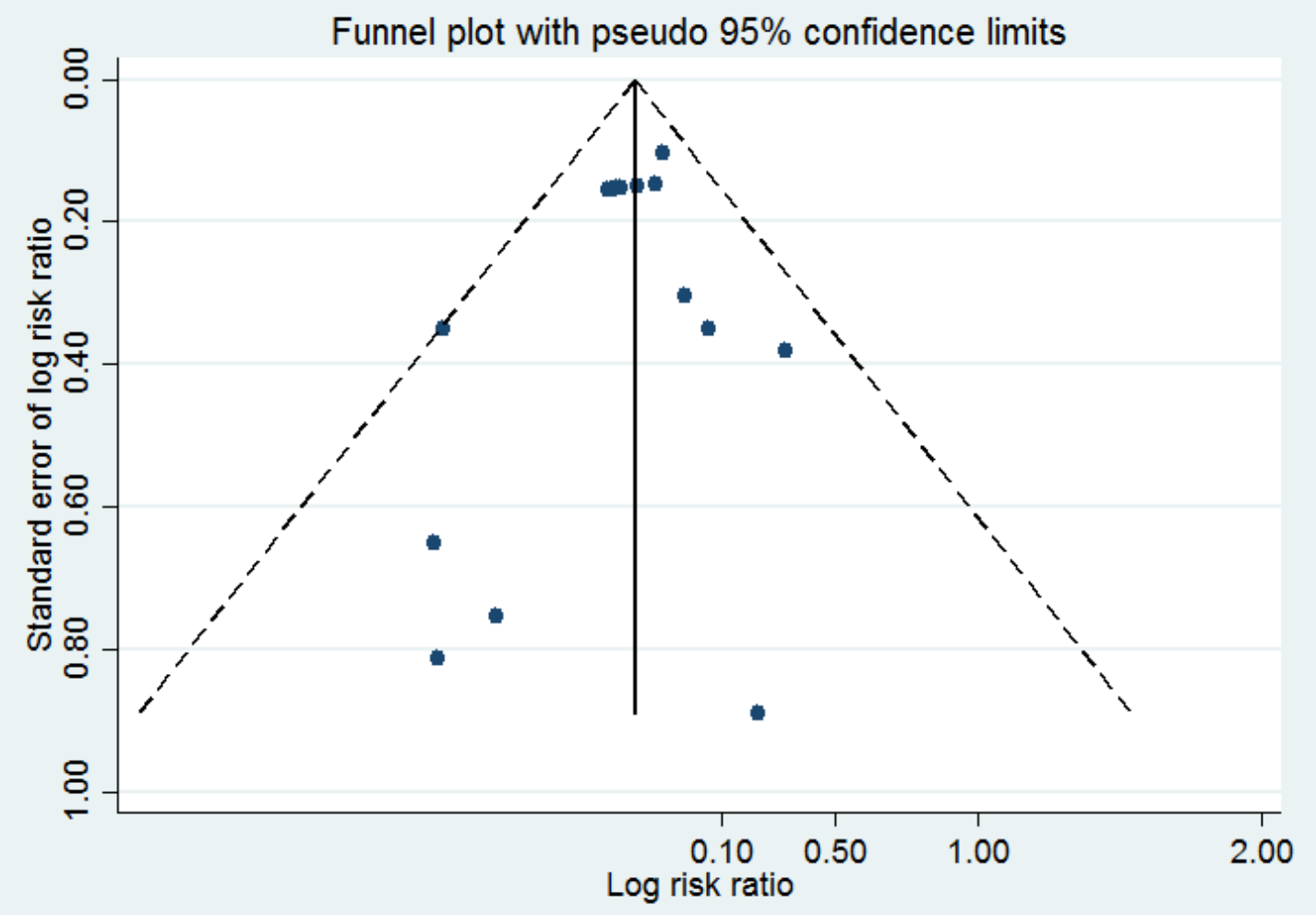

For all analyses the $\mathrm{P}$ value was greater than 0.1 , which indicates, although not conclusively, a lack of publication bias for these outcomes.

\section{Effects of interventions}

See: Summary of findings for the main comparison Multifactorial interventions compared with usual care or attention

Multifactorial and multiple component interventions for preventing falls in older people living in the community (Review) 
control for preventing falls in older people living in the community; Summary of findings $\mathbf{2}$ Multifactorial interventions compared with exercise for preventing falls in older people living in the community; Summary of findings 3 Multiple component interventions compared with usual care or attention control for preventing falls in older people living in the community; Summary of findings 4 Multiple component interventions compared with exercise for preventing falls in older people living in the community

The raw data available for rate of falls, number of fallers, recurrent fallers, and numbers of people sustaining fractures, being admitted to hospital or requiring medical attention are presented respectively in Appendix 3; Appendix 4; Appendix 5; Appendix 6; Appendix 7; Appendix 8.

\section{Multifactorial interventions}

\section{Multifactorial interventions versus usual care or attention control}

Forty-three trials compared multifactorial interventions with 'usual care' (i.e. no change in usual activities), or an attention control intervention (i.e. an intervention that is not thought to reduce falls; e.g. general health education or social visits).

\section{Primary outcomes}

\section{Rate of falls}

Of 22 trials that assessed the rate of falls, we could pool data from 19 trials. Multifactorial interventions may reduce the rate of falls compared with those who receive usual care or an attention control: RaR $0.77,95 \%$ CI 0.67 to $0.87 ; 19$ trials; 5853 participants; $\mathrm{I}^{2}=88 \%$; low-quality evidence; Analysis 1.1; Figure 6). There was considerable heterogeneity that could not be explained based on our prespecified sensitivity and subgroup analyses, shown below. However, despite this high level of unexplained heterogeneity, we considered it is still appropriate to pool the data for these trials. Multifactorial interventions are a specific type of intervention, whereby their definition means that the individual components of the intervention will differ (based on an individual's risk profile), both within an individual trial and across trials. Despite the high level of heterogeneity, the direction of the treatment effect was fairly consistent across trials. As such, we believe it is clinically useful to pool the data, but have downgraded our confidence in the results to low, reflecting our uncertainty around the treatment effect.

Figure 6. Forest plot of comparison: I Multifactorial intervention vs usual care or attention control, outcome: I.I Rate of falls (falls per person years).

\begin{tabular}{|c|c|c|c|c|c|c|c|c|c|}
\hline Study or Subgroup & log[Rate Ratio] & SE & $\begin{array}{r}\text { Multifactorial } \\
\text { Total }\end{array}$ & $\begin{array}{c}\text { Control } \\
\text { Total } \\
\end{array}$ & Weight & $\begin{array}{c}\text { Rate Ratio } \\
\text { IV, Random, } 95 \% \mathrm{Cl} \\
\end{array}$ & \multicolumn{3}{|c|}{$\begin{array}{c}\text { Rate Ratio } \\
\text { IV, Random, } 95 \% \mathrm{Cl} \\
\end{array}$} \\
\hline Beling 2009 & -1.7 & 1.12 & 11 & 8 & $0.3 \%$ & $0.18[0.02,1.64]$ & 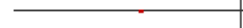 & & \\
\hline Davison 2005 & -0.45 & 0.06 & 144 & 149 & $6.7 \%$ & $0.64[0.57,0.72]$ & - & & \\
\hline Elley 2008 & -0.04 & 0.08 & 155 & 157 & $6.5 \%$ & $0.96[0.82,1.12]$ & & & \\
\hline Fairhall 2014 & 0.11 & 0.19 & 107 & 109 & $4.5 \%$ & $1.12[0.77,1.62]$ & & & \\
\hline Ferrer 2014 & -0.16 & 0.26 & 142 & 131 & $3.4 \%$ & $0.85[0.51,1.42]$ & $\rightarrow$ & & \\
\hline Gallagher 1996 & -0.21 & 0.15 & 50 & 50 & $5.2 \%$ & $0.81[0.60,1.09]$ & $\rightarrow$ & & \\
\hline Hogan 2001 & -0.23 & 0.09 & 75 & 77 & $6.3 \%$ & $0.79[0.67,0.95]$ & $\rightarrow$ & & \\
\hline Lightbody 2002 & -0.16 & 0.11 & 155 & 159 & $6.0 \%$ & $0.85[0.69,1.06]$ & $\rightarrow$ & & \\
\hline Logan 2010 & -0.8 & 0.07 & 98 & 99 & $6.6 \%$ & $0.45[0.39,0.52]$ & $\rightarrow$ & & \\
\hline Lord 2005 & 0.04 & 0.11 & 192 & 197 & $6.0 \%$ & $1.04[0.84,1.29]$ & & & \\
\hline Luck 2013 & -1.14 & 0.2 & 118 & 112 & $4.3 \%$ & $0.32[0.22,0.47]$ & - & & \\
\hline Markle-Reid 2010 & 0.09 & 0.18 & 49 & 43 & $4.7 \%$ & $1.09[0.77,1.56]$ & & & \\
\hline Möller 2014 & 0.03 & 0.15 & 56 & 50 & $5.2 \%$ & $1.03[0.77,1.38]$ & - & & \\
\hline Palvanen 2014 & -0.32 & 0.05 & 661 & 653 & $6.9 \%$ & $0.73[0.66,0.80]$ & - & & \\
\hline Pardessus 2002 & -0.22 & 0.3 & 30 & 30 & $2.9 \%$ & $0.80[0.45,1.44]$ & & & \\
\hline Russell 2010 & -0.44 & 0.04 & 344 & 354 & $7.0 \%$ & $0.64[0.60,0.70]$ & - & & \\
\hline Tinetti 1994 & -0.57 & 0.14 & 147 & 144 & $5.4 \%$ & $0.57[0.43,0.74]$ & $\rightarrow$ & & \\
\hline Vind 2009 & 0.02 & 0.07 & 196 & 196 & $6.6 \%$ & $1.02[0.89,1.17]$ & & & \\
\hline Zijlstra 2009 & -0.15 & 0.14 & 196 & 209 & $5.4 \%$ & $0.86[0.65,1.13]$ & $\rightarrow$ & & \\
\hline Total $(95 \% \mathrm{Cl})$ & & & 2926 & 2927 & $100.0 \%$ & $0.77[0.67,0.87]$ & $\bullet$ & & \\
\hline \multicolumn{7}{|c|}{$\begin{array}{l}\text { Heterogeneity: } \text { Tau }^{2}=0.06 ; \mathrm{Chi}^{2}=150.01, \mathrm{df}=18(\mathrm{P}<0.00001) ;\left.\right|^{2}=88 \% \\
\text { Test for overall effect: } Z=3.99(P<0.0001)\end{array}$} & 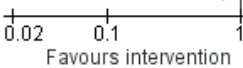 & Favours & $\begin{array}{c}1 \\
10 \\
\text { ntrol }\end{array}$ \\
\hline
\end{tabular}

Multifactorial and multiple component interventions for preventing falls in older people living in the community (Review) 


\section{Subgroup analyses}

- Subgroup analysis by intensity of intervention (assessed and active intervention versus assessed and referral and/or provision of information) showed no evidence of a difference in treatment effect between subgroups for rate of falls $\left(\mathrm{Chi}^{2}=0.15, \mathrm{df}=1, \mathrm{P}\right.$ $=0.70, \mathrm{I}^{2}=0 \%$; Analysis 5.1). Of note is the considerable statistical heterogeneity in both subgroups: active intervention (RaR $0.74,95 \%$ CI 0.58 to $0.95 ; 11$ trials; 2630 participants; $\mathrm{I}^{2}$ = 92\%); referral $(\mathrm{RaR} 0.78,95 \%$ CI 0.69 to $0.88 ; 8$ trials; 3223 participants; $\left.\mathrm{I}^{2}=72 \%\right)$.

- Subgroup analysis by falls risk at baseline showed no evidence of a difference in treatment effect between subgroups for rate of falls $\left(\mathrm{Chi}^{2}=0.24, \mathrm{df}=1, \mathrm{P}=0.63, \mathrm{I}^{2}=0 \%\right.$; Analysis 6.1). Of note is the considerable statistical heterogeneity in both subgroups: selected for higher risk of falling $(\mathrm{RaR} 0.78,95 \% \mathrm{CI}$ 0.68 to $0.89 ; 16$ trials; 5112 participants; $\mathrm{I}^{2}=88 \%$ ); not selected for higher risk of falling ( $\operatorname{RaR} 0.67,95 \%$ CI 0.36 to $1.25 ; 3$ trials; 741 participants; $\left.I^{2}=92 \%\right)$.

\section{Sensitivity analyses}

There was no important change to the overall effect estimate when the analysis was restricted to trials with the following characteristics:
- Trials at low risk of selection bias (RaR 0.80, 95\% CI 0.66 to $0.98 ; 8$ trials; 3516 participants; $\mathrm{I}^{2}=93 \%$; Analysis 8.1 ).

- Trials at low risk of detection bias (RaR 0.78, 95\% CI 0.66 to $0.91 ; 12$ trials; 3718 participants; $\mathrm{I}^{2}=91 \%$; Analysis 9.1 ).

- Trials at low risk of attrition bias ( RaR 0.77, 95\% CI 0.66 to 0.89 ; 11 trials; 4125 participants; $\mathrm{I}^{2}=90 \%$; Analysis 10.1).

- Individually randomised trials (excluding cluster trials) (RaR $0.78,95 \%$ CI 0.68 to $0.89 ; 18$ trials; 5562 participants; $\mathrm{I}^{2}$ $=88 \%$; Analysis 11.1).

\section{Number of people who sustained one or more falls}

Of 34 trials that assessed the number of people sustaining one or more falls, we could pool data from 29 trials. There may be little or no difference in the risk of people sustaining one or more falls between recipients of multifactorial interventions compared with those who received usual care or an attention control (RR 0.96 , $95 \%$ CI 0.90 to 1.03 ; 29 trials; 9637 participants; $\mathrm{I}^{2}=$ 60\%; low-quality evidence; Analysis 1.2; Figure 7). (Spice 2009 contributed data from two different multifactorial interventions: one in primary care and one in secondary care, and so is included in the pooled analysis twice by splitting data from the control group. We used the data from the conservative analysis presented in the main report of this trial, which assumed that all who were lost to follow-up had fallen during follow-up). 
Figure 7. Forest plot of comparison: I Multifactorial intervention vs usual care or attention control, outcome: I.2 Number of people sustaining one or more falls.

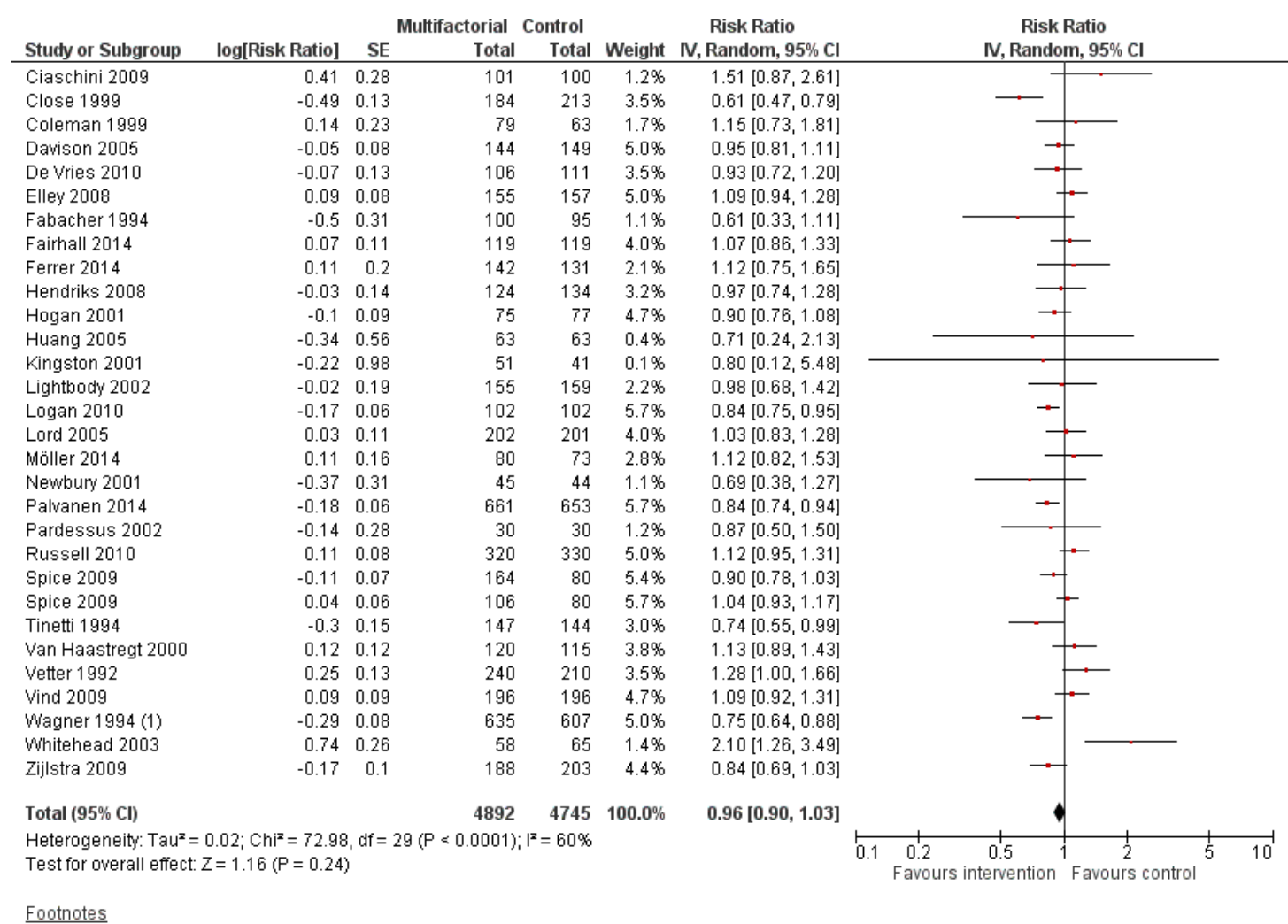

(1) Multifactorial arm vs control

\section{Subgroup analyses}

- Subgroup analysis by intensity of intervention (assessed and active intervention versus assessed and referral and/or provision of information) showed no evidence of a difference in treatment effect between subgroups for number of fallers $\left(\mathrm{Chi}^{2}=1.10, \mathrm{df}=\right.$ $1, \mathrm{P}=0.29, \mathrm{I}^{2}=9.5 \%$; Analysis 5.2). Of note is that both groups continued to show similar statistical heterogeneity to the overall analysis: active intervention (RR $0.93,95 \%$ CI 0.86 to $1.01 ; 13$ trials; 3677 participants; $\mathrm{I}^{2}=54 \%$ ); referral (RR 1.00, 95\% CI 0.89 to $1.13 ; 16$ trials; 5960 participants; $I^{2}=66 \%$ ).

- Subgroup analysis by falls risk at baseline (selected for higher risk of fallings; not selected) showed no evidence of a difference in treatment effect between subgroups for number of fallers $\left(\mathrm{Chi}^{2}=0.26, \mathrm{df}=1, \mathrm{P}=0.61, \mathrm{I}^{2}=0 \%\right.$; Analysis 6.2). Once again, the results in the two groups were statistically heterogeneous: selected for high risk of falls (RR 0.97, 95\% CI 0.90 to $1.04 ; 22$ trials; 6975 participants; $\mathrm{I}^{2}=58 \%$ ); not selected (RR $0.92,95 \%$ CI 0.75 to $1.12 ; 7$ trials; 2662 participants; $I^{2}=$
$67 \%)$

Sensitivity analyses

There was no important change to the overall effect estimate when the analysis was restricted to trials with the following characteristics:

- Trials at low risk of selection bias (RR 0.98, 95\% CI 0.86 to 1.10; 12 trials; 4692 participants; $\mathrm{I}^{2}=77 \%$; Analysis 8.2).

- Trials at low risk of detection bias (RR 0.97, 95\% CI 0.88 to $1.07 ; 16$ trials; 4380 participants; $\mathrm{I}^{2}=64 \%$; Analysis 9.2).

- Trials at low risk of attrition bias (RR $0.95,95 \%$ CI 0.88 to 1.02; 13 trials; 4452 participants; $\mathrm{I}^{2}=34 \%$; Analysis 10.2).

- Individually-randomised trials (excluding cluster trials) (RR $0.97,95 \%$ CI 0.89 to $1.04 ; 26$ trials; 8774 participants; $\mathrm{I}^{2}=$ 62\%; Analysis 11.2).

\section{Number of people who sustained recurrent falls}

Of 13 trials that assessed the number of people sustaining recurrent falls, we could pool data from 12 . There may be a little or 
no difference in the risk of people sustaining recurrent falls between recipients of multifactorial interventions compared with those who received usual care or an attention control (RR 0.87 , $95 \%$ CI 0.74 to 1.03 ; 12 trials; 3368 participants; $\mathrm{I}^{2}=53 \%$; lowquality evidence; Analysis 1.3).

\section{Subgroup analyses}

- Subgroup analysis by intensity of intervention (assessed and active intervention tested in seven trials (2191 participants) versus assessed and referral and/or provision of information tested in five trials (1177 participants)) showed no evidence of a difference in treatment effect between subgroups for number of recurrent fallers $\left(\mathrm{Chi}^{2}=0.76, \mathrm{df}=1, \mathrm{P}=0.38, \mathrm{I}^{2}=0 \%\right.$; Analysis 5.3).

- Subgroup analysis by falls risk at baseline (selected for higher risk of fallings tested in 10 trials (2824 participants); not selected tested in only two trials (544 participants)) did not show evidence of a difference in treatment effect between subgroups $\left(\mathrm{Chi}^{2}=2.63, \mathrm{df}=1, \mathrm{P}=0.11, \mathrm{I}^{2}=62 \%\right.$; Analysis 6.3 $)$.

\section{Sensitivity analyses}

There was no important change to the overall effect estimate when the analysis was restricted to trials with the following characteristics:

- Trials at low risk of selection bias (RR 0.85, 95\% CI 0.62 to

1.15; 6 trials; 1862 participants; $\mathrm{I}^{2}=76 \%$; Analysis 8.3).

- Trials at low risk of detection bias (RR 0.89, 95\% CI 0.73

to 1.08 ; 10 trials; 3033 participants; $\mathrm{I}^{2}=60 \%$; Analysis 9.3).

- Trials at low risk of attrition bias (RR 0.96, 95\% CI 0.81 to 1.13; 5 trials; 1402 participants; $\mathrm{I}^{2}=0 \%$; Analysis 10.3).

- Individually-randomised trials (excluding cluster trials) (no change: RR $0.87,95 \%$ CI 0.74 to $1.03 ; 12$ trials; 3368 participants; $\mathrm{I}^{2}=53 \%$; Analysis 11.3).

\section{Secondary outcomes}

\section{Number of people who sustained one or more fall-related fractures}

We could pool data from all nine trials that assessed the number of people sustaining one or more fall-related fractures. The pooled results showed that multifactorial interventions, compared with usual care or attention control, may reduce but also may make no difference to the risk of people sustaining one or more fallrelated fractures (RR $0.73,95 \%$ CI 0.53 to 1.01 ; 9 trials; 2850 participants; $\mathrm{I}^{2}=0 \%$; low-quality evidence; Analysis 1.4 ). (Spice 2009 contributed data from two different multifactorial interventions: one in primary care and one in secondary care, and so is included in the pooled analysis twice by splitting data from the control group. The observed fracture data in Spice 2009 are used for this outcome).

Sensitivity analyses

The overall effect estimate became more conservative, indicating little or no difference between the two groups, when we restricted the analysis to: trials at low risk of selection bias (RR 0.78, 95\% CI 0.49 to 1.23 ; 4 trials; 1521 participants; $\mathrm{I}^{2}=0 \%$ ); trials at low risk of attrition bias (RR $0.72,95 \%$ CI 0.48 to 1.08 ; 6 trials; 1774 participants; $\mathrm{I}^{2}=0 \%$ ); or individually-randomised trials (excluding cluster trials) (RR 0.75, 95\% CI 0.53 to $1.06 ; 8$ trials; 2425 participants; $\left.I^{2}=0 \%\right)$. When we restricted the analysis to the three trials at low risk of detection bias for fractures, the results became strongly in favour of a multifactorial intervention reducing the risk of fall-related fractures (RR $0.47,95 \%$ CI 0.24 to 0.93 ; 3 trials; 1055 participants; $\mathrm{I}^{2}=0 \%$ ). The results of all sensitivity analyses for this comparison are presented in Table 9.

\section{Number of people who experienced a fall that required hospital admission}

We could pool data from 15 of the 16 trials that assessed the number of people who experienced a fall that required hospital admission. There may be little or no difference in the risk of people who experienced a fall that required hospital admission between recipients of multifactorial interventions compared with those who received usual care or an attention control (RR 1.00, 95\% CI 0.92 to $1.07 ; 15$ trials; 5227 participants; $\mathrm{I}^{2}=0 \%$; low-quality evidence; Analysis 1.5). (Spice 2009 contributed data from two different multifactorial interventions: one in primary care and one in secondary care, and so is included in the pooled analysis twice by splitting data from the control group). We downgraded the evidence one level for serious risk of bias and one level for indirectness, given that poor reporting meant that it was not always possible to specifically determine that the cause of hospital admission was always due to fall.

Sensitivity analyses

There was no important change to the overall effect estimate when the analysis was restricted to: trials at low risk of selection bias (RR 0.98 , 95\% CI 0.76 to 1.26 ; 1 trial; 204 participants); trials at low risk of detection bias (RR $0.94,95 \%$ CI 0.74 to $1.18 ; 4$ trials; 1960 participants; $\mathrm{I}^{2}=0 \%$ ); trials at low risk of attrition bias (RR 1.03, 95\% CI 0.92 to 1.14; 7 trials; 2099 participants; $I^{2}$ $=7 \%$ ); or individually-randomised trials (excluding cluster trials) (RR 0.99, 95\% CI 0.92 to $1.08 ; 12$ trials; 4433 participants; $\mathrm{I}^{2}=$ $0 \%$ ) (Table 9).

\section{Number of people who experienced a fall that required medical attention}

We could pool data from eight of the 11 trials that assessed the number of people who experienced a fall that required medical attention. There may be little or no difference in the risk of people 
who experienced a fall that required medical attention between recipients of multifactorial interventions compared with those who received usual care or an attention control (RR 0.91, 95\% CI 0.75 to $1.10 ; 8$ trials; 3078 participants; $\mathrm{I}^{2}=0 \%$; low-quality evidence; Analysis 1.6).

\section{Sensitivity analyses}

There was no important change to the overall effect estimate when we restricted the analysis to: trials at low risk of selection bias (RR 1.08 , $95 \%$ CI 0.74 to $1.58 ; 2$ trials; 545 participants; $\mathrm{I}^{2}=1.0 \%$ ); trials at low risk of detection bias (RR 0.83, 95\% CI 0.65 to 1.07 ; 3 trials; 1947 participants; $\mathrm{I}^{2}=0 \%$ ); trials at low risk of attrition bias (RR 0.96, 95\% CI 0.71 to $1.31 ; 3$ trials; 868 participants; $\mathrm{I}^{2}$ $=0 \%$ ); or individually-randomised trials (excluding cluster trials) (RR 0.93, 95\% CI 0.75 to $1.15 ; 7$ trials; 2831 participants; $\mathrm{I}^{2}=$ 6\%) (Table 9).

\section{Health-related quality of life}

We could pool data from 11 of the 19 trials that assessed healthrelated quality of life. Based on pooled SMD results from the nine trials that reported final scores, multifactorial interventions may slightly improve people's reported health-related quality of life compared with those who received usual care or an attention control (SMD 0.19, 95\% CI 0.03 to 0.35; 9 trials; 2373 participants; $\mathrm{I}^{2}=70 \%$; low-quality evidence; Analysis 1.7$)$. However, converting these data to the SF-36 scale ( 0 worst to 100 best) indicates that this difference may not correspond to a clinically-important difference (e.g. minimal important difference (MID) is typically 3 to 5; Walters 2003). Hence, multifactorial interventions may make little or no difference to health-related quality of life (SF36: MD 2.47, 95\% CI 0.39 to 4.55). One trial (De Vries 2010) found no important between-group difference in EQ-5D change scores ( 0 to 1 scale; higher scores are better) (MD -0.06, 95\% CI -0.10 to -0.02 ; 1 trial; 212 participants; low-quality evidence). In addition, several trials reported data separately for the different components of health-related quality of life and showed little or no difference in people's mental health-related quality of life (SMD $0.27,95 \% \mathrm{CI}-0.03$ to $0.56 ; 3$ trials; 376 participants; $\mathrm{I}^{2}=50 \%$; Analysis 1.8), or physical health-related quality of life (SMD 0.39, 95\% CI 0.00 to 0.79 ; 3 trials; 376 participants; $I^{2}=72 \%$; Analysis 1.9), based on data for three trials reporting endpoint scores. There was also no difference in SF-36 physical health-related quality of life (0 to 100; best score) in the one trial (Clemson 2004) reporting change scores (MD 0.74, 95\% CI -1.61 to 3.09). Appendix 9 provides summary information for all 19 trials including those which we could not include in the meta-analysis (e.g. because they reported median, IQR or P value), the results of which are similar to the above.

\section{Sensitivity analyses}

Based on data from trials reporting end point scores, there was no important change to the overall effect estimate when we restricted the analysis to: trials at low risk of selection bias (SMD 0.32, 95\%
CI 0.08 to $0.55 ; 2$ trials; 554 participants; $\mathrm{I}^{2}=43 \%$ ); trials at low risk of attrition bias (SMD 0.20, 95\% CI 0.00 to $0.41 ; 6$ trials; 1602 participants; $\mathrm{I}^{2}=72 \%$ ) (Table 9). All trials were individually randomised and at high risk for detection bias.

\section{Adverse effects of the intervention}

Only three trials reported on adverse events that may have been related to the intervention (Fairhall 2014; Tinetti 1994; Zijlstra 2009). Fairhall 2014 reported back pain in two participants $(2 \%$ of 107), which resolved after modification of the exercise programme, and Tinetti 1994 reported musculoskeletal symptoms in 10 participants (7\% of 147), which were "self-limited" and again probably related to the exercise programme. Zijlstra 2009 reported there had been no adverse events.

\section{Multifactorial interventions versus exercise}

One trial (Ueda 2017) compared a multifactorial intervention (tailored education programme using home floor plans in Japan) with exercise as a single active falls prevention intervention.

\section{Primary outcomes}

\section{Rate of falls}

Ueda 2017 provided very low-quality evidence of little or no difference in the rate of falls between multifactorial intervention and exercise (RR $0.13,95 \%$ CI 0.01 to 2.46 ; 1 trial; 51 participants) Analysis 2.1.

Number of people who sustained one or more falls

Ueda 2017 provided very low-quality evidence of little or no difference in the risk of falling between multifactorial intervention and exercise (RR $0.26,95 \%$ CI 0.01 to 5.52 ; 1 trial; 51 participants) Analysis 2.2.

Number of people who sustained recurrent falls

Ueda 2017 did not report on the risk of recurrent falls.

\section{Secondary outcomes}

Ueda 2017 did not report on any of the secondary outcomes: number of people who have sustained one or more fall-related fractures; number of people who experienced a fall that required hospital admission; number of people who experienced a fall that required medical attention; health-related quality of life; or adverse events.

\section{Multiple component interventions}

Multiple component interventions versus usual care or attention control 
Seventeen trials compared multiple component interventions with 'usual care' (i.e. no change in usual activities), or an attention control intervention (i.e. an intervention that is not thought to reduce falls, e.g. general health education or social visits). Exercise was one of the component interventions in 16 of the 17 trials.

\section{Primary outcomes}

\section{Rate of falls}

We could pool data from six of the eight trials that assessed the rate of falls. Multiple component interventions probably reduce the rate of falls compared with those who receive usual care or an attention control (RaR $0.74,95 \%$ CI 0.60 to $0.91 ; 6$ trials; 1085 participants; $\mathrm{I}^{2}=45 \%$; moderate-quality evidence; Analysis 3.1). (Campbell 2005 contributed data from two different multiple component interventions and so is included in the pooled analysis twice, by splitting data from the control group). The overall effect remained the same when excluding Neelemaat 2012, the one trials that did not include exercise as a component of the intervention (RaR $0.79,95 \%$ CI 0.67 to $0.92 ; 6$ trials; 934 participants; $I^{2}$ $=5 \%$ ); however, the amount of heterogeneity between trials was reduced.

\section{Subgroup analysis}

When we subgrouped trials by falls risk at baseline, the statisticallysignificant test for subgroup differences $\left(\mathrm{Chi}^{2}=6.56, \mathrm{df}=1, \mathrm{P}=\right.$ $0.01, \mathrm{I}^{2}=84.8 \%$; Analysis 7.1$)$ indicated a larger protective effect of multiple component interventions where participants were not selected for higher risk of falls ( $\mathrm{RaR} 0.39,95 \%$ CI 0.23 to $0.66 ; 2$ trials; 267 participants; $\mathrm{I}^{2}=0 \%$ ) compared with when they were (RaR $0.79,95 \%$ CI 0.68 to 0.93 ; 4 trials; 818 participants; $\mathrm{I}^{2}=$ $7 \%)$. However, it is important to note that there were only two trials in the smaller subgroup.

Sensitivity analyses

There was no important change to the overall effect estimate when we restricted the analysis to trials with the following characteristics:

- Trials at low risk of selection bias (RaR 0.68, 95\% CI 0.51 to 0.92 ; 4 trials; 584 participants; $\mathrm{I}^{2}=47 \%$; Analysis 12.1 ).

- Trials at low risk of detection bias (RaR 0.75, 95\% CI 0.60 to 0.93 ; 5 trials; 969 participants; $\mathrm{I}^{2}=50 \%$; Analysis 13.1 ).

- Trials at low risk of attrition bias (RaR 0.79, 95\% CI 0.66

to 0.96 ; 3 trials; 596 participants; $\mathrm{I}^{2}=10 \%$; Analysis 14.1).

- Individually-randomised trials (excluding cluster trials) (no change: RaR $0.74,95 \%$ CI 0.60 to $0.91 ; 6$ trials; 1085 participants; $\mathrm{I}^{2}=45 \%$; Analysis 15.1).

\section{Number of people who sustained one or more falls}

We could pool data from 11 of the 14 trials that assessed the number of people sustaining one or more falls. Multiple component interventions probably reduce the risk of sustaining one or more falls compared to those who receive usual care or an attention control
(RR 0.82, 95\% CI 0.74 to $0.90 ; 11$ trials; 1980 participants; I $^{2}$ = 0\%; moderate-quality evidence; Analysis 3.2). (Campbell 2005 and Day 2002 contributed data from different multiple component interventions and so are included in the pooled analysis more than once, by splitting data from the control group). The overall effect remained the same when we excluded data from the two trials (Day 2002; Neelemaat 2012) which did not include exercise as a component of the intervention (RR $0.82,95 \%$ CI 0.74 to 0.91; 9 trials; 1599 participants; $\mathrm{I}^{2}=0 \%$ ).

\section{Subgroup analysis}

Subgroup analysis by falls risk at baseline (selected for higher risk of falls tested in 7 trials (872 participants); not selected tested in 4 trials (1108 participants)) showed no evidence of a difference in treatment effect between subgroups for rate of falls $\left(\mathrm{Chi}^{2}=1.14\right.$, $\mathrm{df}=1, \mathrm{P}=0.29, \mathrm{I}^{2}=12.5 \%$; Analysis 7.2 ).

\section{Sensitivity analyses}

There was no important change to the overall effect estimate when we restricted the analysis to trials with the following characteristics:

- Trials at low risk of selection bias (RR $0.78,95 \%$ CI 0.70 to 0.88; 8 trials; 1478 participants; $\mathrm{I}^{2}=0 \%$; Analysis 12.2).

- Trials at low risk of detection bias (RR 0.81, 95\% CI 0.73 to $0.89 ; 5$ trials; 1518 participants; $\mathrm{I}^{2}=0 \%$; Analysis 13.2).

- Trials at low risk of attrition bias (RR $0.75,95 \%$ CI 0.62 to 0.92; 3 trials; 506 participants; $\mathrm{I}^{2}=0 \%$; Analysis 14.2).

- Individually-randomised trials (excluding cluster trials) (RR $0.81,95 \%$ CI 0.74 to $0.90 ; 10$ trials; 1877 participants; $\mathrm{I}^{2}=0 \%$; Analysis 15.2).

\section{Number of people who sustained recurrent falls}

We could pool data from all four trials that assessed the number of people sustaining recurrent falls. Multiple component interventions may reduce but may also slightly increase the risk of people sustaining recurrent falls compared with those who received usual care or an attention control (RR $0.81,95 \%$ CI 0.63 to $1.05 ; 4$ trials; 662 participants; $\mathrm{I}^{2}=0 \%$; low-quality evidence; Analysis 3.3). (Campbell 2005 contributed data from two different multiple component interventions and so is included in the pooled analysis twice, by splitting data from the control group). Subgroup analysis by baseline risk of falls was not possible because all four trials included participants selected at higher risk of falls (Analysis 7.3).

\section{Sensitivity analyses}


There was no important change to the overall effect estimate when we restricted the analysis to trials with the following characteristics:

- Trials at low risk of selection bias (RR 0.90, 95\% CI 0.62 to

1.30; 3 trials; 352 participants; $\mathrm{I}^{2}=1 \%$; Analysis 12.3).

- Trials at low risk of detection bias (RR 0.79, 95\% CI 0.61

to $1.02 ; 3$ trials; 629 participants; $\mathrm{I}^{2}=0 \%$; Analysis 13.3 ).

- Trials at low risk of attrition bias (RR 0.84, 95\% CI 0.57 to 1.23; 1 trial; 291 participants; Analysis 14.3).

- Individually-randomised trials (excluding cluster trials) (No change: RR $0.81,95 \%$ CI 0.63 to $1.05 ; 4$ trials; 662 participants; $\mathrm{I}^{2}=0 \%$; Analysis 15.3).

\section{Secondary outcomes}

\section{Number of people who sustained one or more fall-related fractures}

We could pool data from both trials that assessed the number of people sustaining one or more fall-related fractures. Given the very few fracture events (one in each trial), we are uncertain of the effects of multiple component interventions on the risk of people sustaining one or more fall-related fractures compared with those who received usual care or an attention control (RR 0.50, 95\% CI 0.05 to $5.32 ; 2$ trials; 232 participants; $\mathrm{I}^{2}=0 \%$; very low-quality evidence; Analysis 3.4).

\section{Sensitivity analyses}

There was no important change to the overall effect estimate when we restricted the analysis to:

- Trials at low risk of selection bias (RR 0.50, 95\% CI 0.05 to

5.32; 2 trials; 232 participants; $\mathrm{I}^{2}=0 \%$ ).

- Trials at low risk of detection bias (RR 0.50, 95\% CI 0.02

to 1.73 ; 1 trial; 210 participants).

- Individually-randomised trials (excluding cluster trials) (RR $0.50,95 \%$ CI 0.05 to $5.32 ; 2$ trials; 232 participants; $\mathrm{I}^{2}=0 \%$ ).

Both trials were at unclear or high risk of attrition bias. The results of all sensitivity analyses are presented in Table 10.

\section{Number of people who experienced a fall that required hospital admission}

Only $\mathrm{Ng} 2015$ assessed the number of people who required a hospital admission, some of which may have been fall-related. Given the few events, we are uncertain of the effects of multiple component interventions on the risk of experiencing a fall that required hospital admission (RR 3.06, 95\% CI 0.65 to 14.42 ; 1 trial; 99 participants; very low-quality evidence; Analysis 3.5). Ng 2015 included participants selected for higher risk of falls.

\section{Number of people who experienced a fall that required medical attention}

One trial assessed the number of people who experienced a fall that required medical attention (Campbell 2005); this trial contributed data from two different multiple interventions and so is included in the pooled analysis twice, by splitting data from the control group. Multiple component interventions may have little or no difference in the risk of people who experienced a fall that required medical attention compared to those who received usual care or an attention control (RR $0.95,95 \%$ CI 0.67 to 1.35 ; 1 trial; 291 participants; low-quality evidence; Analysis 3.6). Campbell 2005 included participants selected for higher risk of falls.

\section{Health-related quality of life}

We could pool data for six of the seven trials that assessed healthrelated quality of life. Multiple component interventions may slightly improve people's reported health-related quality of life compared with those who received usual care or an attention control (SMD 0.77, 95\% CI 0.16 to 1.39 ; 4 trials reporting final scores; 391 participants; $\mathrm{I}^{2}=88 \%$; low-quality evidence; Analysis 3.7). When converted to the SF- 36 scale ( 0 worst to 100 best), the result indicates that this may include a clinically-important difference (MD 9.12, 95\% CI 1.89 to 16.46). One small trial reported change scores using EQ-5D VAS 0 - 100 in favour (MD -19.73, 95\% CI -30.94 to -8.52 ; 1 trial; 33 participants; very low-quality evidence). Several trials reported separate final-score data for different components of health-related quality of life scores. These showed that multiple component interventions may slightly improve people's mental health-related quality of life (SMD 0.69, 95\% CI 0.26 to 1.11 ; 2 trials; 92 participants; I ${ }^{2}=0 \%$; Analysis 3.8 ), but not physical health-related quality of life (SMD 0.12, $95 \% \mathrm{CI}-0.53$ to $0.77 ; 2$ trials; 92 participants; $\mathrm{I}^{2}=54 \%$; Analysis 3.9). There was no difference in mental (MD -0.53 , $95 \% \mathrm{CI}$ -2.93 to 1.87 ; 1 trial; 258 participants) or physical health-related quality of life (MD $0.70,95 \% \mathrm{CI}-1.43$ to 2.83 ; 1 trial; 258 participants) in the one trial reporting change scores. Appendix 10 provides summary information for all seven trials.

\section{Sensitivity analyses}

There was no important change to the overall effect estimate when we restricted the analysis to:

- Trials at low risk of selection bias (SMD 0.84, 95\% CI 0.02 to $1.67 ; 3$ trials; 327 participants; $\mathrm{I}^{2}=92 \%$ ).

- One trial at low risk of attrition bias (SMD 1.15, 95\% CI

0.75 to $1.54 ; 1$ trial; 116 participants).

- Individually-randomised trials (excluding cluster trials) (SMD $0.77,95 \%$ CI 0.16 to 1.39 ; 4 trials; 391 participants; $\mathrm{I}^{2}=$ $88 \%$ ) (Table 10).

- All trials were at high risk for detection bias. 


\section{Adverse effects of the intervention}

Seven trials reported on adverse events that may have been related to the intervention. One trial $(\mathrm{Ng} 2015)$ reported resolvable joint pain in two participants undergoing exercise (2\% of 97 ; it is unclear whether this included the multiple component group) and one trial (Wesson 2013) reported minor complaints in four participants (36\% of 11$)$ relating to stiffness, dizziness and mild joint pain. Five trials reported no adverse events (Campbell 2005; Freiberger 2012; Olsen 2014; Serra-Prat 2017; Waterman 2016).

\section{Multiple component interventions versus exercise}

Five trials compared multiple component interventions (Day 2002; Huang 2010; Ng 2015; Sosnoff 2015; Uusi-Rasi 2015) with exercise as a single active falls-prevention intervention.

\section{Primary outcomes}

\section{Rate of falls}

Of the two trials assessing the rate of falls, we could analyse data for one trial (Uusi-Rasi 2015), which found there was little or no difference in the rate of falls between a multiple component intervention versus exercise ( $\mathrm{RaR} 0.92,95 \%$ CI 0.77 to $1.10 ; 1$ trial; 191 participants; low quality evidence; Analysis 4.1).

Number of people who sustained one or more falls

We could pool data from three of the four trials that assessed the number of people sustaining one or more falls. There may be little or no difference in the risk of sustaining one or more falls between multiple component interventions versus exercise (RR 0.93, 95\%
CI 0.78 to $1.10 ; 3$ trials; 863 participants; low-quality evidence; Analysis 4.2). (Day 2002 contributed data from four different multiple component interventions and so is included in the pooled analysis four times, by splitting data from the exercise group).

\section{Number of people who sustained recurrent falls}

No trials comparing multiple component interventions versus exercise reported on the risk of recurrent falls.

\section{Secondary outcomes}

No trials comparing multiple component interventions versus exercise reported on the number of people who sustained one or more fall-related fractures; the number of people who experienced a fall that required medical attention; or health-related quality of life.

\section{Number of people who experienced a fall that required hospital admission}

Only Ng 2015 assessed the number of people who required a hospital admission, some of which may have been fall-related. This found very low-quality evidence, which means we are uncertain of whether there is any difference in the risk for people who experienced a fall that required hospital admission between a multiple component intervention versus exercise (RR 1.95, 95\% CI 0.52 to $7.41 ; 1$ trial; 97 participants; Analysis 4.3).

\section{Adverse events}

Two trials reported on adverse events that may be related to the intervention: $\mathrm{Ng} 2015$ reported joint pain in two participants (2\% of 97) and Uusi-Rasi 2015 reported no adverse events. 


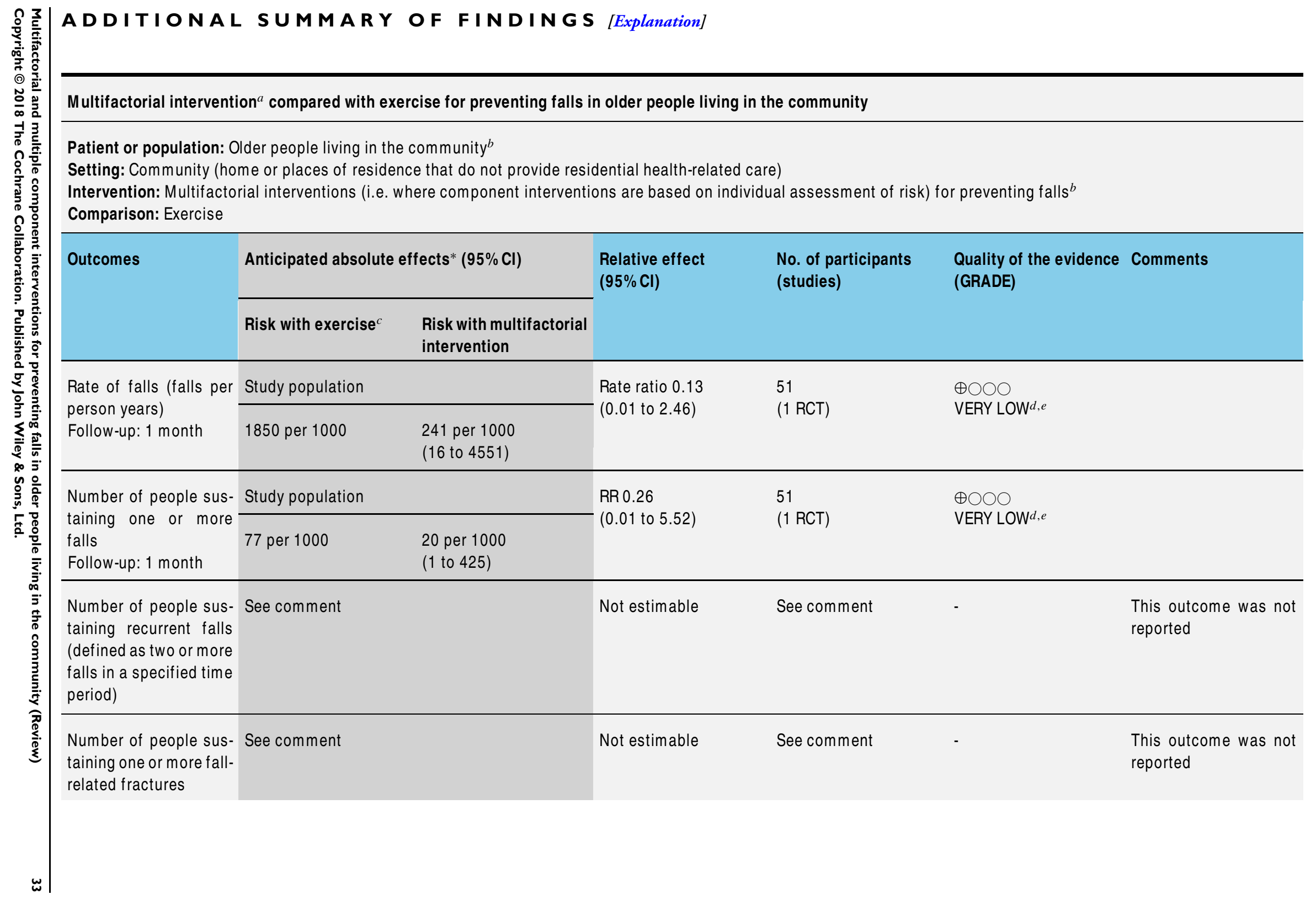




\begin{tabular}{|c|c|c|c|c|}
\hline $\begin{array}{l}\text { Number of people who } \\
\text { experience a fall that re- } \\
\text { quired hospital admis- } \\
\text { sion }\end{array}$ & See comment & Not estimable & See comment & $\begin{array}{l}\text { This outcome was not } \\
\text { reported }\end{array}$ \\
\hline $\begin{array}{l}\text { Number of people who } \\
\text { experience a fall that } \\
\text { required medical atten- } \\
\text { tion }\end{array}$ & See comment & Not estimable & See comment & $\begin{array}{l}\text { This outcome was not } \\
\text { reported }\end{array}$ \\
\hline $\begin{array}{l}\text { Health-related quality } \\
\text { of life }\end{array}$ & See comment & Not estimable & See comment & $\begin{array}{l}\text { This outcome was not } \\
\text { reported }\end{array}$ \\
\hline Adverse effects & See comment & Not estimable & See comment & $\begin{array}{l}\text { This outcome was not } \\
\text { reported }\end{array}$ \\
\hline
\end{tabular}

${ }^{*}$ The risk in the intervention group (and its $95 \%$ confidence interval) is based on the assumed risk in the comparison group and the relative effect of the intervention (and its $95 \% \mathrm{Cl})$.

Cl: Confidence interval; RR: Risk ratio

\section{GRADE Working Group grades of evidence}

High quality: We are very confident that the true effect lies close to that of the estimate of the effect

Moderate quality: We are moderately confident in the effect estimate: The true effect is likely to be close to the estimate of the effect, but there is a possibility that it is substantially different

Low quality: Our confidence in the effect estimate is limited: The true effect may be substantially different from the estimate of the effect

Very low quality: We have very little confidence in the effect estimate: The true effect is likely to be substantially different from the estimate of effect

${ }^{a} \mathrm{~A}$ multifactorial intervention is one in which the selection of falls prevention interventions (such as exercise, home-hazard modification or medication review) prescribed or provided to each individual is matched to their risk-of-falls profile, which is assessed beforehand. This individually-tailored intervention means that after receiving an assessment of known risk factors for falling, individuals are likely to received different combinations of interventions: i.e. one person may receive supervised exercise and home-hazard modification whereas another may receive home-hazard modification and medication review.

${ }^{b}$ The participants in the only trial testing this comparison were recently-discharged orthopaedic patients in Japan. The specific multifactorial intervention comprised a tailored education programme using home floor plans.

${ }^{c}$ We calculated the risk in the exercise group based on the number of events and the total number of participants in the exercise group for each outcome.

${ }^{d}$ Downgraded one level for risk of bias (more than one domain is at high or unclear risk of bias).

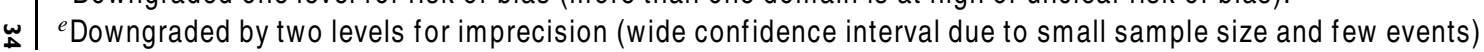


Multiple component intervention ${ }^{a}$ compared to usual care or attention control for preventing falls in older people living in the community

Patient or population: Older people living in the community

Setting: Community (home or places of residence that do not provide residential health-related care)

Intervention: Multiple component interventions (i.e. where the same component interventions are provided to all people) for preventing falls ${ }^{b}$

Comparison: Usual care or attention control

\begin{tabular}{|c|c|c|c|c|c|c|}
\hline \multirow[t]{2}{*}{ Outcomes } & \multicolumn{2}{|c|}{ Anticipated absolute effects* $(95 \% \mathrm{Cl})$} & \multirow{2}{*}{$\begin{array}{l}\text { Relative effect } \\
(95 \% \mathrm{Cl})\end{array}$} & \multirow{2}{*}{$\begin{array}{l}\text { No. of participants } \\
\text { (studies) }\end{array}$} & \multirow{2}{*}{$\begin{array}{l}\text { Quality of the evidence } \\
\text { (GRADE) }\end{array}$} & \multirow[t]{2}{*}{ Comments } \\
\hline & $\begin{array}{l}\text { Risk with usual care or } \\
\text { attention control }^{c}\end{array}$ & $\begin{array}{l}\text { Risk with Multiple in- } \\
\text { tervention }\end{array}$ & & & & \\
\hline \multirow{2}{*}{$\begin{array}{l}\text { Rate of falls (falls per } \\
\text { person years) } \\
\text { Follow-up: range } 3 \text { to } 24 \\
\text { months }\end{array}$} & Study population & & \multirow[t]{2}{*}{$\begin{array}{l}\text { Rate ratio } 0.74 \\
(0.60 \text { to } 0.91)\end{array}$} & \multirow[t]{2}{*}{$\begin{array}{l}1085 \\
\text { (6 RCTs) }\end{array}$} & \multirow[t]{2}{*}{$\begin{array}{l}\oplus \oplus \oplus \bigcirc \\
\text { MODERATE }^{d}\end{array}$} & \multirow{2}{*}{$\begin{array}{l}\text { This is just a guide to } \\
\text { the data. } \\
\text { If } 1000 \text { people were fol- } \\
\text { lowed over } 1 \text { year, the } \\
\text { number of falls would } \\
\text { be } 1206 \text { ( } 95 \% 978 \text { to } \\
1483 \text { ) compared with } \\
1630 \text { in the group re- } \\
\text { ceiving usual care or at- } \\
\text { tention control }\end{array}$} \\
\hline & 1630 per 1000 & $\begin{array}{l}1206 \text { per } 1000 \\
(978 \text { to } 1483 \text { ) }\end{array}$ & & & & \\
\hline \multirow[t]{2}{*}{$\begin{array}{l}\text { Number of people sus- } \\
\text { taining one or more } \\
\text { falls } \\
\text { Follow-up: range } 3 \text { to } 18 \\
\text { months }\end{array}$} & Study population & & \multirow[t]{2}{*}{$\begin{array}{l}\text { RR } 0.82 \\
(0.74 \text { to } 0.90)\end{array}$} & \multirow[t]{2}{*}{$\begin{array}{l}1980 \\
\text { (11 RCTs) }\end{array}$} & \multirow[t]{2}{*}{$\begin{array}{l}\oplus \oplus \oplus \bigcirc \\
\text { MODERATE }^{d}\end{array}$} & \multirow{2}{*}{$\begin{array}{l}\text { This is just a guide to } \\
\text { the data. } \\
\text { If } 1000 \text { people were fol- } \\
\text { lowed over } 1 \text { year, the } \\
\text { number of fallers would } \\
\text { be } 243 \text { ( } 95 \% \mathrm{Cl} 220 \\
\text { to } 267) \text { compared with } \\
297 \text { in the group receiv- } \\
\text { ing usual care or atten- } \\
\text { tion control }\end{array}$} \\
\hline & 297 per 1000 & $\begin{array}{l}243 \text { per } 1000 \\
(220 \text { to } 267)\end{array}$ & & & & \\
\hline
\end{tabular}




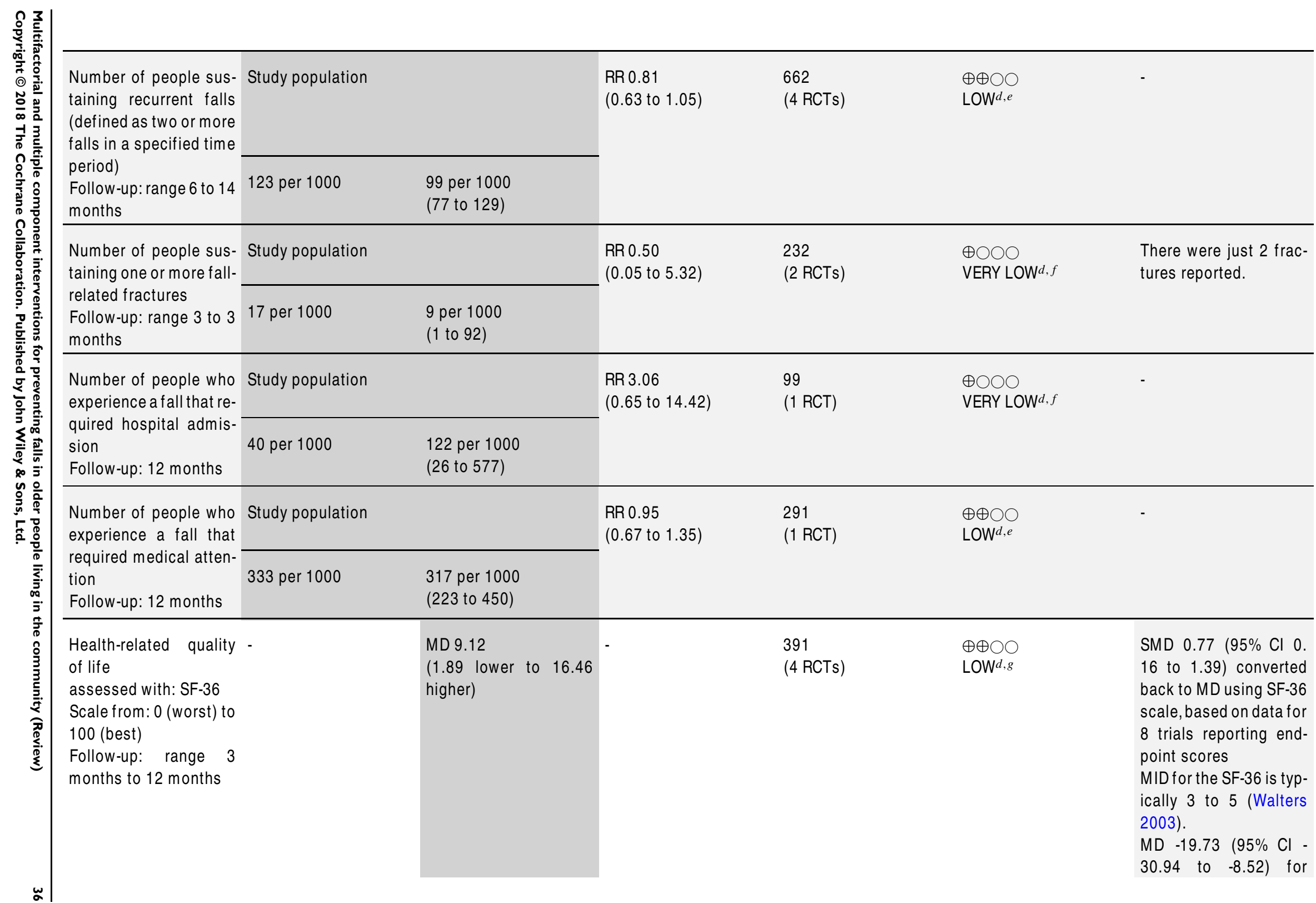




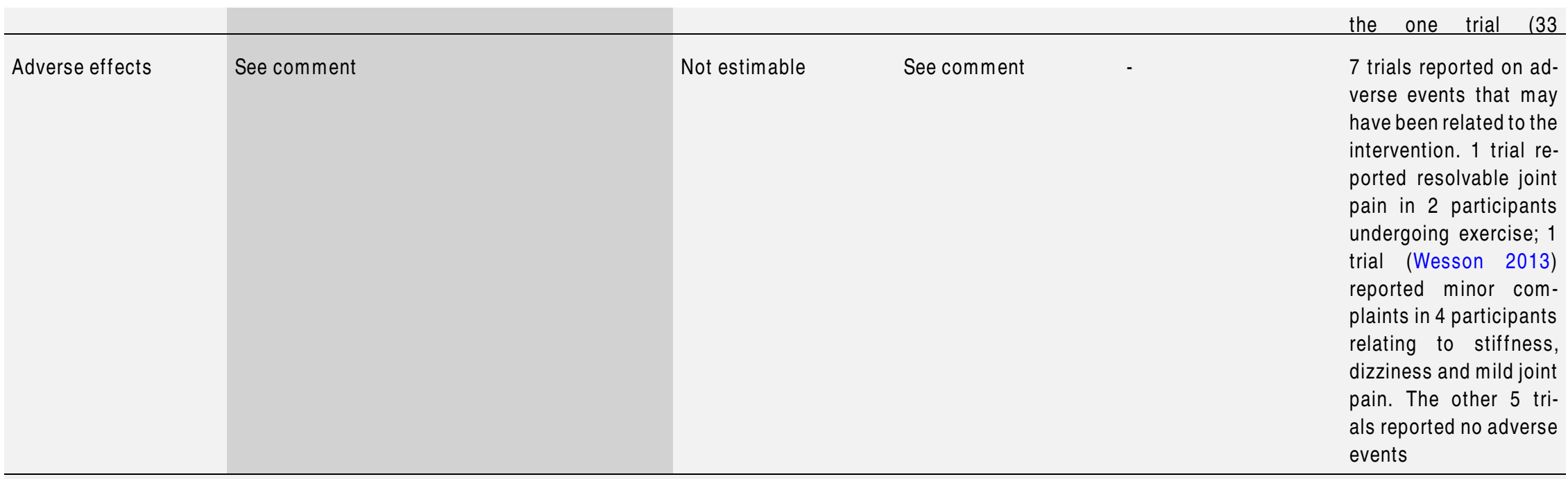

* The risk in the intervention group (and its $95 \%$ confidence interval) is based on the assumed risk in the comparison group and the relative effect of the intervention (and its $95 \% \mathrm{Cl})$.

Cl: Confidence interval; RR: Risk ratio MID: minimal important difference

\section{GRADE Working Group grades of evidence}

High quality: We are very confident that the true effect lies close to that of the estimate of the effect

Moderate quality: We are moderately confident in the effect estimate: The true effect is likely to be close to the estimate of the effect, but there is a possibility that it is substantially different

Low quality: Our confidence in the effect estimate is limited: The true effect may be substantially different from the estimate of the effect

Very low quality: We have very little confidence in the effect estimate: The true effect is likely to be substantially different from the estimate of effect

${ }^{a}$ Multiple component interventions are those where people receive a fixed combination of two or more fall prevention interventions selected from different categories of intervention (e.g. exercises, medication review, environment/assistive technology).

${ }^{b}$ The multiple component interventions used in the 17 trials testing this comparison were: exercise and education ( 2 trials); exercise and home safety (4 trials); exercise and nutrition (2 trials); exercise and psychological intervention (2 trials); exercise and home safety and nutrition ( 1 trial); exercise and home safety and vision assessment ( 3 trials); or exercise and nutrition and psychological intervention ( 1 trial), home safety and vision ( 1 trial) and nutrition and psychological intervention ( 1 trial).

${ }^{c}$ We calculated the risk in the control group based on the number of events and the total number of participants in the control

$\omega$ group for each outcome 


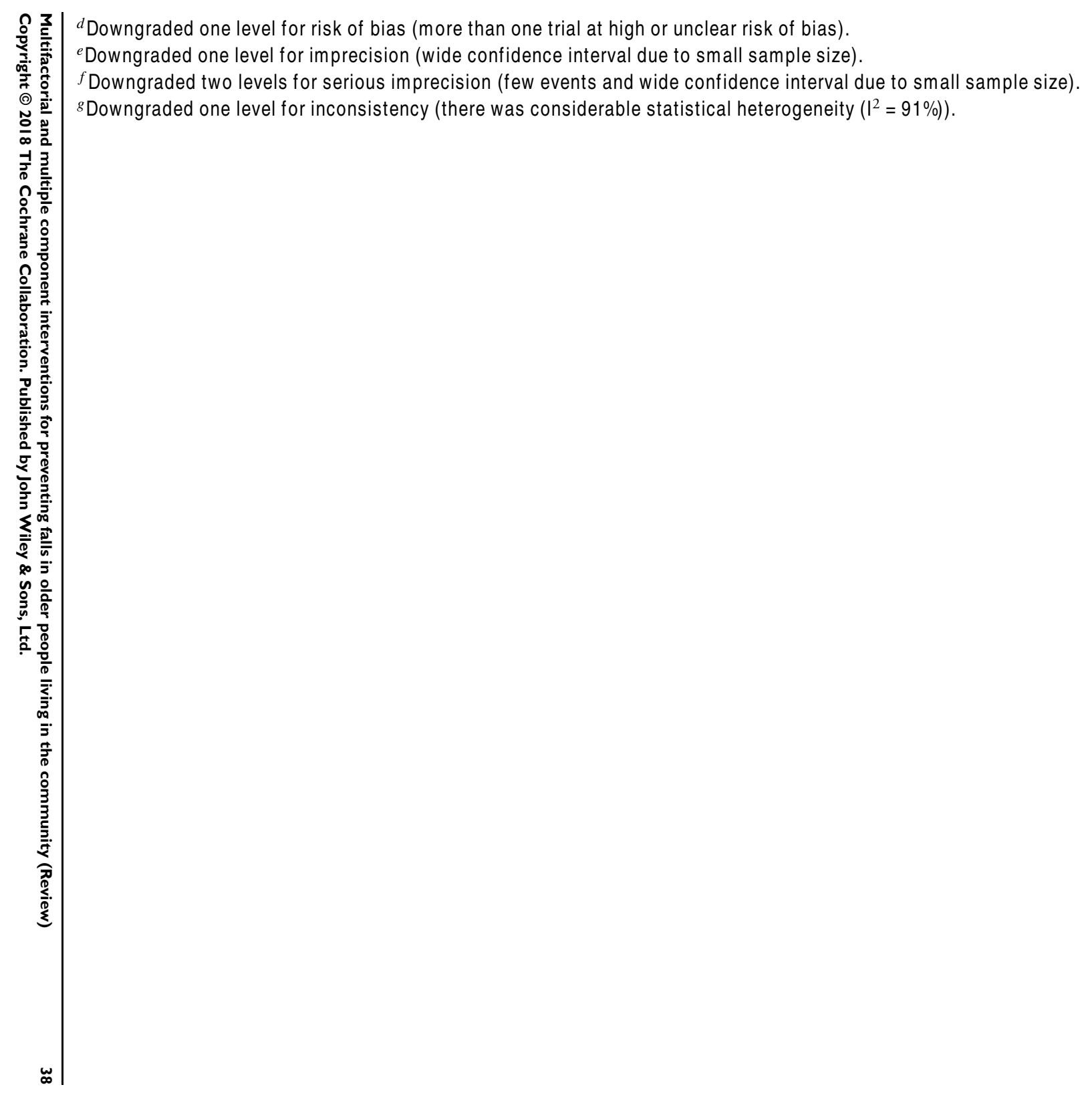




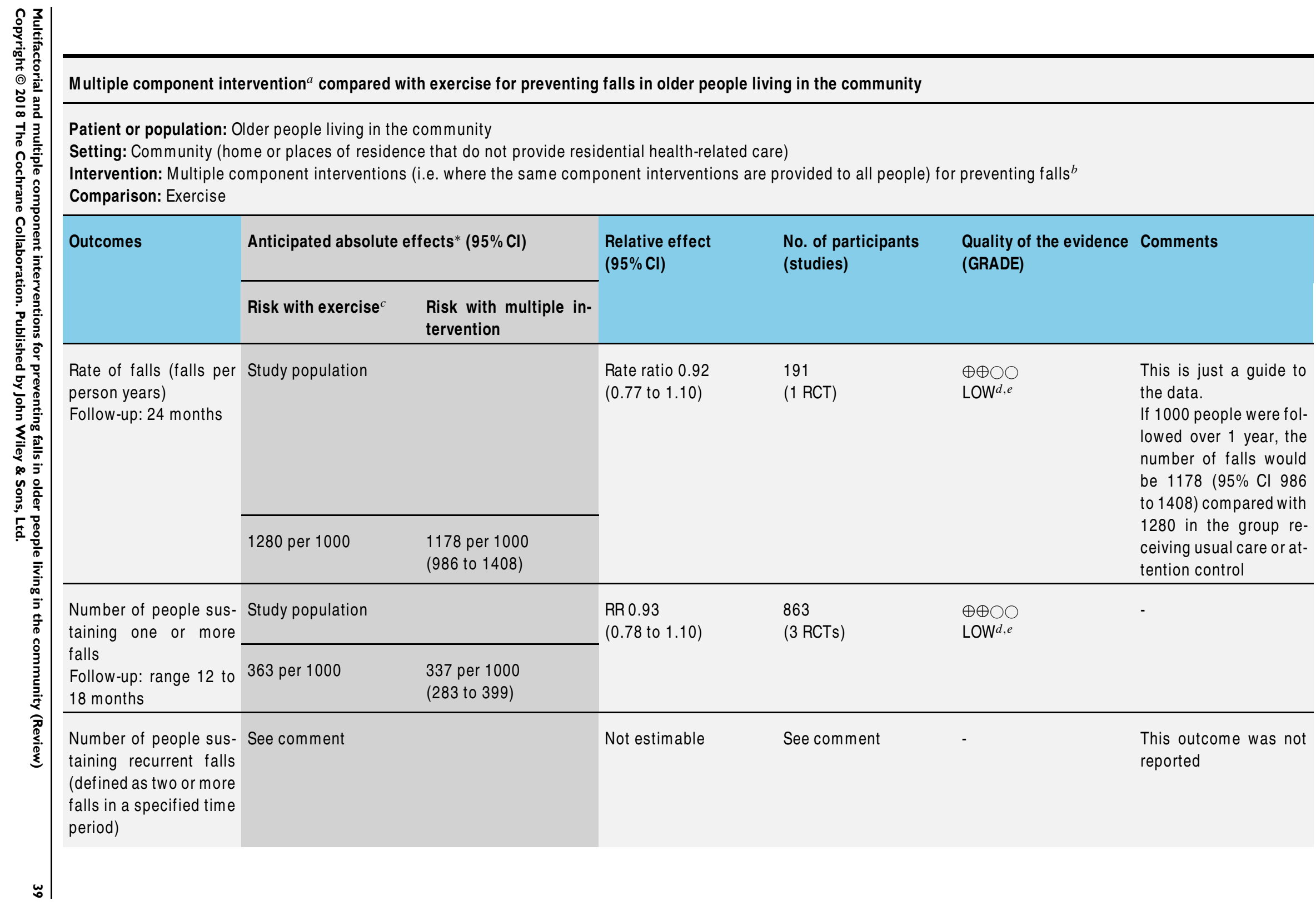




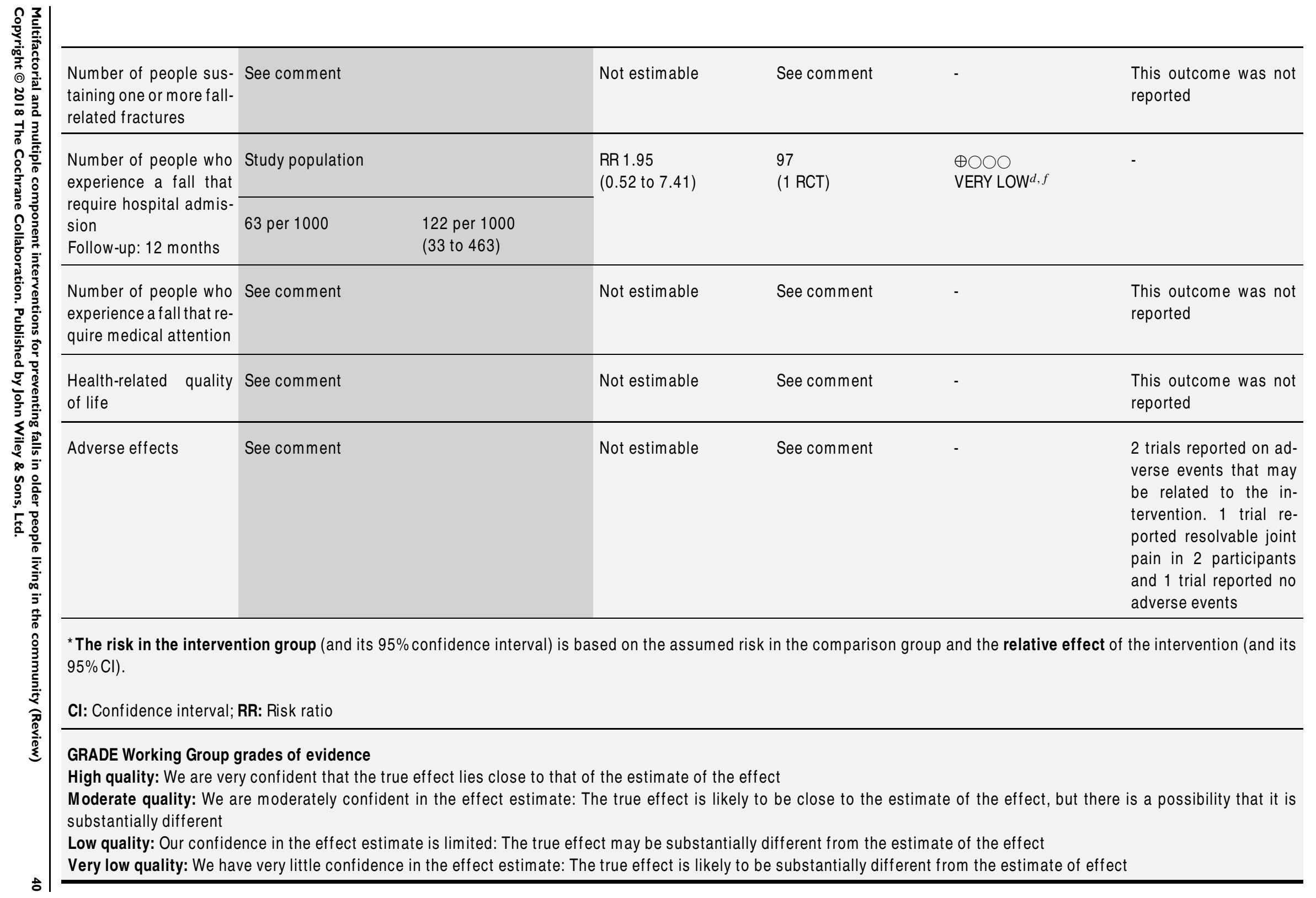


Multiple component interventions are those where people receive a fixed combination of two or more fall prevention interventions selected from different categories of intervention (e.g. exercises, medication review, environment/assistive technology).

The multiple component interventions used in the five trials testing this comparison were: exercise and education ( 1 trial) exercise and nutrition ( 1 trial); exercise, nutrition and psychological ( 1 trial); exercise, home safety and vision assessment (1 trial); and exercise, nutrition and psychological intervention (1 trial).

We calculated the risk in the exercise group based on the number of events and the total number of participants in the exercise group for each outcome.

${ }^{d}$ Downgraded one level for risk of bias (more than one trial at high or unclear risk of bias).

Downgraded one level for imprecision (wide confidence interval due to small sample size).

Downgraded two levels for serious imprecision (very wide confidence interval due to small sample size). 


\section{DISCUSSION}

\section{Summary of main results}

\section{Multifactorial interventions}

Forty-four trials assessed the effects of multifactorial interventions (where the different components of the intervention are linked to each individual's risk profile) for preventing falls in older people living in the community. Of these, 43 trials compared a multifactorial intervention with usual care or attention control, and one compared a multifactorial intervention with exercise as a single intervention. The trials included a range of multifactorial interventions, most involving assessment by registered medical or health professionals, but not all trials used this method. Commonly-used component interventions included exercise, applied in 37 trials; environment/assistive technologies, applied in 34 trials; medication review, applied in 28 trials; and psychological interventions, applied in 19 trials. In 21 trials, the intervention was designed to actively provide treatment to address identified risk factors as opposed to where the intervention consisted mainly of referral to other services or the provision of information on falls prevention.

\section{Multifactorial interventions versus usual care or attention control}

We summarise the evidence for this comparison, tested by 43 trials, in Summary of findings for the main comparison. Results show that multifactorial interventions may reduce the rate of falls compared with those who receive usual care or an attention control (Analysis 1.1). There was considerable heterogeneity that could not be explained based on our prespecified sensitivity and subgroup analyses, but we nonetheless pooled data because the nature of a multifactorial intervention means that, even within a single trial, different participants will receive different combinations of treatment based on their risk profile and so we would expect a certain amount of heterogeneity. There may be little or no difference between recipients of multifactorial interventions compared with those who received usual care or an attention control and the risk of people sustaining one or more falls; sustaining recurrent falls; experiencing a fall that required hospital admission or experiencing a fall that required medical attention. There is low-quality evidence that multifactorial interventions may reduce the risk of sustaining one or more fall-related fractures, although it also supports a conclusion of little or no difference in effect. Multifactorial interventions may slightly improve a person's health-related quality of life, but the effect size may be too small to be noticeable. Of the three trials reporting on adverse events potentially relating to the interventions, one trial reported back pain in two participants, one reported musculoskeletal symptoms in 10 participants, and the third trial stated that no adverse events had been reported. All 12 adverse events were self-limiting.

\section{Multifactorial interventions versus exercise}

The evidence for this comparison, which is summarised in Summary of findings 2, was from one small trial that tested a multifactorial intervention, centred on Japanese home floor plans, in recently-discharged orthopaedic patients. Very low-quality evidence means that we are uncertain of the effects on the rate of falls or the risk of people sustaining falls of multifactorial interventions versus exercise as a single intervention. Other outcomes were not reported.

\section{Multiple component interventions}

Eighteen trials assessed the effects of multiple component interventions (where two or more main categories of intervention are given to all participants) for preventing falls in older people living in the community. Seventeen were compared with usual care or attention control, and five were compared with exercise as a single intervention. Exercise was an almost universal component of multiple interventions in 17 of the 18 trials and statistical heterogeneity was generally low. Given these, we made the post hoc decision to present the results for the pooled analyses in addition to subgrouping trials by the different combination of interventions. This enabled us to examine the effect of using different combinations of treatment compared with usual care or exercise alone. Popular combinations of interventions were exercise with home safety (5 of 18) and exercise with education ( 4 of 18). Eleven of the 18 trials included participants at higher risk of falls at baseline.

\section{Multiple component interventions versus usual care or attention control}

We summarise the evidence for this comparison in Summary of findings 3. There is moderate-quality evidence that multiple component interventions probably reduce the rate of falls and the risk of sustaining one or more falls compared with usual care or an attention control. There is low-quality evidence that multiple component interventions may reduce the risk of people sustaining recurrent falls, but the $95 \%$ confidence interval also included the possibility of no difference or a slight increase in this risk. Very lowquality evidence means that we are uncertain of the effects of multiple component interventions compared with usual care or an attention control on the risk of fall-related fractures or of experiencing a fall that required hospital admission. There was low-quality evidence that there may be little or no difference between multiple component interventions and usual care or an attention control and the risk of people experiencing a fall that required medical attention. There is some low-quality evidence that multiple component interventions may slightly improve a person's health-related quality of life, but the limited available evidence for this outcome was also heterogeneous. Seven trials reported on adverse events. Of the seven trials reporting on adverse events potentially relating to the interventions, one trial reported resolvable joint pain in two participants and one trial reported four participants with minor 
complaints relating to stiffness, dizziness and mild joint pain; the remaining five trials reported no adverse events.

\section{Multiple component interventions versus exercise}

We summarise the evidence for this comparison, tested in five trials, in Summary of findings 4. Compared with exercise as a single intervention, multiple component interventions may have little or no difference in the rate of falls (one trial) or on the risk of sustaining one or more falls (three trials). We are uncertain whether there is a difference between the two interventions for the risk of experiencing a fall that required hospital admission (one trial). None of the five trials reported on the risk of recurrent falls, fall-related fractures, falls requiring medical attention, or healthrelated quality of life. Two trials reported on adverse events. One trial reported joint pain (in two participants) as an adverse event which may have been related to the intervention; the remaining trial stated that no adverse events were reported.

\section{Overall completeness and applicability of evidence}

This review provides the most up-to-date evidence for the effects of multifactorial and multiple component intervention for the prevention of falls in older people living in the community, compared with either usual care (or attention control) or exercise as a single intervention.

\section{Participants}

We included 44 trials assessing the effects of multifactorial interventions with a total of 15,733 participants. Most trials were moderately small (median = 303 participants; IQR 156 to 489), with a mean age of participants ranging from 72 to a maximum of 85 years. Trials were performed over 20 years from 1992 to 2014. Only one of the 44 trials included participants from a lowor middle-income country (Thailand), suggesting the findings of this review may not be applicable to those settings. In addition, most trials made a purposeful attempt to select samples who were at higher risk of falls, with 31 of the 44 trials including participants at higher risk of falls at baseline. The age range of participants and the rate of falls in the control arm also indicate that trials of multifactorial interventions may have selected populations who were experiencing more falls.

The total number of participants included in the 18 trials of multiple component interventions was smaller (total $=4202$ ), as was the size of the individual trials (median = 179; IQR 72 to 310). The mean age range of participants included in the trials was 62 to 84 years, suggesting that, for a few trials at least, the average age of participants included in the multiple component intervention trials was slightly less than for trials of multifactorial interventions. Trials were also performed more recently, from 2001 to
2017. Again, only one of the 18 trials included participants from a low- or middle-income country (Mexico), suggesting the findings of this review may not be applicable to those settings.

Most trials specifically excluded older people who were cognitively impaired, indicating that the results of this review may not be applicable to this important group of people at risk of falls. We excluded trials recruiting people with Parkinson's disease and poststroke, as we consider the results of interventions for those neurological conditions are not necessarily applicable to older people as a whole; these topic areas are covered by other Cochrane Reviews (Canning 2015; Verheyden 2013).

\section{Interventions}

Evidence is limited for the effects of multifactorial interventions compared with those who receive usual care or an attention control, showing that they may reduce the rate of falls but may have little or no effect on other fall-related outcomes. This is despite multifactorial interventions being the recommended approach for falls prevention in the UK (NICE 2013), and recommended as a primary treatment strategy in guidelines for prevention of falls published by the American Geriatrics Society, British Geriatrics Society and Australian Commission on Safety and Quality in Healthcare (ACSQH 2009; American Geriatrics Society 2011).

Evidence for the effects of multiple component interventions compared with those who receive usual care or an attention control show that they probably reduce the rate of falls and the risk of sustaining one or more falls. The multiple component interventions included in this review were heterogeneous. Often, only a single trial examined the effectiveness of each combination of components; however, exercise was a key component in all but one of the 18 multiple component interventions. In this review, we did not investigate which combinations of multiple component interventions were most effective, but we conclude that providing two or more interventions may be more effective in comparison with usual care in reducing the rate and risk of falls, and noting that most combinations included an exercise programme.

The included trials were conducted in over 20 countries, using a variety of different healthcare models. The extent to which the effectiveness of some interventions may be sensitive to differences between healthcare systems and structures at a local and national level is unclear. For example, Hendriks 2008 reported the results of a study which aimed to reproduce in The Netherlands the successful multifactorial intervention reported by Close 1999 from the UK. Major differences in the health systems in The Netherlands may be one reason why Hendriks 2008 found no difference in the number of people sustaining one or more falls, whereas the study by Close 1999 did.

We decide a priori to only include trials where the intervention was compared with usual care (i.e. no change in usual activities), an attention control (i.e. an intervention that is not thought to reduce falls such as general health information or social visits) or exercise as a single intervention. When defining usual care (i.e. no change 
in usual activities) we used the definition of usual care as defined by the trial as meaning no active treatment (i.e. no intervention). Several large trials excluded from this review included a falls prevention leaflet as the comparator intervention (Bruce 2016; Conroy 2010; Perula 2012; Salminen 2009; Shumway-Cook 2007). The inclusion of trials with a structured advice leaflet for the prevention of falls as the comparator intervention could potentially reduce the effectiveness of a multifactorial and multiple component intervention, although this would need to be proved empirically and warrants further investigation.

\section{Outcomes}

We sought data on the rate of falls, the number of people sustaining one or more falls, recurrent falls, fall-related fractures, hospital admission following a fall, medical attention, health-related quality of life and adverse events. Data for adverse events were sparse and are discussed separately. For multifactorial interventions compared with control, there was low quality of evidence for both primary and secondary outcomes (between 8 and 29 trials per outcome). However, the evidence was more limited for multiple component interventions versus control; for example, just two trials provided data on fall-related fractures, and single trials provided data on medical attention and hospital admission. The evidence was also limited for the effects of multifactorial (one trial) or multiple component interventions (five trials) compared with exercise as a single intervention.

Prospective daily calendars returned monthly for at least one year from randomisation were the preferred method for recording falls (Lamb 2005). However, we also included studies where falls were recorded retrospectively, or not monitored continuously throughout the trial, as this is still common practice and increases the applicability of our findings and avoids potential bias, as it would have resulted in the exclusion of a number of trials.

There was limited evidence available on adverse events occurring as a result of the interventions tested in this review; while some trials did report on whether adverse events occurred, it was not always clear whether this was specifically due to the intervention. Trials which did report information on adverse events were more likely to have been published more recently. Inspection of the reasons for loss to follow-up did not reveal withdrawal explicitly due to adverse events; however, reasons for loss to follow-up were not reported consistently across studies. While we did not specifically assess this in our review, it is noteworthy that none of the trials assessed mortality due to falls as an outcome measure. Over half of the trials reported death as a reason for loss to follow-up. In some trials, mortality was the main reason for loss to follow-up; for example, in Carpenter 1990 (120 deaths, 22\% of 539 participants, occurred over the three-year follow-up) and in Vetter 1992 (194 deaths, $29 \%$ of 674 participants, occurred over the four-year follow-up).

\section{Quality of the evidence}

We have summarised the GRADE quality of evidence in Summary of findings for the main comparison (Multifactorial interventions versus control), Summary of findings 3 (Multiple interventions versus control), Summary of findings 2 (Multifactorial interventions versus exercise) and Summary of findings 4 (Multiple interventions versus exercise).

Overall the quality of the evidence ranged from moderate to very low. We downgraded all outcomes by one level for risk of bias, as more than one trial was at unclear or high risk of bias in all domains. We also downgraded one level for inconsistency where heterogeneity was a significant problem, such as for 'Rate of falls' for the comparison of multifactorial interventions versus control, which could not be explained by prespecified subgroup and sensitivity analyses. We downgraded one level for indirectness for fallrelated hospital admission for the first comparison because poor reporting meant that it was not always possible to specifically determine that the cause of hospital admission was always due to a fall.

We also downgraded the level of evidence for imprecision by one or two levels due to the wide confidence intervals, often reflecting the small number of trials, participants and sometimes events for some outcomes such as fall-related fractures.

\section{Potential biases in the review process}

We are not aware of any obvious biases within the review process. We conducted a comprehensive search, which was not restricted by language or by full-text publication, to optimise the chances of identifying all relevant trials. Two review authors who were blinded to each other's results performed screening and data extraction in duplicate to minimise bias. A limitation of this review is that for some outcomes the original authors published results in a format that did not allow for inclusion in meta-analysis and therefore could not contribute data for these outcomes; this was particularly the case when analysing the rate of falls and health-related quality of life. Additionally, several subgroup analyses have limited power due to the small number of studies within subgroups. We were therefore cautious in our interpretation of subgroup analyses where there was a limited number of studies within a subgroup.

\section{Agreements and disagreements with other studies or reviews}

This review provides updated evidence for two of the intervention categories (multifactorial and multiple component interventions) covered in the Cochrane Review of Interventions for preventing falls in older people living in the community, published in 2012 (Gillespie 2012). We have excluded several trials included in Gillespie 2012 from this review because the comparator was either a different 
multifactorial intervention or different multiple component intervention, or because the comparator was a single active intervention (apart from exercise) or included a falls prevention leaflet. Updated evidence for the category of 'Exercise interventions' is being covered in Sherrington 2016a.

Our review adds to this existing body of evidence and supports the findings of Gillespie 2012, where multifactorial interventions were found to reduce the rate of falls (RaR $0.76,95 \%$ CI 0.67 to 0.86; 19 trials; 9503 participants) but not the risk of falling (RR $0.93,95 \%$ CI 0.86 to 1.02 ; 34 trials; 13,617 participants) or the risk of fall-related fractures. However, as in our review, there was significant unexplained statistical heterogeneity in the rate of falls, thus weakening our confidence in the observed treatment effect. In Gillespie 2012, exercise, whether group- or home-based, saw the greatest reduction in the rate of falls, risk of falling and the risk of fall-related fracture. This is supported by a recent review using network meta-analysis by Tricco 2017 of 54 trials (41,596 participants), across a range of acute and community settings, showing that exercise alone or exercise combined with various combinations of interventions was associated with lower risk of injurious falls compared with usual care.

A Cochrane Review of interventions for preventing falls in older people in care facilities and hospitals (Cameron 2012) found evidence that, as in our review, multifactorial interventions also reduced the rate of falls ( $\mathrm{RaR} 0.69,95 \%$ CI 0.49 to $0.96 ; 4$ trials; 6478 participants) in older people in hospitals but not the risk of falling. However, there was no difference in the rate of falls and risk of falling between multifactorial interventions and control in older people living in care facilities, or comparing exercise interventions to a control intervention.

Gillespie 2012 did not pool the results of individual trials comparing different multiple component interventions and also included other fall prevention interventions as a comparator. In our review, we decided a priori to limit the choice of comparator to either usual care (or attention control) or to exercise as a single intervention, in order to be able to compare outcomes more consistently across trials. Goodwin 2014 carried out a systematic review of trials evaluating the effects of multiple component interventions in adults aged over 60 years, with any medical condition or in any setting. As in our review, they found that multiple component interventions reduced the rate of falls ( $\mathrm{RaR} 0.80,95 \% \mathrm{CI} 0.72$ to 0.89 ) and the risk of falling (RR $0.85,95 \%$ CI 0.80 to 0.91 ) compared with those who received a control intervention, suggesting that offering multiple component treatments, regardless of risk profile, could be considered an option for service delivery.

\section{A U THORS' CONCLUSIONS}

\section{Implications for practice}

Despite their appeal as a strategy to prevent falls in older people living in the community, the findings from our review show that while multifactorial interventions may reduce the rate of falls compared with those who receive usual care or an attention control, there may be little or no difference in other fall-related outcomes. An exception may be that these interventions reduce the risk of fall-related fractures, but the low-quality evidence also supports a conclusion of little or no difference in effect. There was very limited evidence available on adverse events occurring as a result of the intervention; all 12 reported musculoskeletal events resolved.

Very low-quality evidence from one small trial means that we are uncertain of the effects on rate of falls or the risk of people sustaining falls of multifactorial interventions versus exercise as a single intervention. Other fall-related outcomes were not assessed.

Multiple component interventions, where exercise was a key component, probably reduce the rate of falls and the risk of sustaining one or more falls and may reduce the risk of recurrent falls. Such interventions may make little or no difference to the number of people requiring medical attention but may slightly improve quality of life. There was insufficient evidence to determine the effects on fall-related fracture or hospital admission. There was limited evidence available on adverse events occurring as a result of the intervention; all six adverse events were minor.

The few trials comparing multiple component interventions with exercise as a single intervention provided low-quality evidence that there may be little or no difference between the interventions in the rate of falls or the risk of sustaining one or more falls. The very low-quality evidence from one small trial means that we are uncertain of the relative effects on hospital admission. The two reported adverse events were minor. Other fall-related outcomes were not reported.

\section{Implications for research}

Exercise is one of the most common elements of both multifactorial and multiple component interventions and is an effective single intervention. Future research should build on this and establish a better picture of the added benefit of including co-interventions alongside exercise. Many of the types of intervention added to exercise as part of a multifactorial or multiple component interventions are expensive and the additional benefits are unclear. The addition of health economic data would help aid decisionmaking and provide a greater understanding of the broader impacts of these and other similar interventions.

Given that exercise is an effective and well-established intervention in community-dwelling populations, we believe this should be considered as the comparator intervention for new research, as opposed to usual care (i.e. no change in usual activities) or an attention control (e.g. social visits) comparator, as was the case for most of the trials included in this review. Measuring adherence to interventions is also important. Only half of the trials included in this review reported that they assessed adherence to the

Multifactorial and multiple component interventions for preventing falls in older people living in the community (Review) 
intervention, and the extent to which participants within the trials complied with the individual treatment components was unclear. We would recommend that future trials look at ways to maximise adherence and measure its impact on the trial findings. Another potential area for research is to develop interventions for those who are either unable or unwilling to engage with exercise or to adhere in the longer term.

The underlying quality of the research evidence also remains a concern. Nearly all the trials in this review depended on the participants or observers reporting falls either prospectively in diaries, or through recollection. The obvious drawback is that participants, care providers and/or carers cannot be blinded from the treatment received.The degree to which knowledge of the treatment as opposed to treatment received influences reporting of falls is not known. Wearable sensors are evolving and will soon offer the possibility for monitoring falls independently of self-report. This is an important aspect of methodology that should be pursued in future trials. In this review, few trials reported outcomes that can be independently verified, for example, falls resulting in fracture or hospital admission. As the event rates are much lower for these outcomes (Campbell 1990; Tinetti 1988), trials which use injurious or fracture falls need to be substantially larger than those reported to date (Bruce 2016; Bhasin 2018). We suggest that robust data on a larger number of people offers better value for money in terms of research investment.

Use of core data sets has improved over the last decade after the ProFANE consensus (Copsey 2016). We encourage trialists to adopt the consensus and to use a unified approach to defining and reporting outcomes. There is a paucity of data on health-related quality of life and future trials should include this. The types of interventions being tested potentially have much broader effects than a reduction in falls. For example, improving mobility without changing falls, improving depression and pain, better management of chronic disease. Measurement of health-related quality of life would capture these potential benefits.

\section{ACKNOWLEDGEMENTS}

We are very grateful to Kirsti Uusi-Rasi and Pip Logan (via statistician Carol Coupland) for providing additional study data. We are also grateful to Debbie Brown for her help in screening studies for eligibility and data extraction. We also thank Helen Handoll, Barbara Resnick and Janet Wale for helpful comments on drafts of the protocol, Joanne Elliott for her assistance with developing the search strategy and Lindsey Elstub for editorial support on the protocol.

We thank Liz Bickerdike, Helen Handoll, Anne-Marie Hill, Lynette MacKenzie, Barbara Resnick, Helen Wakeford and Janet Wale for their helpful comments on drafts of the review. We also thank Joanne Elliott, Lindsey Elstub and Helen Wakeford for editorial support on the review.

This project was supported by the National Institute for Health Research (NIHR) via Cochrane Infrastructure funding to the Cochrane Bone, Joint and Muscle Trauma Group. The views and opinions expressed therein are those of the review authors and do not necessarily reflect those of the Systematic Reviews Programme, the NIHR, the National Health Service (NHS) or the Department of Health.

\section{R E F E R E N C E S}

\section{References to studies included in this review}

Beling 2009 \{published data only\}

Beling J, Roller M. Multifactorial intervention with balance training as a core component among fall-prone older adults. Journal of Geriatric Physical Therapy 2009;32(3):125-33. MEDLINE: 20128337

Campbell 2005 \{published data only\}

* Campbell AJ, Robertson MC, La Grow SJ, Kerse NM, Sanderson GF, Jacobs RJ, et al. Randomised controlled trial of prevention of falls in people aged $>$ or $=75$ with severe visual impairment: the VIP trial. BMJ 2005;331(7520): 817. MEDLINE: 16183652

Jacobs R, Campbell AJ, Robertson MC. Randomized controlled trial of falls prevention in people 75 years and older with severe visual impairment [abstract]. American Academy of Optometry Meeting 2005 Dec 8-12; San Diego (CA). 2005. CENTRAL: CN-00634854]

Kiata L, Kerse NM, Hughes WE, Hayman KJ, Robertson
MC, La Grow SJ, et al. Agreement and compliance with advice on removing mats or rugs by older people with visual impairments. Journal of Visual Impairment \& Blindness 2008;102(3):167-72.

La Grow SJ, Robertson MC, Campbell AJ, Clarke GA, Kerse NM. Reducing hazard related falls in people 75 years and older with significant visual impairment: how did a successful program work?. Injury Prevention 2006;12(5): 296-301. MEDLINE: 17018669

Carpenter 1990 \{published data only\}

Carpenter GI, Demopoulos GR. Screening the elderly in the community: controlled trial of dependency surveillance using a questionnaire administered by volunteers. BMJ 1990;300(6734):1253-6. MEDLINE: 2354297

Carter 1997 \{unpublished data only\}

Carter S, Campbell E, Sanson-Fisher R, Tiller K, Gillespie WJ. Trial data (as supplied 1997). Data on file. 
Ciaschini 2009 \{published data only\}

* Ciaschini PM, Straus SE, Dolovich LR, Goeree RA, Leung $\mathrm{KM}$, Woods CR, et al. Community-based intervention to optimise falls risk management: a randomised controlled trial. Age and Ageing 2009;38(6):724-30. MEDLINE: 19767629

Ciaschini PM, Straus SE, Dolovich LR, Goeree RA, Leung $\mathrm{KM}$, Woods CR, et al. Community-based randomised controlled trial evaluating falls and osteoporosis risk management strategies. Trials [Electronic Resource] 2008;9: 62. MEDLINE: 18983670

NCT00465387. FORCE (Falls, Fracture, and Osteoporosis Risk Control Evaluation) study. clinicaltrials.gov/show/ NCT00465387 (accessed 31 August 2011).

\section{Clemson 2004 \{published data only\}}

Clemson L. Stepping On, reducing falls and building confidence: a practical program that works. Falls prevention in older people: from research to practice. 1st Australian falls prevention conference; 2004 Nov 21-23; Sydney, Australia. Randwick, NSW, Australia: Prince of Wales Medical Research Institute, 2004:68.

* Clemson L, Cumming RG, Kendig H, Swann M, Heard $\mathrm{R}$, Taylor K. The effectiveness of a community-based program for reducing the incidence of falls in the elderly: a randomized trial. Journal of the American Geriatrics Society 2004;52(9):1487-94. MEDLINE: 15341550

Clemson L, Taylor K, Kendig H, Cumming RG, Swann M. Recruiting older participants to a randomised trial of a community-based fall prevention program. Australasian Journal on Ageing 2007;26(1):35-9. CINAHL: 2009512824]

Swann M, Clemson L. Evaluating falls efficacy following a community based falls prevention program for older people. Falls prevention in older people: from research to practice. 1st Australian falls prevention conference; 2004 Nov 21-23; Sydney, Australia. Randwick, NSW, Australia: Prince of Wales Medical Research Institute, 2004:34.

Close 1999 \{published and unpublished data\}

Close J. Personal communication. 9 December 2008.

* Close J, Ellis M, Hooper R, Glucksman E, Jackson S, Swift C. Prevention of falls in the elderly trial (PROFET): a randomised controlled trial. Lancet 1999;353(9147):93-7. MEDLINE: 10023893

Close J, Hooper R, Glucksman E, Jackson S, Swift C. Predictors of falls in a high risk population - results from the prevention of falls in the elderly trial (PROFET) [abstract]. Journal of the American Geriatrics Society 2000;48(8):S79. Close JC, Ellis M, Hooper R, Glucksman E, Jackson SH, Swift CG. Predictors of falls - results from prevention of falls in the elderly trial (PROFET). Age and Ageing 1999;28 (Suppl 1):14.

Close JC, Ellis M, Jackson SH, Glucksman E, Swift CG. Interdisciplinary assessment of elderly people presenting to A\&E with a fall [abstract]. Age and Ageing 1998;27(Suppl 1):20.

Close JC, Patel A, Hooper R, Glucksman E, Jackson SH, Swift CG. PROFET - Improved clinical outcomes at no additional cost. Age and Ageing 2000;29(Suppl 1):48. F0300115. Can the incidence of falls in the elderly be reduced by a secondary prevention protocol?. National Research Register (NRR) Archive. www.nihr.ac.uk/Pages/ NRRArchiveSearch.aspx (accessed 23 February 2011). NRR Publication ID: F0300115]

\section{Coleman 1999 \{published data only\}}

Coleman EA, Grothaus LC, Sandhu N, Wagner EH. Chronic care clinics: a randomized controlled trial of a new model of primary care for frail older adults. Journal of the American Geriatrics Society 1999;47(7):775-83. MEDLINE: 10404919

Davison 2005 \{published data only\}

* Davison J, Bond J, Dawson P, Steen IN, Kenny RA. Patients with recurrent falls attending Accident \& Emergency benefit from multifactorial intervention - a randomised controlled trial. Age and Ageing 2005;34(2): 162-8. MEDLINE: 15716246

Kenny R. Syncope and Falls in the Emergency Room (SAFER 2) - A multidisciplinary post-fall assessment and intervention strategy for elderly recurrent fallers attending casualty (RRCC53R). The Research Findings Register. Summary number 1174. www.ReFeR.nhs.uk/ ViewRecord.asp?ID=1174 (accessed 24 August 2006). N0009027144. SAFER2 - Syncope and falls in the emergency room - an explanatory randomised controlled trial of a multidisciplinary post-fall assessment and intervention strategy in elderly recurrent fallers attending casualty. National Research Register (NRR) Archive. www.nihr.ac.uk/Pages/NRRArchiveSearch.aspx (accessed 23 February 2011). NRR Publication ID: N0009027144] N0116069489. Can the incidence of falls in the elderly be reduced by a secondary falls prevention protocol. National Research Register (NRR) Archive. www.nihr.ac.uk/Pages/ NRRArchiveSearch.aspx (accessed 23 February 2011). NRR Publication ID: N0116069489] N0145036249. A post-fall intervention strategy after presentation to casualty. National Research Register (NRR) Archive. www.nihr.ac.uk/Pages/NRRArchiveSearch.aspx (accessed 23 February 2011). NRR Publication ID: N0145036249]

N0145049230. A post-fall intervention strategy after presentation to casualty - Safer 2. National Research Register (NRR) Archive. www.nihr.ac.uk/Pages/ NRRArchiveSearch.aspx (accessed 23 February 2011). NRR Publication ID: N0145049230]

N0503055776. SAFER 2 - Syncope and falls in the emergency room - The Tyneside casualty falls intervention project. National Research Register (NRR) Archive. www.nihr.ac.uk/Pages/NRRArchiveSearch.aspx (accessed 23 February 2011). NRR Publication ID: N0503055776]

\section{Day 2002 \{published and unpublished data\}}

* Day L, Fildes B, Gordon I, Fitzharris M, Flamer H, Lord $S$. Randomised factorial trial of falls prevention among older people living in their own homes. BMJ 2002;325(7356): 128-31. MEDLINE: 12130606

Fitzharris MP, Day L, Lord SR, Gordon I, Fildes B. The 
Whitehorse NoFalls trial: effects on fall rates and injurious fall rates. Age \& Ageing 2010;39(6):728-33. MEDLINE: 20817936

De Vries 2010 \{published data only\}

* De Vries OJ, Peeters GM, Elders PJ, Muller M, Knol DL, Danner SA, et al. Multifactorial intervention to reduce falls in older people at high risk of recurrent falls: a randomized controlled trial. Archives of Internal Medicine 2010;170(13): 1110-7. MEDLINE: 20625015

ISRCTN11546541. Prevention of fall incidents in patients with a high risk of falling; a multidisciplinary study on the effects of transmural health care compared to usual care. controlled-trials.com/ISRCTN11546541 (accessed 14 September 2011).

Peeters GM, De Vries OJ, Elders PJ, Pluijm SM, Bouter LM, Lips P. Prevention of fall incidents in patients with a high risk of falling: design of a randomised controlled trial with an economic evaluation of the effect of multidisciplinary transmural care. BMC Geriatrics 2007;7:15. MEDLINE: 17605771

Peeters GM, Heymans MW, De Vries OJ, Bouter LM, Lips P, Van Tulder MW. Multifactorial evaluation and treatment of persons with a high risk of recurrent falling was not costeffective. Osteoporosis International 2011;22(7):2187-96.

Elley 2008 \{published data only\}

ACTRN12605000054617. Falls Assessment Clinical

Trial: randomised controlled trial of a multi-component intervention in primary health care to reduce falls amongst over 75 year old adults with a history of falling. www.anzctr.org.au/trial_view.aspx?ID=85 (accessed 30 November 2011).

* Elley CR, Robertson MC, Garrett S, Kerse NM, MacKinlay E, Lawton B, et al. Effectiveness of a falls-and-fracture nurse coordinator to reduce falls: a randomized, controlled trial of at-risk older adults. Journal of the American Geriatrics Society 2008;56(8):1383-9. MEDLINE: 18808597

Elley CR, Robertson MC, Kerse NM, Garrett S, McKinlay E, Lawton B, et al. Falls Assessment Clinical Trial (FACT): design, interventions, recruitment strategies and participant characteristics. BMC Public Health 2007;7:185. MEDLINE: 17662156

Fabacher 1994 \{published data only\}

Fabacher D, Josephson K, Pietruszka F, Linderborn K, Morley JE, Rubenstein LZ. An in-home preventive assessment program for independent older adults: a randomized controlled trial. Journal of the American Geriatrics Society 1994;42(6):630-8. MEDLINE: 8201149

Faes 2011 \{published data only\}

Faes MC, Reelick MF, Esselink RA, Rikkert MG.

Developing and evaluating complex healthcare interventions in geriatrics: the use of the medical research council framework exemplified on a complex fall prevention intervention. Journal of the American Geriatrics Society 2010; 58(11):2212-21. MEDLINE: 21039367

* Faes MC, Reelick MF, Melis RJ, Borm GF, Esselink RA, Olde Rikkert MG. Multifactorial fall prevention for pairs of frail community-dwelling older fallers and their informal caregivers: a dead end for complex interventions in the frailest fallers. Journal of the American Medical Directors Association 2011; Vol. 12, issue 6:451-8.

NCT00512655. Trial to reduce falls incidence rate in frail elderly (CP). clinicaltrials.gov/show/NCT00512655 (accessed 31 August 2011).

Reelick MF, Faes MC, Esselink RA, Kessels RP, Olde Rikkert MG. How to perform a preplanned process evaluation for complex interventions in geriatric medicine: exemplified with the process evaluation of a complex falls-prevention program for community-dwelling frail older fallers. Journal of the American Medical Directors Association 2011;12(5): 331-6.

\section{Fairhall 2014 \{published data only\}}

Cameron ID, Fairhall N, Langron C, Lockwood

K, Monaghan N, Aggar C, et al. A multifactorial interdisciplinary intervention reduces frailty in older people: randomized trial. BMC Medicine 2013;11:65. PUBMED: 23497404]

Fairhall N, Aggar C, Kurrle SE, Sherrington C, Lord S, Lockwood K, et al. Frailty Intervention Trial (FIT). BMC Geriatrics 2008;8:27. PUBMED: 18851754]

Fairhall N, Sherrington C, Kurrle SE, Lord SR, Lockwood K, Howard K, et al. Economic evaluation of a multifactorial, interdisciplinary intervention versus usual care to reduce frailty in frail older people. Journal of the American Medical Directors Association 2015;16(1):41-8. PUBMED: 25239014]

* Fairhall N, Sherrington C, Lord SR, Kurrle SE, Langron C, Lockwood K, et al. Effect of a multifactorial, interdisciplinary intervention on risk factors for falls and fall rate in frail older people: a randomised controlled trial. Age and Ageing 2014;43(5):616-22. PUBMED: 24381025]

\section{Ferrer 2014 \{published data only\}}

Badia T, Formiga F, Ferrer A, Sanz H, Hurtos L, Pujol R. Multifactorial assessment and targeted intervention in nutritional status among the older adults: a randomized controlled trial: the Octabaix study. BMC Geriatrics 2015; 15:45. PUBMED: 25887312]

Ferrer A, Badia T, Formiga F, Almeda J, Fernandez C, Pujol $\mathrm{R}$. Gender differences in health status in a population of over 85 year-olds. The Octabaix study [Diferencias de genero en el perfil de salud de una cohorte de 85 años. Estudio Octabaix]. Atencion Primaria 2011;43(11):577-84. EMBASE: 2011609254]

Ferrer A, Badia T, Formiga F, Gil A, Padros G, Sarro M, et al. A randomized clinical trial of falls and malnutrition prevention in community-dwelling elders aged 85 years old. The OCTABAIX study [Ensayo clinico aleatorizado de prevencion de caidas y malnutricion en personas de 85 años en la comunidad. Estudio OCTABAIX]. Revista Espanola de Geriatria y Gerontologia 2010;45(2):79-85.

* Ferrer A, Formiga F, Sanz H, De Vries OJ, Badia T, Pujol R. Multifactorial assessment and targeted intervention to reduce falls among the oldest-old: a randomized controlled 
trial. Clinical Interventions in Aging 2014;9:383-93. PUBMED: 24596458]

\section{Freiberger 2012 \{published and unpublished data\}}

Freiberber E. Personal communication. 25 October 2007.

Freiberger E. Personal communication. 12 December 2007.

* Freiberger E, Haberle L, Spirduso WW, Zijlstra GA.

Long-term effects of three multicomponent exercise interventions on physical performance and fall-related psychological outcomes in community-dwelling older adults: a randomized controlled trial. Journal of the American Geriatrics Society 2012;30(3):437-46. DOI: 10.1111/j.1532-5415.2011.03859.x

Freiberger E, Menz HB. Characteristics of falls in physically active community-dwelling older people: Findings from the 'Standfest im Alter' study. Zeitschrift fur Gerontologie und Geriatrie 2006;39(4):261-7.

Freiberger E, Menz HB, Abu-Omar K, Rutten A. Preventing falls in physically active community-dwelling older people: a comparison of two intervention techniques. Gerontology 2007;53(5):298-305.

Gallagher 1996 \{published data only\}

Gallagher EM, Brunt H. Head over heels: impact of a health promotion program to reduce falls in the elderly. Canadian Journal on Aging 1996;15(1):84-96. EMBASE 1996164172]

Hagovska 2016 \{published data only\}

Hagovska M, Olekszyova Z. Impact of the combination of cognitive and balance training on gait, fear and risk of falling and quality of life in seniors with mild cognitive impairment. Geriatrics and Gerontology International 2016; 16(9):1043-50. PUBMED: 26338465]

Hendriks 2008 \{published data only\}

Bleijlevens MH, Hendriks MR, Van Haastregt JC, Crebolder HF, Van Eijk JT. Lessons learned from a multidisciplinary fall-prevention programme: the occupational-therapy element. Scandinavian Journal of Occupational Therapy 2010;17(4):319-25.

Bleijlevens MH, Hendriks MR, Van Haastregt JC, Van Rossum E, Kempen GI, et al. Process factors explaining the ineffectiveness of a multidisciplinary fall prevention programme: a process evaluation. BMC Public Health 2008;8:332.

Hendriks MR, Bleijlevens MH, Van Haastregt JC, Crebolder HF, Diederiks JP, Evers SM, et al. Lack of effectiveness of a multidisciplinary fall-prevention program in elderly people at risk: a randomized controlled trial. Journal of the American Geriatrics Society 2008;56(8):13907. MEDLINE: 18662214

Hendriks MR, Bleijlevens MH, Van Haastregt JC, De Bruijn FH, Diederiks JP, Mulder WJ, et al. A multidisciplinary fall prevention program for elderly persons: a feasibility study. Geriatric Nursing 2008;29(3):186-96. MEDLINE: 18555160

* Hendriks MR, Evers SM, Bleijlevens MH, Van Haastregt JC, Crebolder HF, Van Eijk JT. Cost-effectiveness of a multidisciplinary fall prevention program in communitydwelling elderly people: A randomized controlled trial (ISRCTN 64716113). International Journal of Technology Assessment in Health Care 2008;24(2):193-202. MEDLINE: 18400123

Hendriks MR, Van Haastregt JC, Diederiks JP, Evers SM, Crebolder HF, Van Eijk JT. Effectiveness and costeffectiveness of a multidisciplinary intervention programme to prevent new falls and functional decline among elderly persons at risk: design of a replicated randomised controlled trial [ISRCTN64716113]. BMC Public Health 2005;5:6. MEDLINE: 15651990

ISRCTN64716113. Preventing further falls and functional decline among elderly persons presented to the Accident and Emergency (A\&E) department with a fall: randomised controlled trial. controlled-trials.com/ISRCTN64716113 (accessed 30 November 2011).

\section{Hogan 2001 \{published data only\}}

Hogan DB, MacDonald FA, Betts J, Bricker S, Ebly EM, Delarue B, et al. A randomized controlled trial of a community-based consultation service to prevent falls. CMAJ: Canadian Medical Association Journal 2001;165(5): 537-43. MEDLINE: 11563205

\section{Huang 2005 \{published data only\}}

Huang TT, Liang SH. A randomized clinical trial of the effectiveness of a discharge planning intervention in hospitalized elders with hip fracture due to falling. Journal of Clinical Nursing 2005;14(10):1193-201. MEDLINE: 16238765

Huang 2010 \{published data only\} Huang HC, Liu CY, Huang YT, Kernohan WG. Community-based interventions to reduce falls among older adults in Taiwan - long time follow-up randomised controlled study. Journal of Clinical Nursing 2010;19(7-8): 959-68.

\section{Huang 2011 \{published data only\}}

Huang TT, Wang WS. Comparison of three established measures of fear of falling in community-dwelling older adults: psychometric testing. International Journal of Nursing Studies 2009;46(10):1313-9.

* Huang TT, Yang LH, Liu CY. Reducing the fear of falling among community-dwelling elderly adults through cognitive-behavioural strategies and intense Tai Chi exercise: a randomized controlled trial. Journal of Advanced Nursing 2011; Vol. 67, issue 5:961-71.

Imhof 2012 \{published data only\} Imhof L, Naef R, Wallhagen MI, Schwarz J, Mahrer-Imhof $R$. Effects of an advanced practice nurse in-home health consultation program for community-dwelling persons aged 80 and older. Journal of the American Geriatrics Society 2012;60(12):2223-31. PUBMED: 23194103]

Jitapunkul 1998 \{published data only\} Jitapunkul S. A randomised controlled trial of regular surveillance in Thai elderly using a simple questionnaire administered by non-professional personnel. Journal of the Medical Association of Thailand 1998;81(5):352-6. MEDLINE: 9623035 
Kingston 2001 \{published data only\}

Kingston P, Jones M, Crome P. A RCT of health visitor (HV) intervention in falls. Age and Ageing 2001;30(Suppl 1): 40

* Kingston P, Jones M, Lally F, Crome P. Older people and falls: A randomized controlled trial of a health visitor (HV) intervention. Reviews in Clinical Gerontology 2001;11(3): 209-14. EMBASE: 2002061828]

Kingston PA. Older People and 'Falls' A Randomised Control Trial of Health Visitor Intervention [thesis]. Stoke-on-Trent

(UK): Keele University, 1998.

N0498009612. Elderly people and accidents: a prospective analysis of accidental causation among elderly populations and their post discharge requirements. National

Research Register (NRR) Archive. www.nihr.ac.uk/Pages/ NRRArchiveSearch.aspx (accessed 23 February 2011). NRR Publication ID: N0498009612]

Lightbody 2002 \{published data only\}

* Lightbody E, Watkins C, Leathley M, Sharma A, Lye M. Evaluation of a nurse-led falls prevention programme versus usual care: a randomized controlled trial. Age and Ageing 2002;31(3):203-10. MEDLINE: 12006310

N0500000414. Fallers attending casualty. National Research Register (NRR) Archive. www.nihr.ac.uk/Pages/ NRRArchiveSearch.aspx (accessed 23 February 2011). NRR Publication ID: N0500000414]

Logan 2010 \{published data only\}

ISRCTN10538608. Evaluation of the costs and benefits of computerised on-scene decision support for emergency ambulance personnel to assess and plan appropriate care for older people who have fallen: a randomised controlled trial. controlled-trials.com/ISRCTN10538608 (accessed 23 September 2011).

* Logan PA, Coupland CA, Gladman JR, Sahota O, Stoner-Hobbs V, Robertson K, et al. Community falls prevention for people who call an emergency ambulance after a fall: randomised controlled trial. BMJ 2010;340: c2102. MEDLINE: 20460331

N0171168738. An evaluation of the Primary Care falls prevention services for older fallers presenting to the ambulance service. National Research Register (NRR) Archive. www.nihr.ac.uk/Pages/NRRArchiveSearch.aspx (accessed 30 November 2011). NRR Publication ID: N0171168738]

Sach TH, Logan PA, Coupland CA, Gladman JR, Sahota $\mathrm{O}$, Stoner-Hobbs V, et al. Community falls prevention for people who call an emergency ambulance after a fall: an economic evaluation alongside a randomised controlled trial. Age and Ageing 2012; Vol. 41, issue 5:635-41. DOI: 10.1093/ageing/afs071

Lord 2005 \{published data only\}

Lord SR, Tiedemann A, Chapman K, Munro B, Murray SM, Gerontology M, et al. The effect of an individualized fall prevention program on fall risk and falls in older people: a randomized, controlled trial. Journal of the American Geriatrics Society 2005;53(8):1296-304. MEDLINE: 16078954
Luck 2013 \{published data only\}

Luck T, Motzek T, Luppa M, Matschinger H, Fleischer $S$, Sesselmann Y, et al. Effectiveness of preventive home visits in reducing the risk of falls in old age: a randomized controlled trial. Clinical Interventions in Aging 2013;8: 697-702. PUBMED: 23788832]

Markle-Reid 2010 \{published data only\}

* Markle-Reid M, Browne G, Gafni A, Roberts J, Weir R, Thabane $\mathrm{L}$, et al. The effects and costs of a multifactorial and interdisciplinary team approach to falls prevention for older home care clients 'at risk' for falling: a randomized controlled trial. Canadian Journal of Aging 2010;29(1): 139-61.

Markle-Reid M, Miles M, Vaitonis V, Henderson S, Browne Weir R, et al. From Isolation to Integration: the Effects and Costs of a Multifactorial and Interdisciplinary Team Approach to Falls Prevention for Frail Older Home Care Clients. Final Research Report. Edmonton: Canadian Patient Safety Institute, 2008.

NCT00463658. Interdisciplinary falls prevention for seniors. clinicaltrials.gov/show/NCT00463658 (accessed 31 August 2011).

Mendoza-Ruvalcaba 2015 \{published data only\} Mendoza-Ruvalcaba NM, Arias-Merino ED. "I am active": effects of a program to promote active aging. Clinical Interventions in Aging 2015;10:829-37. PUBMED: 26005337]

Metzelthin 2013 \{published data only\}

* Metzelthin SF, Van Rossum E, De Witte LP, Ambergen AW, Hobma SO, Sipers W, et al. Effectiveness of interdisciplinary primary care approach to reduce disability in community dwelling frail older people: cluster randomised controlled trial. BMJ (Clinical research ed.) 2013;347:f5264. PUBMED: 24022033]

Metzelthin SF, Van Rossum E, Hendriks MR, De Witte LP, Hobma SO, Sipers W, et al. Reducing disability in community-dwelling frail older people: cost-effectiveness study alongside a cluster randomised controlled trial. Age and Ageing 2015;44(3):390-6. PUBMED: 25566783]

\section{Möller 2014 \{published data only\}}

Möller UO, Kristensson J, Midlöv P, Ekdahl C, Jakobsson $\mathrm{U}$. Effects of a one-year home-based case management intervention on falls in older people: a randomized controlled trial. Journal of Aging and Physical Activity 2014; 22(4):457-64. PUBMED: 24152667]

\section{Neelemaat 2012 \{published data only\}}

* Neelemaat F, Bosmans JE, Thijs A, Seidell JC, Van Bokhorst-de van der Schueren MA. Post-discharge nutritional support in malnourished elderly individuals improves functional limitations. Journal of the American Medical Directors Association 2011;12(4):295-301. Neelemaat F, Lips P, Bosmans JE, Thijs A, Seidell JC, Van Bokhorst-de van der Schueren MA. Short-term oral nutritional intervention with protein and vitamin $\mathrm{D}$ decreases falls in malnourished older adults. Journal of 
the American Geriatrics Society 2012;60(4):691-9. DOI: 10.1111/j.1532-5415.2011.03888.x

Neelemaat F, Thijs A, Seidell JC, Bosmans JE, Van Bokhorst-de van der Schueren MA. Study protocol: Cost-effectiveness of transmural nutritional support in malnourished elderly patients in comparison with usual care. Nutrition Journal 2010;9(1):Article number: 6.

Newbury 2001 \{published data only\}

Newbury J, Marley J. Preventive home visits to elderly people in the community. Visits are most useful for people aged >75 [letter]. BMJ 2000;321(7529):512.

* Newbury JW, Marley JE, Beilby J. A randomised controlled trial of the outcome of health assessment of people aged 75 years and over. Medical Journal of Australia 2001;175(2):104-7. MEDLINE: 11556409

Ng 2015 \{published data only\}

Ng TP, Feng L, Nyunt MS, Feng L, Niti M, Tan BY, et al. Nutritional, physical, cognitive, and combination interventions and frailty reversal among older adults: a randomized controlled trial. American Journal of Medicine 2015;128(11):1225-36.e1. PUBMED: 26159634]

Olsen 2014 \{published data only\}

Bergland A, Thorsen H, Karesen R. Effect of exercise on mobility, balance, and health-related quality of life in osteoporotic women with a history of vertebral fracture: a randomized, controlled trial. Osteoporosis International 2011;22(6):1863-71. PUBMED: 21060992]

* Olsen CF, Bergland A. The effect of exercise and education on fear of falling in elderly women with osteoporosis and a history of vertebral fracture: results of a randomized controlled trial. Osteoporosis International 2014;25(8): 2017-25. PUBMED: 24807628]

Palvanen 2014 \{published data only\} ISRCTN48015966. The Chaos Clinic for prevention of falls and related injuries: a randomised, controlled trial. www.controlled-trials.com/ISRCTN48015966 (accessed 2 September 2011).

* Palvanen M, Kannus P, Piirtola M, Niemi S, Parkkari J, Jarvinen M. Effectiveness of the Chaos Falls Clinic in preventing falls and injuries of home-dwelling older adults: a randomised controlled trial. Injury 2014;45(1):265-71. PUBMED: 23579066]

Pardessus 2002 \{published data only\}

Pardessus V, Puisieux F, Di Pompeo C, Gaudefroy C, Thevenon A, Dewailly P. Benefits of home visits for falls and autonomy in the elderly: a randomized trial study. American Journal of Physical Medicine and Rehabilitation 2002;81(4):247-52. PUBMED: 11953541]

Rubenstein 2007 \{published and unpublished data\} Alessi C. Personal communication. 10 June 2007. Josephson K. Personal communication. 20 November 2007.

* Rubenstein LZ, Alessi CA, Josephson KR, Trinidad Hoyl M, Harker JO, Pietruszka FM. A randomized trial of a screening, case finding, and referral system for older veterans in primary care. Journal of the American Geriatrics Society 2007;55(2):166-74. MEDLINE: 17302651
Russell 2010 \{published data only\}

ACTRN12605000672651. A randomised controlled trial to reduce further falls and injuries for older fallers presenting to an Emergency Department. www.anzctr.org.au/ trial_view.aspx?ID=849 (accessed 14 September 2011). Hill K, Womer M, Russell M, Blackberry I, McGann A. Fear of falling in older fallers presenting at emergency departments. Journal of Advanced Nursing 2010;66(8): 1769-79.

NCT00217360. RCT to reduce further falls and injuries for older fallers presenting to an Emergency Department. clinicaltrials.gov/ct2/show/NCT00217360 (accessed 14 September 2011).

* Russell MA, Hill KD, Day LM, Blackberry I, Schwartz J, Giummarra MJ, et al. A randomized controlled trial of a multifactorial falls prevention intervention for older fallers presenting to Emergency Departments. Journal of the American Geriatrics Society 2010;58:2265-74.

Schrijnemaekers 1995 \{published data only\} Schrijnemaekers VJ, Haveman MJ. Effects of preventive outpatient geriatric assessment: short-term results of a randomized controlled study. Home Health Care Services Quarterly 1995;15(2):81-97. MEDLINE: 10143898

Serra-Prat 2017 \{published data only\} Serra-Prat M, Sist X, Domenich R, Jurado L, Saiz A, Roces A, et al. Effectiveness of an intervention to prevent frailty in pre-frail community-dwelling older people consulting in primary care: a randomised controlled trial. Age and Ageing 2017;46(3):401-7. PUBMED: 28064172]

\section{Sheffield 2013 \{published data only\}}

Sheffield C, Smith CA, Becker M. Evaluation of an agencybased occupational therapy intervention to facilitate aging in place. Gerontologist 2013;53(6):907-18. PUBMED: 23213082]

Shyu 2010 \{published data only\}

Shyu YI, Liang J, Wu CC, Cheng HS, Chen MC. An interdisciplinary intervention for older Taiwanese patients after surgery for hip fracture improves health-related quality of life. BMC Musculoskeletal Disorders 2010;11:225. MEDLINE: 20920220

Shyu YI, Liang J, Wu CC, Su JY, Cheng HS, Chou SW, et al. Interdisciplinary intervention for hip fracture in older Taiwanese: benefits last for 1 year. Journals of Gerontology Series A-Biological Sciences \& Medical Sciences 2008;63(1): 92-7. MEDLINE: 18245766

* Shyu YI, Liang J, Wu CC, Su JY, Cheng HS, Chou SW, et al. Two-year effects of interdisciplinary intervention for hip fracture in older Taiwanese. Journal of the American Geriatrics Society 2010;58(6):1081-9. MEDLINE: 20722845

\section{Sosnoff 2015 \{published data only\}}

Sosnoff JJ, Moon Y, Wajda DA, Finlayson ML, McAuley E, Peterson EW, et al. Fall risk and incidence reduction in high risk individuals with multiple sclerosis: a pilot randomized control trial. Clinical Rehabilitation 2015;29(10):952-60. PUBMED: 25540170] 
Spice 2009 \{published and unpublished data\}

George S, Spice C, Morotti W, Rose J, Harris S, Gordon C. The Winchester Falls Project: a cluster randomised community intervention trial of secondary prevention of falls in community dwelling older people. Journal of Epidemiology and Community Health 2006;60(Suppl 1): A17.

N0278078805. The Winchester Falls Project: A randomised controlled trial of multidisciplinary assessment in the secondary prevention of falls. National Research Register (NRR) Archive. www.nihr.ac.uk/Pages/ NRRArchiveSearch.aspx (accessed 23 February 2011). NRR Publication ID: N0278078805]

NCT00130624. The Winchester Falls Project: A cluster randomised community intervention trial of secondary prevention of falls in community-dwelling older people. clinicaltrials.gov/show/NCT00130624 (accessed 30 November 2011)

Spice C. Personal communication. 24 December 2006. Spice C, Morotti W, Dent T, George S, Rose J, Gordon C. The Winchester Falls Project: A randomised controlled trial of secondary falls prevention. Age and Ageing 2005;34 (Suppl 2):ii18.

* Spice C, Morotti W, George S, Dent T, Rose J, Harris S, et al. The Winchester falls project: a randomised controlled trial of secondary prevention of falls in older people. Age and Ageing 2009; Vol. 38, issue 1:33-40. MEDLINE: 18829689

\section{Tinetti 1994 \{published data only\}}

King MB, Tinetti ME. A multifactorial approach to reducing injurious falls. Clinics in Geriatric Medicine 1996; 12(4):745-59. MEDLINE: 8890114

Koch M, Gottschalk M, Baker DI, Palumbo S, Tinetti ME. An impairment and disability assessment and treatment protocol for community-living elderly persons. Physical Therapy 1994;74:286-94; discussion 295-8.

Rizzo JA, Baker DI, McAvay G, Tinetti ME. The costeffectiveness of a multifactorial targeted prevention program for falls among community elderly persons. Medical Care 1996;34(9):954-69. MEDLINE: 8792783

Tinetti ME. Prevention of falls and fall injuries in elderly persons: a research agenda. Preventive Medicine 1994;23(5): 756-62. MEDLINE: 7845954

Tinetti ME, Baker DI, Garrett PA, Gottschalk M, Koch ML, Horwitz RI. Yale FICSIT: risk factor abatement strategy for fall prevention. Journal of the American Geriatrics Society 1993;41:315-20. MEDLINE: 8440856

* Tinetti ME, Baker DI, McAvay G, Claus EB, Garrett P, Gottschalk $\mathrm{M}$, et al. A multifactorial intervention to reduce the risk of falling among elderly people living in the community. New England Journal of Medicine 1994;331 (13):821-7. MEDLINE: 8078528

Tinetti ME, McAvay G, Claus E. Does multiple risk factor reduction explain the reduction in fall rate in the Yale FICSIT Trial? Frailty and Injuries Cooperative Studies of Intervention Techniques. American Journal of Epidemiology 1996;144(4):389-99. MEDLINE: 8712196
Ueda 2017 \{published data only\}

Ueda T, Higuchi Y, Imaoka M, Todo E, Kitagawa T, Ando $\mathrm{S}$. Tailored education program using home floor plans for falls prevention in discharged older patients: A pilot randomized controlled trial. Archives of Gerontology and Geriatrics 2017;71:9-13. PUBMED: 28242580]

Uusi-Rasi 2015 \{published data only\}

NCT00986466. Vitamin D and exercise in falls prevention (DEX). clinicaltrials.gov/show/NCT00986466 (accessed 31 August 2011)

Patil R, Kolu P, Raitanen J, Valvanne J, Kannus P, Karinkanta $\mathrm{S}$, et al. Cost-effectiveness of vitamin $\mathrm{D}$ supplementation and exercise in preventing injurious falls among older home-dwelling women: findings from an RCT. Osteoporosis International 2016;27(1):193-201. PUBMED: 26205890] Patil R, Uusi-Rasi K, Tokola K, Karinkanta S, Kannus P, Sievanen H. Effects of a multimodal exercise program on physical function, falls, and injuries in older women: A 2year community-based, randomized controlled trial. Journal of the American Geriatrics Society 2015;63(7):1306-13. PUBMED: 26115073]

Uusi-Rasi K, Kannus P, Karinkanta S, Pasanen M, Patil R, Lamberg-Allardt C, et al. Study protocol for prevention of falls: a randomized controlled trial of effects of vitamin D and exercise on falls prevention. BMC Geriatrics 2012;12: 12. PUBMED: 22448872]

Uusi-Rasi K, Patil R, Karinkanta S, Kannus P, Tokola K, Lamberg-Allardt C, et al. A 2-year follow-up after a 2-year RCT with vitamin D and exercise: effects on falls, injurious falls and physical functioning among older women. Journals of Gerontology. Series A, Biological Sciences and Medical Sciences 2017;72(9):1239-45. PUBMED: 28369286]

* Uusi-Rasi K, Patil R, Karinkanta S, Kannus P, Tokola K, Lamberg-Allardt C, et al. Exercise and vitamin D in fall prevention among older women: a randomized clinical trial. JAMA Internal Medicine 2015;175(5):703-11. PUBMED: 25799402]

Van Haastregt 2000 \{published data only\}

* Van Haastregt JC, Diederiks JP, Van Rossum E, De Witte LP, Voorhoeve PM, Crebolder HF. Effects of a programme of multifactorial home visits on falls and mobility impairments in elderly people at risk: randomised controlled trial. BMJ 2000;321(7267):994-8. MEDLINE: 11039967

Van Haastregt JC, Van Rossum E, Diederiks JP, De Witte LP, Voorhoeve PM, Crebolder HF. Process-evaluation of a home visit programme to prevent falls and mobility impairments among elderly people at risk. Patient Education and Counseling 2002;47(4):301-9. MEDLINE: 12135821 Van Haastregt JC, Van Rossum E, Diederiks JP, Voorhoeve PM, De Witte LP, Crebolder HF. Preventing falls and mobility problems in community-dwelling elders: the process of creating a new intervention. Geriatric Nursing 2000;21(6):309-14. MEDLINE: 11135129

Van Rossum 1993 \{published data only\} Van Rossum E, Frederiks CM, Philipsen H, Portengen $\mathrm{K}$, Wiskerke J, Knipschild P. Effects of preventive home 
visits to elderly people. BMJ 1993;307(6895):27-32. MEDLINE: 8343668

\section{Vetter 1992 \{published data only\}}

Vetter NJ, Lewis PA, Ford D. Can health visitors prevent fractures in elderly people?. BMJ 1992;304(6831):888-90. MEDLINE: 1392755

\section{Vind 2009 \{published data only\}}

NCT00226486. Examination and treatment of elderly after a fall. clinicaltrials.gov/show/NCT00226486 (accessed 31 August 2011).

Vind AB. Personal communication. 30 March 2006. Vind $\mathrm{AB}$, Andersen HE, Pedersen KD, Joergensen T, Schwarz P. Effect of a program of multifactorial fall prevention on health-related quality of life, functional ability, fear of falling and psychological well-being. A randomized controlled trial. Aging - Clinical and Experimental Research 2010;22(3):249-54.

* Vind AB, Andersen HE, Pedersen KD, Jorgensen T, Schwarz P. An outpatient multifactorial falls prevention intervention does not reduce falls in high-risk elderly Danes. Journal of the American Geriatrics Society 2009;57(6):971-7. MEDLINE: 19507291

Vind AB, Andersen HE, Pedersen KD, Jorgensen T, Schwarz P. Baseline and follow-up characteristics of participants and nonparticipants in a randomized clinical trial of multifactorial fall prevention in Denmark. Journal of the American Geriatrics Society 2009;57(10):1844-9. MEDLINE: 19682128

Wagner 1994 \{published data only\}

Wagner EH, LaCroix AZ, Grothaus L, Leveille SG, Hecht JA, Artz K, et al. Preventing disability and falls in older adults: a population-based randomized trial. American Journal of Public Health 1994;84(11):1800-6. MEDLINE: 7977921

Waterman 2016 \{published data only\} Waterman H, Ballinger C, Brundle C, Chastin S, Gage H, Harper R, et al. A feasibility study to prevent falls in older people who are sight impaired: the VIP2UK randomised controlled trial. Trials 2016;17(1):464. PUBMED: 27671540]

Wesson 2013 \{published data only\}

Wesson J, Clemson L, Brodaty H, Lord S, Taylor M, Gitlin $\mathrm{L}$, et al. A feasibility study and pilot randomised trial of a tailored prevention program to reduce falls in older people with mild dementia. BMC Geriatrics 2013;13:89. PUBMED: 24004682]

Whitehead 2003 \{published data only\} Whitehead C, Wundke R, Crotty M, Finucane P. Evidencebased clinical practice in falls prevention: a randomised controlled trial of a falls prevention service. Australian Health Review 2003;26(3):88-96. MEDLINE: 15368824

Wilder 2001 \{published data only\}

Wilder P. Seniors to seniors exercise program: a cost effective way to prevent falls in the frail elderly living at home. Journal of Geriatric Physical Therapy 2001;24(3):13.
Zijlstra 2009 \{published data only\}

Van Haastregt JC, Zijlstra GA, Hendriks MR, Goossens ME, Van Eijk JT, Kempen GI. Cost-effectiveness of an intervention to reduce fear of falling. International Journal of Technology Assessment in Health Care 2013;29(3):219-26. PUBMED: 23778198]

Zijlstra G, Van Haastregt JC, Van Eijk JT, Kempen GI. Evaluating an intervention to reduce fear of falling and associated activity restriction in elderly persons: design of a randomised controlled trial [ISRCTN43792817]. BMC Public Health 2005;5:26. PUBMED: 15780139] * Zijlstra GA, Van Haastregt JC, Ambergen T, Van Rossum E, Van Eijk JT, Tennstedt SL, et al. Effects of a multicomponent cognitive behavioral group intervention on fear of falling and activity avoidance in communitydwelling older adults: results of a randomized controlled trial. Journal of the American Geriatrics Society 2009;57(11): 2020-8. PUBMED: 19793161]

\section{References to studies excluded from this review}

\section{ACTRN12610000838011 \{published data only\}}

ACTRN12610000838011. In community-dwelling older people who have fallen, does the provision of falls prevention interventions and osteoporosis treatment through a specialist Falls and Bone service prevent more falls than care coordinated by General Practitioners. www.anzctr.org.au/ trial_view.aspx?ID=335807 (accessed 30 November 2011)

Alexander 2003 \{published data only\}

Alexander N. Personal communication. 23 August 2006. * Alexander NB, Bentur N, Strasburg D, Nyquist LV. Fall risk reduction in Israeli day care center attendees using exercise and behavior strategies. Journal of the American Geriatrics Society 2003;51(Suppl 4):S117.

Assantachai 2002 \{published and unpublished data\} Assantachai P. Personal communication. 11 June 2007. * Assantachai P, Chatthanawaree W, Thamlikitkul V, Praditsuwan R, Pisalsarakij D. Strategy to prevent falls in the Thai elderly: a controlled study integrated health research program for the Thai elderly. Journal of the Medical Association of Thailand 2002;85(2):215-22. MEDLINE: 12081122

\section{Bruce 2016 \{published data only\}}

* Bruce J, Lall R, Withers EJ, Finnegan S, Underwood M, Hulme C, et al. A cluster randomised controlled trial of advice, exercise or multifactorial assessment to prevent falls and fractures in community-dwelling older adults: protocol for the prevention of falls injury trial (PreFIT). BMJ Open 2016;6(1):e009362. PUBMED: 26781504]

Bruce J, Ralhan S, Sheridan R, Westacott K, Withers E, Finnegan $S$, et al. The design and development of a complex multifactorial falls assessment intervention for falls prevention: The Prevention of Falls Injury Trial (PreFIT). BMC Geriatrics 2017;17(1):116. PUBMED: 28571563]

Chu 2017 \{published data only\} Chu MM, Fong KN, Lit AC, Rainer TH, Cheng SW, Au FL, et al. An occupational therapy fall reduction home visit 
program for community-dwelling older adults in Hong Kong after an emergency department visit for a fall. Journal of the American Geriatrics Society 2017;65(2):364-72. PUBMED: 27858951]

Clemson 2012 \{published data only\} ACTRN12606000025538. Lifestyle (LIFE) versus structured balance and strength training to reduce falls in the elderly: a randomized trial. www.anzctr.org.au/ trial_view.aspx?ID=1007 (accessed 15 June 2012). * Clemson L, Fiatarone Singh MA, Bundy A, Cumming RG, Manollaras K, O'Loughlin P, et al. Integration of balance and strength training into daily life activity to reduce rate of falls in older people (the LiFE study): randomised parallel trial. BMJ 2012; Vol. 345, issue 7870: Article Number: e4547.

\section{Cockayne 2014 \{published data only\}}

Cockayne S, Adamson J, Corbacho Martin B, Fairhurst C, Hewitt C, Hicks K, et al. The REFORM study protocol: a cohort randomised controlled trial of a multifaceted podiatry intervention for the prevention of falls in older people. BMJ Open 2014;4(12):e006977. PUBMED: 25518875]

Cohen 2015 \{published data only\} Cohen MA, Miller J, Shi X, Sandhu J, Lipsitz LA. Prevention program lowered the risk of falls and decreased claims for long-term services among elder participants. Health Affairs 2015;34(6):971-7. PUBMED: 26056202]

Comans 2010 \{published data only\} ACTRN12605000056695. Domiciliary versus centre-based rehabilitation of older community dwellers: Randomised trial with economic evaluation. www.anzctr.org.au/ trial_view.aspx?ID=52 (accessed 30 November 2011). Comans TA, Brauer S, Haines T. Domiciliary vs centrebased rehabilitation of older community dwellers: randomised trial with economic evaluation. Open Geriatric Medicine Journal 2008;1(6):62-7.

* Comans TA, Brauer SG, Haines TP. Randomized trial of domiciliary versus center-based rehabilitation: which is more effective in reducing falls and improving quality of life in older fallers?. Journals of Gerontology Series A-Biological Sciences and Medical Sciences 2010;65(6):672-9.

Comans TA, Currin ML, Brauer SG, Haines TP. Factors associated with quality of life and caregiver strain amongst frail older adults referred to a community rehabilitation service: implications for service delivery. Disability and Rehabilitation 2011;33(13-14):1215-21.

Conroy 2010 \{published data only\}

* Conroy S, Kendrick D, Harwood R, Gladman J, Coupland C, Sach T, et al. A multicentre randomised controlled trial of day hospital-based falls prevention programme for a screened population of communitydwelling older people at high risk of falls. Age and Ageing 2010;39(6):704-10.

Conroy S, Morris R, Masud T. Multifactorial day hospital intervention to reduce falls in high risk older people in primary care: a multi-centre randomised controlled trial.
ProFaNE (Prevention of Falls Network Europe) meeting; 2004 June 11-13; Manchester (UK). 2004.

ISRCTN46584556. Multifactorial day hospital intervention to reduce falls in high risk older people in primary care: a multi-centre randomised controlled trial. controlled-trials.com/ISRCTN46584556 (accessed 14 September 2011).

Irvine L, Conroy SP, Sach T, Gladman JR, Harwood RH, Kendrick D, et al. Cost-effectiveness of a day hospital falls prevention programme for screened community-dwelling older people at high risk of falls. Age and Ageing 2010;39 (6):710-6.

Masud T, Coupland C, Drummond A, Gladman J, Kendrick D, Sach T, et al. Multifactorial day hospital intervention to reduce falls in high risk older people in primary care: a multi-centre randomised controlled trial [ISRCTN46584556]. Trials 2006;7:5-10.

De Negreiros 2013 \{published data only\}

De Negreiros Cabral K, Perracini MR, Soares AT, De Cristo Stein F, Sera CT, Tiedemann A, et al. Effectiveness of a multifactorial falls prevention program in communitydwelling older people when compared to usual care: study protocol for a randomised controlled trial (Prevquedas Brazil). BMC Geriatrics 2013;13:27. PUBMED: 23497000]

Di Monaco 2008 \{published data only\} Di Monaco M, Vallero F, De Toma E, De Lauso L, Tappero $\mathrm{R}$, Cavanna A. A single home visit by an occupational therapist reduces the risk of falling after hip fracture in elderly women: a quasi-randomized controlled trial. Journal of Rehabilitation Medicine 2008;40(6):446-50. MEDLINE: 18509559

Fox 2010 \{published data only\}

Fox PJ, Vazquez L, Tonner C, Stevens JA, Fineman N, Ross LK. A randomized trial of a multifaceted intervention to reduce falls among community-dwelling adults. Health Education and Behavior 2010;37(6):831-48.

Gianoudis 2014 \{published data only\} Gianoudis J, Bailey CA, Ebeling PR, Nowson CA, Sanders $\mathrm{KM}$, Hill $\mathrm{K}$, et al. Effects of a targeted multimodal exercise program incorporating high-speed power training on falls and fracture risk factors in older adults: a community-based randomized controlled trial. Journal of Bone and Mineral Research 2014;29(1):182-91. PUBMED: 23775701]

Gill 2008 \{published and unpublished data\}

* Gill DP, Zou GY, Jones GR, Speechley M. Injurious falls are associated with lower household but higher recreational physical activities in community-dwelling older male veterans. Gerontology 2008;54(2):106-15. MEDLINE: 18259094

Speechley M. Falls data (as supplied 03 June 2008). Data on file.

\section{Giordano 2016 \{published data only\}}

Giordano A, Bonometti GP, Vanoglio F, Paneroni M, Bernocchi P, Comini L, et al. Feasibility and costeffectiveness of a multidisciplinary home-telehealth intervention programme to reduce falls among elderly 
discharged from hospital: study protocol for a randomized controlled trial. BMC Geriatrics 2016;16(1):209.

PUBMED: 27923343]

\section{Hill 2000 \{published data only\}}

Crome P. Personal communication. 29 August 2006 Crome P, Hill S, Mossman J, Stockdale P. A randomised controlled trial of a nurse led falls prevention clinic. Journal of the American Geriatrics Society 2000;48(8):S78.

* Hill S, Mossman J, Stockdale P, Crome P. A randomised controlled trial of a nurse-led falls prevention clinic. Age and Ageing 2000;29(Suppl 2):20.

Hornbrook 1994 \{published data only\} Hornbrook MC, Stevens VJ, Wingfield DJ. Seniors' program for injury control and education. Journal of the American Geriatrics Society 1993;41(3):309-14. MEDLINE: 8440855

* Hornbrook MC, Stevens VJ, Wingfield DJ, Hollis JF, Greenlick MR, Ory MG. Preventing falls among community-dwelling older persons: results from a randomized trial. Gerontologist 1994;34(1):16-23. MEDLINE: 8150304

Stevens VJ, Hornbrook MC, Wingfield DJ, Hollis JF, Greenlick MR, Ory MG. Design and implementation of a falls prevention intervention for community-dwelling older persons. Behavior, Health, and Aging 1991/92;2(1):57-73.

Huang 2004 \{published data only\}

Huang TT, Acton GJ. Effectiveness of home visit falls prevention strategy for Taiwanese community-dwelling elders: randomized trial. Public Health Nursing 2004;21(3): 247-56. MEDLINE: 15144369

Lamb 2010 \{published data only\}

Bruce J, Lall R, Withers EJ, Finnegan S, Underwood M, Hulme C, et al. A cluster randomised controlled trial of advice, exercise or multifactorial assessment to prevent falls and fractures in community-dwelling older adults: protocol for the prevention of falls injury trial (PreFIT). BMJ Open 2016;6(1):e009362. PUBMED: 26781504]

ISRCTN71002650. Prevention of Fall Injury Trial: a parallel group cluster randomised controlled trial and economic evaluation. www.controlled-trials.com/ ISRCTN71002650 (accessed 22 Sept 2010).

Lamb S. Prevention of fall related injuries trial (Pre-FIT). www.hta.ac.uk/2146 (accessed 22 Sept 2010).

Lee 2013 \{published data only\}

Lee HC, Chang KC, Tsauo JY, Hung JW, Huang YC, Lin SI. Effects of a multifactorial fall prevention program on fall incidence and physical function in community-dwelling older adults with risk of falls. Archives of Physical Medicine and Rehabilitation 2013;94(4):606-15, 615.e1. PUBMED: 23220343]

Mahoney 2007 \{published data only\}

Gleason CE, Gangnon RE, Fischer BL, Mahoney JE. Increased risk for falling associated with subtle cognitive impairment: secondary analysis of a randomized clinical trial. Dementia and Geriatric Cognitive Disorders 2009;27 (6):557-63. MEDLINE: 19602883

* Mahoney JE, Shea TA, Przybelski R, Jaros L, Gangnon $\mathrm{R}$, Cech S, et al. Kenosha County falls prevention study: a randomized, controlled trial of an intermediate-intensity, community-based multifactorial falls intervention. Journal of the American Geriatrics Society 2007;55(4):489-98. MEDLINE: 17397425

Matchar 2017 \{published data only\}

Matchar DB, Duncan PW, Lien CT, Ong MEH, Lee M, Gao F, et al. Randomized controlled trial of screening, risk modification, and physical therapy to prevent falls among the elderly recently discharged from the emergency department to the community: the steps to avoid falls in the elderly study. Archives of Physical Medicine and Rehabilitation 2017;98(6):1086-96. PUBMED: 28202383]

Mikolaizak 2017 \{published data only\}

Mikolaizak AS, Lord SR, Tiedemann A, Simpson P, Caplan GA, Bendall J, et al. A multidisciplinary intervention to prevent subsequent falls and health service use following fall-related paramedic care: a randomised controlled trial. Age and Ageing 2017;46(2):200-7. PUBMED: 28399219]

NCT00126152 \{published data only\}

NCT00126152. Senior Falls Prevention Study. clinicaltrials.gov/show/NCT00126152 (accessed 13 June 2017).

\section{NCT00483275 \{published data only\}} NCT00483275. Fall prevention by alfacalcidol and training (SPALT). clinicaltrials.gov/show/NCT00483275 (accessed 23 February 2011).

Perula 2012 \{published data only\} Perula LA, Varas-Fabra F, Rodriguez V, Ruiz-Moral R, Fernandez JA, Gonzalez J, et al. Effectiveness of a multifactorial intervention program to reduce falls incidence among community-living older adults: a randomized controlled trial. Archives of Physical Medicine and Rehabilitation 2012;93(10):1677-84. PUBMED: 22609117]

Salminen 2009 \{published and unpublished data\} Kivela S-L, Aarnio P, Asikainen E, Hyttinen H, Isoaho R, Karra E, et al. Prevention of injurious falls and fractures in ageing and aged population. ProFaNE (Prevention of Falls Network Europe) meeting; 2004 June 11-13; Manchester (UK). 2004.

NCT00247546. Prevention of falls and injurious falls among elderly people. clinicaltrials.gov/ct $2 /$ show/ NCT00247546 (accessed 14 September 2011).

Salminen M, Vahlberg T, Kivela SL. The long-term effect of a multifactorial fall prevention programme on the incidence of falls requiring medical treatment. Public Health 2009; 123(12):809-13. MEDLINE: 19958918 Salminen M, Vahlberg T, Sihvonen S, Piirtola M, Isoaho R, Aarnio P, et al. Effects of risk-based multifactorial fall prevention program on maximal isometric muscle strength in community-dwelling aged: a randomized controlled 
trial. Aging-Clinical and Experimental Research 2008;20(5): 487-93. MEDLINE: 19039292

Salminen M, Vahlberg T, Sihvonen S, Sjosten N, Piirtola $\mathrm{M}$, Isoaho $\mathrm{R}$, et al. Effects of risk-based multifactorial fall prevention on postural balance in the community-dwelling aged: a randomized controlled trial. Archives of Gerontology and Geriatrics 2009;48(1):22-7. MEDLINE: 17950944

* Salminen MJ, Vahlberg TJ, Salonoja MT, Aarnio PT, Kivela SL. Effect of a risk-based multifactorial fall prevention program on the incidence of falls. Journal of the American Geriatrics Society 2009;57(4):612-9. MEDLINE: 19392952

Salminen MJ, Vahlberg TJ, Salonoja MT, Aarnio PT, Kivelä S-L. Falls data (as supplied 20 May 2008). Data on file. Salonoja M, Salminen M, Aarnio P, Vahlberg T, Kivela SL. One-time counselling decreases the use of benzodiazepines and related drugs among community-dwelling older persons. Age and Ageing 2010;39(3):313-9. MEDLINE: 20089547

Salonoja M, Salminen M, Vahlberg T, Aarnio P, Kivela S-L. Withdrawal of psychotropic drugs decreases the risk of falls requiring treatment. Archives of Gerontology and Geriatrics 2011;54(1):160-7.

Sjosten NM, Salonoja M, Piirtola M, Vahlberg T, Isoaho $\mathrm{R}$, Hyttinen $\mathrm{H}$, et al. A multifactorial fall prevention programme in home-dwelling elderly people: A randomized-controlled trial. Public Health 2007;121(4): 308-18. MEDLINE: 17320125

Sjosten NM, Salonoja M, Piirtola M, Vahlberg TJ, Isoaho $\mathrm{R}$, Hyttinen $\mathrm{HK}$, et al. A multifactorial fall prevention programme in the community-dwelling aged: predictors of adherence. European Journal of Public Health 2007;17(5): 464-70. MEDLINE: 17208952

Sjosten NM, Vahlberg TJ, Kivela S-L. The effects of multifactorial fall prevention on depressive symptoms among the aged at increased risk of falling. International Journal of Geriatric Psychiatry 2008;23(5):504-10. EMBASE: 2008251008]

Vaapio S, Salminen M, Vahlberg T, Kivela SL. Increased muscle strength improves managing in activities of daily living in fall-prone community-dwelling older women. Aging-Clinical and Experimental Research 2011;23(1):42-8. Vaapio S, Salminen M, Vahlberg T, Sjosten N, Isoaho R, Aarnio P, et al. Effects of risk-based multifactorial fall prevention on health-related quality of life among the community-dwelling aged: a randomized controlled trial. Health and Quality of Life Outcomes 2007;5:20. MEDLINE: 17462083

\section{Shaw 2003 \{published data only\}}

Dawson P, Chapman KL, Shaw FE, Kenny RA. Measuring the outcome of physiotherapy in cognitively impaired elderly patients who fall. Physiotherapy 1997;83(7):352. N0461021713. Physiotherapy intervention for cognitively impaired elderly fallers attending casualty. National Research Register (NRR) Archive. www.nihr.ac.uk/Pages/ NRRArchiveSearch.aspx (accessed 30 November 2011).
NRR Publication ID: N0461021713]

N0461044514. Risk modification of falls in cognitively impaired elderly patients attending a casualty department. A randomised controlled explanatory study. National Research Register (NRR) Archive. www.nihr.ac.uk/Pages/ NRRArchiveSearch.aspx (accessed 30 November 2011). NRR Publication ID: N0461044514]

* Shaw FE, Bond J, Richardson DA, Dawson P, Steen IN McKeith IG, et al. Multifactorial intervention after a fall in older people with cognitive impairment and dementia presenting to the accident and emergency department: randomised controlled trial. BMJ 2003;326(7380):73-5. Shaw FE, Richardson DA, Dawson P, Steen IN, McKeith IG, Bond J, et al. Can multidisciplinary intervention prevent falls in patients with cognitive impairment and dementia attending a casualty department. Age and Ageing 2000;29(Suppl 1):47.

\section{Sherrington 2014 \{published data only\}}

ACTRN12607000563460. Minimising disability and falls in older people through a post-hospital individualised exercise program. www.anzctr.org.au/trial_view.aspx?ID= 82384 (accessed 2 September 2011).

* Sherrington C, Lord SR, Vogler CM, Close JC, Howard $\mathrm{K}$, Dean CM, et al. A post-hospital home exercise program improved mobility but increased falls in older people: a randomised controlled trial. PloS One 2014;9(9):e104412. PUBMED: 25180702]

Sherrington C, Lord SR, Vogler CM, Close JC, Howard $\mathrm{K}$, Dean CM, et al. Minimising disability and falls in older people through a post-hospital exercise program: a protocol for a randomised controlled trial and economic evaluation. BMC Geriatrics. England: Musculoskeletal Division, The George Institute for International Health, The University of Sydney, Sydney, NSW, Australia. csherrington@george.org.au, 2009; Vol. 9:8. MEDLINE: 19245697

\section{Shumway-Cook 2007 \{published data only\}}

Shumway-Cook A, Silver I, Mary L, York S, Cummings P, Koepsell T. The effectiveness of a community-based multifactorial intervention on falls and fall risk factors in community living older adults: a randomized, controlled trial. CSM 2007. Journal of Geriatric Physical Therapy 2006;29(3):117.

* Shumway-Cook A, Silver IF, LeMier M, York S, Cummings P, Koepsell TD. Effectiveness of a communitybased multifactorial intervention on falls and fall risk factors in community-living older adults: a randomized, controlled trial. Journals of Gerontology. Series A, Biological Sciences and Medical Sciences 2007; Vol. 62, issue 12:1420-7. MEDLINE: 18166695

\section{Snooks 2010 \{published data only\}} ISRCTN10538608. SAFER (Support and Assessment for Fall Emergency Referrals) Trial: Evaluation of the costs and benefits of computerised on-scene decision support for emergency ambulance personnel to assess and plan appropriate care for older people who have fallen: a 
randomised controlled trial. www.controlled-trials.com/ ISRCTN10538608 (accessed 2 September 2011).

* Snooks H, Cheung WY, Close J, Dale J, Gaze S, Humphreys I, et al. Support and Assessment for Fall Emergency Referrals (SAFER 1) trial protocol. Computerised on-scene decision support for emergency ambulance staff to assess and plan care for older people who have fallen: evaluation of costs and benefits using a pragmatic cluster randomised trial. BMC Emergency Medicine 2010;10:2.

Thomas G, Sanchez A, Snooks H. Before the fall: A discussion paper on the organisational difficulties of setting up complex randomised control trials in emergency prehospital care. Emergency Medicine Journal 2011;28(3):2.

\section{Snooks 2017 \{published data only\}}

Care of older people who fall: evaluation of the clinical and cost effectiveness of new protocols for emergency ambulance personnel to assess and refer to appropriate community based care. www.hta.ac.uk/1802 (accessed 2 May 2011). Gaze S. SAFER 2: Support and assessment for fall emergency referrals (part of the SAFER research programme. Emergency Medicine Journal 2011;28(3):3.

ISRCTN60481756. Care of older people who fall: evaluation of the clinical and cost effectiveness of new protocols for emergency ambulance personnel to assess and refer to appropriate community based care - a cluster randomised controlled trial. www.controlled-trials.com/ ISRCTN60481756 (accessed 2 May 2011). * Snooks HA, Anthony R, Chatters R, Dale J, Fothergill $\mathrm{R}$, Gaze $\mathrm{S}$, et al. Support and Assessment for Fall Emergency Referrals (SAFER) 2: a cluster randomised trial and systematic review of clinical effectiveness and costeffectiveness of new protocols for emergency ambulance paramedics to assess older people following a fall with referral to community-based care when appropriate. Health Technology Assessment 2017;21(13):1-218. PUBMED: 28397649]

\section{Spink 2011 \{published data only\}}

ACTRN12608000065392. Podiatry treatment to improve balance and prevent falls in older people. www.anzctr.org.au/trial_view.aspx?ID=82558 (accessed 30 November 2011).

Spink MJ, Fotoohabadi MR, Wee E, Landorf KB, Hill KD, Lord SR, et al. Predictors of adherence to a multifaceted podiatry intervention for the prevention of falls in older people. BMC Geriatrics 2011;11:51.

* Spink MJ, Menz HB, Fotoohabadi MR, Wee E, Landorf $\mathrm{KB}$, Hill KD, et al. Effectiveness of a multifaceted podiatry intervention to prevent falls in community dwelling older people with disabling foot pain: randomised controlled trial. BMJ 2011;342:d3411.

Spink MJ, Menz HB, Lord SR. Efficacy of a multifaceted podiatry intervention to improve balance and prevent falls in older people: study protocol for a randomised trial. $B M C$ Geriatrics 2008;8:30. MEDLINE: 19025668
Steinberg 2000 \{published and unpublished data\}

Peel N. Personal communication. 10 October 2007. Peel N, Cartwright C, Steinberg M. Monitoring slips, trips and falls in the older community: preliminary results. Health Promotion Journal of Australia 1998;8(2):148-50. Peel N, Steinberg M, Williams G. Home safety assessment in the prevention of falls among older people. Australian and New Zealand Journal of Public Health 2000;24(5): 536-9. MEDLINE: 11109693

* Steinberg M, Cartwright C, Peel N, Williams G. A sustainable programme to prevent falls and near falls in community dwelling older people: results of a randomised trial. Journal of Epidemiology and Community Health 2000; 54(3):227-32. MEDLINE: 10746118

Suman 2011 \{published data only\}

Suman S, Myint PK, Clark A, Das P, Ring L, Trepte NJ. Community-based fall assessment compared with hospitalbased assessment in community-dwelling older people over 65 at high risk of falling: a randomized study. AgingClinical and Experimental Research 2011;23(1):35-41.

Swanenburg 2007 \{published data only\}

Swanenburg J, De Bruin ED, Stauffacher M, Mulder T, Uebelhart D. Effects of exercise and nutrition on postural balance and risk of falling in elderly people with decreased bone mineral density: randomized controlled trial pilot study. Clinical Rehabilitation 2007;21(6):523-34. MEDLINE: 17613583

Tiedemann 2015 \{published data only\}

Tiedemann A, Paul S, Ramsay E, O'Rourke SD Chamberlain K, Kirkham C, et al. What is the effect of a combined physical activity and fall prevention intervention enhanced with health coaching and pedometers on older adults' physical activity levels and mobility-related goals? Study protocol for a randomised controlled trial. BMC Public Health 2015;15:477. PUBMED: 25956926]

Von Stengel 2011 \{published and unpublished data\} Kemmler W, Von Stengel S, Mayer S, Niedermayer M, Hentschke C, Kalender WA. Effect of whole body vibration on the neuromuscular performance of females 65 years and older. One-year results of the controlled randomized ELVIS study [Effekte von ganzkorpervibrationen auf die neuromuskulare leistungsfahigkeit von frauen uber dem 65. Lebensjahr. Einjahresergebnisse der kontrollierten randomisierten ELVIS-Studie]. Zeitschrift fur Gerontologie und Geriatrie 2010;43(2):125-32. MEDLINE: 19789832 NCT00292916. Effect of whole body vibration on bone and fall related parameters. clinicaltrials.gov/show/ NCT00292916 (accessed 31 August 2011).

Von Stengel S, Kemmler W, Engelke K, Bebenek M, Mayhew JL, Kalender WA. Effects of whole body vibration training on the fracture risk of postmenopausal women: preliminary results of the Erlangen Longitudinal Vibration Study (ELVIS). Medicine and Science in Sports and Exercise 2008;40(5 Suppl 1):S313.

Von Stengel S, Kemmler W, Engelke K, Kalender WA. Effect of whole-body vibration on neuromuscular performance and body composition for females 65 years 
and older: a randomized-controlled trial. Scandinavian Journal of Medicine \& Science in Sports 2012;22(1):119-27. MEDLINE: 20500555

* Von Stengel S, Kemmler W, Engelke K, Kalender WA. Effects of whole body vibration on bone mineral density and falls: results of the randomized controlled ELVIS study with postmenopausal women. Osteoporosis International 2011;22(1):317-25. MEDLINE: 20306017

Von Stengel S, Kemmler W, Mayer S, Engelke K, Klarner A, Kalender WA. Effect of whole body vibration exercise on osteoporotic risk factors [Effekte eines ganzkorpervibrationstrainings auf parameter des frakturrisikos]. Deutsche Medizinische Wochenschrift 2009; 134(30):1511-6. MEDLINE: 19603365

Wyman 2005 \{published and unpublished data\} Findorff MJ, Stock HH, Gross CR, Wyman JF. Does the Transtheoretical Model (TTM) explain exercise behavior in a community-based sample of older women?. Journal of Aging and Health 2007;19(6):985-1003. MEDLINE: 18165292

Findorff MJ, Wyman JF, Nyman JA, Croghan CF. Measuring the direct healthcare costs of a fall injury event. Nursing Research 2007;56(4):283-7. MEDLINE: 17625468

Lindquist R, Wyman JF, Talley KM, Findorff M, Gross CR. Design of control-group conditions in clinical trials of behavioral interventions. Journal of Nursing Scholarship 2007;39(3):214-21. MEDLINE: 17760793

Nachreiner NM, Findorff MJ, Wyman JF, McCarthy TC. Circumstances and consequences of falls in communitydwelling older women. Journal of Women's Health 2007;16 (10):1437-46. MEDLINE: 18062759

Wyman J. A home-based fall prevention intervention for high risk older women. www.dhs.state.mn.us/main/groups/ aging/documents/pub/dhs16_137823.pdf (accessed 14/10/ 07).

Wyman J. Design of the Fall Evaluation and Prevention Program (FEPP): a randomized trial of exercise and risk reduction education in high-risk older women. ICADI: International conference on aging, disability and independence; 2003 Dec 4-6; Washington (DC) www.icadi.phhp.ufl.edu/2003/presentation.php?PresID= 151. (accessed 30 November 2011).

* Wyman J, Gross C, DiFabio R, Nyman J, Lindquist $\mathrm{R}$, McCarthy T, et al. A randomized trial of exercise, education, and risk reduction counseling to prevent falls in population-based sample of older women. Gerontologist 2005;45(Special Issue II):297.

Wyman J, Gross C, DiFabio R, Nyman J, Lindquist R, McCarthy T, et al. Efficacy of exercise, education, and tailored counseling in reducing falls at 1- and 2-years in older women. Gerontologist 2006;46(Special Issue I):141. Wyman JF. Personal communication. 10 November 2007. Wyman JF, Croghan CF, Nachreiner NM, Gross CR, Stock HH, Talley K, et al. Effectiveness of education and individualized counseling in reducing environmental hazards in the homes of community-dwelling older women.
Journal of the American Geriatrics Society 2007;55(10): 1548-56. MEDLINE: 17908058

Xia 2009 \{published data only\}

Xia QH, Jiang Y, Niu CJ, Tang CX, Xia ZL. Effectiveness of a community-based multifaceted fall-prevention intervention in active and independent older Chinese adults. Injury Prevention 2009;15(4):248-51.

\section{Yamada 2013 \{published data only\}}

Yamada M, Higuchi T, Nishiguchi S, Yoshimura K, Kajiwara Y, Aoyama T. Multitarget stepping program in combination with a standardized multicomponent exercise program can prevent falls in community-dwelling older adults: a randomized, controlled trial. Journal of the American Geriatrics Society 2013;61(10):1669-75. PUBMED: 24001116]

\section{References to ongoing studies}

\section{ACTRN12607000206426 \{published data only\}} ACTRN12607000206426. Community care and hospital based collaborative falls prevention project. www.anzctr.org.au/trial_view.aspx?ID=81955 (accessed 12 June 2017).

\section{ACTRN12614000827639 \{published data only\}} ACTRN12614000827639. Does nutrition and exercise prevent frailty and reduce falls in pre-frail older adults in New Zealand?. www.anzctr.org.au/Trial/Registration/ TrialReview.aspx?id=366773 (accessed 12 June 2017).

\section{ACTRN12615001326583 \{published data only\}} ACTRN12615001326583. Preventing falls in older people after discharge from hospital as a result of a fall. anzctr.org.au/Trial/Registration/TrialReview.aspx?id= 369603 (accessed 12 June 2017).

\section{Barker 2015 \{published data only\}}

* Barker AL, Cameron PA, Hill KD, Flicker L, Haines TP, Lowthian JA, et al. RESPOND--A patient-centred programme to prevent secondary falls in older people presenting to the emergency department with a fall: protocol for a multicentre randomised controlled trial. Injury prevention: Journal of the International Society for Child and Adolescent Injury Prevention 2015;21(1):e1. PUBMED: 24958769]

Morello RT, Morris RL, Hill KD, Haines TP, Arendts G, Redfern J, et al. RESPOND: a programme to prevent secondary falls in older people presenting to the emergency department with a fall: protocol for an economic evaluation. Injury prevention : journal of the International Society for Child and Adolescent Injury Prevention 2017;23(2):124-30. PUBMED: 28330932]

\section{Blank 2011 \{published data only\}}

Blank WA, Freiberger E, Siegrist M, Landendoerfer P, Linde $\mathrm{K}$, Schuster T, et al. An interdisciplinary intervention to prevent falls in community-dwelling elderly persons: protocol of a cluster-randomized trial [PreFalls]. BMC Geriatrics 2011;11:7. MEDLINE: 21329525

NCT01032252. Prevention of falls in general practitioner for community-dwelling older adults [PreFalls]. 
clinicaltrials.gov/show/NCT01032252 (accessed August 2011).

Close 2014 \{published data only\}

Close JC, Wesson J, Sherrington C, Hill KD, Kurrle S, Lord SR, et al. Can a tailored exercise and home hazard reduction program reduce the rate of falls in community dwelling older people with cognitive impairment: protocol paper for the i-FOCIS randomised controlled trial. $B M C$ geriatrics 2014;14:89. PUBMED: 25128411]

Hill 2017 \{published data only\}

Hill AM, Etherton-Beer C, McPhail SM, Morris ME, Flicker L, Shorr R, et al. Reducing falls after hospital discharge: a protocol for a randomised controlled trial evaluating an individualised multimodal falls education programme for older adults. BMJ Open 2017;7(2):e013931. PUBMED: 28153933]

ISRCTN21120199 \{published data only\}

ISRCTN21120199. The effect of an assessment-based falls prevention programme in elderly people utilising day-care services. www.isrctn.com/ISRCTN21120199 (accessed 13 June 2017).

Landi 2017 \{published data only\} Landi F, Cesari M, Calvani R, Cherubini A, Di Bari M, Bejuit R, et al. The "Sarcopenia and Physical fRailty IN older people: multi-componenT Treatment strategies" (SPRINTT) randomized controlled trial: design and methods. Aging Clinical and Experimental Research 2017;29 (1):89-100. PUBMED: 28144914]

NCT01080196 \{published data only\} NCT01080196. Reducing falls with RENEW in older adults who have fallen. clinicaltrials.gov/show/ NCT01080196 (accessed 31 August 2011).

NCT01552551 \{published data only\}

NCT01552551. Assessment and referral versus exercise in primary prevention of falls: PA Healthy Steps Program. clinicaltrials.gov/ct2/show/record/NCT01552551 (accessed 13 June 2017).

NCT01713543 \{published data only\} NCT01713543. Community-based falls prevention program for the elderly. clinicaltrials.gov/show/ NCT01713543 (accessed 12 June 2017).

NCT02374307 \{published data only\}

NCT02374307. Falls-prevention in older people receiving home-help services. clinicaltrials.gov/show/NCT02374307 (accessed 12 June 2017).

NCT02631330 \{published data only\}

NCT02631330. Effect on falls reduction of a multimodal intervention in frail and pre-frail elderly communitydwelling people (FAREMAVA). clinicaltrials.gov/show/ NCT02631330 (accessed 12 June 2017).

Sherrington 2016 \{published data only\} ACTRN12610000805077. RESTORE: Recovery exercises and Stepping On after fracture. www.anzctr.org.au/ trial_view.aspx?ID=335781 (accessed 12 June 2017). Sherrington C, Fairhall N, Kirkham C, Clemson L, Howard K, Vogler C, et al. Exercise and fall prevention self-management to reduce mobility-related disability and falls after fall-related lower limb fracture in older people: protocol for the RESTORE (Recovery Exercises and STepping On afteR fracturE) randomised controlled trial. BMC Geriatrics 2016;16:34. PUBMED: 26838998]

Tan 2014 \{published data only\}

Tan PJ, Khoo EM, Chinna K, Hill KD, Poi PJ, Tan MP. An individually-tailored multifactorial intervention program for older fallers in a middle-income developing country: Malaysian Falls Assessment and Intervention Trial (MyFAIT). BMC Geriatrics 2014;14:78. PUBMED: 24951180]

\section{Additional references}

\section{ACSQH 2009}

Australian Commission on Safety and Quality in Healthcare. Preventing falls and harm from falls in older people. Best Practice Guidelines for Australian Community Care 2009. www.safetyandquality.gov.au/wp-content/uploads/2012/ 01/Guidelines-COMM.pdf (accessed 27 May 2016).

American Geriatrics Society 2011

Panel on Prevention of Falls in Older Persons, American Geriatrics Society and British Geriatrics Society. Summary of the updated American Geriatrics Society/British Geriatrics Society clinical practice guideline for prevention of falls in older persons. Journal of the American Geriatrics Society 2011;59(1):148-57. [PUBMED: 21226685]

Berry 2008

Berry SD, Miller RR. Falls: epidemiology, pathophysiology, and relationship to fracture. Current Osteoporosis Reports 2008;6(4):149-54. [PUBMED: 19032925]

Bhasin 2018

Bhasin S, Gill TM, Reuben DB, Latham NK, Gurwitz JH, Dykes $\mathrm{P}$, et al. Strategies to reduce injuries and develop confidence in elders (STRIDE): a cluster-randomized pragmatic trial of a multifactorial fall injury prevention strategy: design and methods. Journals of Gerontology. Series A, Biological Sciences and Medical Sciences 2018;73 (8):1053-61. DOI: 10.1093/gerona/glx190; PUBMED: 29045582

\section{Burns 2016}

Burns ER, Stevens JA, Lee R. The direct costs of fatal and non-fatal falls among older adults - United States. Journal of Safety Research 2016;58:99-103. [PUBMED: 27620939]

\section{Cameron 2012}

Cameron ID, Gillespie LD, Robertson MC, Murray GR, Hill KD, Cumming RG, et al. Interventions for preventing falls in older people in care facilities and hospitals. Cochrane Database of Systematic Reviews 2012, Issue 12. DOI: 10.1002/14651858.CD005465.pub3; PUBMED: 23235623

\section{Campbell 1990}

Campbell AJ, Borrie MJ, Spears GF, Jackson SL, Brown JS, Fitzgerald JL. Circumstances and consequences of falls experienced by a community population 70 years and over 
during a prospective study. Age and Ageing 1990;19(2): 136-41. [PUBMED: 2337010]

\section{Campbell 2006}

Campbell AJ, Robertson MC. Implementation of multifactorial interventions for fall and fracture prevention. Age and Ageing 2006;35 Suppl 2:ii60-4. [PUBMED: 16926208]

\section{Canning 2015}

Canning CG, Allen NE, Bloem BR, Keus SHJ, Munneke $\mathrm{M}$, Nieuwboer A, et al. Interventions for preventing falls in Parkinson's disease. Cochrane Database of Systematic Reviews 2015, Issue 3. DOI: 10.1002/14651858.CD011574

\section{Copsey 2016}

Copsey B, Hopewell S, Becker C, Cameron ID, Lamb SE. Appraising the uptake and use of recommendations for a common outcome data set for clinical trials: a case study in fall injury prevention. Trials 2016;17(1):131. [PUBMED: 26965046]

\section{Deandrea 2010}

Deandrea S, Lucenteforte E, Bravi F, Foschi R, La Vecchia C, Negri E. Risk factors for falls in community-dwelling older people: a systematic review and meta-analysis. Epidemiology 2010;21(5):658-68. [PUBMED: 20585256]

\section{Gates 2008}

Gates S, Fisher JD, Cooke MW, Carter YH, Lamb SE. Multifactorial assessment and targeted intervention for preventing falls and injuries among older people in community and emergency care settings: systematic review and meta-analysis. BMJ 2008;336(7636):130-3. [PUBMED: 18089892]

\section{Goodwin 2014}

Goodwin VA, Abbott RA, Whear R, Bethel A, Ukoumunne OC, Thompson-Coon J, et al. Multiple component interventions for preventing falls and fall-related injuries among older people: systematic review and meta-analysis. BMC Geriatrics 2014;14:15. [PUBMED: 24495705]

\section{Hannan 2010}

Hannan MT, Gagnon MM, Aneja J, Jones RN, Cupples LA, Lipsitz LA, et al. Optimizing the tracking of falls in studies of older participants: comparison of quarterly telephone recall with monthly falls calendars in the MOBILIZE Boston Study. American Journal of Epidemiology 2010;171 (9):1031-6. [PUBMED: 20360242]

\section{Higgins 2011a}

Higgins JPT, Altman DG, Sterne JAC (editors). Chapter 8: Assessing risk of bias in included studies. In: Higgins JPT, Green S (editors). Cochrane Handbook of Systematic Reviews of Interventions Version 5.1.0 [updated March 2011]. The Cochrane Collaboration, 2011. Available from www.cochrane-handbook.org.

Higgins 2011b

Higgins JPT, Deeks JJ, Altman DG (editors). Chapter 16.3.4: Approximate analyses of cluster-randomized trials for meta-analysis: effective sample sizes. In: Higgins JPT, Green S (editors). Cochrane Handbook of Systematic Reviews of Interventions Version 5.1.0 [updated March
2011]. The Cochrane Collaboration, 2011. Available from www.cochrane-handbook.org.

\section{Higgins 2011c}

Deeks JJ, Higgins JPT, Altman DG (editors). Chapter 9. Analysing data and undertaking meta-analyses. In: Higgins JPT, Green S (editors). Cochrane Handbook of Systematic Reviews of Interventions Version 5.1.0 [updated March 2011]. The Cochrane Collaboration, 2011. Available from www.cochrane-handbook.org.

\section{Higgins 2011d}

Higgins JPT, Deeks JJ, Altman DG (editors). Chapter 16.5.4 How to include multiple groups from one study. In: Higgins JPT, Green S (editors). Cochrane Handbook of Systematic Reviews of Interventions Version 5.1.0 [updated March 2011]. The Cochrane Collaboration, 2011. Available from www.cochrane-handbook.org.

\section{Kellogg 1987}

Anonymous. The prevention of falls in later life. A report of the Kellogg International Work Group on the Prevention of Falls by the Elderly. Danish Medical Bulletin 1987;34 Suppl 4:1-24.

\section{Kendrick 2014}

Kendrick D, Kumar A, Carpenter H, Zijlstra GA, Skelton DA, Cook JR, et al. Exercise for reducing fear of falling in older people living in the community. Cochrane Database of Systematic Reviews 2014, Issue 11. DOI: 10.1002/ 14651858.CD009848.pub2; PUBMED: 25432016

\section{Laird 2001}

Laird RD, Studenski S, Perera S, Wallace D. Fall history is an independent predictor of adverse health outcomes and utilization in the elderly. American Journal of Managed Care 2001;7(12):1133-8. [PUBMED: 11767299]

\section{Lamb 2005}

Lamb SE, Jørstad-Stein EC, Hauer K, Becker C, Prevention of Falls Network Europe and Outcomes Consensus Group. Development of a common outcome data set for fall injury prevention trials: the Prevention of Falls Network Europe consensus. Journal of the American Geriatrics Society 2005; 53(9):1618-22. [PUBMED: 16137297]

\section{Lamb 2007}

Lamb SE, Hauer K, Becker C. Manual for the fall prevention classification system. Version 1 (4 April 2007). www.profane.eu.org/documents/Falls_Taxonomy.pdf (accessed 17 May 2016).

\section{Lamb 2011}

Lamb SE, Becker C, Gillespie LD, Smith JL, Finnegan $\mathrm{S}$, Potter R, et al. Reporting of complex interventions in clinical trials: development of a taxonomy to classify and describe fall-prevention interventions. Trials 2011;12:125.

\section{Lefebvre 2011}

Lefebvre C, Manheimer E, Glanville J. Chapter 6.4.11.1 The Cochrane Highly Sensitive Search Strategies for identifying randomized trials in MEDLINE. In: Higgins JPT, Green S (editors). Cochrane Handbook for Systematic Reviews of Interventions Version 5.1.0 [updated March 
2011]. The Cochrane Collaboration, 2011. Available from www.cochrane-handbook.org.

\section{NICE 2013}

National Institute for Health and Clinical Excellence. Falls in older people: assessing risk and prevention 2013 [CG161]. www.nice.org.uk/guidance/cg161/chapter/1recommendations (accessed 27 May 2016).

\section{Peel 2002}

Peel NM, Kassulke DJ, McClure RJ. Population based study of hospitalised fall related injuries in older people. Injury Prevention 2002;8(4):280-3. [PUBMED: 12460962]

\section{RevMan 2014 [Computer program]}

The Nordic Cochrane Centre, The Cochrane Collaboration. Review Manager (RevMan). Version 5.3. Copenhagen: The Nordic Cochrane Centre, The Cochrane Collaboration, 2014.

\section{Rizzo 1996}

Rizzo JA, Baker DI, McAvay G, Tinetti ME. The costeffectiveness of a multifactorial targeted prevention program for falls among community elderly persons. Medical Care 1996;34(9):954-69. MEDLINE: 8792783

\section{Sach 2012}

Sach TH, Logan PA, Coupland CA, Gladman JR, Sahota $\mathrm{O}$, Stoner-Hobbs V, et al. Community falls prevention for people who call an emergency ambulance after a fall: an economic evaluation alongside a randomised controlled trial. Age and Ageing 2012; Vol. 41, issue 5:635-41. DOI: 10.1093/ageing/afs071

\section{Schünemann 2011}

Schünemann HJ, Oxman AD, Higgins JPT, Vist GE, Glasziou P, Guyatt GH. Chapter 11: Presenting results and 'Summary of findings' tables. In: Higgins JPT, Green $S$ (editors), Cochrane Handbook for Systematic Reviews of Interventions Version 5.1.0 [updated March 2011]. The Cochrane Collaboration, 2011. Available from www.cochrane-handbook.org.

\section{Scuffham 2003}

Scuffham P, Chaplin S, Legood R. Incidence and costs of unintentional falls in older people in the United Kingdom. Journal of Epidemiology and Community Health 2003;57(9): 740-4. [PUBMED: 12933783]

\section{Sherrington 2016a}

Sherrington C, Tiedemann A, Fairhall NJ, Hopewell S, Michaleff ZA, Howard K, et al. Exercise for preventing falls in older people living in the community [protocol]. Cochrane Database of Systematic Reviews 2016, Issue 11. Art. No.: CD012424.

\section{Sherrington 2016b}

Sherrington C, Michaleff ZA, Fairhall N, Paul SS, Tiedemann A, Whitney J, et al. Exercise to prevent falls in older adults: an updated systematic review and metaanalysis. British Journal of Sports Medicine 2016;51(24): 1750-8. [PUBMED: 27707740]

\section{Smeeth 2002}

Smeeth L, Ng ES. Intraclass correlation coefficients for cluster randomized trials in primary care: data from the MRC Trial of the Assessment and Management of Older People in the Community. Controlled Clinical Trials 2002; 23(4):409-21.

\section{Stata 2015}

StataCorp. 2015. Stata Statistical Software: Release 14. College Station, TX: StataCorp LP.

\section{Tinetti 1988}

Tinetti ME, Speechley M, Ginter SF. Risk factors for falls among elderly persons living in the community. New England Journal of Medicine 1988;319(26):1701-7. [PUBMED: 3205267]

\section{Tinetti 1997}

Tinetti ME, Williams CS. Falls, injuries due to falls, and the risk of admission to a nursing home. New England Journal of Medicine 1997;337(18):1279-84. [PUBMED: 9345078]

Tinetti 2003

Tinetti ME. Clinical practice. Preventing falls in elderly persons. New England Journal of Medicine 2003;348(1): 42-9. [PUBMED: 12510042]

\section{Todd 2004}

Todd C, Skelton D. What are the main risk factors for falls among older people and what are the most effective interventions to prevent these falls? March 2004 Copenhagen, WHO Regional Office for Europe (Health Evidence Network report). www.euro.who.int/document/ E82552.pdf (accessed 28 April 2016)

\section{Tricco 2017}

Tricco AC, Thomas SM, Veroniki AA, Hamid JS, Cogo E, Strifler $\mathrm{L}$, et al. Comparisons of interventions for preventing falls in older adults: a systematic review and meta-analysis. JAMA 2017;318(17):1687-99. [PUBMED: 29114830]

Verheyden 2013

Verheyden GS, Weerdesteyn V, Pickering RM, Kunkel $D$, Lennon $S$, Geurts AC, et al. Interventions for preventing falls in people after stroke. Cochrane Database of Systematic Reviews 2013, Issue 5. DOI: 10.1002/ 14651858.CD008728.pub2; PUBMED: 23728680

\section{Vieira 2016}

Vieira ER, Palmer RC, Chaves PH. Prevention of falls in older people living in the community. BMJ 2016;353: i1419.

Walters 2003

Walters SJ, Brazier JE. What is the relationship between the minimally important difference and health state utility values? The case of the SF-6D. Health and Quality of Life Outcomes 2003;1:4. [PUBMED: 12737635]

\section{Yardley 2002}

Yardley L, Smith H. A prospective study of the relationship between feared consequences of falling and avoidance of activity in community-living older people. Gerontologist 2002;42(1):17-23. [PUBMED: 11815695] 


\section{Zecevic 2006}

Zecevic AA, Salmoni AW, Speechley M, Vandervoort AA.

Defining a fall and reasons for falling: comparisons among the views of seniors, health care providers, and the research literature. Gerontologist 2006;46(3):367-76. [PUBMED: 16731875]

\section{References to other published versions of this review}

\section{Gillespie 2012}

Gillespie LD, Robertson MC, Gillespie WJ, Sherrington C, Gates S, Clemson LM, et al. Interventions for preventing falls in older people living in the community. Cochrane Database of Systematic Reviews 2012, Issue 9. DOI: 10.1002/ 14651858.CD007146.pub3; PUBMED: 22972103

* Indicates the major publication for the study 


\section{CHARACTERISTICS OF STUDIES}

\section{Characteristics of included studies [ordered by study ID]}

Beling 2009

Methods

Study Design: RCT (parallel design)

Number of study arms: 2

Study centres: Single centre

Length of follow-up: 3 months

Participants

Setting: United States of America

Number randomised: 23

Number analysed: 19

Number lost to follow-up: 4

Sample: Volunteers were recruited through press releases, newspaper advertisements and university website

Age (years): mean 80 (SD 5.7)

Sex: $42 \%$ women

Ethnicity : $78 \%$ white, $15.8 \%$ Hispanic, $5.3 \%$ Asian

Inclusion criteria: $\geq 65$ years; community-dwelling; English-speaking; minimal vision and hearing deficit; access to transportation; consenting; with physician approval to participate; MMSE $\geq 24 / 30 ; 3$ metre TUG test $\geq 13.5 \mathrm{sec}$ and/or to have $\geq 2$ falls in past year and/or 1 injurious fall in the past year

Exclusion criteria: cardiac conditions; musculoskeletal and/or neurological impairment that could result in falls, e.g. stroke, Parkinson's disease, lower extremity joint replacement, fracture in last year

Interventions

Type of intervention: Multifactorial intervention

1. The Matter of Balance programme: 12-week small-group-based balance programme and falls home-based risk assessment $(\mathrm{n}=12)$

2. Control: Usual care $(\mathrm{n}=11)$

Who delivered the intervention: Physical therapists, teams of physical therapy students enrolled in the last semester of their curriculum

Compliance assessed: Not reported

\begin{tabular}{ll} 
Outcomes & 1. Rate of falls \\
\hline \multirow{2}{*}{ Notes } & Source of Funding: Supported by a grant from Unihealth Foundation \\
& Conflicts of interest: None \\
& Economic information: Not reported
\end{tabular}

Risk of bias

\begin{tabular}{|c|c|c|}
\hline Bias & Authors' judgement & Support for judgement \\
\hline
\end{tabular}

Random sequence generation (selection Unclear risk bias)

Quote: “Twelve subjects were randomly assigned to the experimental group and 11 subjects were assigned to the control group. 
Beling 2009 (Continued)

Allocation concealment (selection bias) Unclear risk

Blinding of participants and personnel Unclear risk (performance bias)

All outcomes

Blinding of outcome assessment (detection Unclear risk bias)

Falls and fallers

Blinding of outcome assessment (detection Unclear risk bias)

Fractures

Blinding of outcome assessment (detection Unclear risk bias)

Hospital admission \& medical attention

Incomplete outcome data (attrition bias) High risk All outcomes
Quote: "randomly assigned" but no further information on allocation schedule. Insufficient information to permit judgement

Participants and personnel not blind to allocated group but impact of non-blinding unclear

Quote “relied on each participant's self-reported fall history over time". Insufficient information to permit judgement

Not Applicable

Not Applicable

Less than $20 \%$ missing outcome data with imbalanced losses groups

1. The Matter of Balance programme: randomised $\mathrm{n}=12$, analysed $\mathrm{n}=11$ (1 participant dropped out due to unrelated hospitalisation and deteriorating health)

2. Usual care: randomised $\mathrm{n}=11$, analysed $n=8$ (3 participants excluded from analysis: 1 demonstrated prolonged latencies of motor responses during the Motor Control Test, 1 refused further participation because of unrelated health problems, and 1 enrolled in a Tai Chi course during the study to improve balance)

\begin{tabular}{|c|c|c|}
\hline Selective reporting (reporting bias) & Low risk & All prespecified outcomes were reported \\
\hline Method of ascertaining falls & Unclear risk & $\begin{array}{l}\text { Data gathering was prospective, and study } \\
\text { relied on each participant's self-reported fall } \\
\text { history over time. Insufficient information } \\
\text { to permit judgement }\end{array}$ \\
\hline
\end{tabular}




\begin{tabular}{|c|c|}
\hline Methods & $\begin{array}{l}\text { Study design: RCT ( } 2 \text { × } 2 \text { factorial design }) \\
\text { Number of study arms: } 4 \\
\text { Study centres: Multiple centres } \\
\text { Length of follow-up: } 12 \text { months }\end{array}$ \\
\hline Participants & $\begin{array}{l}\text { Setting: New Zealand } \\
\text { Number randomised: } 391 \\
\text { Number analysed: } 360 \\
\text { Number lost to follow-up: } 30 \\
\text { Sample: Men and women with severe visual impairment (visual acuity } 6 / 24 \text { or worse) } \\
\text { identified in blind register, university and hospital outpatient clinics, and private oph- } \\
\text { thalmology practice } \\
\text { Age (years): Mean } 83.6 \text { (SD 4.8), range } 75 \text { to } 96 \\
\text { Sex: } 68 \% \text { women } \\
\text { Ethnicity: Not reported } \\
\text { Inclusion criteria: Vision worse than } 6 / 24 \text { in better eye; age } \geq 75 \text { years } \\
\text { Exclusion criteria: Unable to walk around at home }\end{array}$ \\
\hline Interventions & $\begin{array}{l}\text { Type of intervention: Multiple intervention } \\
\text { 1. Home safety programme }(\mathrm{n}=100) \\
\text { 2. Otago Exercise Programme plus vitamin D supplements }(\mathrm{n}=97) \\
\text { 3. Home safety programme plus Otago Exercise Programme plus vitamin D supplement } \\
(\mathrm{n}=98) \\
\text { 4. Social visits }(\mathrm{n}=96) \\
\text { Who delivered intervention: Occupational therapists and physiotherapists } \\
\text { Compliance assessed: Yes, OTs evaluated adherence to home-safety programme during } \\
\text { phone interviews, exercise compliance assessed using participant-completed monthly } \\
\text { postcard reminders, physiotherapy compliance assessed by twice-yearly monitoring }\end{array}$ \\
\hline Outcomes & $\begin{array}{l}\text { 1. Rate of falls } \\
\text { 2. Number of people sustaining one or more falls } \\
\text { 3. Number of people sustaining recurrent falls } \\
\text { 4. Number of people who experienced a fall that required medical attention } \\
\text { 5. Adverse events of the intervention }\end{array}$ \\
\hline Notes & $\begin{array}{l}\text { Source of funding: Health Research Council of New Zealand } \\
\text { Conflicts of interest: None } \\
\text { Economic information:The programme cost NZD 64,337 to deliver to the } 198 \text { partic- } \\
\text { ipants in the } 2 \text { centres, or NZD } 325 \text { (SD NZD 292) per person } \\
\text { Otago Exercise Programme manual can be obtained from: www.cdc.gov/ } \\
\text { HomeandRecreationalSafety/Falls/compendium/1.2_otago.html. } \\
\text { Adverse events: "No significant adverse events were reported during the study" }\end{array}$ \\
\hline
\end{tabular}

\section{Risk of bias}

Bias

Authors' judgement

Random sequence generation (selection Low risk bias)

Computer-generated random numbers

Multifactorial and multiple component interventions for preventing falls in older people living in the community (Review) 


\begin{tabular}{|c|c|c|}
\hline Allocation concealment (selection bias) & Low risk & $\begin{array}{l}\text { Schedule held by independent person at } \\
\text { separate site, telephone access }\end{array}$ \\
\hline $\begin{array}{l}\text { Blinding of participants and personnel } \\
\text { (performance bias) } \\
\text { All outcomes }\end{array}$ & Unclear risk & $\begin{array}{l}\text { Participants and personnel not blind to al- } \\
\text { located group but impact of non-blinding } \\
\text { unclear }\end{array}$ \\
\hline $\begin{array}{l}\text { Blinding of outcome assessment (detection } \\
\text { bias) } \\
\text { Falls and fallers }\end{array}$ & Low risk & $\begin{array}{l}\text { Falls recorded monthly by patients return- } \\
\text { ing postcard calenders }\end{array}$ \\
\hline $\begin{array}{l}\text { Blinding of outcome assessment (detection } \\
\text { bias) } \\
\text { Fractures }\end{array}$ & Unclear risk & Not applicable \\
\hline $\begin{array}{l}\text { Blinding of outcome assessment (detection } \\
\text { bias) } \\
\text { Hospital admission \& medical attention }\end{array}$ & Low risk & $\begin{array}{l}\text { Requiring medical attention confirmed by } \\
\text { GP and hospital records }\end{array}$ \\
\hline $\begin{array}{l}\text { Incomplete outcome data (attrition bias) } \\
\text { All outcomes }\end{array}$ & Low risk & $\begin{array}{l}\text { Less than } 20 \% \text { missing outcome data, losses } \\
\text { balanced across groups with similar reasons } \\
\text { for missing data } \\
\text { 1. Otago Exercise Programme plus vitamin } \\
D \text { supplements: randomised } n=97 \text {, anal- } \\
\text { ysed } n=90 \text { ( } 2 \text { died, } 2 \text { withdrew, } 3 \text { unspec- } \\
\text { ified) } \\
\text { 2. Home-safety programme plus Otago Ex- } \\
\text { ercise Programme plus vitamin D supple- } \\
\text { ments: randomised } n=98 \text {, analysed } n=87 \\
\text { (4 died, } 6 \text { withdrew, } 1 \text { unspecified) } \\
3 . \text { Social visits: randomised } n=96 \text {, analysed } \\
n=87 \text { ( } 7 \text { died, } 2 \text { withdrew) }\end{array}$ \\
\hline Selective reporting (reporting bias) & Low risk & All prespecified outcomes were reported. \\
\hline Method of ascertaining falls & Low risk & $\begin{array}{l}\text { Falls recorded monthly by patients return- } \\
\text { ing postcard calenders }\end{array}$ \\
\hline
\end{tabular}

\section{Carpenter 1990}

Methods
Study design: RCT (parallel design)

Number of study arms: 2

Study centres: Multiple centres

Length of follow-up: 36 months
Setting: United Kingdom

Number randomised: 539

Number analysed: 367 


\begin{tabular}{|c|c|}
\hline & $\begin{array}{l}\text { Number lost to follow-up: } 172 \\
\text { Sample: women and men recruited from patient lists of } 2 \text { general medical practices } \\
\text { Age (years): } \geq 75 \text { years } \\
\text { Sex: } 65 \% \text { women } \\
\text { Ethnicity: Not reported } \\
\text { Inclusion criteria: aged } \geq 75 \text {; living in Andover town, including the surrounding house } \\
\text { estates } \\
\text { Exclusion criteria: living in residential care; living in surrounding villages }\end{array}$ \\
\hline Interventions & $\begin{array}{l}\text { Type of intervention: Multifactorial intervention } \\
\text { 1. Visit by trained volunteers for dependency surveillance using Winchester disability } \\
\text { rating scale. The intervention was stratified by degree of disability on the entry evaluation. } \\
\text { For those with no disability, the visit was every } 6 \text { months; for those with disability, } 3 \\
\text { months. Scores compared with previous assessment and referral to GP if score increased } \\
\text { by } 5 \text { or more. }(\mathrm{n}=272) \\
\text { 2. Control: no disability surveillance between initial and final evaluation }(\mathrm{n}=267) \\
\text { Who delivered the intervention: Unskilled volunteers and general practitioners } \\
\text { Compliance assessed: Not reported }\end{array}$ \\
\hline Outcomes & $\begin{array}{l}\text { 1. Rate of falls } \\
\text { 2. Number of people who experienced a fall that required hospital admission }\end{array}$ \\
\hline Notes & $\begin{array}{l}\text { Source of funding: Wessex Regional Health Authority } \\
\text { Conflicts of interest: None } \\
\text { Economic information: Quote "The running costs of the project were low, the only } \\
\text { expenses incurred were costs of printing questionnaires, salary, and travel expenses for } \\
\text { half term research assistant and purchase of statistical software for the data analysis" }\end{array}$ \\
\hline
\end{tabular}

\section{Risk of bias}

\begin{tabular}{l|l|l}
\hline Bias & Authors' judgement & Support for judgement \\
\hline $\begin{array}{l}\text { Random sequence generation (selection } \\
\text { bias) }\end{array}$ & Low risk & Randomised by random-number tables \\
\hline $\begin{array}{l}\text { Allocation concealment (selection bias) } \\
\text { Blinding of participants and personnel }\end{array}$ & Unclear risk & Unclear risk \\
$\begin{array}{l}\text { All outcomes } \\
\text { Blinding of outcome assessment (detection } \\
\text { bias) } \\
\text { Falls and fallers }\end{array}$ & High risk & $\begin{array}{l}\text { No information on allocation schedule. In- } \\
\text { sufficient information to permit judgement }\end{array}$ \\
\hline
\end{tabular}

Blinding of outcome assessment (detection Unclear risk

Not applicable

bias)

Fractures

Multifactorial and multiple component interventions for preventing falls in older people living in the community (Review) 


\section{Carpenter 1990 (Continued)}

Blinding of outcome assessment (detection High risk

bias)

Hospital admission \& medical attention

Incomplete outcome data (attrition bias) High risk

All outcomes
Self-report by participants

More than 20\% missing outcome data, losses balanced across groups with similar reasons for loss to follow-up

1. Home visits for dependency surveillance: randomised $\mathrm{n}=272$, analysed $\mathrm{n}=181(66$ died, 14 withdrew from project, 11 moved out of area)

2. No disability surveillance: randomised $\mathrm{n}$ $=267$, analysed $\mathrm{n}=186$ (54 died, 11 withdrew from project, 11 moved out of area, 2 changed doctors to a different practice, 3 moved into long-term nursing care)

All prespecified outcomes were reported

Selective reporting (reporting bias)

Low risk

Falls were reported by participants retrospectively by interview at the end of the study

Carter 1997

\begin{tabular}{ll} 
Methods & Study design: RCT (parallel design) \\
Number of study arms: 3 \\
Study centre: unclear \\
Length of follow-up: 12 months \\
\hline Participants & Setting: Australia \\
& Number randomised: 657 \\
& Number analysed: 457 \\
& Number lost to follow-up: 200 \\
& Sample: All full-time general practitioners in the Lower Hunter Region of NSW, Australia \\
& were approached and asked to generate lists of their patients who fulfilled eligibility \\
Age (years) 80 years+: Mean $34 \%$ & Sex: $66 \%$ women \\
Ethnicity: Not reported & Inclusion criteria: Aged 70 years and over, ability to speak and understand English, living \\
independently at home, in hostel or retirement village, not suffering from psychiatric \\
disturbance \\
Exclusion criteria: Those who were listed as living outside the region, those with no \\
phone
\end{tabular}


hazards, pamphlet on home safety, pamphlet on the wise use of medicines for older people $(\mathrm{n}=220)$

2. Action plan for home safety plus medication review: House check with more comprehensive feedback including how it could be fixed Could arrange local service club to do the work. Pamphlet on safety $(\mathrm{n}=205)$

3. Control: No intervention $(n=232)$

Who delivered the intervention: Trained project officer

Compliance assessed: Yes, approximately 3 months after, participants were sent the letter recommending medication review, a member of the research team rang them and asked if they had been to their doctor for medication review and if their medication use had altered as a result

Outcomes

1) Number of people sustaining one or more falls

2) Number of people sustaining recurrent falls

3) Number of people requiring medical attention (e.g. attendance at emergency department, requiring GP consultation)

Notes

Source of funding: Australian Rotary Health Research Fund

Conflicts of interest: Not reported

Economic information: Not reported

\section{Risk of bias}

Bias

Random sequence generation (selection Low risk bias)
Authors' judgement

\begin{tabular}{|c|c|c|}
\hline Allocation concealment (selection bias) & Unclear risk & Not reported \\
\hline $\begin{array}{l}\text { Blinding of participants and personnel } \\
\text { (performance bias) } \\
\text { All outcomes }\end{array}$ & Unclear risk & $\begin{array}{l}\text { Participants and provider not blinded to al- } \\
\text { location group but impact of non-blinding } \\
\text { unclear }\end{array}$ \\
\hline $\begin{array}{l}\text { Blinding of outcome assessment (detection } \\
\text { bias) } \\
\text { Falls and fallers }\end{array}$ & High risk & $\begin{array}{l}\text { Falls were self-reported and participants } \\
\text { were unblinded }\end{array}$ \\
\hline $\begin{array}{l}\text { Blinding of outcome assessment (detection } \\
\text { bias) } \\
\text { Fractures }\end{array}$ & Unclear risk & Not applicable \\
\hline $\begin{array}{l}\text { Blinding of outcome assessment (detection } \\
\text { bias) } \\
\text { Hospital admission \& medical attention }\end{array}$ & High risk & $\begin{array}{l}\text { Falls were self-reported and participants } \\
\text { were unblinded }\end{array}$ \\
\hline $\begin{array}{l}\text { Incomplete outcome data (attrition bias) } \\
\text { All outcomes }\end{array}$ & High risk & $\begin{array}{l}\text { More than } 20 \% \text { missing outcome data, } \\
\text { losses were unbalanced across groups with }\end{array}$ \\
\hline
\end{tabular}

Quote: “Subjects were randomised to one of the three groups using a random generator in SAS software"

\section{Support for judgement} location group but impact of non-blinding Falls were self-reported and participants were unblinded

Not applicable

Falls were self-reported and participants were unblinded

More than $20 \%$ missing outcome data, losses were unbalanced across groups with 
Carter 1997 (Continued)

no reasons given for loss to follow-up

1. Brief feedback on home safety plus pam-

phlets on home safety: randomised $n=220$, analysed $n=163$ (57, no reasons)

2. Action plan for home safety plus medication review: randomised $\mathrm{n}=205$, analysed $\mathrm{n}=133$ (72, no reasons)

3. Control: no intervention: randomised $n$ $=232$, analysed $\mathrm{n}=161$ (71, no reasons )

\begin{tabular}{lll}
\hline Selective reporting (reporting bias) & Unclear risk & Unpublished study \\
\hline Method of ascertaining falls & High risk & Falls were recorded retrospectively
\end{tabular}

Ciaschini 2009

\begin{tabular}{ll}
\hline Methods & Study design: RCT (parallel design) \\
Number of study arms: 2 \\
Study centres: Single centre \\
Length of follow-up: 12 months \\
\hline Participants & Setting: Canada \\
& Number randomised: 201 \\
& Number analysed: 176 \\
& Number lost to follow-up: 25 \\
& Sample: Community-dwelling people at risk of a fall-related fracture \\
& Age (years): mean 72 (SD 8.4 ), range $65-79$ \\
& Sex: $94 \%$ women \\
Ethnicity: 11 of aboriginal origin: $5.5 \%$ & Inclusion criteria: Community-dwelling; age $>55$ years old; able to consent; at risk of \\
fracture (non-pathological fracture in past year with T-score < 2.0 ; attended ED with a \\
fall, self-referred, or referred by health professional and at high risk of falls (TUG test $>$ \\
14 sec) \\
Exclusion criteria: If already receiving therapy for osteoporosis as per Osteoporosis \\
Canada guidelines
\end{tabular}

1. Multifactorial falls risk assessment by nurse + counselling and referral for PT and OT and interventions, plus recommendations for osteoporosis therapy targeting physicians and their patients $(\mathrm{n}=101)$

2. Control: usual care until 6 months, then same as intervention group $(\mathrm{n}=100)$

Who delivered the intervention: Research nurse, physiotherapist, occupational therapist Compliance assessed: Yes. Adherance of participants to intervention was assessed as changes to medication were reviewed at 6 months

Outcomes

1. Number of people sustaining 1 or more falls

2. Number of people of sustaining 1 or more fall-related fractures

3. Number of people who experienced a fall that required hospital admissions

Multifactorial and multiple component interventions for preventing falls in older people living in the community (Review) 
Notes

Risk of bias

Source of funding: Financial support for the completion of the study was given by Merck Frosst Canada Ltd, Sanofi-Aventis Pharma Inc., Proctor \& Gamble Pharmaceuticals Canada Inc., Eli Lilly Canada Inc., and the Greenshield Foundation. Equipment (e. g. office space, computers, telephones) was contributed in-kind by the Group Health Centre, Algoma Public Health, Sault Area Hospital, AlgomaCommunity Care AccessCentre, and the Slips, Trips and Falls Committee of Sault Ste. Marie Safe Communities Partnership, all located in Sault Ste.Marie, Ontario, Canada

Conflicts of interest: None

Economic information: Not reported

12-month study but 6-month data used in review analysis as control group participants were offered the intervention after 6 months

Bias

Random sequence generation (selection Low risk bias)
Authors' judgement

\section{Support for judgement}

Quote: "Eligible patients were randomized using a computer generated randomization scheme under supervision of the study biostatistician, into an immediate intervention protocol (IP) group or to a delayed intervention protocol (DP) group"

Insufficient information to permit judgement (see above)

Quote: "The patients, treating physicians and outcomes collectors could not be blinded to the intervention status." but impact of non-blinding unclear

Falls and fall-related injuries were obtained from electronic medical records as well as patient diaries

bias)

Falls and fallers

Falls and fall-related injuries were obtained from electronic medical records as well as patient diaries

Measurement of outcomes was obtained through patient records (electronic medical records)

Hospital admission \& medical attention

Less than $20 \%$ missing outcome data, intervention arm records a higher loss to follow-up than control. Similar reasons for missing data in both arms

1. Multifactorial assessment: referral and 


\begin{tabular}{|c|c|c|}
\hline & & $\begin{array}{l}\text { counselling: randomised } \mathrm{n}=101 \text {, analysed } \\
\mathrm{n}=85 \text { ( } 1 \text { withdrew, } 6 \text { died, } 9 \text { other reasons) } \\
\text { 2. Control: usual care until } 6 \text { months, then } \\
\text { same as intervention group: randomised } \mathrm{n} \\
=100 \text {, analysed } \mathrm{n}=91 \text { ( } 4 \text { died, } 5 \text { other } \\
\text { reasons) }\end{array}$ \\
\hline Selective reporting (reporting bias) & Low risk & All prespecified outcomes were reported \\
\hline Method of ascertaining falls & Low risk & $\begin{array}{l}\text { Falls and fall-related injuries were obtained } \\
\text { from electronic medical records as well as } \\
\text { patient diaries }\end{array}$ \\
\hline
\end{tabular}

Clemson 2004

\begin{tabular}{ll} 
Methods & $\begin{array}{l}\text { Study design: RCT (parallel design) } \\
\text { Number of study arms: } 2 \\
\text { Study centres: Multiple centres } \\
\text { Length of follow-up: } 14 \text { months }\end{array}$ \\
\hline Participants & Setting: Australia \\
& Number randomised: 310 \\
& Number analysed: 285 \\
Number lost to follow-up: 25 \\
Sample: Volunteer community-dwelling men and women recruited by various strategies \\
Age (years): mean 78 (SD 5) \\
Sex: $74 \%$ women \\
Ethnicity: Not reported \\
Inclusion criteria: aged $\geq 70 ;$ community-dwelling; fallen in past year or felt themselves \\
to be at risk of falling \\
Exclusion criteria: dementia (>3 errors on Short Portable Mental Status Questionnaire) \\
; home-bound; unable to independently leave home; unable to speak English
\end{tabular}

Interventions

Type of intervention: Multiple intervention

1. Stepping On programme. Multifaceted small-group learning environment to encourage self-efficacy, behaviour change, and reduce falls using decision-making theory and a variety of learning strategies. 2 hours a week for 7 weeks; taught exercises and practised in classes OT home visit within 6 weeks of final programme session; booster session 3 months after final session. $(\mathrm{n}=157)$

2. Social visits from student OT with no discussion of falls or fall prevention ( $\mathrm{n}=153$ ) Who delivered intervention: OTs experienced in group work with 12 years experience in geriatrics

Compliance assessed: Yes, through home visit by research assistant

Outcomes

1. Rate of falls

2. Number of people sustaining 1 or more falls

3. Number of people sustaining recurrent falls

4. Health-related quality of life (SF-36 $0-100$, mental and physical subscales: change

Multifactorial and multiple component interventions for preventing falls in older people living in the community (Review) 


$\begin{array}{ll}\text { Notes } & \text { Source of funding: National Health and Medical Research Council, Australia } \\ \text { Conflicts of interest: None } \\ \text { Economic information: Not reported }\end{array}$

Risk of bias

\begin{tabular}{|c|c|c|}
\hline Bias & Authors' judgement & Support for judgement \\
\hline $\begin{array}{l}\text { Random sequence generation (selection } \\
\text { bias) }\end{array}$ & Unclear risk & $\begin{array}{l}\text { Quote: "Randomised by researcher not in- } \\
\text { volved in subject screening or assessment". } \\
\text { Method not described }\end{array}$ \\
\hline Allocation concealment (selection bias) & Unclear risk & $\begin{array}{l}\text { Insufficient information to permit judge- } \\
\text { ment }\end{array}$ \\
\hline $\begin{array}{l}\text { Blinding of participants and personnel } \\
\text { (performance bias) } \\
\text { All outcomes }\end{array}$ & Unclear risk & $\begin{array}{l}\text { Participants and personnel not blinded to } \\
\text { allocated group but impact of non-blinding } \\
\text { unclear }\end{array}$ \\
\hline $\begin{array}{l}\text { Blinding of outcome assessment (detection } \\
\text { bias) } \\
\text { Falls and fallers }\end{array}$ & Low risk & $\begin{array}{l}\text { Falls recorded monthly by participants re- } \\
\text { turning postcard calenders }\end{array}$ \\
\hline $\begin{array}{l}\text { Blinding of outcome assessment (detection } \\
\text { bias) } \\
\text { Fractures }\end{array}$ & Unclear risk & Not applicable \\
\hline $\begin{array}{l}\text { Blinding of outcome assessment (detection } \\
\text { bias) } \\
\text { Hospital admission \& medical attention }\end{array}$ & Unclear risk & Not applicable \\
\hline $\begin{array}{l}\text { Incomplete outcome data (attrition bias) } \\
\text { All outcomes }\end{array}$ & Unclear risk & $\begin{array}{l}\text { Less than } 20 \% \text { missing outcome data, losses } \\
\text { balanced across group but reasons not given } \\
\text { 1. Step On Programme: randomised } n= \\
\text { 157, analysed } n=147 \\
\text { 2. Social visits: randomised } n=153 \text {, anal- } \\
\text { ysed } n=138 \\
\text { ( } 7 \text { died, } 6 \text { withdrew, } 5 \text { lost contact, } 6 \text { nurs- } \\
\text { ing home, } 1 \text { cognitive decline) }\end{array}$ \\
\hline Selective reporting (reporting bias) & Low risk & All pre-specified outcomes reported \\
\hline Method of ascertaining falls & Low risk & $\begin{array}{l}\text { Falls recorded monthly by patients return- } \\
\text { ing postcard calenders }\end{array}$ \\
\hline
\end{tabular}




\begin{tabular}{|c|c|c|}
\hline Methods & \multicolumn{2}{|c|}{$\begin{array}{l}\text { Study design: RCT (parallel design) } \\
\text { Number of study arms: } 2 \\
\text { Study centre: Unclear } \\
\text { Length of follow-up: } 12 \text { months }\end{array}$} \\
\hline Participants & \multicolumn{2}{|c|}{$\begin{array}{l}\text { Setting: United Kingdom } \\
\text { Number randomised: } 397 \\
\text { Number analysed: } 304 \\
\text { Number lost to follow-up: } 93 \\
\text { Sample: Community-dwelling individuals presenting at A\&E after a fall. Admitted pa- } \\
\text { tients not recruited until discharge } \\
\text { Age (years): Mean } 78.2 \text { (SD 7.5) } \\
\text { Sex: } 68 \% \text { women } \\
\text { Ethnicity: Not reported } \\
\text { Inclusion criteria: aged } \geq 65 \text {; lived in the community; history of falling } \\
\text { Exclusion criteria: cognitive impairment (AMT < } 7 \text { ) and no regular carer (for informed } \\
\text { consent reasons); speaking little or no English; not living locally }\end{array}$} \\
\hline Interventions & \multicolumn{2}{|c|}{$\begin{array}{l}\text { Type of intervention: Multifactorial intervention } \\
\text { 1. Medical and occupational therapy assessments and interventions: Medical assessment } \\
\text { to identify primary cause of fall and other risk factors present (general examination } \\
\text { and visual acuity, balance, cognition, affect, medications). Intervention and referral as } \\
\text { required. Home visit by OT (functional assessment and environmental hazards). Advice, } \\
\text { equipment, and referrals as required. }(\mathrm{n}=184) \\
\text { 2. Control: usual care only ( } \mathrm{n}=213 \text { ) } \\
\text { Who delivered the intervention: Physician and OT } \\
\text { Compliance assessed: Not reported }\end{array}$} \\
\hline Outcomes & \multicolumn{2}{|c|}{$\begin{array}{l}\text { 1. Rate of falls } \\
\text { 2. Number of people sustaining } 1 \text { or more falls } \\
\text { 3. Number of people sustaining recurrent falls } \\
\text { 4. Health-related quality of life (Barthel Index } 0-20 \text { : endpoint score) }\end{array}$} \\
\hline Notes & \multicolumn{2}{|c|}{$\begin{array}{l}\text { Source of funding: South Thames NHS Research and Development project grant } \\
\text { Conflicts of interest: None } \\
\text { Economic information: Cost analysis reported in Close } 2000\end{array}$} \\
\hline \multicolumn{3}{|l|}{ Risk of bias } \\
\hline Bias & Authors' judgement & Support for judgement \\
\hline $\begin{array}{l}\text { Random sequence generation (selection } \\
\text { bias) }\end{array}$ & Low risk & Randomised by random-numbers table \\
\hline Allocation concealment (selection bias) & Low risk & List held independently of the investigators \\
\hline $\begin{array}{l}\text { Blinding of participants and personnel } \\
\text { (performance bias) } \\
\text { All outcomes }\end{array}$ & Unclear risk & $\begin{array}{l}\text { Participants and personnel not blind to al- } \\
\text { located group but impact of non-blinding } \\
\text { unclear }\end{array}$ \\
\hline
\end{tabular}


Close 1999 (Continued)

Blinding of outcome assessment (detection Low risk bias)

Falls and fallers

Blinding of outcome assessment (detection Unclear risk bias)

Fractures

Blinding of outcome assessment (detection High risk bias)

Hospital admission \& medical attention
Quote "Each participant was given a "falls diary" with 12 monthly sheets to assist with the recall of further falls"

Not applicable
Quote: "follow-up was done by postal questionnaire, which was sent to all participants every 4 months for 1 year after the fall. Information about subsequent falls, fallrelated injury, and details of doctor and hospital visits or admissions and degree of function were requested"

Incomplete outcome data (attrition bias) High risk All outcomes
More than $20 \%$ missing outcome data, losses balanced across groups with similar reasons for missing data

1. Medical and occupational therapy assessments and interventions: randomised $\mathrm{n}=$ 184 , analysed $n=141$ (18 moved to institutional care, 19 died, 6 otherwise lost to follow-up)

2. Control usual care: randomised $n=213$, analysed $\mathrm{n}=163$ (18 moved to institutional care, 27 died, 5 otherwise lost to followup)

All prespecified outcomes reported

Self-reports by study participants through "falls diary"

\section{Coleman 1999}

\begin{tabular}{ll}
\hline Methods & Study design: Cluster-RCT (by physician practice) \\
& Number of study arms: 2 \\
& Number of clusters: 9 \\
& Study centres: Multiple centres \\
& Length of follow-up: 12 months \\
\hline Participants & Setting: United States of America \\
& Number randomised: 169 \\
& Number analysed: 142 \\
& Number lost to follow-up: 27 \\
Sample: Community-dwelling men and women in 9 physician practices in an ambulatory \\
clinic
\end{tabular}




\begin{tabular}{|c|c|}
\hline & $\begin{array}{l}\text { Age (years): mean } 77 \\
\text { Sex: } 49 \% \text { women } \\
\text { Ethnicity: Not reported } \\
\text { Inclusion criteria: Community-dwelling adults aged } \geq 65 \text {; high risk of being hospitalised } \\
\text { or of developing functional decline } \\
\text { Exclusion criteria: Living in nursing home; terminal illness; moderate to severe dementia } \\
\text { or "too ill" (physician's judgement) }\end{array}$ \\
\hline Interventions & $\begin{array}{l}\text { Type of intervention: Multifactorial intervention } \\
\text { 1. Half-day Chronic Care Clinics every } 3 \text { to } 4 \text { months in } 5 \text { practices focusing on planning } \\
\text { chronic disease management (physician and nurse); reducing polypharmacy and high- } \\
\text { risk medications (pharmacist); patient self-management/support group }(\mathrm{n}=73) \\
\text { 2. Control: usual care }(\mathrm{n}=96 \text { ) } \\
\text { Who delivered intervention: Multidisciplinary team } \\
\text { Compliance assessed: Semi-structured interventions with physicians' perceived ability to } \\
\text { provide comprehensive primary care to their frail older patients }\end{array}$ \\
\hline Outcomes & $\begin{array}{l}\text { 1. Number of people sustaining } 1 \text { or more falls } \\
\text { 2. Number of people who experienced a fall that required hospital admission } \\
\text { 3. Health-related quality of life (measured using SF } 36 \text { - physical function score) }\end{array}$ \\
\hline Notes & $\begin{array}{l}\text { Source of funding: Robert Wood Foundation Chronic Care Initiative } \\
\text { Conflicts of interest: Not reported } \\
\text { Economic information: Cost analysis reported as Multifactorial intervention USD } 9535 \\
\text { a year and Usual care USD 10,116 a year }\end{array}$ \\
\hline
\end{tabular}

\section{Risk of bias}

\section{Bias}

Random sequence generation (selection Unclear risk bias)

Allocation concealment (selection bias) Unclear risk

Blinding of participants and personnel Unclear risk (performance bias)

All outcomes

Blinding of outcome assessment (detection Unclear risk bias)

Falls and fallers

Blinding of outcome assessment (detection Low risk bias)

Fractures

\section{Support for judgement}

Quote: "randomized using simple randomization"

\section{Insuffient information}

Participants and personnel not blind to allocated group but impact of non-blinding unclear

Participant self-reported fall information. No further information given

Not applicable 
Coleman 1999 (Continued)

Blinding of outcome assessment (detection Unclear risk

Participant self-reported information

bias)

Hospital admission \& medical attention

Incomplete outcome data (attrition bias) Unclear risk

All outcomes

Less than $20 \%$ missing outcome data, unbalanced losses across groups with similar reasons for missing data

1. Multifactorial intervention: randomised $\mathrm{n}=96$, analysed $\mathrm{n}=79$ (7 refusal, 3 lost to follow-up, 5 died, 2 other)

2. Usual care: randomised $\mathrm{n}=73$, analysed $\mathrm{n}=63$ (2 refusal, 2 lost to follow-up, 5 died, 1 other)

\begin{tabular}{|c|c|c|}
\hline Selective reporting (reporting bias) & Low risk & All prespecified outcomes were reported \\
\hline Method of ascertaining falls & Unclear risk & Participant self-reported fall information \\
\hline Relating to cluster randomisation & High risk & $\begin{array}{l}\text { Recruitment bias: participants were re- } \\
\text { cruited and randomised based on risk score } \\
\text { for all participants at the same time (low } \\
\text { risk) } \\
\text { Baseline imbalance: baseline similar be- } \\
\text { tween intervention arms (low risk) } \\
\text { Loss of clusters: no clusters lost from the } \\
\text { trial (low risk) } \\
\text { Incorrect analysis: the trial did not adjust } \\
\text { for clustering (high risk) } \\
\text { Comparability: results comparable with in- } \\
\text { dividually-randomised trials (low risk) }\end{array}$ \\
\hline
\end{tabular}

Davison 2005

Methods
Study Design: RCT (parallel design)

Number of study arms: 2

Study centres: Unclear

Length of follow-up: 12 months
Setting: United Kingdom

Number randomised: 313

Number analysed: 282

Number lost to follow-up: 31

Sample: People presenting at A\&E with a fall or fall-related injury

Age (years): mean 77 (SD 7)

Sex: $72 \%$ women

Ethnicity: Not reported

Inclusion criteria: age $>65$ years, presenting at A\&E with a fall or fall-related injury; history of at least 1 additional fall in previous year; community-dwelling 
Exclusion criteria: cognitively impaired (MMSE < 24); > 1 previous episode of syncope; immobile; live $>15$ miles away from A\&E; registered blind; aphasic; clear medical explanation for their fall, e.g. acute myocardial infarction, stroke, epilepsy; enrolled in another study

Interventions

Notes

Outcomes
Type of intervention: Multifactorial intervention

1. Multifactorial post-fall assessment and intervention: Hospital-based medical assessment and intervention: fall history and examination including medications, vision, cardiovascular assessment, laboratory blood tests, ECG. Home-based physiotherapist assessment and intervention: gait, balance, assistive devices, footwear. Home-based OT home-hazard assessment and interventions. $(\mathrm{n}=159)$

2. Control: usual care $(\mathrm{n}=154)$

Who delivered the intervention: Not reported

Compliance assessed: Yes. It was recorded whether participants followed certain recommendations

1. Rate of falls

2. Number of people sustaining 1 or more falls

3. Number of people sustaining 1 or more fall-related fractures

4. Number of people who experienced a fall that required hospital admissions

5. Number of people who experienced a fall that requires medical attention (e.g. attendance at emergency, requiring GP consultation)

Source of Funding: Wellcome Trust and Northern and Yorkshire NHS Executive

Conflicts of interest: None

Economic information: Not reported

\section{Risk of bias}

Bias Authors' judgement $\quad$ Support for judgement

Random sequence generation (selection Low risk bias)

Randomised by computer-generated block randomisation

Allocation concealment (selection bias) Unclear risk Insufficient information to permit judgement

Blinding of participants and personnel Unclear risk (performance bias)

All outcomes

Participants and personnel not blind to allocated group but impact of non-blinding unclear

Blinding of outcome assessment (detection Low risk bias)

Falls and fallers
Quote: "Fall data were collected prospectively by fall diaries, with four weekly cards per diary, returned every 4 weeks over 12 months. There was telephone prompting to maximise compliance. Subjects were asked to detail the frequency and circumstances of each fall” 
Davison 2005 (Continued)

Blinding of outcome assessment (detection Unclear risk bias)

Fractures

Blinding of outcome assessment (detection Low risk bias)

Hospital admission \& medical attention
Insufficient information to permit judgement

Quote: "Hospital and A\&E attendances were recorded prospectively,prompted by diary reports, and hospital records were checked retrospectively at 1 year for all participants. For each episode, an independent reviewer determined whether attendances were fall-related"

Incomplete outcome data (attrition bias) Low risk All outcomes

Less than $20 \%$ missing outcome data, losses balanced across groups with similar reasons for missing data

1. Multifactorial post-fall assessment and intervention: randomised $\mathrm{n}=159$, analysed $\mathrm{n}=141$ ( 1 withdrew and died, 2 died, 15 withdrew)

2. Usual care: randomised $\mathrm{n}=154$, analysed $\mathrm{n}=141$ (1 withdrew and died, 4 died, 8 withdrew)

\begin{tabular}{|c|c|c|}
\hline Selective reporting (reporting bias) & Low risk & All prespecified outcomes were reported \\
\hline Method of ascertaining falls & Low risk & $\begin{array}{l}\text { Quote: "Fall data were collected prospec- } \\
\text { tively by fall diaries, with four weekly cards } \\
\text { per diary, returned every } 4 \text { weeks over } 12 \\
\text { months. There was telephone prompting to } \\
\text { maximise compliance. Subjects were asked } \\
\text { to detail the frequency and circumstances } \\
\text { of each fall" }\end{array}$ \\
\hline
\end{tabular}

Day 2002

Methods Study design: RCT ( 2 x 2 factorial design $)$

Number of study arms: 8

Study centres: Multiple centres

Length of follow-up: 18 months

\begin{tabular}{ll}
\hline Participants & Setting: Australia \\
& Number randomised: 1107 \\
& Number analysed: 1090 \\
Number lost to follow-up: 17 \\
Sample: Community-dwelling men and women identified from electoral roll \\
Age (years): mean 76.1 (SD 5.0) \\
Sex: $60 \%$ women \\
Ethnicity: Mainly Australian-born
\end{tabular}

Multifactorial and multiple component interventions for preventing falls in older people living in the community (Review) 
Inclusion criteria: Aged $\geq 70$; community-dwelling and able to make modifications; expected to remain in area for 2 years (except for short absences); have approval of family physician

Exclusion criteria: Undertaken regular to moderate exercise with a balance component in previous 2 months; unable to walk 10 to 20 metres without rest or help or having angina; severe respiratory or cardiac disease; psychiatric illness prohibiting participation; dysphasia; recent major home modifications; education- and language-adjusted score > 4 on the Short Portable Mental Status Questionnaire

Interventions

Type of intervention: Multiple intervention

1. Exercise: 1-hour class a week for 15 weeks, plus daily home exercises. Designed by physiotherapist to improve flexibility, leg strength, and balance (or less demanding routine depending on participant's capability) $(\mathrm{n}=135)$

2. Home hazard management: home assessed by "trained assessor", hazards removed or modified by participants or City of Whitehorse's home maintenance programme. Staff visited home, provided quote for work including free labour and materials up to AUD $100(\mathrm{n}=136)$

3. Vision improvement: assessed at baseline using dual visual acuity chart. Referred to usual eye care provider, general practitioner, or local optometrist if not already receiving treatment for identified impairment $(\mathrm{n}=139)$
4. $(1)+(2)(n=135)$
5. $(1)+(3)(\mathrm{n}=136)$
6. $(3)+(2)(\mathrm{n}=137)$
7. $(1)+(2)+(3)(n=135)$

8. No intervention. Received brochure on eye care for over 40-year olds $(n=137)$

Who delivered interventions: Multidisciplinary team

Compliance assessed: Not reported

Outcomes

1. Rate of falls

2. Number of people sustaining 1 or more falls

Notes

Source of funding: National Health and Medical Research Council, Australia

Conflicts of interest: None

Economic information: Not reported

\section{Risk of bias}

\section{Bias}

Random sequence generation (selection Low risk bias)

\section{Support for judgement}

Randomised by "adaptive biased coin" technique, to ensure balanced group numbers

Computer-generated by an independent third party contacted by telephone

Participants and personnel not blind to allocated group but impact of non-blinding unclear 
Day 2002 (Continued)

Blinding of outcome assessment (detection Low risk bias)

Falls and fallers
Falls recorded monthly by participants returning postcard calenders

\begin{tabular}{|c|c|c|}
\hline $\begin{array}{l}\text { Blinding of outcome assessment (detection } \\
\text { bias) } \\
\text { Fractures }\end{array}$ & Unclear risk & Not applicable \\
\hline $\begin{array}{l}\text { Blinding of outcome assessment (detection } \\
\text { bias) } \\
\text { Hospital admission \& medical attention }\end{array}$ & Unclear risk & Not applicable \\
\hline $\begin{array}{l}\text { Incomplete outcome data (attrition bias) } \\
\text { All outcomes }\end{array}$ & Unclear risk & $\begin{array}{l}\text { Less than } 20 \% \text { missing outcome data, but } \\
\text { distribution across groups and reasons not } \\
\text { reported, randomised } n=1107 \text {, analysed } n \\
=1090\end{array}$ \\
\hline Selective reporting (reporting bias) & Low risk & All prespecified outcomes were reported \\
\hline Method of ascertaining falls & Low risk & $\begin{array}{l}\text { Falls recorded monthly by participants re- } \\
\text { turning postcard calenders }\end{array}$ \\
\hline
\end{tabular}

\section{De Vries 2010}

\begin{tabular}{ll} 
Methods & $\begin{array}{l}\text { Study design: RCT (parallel design) } \\
\text { Number of study arms: } 2 \\
\text { Study centres: Multiple centres } \\
\text { Length of follow-up: } 12 \text { months }\end{array}$ \\
\hline Participants & Setting: The Netherlands \\
& Number randomised: 217 \\
& Number analysed: 187 \\
& Number lost to follow-up: 30 \\
& Sample: People consulting ED or family physician after a fall \\
& Age (years): Mean 79.8 (SD 7.35) \\
Sex: $71 \%$ women & Ethnicity: Not reported \\
Inclusion criteria: Aged $\geq 65$ years; living independently or in assisted living facility; \\
living near University Medical Center; history of falling in previous 3 months \\
Exclusion criteria: Unable to sign informed consent or provide a fall history; cognitive \\
impairment (MMSE < 24); fall due to traffic or occupational accident; living in nursing \\
home; acute pathology requiring long-term rehabilitation, e.g. stroke
\end{tabular}

Interventions

Type of intervention: Multifactorial intervention

1. Multidisciplinary intervention: Multidisciplinary assessment in geriatric outpatient clinic and individually-tailored treatment in collaboration with participant's GP, e.g. withdrawal of psychotropic drugs, balance and strength exercises, home-hazard reduction, referral to specialists $(\mathrm{n}=106)$ 
De Vries 2010 (Continued)

2. Control: usual care $(\mathrm{n}=111)$

Who delivered the intervention: Geriatrician, physical therapist, occupational therapist, opthalmologist, family physician, cardiologist

Compliance assessed: Yes, during the second home visit in the intervention group, adherence to the treatment regimen was evaluated by recommendation given. Questionnaires at 3 and 6 months and interview also provided adherence data

Outcomes

1. Number of people sustaining 1 or more falls

2. Number of people sustaining recurrent falls

3. Number of people sustaining 1 or more fall-related fractures

4. Health-related quality of life (EQ-5D 0 - 1: change score for overall QoL; SF-36 physical subscale 0 - 100: change score for physical QoL)

Notes
Cource of funding: Not reported
Conflicts of interest: Not reported
Economic information: The total mean costs were EUR 7740 (SD 9129) in the inter-
vention group and EUR 6838 (SD 8623) in the usual care group. The intervention and
usual care groups did not differ in total costs (EUR 902, 95\% CI -1534 to 3357).
Also, the mean healthcare costs and the mean participant and family costs did not differ
significantly between the groups
The percentage of fallers was $4.0 \%$ lower in the intervention group as compared with
the usual care group and the costs were EUR 902 higher, resulting in an ICER of 226.
In other words, the costs per percentage decrease in fallers are EUR 226. Since the
percentage of recurrent fallers was higher in the intervention than in the usual care group,
the ICER for recurrent falling was negative (ICER - 280)
This indicates that if EUR 10,000 were invested, the probability that the intervention
would reduce the percentage of fallers by $1 \%$ was 0.80. Likewise, if EUR 300,000 were
invested, the probability that the intervention would improve the quality of life (utility)
by one point was only about 0.30 . Since the costs were higher and effects were smaller
for the outcome recurrent fallers, the intervention was not cost-effective at any given
ceiling ratio

Risk of bias

Bias

Random sequence generation (selection Low risk bias)

Allocation concealment (selection bias) Low risk

\section{Support for judgement}

Quote: “computer-generated random sequence"

Quote: “...opaque envelopes are numbered and filled with group names. When a participant is designated to the high-risk group, the interviewer, who is unaware of the content, opens the envelope with the lowest number." (from protocol paper)

Quote: "Participants, intervention caregivers, and interviewers could not be blinded to group assignment." but impact
Blinding of participants and personnel Unclear risk (performance bias)

All outcomes

Multifactorial and multiple component interventions for preventing falls in older people living in the community (Review)

Copyright ( 2018 The Cochrane Collaboration. Published by John Wiley \& Sons, Ltd. 
De Vries 2010 (Continued)

of non-blinding unclear

\begin{tabular}{|c|c|c|}
\hline $\begin{array}{l}\text { Blinding of outcome assessment (detection } \\
\text { bias) } \\
\text { Falls and fallers }\end{array}$ & Low risk & $\begin{array}{l}\text { Falls recorded weekly by participants by the } \\
\text { use of a falls calender }\end{array}$ \\
\hline $\begin{array}{l}\text { Blinding of outcome assessment (detection } \\
\text { bias) } \\
\text { Fractures }\end{array}$ & High risk & $\begin{array}{l}\text { Quote: "By their response to a question- } \\
\text { naire sent } 1.5 \text { years after the first home visit, } \\
\text { participants were asked to indicate whether } \\
\text { they had sustained a fracture since the first } \\
\text { home visit" }\end{array}$ \\
\hline
\end{tabular}

Blinding of outcome assessment (detection Unclear risk

Not applicable bias)

Hospital admission \& medical attention

Incomplete outcome data (attrition bias) Low risk All outcomes
Less than $20 \%$ missing outcome data, losses balanced across groups

1.Multidisciplinary intervention: randomised $\mathrm{n}=106$, analysed $\mathrm{n}=93$ (1 died, 11 no reasons given, 1 objected to procedure)

2.Usual care: randomised $\mathrm{n}=111$, analysed $\mathrm{n}=94$ (7 died, 9 no reasons given, $1 \mathrm{did}$ not expect to benefit)

\begin{tabular}{|c|c|c|}
\hline Selective reporting (reporting bias) & Low risk & All prespecified outcomes were reported \\
\hline Method of ascertaining falls & Low risk & $\begin{array}{l}\text { Falls recorded weekly by participants by the } \\
\text { use of a falls calender }\end{array}$ \\
\hline
\end{tabular}

Elley 2008

Methods

Study Design: RCT (parallel design)

Number of study arms: 2

Study centres: Multiple centres

Length of follow-up: 12 months

Participants Setting: New Zealand

Number randomised: 312

Number analysed: 280

Number lost to follow-up: 32

Sample: Patients from 19 primary care practices

Age (years): Mean 80.8 (SD 5)

Sex: $69 \%$ women

Ethnicity: 9 participants identified themselves as either Maori or Pacific.

Inclusion criteria: Aged $\geq 75$ ( $>50$ years for Maori and Pacific people), fallen in last year, living independently 
Exclusion criteria: Unable to understand study information and consent processes, unstable or progressive medical condition, severe physical disability, dementia ( $<7$ on AMT Score)

Interventions

Type of intervention: Multifactorial intervention

1. Intervention: Community-based nurse assessment of falls and fracture risk factors, home hazards, referral to appropriate community interventions, and strength and balance exercise programme $(\mathrm{n}=155)$

2. Control: usual care and social visits $(\mathrm{n}=157)$

Who delivered the intervention: Nurse, family physician, OT, optometrist, physiotherapist, podiatrist, physiotherapist, physical therapist, continence nurse

Compliance assessed: Yes, the intervention assessment was usually undertaken at 1 visit. The nurse telephoned 2 - 4 weeks later to ensure referral consultations had taken place

\begin{tabular}{ll} 
Outcomes & $\begin{array}{l}\text { 1. Rate of falls } \\
\text { 2. Number of people sustaining } 1 \text { or more falls } \\
\text { 3. Number of people sustaining recurrent falls } \\
\text { 4. Health-related quality of life }\end{array}$ \\
\hline Notes & $\begin{array}{l}\text { Source of funding: The New Zealand ACC, the New Zealand Lotteries Commission, } \\
\text { the Wellington Medical Research Foundation, the University of Otago, and the Hutt } \\
\text { Valley District Health Board } \\
\text { Conflicts of interest: None } \\
\text { Economic information: Not reported }\end{array}$ \\
\hline
\end{tabular}

\section{Risk of bias}

Bias

Random sequence generation (selection Low risk bias)

Allocation concealment (selection bias) Low risk

Blinding of participants and personnel Unclear risk (performance bias)

All outcomes

Blinding of outcome assessment (detection Low risk bias)

Falls and fallers

Blinding of outcome assessment (detection Unclear risk bias)

Fractures

Blinding of outcome assessment (detection Unclear risk bias)

\section{Support for judgement}

Quote: “computer randomisation"

Quote: “independent researcher at a distant site"

Participants and personnel not blind to allocated group but impact of non-blinding unclear

Falls were recorded daily by participants and posted monthly to the research team

Not applicable

Not applicable 
Elley 2008 (Continued)

Hospital admission \& medical attention

Incomplete outcome data (attrition bias) Unclear risk

All outcomes

Less than $20 \%$ missing outcome data with unbalanced losses across groups. Overall, similar reasons for missing data in both arms

1. Intervention group: randomised $\mathrm{n}=155$, analysed $n=135$ ( 4 unwell or cognitive decline, 5 admitted to rest home or hospital, 2 moved away, 2 declined, 7 died)

2. Usual care and social visits: randomised $\mathrm{n}=157$, analysed $\mathrm{n}=145$ (5 admitted to rest home or hospital, 1 unwell or cognitive decline, 1 moved away, 1 declined, 4 died)

\begin{tabular}{|c|c|c|}
\hline Selective reporting (reporting bias) & Low risk & All prespecified outcomes were reported \\
\hline Method of ascertaining falls & Low risk & $\begin{array}{l}\text { Falls were recorded daily by participants } \\
\text { and posted monthly to the research team }\end{array}$ \\
\hline
\end{tabular}

Fabacher 1994

\begin{tabular}{ll} 
Methods & Study design: RCT (parallel design) \\
& Number of study arms: 2 \\
& Study centres: Single centre \\
& Length of follow-up: 12 months \\
\hline Participants & Setting: United States of America \\
& Number randomised: 254 \\
& Number analysed: 195 \\
& Number lost to follow-up: 59 \\
& Sample: Men and women aged $>70$ years and eligible for veterans medical care. Identified \\
& from voter registration lists and membership lists of service organisations \\
& Age (years): Mean 73 \\
Sex: $2 \%$ women & Ethnicity: Participants were predominantly white men (98\%) \\
Inclusion criteria: Aged $\geq 70 ;$ not receiving health care at Veterans Administration \\
Medical Centre \\
Exclusion criteria: Known terminal disease, dementia \\
\hline
\end{tabular}

Interventions

Type of intervention: Multifactorial intervention

1. HAPSA: Home visit by health professional to screen for medical, functional, and psychosocial problems, followed by a letter for participants to show to their personal physician. Targeted recommendations for individual disease states, preventive health practices $(\mathrm{n}=131)$

2. Control: follow-up telephone calls for outcome data only $(\mathrm{n}=123)$

Who delivered the intervention: Physician assistant, nurses, trained volunteers

Compliance assessed: Yes, information on compliance with recommendations was ob-

Multifactorial and multiple component interventions for preventing falls in older people living in the community (Review) 
Fabacher 1994 (Continued)

tained from participants during the follow-up visits

\begin{tabular}{ll} 
Outcomes & $\begin{array}{l}\text { 1. Number of people sustaining } 1 \text { or more falls } \\
\text { 2. Number of people who experienced a fall that required hospital admissions }\end{array}$ \\
\hline Notes & $\begin{array}{l}\text { Source of funding: Disabled American Veterans Charities of Greater Los Angeles and } \\
\text { the Disability American Veterans California Rehabilitation Foundation Inc } \\
\text { Conflicts of interest: Not reported } \\
\text { Economic information: Not reported }\end{array}$ \\
\hline
\end{tabular}

\section{Risk of bias}

\begin{tabular}{ll|l}
\hline Bias & Authors' judgement & Support for judgement \\
\hline $\begin{array}{l}\text { Random sequence generation (selection } \\
\text { bias) }\end{array}$ & Unclear risk & $\begin{array}{l}\text { Quote: "randomly assigned ... using ran- } \\
\text { domly generated assignment cards in sealed } \\
\text { envelopes". Judged to be unclear }\end{array}$ \\
\hline Allocation concealment (selection bias) & Unclear risk & $\begin{array}{l}\text { Quote: "randomly assigned ... using ran- } \\
\text { domly generated assignment cards in sealed } \\
\text { envelopes". Judged to be unclear }\end{array}$ \\
\hline
\end{tabular}

Blinding of participants and personnel Unclear risk (performance bias)

All outcomes

Participants and personnel not blind to allocated group but impact of non-blinding unclear

Blinding of outcome assessment (detection High risk bias)

Falls data collected by self-reports at 12

Falls and fallers month follow-up interview

Blinding of outcome assessment (detection Unclear risk

bias)

Not applicable

Fractures

Blinding of outcome assessment (detection High risk bias)

Hospital admission \& medical attention

Incomplete outcome data (attrition bias) High risk All outcomes
More than $20 \%$ missing outcome data with losses balanced across both groups

1. HAPSA: randomised $\mathrm{n}=131$, analysed $\mathrm{n}=100$ (13 refused initial assessment, 5 refused follow-up visits, 3 moved, 4 died, 6 logistic reasons)

2. Control: randomised $\mathrm{n}=123$, analysed $n=95$ (15 refused follow-up visits, 9 moved, 4 died) 
Fabacher 1994 (Continued)

\begin{tabular}{l|ll}
\hline Selective reporting (reporting bias) & Unclear risk & $\begin{array}{l}\text { Fall rates reported to be similar across } \\
\text { groups but numerical values were not given }\end{array}$ \\
\hline Method of ascertaining falls & High risk & $\begin{array}{l}\text { Falls were self-reported at 12-month fol- } \\
\text { low-up interview }\end{array}$ \\
\hline
\end{tabular}

Faes 2011

Methods

Study design: Parallel RCT

Number of study arms: 2

Study centres: Multiple centres

Length of follow-up: Trial terminated due to "Extremely difficult recruitment"

Setting: The Netherlands
Pumber randomised: Not reported - target sample 160 people plus their carer (N = 320)
Number analysed: Not reported
NUmber lost to follow-up: Not reported
Sample: Patients recruited from 3 geriatric outpatient clinics
Age (years): mean 78.3 (SD 7)
Sex: 70\% women
Inclusion criteria: Fallen in previous 6 months; able to walk 15 metres independently
(with or without walking aid); had a primary informal caregiver; community-dwelling;
life expectancy > 1 year; frail ( $\geq 2$ frailty indicators)
Exclusion criteria: Awaiting nursing home admission; MMSE $<15$

Interventions $\quad$ Type of intervention: Multiple intervention

1. Psychological teaching and training + physical training in small groups. $10 \times 2$-hour sessions twice a week + booster session 6 weeks later. Caregivers trained in autonomyboosting strategies, and being co-therapist at home

2. Control: usual care

Who delivered intervention: Not reported

Compliance assessed: Not reported

Outcomes

1. Number of people sustaining 1 or more falls

2. Number of people sustaining recurrent falls

3. Health-related quality of life (EQ-5D VAS 0 - 100: change score)

Notes

Source of funding: The Netherlands Organisation for Health Research and Development (920-03-457) and the NUTS-Ohra Fund (0601-60) and a career development sponsorship acquired from the Radboud University Nijmegen Medical Centre, The Netherlands Conflicts of interest: None

Economic information: Not reported

\section{Risk of bias}

Bias

Authors' judgement

Support for judgement

Multifactorial and multiple component interventions for preventing falls in older people living in the community (Review) 
Faes 2011 (Continued)

\begin{tabular}{|c|c|c|}
\hline $\begin{array}{l}\text { Random sequence generation (selection } \\
\text { bias) }\end{array}$ & Low risk & $\begin{array}{l}\text { Quote: "Treatment allocation... was based } \\
\text { on a minimization algorithm that balanced } \\
\text { for the minimization factors" }\end{array}$ \\
\hline Allocation concealment (selection bias) & Low risk & $\begin{array}{l}\text { Quote: "allocation, carried out by an inde- } \\
\text { pendent statistician" }\end{array}$ \\
\hline $\begin{array}{l}\text { Blinding of participants and personnel } \\
\text { (performance bias) } \\
\text { All outcomes }\end{array}$ & Unclear risk & $\begin{array}{l}\text { Participants and personnel not blind to al- } \\
\text { located group but impact of non-blinding } \\
\text { unclear }\end{array}$ \\
\hline $\begin{array}{l}\text { Blinding of outcome assessment (detection } \\
\text { bias) } \\
\text { Falls and fallers }\end{array}$ & Unclear risk & $\begin{array}{l}\text { Insufficent information to provide judge- } \\
\text { ment }\end{array}$ \\
\hline $\begin{array}{l}\text { Blinding of outcome assessment (detection } \\
\text { bias) } \\
\text { Fractures }\end{array}$ & Unclear risk & Not applicable \\
\hline $\begin{array}{l}\text { Blinding of outcome assessment (detection } \\
\text { bias) } \\
\text { Hospital admission \& medical attention }\end{array}$ & Unclear risk & Not applicable \\
\hline $\begin{array}{l}\text { Incomplete outcome data (attrition bias) } \\
\text { All outcomes }\end{array}$ & High risk & $\begin{array}{l}\text { The trial was terminated due to "Extremely } \\
\text { difficult recruitment". No data are pro- } \\
\text { vided on the number of participants anal- } \\
\text { ysed }\end{array}$ \\
\hline Selective reporting (reporting bias) & High risk & $\begin{array}{l}\text { The trial was terminated due to "Extremely } \\
\text { difficult recruitment". No data are pro- } \\
\text { vided on the number of participants anal- } \\
\text { ysed }\end{array}$ \\
\hline Method of ascertaining falls & Unclear risk & $\begin{array}{l}\text { Insufficent information to provide judge- } \\
\text { ment }\end{array}$ \\
\hline
\end{tabular}

Fairhall 2014

\begin{tabular}{ll} 
Methods & Study Design: RCT (parallel design) \\
& Number of study arms: 2 \\
& Study centres: Single centre \\
& Length of follow-up: 12 months \\
\hline Participants & Setting: Australia \\
& Number randomised: 241 \\
& Number analysed: 216 \\
& Number lost to follow-up: 25 \\
& Sample: Potential participants were identified from older people being discharged from
\end{tabular}


Fairhall 2014 (Continued)

the Division of Rehabilitation and Aged care services at Hornsby Ku-ring-gai Health Service, Sydney, Australia

Age (years): Mean 83.3 (SD 5.9)

Sex: $67 \%$ women

Ethnicity: Not reported

Inclusion criteria: 70 years or older, frail (met specified cut-offs for 3 or more of the CHS frailty criteria : slow gait, weak grip, exhaustion, low energy expenditure, and weight loss), did not live in a residential aged care facility, had a MMSE score $>18$ and life expectancy of at least 12 months (a modified Implicit illness Severity Scale score $\leq 3$ )

Exclusion criteria: Lives in residential aged care facility

Interventions

Outcomes
Type of intervention: Multifactorial intervention

1. Multifactorial intervention: An individualised home-exercise programme prescribed in 10 home visits from a physiotherapist and interdisciplinary management of medical, psychological and social problems $(\mathrm{n}=120)$

2. Control: usual care $(\mathrm{n}=121)$

Who delivered the intervention: Physiotherapists, geriatrician, rehabilitation physician, dietician, nurses, OTs

Compliance assessed: Yes, adherence to home-exercise sessions

\begin{tabular}{ll}
\hline Outcomes & 1. Rate of falls \\
2. Number of people sustaining 1 or more falls \\
3. Number of people sustaining recurrent falls \\
4. Number of people sustaining 1 or more fall-related fracture \\
5. Health-related quality of life \\
6. Adverse effects of the intervention \\
\hline
\end{tabular}

Source of Funding: Supported by Australian National Health and Medical Research Council Health Services Research Grant

Conflicts of interest: None

Economic information: Not reported

Adverse events: "Two intervention group participants experienced back pain consistent with the study definition of an adverse event: a medical event or injury that restricted activities of daily living for more than 2 days or resulted in medical attention [26]. Both participants recommenced exercise following modification of the exercise program.”

Risk of bias

\begin{tabular}{l|l|l} 
Bias & Authors' judgement & Support for judgement \\
\hline $\begin{array}{l}\text { Random sequence generation (selection } \\
\text { bias) }\end{array}$ & Low risk & $\begin{array}{l}\text { Quote: "The group allocation schedule was } \\
\text { generated and managed by an investigator } \\
\text { independent of participant recruitment us- } \\
\text { ing a computer generated random number } \\
\text { schedule with varying block sizes." }\end{array}$ \\
\hline
\end{tabular}

$\begin{array}{lll}\text { Allocation concealment (selection bias) } \quad \text { Unclear risk } & \text { Not reported }\end{array}$ 
Fairhall 2014 (Continued)

Blinding of participants and personnel Unclear risk (performance bias)

All outcomes

Blinding of outcome assessment (detection Low risk

bias)

Falls and fallers
Participants and personnel not blinded to allocated group but impact on blinding unclear

Falls were monitored prospectively using monthly calender with follow-up telephone call

Not reported

Blinding of outcome assessment (detection Unclear risk

bias)

Fractures

Blinding of outcome assessment (detection Unclear risk bias)

Hospital admission \& medical attention

Incomplete outcome data (attrition bias) Low risk All outcomes

Less than $20 \%$ missing outcome data, losses are balanced across groups with similar reasons for missing data

1. Multifactorial intervention: randomised $\mathrm{n}=120$, analysed $\mathrm{n}=107$ (12 died unrelated to trial protocol, 1 withdrew)

2. Usual care: randomised $n=121$, analysed $\mathrm{n}=109$ (10 died unrelated to trial protocol, 2 withdrew)

\begin{tabular}{|c|c|c|}
\hline Selective reporting (reporting bias) & Low risk & All outcomes listed were reported \\
\hline Method of ascertaining falls & Low risk & $\begin{array}{l}\text { Falls data were collected by monthly calen- } \\
\text { ders and telephone calls }\end{array}$ \\
\hline
\end{tabular}

Ferrer 2014

Methods

Study Design: RCT (parallel design)

Number of study arms: 2

Study centres: Single centre

Length of follow-up: 12 months
Setting: Barcelona, Spain

Number randomised: 328

Number analysed: 273

Number lost to follow-up: 55

Sample: All community-dwelling individuals born in 1924, and registered at 1 of the 7 healthcare centres in Baix Llobregrat, Barcelona

Age (years): Mean 81

Sex: $61.6 \%$ female

Ethnicity: Not reported

Inclusion criteria: Age of 85 
Ferrer 2014 (Continued)

Exclusion criteria: Being institutionalised

\begin{tabular}{|c|c|}
\hline Interventions & $\begin{array}{l}\text { Type of intervention: Multifactorial intervention } \\
\text { 1. Multifactorial intervention: Specific algorithm identifying } 9 \text { areas of potentially mod- } \\
\text { ifiable risk factors for falls, including psychotropic and cardiovascular use, auditory acu- } \\
\text { ity, visual acuity, balance and gait disorders, cognitive impairment, risk of malnutrition, } \\
\text { disability, social risk and home safety }(\mathrm{n}=164) \\
\text { 2. Control: Usual care }(\mathrm{n}=164) \\
\text { Who delivered the intervention: Physician, opthalmologist, physical therapist, physio- } \\
\text { therapist, dietician, healthcare professional with specialised training in geriatrics } \\
\text { Compliance assessed: Yes, adherence to recommendations was monitored by quarterly } \\
\text { visits or telephone calls made by the therapist during the first and second years }\end{array}$ \\
\hline Outcomes & $\begin{array}{l}\text { 1. Rate of falls } \\
\text { 2. Number of people sustaining } 1 \text { or more falls } \\
\text { 3. Number of people sustaining recurrent falls }\end{array}$ \\
\hline Notes & $\begin{array}{l}\text { Source of Funding: Fond de Investigation Sanitaria-Institute de Salud Carlos III Spain } \\
\text { Conflicts of interest: None } \\
\text { Economic information: Not reported }\end{array}$ \\
\hline
\end{tabular}

Risk of bias

\begin{tabular}{|c|c|c|}
\hline Bias & Authors' judgement & Support for judgement \\
\hline $\begin{array}{l}\text { Random sequence generation (selection } \\
\text { bias) }\end{array}$ & Low risk & $\begin{array}{l}\text { Quote: "After the baseline questionnaire } \\
\text { had been questionnaire had been adminis- } \\
\text { tered, the subjects were randomised to an } \\
\text { intervention or control group using a com- } \\
\text { puter-generated randomization table" }\end{array}$ \\
\hline Allocation concealment (selection bias) & Unclear risk & No mention of allocation concealment \\
\hline $\begin{array}{l}\text { Blinding of participants and personnel } \\
\text { (performance bias) } \\
\text { All outcomes }\end{array}$ & Unclear risk & $\begin{array}{l}\text { Participants and personnel not blinded to } \\
\text { allocated group but impact on blinding un- } \\
\text { clear }\end{array}$ \\
\hline
\end{tabular}

Blinding of outcome assessment (detection Low risk bias)

Falls and fallers

Blinding of outcome assessment (detection Unclear risk bias)

Fractures

Blinding of outcome assessment (detection Unclear risk bias)

Hospital admission \& medical attention
Falls were monitored prospectively using a monthly calender with a 3-monthly followup telephone call

Not applicable

Not applicable

Multifactorial and multiple component interventions for preventing falls in older people living in the community (Review) 
Incomplete outcome data (attrition bias) Unclear risk All outcomes
Less than $20 \%$ missing outcome data, unbalanced losses across groups with similar reasons for missing data

1. Multifactorial intervention: randomised $\mathrm{n}=164$, analysed $\mathrm{n}=142$ (9 died, 3 moved, 3 nursing home, 7 other)

2. Usual care: randomised $\mathrm{n}=164$, analysed $\mathrm{n}=131$ (8 died, 7 moved, 7 nursing home, 11 other)

All outcomes stated in the Methods were reported

Fall data were collected by monthly selfreports and telephone calls every 3 months
Freiberger 2012

Methods

Method of ascertaining falls

Low risk
Low risk

Study Design: RCT (parallel design)

Number of study arms: 4

Study centre: Single centre

Length of follow-up: 24 months

\section{Setting: Germany}

Number randomised: 280

Number analysed: 201

Number lost to follow-up: 79

Sample: Recruited from health insurance company membership database

Age (years): Mean 76.1 (SD 4.1)

Sex: $44 \%$ women

Ethnicity: Not reported

Inclusion criteria: Community-dwelling adults; aged 70 to 90 ; fallen in the past 6 months or reported fear of falling

Exclusion criteria: Unable to walk independently; cognitive impairment $(<25$ on the DSST)

Interventions

Type of intervention: Multiple intervention

1. "Strength and balance group": strength and balance exercises only $(\mathrm{n}=63)$

2. "Fitness group": strength and balance plus endurance training $(n=64)$

3. "Multifaceted group": strength and balance plus fall-risk education $(n=73)$

4. Control group: No intervention $(n=80)$

Who delivered the intervention: Falls-prevention instructors

Compliance assessed: Yes, session observations and monthly supervision meetings

Outcomes
1. Rate of falls

2. Adverse events of the intervention 
Freiberger 2012 (Continued)

\begin{tabular}{|c|c|c|}
\hline Notes & \multicolumn{2}{|c|}{$\begin{array}{l}\text { Source of funding: Robert Bosch Foundation and Siemens Health Insurance } \\
\text { Conflicts of interest: None } \\
\text { Economic information: Not reported } \\
\text { Adverse events: "No significant adverse events were reported during the study" }\end{array}$} \\
\hline \multicolumn{3}{|l|}{ Risk of bias } \\
\hline Bias & Authors' judgement & Support for judgement \\
\hline $\begin{array}{l}\text { Random sequence generation (selection } \\
\text { bias) }\end{array}$ & Low risk & $\begin{array}{l}\text { Quote: "A third party not involved in } \\
\text { the study applied a computerised random- } \\
\text { number generator" }\end{array}$ \\
\hline Allocation concealment (selection bias) & Low risk & $\begin{array}{l}\text { Quote: "All randomisations were con- } \\
\text { cealed". "A third party not involved in } \\
\text { the study applied a computerised random- } \\
\text { number generator" }\end{array}$ \\
\hline
\end{tabular}

Blinding of participants and personnel Unclear risk (performance bias)

Participants and personnel not blind to al-

All outcomes located group but impact of non-blinding unclear

Blinding of outcome assessment (detection Unclear risk bias)

Falls and fallers

Quote: "Data on falls were collected prospectively using a monthly fall calendar between months 12 and 24; fall sheets were mailed in at the end of the month. Up to five follow-up telephone calls were made in the event of no response after each month. If falls were reported, details were collected during a structured telephone interview"

Blinding of outcome assessment (detection Unclear risk

Not applicable

bias)

Fractures

Blinding of outcome assessment (detection Unclear risk

bias)

Hospital admission \& medical attention

Incomplete outcome data (attrition bias) High risk

All outcomes

More than 20\% loss to follow-up, losses unbalanced across groups. No reasons included for missing data

1. Multifaceted group: randomised $n=73$, analysed $\mathrm{n}=58$

2. Strength and Balance intervention: randomised $n=63$, analysed $n=49$

3. Fitness intervention: randomised $n=64$, analysed $\mathrm{n}=48$ 
Freiberger 2012 (Continued)

4. Control (no intervention): randomised $\mathrm{n}=80$, analysed $\mathrm{n}=52$

\begin{tabular}{|c|c|c|}
\hline Selective reporting (reporting bias) & Low risk & All prespecified outcomes were reported \\
\hline Method of ascertaining falls & Unclear risk & $\begin{array}{l}\text { Quote: "Data on falls were collected } \\
\text { prospectively using a monthly fall calendar } \\
\text { between months } 12 \text { and } 24 \text {; fall sheets were } \\
\text { mailed in at the end of the month. Up to } \\
\text { five follow-up telephone calls were made in } \\
\text { the event of no response after each month. } \\
\text { If falls were reported, details were collected } \\
\text { during a structured telephone interview" }\end{array}$ \\
\hline
\end{tabular}

Gallagher 1996

\begin{tabular}{|c|c|}
\hline Methods & $\begin{array}{l}\text { Study design: RCT (parallel design) } \\
\text { Number of study arms: } 2 \\
\text { Study centres: Unclear } \\
\text { Length of follow-up: } 6 \text { months }\end{array}$ \\
\hline Participants & $\begin{array}{l}\text { Setting: Canada } \\
\text { Number randomised: } 100 \\
\text { Number analysed: } 100 \\
\text { Number lost to follow-up: } 0 \\
\text { Sample: Community-dwelling volunteers } \\
\text { Age (years): Mean } 74.6 \\
\text { Sex: } 80 \% \text { women } \\
\text { Ethnicity: } 92 \% \text { of participants were white } \\
\text { Inclusion criteria: Aged } \geq 60 \text {; fallen in previous } 3 \text { months } \\
\text { Exclusion criteria: None described }\end{array}$ \\
\hline Interventions & $\begin{array}{l}\text { Type of intervention: Multifactorial intervention } \\
\text { 1. Falls-reduction programme: } 2 \text { risk-assessment interviews of } 45 \text { minutes each. } 1 \text { coun- } \\
\text { selling interview of } 60 \text { minutes showing video and booklet and results of risk assessment } \\
(\mathrm{n}=50) \\
\text { 2. Control: baseline interview and follow-up only. No intervention }(\mathrm{n}=50) \\
\text { Who delivered the intervention: Trained nurses were interviewers } \\
\text { Compliance assessed: Yes, checklist of recommendations re-checked at } 6 \text { months follow- } \\
\text { up }\end{array}$ \\
\hline Outcomes & $\begin{array}{l}\text { 1. Rate of falls } \\
\text { 2. Health-related quality of life (SF-36 } 0 \text { - 100: endpoint score) }\end{array}$ \\
\hline Notes & $\begin{array}{l}\text { Source of funding: Not reported } \\
\text { Conflicts of interest: Not reported } \\
\text { Economic information: Not reported }\end{array}$ \\
\hline
\end{tabular}

Multifactorial and multiple component interventions for preventing falls in older people living in the community (Review) 


\section{Gallagher 1996 (Continued)}

\section{Risk of bias}

\begin{tabular}{l|ll}
\hline Bias & Authors' judgement & Support for judgement \\
\hline $\begin{array}{l}\text { Random sequence generation (selection } \\
\text { bias) }\end{array}$ & Unclear risk & Method of randomisation not described \\
\hline $\begin{array}{l}\text { Allocation concealment (selection bias) } \\
\begin{array}{l}\text { Blinding of participants and personnel } \\
\text { (performance bias) } \\
\text { All outcomes }\end{array}\end{array}$ & Unclear risk & Method of randomisation not described \\
\hline
\end{tabular}

Blinding of outcome assessment (detection Low risk bias)

Falls and fallers

Blinding of outcome assessment (detection Unclear risk bias)

Fractures

Blinding of outcome assessment (detection Unclear risk bias)

Hospital admission \& medical attention

Incomplete outcome data (attrition bias) Low risk All outcomes

\begin{tabular}{|c|c|c|}
\hline 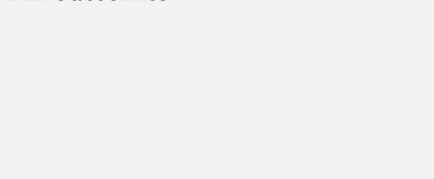 & & $\begin{array}{l}\text { 1. Falls reduction programme: randomised } \\
n=50 \text {, analysed } n=50 \\
\text { 2. Baseline interview and follow-up only: } \\
\text { randomised } n=50 \text {, analysed } n=50\end{array}$ \\
\hline Selective reporting (reporting bias) & Low risk & All prespecified outcomes were reported. \\
\hline Method of ascertaining falls & Low risk & $\begin{array}{l}\text { Falls were monitored prospectively using } \\
\text { a } 2 \text {-week calender with a follow-up tele- } \\
\text { phone call to ascertain details }\end{array}$ \\
\hline
\end{tabular}

\section{Hagovska 2016}

Methods

Study Design: RCT (parallel design)

Number of study arms: 2

Study centres: Single centre

Length of follow-up: 21/2 months
Falls were monitored prospectively using a 2-week calender with a follow-up telephone call to ascertain details

Not applicable
Not applicable

Less than $20 \%$ missing outcome data, as no one dropped out of study

1. Falls reduction programme: randomised $\mathrm{n}=50$, analysed $\mathrm{n}=50$

2. Baseline interview and follow-up only: randomised $n=50$, analysed $n=50$

phone call to ascertain details
Setting: Slovak Republic

Number randomised: 80 
Hagovska 2016 (Continued)

\begin{tabular}{|c|c|}
\hline & $\begin{array}{l}\text { Number analysed: } 78 \\
\text { Number lost to follow-up: } 2 \\
\text { Sample: Elderly patients from the region were referred for diagnosis treatment by psy- } \\
\text { chiatrist/psychologist } \\
\text { Age (years): Mean } 67.07 \\
\text { Sex: } 48.5 \% \text { women } \\
\text { Ethnicity: Not reported } \\
\text { Inclusion criteria: Mild cognitive impairment, encompassing subjective mild decrease in } \\
\text { memory and attention domains, Age } 65 \text { - } 75 \\
\text { Exclusion criteria: Moderate and severe cognitive deficits of MMSE, major depressive } \\
\text { and anxiety disorder, cancer, significant visual and auditory damage, prior history of } \\
\text { neurological disease or brain injury, psychiatric disorders }\end{array}$ \\
\hline Interventions & $\begin{array}{l}\text { Type of intervention: Multiple intervention } \\
\text { 1. Multiple intervention: Cogniplus programme and balance training }(\mathrm{n}=40) \\
\text { 2. Control: usual care }(\mathrm{n}=40) \\
\text { Who delivered the intervention: psychiatrist, psychologist } \\
\text { Compliance assessed: Not reported }\end{array}$ \\
\hline Outcomes & 1. Health-related quality of life (Quality of life assessment 0 - 10: endpoint score) \\
\hline Notes & $\begin{array}{l}\text { Source of Funding: No funding } \\
\text { Conflicts of interest: None } \\
\text { Economic information: Not reported }\end{array}$ \\
\hline
\end{tabular}

Risk of bias

Bias

Random sequence generation (selection Low risk bias)
Authors' judgement

Allocation concealment (selection bias) Low risk

\section{Support for judgement}

Quote: “The project's data analyst generated a random sequence of numbers to arbitrarily select probands for the experimental group and control using Excel 2010"

Quote: “These numbers were put in a subsequently sealed envelope. The project manager opened the envelope and informed participating persons of their assignment to either groups"

Quote: "patients were not told what kind of intervention they would undergo, training staff was not blinded"

Not applicable
Blinding of participants and personnel Unclear risk (performance bias)

All outcomes

Blinding of outcome assessment (detection Unclear risk bias)

Falls and fallers 
Hagovska 2016 (Continued)

\begin{tabular}{|c|c|c|}
\hline $\begin{array}{l}\text { Blinding of outcome assessment (detection } \\
\text { bias) } \\
\text { Fractures }\end{array}$ & Unclear risk & Not applicable \\
\hline $\begin{array}{l}\text { Blinding of outcome assessment (detection } \\
\text { bias) } \\
\text { Hospital admission \& medical attention }\end{array}$ & Unclear risk & Not applicable \\
\hline $\begin{array}{l}\text { Incomplete outcome data (attrition bias) } \\
\text { All outcomes }\end{array}$ & Unclear risk & $\begin{array}{l}\text { Less than } 20 \% \text { missing outcome data, losses } \\
\text { are unbalanced across groups } \\
\text { 1. Cogniplus programme }+ \text { balance train- } \\
\text { ing: randomised } n=40 \text {, analysed } n=40 \\
\text { 2. Usual care: randomised } n=40 \text {, analysed } \\
n=38 \text { ( } 2 \text { did not complete training, respi- } \\
\text { ratory disease) }\end{array}$ \\
\hline Selective reporting (reporting bias) & Low risk & $\begin{array}{l}\text { All outcomes listed in the abstract were re- } \\
\text { ported }\end{array}$ \\
\hline Method of ascertaining falls & Unclear risk & Not applicable \\
\hline
\end{tabular}

\section{Hendriks 2008}

Methods

Study design: RCT with economic evaluation (parallel design)

Number of study arms: 2

Study centres: Single centre

Length of follow-up: 12 months

Participants

Setting: The Netherlands

Number randomised: 333

Number analysed: 258

Number lost to follow-up: 75

Sample: People who have visited an ED or a GP because of a fall

Age (years): Mean 74.8 (SD 6.4)

Sex: $68 \%$ women

Ethnicity: Not reported

Inclusion criteria: $\geq 65$ years; community-dwelling; history of a fall requiring visit to ED or GP; living in Maastricht area

Exclusion criteria: Not able to speak or understand Dutch; unable to complete questionnaires or interviews by telephone; cognitive impairment (< 4 on AMT4); long-term admission to hospital or other institution ( $>4$ weeks from date of inclusion); permanently bedridden; fully dependent on a wheelchair

Interventions

Type of intervention: Multifactorial intervention

1. Multifactorial intervention: Detailed assessment by geriatrician, rehabilitation physician, geriatric nurse; recommendations and indications for referral sent to participants' GPs. GPs could then take action if they agreed with the recommendations and/or referrals. Home assessment by OT; recommendations sent to participants and their GPs, 
and direct referral to social or community services for provision of technical aids and adaptations or additional support $(\mathrm{n}=166)$

2. Control: Usual care $(n=167)$

Who delivered the intervention: GP, OT, geriatrician, geriatric nurse, rehabilitation physician

Compliance assessed: Yes, structured recording forms after each assessment, structured face-to-face interviews and plenary group discussion with practitioners

Outcomes

1. Number of people sustaining 1 or more falls

2. Number of people sustaining recurrent falls

3. Number of people who experienced a fall that required medical attention

4. Health-related quality of life (EQ-5D utilities, range unclear: endpoint score)

Notes

Source of funding: The Netherlands Organisation for Health Research and Development Grants

Conflicts of interest: None

Economic information: Multifactorial intervention cost: EUR 4857; Control cost: EUR 4991

\section{Risk of bias}

Bias

Random sequence generation (selection Unclear risk bias)

Allocation concealment (selection bias)

Unclear risk

Blinding of participants and personnel Unclear risk (performance bias)

All outcomes

Blinding of outcome assessment (detection Low risk bias)

Falls and fallers

Blinding of outcome assessment (detection Unclear risk bias)

Fractures

Blinding of outcome assessment (detection Unclear risk bias)

Hospital admission \& medical attention

\section{Support for judgement}

Quote: "Randomisation was achieved by means of computerised alternative allocation and performed by an external agency"

Quote: "Randomisation was achieved by means of computerised alternative allocation and performed by an external agency"

Participants and personnel not blind to allocation group but effect of non-blinding unclear

Quote: "Falls were recorded continuously by means of a falls calendar for 12 months after baseline"

Not applicable

Quote: "Falls were recorded continuously by means of a falls calendar for 12 months after baseline" 
Incomplete outcome data (attrition bias) High risk All outcomes
More than $20 \%$ missing outcome data, losses are unbalanced across groups with similar reasons for missing data

1. Multifactorial intervention: randomised $\mathrm{n}=166$, analysed $\mathrm{n}=124$ (16 health problems, 14 refused to participate, 5 died, 7 dropped out for other reasons)

2. Usual care: randomised $\mathrm{n}=167$, analysed $\mathrm{n}=134$ (21 health problems, 10 refused to participate, 1 died, 1 dropped out for other reasons)

All prespecified outcomes were reported

Quote: "Falls were recorded continuously by means of a falls calendar for 12 months after baseline"

Hogan 2001

\begin{tabular}{ll} 
Methods & Study design: RCT (parallel design) \\
Number of study arms: 2 \\
Study centres: Unclear \\
Length of follow-up: 24 months \\
\hline Participants & Setting: Canada \\
& Number randomised: 163 \\
& Number analysed: 139 \\
& Number lost to follow-up: 24 \\
& Sample: High-risk community-dwelling men and women \\
& Age (years): Mean 77.6 (SD 6.8) \\
& Sex: $72 \%$ women \\
& Ethnicity: Not reported \\
& Inclusion criteria: Aged $\geq 65 ;$ fallen in previous 3 months; community-dwelling; am- \\
bulatory (with or without aid); mentally intact (able to give consent) & Exclusion criteria: Qualifying fall resulted in lower extremity fracture, resulted from \\
vigorous or high-risk activities, because of syncope or acute stroke, or while undergoing \\
active treatment in hospital
\end{tabular}

Interventions

Type of intervention: Multifactorial intervention

1. Multifactorial intervention: 1 in-home assessment by a geriatric specialist (doctor, nurse, physiotherapist, or OT) lasting 1 to 2 hours. Intrinsic and environmental risk factors assessed. Multidisciplinary case conference (20 minutes). Recommendations sent to participants and participants' doctor for implementation. Participants referred to exercise class if problems with balance or gait and not already attending an exercise programme. Given instructions about exercises to do at home $(\mathrm{n}=79)$

2. Control: usual care: 1 home visit by recreational therapist $(n=84)$

Who delivered intervention: Geriatrician, OT, physiotherapist, recreational therapist,

Multifactorial and multiple component interventions for preventing falls in older people living in the community (Review) 
Hogan 2001 (Continued)

physician, research assistant

Compliance assessed: Yes, assessors documented adherence to recommendations. Adherence was categorised as none, partial, or complete

Outcomes
$\begin{aligned} & \text { 1. Rate of falls } \\ & \text { 2. Number of people sustaining } 1 \text { or more falls } \\ & \text { 3. Number of people sustaining recurrent falls } \\ & \text { 4. Number of people who experience } 1 \text { or more fall-related fractures } \\ & \text { 5. Number of people who experienced a fall that required hospital admission } \\ & \text { 6. Number of people who experienced a fall that required medical attention }\end{aligned}$

Notes

Source of funding: Health Services Research and Innovation Fund of the Alberta Heritage

Foundation for Medical Research

Conflicts of interest: Not reported

Economic information: Not reported

Risk of bias

Bias

Authors' judgement

Support for judgement

Random sequence generation (selection Low risk

Computer-generated. Stratified by number bias) of falls in previous year: 1 or $>1$

Allocation concealment (selection bias)

Unclear risk

Sequence concealed in locked cabinet prior to randomisation

Blinding of participants and personnel Unclear risk (performance bias)

All outcomes

Participants and personnel not blind to allocated group but impact of non-blinding unclear

Blinding of outcome assessment (detection Low risk

bias)

Falls and fallers

Participants were asked to record the date of any falls on a calender which was to be returned monthly in a stamped addressed envelope. A research assistant also visited participants at 3 and 6 months after randomisation, and called them 12 months after randomisation. At these times, the research assistant asked about any more falls since the last contact

Blinding of outcome assessment (detection Unclear risk bias)

A research assistant also visited participants at 3 and 6 months after randomisation, and called them 12 months after randomisation. At these times, the research assistant asked about more falls-related information since the last contact

Multifactorial and multiple component interventions for preventing falls in older people living in the community (Review) 


\section{Hogan 2001 (Continued)}

Blinding of outcome assessment (detection Unclear risk bias)

Hospital admission \& medical attention
Quote: "Data concerning hospital and emergency department use were obtained from the Calgary Regional Health Authority for all subjects for the 6 months before and the 12 months after study entry. ICD-9 codes for classifying external causes of injury (i.e. E codes) for selected accidental falls (E880, E884.2, E885, E886.9, E887, E888) were used to identify fall-related use of hospital services". It does not specify blinding of research assistant

Incomplete outcome data (attrition bias) Low risk All outcomes
Less than $20 \%$ missing outcome data, losses balanced across groups with similar reasons for missing data

1. Multifactorial intervention: randomised $\mathrm{n}=79$, analysed $\mathrm{n}=66$ ( 2 died, 8 withdrew consent/non adherence to protocol, 2 admitted to an institution, 1 moved away) 2. Usual care: randomised $\mathrm{n}=84$, analysed $\mathrm{n}=73$ (5 died, 4 withdrew consent/non adherence to protocol, 1 admitted to an institution, 1 moved away)

All prespecified outcomes were reported

Participants were asked to record the date of any falls on a calender which was to be returned monthly in a stamped addressed envelope. A research assistant also visited participants at 3 and 6 months after randomisation, and called them 12 months after randomisation. At these times, the research assistant asked about any more falls since the last contact

Huang 2005

Methods
Study Design: RCT (parallel design)

Number of study arms: 2

Study centres: Single centre

Length of follow-up: 3 months
Setting:Taiwan

Number randomised: 141

Number analysed: 126

Number lost to follow-up: 15

Sample: People in hospital with a fall-related hip fracture. Most were community- 
dwelling as stated "the majority of older people with hip fracture who are discharged from hospital are at home..." Intervention included a home visit. $91 \%$ living with family Age (years): Mean 77 (SD 7.6)

Sex: $69 \%$ women

Ethnicity: Not reported

Inclusion criteria: In hospital with fall-related hip fracture; aged $\geq 65$; discharged within medical centre catchment area

Exclusion criteria: Cognitively impaired; too ill (comorbidities, unable to communicate or in intensive care unit)

Interventions

Outcomes
Type of intervention: Multifactorial intervention

1. Multifactorial intervention: Discharge planning intervention by masters-level gerontological nurse, from hospital admission until 3 months after discharge (first visit within 48 hours of admission, seen every 48 hours while in hospital, 1 home visit 3 to 7 days after discharge, available by phone $8 \mathrm{am}-8 \mathrm{pm} 7$ days/wk, phoned participant or caregiver once a week). Nurse created individualised discharge plan and facilitated set-up of home-care services etc. Participants provided with brochures on self-care for hip fracture patients and fall prevention (environmental safety and medication issues). Nurse provided direct care and education on correct use of assistive devices, assessed rehabilitation needs, and collaborated with physicians to modify therapies $(\mathrm{n}=70)$

2. Control: usual discharge planning also by nurses, but not specialists. No brochures, written discharge summaries, home visits, or phone calls $(\mathrm{n}=71)$

Who delivered the intervention: Masters-level gerontological nurse

Compliance assessed: No

1. Number of people sustaining 1 or more falls

2. Number of people who experienced a fall that required hospital admission

3. Health-related quality of life (SF-36 0 - 100, overall, mental and physical subscales: endpoint score)

Notes

Source of funding: Funded by the National Science Council, Taiwan and Chung Gung University

Conflicts of interest: Not reported

Economic information: Not reported

\section{Risk of bias}

Bias Authors' judgement

Random sequence generation (selection Low risk bias)

Allocation concealment (selection bias)

Unclear risk

Blinding of participants and personnel Unclear risk (performance bias)

All outcomes

\section{Support for judgement}

Randomly assigned using a computer-generated table

Insufficient information to permit judgement

The discharge planning in the intervention group was conducted by a full-time geriatric nurse. Discharge planning in the control group was conducted by general nurses. 
Huang 2005 (Continued)

Impact of non-blinding of participants and personnel unclear

\begin{tabular}{l|l|l}
\hline $\begin{array}{l}\text { Blinding of outcome assessment (detection } \\
\text { bias) }\end{array}$ & Unclear risk & $\begin{array}{l}\text { Participants kept a falls diary but it was un- } \\
\text { clear if diary was checked every month or } \\
\text { Falls and fallers }\end{array}$ \\
\hline
\end{tabular}

Blinding of outcome assessment (detection Unclear risk

Not applicable

bias)

Fractures

Blinding of outcome assessment (detection Unclear risk bias)

Hospital admission \& medical attention
Incomplete outcome data (attrition bias) Low risk All outcomes
Not applicable

Less than $20 \%$ of missing outcome data, losses balanced across groups with similar reasons for missing data

1. Discharge planning intervention: randomised $n=63$, analysed $n=56$ (7 left the study before discharge due to refusal of participation or changes in health status)

2. Usual discharge planning: randomised $n$ $=63$, analysed $\mathrm{n}=55$ (left the study before discharge due to refusal of participation or changes in health status)

\begin{tabular}{|c|c|c|}
\hline Selective reporting (reporting bias) & Low risk & All prespecified outcomes were reported \\
\hline Method of ascertaining falls & Unclear risk & $\begin{array}{l}\text { Participants kept a falls diary but it was un- } \\
\text { clear if diary was checked every month or } \\
\text { at the end of the month or at end of the 3- } \\
\text { month intervention period }\end{array}$ \\
\hline
\end{tabular}

Huang 2010

Methods Study design: Cluster RCT

Number of study arms: 4

Number of clusters: 4 villages

Study centres: Multiple centres

Length of follow-up: 18 months 
Age (years): Mean 71.5 (SD 0.64)

Sex: $48 \%$ women

Ethnicity: Not reported

Inclusion criteria: Aged > 65 years; living in a non-organised community of Taiwan

Exclusion criteria: Immobile; living outside registered living area

Type of intervention: Multiple intervention
1. Education: 5 group teaching sessions over 5 months (medications, nutrition, environ-
ment (inside and outside), footwear) plus discussion $(\mathrm{n}=61)$
2. Tai Chi Chuan: 13 simple movements, 40 minutes, 3 a week for 20 weeks $(\mathrm{n}=65)$
3. Tai Chi Chuan + education $(\mathrm{n}=85)$
4. Control $(\mathrm{n}=50)$
Who delivered intervention: Coaches, community nurses
Compliance assessed: No

$\begin{array}{ll}\text { Outcomes } & \text { 1. Number of people sustaining } 1 \text { or more falls }\end{array}$

Notes

Source of funding: National Science Council, Taiwan

Conflicts of interest: None

Economic information: Not reported

\section{Risk of bias}

\begin{tabular}{l|l|l}
\hline Bias & Authors' judgement & Support for judgement \\
\hline $\begin{array}{l}\text { Random sequence generation (selection } \\
\text { bias) }\end{array}$ & Unclear risk & $\begin{array}{l}\text { Quote: “The three intervention groups and } \\
\text { one control group were then assigned ran- } \\
\text { domly to one each of the four selected vil- } \\
\text { lages." }\end{array}$ \\
\hline $\begin{array}{l}\text { Allocation concealment (selection bias) } \\
\text { Blinding of participants and personnel } \\
\text { (performance bias) } \\
\text { All outcomes }\end{array}$ & Unclear risk & $\begin{array}{l}\text { Insufficent information to make a judge- } \\
\text { ment }\end{array}$ \\
\hline $\begin{array}{l}\text { Blinding of outcome assessment (detection } \\
\text { bias) } \\
\text { Falls and fallers }\end{array}$ & Unclear risk & $\begin{array}{l}\text { Participants and personnel not blind to al- } \\
\text { located group but impact on non-blinding } \\
\text { unclear }\end{array}$ \\
\hline $\begin{array}{l}\text { Blinding of outcome assessment (detection } \\
\text { bias) } \\
\text { Fractures }\end{array}$ & Unclear risk & $\begin{array}{l}\text { No information provided on how falls were } \\
\text { recorded }\end{array}$ \\
\hline
\end{tabular}

Blinding of outcome assessment (detection Unclear risk

bias)

Hospital admission \& medical attention

Not applicable 
Huang 2010 (Continued)

\begin{tabular}{|c|c|c|}
\hline $\begin{array}{l}\text { Incomplete outcome data (attrition bias) } \\
\text { All outcomes }\end{array}$ & High risk & $\begin{array}{l}\text { More than } 20 \% \text { missing outcome data, due } \\
\text { to participants moving, hospitalisation or } \\
\text { they had died } \\
\text { 1. Education: randomised } n=61 \text {, analysed } \\
n=29 \\
\text { 2. Tai Chi Chuan: randomised } n=65 \text {, anal- } \\
\text { ysed } n=31 \\
\text { 3. Tai Chi Chuan }+ \text { education: randomised } \\
n=85 \text {, analysed } n=56 \\
\text { 4. Control: randomised: randomised } n= \\
\text { 50, analysed } n=47\end{array}$ \\
\hline Selective reporting (reporting bias) & Unclear risk & $\begin{array}{l}\text { Insufficent information to make a judge- } \\
\text { ment }\end{array}$ \\
\hline Method of ascertaining falls & Unclear risk & $\begin{array}{l}\text { No information provided on how falls were } \\
\text { recorded }\end{array}$ \\
\hline Relating to cluster randomisation & High risk & $\begin{array}{l}\text { Recruitment bias: villages were randomised } \\
\text { prior to screening, however, all eligible par- } \\
\text { ticipants within a cluster were invited to } \\
\text { participate (low risk) } \\
\text { Baseline imbalance: baseline imbalance be- } \\
\text { tween intervention arms (high risk) } \\
\text { Loss of clusters: no clusters lost from the } \\
\text { trial (low risk) } \\
\text { Incorrect analysis: the trial did not adjust } \\
\text { for clustering (high risk) } \\
\text { Comparability: only } 1 \text { trial for this com- } \\
\text { parison (unclear risk) }\end{array}$ \\
\hline
\end{tabular}

Huang 2011

\begin{tabular}{ll} 
Methods & Study design: RCT (parallel design) \\
& Number of study arms: 3 \\
& Study centres: unclear \\
& Length of follow-up: 5 months \\
\hline Participants & Setting: Taiwan \\
& Number randomised: 186 \\
& Number analysed: 176 \\
& Number lost to follow-up: 10 \\
& Sample: Randomly-selected sample of registered households in Yi-Lan county \\
& Age (years): Not reported \\
& Sex: $59 \%$ women \\
& Ethnicity: Taiwanese \\
Inclusion criteria: Aged $\geq 60 ;$ community-dwelling; able to communicate in Mandarin \\
or Taiwanese
\end{tabular}

Multifactorial and multiple component interventions for preventing falls in older people living in the community (Review) 
Exclusion criteria: Cognitively impaired; artificial leg or leg brace; unstable health problems or terminally ill

Interventions

Type of intervention: Multiple intervention

1. Cognitive behavioural intervention: 60 to 90 minutes once a week for 8 weeks, in groups of 8 to 12 . Promoting view that fall risk and fear of falling is controllable ( $\mathrm{n}=$ 62)

2. Cognitive behavioural intervention + intense Tai Chi: as above plus Tai Chi 60 minutes,

5 times a week for 8 weeks, in groups of 10 to $16(n=62)$

3. Control: no intervention $(n=62)$

Who delivered intervention: 2 professional Tai Chi instructors and nurse with CB experience

Compliance assessed: No

1. Rate of falls
2. Number of people who experienced 1 or more falls
3. Health-related quality of life (WHOQOL-BREF 16 - 80: endpoint score)

Notes

Source of funding: National Science Council, Taiwan

Conflicts of interest: None

Economic information: Not reported

\section{Risk of bias}

\section{Bias}

Random sequence generation (selection Low risk bias)

Allocation concealment (selection bias) Low risk

\section{Support for judgement}

Quote: “The first author used a computerdeveloped random table to randomly assign patients to three intervention groups ..."

Quote: "Allocation was concealed from the recruiting RA"

Participants and personnel not blind to allocated group but impact of non-blinding unclear

Insufficent information to make a judgement bias)

Falls and fallers

Blinding of outcome assessment (detection Unclear risk bias)

Fractures

Blinding of outcome assessment (detection Unclear risk bias)

Hospital admission \& medical attention
Not applicable

Not applicable 
Huang 2011 (Continued)

\begin{tabular}{|c|c|c|}
\hline $\begin{array}{l}\text { Incomplete outcome data (attrition bias) } \\
\text { All outcomes }\end{array}$ & Low risk & $\begin{array}{l}\text { Less than } 20 \% \text { missing outcome data, losses } \\
\text { balanced across groups with similar reasons } \\
\text { for missing data } \\
\text { 1. Cognitive behavioural intervention: ran- } \\
\text { domised } n=62 \text {, analysed } n=60 \text { ( } 2 \text { did not } \\
\text { complete intervention) } \\
\text { 2. Cognitive behavioural intervention + in- } \\
\text { tense Tai Chi: randomised } n=62 \text {, analysed } \\
n=60 \text { ( } 2 \text { did not complete intervention) } \\
\text { 3. Control: no intervention: randomised } n \\
=62 \text {, analysed } n=56 \text { ( } 6 \text { did not complete } \\
\text { intervention) }\end{array}$ \\
\hline Selective reporting (reporting bias) & Unclear risk & $\begin{array}{l}\text { Insufficent information to make a judge- } \\
\text { ment }\end{array}$ \\
\hline Method of ascertaining falls & Unclear risk & $\begin{array}{l}\text { No information provided on how falls were } \\
\text { recorded }\end{array}$ \\
\hline
\end{tabular}

Imhof 2012

\begin{tabular}{|c|c|}
\hline Methods & $\begin{array}{l}\text { Study Design: RCT (parallel design) } \\
\text { Number of study arms: } 2 \\
\text { Study centres: Single centre } \\
\text { Length of follow-up: } 9 \text { months }\end{array}$ \\
\hline Participants & $\begin{array}{l}\text { Setting: Switzerland } \\
\text { Number randomised: } 461 \\
\text { Number analysed: } 413 \\
\text { Number lost to follow-up: } 48 \\
\text { Sample: Various health organisations such as local hospitals, home care organisations } \\
\text { and church social services, and by community nurses and family physicians extended } \\
\text { the invitation to } 1182 \text { participants } \\
\text { Age (years): Mean } 85 \\
\text { Sex: } 73 \% \\
\text { Ethnicity: All white } \\
\text { Inclusion criteria: Community-dwelling individuals } \\
\text { Exclusion criteria: Individuals aged } 80 \text { years or older }\end{array}$ \\
\hline Interventions & $\begin{array}{l}\text { Type of intervention: Multifactorial intervention } \\
\text { 1. Usual care and advanced practice nurse home consultation programme: individualised } \\
\text { interventions, } 4 \text { home visits, } 3 \text { follow-up telephone calls }(\mathrm{n}=231) \\
\text { 2. Control: standard care ( } \mathrm{n}=230) \\
\text { Who delivered the intervention: Community health nurses, physicians, physiotherapists, } \\
\text { OTs } \\
\text { Compliance assessed: Not reported }\end{array}$ \\
\hline
\end{tabular}

Multifactorial and multiple component interventions for preventing falls in older people living in the community (Review) 
Imhof 2012 (Continued)

\begin{tabular}{l|l}
\hline Outcomes & $\begin{array}{l}\text { 1. Number of people who experienced } 1 \text { or more falls } \\
\text { 2. Number of people who experienced a fall that required hospital admission } \\
\text { 3. Health-related quality of life }\end{array}$ \\
\hline Notes & $\begin{array}{l}\text { Source of Funding: Age Foundation Zurich, Ebnet foundation Teufen, Heinrich and } \\
\text { Ema Walder Foundation Zurich and City of Winterthur } \\
\text { Conflicts of interest: Conflict of interest acknowledged as study was funded by Age } \\
\text { foundation Zurich, Ebnet foundation Teufen, Heinrich and Ema Walder Foundation } \\
\text { Zurich and City of Winterthur } \\
\text { Economic information: Intervention cost is approximately USD } 1250 \text { per participant }\end{array}$ \\
\hline
\end{tabular}

Risk of bias

\begin{tabular}{|c|c|c|}
\hline Bias & Authors' judgement & Support for judgement \\
\hline $\begin{array}{l}\text { Random sequence generation (selection } \\
\text { bias) }\end{array}$ & Low risk & $\begin{array}{l}\text { Quote: "After the second assessment visit, } \\
\text { participants were randomly assigned to the } \\
\text { intervention or control group using a com- } \\
\text { puter generated list of random numbers } \\
\text { with a one to one sequence" }\end{array}$ \\
\hline Allocation concealment (selection bias) & Low risk & $\begin{array}{l}\text { Quote: "A person who was not involved } \\
\text { in the recruitment of study participants or } \\
\text { data collection prepared sealed envelopes } \\
\text { with group assignment. The APN opened } \\
\text { the envelope at the end of the visit, and } \\
\text { the participant was informed about group } \\
\text { allocation" }\end{array}$ \\
\hline
\end{tabular}

Blinding of participants and personnel Unclear risk (performance bias)

Particants and personnel not blind to alAll outcomes located group but impact of non-blinding unclear

Blinding of outcome assessment (detection Unclear risk Not applicable

bias)

Falls and fallers

Blinding of outcome assessment (detection Unclear risk bias)

Fractures

Blinding of outcome assessment (detection Unclear risk bias)

Hospital admission \& medical attention

Quote: "Particpants were asked have you had a fall and been in hospital in the last 3 months"

Incomplete outcome data (attrition bias) Low risk All outcomes

Less than $20 \%$ missing outcome data, balanced losses across groups with similar reasons for missing data

1. Usual care and advanced practice

Multifactorial and multiple component interventions for preventing falls in older people living in the community (Review) 
Imhof 2012 (Continued)

nurse home consultation programme: randomised $\mathrm{n}=231$, analysed $\mathrm{n}=207$, (12 withdrew participation, 4 admitted to long-term care, 8 died)

2. Standard care: randomised $\mathrm{n}=230$, analysed $\mathrm{n}=206$, (10 withdrew participation, 7 admitted into long-term care, 7 died )

Selective reporting (reporting bias) Low risk

All outcomes listed in the Methods were reported

Method of ascertaining falls

High risk

Retrospective by 3-month period

\section{Jitapunkul 1998}

Methods

Study design: RCT (parallel design)

Number of study arms: 2

Study centres: Unclear

Length of follow-up: 36 months

Setting: Thailand
Number randomised: 160
Number analysed: 116
Number lost to follow-up: 44
Sample: People recruited from a sample for a previous study in Thai elderly persons
Age (years): Mean 75.6 (SD 5.8)
Sex: $65 \%$ women
Ethnicity: Thai
Inclusion criteria: Aged $\geq 70$; living at home
Exclusion criteria: None stated

Interventions

Type of intervention: Multifactorial intervention

1. Home visit group: Home visit from non-professional personnel with structured questionnaire. 3-monthly visits for 3 years. Referred to nurse/geriatrician (community-based) if Barthel ADL index and/or Chula ADL index declined $\geq 2$ points, or $\geq 1$ fall in previous 3 months. Nurse/geriatrician would visit, assess, educate, prescribe drugs/aids, provide rehabilitation programme, make referrals $(n=80)$

2. Control: no intervention. Visit at the end of 3 years $(n=80)$

Who delivered the intervention: Non-professional personnel, nurses, geriatrician

Compliance assessed: No

Outcomes

1. Number of people sustaining 1 or more falls

2. Number of people who experienced a fall that required hospital admission

3. Number of people who experienced a fall that required medical attention

4. Health-related quality of life (Barthel Index 0 - 20: endpoint score)

Multifactorial and multiple component interventions for preventing falls in older people living in the community (Review) 


\section{Risk of bias}

\begin{tabular}{|c|c|c|}
\hline Bias & Authors' judgement & Support for judgement \\
\hline $\begin{array}{l}\text { Random sequence generation (selection } \\
\text { bias) }\end{array}$ & Unclear risk & $\begin{array}{l}\text { Quote: “... divided into case group }(\mathrm{n}=80) \\
\text { and control group }(\mathrm{n}=80) \text { at random.” In- } \\
\text { sufficient information to permit judgement }\end{array}$ \\
\hline Allocation concealment (selection bias) & Unclear risk & $\begin{array}{l}\text { Insufficient information to permit judge- } \\
\text { ment }\end{array}$ \\
\hline $\begin{array}{l}\text { Blinding of participants and personnel } \\
\text { (performance bias) } \\
\text { All outcomes }\end{array}$ & Unclear risk & $\begin{array}{l}\text { Participants and personnel not blind to al- } \\
\text { located group but impact of non-blinding } \\
\text { unclear }\end{array}$ \\
\hline $\begin{array}{l}\text { Blinding of outcome assessment (detection } \\
\text { bias) } \\
\text { Falls and fallers }\end{array}$ & High risk & $\begin{array}{l}\text { Self-reports by study participants and visits } \\
\text { by non-professional personnel once every } \\
3 \text { months }\end{array}$ \\
\hline $\begin{array}{l}\text { Blinding of outcome assessment (detection } \\
\text { bias) } \\
\text { Fractures }\end{array}$ & Unclear risk & Not applicable \\
\hline $\begin{array}{l}\text { Blinding of outcome assessment (detection } \\
\text { bias) } \\
\text { Hospital admission \& medical attention }\end{array}$ & Unclear risk & $\begin{array}{l}\text { Insufficient information to permit judge- } \\
\text { ment }\end{array}$ \\
\hline $\begin{array}{l}\text { Incomplete outcome data (attrition bias) } \\
\text { All outcomes }\end{array}$ & High risk & $\begin{array}{l}\text { More than } 20 \% \text { missing outcome data, } \\
\text { losses balanced across groups with similar } \\
\text { reasons for missing data } \\
\text { 1. Home visit group: randomised } n=80 \text {, } \\
\text { analysed } n=57 \text { ( } 10 \text { moved elsewhere, } 13 \\
\text { died) } \\
\text { 2. Control: randomised } n=80 \text {, analysed } n \\
=59(8 \text { moved elsewhere, } 13 \text { died })\end{array}$ \\
\hline Selective reporting (reporting bias) & Unclear risk & $\begin{array}{l}\text { Insufficient information to permit judge- } \\
\text { ment }\end{array}$ \\
\hline Method of ascertaining falls & High risk & $\begin{array}{l}\text { Self-reports by study participants and visits } \\
\text { by non-professional personnel once every } \\
3 \text { months }\end{array}$ \\
\hline
\end{tabular}




\begin{tabular}{|c|c|}
\hline Methods & $\begin{array}{l}\text { Study design: RCT (parallel design) } \\
\text { Number of study arms: } 2 \\
\text { Study centres: Single centre } \\
\text { Length of follow-up: } 3 \text { months }\end{array}$ \\
\hline Participants & $\begin{array}{l}\text { Setting: United Kingdom } \\
\text { Number randomised: } 109 \\
\text { Number analysed: } 92 \\
\text { Number lost to follow-up: } 17 \\
\text { Sample: Community-dwelling women attending A\&E with a fall } \\
\text { Age (years): Mean } 71.9 \\
\text { Sex: } 100 \% \text { women } \\
\text { Ethnicity: Not reported } \\
\text { Inclusion criteria: Female; aged } 65 \text { to } 79 \text {; history of a fall; discharged directly to own } \\
\text { home } \\
\text { Exclusion criteria: Admitted from A\&E to hospital or any form of institutional care }\end{array}$ \\
\hline Interventions & $\begin{array}{l}\text { Type of intervention: Multifactorial intervention } \\
\text { 1.Health Visitor intervention: Rapid Health Visitor intervention within } 5 \text { working days } \\
\text { of index fall: pain control and medication, how to get up after a fall, education about risk } \\
\text { factors (environmental and drugs, alcohol etc), advice on diet and exercise to strengthen } \\
\text { muscles and joints. ( } \mathrm{n}=60) \\
\text { 2. Control: usual post-fall treatment, i.e. letter to GP from A\&E detailing the clinical } \\
\text { event, any interventions carried out in hospital and recommendations about follow-up } \\
(\mathrm{n}=49 \text { ) } \\
\text { Who delivered intervention: Health visitor, physician } \\
\text { Compliance assessed: Not reported }\end{array}$ \\
\hline Outcomes & $\begin{array}{l}\text { 1. Number of people sustaining } 1 \text { or more falls } \\
\text { 2. Health-related quality of life }\end{array}$ \\
\hline Notes & $\begin{array}{l}\text { Source of funding: Not reported } \\
\text { Conflicts of interest: Not reported } \\
\text { Economic information: Not reported }\end{array}$ \\
\hline
\end{tabular}

\section{Risk of bias}

\begin{tabular}{l|l|l}
\hline Bias & Authors' judgement & Support for judgement \\
\hline $\begin{array}{l}\text { Random sequence generation (selection } \\
\text { bias) }\end{array}$ & Unclear risk & Quote: "randomly allocated" \\
\hline $\begin{array}{l}\text { Allocation concealment (selection bias) } \\
\text { Blinding of participants and personnel } \\
\text { (performance bias) } \\
\text { All outcomes }\end{array}$ & Unclear risk & $\begin{array}{l}\text { Quote: "randomly allocated”. Insufficient } \\
\text { information to permit judgement }\end{array}$ \\
\hline
\end{tabular}


Kingston 2001 (Continued)

Blinding of outcome assessment (detection Unclear risk

bias)

Falls and fallers
Insufficient information to permit judgement

Not applicable

Blinding of outcome assessment (detection Unclear risk

bias)

Fractures

Blinding of outcome assessment (detection Unclear risk

Not applicable

bias)

Hospital admission \& medical attention

Incomplete outcome data (attrition bias) High risk All outcomes

More than 20\% missing outcome data, unbalanced losses across groups with unspecified reasons for missing data

1. Health Visitor intervention: randomised $\mathrm{n}=60$, analysed $\mathrm{n}=51$ (unspecified reasons for lost to follow-up)

2. Usual post-fall treatment : randomised $n$ $=49$, analysed $n=41$ (unspecified reasons for lost to follow-up)

\begin{tabular}{ll|l} 
Selective reporting (reporting bias) & Low risk & All prespecified outcomes were reported \\
\hline Method of ascertaining falls & Unclear risk & $\begin{array}{l}\text { Insufficient information to permit judge- } \\
\text { ment }\end{array}$
\end{tabular}

Lightbody 2002

Methods

Study Design: RCT (parallel design)

Number of study arms: 2

Study centres: Single centre

Length of follow-up: 6 months

Participants

Setting: United Kingdom

Number randomised: 348

Number analysed: 314

Number lost to follow-up: 34

Subjects: Consecutive patients attending A\&E with a fall

Age (years): Median (IQR) 75 (70 to 81 )

Sex: $74 \%$ women

Ethnicity: Not reported, but all participants resided in Liverpool, U.K

Inclusion criteria: Aged $>65$, patients attending A\&E with a fall

Exclusion criteria: Admitted to hospital as result of index fall, living in institutional care, refused or unable to consent, lived out of the area 
Lightbody 2002 (Continued)

\begin{tabular}{ll} 
Interventions & $\begin{array}{l}\text { Type of intervention: Multifactorial intervention } \\
\text { 1. Multifactorial assessment: Multifactorial assessment by falls nurse at } 1 \text { home visit } \\
\text { (medication, ECG, blood pressure, cognition, visual acuity, hearing, vestibular dysfunc- } \\
\text { tion, balance, mobility, feet and footwear, environmental assessment). Referral for spe- } \\
\text { cialist assessment or further action (relatives, community therapy services, social services, } \\
\text { primary care team. No referrals to day hospital or hospital outpatients). Advice and ed- } \\
\text { ucation about home safety and simple modifications, e.g. mat removal (n = 171) } \\
\text { 2. Control: usual care (n = 177) } \\
\text { Who delivered intervention: Therapists, clinicians, nurse, relatives, community therapy } \\
\text { services, social services, primary care team } \\
\text { Compliance assessed: No }\end{array}$ \\
\hline $\begin{array}{l}\text { 1. Rate of falls } \\
\text { 2. Number of people sustaining } 1 \text { or more falls } \\
\text { 3. Health-related quality of life (Barthel Index } 0 \text { - 20: endpoint score) }\end{array}$ \\
\hline $\begin{array}{l}\text { Source of funding: North West Region NHS Executive and supported by Liverpool and } \\
\text { Wirral Research and Development Liason Group } \\
\text { Conflicts of interest: Not reported } \\
\text { Economic information: Cost savings of GBP } 11,719 \text { in intervention group and GBP } \\
\text { 37,951 in control group was reported in the cost evaluation of falls-related bed days }\end{array}$ \\
\hline
\end{tabular}

\section{Risk of bias}

\section{Bias}

Random sequence generation (selection Unclear risk bias)

\section{Support for judgement}

Quote: "Patients were block-randomized consecutively to groups". Insufficient information to permit judgement

Insufficient information to permit judgement

Participants and personnel not blind to allocated group but impact of non-blinding unclear

Falls detection was by daily falls diary, and retrospective questionnaire at 6 months

Not applicable

Blinding of outcome assessment (detection Unclear risk bias)

Fractures

Blinding of outcome assessment (detection Low risk bias)

Hospital admission \& medical attention
GP records were reviewed and hospital databases interrogated for attendances and admissions 
Lightbody 2002 (Continued)

\begin{tabular}{|c|c|c|}
\hline $\begin{array}{l}\text { Incomplete outcome data (attrition bias) } \\
\text { All outcomes }\end{array}$ & Low risk & $\begin{array}{l}\text { Less than } 20 \% \text { missing outcome data, losses } \\
\text { balanced across groups with similar reasons } \\
\text { for missing data } \\
\text { 1. Multifactorial assessment: randomised } n \\
=171 \text {, analysed } n=155 \text { ( } 2 \text { withdrew, } 11 \\
\text { died, } 3 \text { lost to follow-up) } \\
\text { 2. Usual care: randomised } n=177 \text {, analysed } \\
n=159 \text { ( } 10 \text { withdrew, } 7 \text { died, } 1 \text { lost to } \\
\text { follow-up) }\end{array}$ \\
\hline Selective reporting (reporting bias) & Low risk & All prespecified outcomes were reported \\
\hline Method of ascertaining falls & Unclear risk & $\begin{array}{l}\text { Falls detection was by daily falls diary, and } \\
\text { retrospective questionnaire at } 6 \text { months }\end{array}$ \\
\hline
\end{tabular}

Logan 2010

\begin{tabular}{ll} 
Methods & $\begin{array}{l}\text { Study design: RCT (parallel design) } \\
\text { Number of study arms: } 2 \\
\text { Study centres: Unclear } \\
\text { Length of follow-up: } 12 \text { months }\end{array}$ \\
\hline Participants & Setting: United Kingdom \\
& Number randomised: 204 \\
& Number analysed: 157 \\
& Number lost to follow-up: 47 \\
& Sample: People living in the 4 primary care trust areas \\
& Age (years): Median (IQR) 83 (77 to 86 ) \\
& Sex: $65 \%$ women \\
Ethnicity: Not reported \\
Inclusion criteria: Aged $\geq 60$; living at home or in a care home (participants were \\
predominantly community-dwelling - only $5 \%$ in care home or hospital); called for an \\
ambulance after a fall and not taken to hospital, or taken to hospital but not admitted \\
Exclusion criteria: Receiving a falls prevention services (in geriatric day hospitals or \\
hospital outpatient departments)
\end{tabular}

Interventions

Type of intervention: Multifactorial intervention

1. Individualised Multifactorial Intervention Programme: Referred to multidisciplinary falls-prevention service for assessment and interventions. Tailored interventions including balance training, muscle strengthening, reduction of environmental hazards, education about how to get off the floor, and provision of equipment. If medical assessment required for medication check or visual problems, referred to GP in first instance and then to the community geriatrician if necessary $(n=102)$

2. Control: No intervention by falls-prevention service $(n=102)$

Who delivered the intervention: Physiotherapists, OTs, social care workers, nurses, doctors

Compliance assessed: No 


\begin{tabular}{|c|c|}
\hline Outcomes & $\begin{array}{l}\text { 1. Rate of falls } \\
\text { 2. Number of people sustaining } 1 \text { or more falls } \\
\text { 3. Number of people sustaining } 1 \text { or more fall-related fractures } \\
\text { 4. Number of people who experienced a fall that required hospital admission } \\
\text { 5. Health-related quality of life (Barthel Index } 0 \text { - 20: endpoint score) }\end{array}$ \\
\hline Notes & $\begin{array}{l}\text { Source of funding: Postdoctoral training scholarship awarded to principal investigator } \\
\text { from the UK NHS National Institute of Health Research } \\
\text { Conflicts of interest: None } \\
\text { Economic information: Reported in a separate publication (Sach 2012). The mean total } \\
\text { NHS and personal social service cost per participant (mean and SD) during the 12- } \\
\text { month follow-up period (excluding participant and carer costs) was Intervention: GBP } \\
\text { 15,266 (SD GBP 13,504); Control: GBP 16,818 (SD GBP 14,210) giving an MD } \\
\text { of GBP - } 1551 \text { (95\% CI: GBP -5932 to GBP 2829). Total costs Intervention: GBP } \\
\text { 19,032.9 (17,055.79); Control: GBP 19,129.83 (14,930.35); MD -96.92 (95\% CI } \\
\text {-5140.92 to 4947.07) }\end{array}$ \\
\hline
\end{tabular}

Risk of bias

\begin{tabular}{|c|c|c|}
\hline Bias & Authors' judgement & Support for judgement \\
\hline $\begin{array}{l}\text { Random sequence generation (selection } \\
\text { bias) }\end{array}$ & Low risk & $\begin{array}{l}\text { Quote: "Nottingham Clinical Trials Unit } \\
\text { produced a computer generated randomi- } \\
\text { sation scheme with stratification by pri- } \\
\text { mary care trust" }\end{array}$ \\
\hline Allocation concealment (selection bias) & Low risk & $\begin{array}{l}\text { Quote: "The allocation sequence was con- } \\
\text { cealed until allocation. After written con- } \\
\text { sent had been obtained, PAL accessed the } \\
\text { randomisation sequence through the inter- } \\
\text { net and assigned the participants to their } \\
\text { group." }\end{array}$ \\
\hline
\end{tabular}

Blinding of participants and personnel Unclear risk (performance bias)

All outcomes

Blinding of outcome assessment (detection Low risk bias)

Falls and fallers

Blinding of outcome assessment (detection Low risk bias)

Fractures
Quote: "It was not possible to blind the participants and treating therapists to allocation group as they would be aware of receiving or giving falls rehabilitation.” Impact of non-blinding unclear

Quote: "Data on falls were recorded monthly using a diary"

Additional outcome measures in this study included falls-related fractures over 12 months which were determined by a researcher blind to allocation by checking the Nottingham University Hospital computer 
Logan 2010 (Continued)

\section{system}

Blinding of outcome assessment (detection Low risk bias)

Hospital admission \& medical attention
Requiring hospitalisation and medical attention was determined by a researcher blind to allocation by checking the Nottingham University Hospital computer system. The East Midlands Ambulance Service computer system was also checked to determine the number of emergency ambulance calls received for falls over 12 months and the number of such participants taken to an accident and emergency

Less than $20 \%$ missing outcome data, losses balanced across groups with similar reasons for missing data

1. Individualised Multifactorial Intervention Programme: randomised $\mathrm{n}=102$, analysed $\mathrm{n}=82$ ( 4 withdrew, 16 died)

2. No intervention: randomised $n=102$, analysed $n=75$ ( 8 withdrew, 19 died)

\begin{tabular}{|c|c|c|}
\hline Selective reporting (reporting bias) & Low risk & All prespecified outcomes were reported \\
\hline Method of ascertaining falls & Low risk & $\begin{array}{l}\text { Quote: "Data on falls were recorded } \\
\text { monthly using a diary" }\end{array}$ \\
\hline
\end{tabular}

Lord 2005

Methods

Study design: RCT (parallel design)

Number of study arms: 3

Study centres: Single centre

Length of follow-up: 12 months
Setting: Australia

Number randomised: 620

Number analysed: 578

Number lost to follow-up: 42

Sample: Health insurance membership database

Age (years): Mean 80.4 (SD 4.5)

Sex: $66 \%$ women

Ethnicity: Not reported

Inclusion criteria: Low score on PPA test; community-dwelling; $\geq 75$ years

Exclusion criteria: Minimal English language skills; blind; Parkinson's disease; cognitive impairment 
Interventions

Outcomes

Notes

\section{Risk of bias}

Type of intervention: Multifactorial intervention

1. Extensive intervention: Individualised exercise intervention ( 2 a week for 12 months) , visual intervention, peripheral sensation counselling intervention $(n=210)$

2. Minimal intervention. Participants received a report outlining their falls risk, a profile of their test results, and specific recommendations on preventing falls based on their test performances $(\mathrm{n}=206)$

3. Control: no intervention (received minimal intervention after 12-month follow-up) $(\mathrm{n}=204)$

Who delivered the intervention: Eye specialist, fitness instructors and primary care physicians

Compliance assessed: Yes, self-reported participant compliance at 6 months

1. Rate of falls

2. Number of people who sustained 1 or more falls

3. Number of people who sustained recurrent falls

Source of funding: The National Health and Medical Research Council (POPI Partnership in Injury and Project Grants), MBF Australia, and the Vincent Fairfax Family Foundation

Conflicts of interest: Not reported

Economic information: Not reported

Bias Authors' judgement

Random sequence generation (selection Low risk bias)

\begin{tabular}{l|l} 
Allocation concealment (selection bias) & Unclear risk \\
\hline $\begin{array}{l}\text { Blinding of participants and personnel } \\
\text { (performance bias) }\end{array}$ & Unclear risk \\
All outcomes &
\end{tabular}

Blinding of outcome assessment (detection Low risk bias)

Falls and fallers

Blinding of outcome assessment (detection Unclear risk

bias)

Fractures

\section{Support for judgement}

Quote: "randomised in matched blocks N $=20 \ldots$ using concealed allocation (drawing lots)"

Quote: "concealed allocation"

Blinding of participants and treatment personnel not mentioned in report, but unlikely. Insufficient evidence to make judgement on impact of lack of blinding

Falls were monitored for 1 year using monthly fall calendars. When a fall occurred, specific details about fall injuries were obtained from telephone interviews

Not applicable 


\begin{tabular}{|c|c|c|}
\hline $\begin{array}{l}\text { Blinding of outcome assessment (detection } \\
\text { bias) } \\
\text { Hospital admission \& medical attention }\end{array}$ & Unclear risk & Not applicable \\
\hline $\begin{array}{l}\text { Incomplete outcome data (attrition bias) } \\
\text { All outcomes }\end{array}$ & Low risk & $\begin{array}{l}\text { Less than } 20 \% \text { missing outcome data, losses } \\
\text { balanced across groups with similar reasons } \\
\text { for missing data } \\
\text { 1. Extensive intervention group: ran- } \\
\text { domised } \mathrm{n}=210 \text {, analysed } \mathrm{n}=192 \text { ( } 4 \\
\text { dropped out due to ill health, } 1 \text { died, } 1 \\
\text { moved residence, } 12 \text { withdrew consent) } \\
\text { 2. Minimal intervention group: ran- } \\
\text { domised } \mathrm{n}=206 \text {, analysed } \mathrm{n}=189 \text { ( } 1 \\
\text { dropped out due to ill health, } 1 \text { moved res- } \\
\text { idence, } 15 \text { withdrew consent) } \\
\text { 3. Control: randomised } \mathrm{n}=204 \text {, analysed } \\
\mathrm{n}=197 \text { ( } 1 \text { dropped out due to ill health, } 3 \\
\text { died, } 3 \text { withdrew consent) }\end{array}$ \\
\hline Selective reporting (reporting bias) & Unclear risk & $\begin{array}{l}\text { Methods state that a short-Form } 12 \text { Health } \\
\text { Status Questionnaire was used to provide } \\
\text { validated assessments of physical and men- } \\
\text { tal health but not reported in Results }\end{array}$ \\
\hline Method of ascertaining falls & Low risk & $\begin{array}{l}\text { Falls were monitored for } 1 \text { year using } \\
\text { monthly fall calendars. When a fall oc- } \\
\text { curred, specific details about fall injuries } \\
\text { were obtained from telephone interviews }\end{array}$ \\
\hline
\end{tabular}

Luck 2013

\begin{tabular}{ll}
\hline Methods & Study Design: RCT (parallel design) \\
& Number of study arms: 2 \\
& Study centres: Multiple centres \\
& Length of follow-up: 18 months \\
\hline Participants & Setting: Germany \\
& Number randomised: 305 \\
& Number analysed: 230 \\
& Number lost to follow-up: 75 \\
& Sample: Participants were recruited from healthcare settings (general practices, general \\
hospitals) and by mail (general population with addresses provided by local registration) & Age (years): 85.3 \\
Sex: $68.5 \%$ & Ethnicity : Not reported \\
Inclusion criteria: Living at home, aged 80 years or older, functional impairment in at \\
least 3 activities of daily living \\
Exclusion criteria: People with insufficient knowledge of German language, cognitive
\end{tabular}

Multifactorial and multiple component interventions for preventing falls in older people living in the community (Review) 
Luck 2013 (Continued)

impairment, an inability to give informed consent, a level of care higher than 1 (according to German long-term care insurance)

\begin{tabular}{ll} 
Interventions & $\begin{array}{l}\text { Type of intervention: Multifactorial intervention } \\
\text { 1. Multifactorial intervention: Multidimensional geriatric assessment, case review (indi- } \\
\text { vidualised intervention and recommendation), home counselling visit, booster session, } \\
\text { falls prevention }(\mathrm{n}=150) \\
\text { 2. Control: No preventive home visits }(\mathrm{n}=155) \\
\text { Who delivered the intervention: Multidisciplinary team (nurse, scientist, psychologist, } \\
\text { geronto-psychiatrist), nutritionist, social worker } \\
\text { Compliance assessed: Yes, obstacles and facilitators to adherence were assessed at booster } \\
\text { sessions, recommendations were re-emphasised and further assistance was provided }\end{array}$ \\
\hline Outcomes & $\begin{array}{l}\text { 1. Rate of falls } \\
\text { Notes }\end{array}$ \\
$\begin{array}{l}\text { Source of funding: Supported by grants from the German Federal Ministry of Education } \\
\text { and Research (01GT0601,01GT0604) as part of the German Nursing Research Network } \\
\text { Conflicts of interest: None } \\
\text { Economic information: Not reported }\end{array}$ \\
\hline
\end{tabular}

\section{Risk of bias}

\section{Bias}

Random sequence generation (selection Unclear risk bias)

\section{Authors' judgement}

\section{Support for judgement}

Quote: "Participants were randomised to an intervention group or to a control group using balanced blockwise randomization stratified by center". Insufficent information but likely to be computer-generated

\begin{tabular}{l|l|l}
\hline Allocation concealment (selection bias) & Unclear risk & Insufficent information \\
\hline $\begin{array}{l}\text { Blinding of participants and personnel } \\
\text { (performance bias) } \\
\text { All outcomes }\end{array}$ & Unclear risk & $\begin{array}{l}\text { Participants and personnel not blind to al- } \\
\text { located group but impact of non-blinding } \\
\text { unclear }\end{array}$ \\
\hline
\end{tabular}

Blinding of outcome assessment (detection High risk bias)

Falls and fallers

Blinding of outcome assessment (detection Unclear risk bias)

Fractures

Blinding of outcome assessment (detection Unclear risk bias)

Hospital admission \& medical attention
Falls were assessed retrospectively by asking questions, no use of diary or postcards
Not applicable 
Luck 2013 (Continued)

\begin{tabular}{|c|c|c|}
\hline $\begin{array}{l}\text { Incomplete outcome data (attrition bias) } \\
\text { All outcomes }\end{array}$ & High risk & $\begin{array}{l}\text { More than } 20 \% \text { missing outcome data, un- } \\
\text { balanced losses across groups with no rea- } \\
\text { sons for missing data } \\
\text { 1. Multifactorial intervention: randomised } \\
\mathrm{n}=150 \text { analysed } \mathrm{n}=118,(32 \text {, no reasons) } \\
\text { 2. No preventive home visits: randomised } \\
\mathrm{n}=155 \text {, analysed } \mathrm{n}=112 \text {, (43, no reasons) }\end{array}$ \\
\hline Selective reporting (reporting bias) & Low risk & $\begin{array}{l}\text { All outcomes listed in the Methods section } \\
\text { were reported }\end{array}$ \\
\hline Method of ascertaining falls & High risk & $\begin{array}{l}\text { Falls were assessed retrospectively by asking } \\
\text { questions, without use of diary or postcards }\end{array}$ \\
\hline
\end{tabular}

Markle-Reid 2010

\begin{tabular}{|c|c|}
\hline Methods & $\begin{array}{l}\text { Study Design: RCT (parallel design) } \\
\text { Number of study arms: } 4 \\
\text { Study centres: Multiple centres } \\
\text { Length of follow-up: } 6 \text { months }\end{array}$ \\
\hline Participants & $\begin{array}{l}\text { Setting: Canada } \\
\text { Number randomised: } 109 \\
\text { Number analysed: } 92 \\
\text { Number lost to follow-up: } 17 \\
\text { Sample: Adults newly-referred to, and eligible for, home support services } \\
\text { Age: Range } 75 \text { to } 84 \\
\text { Sex: } 72 \% \text { women } \\
\text { Ethnicity: Not reported } \\
\text { Inclusion criteria: Aged } \geq 75 \text {; community-dwelling (not in nursing home or long-term } \\
\text { care facility); "at risk of falls" (fallen in past } 12 \text { month, fear of falling, unsteady on feet) } \\
\text { Exclusion criteria: Not mentally competent; not competent in English or with a translator } \\
\text { available }\end{array}$ \\
\hline Interventions & $\begin{array}{l}\text { Type of intervention: Multifactorial intervention } \\
\text { 1. Multifactorial and Interdisciplinary Team Approach: Standard home services + home } \\
\text { visits by health professionals }(\mathrm{n}=54) \\
\text { 2. Control usual care: standard home services }(\mathrm{n}=55) \\
\text { Who delivered intervention: Community care access centre (CCAC) case manager, reg- } \\
\text { istered nurse, OT, physiotherapist, registered dietician } \\
\text { Compliance assessed: Yes, monitoring and evaluating the plan of care on an ongoing } \\
\text { basis through in-home assessments with clients }\end{array}$ \\
\hline Outcomes & $\begin{array}{l}\text { 1. Rate of falls } \\
\text { 2. Health-related quality of life (SF-36 } 0 \text { - 100, mental and physical subscales: endpoint } \\
\text { score) }\end{array}$ \\
\hline
\end{tabular}

Multifactorial and multiple component interventions for preventing falls in older people living in the community (Review) 
Notes

\section{Risk of bias}

\section{Bias}

Authors' judgement

Random sequence generation (selection Low risk bias)

Allocation concealment (selection bias) Low risk

Blinding of participants and personnel Unclear risk

(performance bias)

All outcomes

Blinding of outcome assessment (detection Unclear risk

bias)

Falls and fallers

Blinding of outcome assessment (detection Unclear risk

bias)

Fractures

Blinding of outcome assessment (detection Unclear risk

bias)

Hospital admission \& medical attention

Incomplete outcome data (attrition bias) Unclear risk All outcomes

\section{Support for judgement}

Quote: "randomly generated numbers constructed by a biostatistician who was not involved in the recruitment process"

Quote: “Randomization was achieved using consecutively numbered, sealed, opaque envelopes containing randomly generated numbers constructed by a biostatistician who was not involved in the recruitment process."

Participants and personnel implementing the intervention not blind to allocated group, but impact of non-blinding unclear

Not applicable

Not applicable

Not applicable

Less than $20 \%$ missing outcome data, unbalanced losses across groups with similar reasons for missing data

1. Multifactorial and Interdisciplinary Team Approach: randomised $\mathrm{n}=54$, analysed $\mathrm{n}=49$, (3 died, 2 refused treatment) 
Markle-Reid 2010 (Continued)

2. Usual care: randomised $\mathrm{n}=55$, analysed $\mathrm{n}=43$, (4 died, 8 refused treatment)

Selective reporting (reporting bias) High risk

Different outcomes stated in clinical trials register compared to full-text publication

$\begin{array}{lll}\text { Method of ascertaining falls } & \text { Unclear risk } & \text { Not applicable }\end{array}$

Mendoza-Ruvalcaba 2015

Methods

Study Design: RCT (parallel design)

Number of study arms: 2

Study centres: Multiple centres

Length of follow-up: 6 months

\section{Participants}

Setting: Mexico

Number randomised: 72

Number analysed: 64

Number lost to follow-up: 8

Sample: From senior centre

Age (years): 70.6

Sex: $89 \%$ women

Ethnicity : Not reported

Inclusion criteria: Age 60 years or older, availability to attend sessions at least twice a week, willingness to participate in the programme, and being literate

Exclusion criteria: Depressive symptomatology measured by the Spanish version of the Geriatric Depression Scale and cognitive impairment determined by the Mini-Mental State Examination

Interventions

Type of intervention: Multiple intervention

1. I am active programme: reality orientation, physical activity, nutritional education, cognitive exercises $(\mathrm{n}=36)$

2. Waitlist: $(\mathrm{n}=36)$

Who delivered the intervention: Trainer

Compliance assessed: Not reported

Outcomes

1. Health-related quality of life (Spanish version of Quality of Life Index 0 - 30, overall, psychological, and health and functionality subscales: endpoint score)

\begin{tabular}{|c|c|}
\hline Notes & $\begin{array}{l}\text { Source of funding: Not reported } \\
\text { Conflicts of interest: None } \\
\text { Economic information: It was found that participants in the programme showed im- } \\
\text { provements after the intervention (post-test) in social and economic status }(\mathrm{P}<0.05, \mathrm{~d} \\
=0.59) \text {, with medium effect sizes of } \mathrm{d}=0.59 \text {, respectively, which declined at follow-up } \\
\text { to small effect sizes }(\mathrm{d}=0.27 \text { ) }\end{array}$ \\
\hline
\end{tabular}

Risk of bias

Multifactorial and multiple component interventions for preventing falls in older people living in the community (Review) 
Mendoza-Ruvalcaba 2015 (Continued)

\begin{tabular}{|c|c|c|}
\hline Bias & Authors' judgement & Support for judgement \\
\hline $\begin{array}{l}\text { Random sequence generation (selection } \\
\text { bias) }\end{array}$ & Unclear risk & Insufficient information \\
\hline Allocation concealment (selection bias) & Unclear risk & Not reported \\
\hline $\begin{array}{l}\text { Blinding of participants and personnel } \\
\text { (performance bias) } \\
\text { All outcomes }\end{array}$ & Unclear risk & $\begin{array}{l}\text { Participants and personnel not blind to al- } \\
\text { located group but impact on blinding un- } \\
\text { clear }\end{array}$ \\
\hline $\begin{array}{l}\text { Blinding of outcome assessment (detection } \\
\text { bias) } \\
\text { Falls and fallers }\end{array}$ & Unclear risk & Not applicable \\
\hline $\begin{array}{l}\text { Blinding of outcome assessment (detection } \\
\text { bias) } \\
\text { Fractures }\end{array}$ & Unclear risk & Not applicable \\
\hline $\begin{array}{l}\text { Blinding of outcome assessment (detection } \\
\text { bias) } \\
\text { Hospital admission \& medical attention }\end{array}$ & Unclear risk & Not applicable \\
\hline $\begin{array}{l}\text { Incomplete outcome data (attrition bias) } \\
\text { All outcomes }\end{array}$ & Unclear risk & $\begin{array}{l}\text { Less than } 20 \% \text { missing outcome data, un- } \\
\text { balanced losses across groups with no rea- } \\
\text { sons for missing data } \\
\text { 1. I am active programme: randomised } n= \\
36 \text {, analysed } n=31,(5 \text { missing, no reasons) } \\
\text { 2. Waitlist: randomised } n=36 \text {, analysed } n \\
=33 \text {, ( } 3 \text { missing, no reasons) }\end{array}$ \\
\hline Selective reporting (reporting bias) & Low risk & $\begin{array}{l}\text { All outcomes listed in Methods are reported } \\
\text { in Results }\end{array}$ \\
\hline Method of ascertaining falls & Unclear risk & Not applicable \\
\hline
\end{tabular}

Metzelthin 2013

Methods

Study Design: Cluster RCT

Number of study arms: 2

Number of clusters: 12

Study centres: Multiple centres

Length of follow-up: 24 months

Participants

Setting: The Netherlands

Number randomised: 346

Number analysed: 270

Number lost to follow-up: 76 
Sample: They invited all general practices in the region of Sittard, The Netherlands and its surrounding area that had no current active and systematic policy for the detection and follow-up of frail older people to take part in the study

Age (years): Mean 77.2 (S.D, 5.1)

Sex: $58 \%$ women

Ethnicity : Not reported

Inclusion criteria: Community-dwelling frail older patients (70 years or older)

Exclusion criteria: Terminally ill, confined to bed, had severe cognitive or psychological impairments, unable to communicate in Dutch

Interventions

Type of intervention: Multifactorial intervention

1. Prevention of care approach: Frailty screening, assessment, analysis and preliminary treatment plan, agreement on treatment plan, executing treatment plan, evaluation and follow-up $(\mathrm{n}=193)$

2. Control: usual care $(\mathrm{n}=153)$

Who delivered the intervention: Practice nurses, general practitioner, occupational therapist, physical therapist, pharmacist geriatrician

Compliance assessed: Not reported

Outcomes

Notes
1. Health-related quality of life

Source of Funding: Funded by the Dutch National care for the elderly programme by The Netherlands Organisation for Health Research and Development

Conflicts of interest: Not reported

Economic information: mean total healthcare costs Intervention group: GBP 26,503;

Control: GBP 20,550

\section{Risk of bias}

\section{Bias}

Random sequence generation (selection Low risk bias)

\section{Support for judgement}

Quote: "stratified the practices in pairs and used a computer generated randomisation list to randomise into intervention or control"

Insufficent information

Participants and personnel not blind to allocated group but impact of non-blinding unclear

Not applicable

bias)

Falls and fallers

Blinding of outcome assessment (detection Unclear risk bias)

Fractures 
Metzelthin 2013 (Continued)

\begin{tabular}{|c|c|c|}
\hline $\begin{array}{l}\text { Blinding of outcome assessment (detection } \\
\text { bias) } \\
\text { Hospital admission \& medical attention }\end{array}$ & Unclear risk & Not applicable \\
\hline $\begin{array}{l}\text { Incomplete outcome data (attrition bias) } \\
\text { All outcomes }\end{array}$ & High risk & $\begin{array}{l}\text { More than } 20 \% \text { missing outcome data,un- } \\
\text { balanced losses across groups with similar } \\
\text { reasons for missing data } \\
\text { 1. Prevention of care approach: randomised } \\
\mathrm{n}=193 \text {, analysed } \mathrm{n}=143 \text {, ( } 15 \text { died, } 8 \text { ad- } \\
\text { mitted, } 12 \text { health problems, } 8 \text { lost interest, } \\
7 \text { other reasons) } \\
\text { 2. Usual care: randomised } \mathrm{n}=153 \text {, anal- } \\
\text { ysed } \mathrm{n}=127 \text { ( } 10 \text { died, } 5 \text { admitted, } 4 \text { health } \\
\text { problems, } 6 \text { lost interest, } 1 \text { other reasons) }\end{array}$ \\
\hline Selective reporting (reporting bias) & Low risk & $\begin{array}{l}\text { All outcomes listed in the abstract were re- } \\
\text { ported }\end{array}$ \\
\hline Method of ascertaining falls & Unclear risk & Not applicable \\
\hline Relating to cluster randomisation & Low risk & $\begin{array}{l}\text { Recruitment bias: GP practices were ran- } \\
\text { domised prior to screening, but all eligible } \\
\text { participants within a cluster were invited to } \\
\text { participate (low risk) } \\
\text { Baseline imbalance: baseline similar be- } \\
\text { tween intervention arms (low risk) } \\
\text { Loss of clusters: no clusters lost from the } \\
\text { trial (low risk) } \\
\text { Incorrect analysis: the trial adjusted for } \\
\text { clustering (low risk) } \\
\text { Comparability: results comparable with in- } \\
\text { dividually-randomised trials (low risk) }\end{array}$ \\
\hline
\end{tabular}

Möller 2014

Methods

Study Design: RCT (parallel design)

Number of study arms: 2

Study centres: Multiple centres

Length of follow-up: 12 months

Participants

Setting: Sweden

Number randomised: 153

Number analysed: 106

Number lost to follow-up: 47

Sample: The sample was recruited through the municipal home care organization $(\mathrm{n}=$ 13), from 3 care centres in the municipality $(n=117), 3$ clinics at a nearby University hospital $(\mathrm{n}=20)$, or by own referral $(\mathrm{n}=3)$

Age (years): Mean 81.5 (S.D, 6.4)

Multifactorial and multiple component interventions for preventing falls in older people living in the community (Review) 


\begin{tabular}{|c|c|}
\hline & $\begin{array}{l}\text { Sex: } 67 \% \text { women } \\
\text { Ethnicity : Not reported } \\
\text { Inclusion criteria: Aged } 65 \text { years or older, resident in the study municipality, need of } \\
\text { help with at least } 2 \text { activities of daily living, admitted to hospital at least twice or have } \\
\text { had at least } 4 \text { outpatient contacts during the previous } 12 \text { months. The participants had } \\
\text { to be able to communicate verbally and to have no cognitive impairments (i.e. a score } \\
\text { of } \geq 25 \text { in MMSE) } \\
\text { Exclusion criteria: None }\end{array}$ \\
\hline Interventions & $\begin{array}{l}\text { Type of intervention: Multifactorial intervention } \\
\text { 1. Home-based case management intervention: Falls risk assessment, tailored exercise } \\
\text { programme, referral to physical therapist, home safety assessment with corrections ( } \mathrm{n}= \\
\text { 80) } \\
\text { 2. Control: Usual care }(\mathrm{n}=73) \\
\text { Who delivered the intervention: Nurses, physiotherapists } \\
\text { Compliance assessed: No }\end{array}$ \\
\hline Outcomes & $\begin{array}{l}\text { 1. Rate of falls } \\
\text { 2. Number of people sustaining } 1 \text { or more falls } \\
\text { 3. Number of people sustaining recurrent falls } \\
\text { 4. Number of people requiring medical attention (e.g. attendance at emergency depart- } \\
\text { ment, requiring GP consultation) }\end{array}$ \\
\hline Notes & $\begin{array}{l}\text { Source of Funding: Faculty of Medicine at Lund University, the Swedish Institute for } \\
\text { Health Sciences, Region Skane, the Governmental Funding of Clinical Research within } \\
\text { the NHS (ALF), the Swedish Research Council, the Greta and Johan Kock Foundation, } \\
\text { and the Magnus Bergval Foundation } \\
\text { Conflicts of interest: Not reported } \\
\text { Economic information: Not reported }\end{array}$ \\
\hline
\end{tabular}

Risk of bias

\begin{tabular}{l|ll}
\hline Bias & Authors' judgement & Support for judgement \\
\hline $\begin{array}{l}\text { Random sequence generation (selection } \\
\text { bias) }\end{array}$ & Unclear risk & Not reported \\
\hline $\begin{array}{l}\text { Allocation concealment (selection bias) } \\
\begin{array}{l}\text { Blinding of participants and personnel } \\
\text { (performance bias) } \\
\text { All outcomes }\end{array}\end{array}$ & Lnclear risk & Sealed envelopes \\
\hline
\end{tabular}

Blinding of outcome assessment (detection High risk

Falls were self-reported in the last 3 months bias)

Falls and fallers 
Blinding of outcome assessment (detection Unclear risk

Not applicable

bias)

Fractures

Blinding of outcome assessment (detection High risk bias)

Hospital admission \& medical attention

Incomplete outcome data (attrition bias) High risk All outcomes
More than 20\% missing outcome data, balanced losses across groups with similar reasons for missing data

1. Home-based case management intervention: randomised $\mathrm{n}=80$, analysed $\mathrm{n}=56$, (9 died, 15 declined to participate)

2. Usual care: randomised $n=73$, analysed $\mathrm{n}=50$ (3 died, 18 declined to participate, 2 lost to follow-up)

\begin{tabular}{l|l|l}
\hline Selective reporting (reporting bias) & Low risk & All in Methods section reported in Results \\
\hline Method of ascertaining falls & High risk & $\begin{array}{l}\text { Retrospective self-report in the last 3 } \\
\text { months }\end{array}$ \\
\hline
\end{tabular}

Neelemaat 2012

Methods

Study design: RCT (parallel design)

Number of study arms: 2

Study centres: Multiple centres

Length of follow-up: 3 months
Setting: The Netherlands

Number randomised: 210

Number analysed: 150

Number lost to follow-up: 60

Sample: Malnourished older adults newly admitted to an acute hospital (general internal medicine, rheumatology, gastroenterology, dermatology, nephrology, orthopaedics, traumatology, or vascular surgery) and discharged into the community (not all communitydwelling, but $88 \%$ were prior to admission)

Age (years): Mean 74.5 (SD 9.5)

Sex: Not reported

Ethnicity: Not reported

Inclusion criteria: Aged $\geq 60$; expected length of hospital stay $>2$ days; malnourished $\left(\mathrm{BMI} \leq 20.0 \mathrm{~kg} / \mathrm{m}^{2}, 5 \%\right.$ or more self-reported unintentional weight loss in the previous month, or $10 \%$ or more self-reported unintentional weight loss in the previous 6 months) Exclusion criteria: Dementia 
Neelemaat 2012 (Continued)

\begin{tabular}{|c|c|}
\hline Interventions & $\begin{array}{l}\text { Type of intervention: Multiple intervention } \\
\text { 1. Nutritional intervention (energy- and protein-enriched diet, oral nutritional supple- } \\
\text { ments, calcium-vitamin D supplement, telephone counselling by a dietitian }(\mathrm{n}=105) \\
\text { 2. Control: usual care ( } \mathrm{n}=105) \\
\text { Who delivered the intervention: Dietician } \\
\text { Compliance assessed: Yes. "The dietitian contacted participants by telephone" }\end{array}$ \\
\hline Outcomes & $\begin{array}{l}\text { 1. Rate of falls } \\
\text { 2. Number of people sustaining } 1 \text { or more falls } \\
\text { 3. Number of people who experienced } 1 \text { or more fall-related fractures }\end{array}$ \\
\hline Notes & $\begin{array}{l}\text { Source of funding: The Netherlands Organisation for Health Research and Development } \\
\text { (ZonMw) } \\
\text { Conflicts of interest: None } \\
\text { Economic information: Not reported }\end{array}$ \\
\hline
\end{tabular}

Risk of bias

\begin{tabular}{lll}
\hline Bias & Authors' judgement & Support for judgement \\
\hline $\begin{array}{l}\text { Random sequence generation (selection } \\
\text { bias) }\end{array}$ & Low risk & $\begin{array}{l}\text { Quote: "A computerized random number } \\
\text { generator was used to assign participants in } \\
\text { blocks of } 10 \text { to the control or intervention } \\
\text { group" }\end{array}$ \\
\hline Allocation concealment (selection bias) & Low risk & $\begin{array}{l}\text { Quote: “ the primary investigator (FN) } \\
\text { opened a consecutively numbered opaque } \\
\text { envelope containing the participant's group } \\
\text { assignment" }\end{array}$ \\
\hline
\end{tabular}

Blinding of participants and personnel Unclear risk (performance bias)

All outcomes

Participants and personnel not blind to allocated group but impact of non-blinding unclear

Blinding of outcome assessment (detection Low risk bias)

Falls and fallers

Quote: "Participants recorded their falls weekly, and were asked to return their first diary by mail 6 weeks after discharge from hospital. In a few cases, sending back the diary was not possible, and the information on falls was obtained over the telephone"

Blinding of outcome assessment (detection Low risk

Not applicable

bias)

Fractures

Blinding of outcome assessment (detection Low risk bias)

Hospital admission \& medical attention 
Neelemaat 2012 (Continued)

\begin{tabular}{|c|c|c|}
\hline $\begin{array}{l}\text { Incomplete outcome data (attrition bias) } \\
\text { All outcomes }\end{array}$ & High risk & $\begin{array}{l}\text { More than } 20 \% \text { missing outcome data, } \\
\text { losses balanced across groups with similar } \\
\text { reasons for missing data } \\
\text { 1. Nutritional intervention: randomised } n \\
=105 \text {, analysed } n=75 \text { ( } 16 \text { withdrew, } 14 \\
\text { died during the study) } \\
\text { 2. Usual care: randomised } n=105 \text {, analysed } \\
n=75 \text { ( } 19 \text { withdrew, } 11 \text { died during the } \\
\text { study) }\end{array}$ \\
\hline Selective reporting (reporting bias) & Low risk & All prespecified outcomes were reported. \\
\hline Method of ascertaining falls & Low risk & $\begin{array}{l}\text { Prospective weekly recording, and tele- } \\
\text { phone call }\end{array}$ \\
\hline
\end{tabular}

Newbury 2001

\begin{tabular}{ll}
\hline Methods & Study Design: RCT (parallel design) \\
& Number of study arms: 2 \\
& Study centres: Multiple centres \\
& Length of follow-up: 12 months \\
\hline Participants & Setting: Australia \\
& Number randomised: 100 \\
& Number analysed: 89 \\
& Number lost to follow-up: 11 \\
& Sample: Every 20 th name in an age-sex register of community-dwelling patients regis- \\
& tered with 6 general practices $(63 \%$ women) \\
& Age: Median (intervention group) 78.5; (control group) 80, range $75-91$ \\
& Sex: $63 \%$ women \\
& Ethnicity: Not reported \\
& Inclusion criteria: Aged $\geq 75 ;$ independently community-dwelling \\
& Exclusion criteria: None reported \\
\hline
\end{tabular}

Interventions

Type of intervention: Multifactorial intervention

1. Health assessment of people aged 75 years or older by nurse $(75+\mathrm{HA})$. Problems identified were counted and reported to participant's GP. No reminders or other intervention for 12 months $(\mathrm{n}=50)$

2. No 75+HA until 12 months after randomisation $(n=50)$

Who delivered intervention: Nurse

Complaince assessed: Not reported

Outcomes

1. Number of people sustaining 1 or more falls

2. Health-related quality of life

Notes

Source of funding: General Practice Evaluation Prgram, Commonwealth Dept of Health and Aged Care

Multifactorial and multiple component interventions for preventing falls in older people living in the community (Review) 
Newbury 2001 (Continued)

Conflicts of interest: None

Economic information: Not reported

\section{Risk of bias}

\begin{tabular}{|c|c|c|}
\hline Bias & Authors' judgement & Support for judgement \\
\hline $\begin{array}{l}\text { Random sequence generation (selection } \\
\text { bias) }\end{array}$ & Low risk & Randomisation by random numbers \\
\hline Allocation concealment (selection bias) & Low risk & Sequentially-numbered sealed envelopes \\
\hline $\begin{array}{l}\text { Blinding of participants and personnel } \\
\text { (performance bias) } \\
\text { All outcomes }\end{array}$ & Unclear risk & $\begin{array}{l}\text { Participants and personnel not blind to al- } \\
\text { located group but impact of non-blinding } \\
\text { unclear }\end{array}$ \\
\hline $\begin{array}{l}\text { Blinding of outcome assessment (detection } \\
\text { bias) } \\
\text { Falls and fallers }\end{array}$ & Unclear risk & $\begin{array}{l}\text { Self-report by participant, timeframe not } \\
\text { specified }\end{array}$ \\
\hline $\begin{array}{l}\text { Blinding of outcome assessment (detection } \\
\text { bias) } \\
\text { Fractures }\end{array}$ & Unclear risk & Not applicable \\
\hline $\begin{array}{l}\text { Blinding of outcome assessment (detection } \\
\text { bias) } \\
\text { Hospital admission \& medical attention }\end{array}$ & Unclear risk & Not applicable \\
\hline $\begin{array}{l}\text { Incomplete outcome data (attrition bias) } \\
\text { All outcomes }\end{array}$ & Low risk & $\begin{array}{l}\text { Less than } 20 \% \text { missing outcome data, losses } \\
\text { balanced across groups with similar reasons } \\
\text { for missing data } \\
\text { 1. } 75+\text { HA: randomised } n=50 \text {, analysed } n \\
=45 \text { ( } 1 \text { died, } 1 \text { too unwell, } 3 \text { discontinued) } \\
\text { 2. No } 75+\text { HA: randomised } n=50 \text {, analysed } \\
44 \text { ( } 5 \text { died, } 1 \text { declined) }\end{array}$ \\
\hline Selective reporting (reporting bias) & Low risk & All prespecified outcomes were reported \\
\hline Method of ascertaining falls & Unclear risk & $\begin{array}{l}\text { Self-report by participant, time frame not } \\
\text { specified }\end{array}$ \\
\hline
\end{tabular}


Methods
Study Design: RCT (parallel design)

Number of study arms: 5 (3 eligible)

Study centres: Single centre

Length of follow-up: 12 months
Participants
Setting: Singapore

Number randomised: 246 (147 eligible)

Number analysed: 228

Number lost to follow-up: 18

Sample: Community

Age (years): Mean 70 (SD 4.7)

Sex: $61 \%$ women

Ethnicity: Not reported

Inclusion criteria: Aged 65 years and above, able to walk without personal assistance, and living at home. Pre-frail or frail defined as at least 1 of: unintentional weight loss, slowness, weakness, exhaustion, and low activity

Exclusion criteria: Significant cognitive impairment (MMSE score $\leq 23$ ), major depression, severe audiovisual impairment, any progressive degenerative neurologic disease, terminal illness with life expectancy $<12$ months; were participating in other interventional studies, or were unavailable to participate for the full duration of the study

\section{Interventions}

Type of intervention: Multiple intervention

1. Combination: Physical activity, nutritional supplements, cognitive training $(\mathrm{n}=49)$

2. Physical exercise: Resistance exercises (integrated with functional tasks); and balance training exercises (involving functional strength and sensory input) $(\mathrm{n}=48)$

3. Usual care: Placebo $(n=50)$

Who delivered the intervention: Interventional nurses

Compliance assessed: Yes, adherence of participants to the intervention was determined by averaged proportion of supplements consumed and sessions completed

Outcomes

1. Number of people sustaining 1 or more falls

2. Number of people who experience a fall that required hospital admission

3. Adverse events of the intervention

Source of Funding: National Medical Research Council (Singapore)
Conflicts of interest: None
Economic information: Not reported
Adverse events: "2 subjects who participated in exercise training had joint pain (hip and
knee) initially, that was relieved after adjusting the training regime. No other adverse
events occurred during the study"

\section{Risk of bias}

\begin{tabular}{l|l|l} 
Bias & Authors' judgement & Support for judgement \\
\hline $\begin{array}{l}\text { Random sequence generation (selection } \\
\text { bias) }\end{array}$ & Low risk & $\begin{array}{l}\text { Central computerised randomisation pro- } \\
\text { cedure }\end{array}$
\end{tabular}

Multifactorial and multiple component interventions for preventing falls in older people living in the community (Review) 
$\mathrm{Ng} 2015$ (Continued)

\begin{tabular}{|c|c|c|}
\hline Allocation concealment (selection bias) & Low risk & $\begin{array}{l}\text { Treatment was allocated by a project man- } \\
\text { ager not involved in the enrolment, inter- } \\
\text { vention or assessment }\end{array}$ \\
\hline $\begin{array}{l}\text { Blinding of participants and personnel } \\
\text { (performance bias) } \\
\text { All outcomes }\end{array}$ & Unclear risk & $\begin{array}{l}\text { Participants and personnel not blind to al- } \\
\text { located group but impact of non-blinding } \\
\text { unclear }\end{array}$ \\
\hline $\begin{array}{l}\text { Blinding of outcome assessment (detection } \\
\text { bias) } \\
\text { Falls and fallers }\end{array}$ & Unclear risk & $\begin{array}{l}\text { Self-reported by participant, time frame } \\
\text { not specified }\end{array}$ \\
\hline $\begin{array}{l}\text { Blinding of outcome assessment (detection } \\
\text { bias) } \\
\text { Fractures }\end{array}$ & Unclear risk & Not applicable \\
\hline $\begin{array}{l}\text { Blinding of outcome assessment (detection } \\
\text { bias) } \\
\text { Hospital admission \& medical attention }\end{array}$ & High risk & Self-reported by participant \\
\hline $\begin{array}{l}\text { Incomplete outcome data (attrition bias) } \\
\text { All outcomes }\end{array}$ & Low risk & $\begin{array}{l}\text { Less than } 20 \% \text { missing outcome data, losses } \\
\text { balanced across groups with similar reasons } \\
\text { for missing data } \\
\text { 1. Combination: randomised } n=49 \text {, anal- } \\
\text { ysed } n=46 \text { ( } 3 \text { withdrew) } \\
\text { 2. Physical exercise: randomised } n=48 \text {, } \\
\text { analysed } n=46 \text { ( } 1 \text { withdrew, } 1 \text { unable to } \\
\text { contact) } \\
\text { 3. Placebo: randomised } n=50 \text {, analysed } n \\
=46 \text { ( } 3 \text { withdrew, } 1 \text { died) }\end{array}$ \\
\hline Selective reporting (reporting bias) & Low risk & $\begin{array}{l}\text { All outcomes given in Methods are reported } \\
\text { in Results }\end{array}$ \\
\hline Method of ascertaining falls & Unclear risk & $\begin{array}{l}\text { Self-reported by participant, time frame } \\
\text { not specified }\end{array}$ \\
\hline
\end{tabular}

Olsen 2014

Methods

Study Design: RCT(parallel design)

Number of study arms: 2

Study centres: Single centre

Length of follow-up: 12 months

\begin{tabular}{ll}
\hline Participants & Setting: Norway \\
& Number randomised: 89 \\
& Number analysed: 70 \\
& Number lost to follow-up: 19
\end{tabular}

Multifactorial and multiple component interventions for preventing falls in older people living in the community (Review)

Copyright ( 2018 The Cochrane Collaboration. Published by John Wiley \& Sons, Ltd. 
Sample: Participants were recruited from the osteoporosis outpatient clinic at the Ostfold Hospital, Sarpsborg, Norway

Age (years): Mean 71

Sex: $100 \%$ women

Ethnicity: Not reported

Inclusion criteria: Established osteoporosis by means of dual energy $\mathrm{x}$-ray absorptiometry using WHO criteria for osteoporosis, history of 1 or more vertebral fractures verified by radiography, aged 60 years or older, living at home and ambulatory

Exclusion criteria: Major cognitive impairments (MMSE), recent vertebral fractures, inability to complete questionnaires

Interventions

Outcomes

Notes
Type of intervention: Multiple intervention

1. Multiple intervention: 3-month group-based circuit exercise programme and 3-hour educational session focusing on the reduction of the risk of falls and challenges specific to osteoporosis and vertebral fractures. $(n=47)$

2. Control: Usual care $(\mathrm{n}=42)$

Who delivered the intervention: Physiotherapist

Compliance assessed: Yes, session attendance

Number of people sustaining 1 or more falls

2. Adverse events of the intervention

Source of Funding: The Norwegian Fund for postgraduate training in physiotherapy
Conflicts of interest: None
Economic information: Not reported
Adverse events: "No adverse events or side effects associated with the exercise program
were reported by the intervention group participants"

\section{Risk of bias}

\begin{tabular}{|c|c|c|}
\hline Bias & Authors' judgement & Support for judgement \\
\hline $\begin{array}{l}\text { Random sequence generation (selection } \\
\text { bias) }\end{array}$ & Unclear risk & $\begin{array}{l}\text { Quote: " the subjects were randomly as- } \\
\text { signed by a computer generated list in two } \\
\text { groups, intervention and control" }\end{array}$ \\
\hline Allocation concealment (selection bias) & Unclear risk & $\begin{array}{l}\text { Quote: "Researchers not involved in the } \\
\text { study performed the randomization by } \\
\text { drawing lots concealed in sealed opaque en- } \\
\text { velopes" }\end{array}$ \\
\hline
\end{tabular}

Blinding of participants and personnel Unclear risk (performance bias)

All outcomes

Blinding of outcome assessment (detection Unclear risk bias)

Falls and fallers
Participants and personnel not blind to allocated group but impact of non-blinding unclear

Self-reported by participant, time frame not specified 
Blinding of outcome assessment (detection Unclear risk

Not applicable

bias)

Fractures

Blinding of outcome assessment (detection Unclear risk bias)

Hospital admission \& medical attention

Incomplete outcome data (attrition bias) Unclear risk All outcomes
Not applicable

Less than $20 \%$ missing outcome data,unbalanced losses across groups with similar reasons for missing data

1. Multiple intervention: randomised $\mathrm{n}=$ 47, analysed $\mathrm{n}=38$ ( 2 did not receive allocated intervention, 7 lost to follow-up)

2. Usual care: randomised $n=42$, analysed $\mathrm{n}=32$ (10 lost to follow-up)

All outcomes stated in the Methods section were reported

Falls were recorded retrospectively by selfreport, time frame not specified

Palvanen 2014

Methods

Number of study centres: 2
Study centres: Multiple centres
Length of follow-up: 12 months

Setting: Finland

Number randomised: 1314

Number analysed: 1145

Number lost to follow-up: 169

Sample: Home-dwelling persons, aged $>70$ with increased risk of falling and fall-induced injuries

Age (years): Mean 77 (SD 5.7)

Sex: $86 \%$ women

Ethnicity: Not reported

Inclusion criteria: Home-dwelling; aged $\geq 70$; problems in mobility or every day function, 3 or more falls in last 12 months, high risk for falling and fall-induced injuries and fractures

Exclusion criteria: Inability to consent, disabilities or illness preventing physical activity, inability to move
Type of intervention: Multifactorial intervention

1. Chaos clinic intervention: Baseline assessment and general injury-prevention brochure plus individual preventive measures by Chaos Clinic staff based on baseline assessment: 


\begin{tabular}{|c|c|}
\hline & $\begin{array}{l}\text { physical activity prescription, nutritional advice, individually-tailored or group exercises, } \\
\text { treatment of conditions, medication review, alcohol reduction, smoking cessation, hip } \\
\text { protectors, osteoporosis treatment, home hazard assessment and modification ( } \mathrm{n}=661) \\
\text { 2. Control: Baseline assessment and general injury prevention brochure alone (not falls- } \\
\text { specific) ( } \mathrm{n}=653 \text { ) } \\
\text { Who delivered intervention: Nurse, physiotherapist and physician } \\
\text { Compliance assessed: Yes, adherence was 'checked' at each contact session with the } \\
\text { therapist }\end{array}$ \\
\hline Outcomes & $\begin{array}{l}\text { 1. Rate of falls } \\
\text { 2. Number of people sustaining } 1 \text { or more falls }\end{array}$ \\
\hline
\end{tabular}

Notes

Source of funding: multiple sources of Finnish government bodies

Conflicts of interest: None

Economic information: Not reported

Risk of bias

\begin{tabular}{|c|c|c|}
\hline Bias & Authors' judgement & Support for judgement \\
\hline $\begin{array}{l}\text { Random sequence generation (selection } \\
\text { bias) }\end{array}$ & Unclear risk & Insufficient information \\
\hline Allocation concealment (selection bias) & Low risk & Sealed opaque envelopes \\
\hline $\begin{array}{l}\text { Blinding of participants and personnel } \\
\text { (performance bias) } \\
\text { All outcomes }\end{array}$ & Unclear risk & $\begin{array}{l}\text { Participants and personnel not blind to al- } \\
\text { located group but impact of non-blinding } \\
\text { unclear }\end{array}$ \\
\hline $\begin{array}{l}\text { Blinding of outcome assessment (detection } \\
\text { bias) } \\
\text { Falls and fallers }\end{array}$ & High risk & $\begin{array}{l}\text { Falls measured by phone calls at } 3 \text { and } 9 \\
\text { months, and on follow-up visits at } 6 \text { and } \\
12 \text { months }\end{array}$ \\
\hline $\begin{array}{l}\text { Blinding of outcome assessment (detection } \\
\text { bias) } \\
\text { Fractures }\end{array}$ & Unclear risk & Not applicable \\
\hline $\begin{array}{l}\text { Blinding of outcome assessment (detection } \\
\text { bias) } \\
\text { Hospital admission \& medical attention }\end{array}$ & Unclear risk & Not applicable \\
\hline $\begin{array}{l}\text { Incomplete outcome data (attrition bias) } \\
\text { All outcomes }\end{array}$ & Low risk & $\begin{array}{l}\text { Less than } 20 \% \text { missing outcome data, losses } \\
\text { balanced across groups with similar reasons } \\
\text { for missing data } \\
1 \text {. Chaos clinic intervention: randomised } \\
\mathrm{n}=661 \text {, analysed } \mathrm{n}=589 \text { ( } 35 \text { illness, } 31 \\
\text { refusal to continue, } 3 \text { died, } 3 \text { other) } \\
\text { 2. Control: randomised } \mathrm{n}=653 \text {, analysed } \\
\mathrm{n}=556 \text { (54 illness, } 29 \text { refusal to continue, }\end{array}$ \\
\hline
\end{tabular}


Palvanen 2014 (Continued)

8 died, 4 moved, 2 other)

\begin{tabular}{|c|c|c|}
\hline Selective reporting (reporting bias) & Low risk & $\begin{array}{l}\text { All outcomes stated in the Methods section } \\
\text { were reported }\end{array}$ \\
\hline Method of ascertaining falls & High risk & $\begin{array}{l}\text { Measured by phone calls at } 3 \text { and } 9 \text { months, } \\
\text { and on follow-up visits at } 6 \text { and } 12 \text { months } \\
\text { from the beginning }\end{array}$ \\
\hline
\end{tabular}

Pardessus 2002

\begin{tabular}{ll} 
Methods & $\begin{array}{l}\text { Study Design: RCT (parallel design) } \\
\text { Number of study arms: } 2 \\
\text { Study centres: Single centre } \\
\text { Length of follow-up: } 12 \text { months }\end{array}$ \\
\hline Participants & Setting: France \\
& Number randomised: 60 \\
& Number analysed: 51 \\
& Number lost to follow-up: 9 \\
& Sample: Recruited from acute geriatric department of the geriatric hospital \\
& Age (years): Mean 83.2 (SD 7.7) \\
Sex: $78.3 \%$ female \\
Ethnicity: Not reported \\
Inclusion criteria: Age 65 years or older, hospitalised for falling, able to return home after \\
hospitalisation, and gave informed consent for participation \\
Exclusion criteria: Patients with cognitive impairment (MMSE < 24), without phone, \\
patients who lived further than 30 km from the hospital, those whose falls were secondary \\
to cardiac, neurologic, vascular, or therapeutic problems
\end{tabular}

Interventions

Type of intervention: Multifactorial intervention

1. Home visits: Home visit to evaluate the participant's abilities in his/her real-life environment. Modifications made or advice provided. $(\mathrm{n}=30)$

2. Control: Usual care $(\mathrm{n}=30)$

Who delivered the intervention: Physical medicine and rehabilitation doctor, ergo-therapist, hospital social worker

Compliance assessed: Yes, OT checked if the home modifications had been made or encouraged their implementation

Outcomes

1. Rate of falls

2. Number of people sustaining 1 or more falls

3. Number of people who experienced a fall that required hospital admission

Notes

Source of Funding: Not reported

Conflicts of interest: Not reported

Economic information: Not reported

\section{Risk of bias}

Multifactorial and multiple component interventions for preventing falls in older people living in the community (Review) 
Pardessus 2002 (Continued)

\begin{tabular}{|c|c|c|}
\hline Bias & Authors' judgement & Support for judgement \\
\hline $\begin{array}{l}\text { Random sequence generation (selection } \\
\text { bias) }\end{array}$ & Low risk & A random number table was used. \\
\hline Allocation concealment (selection bias) & Unclear risk & Not reported \\
\hline $\begin{array}{l}\text { Blinding of participants and personnel } \\
\text { (performance bias) } \\
\text { All outcomes }\end{array}$ & Unclear risk & $\begin{array}{l}\text { Participants and personnel not blind to al- } \\
\text { located group but impact of non-blinding } \\
\text { unclear }\end{array}$ \\
\hline $\begin{array}{l}\text { Blinding of outcome assessment (detection } \\
\text { bias) } \\
\text { Falls and fallers }\end{array}$ & Unclear risk & $\begin{array}{l}\text { Self-report by participant based on } \\
\text { monthly telephone call }\end{array}$ \\
\hline $\begin{array}{l}\text { Blinding of outcome assessment (detection } \\
\text { bias) } \\
\text { Fractures }\end{array}$ & Unclear risk & Not applicable \\
\hline $\begin{array}{l}\text { Blinding of outcome assessment (detection } \\
\text { bias) } \\
\text { Hospital admission \& medical attention }\end{array}$ & High risk & $\begin{array}{l}\text { Self-report by participant based on } \\
\text { monthly telephone call }\end{array}$ \\
\hline $\begin{array}{l}\text { Incomplete outcome data (attrition bias) } \\
\text { All outcomes }\end{array}$ & Low risk & $\begin{array}{l}\text { Less than } 20 \% \text { missing outcome data, losses } \\
\text { unbalanced across groups with similar rea- } \\
\text { sons for missing data. ( } 20 \% \text { died in inter- } \\
\text { vention group and } 10 \% \text { died in control } \\
\text { group) } \\
\text { 1. Home visits: randomised } n=30 \text {, anal- } \\
\text { ysed } n=24 \text { ( } 6 \text { died) } \\
2 \text {. Usual care: randomised } n=30 \text {, analysed } \\
n=27 \text { ( } 3 \text { died) }\end{array}$ \\
\hline Selective reporting (reporting bias) & Low risk & $\begin{array}{l}\text { All outcomes reported in methods were } \\
\text { given in results }\end{array}$ \\
\hline Method of ascertaining falls & Unclear risk & $\begin{array}{l}\text { Falls were recorded by self-report by partic- } \\
\text { ipant based on monthly telephone call }\end{array}$ \\
\hline
\end{tabular}

Rubenstein 2007

Methods

Study design: RCT (parallel design)

Number of study arms: 2

Study centres: Single centre

Length of follow-up: 12 months 
Number analysed: 694

Number lost to follow-up: 98

Sample: Patients receiving care at ambulatory care centre

Age (years): Mean 74.5 (SD 6)

Sex: 3\% women

Ethnicity: Not reported

Inclusion criteria: Aged $\geq 65$; previously randomised to either of the 2 practice groups involved in the trial; $\geq 1$ clinic visit in previous 18 months; scoring $\geq 4$ on GPSS

Exclusion criteria: Living over 30 miles from care centre; already enrolled in outpatient geriatric services at care centre; living in long-term care facility; scoring less than 4 GPSS

Interventions

Type of intervention: Multifactorial intervention

1. Multifactorial intervention: Structured risk and needs assessment and referral algorithm implemented by case manager (physician assistant). Targeting 5 geriatric conditions including falls. Assessment followed by referrals and recommendations for further assessment or treatment. 3-monthly telephone contact with case manager $(\mathrm{n}=380)$

2. Control: usual care $(\mathrm{n}=412)$

Who delivered intervention: Physician assistant, case manager, geriatricians, internal medicine home staff, geriatric psychiatrist, physical therapist

Compliance assessed: Yes, the case manager phoned intervention participants 1 month after the first telephone contact, and again every 3 months over the 3-year study period. The purpose of these follow-up falls was to encourage participants to adhere to referrals and recommendations, and also to monitor changes in health

Outcomes

1. Rate of falls

2. Number of people sustaining 1 or more falls

3. Number of people who experience a fall that require hospital admission

4. Health-related quality of life (SF-36 0 - 100: endpoint score)

Notes

Source of funding: The research was supported by the Department of Veterans Affairs, Veterans Health Administration, Health Services Research and Development Service (HSR\&D), and the VA Greater Los Angeles Geriatric Research, Education and Clinical Center

Conflicts of interest: Not reported

Economic information: Not reported

\section{Risk of bias}

\begin{tabular}{l|l|l} 
Bias & Authors' judgement & Support for judgement \\
\hline $\begin{array}{l}\text { Random sequence generation (selection } \\
\text { bias) }\end{array}$ & Unclear risk & $\begin{array}{l}\text { Participants “previously" randomly as- } \\
\text { signed by Social Security number to one of } \\
3 \text { primary care practice groups. One prac- } \\
\text { tice was assigned to intervention and one } \\
\text { to control; the third practice group was not } \\
\text { included in this study because it was in- }\end{array}$ \\
\hline
\end{tabular}


Rubenstein 2007 (Continued)

volved in the pilot study

\begin{tabular}{|c|c|c|}
\hline Allocation concealment (selection bias) & Unclear risk & $\begin{array}{l}\text { Insufficent information to permit judge- } \\
\text { ment }\end{array}$ \\
\hline $\begin{array}{l}\text { Blinding of participants and personnel } \\
\text { (performance bias) } \\
\text { All outcomes }\end{array}$ & Unclear risk & $\begin{array}{l}\text { Participants and personnel implementing } \\
\text { the intervention not blind to allocated } \\
\text { group, but impact of non-blinding unclear }\end{array}$ \\
\hline $\begin{array}{l}\text { Blinding of outcome assessment (detection } \\
\text { bias) } \\
\text { Falls and fallers }\end{array}$ & High risk & $\begin{array}{l}\text { Data were collected by telephone at } 12 \\
\text { months. Participants unwilling to be sur- } \\
\text { veyed by telephone were mailed question- } \\
\text { naires }\end{array}$ \\
\hline $\begin{array}{l}\text { Blinding of outcome assessment (detection } \\
\text { bias) } \\
\text { Fractures }\end{array}$ & Unclear risk & Not applicable \\
\hline $\begin{array}{l}\text { Blinding of outcome assessment (detection } \\
\text { bias) } \\
\text { Hospital admission \& medical attention }\end{array}$ & Unclear risk & Not applicable \\
\hline $\begin{array}{l}\text { Incomplete outcome data (attrition bias) } \\
\text { All outcomes }\end{array}$ & Low risk & $\begin{array}{l}\text { Less than } 20 \% \text { missing outcome data, losses } \\
\text { balanced across groups with similar reasons } \\
\text { for missing data } \\
\text { 1. Multifactorial intervention: randomised } \\
n=380 \text {, analysed } n=334 \text { ( } 8 \text { refused, } 9 \\
\text { unable to contact, } 29 \text { died) } \\
\text { 2. Usual care: randomised } n=412 \text {, analysed } \\
n=360 \text { ( } 11 \text { refused, } 17 \text { unable to contact, } \\
24 \text { died) }\end{array}$ \\
\hline Selective reporting (reporting bias) & Low risk & All prespecified outcomes were reported \\
\hline Method of ascertaining falls & High risk & $\begin{array}{l}\text { Data were collected by telephone at } 12 \\
\text { months. Participants unwilling to be sur- } \\
\text { veyed by telephone were mailed question- } \\
\text { naires }\end{array}$ \\
\hline
\end{tabular}

\section{Russell 2010}

Methods

Study design: RCT (parallel design)

Number of study arms: 2

Study centres: Multiple centres

Length of follow-up: 12 months

Participants

Setting: Australia

Number randomised: 712

Number analysed: 650 
Number lost to follow-up: 62

Sample: People presenting to ED after a fall

Age (years): $13 \% 60$ to $64 ; 17 \% 65$ to $70 ; 19 \% 70$ to $74 ; 19 \% 75$ to $79 ; 32 \% \geq 80$

Sex: $70 \%$ women

Ethnicity: Not reported

Inclusion criteria: Aged $\geq 60$; community-dwelling; presenting to ED after a fall and discharged straight home

Exclusion criteria: Unable to comply with simple instructions; unable to walk independently indoors (with or without walking aids)

Type of intervention: Multifactorial intervention

1. Multifactorial falls prevention programme: standard care in ED + assessed (FROPCom) and offered multifactorial falls prevention programme consisting of referrals to existing community services and health promotion recommendations. Participants at high risk of falls (FROP-Com score $\geq 25$ ) referred to falls clinic for comprehensive multidisciplinary assessment $(\mathrm{n}=351)$

2. Control: standard care in ED + letter to participants informing them of level of falls risk (FROP-Com), recommendation to speak to GP $(\mathrm{n}=361)$

Who delivered intervention: Baseline assessor, physiotherapist, OT, podiatrist, dietitian, family physician, research fellow

Compliance assessed: Yes, the research officer who collected the 12-month falls and fallinjury data also collected adherence data 4 and 6 months after the baseline assessment. Participants were questioned about all referrals and recommendations made by the study assessors and the ED. They were asked whether they attended the appointment, what recommendations the service made, and whether they had followed the recommendations

$\begin{array}{ll}\text { Outcomes } & \text { 1. Rate of falls } \\ \text { 2. Number of people sustaining } 1 \text { or more falls } \\ \text { 3. Number of people sustaining } 1 \text { or more fall-related fractures }\end{array}$

Notes

Source of funding: Australian Government Department of Veterans' Affairs and the Victorian Department of Human Services

Conflicts of interest: None

Economic information: Not reported

\section{Risk of bias}

\begin{tabular}{l|l|l} 
Bias & Authors' judgement & Support for judgement \\
\hline $\begin{array}{l}\text { Random sequence generation (selection } \\
\text { bias) }\end{array}$ & Low risk & $\begin{array}{l}\text { Quote: "Randomization was performed us- } \\
\text { ing a computer-generated randomization } \\
\text { list." }\end{array}$ \\
\hline Allocation concealment (selection bias) & Low risk & $\begin{array}{l}\text { Quote: "A researcher otherwise not in- } \\
\text { volved in the project generated and held the } \\
\text { randomization sequence." }\end{array}$
\end{tabular}


Blinding of participants and personnel Unclear risk (performance bias)

All outcomes

Blinding of outcome assessment (detection Low risk bias)

Falls and fallers

Blinding of outcome assessment (detection Low risk bias)

Fractures

Blinding of outcome assessment (detection Low risk bias)

Hospital admission \& medical attention
Participants and personnel not blind to allocated group but impact of non-blinding unclear

Participants recorded falls and injuries on a falls calender which they were asked to return monthly using postage-paid mail. Participants were also telephoned every 2 months to confirm details in the calender

Participants recorded falls and injuries on a falls calender which they were asked to return monthly using postage-paid mail. Participants were also telephoned every 2 months to confirm details in the calender

Quote: “After each participant's 12-month follow-up period, his or her hospital medical record was reviewed to verify ED presentations, days in the hospital, and when available, falls and fall injuries. The medical record reviewed in each case was that held at the hospital to which the participant presented after the initial fall". However, medical record information was unavailable for $10.6 \%$ of participants

Incomplete outcome data (attrition bias) Low risk All outcomes

Less than $20 \%$ of missing outcome, losses balanced across groups with similar reasons for missing data

1. Multifactorial falls prevention program: randomised $\mathrm{n}=351$, analysed $\mathrm{n}=344(4$ withdrew, 3 died)

2. Standard care: randomised $\mathrm{n}=361$, analysed $\mathrm{n}=354$ (7 withdrew)

\begin{tabular}{ll} 
Selective reporting (reporting bias) Low risk \\
\hline
\end{tabular}

All prespecified outcomes were reported

Method of ascertaining falls

Low risk

Participants recorded falls and injuries on a falls calender which they were asked to return monthly using postage-paid mail. Participants were also telephoned every 2 months to confirm details in the calender 


\begin{tabular}{ll}
\hline Methods & $\begin{array}{l}\text { Study design: RCT (parallel design) } \\
\text { Number of study arms: } 2\end{array}$ \\
& $\begin{array}{l}\text { Study centres: Single centre } \\
\text { Length of follow-up: } 36 \text { months }\end{array}$ \\
\hline Participants & Setting: The Netherlands \\
& Number randomised: 222 \\
& Number analysed: 182 \\
& Number lost to follow-up: 40 \\
& Sample: People living at home (N = 146) or in residential homes (N = 76) \\
& Age (years): $70 \%$ aged 77 to $84,30 \% \geq 85$ \\
& Sex: $70 \%$ women \\
Ethnicity: Not reported \\
Inclusion criteria: Aged $\geq 75 ;$ living at home or in 1 of 2 residential homes; having \\
problems with $\geq 1$ of the following: IADL, ADL, toileting, mobility or fallen in last 6 \\
months, serious agitation or confusion; informed consent from participant and their GP \\
Exclusion criteria: Living in nursing home; received outpatient or inpatient care from \\
geriatric unit in previous 2 years
\end{tabular}

Interventions

Type of intervention: Multifactorial intervention

1.Comprehensive assessment: Comprehensive assessment in outpatient geriatric unit (geriatrician, psychologist, social worker); advice to participant and GP about treatment and support $(\mathrm{n}=110)$

2. Control: usual care $(\mathrm{n}=112)$

Who delivered intervention: Geriatrician, psychologist, social worker, physiotherapist Compliance assessed: Yes, a written report was given to the elderly and their GP. GP asked if they followed advice of OGA-unit

Outcomes $\quad$ 1. Number of people sustaining recurrent falls

Notes

Source of funding: The Province of Limburg and the Directorate of Policy for the Elderly of The Netherlands Ministry of Social Welfare. Public Health and Culture

Conflicts of interest: Not reported

Economic information: Not reported

Included in this review as most of the participants were living at home $(\mathrm{N}=146)$

\section{Risk of bias}

\section{Bias}

Random sequence generation (selection Unclear risk bias)

Allocation concealment (selection bias)
Unclear risk

\section{Support for judgement}

Stratified by living condition (home versus home for the elderly) then "randomly allocated" by researcher in blocks of 10

Insufficient information to permit judgement 
Schrijnemaekers 1995 (Continued)

Blinding of participants and personnel Unclear risk (performance bias)

All outcomes

Blinding of outcome assessment (detection Unclear risk bias)

Falls and fallers

Blinding of outcome assessment (detection Unclear risk bias)

Fractures

Blinding of outcome assessment (detection Unclear risk bias)

Hospital admission \& medical attention

Incomplete outcome data (attrition bias) Unclear risk All outcomes

All

\begin{tabular}{lll}
\hline Selective reporting (reporting bias) & Low risk & All prespecified outcomes were reported \\
\hline Method of ascertaining falls & Unclear risk & Method of falls detection not reported \\
\hline
\end{tabular}

\section{Serra-Prat 2017}

Methods

Study Design: RCT (parallel design)

Number of study arms: 2

Study centres: Multiple centres

Length of follow-up: 12 months

Participants

Setting: Spain

Number randomised: 172

Number analysed: 133

Number lost to follow-up: 39

Sample: All non-institutionalised patients aged $\geq 70$ years consulting for any reason at any of the 3 participating primary care centres in Mataro (Barcelona, Spain) were screened for frailty according to Fried criteria

Age (years): Mean 78.3

Sex: $57 \%$ women

Ethnicity : Not reported

Inclusion criteria: Non-institutionalised patients, aged $\geq 70$ years, pre-fail status as defined by one or more of the Fried criteria 
Exclusion criteria: persons unable to stand without assistance, completely blind, previous diagnosis of dementia recorded in clinical notes, receiving palliative care or with life expectancy below 6 months

Interventions

Type of intervention: Multiple intervention

1. Nutritional and physical activity components: Malnutrition screening, dietary recommendations and corrective measures, physical activity programme (aerobic exercise and 15 mixed exercises) $(\mathrm{n}=80)$

2. Control: Usual care $(\mathrm{n}=92)$

Who delivered the intervention: Nurses

Compliance assessed: Yes, a) A nurse monitored compliance by regular telephone contacts with the participants; b) To assess adherence to the study intervention, participants were asked to keep a diary

Outcomes

1. Number of people sustaining 1 or more falls

2. Health-related quality of life (QoL VAS 0 - 10: endpoint score)

3. Adverse events of the intervention

Notes

Source of funding: Partially funded by grants from the Spanish Ministry of Health. Instituto de Salud Carlos III, Fondo de Investigacion Sanitaria FIS programme P113/ 00931

Conflicts of interest: None

Economic information: Not reported

Adverse events: "No adverse events were reported"

\section{Risk of bias}

\begin{tabular}{|c|c|c|}
\hline Bias & Authors' judgement & Support for judgement \\
\hline $\begin{array}{l}\text { Random sequence generation (selection } \\
\text { bias) }\end{array}$ & Low risk & $\begin{array}{l}\text { Quote: "Blocked random code and se- } \\
\text { quentially numbered sealed envelopes were } \\
\text { prepared in the research unit" }\end{array}$ \\
\hline Allocation concealment (selection bias) & Low risk & $\begin{array}{l}\text { Quote: "Randomisation was based on the } \\
\text { opaque envelope method and was stratified } \\
\text { according to } 21 \text { general practitioners par- } \\
\text { ticipating in the study" }\end{array}$ \\
\hline $\begin{array}{l}\text { Blinding of participants and personnel } \\
\text { (performance bias) } \\
\text { All outcomes }\end{array}$ & Unclear risk & $\begin{array}{l}\text { Participants and personnel not blind to al- } \\
\text { located group but impact of non-blinding } \\
\text { unclear }\end{array}$ \\
\hline $\begin{array}{l}\text { Blinding of outcome assessment (detection } \\
\text { bias) } \\
\text { Falls and fallers }\end{array}$ & Unclear risk & Method of fall assessment not reported \\
\hline
\end{tabular}

Blinding of outcome assessment (detection Unclear risk bias)

Fractures

Multifactorial and multiple component interventions for preventing falls in older people living in the community (Review) 


\begin{tabular}{|c|c|c|}
\hline $\begin{array}{l}\text { Blinding of outcome assessment (detection } \\
\text { bias) } \\
\text { Hospital admission \& medical attention }\end{array}$ & Unclear risk & Not applicable \\
\hline $\begin{array}{l}\text { Incomplete outcome data (attrition bias) } \\
\text { All outcomes }\end{array}$ & Unclear risk & $\begin{array}{l}\text { More than } 20 \% \text { missing outcome data, } \\
\text { losses are balanced across groups with sim- } \\
\text { ilar reasons for missing data } \\
\text { 1. Nutritional and physical activity compo- } \\
\text { nents: randomised } n=80 \text {, analysed } n=61 \\
\text { ( } 19 \text { declined, } 0 \text { died }) \\
\text { 2. Usual care: randomised } n=92 \text {, analysed } \\
n=72(18 \text { declined, } 2 \text { died })\end{array}$ \\
\hline Selective reporting (reporting bias) & Low risk & $\begin{array}{l}\text { All outcomes listed in abstract were re- } \\
\text { ported }\end{array}$ \\
\hline Method of ascertaining falls & Unclear risk & Not reported \\
\hline
\end{tabular}

\section{Sheffield 2013}

Methods
Study Design: RCT (parallel design)

Number of study arms: 2

Study centres: Single centre

Length of follow-up: 3 months
Participants
Setting: United States of America

Number randomised: 90

Number analysed: 60

Number lost to follow-up: 30

Sample: Adults over 65 receiving some form of agency care. All participants were known to the 2 local public agencies involved in the study

Age (years): Mean 81.67 (SD 9.46)

Sex: $80 \%$ women

Ethnicity : 58\% white, $41 \%$ non-white, $1 \%$ not disclosed; 7\% Hispanic, 93\% nonHispanic

Inclusion criteria: Community-dwelling over-65s receiving some form of agency care. Additional inclusion criteria included ability to speak English, adequate mobility within the home and sufficient cognitive capacity to participate in the intervention.

Exclusion criteria: None reported
Type of intervention: Multifactorial intervention

1. Home assessment of daily activities in the context of environment, client-family collaboration to achieve mutual goals, provision and training in the use of assistive devices, design and implementation of home modifications, removal of environmental hazards, training in medication management and education in adaptive and compensatory strategies to improve safety and independence: Home assessment, goal-setting, assistive devices, home modification and education $(n=46)$

2. Delayed intervention control group: As above but delayed $(n=44)$ 
Sheffield 2013 (Continued)

Who delivered the intervention: Occupational therapist

Compliance assessed: No

\begin{tabular}{ll}
\hline Outcomes & 1. Health-related quality of life \\
\hline Notes & $\begin{array}{l}\text { Source of Funding: Not reported } \\
\text { Conflicts of interest: None } \\
\text { Economic information: Intervention costs for equipment and home modifications av- } \\
\text { eraged USD 205 per client. Therapy costs inclusive of travel time were USD 940. The } \\
\text { mean intervention costs was USD 1145 per client }\end{array}$ \\
\hline
\end{tabular}

Risk of bias

\begin{tabular}{|c|c|c|}
\hline Bias & Authors' judgement & Support for judgement \\
\hline $\begin{array}{l}\text { Random sequence generation (selection } \\
\text { bias) }\end{array}$ & Low risk & $\begin{array}{l}\text { Quote: "independent researcher blinded to } \\
\text { participant characteristics performed block } \\
\text { randomisation using computer generated } \\
\text { random allocation" }\end{array}$ \\
\hline Allocation concealment (selection bias) & Low risk & $\begin{array}{l}\text { Quote: "independent researcher blinded to } \\
\text { participant characteristics performed block } \\
\text { randomisation using computer generated } \\
\text { random allocation" }\end{array}$ \\
\hline
\end{tabular}

Blinding of participants and personnel Unclear risk (performance bias)

Participants and personnel not blind to All outcomes group assignment but effect of non-blinding unclear

Blinding of outcome assessment (detection Unclear risk

Not applicable

bias)

Falls and fallers

Blinding of outcome assessment (detection Unclear risk bias)

Fractures

Not applicable

Blinding of outcome assessment (detection Unclear risk bias)

Hospital admission \& medical attention

Incomplete outcome data (attrition bias) High risk All outcomes
More than $20 \%$ of missing outcome data, losses are balanced across groups with different reasons for missing data

1. Multifactorial intervention: randomised $\mathrm{n}=46$, analysed $\mathrm{n}=31$ (5 refused followup, 2 unknown reasons, 1 died, 7 found to be ineligible)

2. Delayed intervention : randomised $\mathrm{n}=$ 
Sheffield 2013 (Continued)

44, analysed $\mathrm{n}=29$ (1 moved, 2 institutionalised, 7 found to be ineligible, 5 required emergency intervention)

\begin{tabular}{|l|l|l}
\hline Selective reporting (reporting bias) & High risk & $\begin{array}{l}\text { Not all secondary outcome measures stip- } \\
\text { ulated in protocol paper reported in study } \\
\text { paper }\end{array}$
\end{tabular}

Shyu 2010

\section{Methods}

\begin{tabular}{ll} 
Methods & S \\
& S \\
& L \\
\hline Participants & S \\
& S
\end{tabular}

Study design: RCT (parallel design)

Number of study arms: 2

Study centres: Single centre

Length of follow-up: 12 months

Setting: Taiwan

Number randomised: 162

Number analysed: 122

Number lost to follow-up: 40

Sample: Admitted to hospital for an accidental single side hip fracture

Age (years): Mean 78.2 (SD 7.8)

Sex: $69 \%$ women

Ethnicity: Not reported

Inclusion criteria: Aged $\geq 60$; received hip arthroplasty or internal fixation; able to perform full range of motion; prefracture Chinese Barthel Index $>70$

Exclusion criteria: severely cognitively impaired; terminally ill

\section{Interventions}

Type of intervention: Multifactorial intervention

1. Multidisciplinary programme: geriatric consultation services, a continuous rehabilitation programme, discharge planning services $(n=80)$

2. Control: usual care $(\mathrm{n}=82)$

Who delivered intervention: Geriatric nurses, geriatrician, physical rehabilitation physician, orthopaedists

Compliance assessed: Not reported

Outcomes

1. Number of people who sustained 1 or more falls

2. Number of people who experienced a fall and required hospital admission

3. Number of people who experienced a fall that required medical attention

4. Health-related quality of life (SF-36 0 - 100, mental and physical subscales: endpoint score)

Notes

Source of funding: National Health Research Institute, Taiwan

Conflicts of interest: None

Economic information: The estimated cost added by the intervention program to the current routine care was USD 438

Multifactorial and multiple component interventions for preventing falls in older people living in the community (Review) 
Shyu 2010 (Continued)

\section{Risk of bias}

\begin{tabular}{|c|c|c|}
\hline Bias & Authors' judgement & Support for judgement \\
\hline $\begin{array}{l}\text { Random sequence generation (selection } \\
\text { bias) }\end{array}$ & Low risk & $\begin{array}{l}\text { Quote: "The randomization was con- } \\
\text { ducted using flip of coin by a neutral third } \\
\text { party who was not involved in delivering } \\
\text { the intervention or assessing outcomes" }\end{array}$ \\
\hline Allocation concealment (selection bias) & Unclear risk & $\begin{array}{l}\text { Quote: "Those persons who agreed to par- } \\
\text { ticipate were randomly assigned to an ex- } \\
\text { perimental or control group at the time } \\
\text { of admission. The randomization was con- } \\
\text { ducted using flip of coin by a neutral third } \\
\text { party who was not involved in delivering } \\
\text { the intervention or assessing outcomes" } \\
\text { Insufficient detail to allow a definite judge- } \\
\text { ment }\end{array}$ \\
\hline
\end{tabular}

Blinding of participants and personnel Unclear risk (performance bias)

Participants and personnel not blind to alAll outcomes located group but effect of non-blinding unclear

Blinding of outcome assessment (detection Unclear risk bias)

Self-reports of patients and family caregivers, face-to-face interviews

Falls and fallers

Blinding of outcome assessment (detection Low risk

bias)

Fractures

Blinding of outcome assessment (detection Unclear risk bias)

Hospital admission \& medical attention

Incomplete outcome data (attrition bias) High risk All outcomes

\section{Not applicable}

Self-reports of patients and family caregivers, face-to-face interviews

More than $20 \%$ of missing outcome data, losses are balanced across groups with similar reasons for missing data

1. Multidisciplinary programme: randomised $n=80$, analysed $n=60$ (16 refused to participate, 4 died)

2. Usual care: randomised $\mathrm{n}=82$, analysed $\mathrm{n}=62$ (14 refused to participate, 6 died)

\begin{tabular}{ll|l}
\hline Selective reporting (reporting bias) & Low risk & All prespecified outcomes were reported \\
\hline Method of ascertaining falls & Unclear risk & $\begin{array}{l}\text { Self-reports of patients and family care- } \\
\text { givers, face-to-face interviews }\end{array}$ \\
\hline
\end{tabular}

Multifactorial and multiple component interventions for preventing falls in older people living in the community (Review) 
Methods

Participants
Study Design: RCT ( 2 × 2 factorial design)

Number of study arms: 4

Study centres: Single centre

Length of follow-up: 6 months

\begin{tabular}{ll}
\hline Participants & Setting: Canada \\
& Number randomised: 37 \\
& Number analysed: 34 \\
& Number lost to follow-up: 3 \\
& Sample: Recruited from the North American Research Commitee on Multiple Sclerosis \\
& Patient Registry \\
Age (years): Mean 62.3 (SD 8.7) & Sex: $65 \%$ women \\
Ethnicity : Not reported \\
Inclusion criteria: Neurologist-confirmed diagnosis of Multiple Sclerosis, able to walk \\
with/without aid, demonstrate a comprehension of English, self-reported fall in the last \\
12 months, age between 45 and 75 years old, live within 175-mile radius of testing site, \\
relapse-free for 30 days prior to participation \\
Exclusion criteria: None \\
\hline
\end{tabular}

Interventions

Type of intervention: Multiple intervention

1. Home-Based Exercise: Home-based exercise, focusing on improving balance and lower limb/core muscle strength $(\mathrm{n}=11)$

2. Education group: Visited laboratory at baseline, weeks 2, 4 and 8, groups ranged from 2 to 4 people and lasted approximately 1 hour; education drew on psychoeducational group theory and self-management literature (group brain-storming, problemsolving and action-planning). The programme also applied core principles of self-efficacy enhancement, in particular peer-modelling, vicarious learning, social persuasion and guided mastery $(\mathrm{n}=9)$

3. Exercise and education: Exercise focusing on improving balance and lower limb/core muscle strength

Education drew on psychoeducational group theory and self-management literature (group brain-storming, problem-solving and action-planning). The programme also applied core principles of self-efficacy enhancement, in particular peer-modelling, vicarious learning, social persuasion and guided mastery. $(\mathrm{n}=8)$

4. Waiting List Control: Usual care $(\mathrm{n}=9)$

Who delivered the intervention: 6 trained nurses qualified in the field of geriatrics and working for home-care agencies, trained interventionalist/specialist

Compliance assessed: Yes, Exercise Diary

1. Number of people sustaining 1 or more falls

Notes

Source of Funding: National Multiple Sclerosis Society

Conflicts of interest: None

Economic information: Not reported

\section{Risk of bias}




\begin{tabular}{|c|c|c|}
\hline $\begin{array}{l}\text { Random sequence generation (selection } \\
\text { bias) }\end{array}$ & Low risk & $\begin{array}{l}\text { Quote: "Simple randomization method } \\
\text { with a } 1: 1: 1: 1 \text { allocation ratio (indepen- } \\
\text { dent of baseline assessment) by computer } \\
\text { generated random numbers" }\end{array}$ \\
\hline Allocation concealment (selection bias) & Low risk & $\begin{array}{l}\text { Quote: "Group allocation for each partici- } \\
\text { pant was concealed in opaque envelopes" }\end{array}$ \\
\hline $\begin{array}{l}\text { Blinding of participants and personnel } \\
\text { (performance bias) } \\
\text { All outcomes }\end{array}$ & Unclear risk & $\begin{array}{l}\text { Participants and personnel for blind to al- } \\
\text { located group but effect of non-blinding } \\
\text { unclear }\end{array}$ \\
\hline $\begin{array}{l}\text { Blinding of outcome assessment (detection } \\
\text { bias) } \\
\text { Falls and fallers }\end{array}$ & Low risk & $\begin{array}{l}\text { Monthly falls diary and follow-up tele- } \\
\text { phone call }\end{array}$ \\
\hline $\begin{array}{l}\text { Blinding of outcome assessment (detection } \\
\text { bias) } \\
\text { Fractures }\end{array}$ & Unclear risk & Not applicable \\
\hline $\begin{array}{l}\text { Blinding of outcome assessment (detection } \\
\text { bias) } \\
\text { Hospital admission \& medical attention }\end{array}$ & Unclear risk & Not applicable \\
\hline $\begin{array}{l}\text { Incomplete outcome data (attrition bias) } \\
\text { All outcomes }\end{array}$ & Low risk & $\begin{array}{l}\text { Less than } 20 \% \text { missing outcome data, losses } \\
\text { are balanced across groups with similar rea- } \\
\text { sons for missing data } \\
\text { 1. Home-based exercise group: randomised } \\
\mathrm{n}=11 \text {, analysed } \mathrm{n}=1 \text { (unable to travel) } \\
\text { 2. Education group: randomised } \mathrm{n}=9 \text {, } \\
\text { analysed } \mathrm{n}=1 \text { (lost contact) } \\
\text { 3. Home-based exercise and education } \\
\text { group: randomised } \mathrm{n}=8 \text {, analysed } \mathrm{n}=8 \\
\text { 4. Wait list Control: randomised } \mathrm{n}=9 \text {, } \\
\text { analysed } \mathrm{n}=8 \text { ( } 1 \text { elective spinal surgery) }\end{array}$ \\
\hline Selective reporting (reporting bias) & Low risk & $\begin{array}{l}\text { All outcomes listed in the Methods were } \\
\text { reported }\end{array}$ \\
\hline Method of ascertaining falls & Low risk & $\begin{array}{l}\text { Questionnaire, falls diary and telephone } \\
\text { calls }\end{array}$ \\
\hline
\end{tabular}




\begin{tabular}{|c|c|}
\hline Methods & $\begin{array}{l}\text { Study design: Cluster RCT } \\
\text { Number of study arms: } 3 \\
\text { Number of clusters: } 18 \\
\text { Study centres: Multiple centres } \\
\text { Length of follow-up: } 12 \text { months }\end{array}$ \\
\hline Participants & $\begin{array}{l}\text { Setting: United Kingdom } \\
\text { Number randomised: } 516 \\
\text { Number analysed: } 422 \\
\text { Number lost to follow-up: } 94 \\
\text { Sample: Patients in } 18 \text { general practices } \\
\text { Age (years): Mean } 82 \\
\text { Sex: Not reported } \\
\text { Ethnicity: Not reported } \\
\text { Inclusion criteria: Aged } \geq 65 \text {; community-dwelling; history of at least } 2 \text { falls in previous } \\
\text { year; not presenting to A\&E with index fall } \\
\text { Exclusion criteria: None described }\end{array}$ \\
\hline Interventions & $\begin{array}{l}\text { Type of intervention: Multifactorial intervention } \\
\text { 1. Primary care intervention: health visitor/practice nurse falls risk assessment/referral } \\
\text { ( } \mathrm{n}=141) \\
\text { 2. Secondary care intervention: multidisciplinary day hospital assessment by physician, } \\
\text { OT, and physiotherapist }(\mathrm{n}=213) \\
\text { 3. Control: usual care }(\mathrm{n}=162) \\
\text { Who delivered the intervention: Trained nurses, GP, occupational therapist, physiother- } \\
\text { apist, geriatrician } \\
\text { Compliance assessed: Yes, proportion of different interventions provided such as medi- } \\
\text { cation changes, smoke alarms and duration measured }\end{array}$ \\
\hline Outcomes & $\begin{array}{l}\text { 1. Number of people who sustained } 1 \text { or more falls } \\
\text { 2. Number of people who sustained } 1 \text { or more fall-related fractures } \\
\text { 3. Number of people who experienced a fall that required hospital admission } \\
\text { 4. Health-related quality of life }\end{array}$ \\
\hline Notes & $\begin{array}{l}\text { Source of funding: Grants were received from Winchester Health Promotion Service, } \\
\text { Shire Pharmaceuticals and Proctor and Gamble, with later funding from Mid-Hampshire } \\
\text { Primary Care Trust } \\
\text { Conflicts of interest: None } \\
\text { Economic information: Not reported }\end{array}$ \\
\hline
\end{tabular}

\section{Risk of bias}

Bias Authors' judgement

Random sequence generation (selection Low risk bias)

\section{Support for judgement}

Cluster-randomised.

Quote: "Practices were stratified into urban (three) and rural (fifteen) and randomly allocated to the three arms, in blocks of three, using a random number generator on a Hewlett 
Spice 2009 (Continued)

Packard 21S pocket calculator"

Allocation concealment (selection bias) Unclear risk

Insufficient information to permit judgement

Blinding of participants and personnel Unclear risk (performance bias)

All outcomes

Participants and personnel not blind to allocated group but impact of non-blinding unclear

Blinding of outcome assessment (detection Unclear risk bias)

Falls and fallers

Participants were followed monthly for 12 months, with participants indicating how many falls they had by selecting from the options of 1,2, 3, 4 or $>4$. If the card was not returned, the participant was contacted by telephone. Participants were unblinded to intervention

Blinding of outcome assessment (detection Unclear risk bias)

Participants were followed monthly for 12 months

Fractures

Blinding of outcome assessment (detection Unclear risk bias)

Monthly self-reports from participants

Hospital admission \& medical attention

Incomplete outcome data (attrition bias) Unclear risk All outcomes

Less than $20 \%$ missing outcome data, losses are unbalanced across groups with similar reasons for missing data

1. Primary care intervention group: randomised $\mathrm{n}=141$ ( 8 clusters), analysed $\mathrm{n}=$ 114 (12 died, 10 withdrew, 5 ineligible)

2. Secondary care intervention group: randomised $\mathrm{n}=213$ (4 clusters), analysed $\mathrm{n}=$ 176 (11 died, 23 withdrew, 3 ineligible)

3. Usual care: randomised $n=162$ (6 clusters), analysed $\mathrm{n}=132$ (17 died, 10 withdrew, 3 ineligible)

Selective reporting (reporting bias) Low risk

Method of ascertaining falls
Unclear risk
All prespecified outcomes were reported

Participants were followed monthly for 12 months, with participants indicating how many falls they had by selecting from the options of 1,2, 3, 4 or $>4$. If the card was not returned, the participant was contacted by telephone. Participants were unblinded to intervention 
Spice 2009 (Continued)

Relating to cluster randomisation
Recruitment bias: all GP practices were invited to participate prior to randomisation, but it is unclear how participants were then recruited (unclear risk)

Baseline imbalance: baseline similar between intervention arms (low risk)

Loss of clusters: no clusters lost from the trial (low risk)

Incorrect analysis: the trial adjusted for clustering (low risk)

Comparability: results comparable with individually randomised trials (low risk)

Tinetti 1994

Methods
Participants

Study design: Cluster RCT

Number of study arms: 2

Number of clusters: 16 treating physicians, matched in 4 groups of 4 , into 2 control and 2 intervention in each group; enrolled subjects assigned to same group as their physician Study centres: Multiple centres

Length of follow-up: 12 months

Setting: United States of America

Number randomised: 301

Number analysed: 291

Number lost to follow-up: 10

Sample: People enrolled with participating physicians

Age (years): Mean 77.9 (SD 5.3)

Sex: $69 \%$ women

Ethnicity: Not reported

Inclusion criteria: Aged > 70; community-dwelling; independently ambulant; at least 1 targeted risk factor for falling (postural hypotension, sedative/hypnotic use, use of > 4 medications, inability to transfer, gait impairment, strength or range of motion loss, domestic environmental hazards)

Exclusion criteria: Enrolment in another study; MMSE < 20; current (within last month) participation in vigorous activity

Interventions

Type of intervention: Multifactorial intervention

1. Multifactorial intervention: Interventions targeting individual risk factors, according to decision rules and priority lists. 3-month programme duration $(\mathrm{n}=153)$

2. Control: visits by social work students over same period $(\mathrm{n}=148)$

Who delivered the intervention: Nurse practitioner, physical therapist, physicians, social work students

Compliance assessed: Yes, adherence to exercise programmes as reported by participants was assessed by the physical therapist on weekly basis 
Tinetti 1994 (Continued)

\begin{tabular}{ll}
\hline Outcomes & 1. Rate of falls \\
2. Number of people sustaining 1 or more falls \\
3. Number of people who experienced a fall that required hospital admission \\
4. Number of people who experienced a fall that required medical attention \\
5. Adverse effects of the intervention
\end{tabular}

\section{Risk of bias}

\begin{tabular}{l|l|l}
\hline Bias & Authors' judgement & Support for judgement \\
\hline $\begin{array}{l}\text { Random sequence generation (selection } \\
\text { bias) }\end{array}$ & Low risk & $\begin{array}{l}\text { Quote: “Computerised randomization } \\
\text { program” }\end{array}$ \\
\hline Allocation concealment (selection bias) & Unclear risk & $\begin{array}{l}\text { Insufficient information to permit judge- } \\
\text { ment }\end{array}$ \\
\hline $\begin{array}{l}\text { Blinding of participants and personnel } \\
\text { (performance bias) } \\
\text { All outcomes }\end{array}$ & Unclear risk & $\begin{array}{l}\text { Participants and personnel not blind to al- } \\
\text { located group but effect of non-blinding } \\
\text { unclear }\end{array}$ \\
\hline
\end{tabular}

Blinding of outcome assessment (detection Low risk bias)

Falls and fallers

Falls were recorded on a calender that participants mailed to the research staff monthly

Blinding of outcome assessment (detection Unclear risk bias)

Fractures

Not applicable

Blinding of outcome assessment (detection High risk bias)

Quote: “ during a follow-up telephone interview, research staff asked subjects about

Hospital admission \& medical attention medical care sought after falls and injuries sustained"

Incomplete outcome data (attrition bias) Unclear risk All outcomes

Less than 20\% missing outcome data, with no reasons given for loss to follow-up 1. Multifactorial intervention: randomised 
Tinetti 1994 (Continued)

$\mathrm{n}=153$; analysed $\mathrm{n}=147$

2. Visits by social work students: randomised $\mathrm{n}=148$; analysed $\mathrm{n}=144$

\begin{tabular}{|c|c|c|}
\hline Selective reporting (reporting bias) & Low risk & All prespecified outcomes were reported \\
\hline Method of ascertaining falls & Low risk & $\begin{array}{l}\text { Falls were recorded on a calender that } \\
\text { participants mailed to the research staff } \\
\text { monthly }\end{array}$ \\
\hline
\end{tabular}

Relating to cluster randomisation

High risk

Recruitment bias: participants were recruited and randomised based on risk score for all participants at the same time (low risk)

Baseline imbalance: baseline similar between intervention arms (low risk)

Loss of clusters: no clusters lost from the trial (low risk)

Incorrect analysis: the trial did not adjusted for clustering (high risk)

Comparability: results comparable with individually-randomised trials (low risk)

Ueda 2017

Methods

Study Design: RCT (parallel design)

Number of study arms: 2

Study centres: Single centre

Length of follow-up: 1 month

\begin{tabular}{ll} 
Participants & Setting: Japan \\
& Number randomised: 60 \\
& Number analysed: 51 \\
& Number lost to follow-up: 9 \\
& Sample: All were discharged orthopaedic patients aged $\geq 65$ years who experienced falls \\
in the past year & Age (years): Mean 75.9 \\
Sex: $68.5 \%$ women & Ethnicity : Not reported \\
Inclusion criteria: Discharged orthopedic patients, aged $\geq 65$ years, experienced $\geq 1$ fall \\
in the past year \\
Exclusion criteria: Cognitive impairment - MMSE score 24 , patients without care \\
service, who spoke little or no Japanese, severe neurological visual disorders, who were \\
planning to move within the next month \\
\hline Type of intervention: Multifactorial intervention \\
Interventions \\
gramme using home floor plans/modifying hazards, standard care exercises (as received
\end{tabular}

Multifactorial and multiple component interventions for preventing falls in older people living in the community (Review) 
Ueda 2017 (Continued)

by control arm) $(\mathrm{n}=30)$

2. Exercise $(\mathrm{n}=30)$

Who delivered the intervention: Physical therapist

Compliance assessed: Not reported

Outcomes 1. Rate of falls

2. Number of people sustaining 1 or more falls

\begin{tabular}{ll}
\hline Notes & Source of Funding: Not reported \\
& Conflicts of interest: None \\
& Economic information: Not reported \\
\hline
\end{tabular}

Risk of bias

\begin{tabular}{|c|c|c|}
\hline Bias & Authors' judgement & Support for judgement \\
\hline $\begin{array}{l}\text { Random sequence generation (selection } \\
\text { bias) }\end{array}$ & Low risk & $\begin{array}{l}\text { Quote: "Stratified randomization was con- } \\
\text { ducted using a computer generated random } \\
\text { number schedule with randomly ordered } \\
\text { blocks of 6" }\end{array}$ \\
\hline Allocation concealment (selection bias) & Unclear risk & Insufficent information \\
\hline $\begin{array}{l}\text { Blinding of participants and personnel } \\
\text { (performance bias) } \\
\text { All outcomes }\end{array}$ & Unclear risk & $\begin{array}{l}\text { "Single blind", does not state who was } \\
\text { blinded }\end{array}$ \\
\hline
\end{tabular}

Blinding of outcome assessment (detection Low risk bias)

Prospectively using a monthly falls calender Falls and fallers and contact by telephone

Blinding of outcome assessment (detection Unclear risk bias)

Fractures

Blinding of outcome assessment (detection Unclear risk bias)

Hospital admission \& medical attention

Incomplete outcome data (attrition bias) Low risk All outcomes
Not applicable

Not applicable
Less than $20 \%$ of missing outcome data, losses are balanced across groups with similar reasons for missing data

1. Multifactorial intervention: tailored education programme and standard care exercises: randomised $\mathrm{n}=30$, analysed $\mathrm{n}=25$ (5 withdrew)

2. Usual care: randomised $\mathrm{n}=30$, analysed $\mathrm{n}=26$ (4 withdrew) 
Ueda 2017 (Continued)

\begin{tabular}{l|ll}
\hline Selective reporting (reporting bias) & Low risk & $\begin{array}{l}\text { All outcomes listed in Methods were re- } \\
\text { ported }\end{array}$ \\
\hline Method of ascertaining falls & Low risk & Each participant was given a falls calender \\
\hline
\end{tabular}

Uusi-Rasi 2015

\begin{tabular}{|c|c|}
\hline Methods & $\begin{array}{l}\text { Study Design: RCT ( } 2 \text { × } 2 \text { factorial design }) \\
\text { Number of study arms: } 4 \\
\text { Study centres: Multiple centres } \\
\text { Length of follow-up: } 24 \text { months }\end{array}$ \\
\hline Participants & $\begin{array}{l}\text { Setting: Finland } \\
\text { Number randomised: } 409 \\
\text { Number analysed: } 370 \\
\text { Number lost to follow-up: } 39 \\
\text { Sample: Aged } 70 \text { - } 80 \text { years old, living in Tampere, Finland } \\
\text { Age (years): Mean } 74.2 \\
\text { Sex: } 100 \% \text { women } \\
\text { Ethnicity: Not reported } \\
\text { Inclusion criteria: Women; aged } 70 \text { to } 80 \text { years; independently community-dwelling; } \\
\text { history of at least } 1 \text { fall in previous year; no contraindication to exercise; giving informed } \\
\text { consent } \\
\text { Exclusion criteria: Undertaking moderate-to-vigorous exercise more than } 2 \text { hours a week; } \\
\text { regular user of vitamin D, or calcium + vitamin D supplements; recent fracture (during } \\
\text { preceding } 12 \text { months); contraindication or inability to exercise; marked decline in the } \\
\text { basic activities of daily living (ADL-test); cognitively impaired (MMSE < 18); chronic } \\
\text { conditions, e.g. Parkinson's disease }\end{array}$ \\
\hline Interventions & $\begin{array}{l}\text { Type of intervention: Multiple intervention } \\
\text { 1. Exercise with vitamin D: } 20 \mu \mathrm{g} \text { of vitamin } \mathrm{D} \text { a day for } 2 \text { years supervised training } \\
\text { (twice a week for } 52 \text { weeks), and once a week for next } 52 \text { weeks }(\mathrm{n}=102) \\
\text { 2. Exercise with placebo: as above }(\mathrm{n}=103) \\
\text { 3. No exercise with vitamin D: } 20 \mu \mathrm{g} \text { of vitamin D a day for } 2 \text { years, no supervised } \\
\text { training (maintenance of their current level of physical activity) }(\mathrm{n}=102) \\
\text { 4. No exercise with placebo: placebo once a day for } 2 \text { years, no supervised training } \\
\text { (maintenance of their current level of physical activity) ( } \mathrm{n}=102) \\
\text { Who delivered the intervention: Physiotherapist } \\
\text { Compliance assessed: Yes, adherence was measured by monitored attendance, pill counts, } \\
\text { return of used packs in time of laboratory measurements every } 6 \text { months }\end{array}$ \\
\hline Outcomes & $\begin{array}{l}\text { 1. Rate of falls } \\
\text { 2. Adverse events of the intervention }\end{array}$ \\
\hline Notes & $\begin{array}{l}\text { Source of funding: Academy of Finland, Ministry of Education and Culture, competitive } \\
\text { research, fund of Pirkanmaa Hospital District and Juho Vainio Foundation } \\
\text { Conflicts of interest: None } \\
\text { Economic information (all costs in Euros): The average 2-year cost of vitamin D supple- }\end{array}$ \\
\hline
\end{tabular}

Multifactorial and multiple component interventions for preventing falls in older people living in the community (Review) 
mentation was EUR 73 per participant (EUR 0.10 per pill), while that of implementing the exercise intervention was EUR 47 per participant (EUR 63 per hour). There were no significant between-group differences for mean fall-related healthcare costs. Total costs per person year (including costs of the 2-year intervention) were lowest in the D-Ex group EUR 30.9 (9.5), compared with EUR 73.4 (10.4) in D-Ex+, EUR 188.0 (45.4) in D+Ex+, and EUR 206.9 (80.2) in D+Ex-

Given a willingness to pay EUR 3000 per injurious fall prevented, the exercise intervention had an $86 \%$ probability of being cost-effective in this population. Step-wise calculation of ICERS resulted in exclusion of D+Ex- as more expensive and less effective. Recalculated ICERS were EUR 221 for D-Ex-, EUR 708 for D-Ex+, and EUR 3820 for $\mathrm{D}+\mathrm{Ex}+$; bootstrapping indicated $93 \%$ probability that each injurious fall avoided by D-Ex+ per person year costs EUR 708

The corresponding ICERS per fall prevented (i.e. total number of falls in the comparator group minus total number of falls in the intervention group) were EUR 250 for group $\mathrm{D}-\mathrm{Ex}+$ and EUR 3920 for group $\mathrm{D}+\mathrm{Ex}+$

Adverse events: "In general, the training programme was well tolerated. There were no severe adverse events or injuries due to the training"

\section{Risk of bias}

Bias Authors' judgement

Random sequence generation (selection Low risk bias)
Unclear risk

\section{Support for judgement}

Quote: "409 participants were randomly assigned to 1 of 4 groups using a computer generated list based on simple randomization with random allocation sequence"

Quote: “409 participants were randomly assigned to 1 of 4 groups using a computer generated list based on simple randomization with random allocation sequence"

Participants and personnel not blind to allocated group but effect of non-blinding unclear

Prospectively monthly falls diaries and follow-up telephone call

Not applicable

Blinding of outcome assessment (detection Unclear risk bias)

Fractures

Blinding of outcome assessment (detection Unclear risk bias)

Hospital admission \& medical attention
Not applicable 
Uusi-Rasi 2015 (Continued)

\begin{tabular}{l|l|l}
$\begin{array}{l}\text { Incomplete outcome data (attrition bias) } \\
\text { All outcomes }\end{array}$ & Low risk & $\begin{array}{l}\text { Less than } 20 \% \text { of missing outcome data, } \\
\text { losses are balanced across groups with sim- } \\
\text { ilar reasons for missing data } \\
1 . \text { Vitamin } \mathrm{D}+\text { Exercise: randomised } \mathrm{n}= \\
102, \text { analysed } \mathrm{n}=96(2 \text { lost interest, } 4 \\
\text { health reasons) } \\
\text { 2. Placebo + exercise: randomised } \mathrm{n}=103, \\
\text { analysed } \mathrm{n}=91 \text { (3) lost interest, } 9 \text { health } \\
\text { reasons) }\end{array}$ \\
\hline Selective reporting (reporting bias) & Low risk & $\begin{array}{l}\text { All outcomes listed in Methods were re- } \\
\text { ported }\end{array}$ \\
\hline Method of ascertaining falls & Low risk & $\begin{array}{l}\text { Number of falls were recorded by monthly } \\
\text { prospective recording using a falls diary and } \\
\text { follow-up telephone call }\end{array}$ \\
\hline
\end{tabular}

Van Haastregt 2000

Methods

Study design: RCT (parallel design)

Number of study arms: 2

Study centres: Multiple centres

Length of follow-up: 18 months

Participants

Setting: The Netherlands

Number randomised: 316

Number analysed: 235

Number lost to follow-up: 81

Sample: People registered with 6 general medical practices (66\% women)

Age (years): Mean 77.2 (SD 5.1)

Sex: $66 \%$ women

Inclusion criteria: Aged $\geq 70$; community-dwelling; 2 or more falls in previous 6 months or score 3 or more on mobility scale of Sickness Impact Profile

Exclusion criteria: Bed-ridden; fully wheelchair-dependent; terminally ill; awaiting nursing home placement; receiving regular care from community nurse

Interventions

Type of intervention: Multifactorial intervention

1. Multifactorial intervention: 5 home visits from community nurse over 1 year. Screened for medical, environmental, and behavioural risk factors for falls and mobility impairment; advice, referrals, and "other actions" ( $\mathrm{n}=159)$

2. Control: usual care $(\mathrm{n}=157)$

Who delivered intervention: Community nurse

Compliance assessed: Not reported

Outcomes

1. Number of people sustaining 1 or more falls

2. Number of people who experienced a fall that required medical attention

Multifactorial and multiple component interventions for preventing falls in older people living in the community (Review) 


\begin{tabular}{|c|c|c|}
\hline Notes & \multicolumn{2}{|c|}{$\begin{array}{l}\text { Source of funding: Zorg Onderlock, Netherlands } \\
\text { Conflicts of interest: None } \\
\text { Economic information: Not reported }\end{array}$} \\
\hline \multicolumn{3}{|l|}{ Risk of bias } \\
\hline Bias & Authors' judgement & Support for judgement \\
\hline $\begin{array}{l}\text { Random sequence generation (selection } \\
\text { bias) }\end{array}$ & Low risk & $\begin{array}{l}\text { Randomisation by computer-generated } \\
\text { random numbers }\end{array}$ \\
\hline Allocation concealment (selection bias) & Unclear risk & $\begin{array}{l}\text { Insufficient information to permit judge- } \\
\text { ment }\end{array}$ \\
\hline $\begin{array}{l}\text { Blinding of participants and personnel } \\
\text { (performance bias) } \\
\text { All outcomes }\end{array}$ & Unclear risk & $\begin{array}{l}\text { Quote: "The doctors and healthcare staff } \\
\text { dealing with the participants were not told } \\
\text { which patients were allocated to the usual } \\
\text { care group". Participants and nurses con- } \\
\text { ducting home visits in intervention group } \\
\text { were not blinded. Partial blinding of other } \\
\text { health professionals. Insufficient evidence } \\
\text { to make judgement on impact of lack of } \\
\text { blinding. }\end{array}$ \\
\hline
\end{tabular}

Blinding of outcome assessment (detection Low risk bias)

Falls and fallers

Blinding of outcome assessment (detection Unclear risk bias)

Fractures

Blinding of outcome assessment (detection High risk bias)

Hospital admission \& medical attention

Incomplete outcome data (attrition bias) High risk All outcomes
Falls recorded by the participant using a monthly falls diary

\section{Not applicable}

Assessed by means of self-administered questionnaire at 12 and 18 months followup

More than $20 \%$ missing outcome data 1. Multifactorial intervention: randomised $\mathrm{n}=159$, analysed $\mathrm{n}=120$ (10 died, 14 medical reasons, 15 non medical reasons) 2. Control: randomised $\mathrm{n}=157$, analysed 115 (14 died, 9 medical reasons, 16 non medical reasons, 3 other)

\begin{tabular}{|c|c|c|}
\hline Selective reporting (reporting bias) & Low risk & All prespecified outcomes were reported \\
\hline Method of ascertaining falls & Low risk & $\begin{array}{l}\text { Falls recorded by the participant using a } \\
\text { falls diary }\end{array}$ \\
\hline
\end{tabular}


Methods

Participants

Participants

Study design: RCT (but some clusters as people living together allocated to same group) Number of study arms: 2

Study centres: Unclear

Length of follow-up: 36 months

Setting: The Netherlands

Number randomised: 580

Number analysed: 493

Number lost to follow-up: 87

Sample: General population sampled, not volunteers

Age (years): range 75 to 84

Sex: $58 \%$ women

Ethnicty: not reported

Inclusion criteria: Aged 75 to 84 ; living at home

Exclusion criteria: Patient or partner already receiving regular home-nursing care

Interventions

Type of intervention: Multifactorial intervention

1. Preventive home visits by public health nurse 4 times a year for 3 years. Extra visits/ telephone contact as required. Check list of health topics to discuss. Advice given and referrals to other services $(n=292)$

2. Control: no home visits $(\mathrm{n}=288)$

Who delivered intervention: Nurses and GP

Complaince assessed: Not reported

Outcomes

1. Number of people who experienced a fall that required hospital admission

Notes

Source of funding: Netherlands Ministry of Welfare and Cultural Affairs and Foundation for Research and Development of Social Health care

Conflicts of interest: None

Economic information: Mean total healthcare costs Intervention NLG 20,080 versus 19,321 per person. During the intervention period exchange rate 1 Dutch Guilder = GBP 0.29

\section{Risk of bias}

Bias Authors' judgement

Support for judgement

Random sequence generation (selection Low risk bias)

Low risk

\begin{tabular}{l|l} 
& \\
\hline Allocation concealment (selection bias) & Unclear risk \\
\hline $\begin{array}{l}\text { Blinding of participants and personnel } \\
\text { (performance bias) }\end{array}$ & Unclear risk \\
All outcomes & \\
\hline
\end{tabular}

All outcomes
Stratified by sex, self-rated health, composition of household and social class then randomised by computer-generated random numbers. Participants in intervention group then randomised to nurses

Insufficient information to permit judgement

Participants and nurses conducting home visits in intervention group were not blinded. Insufficient evidence to make 
Van Rossum 1993 (Continued)

judgement on impact of lack of blinding.

\begin{tabular}{l|l|l}
$\begin{array}{l}\text { Blinding of outcome assessment (detection } \\
\text { bias) }\end{array}$ & Unclear risk & Not applicable \\
Falls and fallers & \\
\hline
\end{tabular}

$\begin{array}{lll}\text { Blinding of outcome assessment (detection } & \text { Unclear risk } & \text { Not applicable }\end{array}$

bias)

Fractures

Blinding of outcome assessment (detection Unclear risk

bias)

Hospital admission \& medical attention

Requring hospital admission confirmed by postal questionnaire and personal interview

Incomplete outcome data (attrition bias) Unclear risk All outcomes

Less than $20 \%$ missing outcome data but number analysed per arm and reasons for missing data not reported

\begin{tabular}{lll}
\hline Selective reporting (reporting bias) & Unclear risk & Insufficient information \\
\hline Method of ascertaining falls & Unclear risk & Not applicable \\
\hline
\end{tabular}

Vetter 1992

\begin{tabular}{|c|c|}
\hline Methods & $\begin{array}{l}\text { Study design: RCT (parallel design) } \\
\text { Number of study arms: } 2 \\
\text { Study centres: Single centre } \\
\text { Length of follow-up: } 48 \text { months }\end{array}$ \\
\hline Participants & $\begin{array}{l}\text { Setting: United Kingdom } \\
\text { Number randomised: } 674 \\
\text { Number analysed: } 450 \\
\text { Number lost to follow-up: } 224 \\
\text { Sample: People on } 5 \text { GPs' patient lists } \\
\text { Age (years): > } 70 \\
\text { Sex: Not reported } \\
\text { Ethnicity: Not reported } \\
\text { Inclusion criteria: Aged }>70 \\
\text { Exclusion criteria: None listed }\end{array}$ \\
\hline Interventions & $\begin{array}{l}\text { Type of intervention: Multifactorial intervention } \\
\text { 1. Health visitor visits, minimum yearly, for } 4 \text { years, with advice on nutrition, environ- } \\
\text { mental modification, concomitant medical conditions, and availability of physiotherapy } \\
\text { classes if desired }(\mathrm{n}=350) \\
\text { 2. Control: usual care }(\mathrm{n}=324) \\
\text { Who delivered intervention: Health visitors, physiotherapist } \\
\text { Compliance assessed: Yes, the effectiveness of the health visitor was checked by giving } \\
\text { the respondents a photograph of the health visitor, asking whether the person had visited }\end{array}$ \\
\hline
\end{tabular}


Vetter 1992 (Continued)

them previously, and details of what happened as a result of the visit

$\begin{array}{ll}\text { Outcomes } & \text { 1. Number of people sustaining } 1 \text { or more falls } \\ \text { 2. Number of people sustaining } 1 \text { or more fall-related fractures }\end{array}$

Notes

Source of funding: Grand Charity and Welsh office

Conflicts of interest: Not reported

Economic information: Not reported

Risk of bias

\begin{tabular}{|c|c|c|}
\hline Bias & Authors' judgement & Support for judgement \\
\hline $\begin{array}{l}\text { Random sequence generation (selection } \\
\text { bias) }\end{array}$ & Low risk & $\begin{array}{l}\text { Randomised "using random number tables } \\
\text { with subjects' study numbers and without } \\
\text { direct contact with the subjects" }\end{array}$ \\
\hline Allocation concealment (selection bias) & Low risk & $\begin{array}{l}\text { Randomised "using random number tables } \\
\text { with subjects' study numbers and without } \\
\text { direct contact with the subjects". Introduc- } \\
\text { tion of bias unlikely }\end{array}$ \\
\hline
\end{tabular}

Blinding of participants and personnel Unclear risk (performance bias)

Participants and health visitor conductAll outcomes ing home visits in intervention group were not blinded. Insufficient evidence to make judgement on impact of lack of blinding

Blinding of outcome assessment (detection Unclear risk bias)

Falls and fallers

Self-reported questionnaire and follow-up interview

Blinding of outcome assessment (detection Unclear risk bias)

Fractures

Self-reported questionnaire, and a scheduled interview the questions about fractures were followed up by asking for details of where and when they had occurred and what had caused them. If satisfactory answers were obtained a fracture or fall was counted. In the case of fractures, the case notes were referred to if clear answers were not obtained

Blinding of outcome assessment (detection Unclear risk

Not applicable

bias)

Hospital admission \& medical attention

Incomplete outcome data (attrition bias) High risk All outcomes

More than $20 \%$ missing outcome data, losses balanced across groups with similar reasons for missing data

1. Health visitor visits: randomised $\mathrm{n}=350$,

Multifactorial and multiple component interventions for preventing falls in older people living in the community (Review) 


\begin{tabular}{ll|l}
\hline Selective reporting (reporting bias) & Low risk & All prespecified outcomes were reported \\
\hline Method of ascertaining falls & Unclear risk & $\begin{array}{l}\text { Self-reported questionnaire and follow-up } \\
\text { interview . }\end{array}$ \\
\hline
\end{tabular}

\section{Vind 2009}

\begin{tabular}{|c|c|}
\hline Methods & $\begin{array}{l}\text { Study design: RCT (parallel design) } \\
\text { Number of study arms: } 2 \\
\text { Study centres: Single centre } \\
\text { Length of follow-up: } 12 \text { months }\end{array}$ \\
\hline Participants & $\begin{array}{l}\text { Setting: Denmark } \\
\text { Number randomised: } 392 \\
\text { Number analysed: } 364 \\
\text { Number lost to follow-up: } 28 \\
\text { Sample: People contacted by post after ED treatment or hospital discharge } \\
\text { Age (years): Mean } 74 \text { (SD 6) } \\
\text { Sex: } 74 \% \text { women } \\
\text { Inclusion criteria: Aged } \geq 65 \text {; treated in ED or admitted to hospital because of a fall } \\
\text { Exclusion criteria: Fall caused by external force or alcohol intoxication; not living locally; } \\
\text { institutionalised; unable to walk; terminally ill; impaired communication; described } \\
\text { as suffering from dementia in hospital notes or by staff; having a planned geriatric } \\
\text { intervention }\end{array}$ \\
\hline Interventions & $\begin{array}{l}\text { Type of intervention: Multifactorial intervention } \\
\text { 1. Comprehensive multifactorial intervention. Assessed by doctor ( } 1 \text { hour), and nurse and } \\
\text { PT ( } 11 / 2 \text { hours), during } 2 \text { visits to geriatric outpatient clinic. Team discussion with senior } \\
\text { geriatrician, interventions planned and offered to participants. Carried out in clinic or } \\
\text { referred to specialists. Included progressive, individualised exercise, drug modification, } \\
\text { treatment of untreated disease, advice or referral to ophthalmologist, etc. (see Table } 1 \text { in } \\
\text { Vind } 2009 \text { for details) }(n=196) \\
\text { 2. Usual care as planned in ED or during admission ( } n=196) \\
\text { Who delivered intervention: Multidisiplinary team (Doctor }(A B V) \text {, nurse, physiothera- } \\
\text { pist, geriatrician) } \\
\text { Compliance assessed: Not reported }\end{array}$ \\
\hline Outcomes & $\begin{array}{l}\text { 1. Rate of falls } \\
\text { 2. Number of people sustaining } 1 \text { or more falls } \\
\text { 3. Number of people sustaining recurrent falls (reported as number with }>3 \text { falls) } \\
\text { 4. Number of people who experienced a fall that required medical attention }\end{array}$ \\
\hline
\end{tabular}


Vind 2009 (Continued)

\begin{tabular}{ll}
\hline Notes & $\begin{array}{l}\text { Source of funding: Danish Ministry of Health, Danish Medical Research Council } \\
\text { Conflicts of interest: None } \\
\text { Economic information: Not reported }\end{array}$ \\
\hline
\end{tabular}

\section{Risk of bias}

Bias

Random sequence generation (selection Low risk bias)

Allocation concealment (selection bias) Low risk

Blinding of participants and personnel Unclear risk (performance bias)

All outcomes

Blinding of outcome assessment (detection Low risk bias)

Falls and fallers

Blinding of outcome assessment (detection Unclear risk bias)

Fractures

Blinding of outcome assessment (detection Low risk bias)

Hospital admission \& medical attention

Incomplete outcome data (attrition bias) Low risk All outcomes

\section{Support for judgement}

Quote: "Participants were randomised by simple method, 1:1, using a computer-generated random list and sealed envelopes; a secretary not involved in the intervention performed randomisation."

Quote: “... using a computer-generated random list and sealed envelopes; a secretary not involved in the intervention performed randomisation."

Participants and/or intervention delivery personnel were not blind to group allocation

Falls recorded daily by completion of participant fall diaries

Not applicable

Requiring medical attention confirmed by hospital records

Less than $20 \%$ missing data, losses balanced across groups with similar reasons for missing data

1. Comprehensive multifactorial intervention: randomised $\mathrm{n}=196$, analysed $\mathrm{n}=186$ ( 5 withdrew, 4 died, 1 reason not given) 2. Usual care: randomised $\mathrm{n}=196$, analysed $\mathrm{n}=178$ (12 withdrew, 4 died, 2 reason not given)

Selective reporting (reporting bias) Low risk
All prespecified outcomes reported 
Vind 2009 (Continued)

\begin{tabular}{|c|c|c|}
\hline Tethod of ascertaining falls & Low risk & $\begin{array}{l}\text { Falls were recorded monthly by participants } \\
\text { returning fall diaries }\end{array}$ \\
\hline
\end{tabular}

\section{Wagner 1994}

Methods

Study design: RCT (parallel design)

Number of study arms: 3

Study centres: multiple centres

Length of follow-up: 24 months

Setting: United States of America
Number randomised: 1559
Number analysed: Not reported
Number lost to follow-up: Not reported
Sample: "Healthy elderly" people, HMO enrollees
Age (years): Mean 72
Sex: $59 \%$ women
Ethnicity: Predominantly white
Inclusion criteria: Aged $\geq 65 ;$ HMO members; ambulatory and independent
Exclusion criteria: Too ill to participate as defined by primary care physician

Interventions

Type of intervention: Multifactorial intervention

1. 60- to 90-minute interview with nurse, including review of risk factors, audiometry and blood pressure measurement, development of tailored intervention, motivation to increase physical and social activity $(\mathrm{n}=635)$

2. Chronic disease prevention nurse visit $(n=317)$ [ineligible comparator]

3. Control: usual care $(n=607)$

Who delivered the intervention: Specially-trained nurse, educator, trained volunteer, pharmacist, audiologists

Compliance assessed: Yes, the nurse provided follow-up telephone calls to check attendance and mailed reminders

Outcomes

1. Number of people sustaining 1 or more falls

2. Number of people who experienced a fall that required hospital admission

3. Number of people who experienced a fall that required medical attention

Notes

Source of funding: The Centres for Disease Control and Prevention (CDC)

Conflicts of interest: Not reported

Economic information: Not reported

\section{Risk of bias}

\section{Bias}

Random sequence generation (selection Unclear risk bias)

\section{Support for judgement}

Quote: "Randomized into three groups in a ratio of $2: 1: 2 . "$ 


\begin{tabular}{|c|c|c|}
\hline Allocation concealment (selection bias) & Unclear risk & $\begin{array}{l}\text { Insufficient information to permit judge- } \\
\text { ment }\end{array}$ \\
\hline $\begin{array}{l}\text { Blinding of participants and personnel } \\
\text { (performance bias) } \\
\text { All outcomes }\end{array}$ & Unclear risk & $\begin{array}{l}\text { Participants and personnel implementing } \\
\text { the intervention not blind to allocated } \\
\text { group, but impact of non-blinding unclear }\end{array}$ \\
\hline $\begin{array}{l}\text { Blinding of outcome assessment (detection } \\
\text { bias) } \\
\text { Falls and fallers }\end{array}$ & High risk & $\begin{array}{l}\text { The incidence of falls was assessed from } \\
\text { self-reports of episodes in the previous year }\end{array}$ \\
\hline $\begin{array}{l}\text { Blinding of outcome assessment (detection } \\
\text { bias) } \\
\text { Fractures }\end{array}$ & Low risk & Not applicable \\
\hline $\begin{array}{l}\text { Blinding of outcome assessment (detection } \\
\text { bias) } \\
\text { Hospital admission \& medical attention }\end{array}$ & Low risk & $\begin{array}{l}\text { Self-reports checked against computerised } \\
\text { hospital discharge files }\end{array}$ \\
\hline $\begin{array}{l}\text { Incomplete outcome data (attrition bias) } \\
\text { All outcomes }\end{array}$ & Unclear risk & $\begin{array}{l}\text { It was reported that } 97 \% \text { returned } 1 \text {-year } \\
\text { questionnaire, but the number of partici- } \\
\text { pants analysed and the number lost to fol- } \\
\text { low-up were not reported } \\
\text { 1. Multifactorial intervention: randomised } \\
\mathrm{n}=635 \text {, analysed } \mathrm{n}=\text { Not reported } \\
\text { 2. Chronic disease prevention nurse visit: } \\
\text { randomised } \mathrm{n}=317 \text {, analysed } \mathrm{n}=\text { Not re- } \\
\text { ported } \\
\text { 3. Control-usual care: randomised } \mathrm{n}=607 \text {, } \\
\text { analysed } \mathrm{n}=\text { Not reported }\end{array}$ \\
\hline Selective reporting (reporting bias) & Low risk & All prespecified outcomes were reported \\
\hline Method of ascertaining falls & High risk & $\begin{array}{l}\text { The incidence of falls was assessed from } \\
\text { self-reports of episodes in the previous year }\end{array}$ \\
\hline
\end{tabular}

Waterman 2016

Methods

Study Design: RCT (parallel design)

Number of study arms: 3

Study centres: unclear

Length of follow-up: 6 months

$\begin{array}{ll}\text { Participants } & \text { Setting: United Kingdom } \\ & \text { Number randomised: } 49 \\ & \text { Number analysed: } 43 \\ & \text { Number lost to follow-up: } 6 \\ & \text { Sample: Participants were initially identified from a low-vision clinic by NIHR research }\end{array}$


staff at a hospital in north-west England

Age (years): 81.4 (SD 7.6)

Sex: $61 \%$

Ethnicity: Intervention (94\% white British); Control (100\% white British)

Inclusion criteria: Passed vision-related criteria, aged 65 or over, independently living in the community, able to walk around their own residence, cognitively able to participate and understand study requirements

Exclusion criteria: Receiving an OT or physiotherapist intervention at home, homesafety assessment and modification, or exercise intervention including attendance at a falls clinic, did not achieve between 7 and 10 on abbreviated mental test

Interventions

Outcomes

Notes
Type of intervention: Multiple intervention

1. Home exercise programme and home-safety intervention: Shortened version of Otago exercise programme and Westmead home safety assessment $(\mathrm{n}=17)$

2. Usual care plus social visits: Usual care from NHS, 3 social visits, 2 telephone calls by lay visitors $(\mathrm{n}=16)$

3. Home-safety intervention only $(\mathrm{n}=16)$

Who delivered the intervention: Occupational therapists, peer mentors

Compliance assessed: Yes, the OT visited twice and a peer mentor visited 3 times and rang twice over the 6-month period, to encourage the person to adhere to the exercise programme

1. Rate of falls

2. Number of people sustaining 1 or more falls

3. Number of people sustaining recurrent falls

4. Health-related quality of life (SF-12 0 - 100, mental and physical subscales: endpoint score)

5. Adverse events of the intervention

Source of funding: National Institute for Health Research under the Patient Benefit programme (RfPB)

Conflicts of interest: 2 authors are directors of a not-for-profit training company that runs Otago exercise training for health professionals

Economic information: Intervention cost: GBP 674 per person

Adverse events: "There were no serious adverse events that could be attributed to the interventions of the study"

\section{Risk of bias}

Bias

Authors' judgement

Random sequence generation (selection Low risk bias)

Allocation concealment (selection bias) Low risk

\section{Support for judgement}

Participants were independently randomised by the clinical trials unit by a web-based secure randomisation service

Participants were independently randomised by the clinical trials unit by a web-based secure randomisation service 
Waterman 2016 (Continued)

Blinding of participants and personnel Unclear risk (performance bias)

All outcomes

Blinding of outcome assessment (detection Low risk

bias)

Falls and fallers

Blinding of outcome assessment (detection Unclear risk

bias)

Fractures

Blinding of outcome assessment (detection Unclear risk bias)

Hospital admission \& medical attention

Incomplete outcome data (attrition bias) Unclear risk

All outcomes
Participants and personnel were not blind to allocated group but effect of non-blinding unclear

Participants prospectively completed and returned monthly falls diaries

Not applicable

Not applicable

Less than $20 \%$ missing outcome data, balanced losses across groups with similar reasons for missing data

1. Home exercise programme and home-safety intervention: randomised $\mathrm{n}=17$, analysed $\mathrm{n}=15$ ( 2 withdrew)

2. Usual care and social visits: randomised $\mathrm{n}=16$, analysed $\mathrm{n}=13$ ( 1 withdrew, 2 died)

Selective reporting (reporting bias) Low risk

All outcomes listed in Methods section were reported

Method of ascertaining falls Low risk

Falls calender comprising a single postcard for each month

Wesson 2013

\begin{tabular}{ll} 
Methods & $\begin{array}{l}\text { Study Design: RCT (pilot study) } \\
\text { Number of study arms: } 2\end{array}$ \\
& $\begin{array}{l}\text { Study centres: Single centre } \\
\text { Length of follow-up: } 3 \text { months }\end{array}$ \\
\hline Participants & Setting: Australia \\
& Number randomised: 22 \\
& Number analysed: 22 \\
& Number lost to follow-up: 0 \\
& Sample: Recruited from a Memory Disorders, a Cognitive Disorders and an Aged Care \\
& Clinic, and a clinical dementia service network within the local health network in the \\
& eastern suburbs of Sydney, Australia \\
Age (years): 75.9 & Sex: $41 \%$ women \\
Ethnicity : Not reported
\end{tabular}


Inclusion criteria: Community-dwellings over-65s with a specialist diagnosis of dementia or an Addenbrooke's Cognitive Examination (ACE-R) score $\leq 82$

Exclusion criteria: Deliruim, acute medical condition, severe psychiatric disorder, progressive neurological disorder (except dementia), MMSE $<12$, severe visual impairment, residents in age care facilities

\begin{tabular}{|c|c|}
\hline Interventions & $\begin{array}{l}\text { Type of intervention: Multiple intervention } \\
\text { 1. Strength and balance training exercise and home-hazard reduction: Up to } 6 \text { individ- } \\
\text { ually-tailored strength and balance exercises selected from the Weight-bearing Exercise } \\
\text { for Better Balance (WEBB) programme based on the results of the physical performance } \\
\text { assessment, the Westmead home safety assessment was used to audit the home environ- } \\
\text { ment. A booklet was provided with home safety recommendations which formed the } \\
\text { basis for subsequent occupational therapy visits. }(\mathrm{n}=11) \\
\text { 2. Control: Usual care }(\mathrm{n}=11) \\
\text { Who delivered the intervention: Physiotherapist, occupational therapist } \\
\text { Compliance assessed: Yes, exercise adherence recorded in booklet containing prescribed } \\
\text { strength and balance exercises }\end{array}$ \\
\hline Outcomes & $\begin{array}{l}\text { 1. Number of people sustaining } 1 \text { or more falls } \\
\text { 2. Number of people sustaining } 1 \text { or more fall-related fractures } \\
\text { 3. Adverse events of the intervention }\end{array}$ \\
\hline Notes & $\begin{array}{l}\text { Source of funding: New investigator grant from the Alzeheimer's Association, USA } \\
\text { (Clemson, L) and an Alzheimer's Australia Research (AAR), Dementia Research Grant } \\
\text { for new researchers (Wesson J) } \\
\text { Conflicts of interest: Not reported } \\
\text { Economic information: Not reported } \\
\text { Adverse events: "No serious adverse events related to the intervention were reported } \\
\text { during the study period. Minor complaints relating to stiffness, dizziness and mild joint } \\
\text { pain (n }=4 ; 36 \% \text { ) were reported by participants intermittently and exercises were adjusted } \\
\text { accordingly." }\end{array}$ \\
\hline
\end{tabular}

Risk of bias

\begin{tabular}{l|l|l}
\hline Bias & Authors' judgement & Support for judgement \\
\hline $\begin{array}{l}\text { Random sequence generation (selection } \\
\text { bias) }\end{array}$ & Low risk & $\begin{array}{l}\text { Quote: “Conducted by investigator not in- } \\
\text { volved in assessment or treatment. Used } \\
\text { a random numbers table and permutated } \\
\text { blocks of four and six" }\end{array}$ \\
\hline $\begin{array}{l}\text { Allocation concealment (selection bias) } \\
\text { Low risk }\end{array}$ & Quote: "Allocation concealed using \\
opaque, sealed envelopes with study ID in \\
sequential order"
\end{tabular}

Blinding of participants and personnel Unclear risk (performance bias)

Participants and personnel not blinded to All outcomes group assignment but effect of non-blinding unclear 
Wesson 2013 (Continued)

Blinding of outcome assessment (detection Unclear risk

bias)

Falls and fallers
Falls were recorded by monthly fall diaries completed by the carer. Investigators would ring if diaries were not returned

Not clear how fractures were reported

Blinding of outcome assessment (detection Unclear risk bias)

Fractures

Blinding of outcome assessment (detection Unclear risk bias)

Hospital admission \& medical attention

Incomplete outcome data (attrition bias) Unclear risk All outcomes
No losses across groups

1. Strength and balance training exercise and home hazard reduction: randomised $\mathrm{n}$ $=11$, analysed $\mathrm{n}=11$

2. Control (usual care): randomised $n=11$, analysed $\mathrm{n}=11$

All outcomes listed in the Methods were reported

Monthly fall diaries completed by the carer. If not returned the investigator would ring to obtain details

\section{Whitehead 2003}

\section{Methods}

Study design: RCT (parallel design)

Number of study arms: 2

Study centres: Single centre

Length of follow-up: 6 months
Participants
Setting: Australia

Number randomised: 140

Number analysed: 123

Number lost to follow-up: 17

Sample: Patients presenting with a fall to $A \& E$

Age (years): Mean 77.8 (SD 7.0)

Sex: $71 \%$ women

Ethnicity: Not reported

Inclusion criteria: Aged $\geq 65$; fall-related attendance at $A \& E$; community-dwelling or in low-care residential care (hostel accommodation)

Exclusion criteria: Resident in nursing home; presenting fall-related to a stroke, seizure, cardiac or respiratory arrest, major infection, haemorrhage, motor vehicle accident, or being knocked to the ground by another person; MMSE $<25$; no resident carer; not English-speaking; living out of catchment area; terminal illness 
Interventions

Notes
Type of intervention: Multifactorial intervention

1. Home visit and questionnaire. "Fall risk profile" developed and participant given written care plan itemising elements of intervention. Letter to GP informing him/her of participant's fall, inviting them to review participant, highlighting identified risk factors, suggesting possible strategies (evidence-based). GP also given 1-page evidence summary $(\mathrm{n}=70)$

2. Home visit. No intervention. Standard medical care from GP $(\mathrm{n}=70)$

Who delivered the intervention: General practitioner, specialist geriatrician, occupational therapist, trained health professional

Compliance assessed: Yes, compliance as to whether the GP referred patients to falls clinic if suggested. In addition, at the end of the 6 th month, a research assistant who was blind to participant's allocation undertook a telephone interview with all participants. All falls prevention activities undertaken during the course of the study were recorded

1. Number of people sustaining 1 or more falls

Source of funding: Part of Commonwealth-funded programme aimed at the interface between public hospitals and general practice

Conflicts of interest: Not reported

Economic information: Not reported

\section{Risk of bias}

\section{Bias}

Random sequence generation (selection Unclear risk bias)

Allocation concealment (selection bias)

Low risk

Blinding of participants and personnel Unclear risk (performance bias)

All outcomes

Blinding of outcome assessment (detection Low risk bias)

Falls and fallers

Blinding of outcome assessment (detection Unclear risk bias)

Fractures

\section{Support for judgement}

Randomisation and allocation schedules created by a researcher external to the trial

Randomised by a researcher external to the trial using numbered, sealed opaque envelopes

Participants and personnel implementing the intervention not blind to allocated group, but impact of non-blinding unclear

Falls diary used to $\log$ occurrence of all falls, all participants were contacted by telephone by the principal research officer once every month to monitor any falls, and encourage the use of fall diaries

Not applicable 
Whitehead 2003 (Continued)

\begin{tabular}{l|l|l}
\hline $\begin{array}{l}\text { Blinding of outcome assessment (detection } \\
\text { bias) } \\
\text { Hospital admission \& medical attention }\end{array}$ & Unclear risk & Not applicable \\
\hline $\begin{array}{l}\text { Incomplete outcome data (attrition bias) } \\
\text { All outcomes }\end{array}$ & Unclear risk & $\begin{array}{l}\text { Less than } 20 \% \text { missing outcome data, losses } \\
\text { are unbalanced across groups with no rea- } \\
\text { sons given for missing data } \\
\text { 1. Home visit and questionnaire: } \begin{array}{l}\text { ran- } \\
\text { domised } n=70 \text { analysed } n=58\end{array} \\
\text { 2. Home visit and standard medical care } \\
\text { from GP: randomised n }=70, \text { analysed } n= \\
65\end{array}$ \\
\hline Selective reporting (reporting bias) & High risk & $\begin{array}{l}\text { Modified Barthel Index reported in the } \\
\text { Methods as being collected at } 6 \text { months but } \\
\text { not reported in Results }\end{array}$ \\
\hline Method of ascertaining falls & Low risk & $\begin{array}{l}\text { Falls diary used to log occurrence of all } \\
\text { falls, all participants were contacted by tele- } \\
\text { phone by the principal research officer once } \\
\text { every month to monitor any falls, and en- } \\
\text { courage the use of fall diaries }\end{array}$ \\
\hline
\end{tabular}

Wilder 2001

Methods

Study design: RCT (parallel design)

Number of study arms: 3

Study centres: unclear

Length of follow-up: 9 months

\begin{tabular}{ll} 
Participants & Setting: United States of America \\
Number randomised: 60 \\
Number analysed: Not reported \\
Number lost to follow-up: Not reported \\
Sample: "frail elderly" (proportion of women not stated) \\
Age (years): Not reported \\
Sex: Not reported \\
Inclusion criteria: Aged $\geq 75$ years, living at home, using home services (i.e. Meals on \\
Wheels, Telecare or Lifeline) \\
Exclusion criteria: None described \\
\hline Type of intervention: Multiple intervention \\
1. Home modifications plus home-exercise programme monitored by a "trained volun- \\
teer buddy" \\
2. Simple home modifications \\
$\begin{array}{ll}\text { 3. Control: no intervention } \\
\text { Who delivered intervention: Physiotherapist and buddy volunteer (high school student } \\
\text { or healthy elder) }\end{array}$
\end{tabular}

Multifactorial and multiple component interventions for preventing falls in older people living in the community (Review) 
Wilder 2001 (Continued)

Compliance assessed: Not reported

\begin{tabular}{|c|c|c|}
\hline Outcomes & \multicolumn{2}{|c|}{ 1. Number of people sustaining 1 or more falls (abstract only) } \\
\hline Notes & \multicolumn{2}{|l|}{$\begin{array}{l}\text { Source of funding: Not reported } \\
\text { Conflicts of interest: Not reported } \\
\text { Economic information: Not reported }\end{array}$} \\
\hline \multicolumn{3}{|l|}{ Risk of bias } \\
\hline Bias & Authors' judgement & Support for judgement \\
\hline $\begin{array}{l}\text { Random sequence generation (selection } \\
\text { bias) }\end{array}$ & Unclear risk & $\begin{array}{l}\text { Quote: "randomly assigned" to } 3 \text { arms. } \\
\text { Method not described. }\end{array}$ \\
\hline Allocation concealment (selection bias) & Unclear risk & $\begin{array}{l}\text { Insufficient information to permit judge- } \\
\text { ment }\end{array}$ \\
\hline $\begin{array}{l}\text { Blinding of participants and personnel } \\
\text { (performance bias) } \\
\text { All outcomes }\end{array}$ & Unclear risk & $\begin{array}{l}\text { Participants and personnel implementing } \\
\text { the intervention not blind to allocated } \\
\text { group, but impact of non-blinding unclear }\end{array}$ \\
\hline $\begin{array}{l}\text { Blinding of outcome assessment (detection } \\
\text { bias) } \\
\text { Falls and fallers }\end{array}$ & Unclear risk & Insufficient information \\
\hline $\begin{array}{l}\text { Blinding of outcome assessment (detection } \\
\text { bias) } \\
\text { Fractures }\end{array}$ & Unclear risk & Not applicable \\
\hline $\begin{array}{l}\text { Blinding of outcome assessment (detection } \\
\text { bias) } \\
\text { Hospital admission \& medical attention }\end{array}$ & Unclear risk & Not applicable \\
\hline $\begin{array}{l}\text { Incomplete outcome data (attrition bias) } \\
\text { All outcomes }\end{array}$ & Unclear risk & Insufficient information \\
\hline Selective reporting (reporting bias) & High risk & $\begin{array}{l}\text { Results not published in full, only pub- } \\
\text { lished as conference abstract }\end{array}$ \\
\hline Method of ascertaining falls & Unclear risk & Insufficient information \\
\hline
\end{tabular}




\begin{tabular}{|c|c|}
\hline Methods & $\begin{array}{l}\text { Study Design: RCT (parallel design) } \\
\text { Number of study arms: } 2 \\
\text { Study centres: Multiple centres } \\
\text { Length of follow-up: } 14 \text { months }\end{array}$ \\
\hline Participants & $\begin{array}{l}\text { Setting: The Netherlands } \\
\text { Number randomised: } 540 \\
\text { Number analysed: } 405 \\
\text { Number lost to follow-up: } 135 \\
\text { Sample: Questionnaires were sent out to random samples of } 7341 \text { community-dwelling } \\
\text { adults } \\
\text { Age (years): Control: Mean } 78 \text { (SD 5.0), Intervention: Mean } 77.8 \text { (SD 4.6) } \\
\text { Sex: Control: } 73 \% \text { women, Intervention: } 71 \% \text { women } \\
\text { Ethnicity: Not reported } \\
\text { Inclusion criteria: Community-dwelling adults } 70 \text { years or older reporting at least some } \\
\text { fear of falling } \\
\text { Exclusion criteria: People confined to bed, restricted by permanent use of a wheelchair, } \\
\text { waiting for nursing home admission or participating in other intervention studies }\end{array}$ \\
\hline Interventions & $\begin{array}{l}\text { Type of intervention: Multifactorial intervention } \\
\text { 1. Multicomponent cognitive behavioural group intervention: } 8 \text { weekly sessions of } 2 \\
\text { hours, and booster session } 6 \text { months after the } 8 \text { th session }(n=280) \\
\text { 2. Control: Usual care }(n=260) \\
\text { Who delivered the intervention: Qualified geriatric nurses } \\
\text { Compliance assessed: Yes, method not described }\end{array}$ \\
\hline Outcomes & $\begin{array}{l}\text { 1. Rate of falls } \\
\text { 2. Number of people sustaining } 1 \text { or more falls } \\
\text { 3. Number of people sustaining recurrent falls } \\
\text { 4. Adverse effects of the intervention }\end{array}$ \\
\hline Notes & $\begin{array}{l}\text { Source of funding: CAPHRI - School for Public Health and Primary Care And The } \\
\text { Facility of Health, Medicine and Life Sciences of the Maastricht University } \\
\text { Conflicts of interest: None } \\
\text { Economic information: Not reported } \\
\text { Adverse events: "No adverse events or side effects reported" }\end{array}$ \\
\hline
\end{tabular}

\section{Risk of bias}

\section{Bias}

Authors' judgement

Random sequence generation (selection Low risk bias)

\section{Support for judgement}

Quote: “Independent researcher blinded to participant characteristics performed block randomisation using computer generated random allocation"

Independent researcher was blinded to participant's characteristics 
Blinding of participants and personnel Unclear risk (performance bias)

All outcomes
Participants and personnel were not blinded to group but effect is unclear

Blinding of outcome assessment (detection Low risk

Falls reported by monthly fall diaries

bias)

Falls and fallers

Blinding of outcome assessment (detection Unclear risk

Not applicable

bias)

Fractures

Blinding of outcome assessment (detection Unclear risk bias)

Hospital admission \& medical attention

Incomplete outcome data (attrition bias) High risk

All outcomes

Insufficient information on how medical attention was assessed

\begin{tabular}{|c|c|c|}
\hline & & $\begin{array}{l}\text { similar reasons for missing data } \\
\text { group intervention: randomised } \mathrm{n}=280 \text {, } \\
\text { analysed } \mathrm{n}=196 \text { ( } 6 \text { died, } 36 \text { health prob- } \\
\text { lems, } 21 \text { lost interest, } 12 \text { felt trial too bur- } \\
\text { densome, } 6 \text { life event significant other, } 3 \\
\text { other reasons) } \\
\text { 2. Usual care: randomised } \mathrm{n}=260 \text {, analysed } \\
\mathrm{n}=209 \text { ( } 6 \text { died, } 19 \text { health problems, } 13 \\
\text { lost interest, } 6 \text { felt trial too burdensome, } 1 \\
\text { life event significant other, } 6 \text { other reasons) }\end{array}$ \\
\hline Selective reporting (reporting bias) & High risk & $\begin{array}{l}\text { Not all secondary outcome measures stip- } \\
\text { ulated in protocol paper reported in study } \\
\text { paper }\end{array}$ \\
\hline Method of ascertaining falls & Low risk & $\begin{array}{l}\text { Prospective falls calender returning a page } \\
\text { every } 3 \text { months }\end{array}$ \\
\hline
\end{tabular}

A\&E: accident and emergency; ADL: activities of daily living; AMT: abbreviated mental test; BMI: body mass index; CB: cognitive behavioural; CHS: Cardiovascular Health Study; DSST: digit symbol substitution test; ED: emergency department; GP: general practitioner; GPSS: Geriatric Postal Screening Survey; HMO: health maintenance organisation; ICER: incremental cost-effectiveness ratio; IQR: interquartile range; MMSE: Mini Mental State Examination; OT: occupational therapist; PT: physiotherapist; QoL: quality of life; SD: standard deviation; TUG: timed up and go; VAS: visual analogue scale 
Characteristics of excluded studies [ordered by study ID]

\begin{tabular}{l|l}
\hline Study & Reason for exclusion \\
\hline ACTRN12610000838011 & $\begin{array}{l}\text { A randomised trial comparing } 2 \text { different models of service delivery for falls prevention in older adults } \\
\text { living in the community }\end{array}$ \\
\hline Alexander 2003 & $\begin{array}{l}\text { A quasi-randomised trial assessing the effects of a multifactorial intervention to reduce falls in people } \\
\text { attending daycare centres }\end{array}$ \\
\hline Assantachai 2002 & $\begin{array}{l}\text { A quasi-randomised trial assessing the effects of a multiple intervention versus no intervention to reduce } \\
\text { falls in Thai elderly people living in the community }\end{array}$
\end{tabular}

Bruce 2016

A protocol for a randomised controlled trial comparing a multifactorial intervention versus advice on falls prevention versus advice on falls prevention and exercise in older adults living in the community

Chu 2017

A randomised controlled trial comparing occupational therapy home-hazard modification versus control in older adults in Hong Kong after an emergency visit following a fall

Clemson 2012

A randomised controlled trial comparing Lifestyle integrated Functional Exercise (LiFE), exercises for balance and lower limb strength, versus a sham control programme for the prevention of falls in older people

Cockayne 2014

A randomised controlled trial comparing a multiple intervention of orthotic, foot and ankle exercises and footwear advice for the prevention of falls versus falls prevention advice in older adults living in the community

Cohen 2015

A randomised controlled trial comparing a multifactorial intervention (LIFT - Living Independently and Falls free Together) intervention versus an active falls control group and an administration falls control group in older people living in the community

Comans 2010

A randomised controlled trial comparing an individualised community rehabilitation service versus a group-based community rehabilitation service in community-dwelling older adults

Conroy 2010

A randomised controlled trial comparing a multifactorial intervention versus another falls prevention programme in community-dwelling older people

De Negreiros 2013

A protocol for a randomised controlled trial comparing a multifactorial falls prevention programme versus another active falls prevention intervention in older adults living in the community

Di Monaco 2008

A quasi-randomised trial assessing the effectiveness of a multiple intervention to prevent falls versus usual care in elderly women who sustained a fall-related hip fracture in the community

Fox 2010

A randomised controlled trial comparing the effects of a multifactorial intervention versus another active falls prevention intervention among older adults living in the community

Gianoudis 2014

A randomised controlled trial comparing a multi-modal exercise programme combined with education versus self-management (education only) in older people living in the community

Multifactorial and multiple component interventions for preventing falls in older people living in the community (Review) 

versus a family physician-based multifactorial intervention for the prevention of falls in communitydwelling older male veterans

Giordano 2016

A protocol for a randomised controlled trial comparing a multifactorial falls prevention programme versus another active falls prevention intervention in older adults living in the community

Hill 2000

A randomised controlled trial comparing a nurse-led multifactorial intervention of exercise and individualised falls prevention advice versus standard falls-prevention advice in older people living in the community

Hornbrook 1994

A randomised controlled trial comparing the effects of 2 different types of multifactorial intervention among older people living in the community

Huang 2004

A randomised controlled trial comparing the effects a multifactorial intervention versus standardised fall-prevention information in Taiwanese older people living the community

Lamb 2010

A randomised controlled trial comparing a multifactorial intervention plus advice versus exercise plus advice versus advice on falls prevention only in older adults living in the community

Lee 2013

A randomised controlled trial comparing a multifactorial intervention versus control intervention in older adults living in the community. Included adults with Parkinsons diease and stroke - data were not available separately for analysis

Mahoney 2007

A randomised controlled trial comparing the effects of a community-based multifactorial falls-prevention intervention versus home safety assessments in adults living in the community

Matchar 2017

A randomised controlled trial comparing a multifactorial intervention versus falls-prevention education materials in older adults living in the community

Mikolaizak 2017

A randomised controlled trial comparing a multidisciplinary intervention versus individualised written fall-prevention advice to prevent subsequent falls and health service use using fall-related paramedic care

NCT00126152

A randomised controlled trial comparing a multifactorial intervention versus control in older adults living in the community. The control group also received written information on falls prevention

NCT00483275

A randomised controlled trial comparing alfacalcidol and exercise versus control in older adults. This study was withdrawn prior to enrolment of the first participant

Perula 2012

A randomised controlled trial comparing the effect of a multifactorial intervention to reduce the incidence of falls in older adults versus individual advice and information leaflet on falls prevention

Salminen 2009

A randomised controlled trial comparing the effects of a multifactorial intervention versus counselling and guidance about falls in older people living in the community 

paired people. Most participants not community-dwelling (79\% of participants lived in high and intermediate nursing-care facilities)

Sherrington 2014

A randomised controlled trial comparing exercise and fall-prevention advice materials versus fall-prevention advice materials in older adults post-discharge from hospital

Shumway-Cook 2007 A randomised controlled trial comparing a multiple-component exercise intervention versus written materials on falls prevention in sedentary older adults living in the community

Snooks 2010

A cluster-randomised controlled trial comparing paramedics receiving training and clinical protocols for assessing and referring older people who had fallen versus control paramedics who deliver care as usual

Snooks 2017

A cluster-randomised controlled trial comparing paramedics receiving training and clinical protocols for assessing and referring older people who had fallen versus control paramedics who deliver care as usual

Spink 2011

A randomised controlled trial comparing a multifaceted podiatry intervention versus routine podiatry care in community-dwelling older people with disabling foot pain

Steinberg 2000

A randomised controlled trial assessing the effectiveness of a multiple intervention targeting major risk factors to reduce falls versus another active fall-prevention intervention among older adults living in the community

Suman 2011

A randomised controlled trial assessing the effects of a community-based multifactorial fall-prevention intervention versus a hospital-based multifactorial fall-prevention intervention in older adults living in the community

Swanenburg 2007

A randomised controlled trial assessing the effects of a multiple intervention of exercise and nutrition supplementation versus nutrition supplementation alone in elderly people with decreased bone mineral density

Tiedemann 2015

A randomised controlled trial comparing a falls-prevention brochure plus physical activity promotion and a fall-prevention intervention enhanced with health coaching and a pedometer versus a fall-prevention brochure only in older adults

Von Stengel 2011

A randomised controlled trial comparing the effects of exercise, exercise plus whole-body vibration, and a wellness control group for the prevention of falls in postmenopausal women

Wyman 2005

A randomised controlled trial comparing the effects of a multifactorial intervention versus education and advice about falls prevention among older people living in the community

Xia 2009

A randomised controlled trial of a population-based multifactorial intervention. Falls outcomes were based on a random sample from participating communities 


\section{Characteristics of ongoing studies [ordered by study ID]}

\section{ACTRN12607000206426}

Trial name or title Community care and hospital based collaborative falls prevention project

\begin{tabular}{|c|c|}
\hline Methods & $\begin{array}{l}\text { Study design: RCT } \\
\text { Number of study arms: } 2\end{array}$ \\
\hline Participants & $\begin{array}{l}\text { Setting: Australia } \\
\text { Target sample size: } 200 \\
\text { Inclusion criteria: Male or female, aged } \geq 65 \text {, presenting to A\&E or falls clinic, community-dwelling in Perth } \\
\text { north } \\
\text { Exclusion criteria: Functional cognitive impairment, unable to speak or read English }\end{array}$ \\
\hline Interventions & $\begin{array}{l}\text { Type of intervention: Multifactorial } \\
\text { 1. Community follow-up by support worker ( } 8 \text { hours over } 2 \text { to } 3 \text { weeks) to review risk factors in the home, } \\
\text { strategies to reduce risk factors, assistance to implement Falls Action Plan provided by A\&E or clinic (see } \\
\text { ANZCTR website for further details). } \\
\text { 2. No community follow-up after discharge }\end{array}$ \\
\hline Outcomes & $\begin{array}{l}\text { 1. Number of people sustaining } 1 \text { or more falls } \\
\text { 2. Number of people who experience a fall that requires medical attention }\end{array}$ \\
\hline Starting date & April 2007 \\
\hline Contact information & $\begin{array}{l}\text { J Johnson } \\
\text { Perth Home Care Services } \\
30 \text { Hasler Road } \\
\text { PO Box } 1597 \\
\text { Osborne Park } \\
\text { Western Australia } 6017 \\
\text { Australia }\end{array}$ \\
\hline Notes & Listed as "Not yet recruiting". Emailed author 6 July 2017; no response \\
\hline
\end{tabular}


Trial name or title Does nutrition and exercise prevent frailty and reduce falls in pre-frail older adults in New Zealand?

\begin{tabular}{|c|c|}
\hline Methods & $\begin{array}{l}\text { Study design: RCT } \\
\text { Number of study arms: } 4\end{array}$ \\
\hline Participants & $\begin{array}{l}\text { Setting: New Zealand } \\
\text { Target sample size: } 635 \\
\text { Sample: Older people living in the community } \\
\text { Inclusion criteria: Non-Maori aged } 75 \text { and older, Maori aged } 60 \text { and over; living in the community; pre-fail } \\
\text { criteria of } 1 \text { or } 2 \text { on FRAIL questionnaire; able to stand; able to use kitchen utensils safely } \\
\text { Exclusion criteria: Terminally ill; advanced dementia from GP record }\end{array}$ \\
\hline Interventions & $\begin{array}{l}\text { Type of intervention: Multiple component } \\
\text { 1. Senior Chief Programme consisting of } 8 \text { week programme of } 3 \text {-hour weekly cooking classes followed by } \\
\text { nutrition education } \\
\text { 2. Steady as You Go programme (SAYGO) consisting of } 1 \text { hour weekly for } 10 \text {-week exercise programme based } \\
\text { on adapted Otago Exercise Programme } \\
\text { 3. Senior Chief programme and SAYGO } \\
\text { 4. Control - social activity course }\end{array}$ \\
\hline Outcomes & $\begin{array}{l}\text { 1. Number of people sustaining } 1 \text { or more falls } \\
\text { 2. Health-related quality of life }\end{array}$ \\
\hline Starting date & Auguest 2014 \\
\hline Contact information & $\begin{array}{l}\text { Dr Ruth Teh } \\
\text { The School of Population Health } \\
\text { Tamaki Campus } \\
\text { University of Auckland } \\
261 \text { Morrin Rd St Johns } \\
\text { Auckland } 1072 \\
\text { New Zealand }\end{array}$ \\
\hline Notes & Listed as "Recruiting" as of 6 July 2017 \\
\hline
\end{tabular}

\section{ACTRN12615001326583}

Trial name or title Preventing falls in older people after discharge from hospital as a result of a fall

\begin{tabular}{l|l}
\hline Methods & $\begin{array}{l}\text { Study design: RCT } \\
\text { Number of study arms: } 2\end{array}$ \\
\hline Participants & $\begin{array}{l}\text { Setting: Austrialia } \\
\text { Target sample size: } 30 \\
\text { Sample: Older people after discharge from hospital following admission as a result of a fall } \\
\text { Inclusion criteria: Aged } 65 \text { and older; discharged from hospital into the community following a fall; deemed } \\
\text { medically fit } \\
\text { Exclusion criteria: Weight-bearing restrictions; medically unstable; terminal illness; referred to falls prevention }\end{array}$
\end{tabular}




\section{ACTRN12615001326583 (Continued)}

service on discharge; transition care hospital stay

\begin{tabular}{|c|c|}
\hline Interventions & $\begin{array}{l}\text { Type of intervention: Multiple intervention } \\
\text { 1. Exercise programme based on a modified version of the Otago Exercise Programme }(20-30 \text { minutes } 5 \\
\text { times a week), with medication review component and education on falls-prevention component } \\
\text { 2. Control: usual care }\end{array}$ \\
\hline Outcomes & $\begin{array}{l}\text { 1. Rate of falls } \\
\text { 2. Health-related quality of life }\end{array}$ \\
\hline Starting date & November 2015 to March 2016 (anticipated) \\
\hline Contact information & $\begin{array}{l}\text { Dr Dianne Goeman } \\
\text { Royal District Nursing Service Institute, } \\
31 \text { Alma Road, } \\
\text { St Kilda, Victoria } 3182 \\
\text { Australia }\end{array}$ \\
\hline Notes & Listed as "Recruiting" as of 6 July 2017 \\
\hline
\end{tabular}

\section{Barker 2015}

Trial name or title RESPOND-a patient-centred programme to prevent secondary falls in older people presenting to the emergency department with a fall: protocol for a multicentre randomised controlled trial

\begin{tabular}{ll} 
Methods & $\begin{array}{l}\text { Study design: RCT } \\
\text { Number of study arms: } 2\end{array}$ \\
\hline Participants & $\begin{array}{l}\text { Setting: Australia } \\
\text { Target sample size: } 528 \\
\text { Sample: Older people presenting to the emergency department following a fall } \\
\text { Inclusion criteria: Community-dwelling persons, aged } 60 \text { to } 90 \text { years who present to the Royal Perth and } \\
\text { Alfred Hospital EDs with a fall, and who are planned to be discharged directly home from the hospital within } \\
\text { 72 hours } \\
\text { Exclusion criteria: Live further than } 50 \text { kilometres from the study site, discharged to high-level residential } \\
\text { aged-care, require palliative care or have a terminal illness, require hands-on assistance to walk, are unable to } \\
\text { use a telephone, need an interpreter, have cognitive impairment (MMSE < 23), display social aggression or } \\
\text { have a history of psychoses }\end{array}$ \\
\hline $\begin{array}{l}\text { Type of intervention: Multifactorial } \\
\text { Interventions } \\
\text { goal-setting and } \\
\text { follow-up telephone support for management of } 1 \text { or more of } 4 \text { risk factors with evidence of effective inter- } \\
\text { ventions and (3) } \\
\text { healthcare provider communication and community linkage delivered over } 6 \text { months } \\
\text { 2. Usual care }\end{array}$
\end{tabular}


Barker 2015 (Continued)

\begin{tabular}{l|l}
\hline Outcomes & $\begin{array}{l}\text { 1. Rate of falls } \\
\text { 2. Number of people sustaining } 1 \text { or more falls } \\
\text { 3. Number of people who experience a fall that requires medical attention } \\
\text { 4. Health-related quality of life }\end{array}$ \\
\hline Starting date & March 2014 to July 2016 (actual) \\
\hline Contact information & $\begin{array}{l}\text { Anna Barker } \\
\text { Monash University, The Alfred Centre } \\
\text { DEPM, Level 6, 99 Commercial Road } \\
\text { Melbourne VIC 3004 } \\
\text { Australia }\end{array}$ \\
\hline Notes & \begin{tabular}{l} 
Listed as "Completed" but results not yet published \\
\hline
\end{tabular}
\end{tabular}

Blank 2011

\begin{tabular}{|c|c|}
\hline Trial name or title & Prevent Falls (PreFalls) \\
\hline Methods & $\begin{array}{l}\text { Study design: RCT (cluster-randomised) } \\
\text { Number of study arms: } 2\end{array}$ \\
\hline Participants & $\begin{array}{l}\text { Setting: Germany } \\
\text { Target sample size: } 382 \\
\text { Sample: Community-dwelling people registered with general practices } \\
\text { Inclusion criteria: Aged } 65 \text { and older; with at least } 1 \text { of the following: fall within last } 12 \text { months; fear of } \\
\text { falling; chair stand-ups > } 10 \mathrm{sec} \text {; TUG Test > } 10 \mathrm{sec} \text {; impaired balance; self-reported balance deficits } \\
\text { Exclusion criteria: Not living independently; with physical or mental restrictions which do not allow exercising } \\
\text { or participating in falls risk assessments }\end{array}$ \\
\hline Interventions & $\begin{array}{l}\text { Type of intervention: Multiple component } \\
\text { 1. Group- and home-based exercises (progressive strength and flexibility training; challenging balance; gait } \\
\text { and motor co-ordination training; progressive endurance training). Fear of falling cognitive behavioural } \\
\text { intervention (Matter of Balance programme). } 60 \text { min, } 1 \text { a week for } 16 \text { weeks } \\
\text { 2. Control: no intervention }\end{array}$ \\
\hline Outcomes & $\begin{array}{l}\text { 1. Rate of falls } \\
\text { 2. Number of people sustaining } 1 \text { or more falls }\end{array}$ \\
\hline Starting date & April 2009 to March 2012 \\
\hline Contact information & $\begin{array}{l}\text { Dr. Wolfgang Blank } \\
\text { Institute of General Practice } \\
\text { Klinikum rechts der Isar, Technische Universitaet Muenchen } \\
\text { Orleanstr. } 47 \\
81667 \text { Muenchen } \\
\text { Germany } \\
\text { Telephone: }+4989614658913\end{array}$ \\
\hline
\end{tabular}


Blank 2011 (Continued)

Email: blank@lrz.tum.de

Notes $\quad$ Listed as "Completed" but results not yet published

Close 2014

Trial name or title Can a tailored exercise and home hazard reduction program reduce the rate of falls in community dwelling older people with cognitive impairment: protocol paper for the i-FOCIS randomised controlled trial

\begin{tabular}{|c|c|}
\hline Methods & $\begin{array}{l}\text { Study design: RCT } \\
\text { Number of study arms: } 2\end{array}$ \\
\hline Participants & $\begin{array}{l}\text { Setting: Australia } \\
\text { Target sample size: } 360 \\
\text { Inclusion criteria: Aged } 65 \text { and above living in the community; MMSE }<24 \text { or ACE-R }<83 \text { or specialist } \\
\text { clinical diagnosis of cognitive impairment or dementia; must have an identifiable and consenting person } \\
\text { responsible and a carer (likely to be the person responsible in many cases) who have a minimum of } 3 \frac{1}{2} \text { hours } \\
\text { of face-to-face contact with the participant each week for the purposes of reporting of falls and supervising } \\
\text { the exercise intervention ( } 3 \text { times a week); willingness of participant and carer to give informed consent and } \\
\text { to participate in and comply with the study protocol; proxy consent and participant assent will be used where } \\
\text { participants cannot give informed consent } \\
\text { Exclusion criteria: Participants with a MMSE }<12 / 30 \text {; following medical conditions: delirium, acute medical } \\
\text { illnesses, severe psychiatric disorders, progressive neurological diseases other than dementia and blindness; } \\
\text { residents of residential aged-care facilities; highly dependent on medical care }\end{array}$ \\
\hline Interventions & $\begin{array}{l}\text { Type of intervention: Multifactorial } \\
\text { 1. Individual risk assessment followed by 12-month home-based exercise (based on Weight-bearing Exercise } \\
\text { for Better Balance programme) and home-hazard reduction programme tailored to their cognitive and physical } \\
\text { abilities. Frequency and duration individually prescribed up to } 30 \text { minutes } 3 \text { - } 6 \text { times a week } \\
\text { 2. Control: usual care }\end{array}$ \\
\hline Outcomes & $\begin{array}{l}\text { 1. Rate of falls } \\
\text { 2. Number of people sustaining } 1 \text { or more falls } \\
\text { 3. Health-related quality of life }\end{array}$ \\
\hline Starting date & June 2014 to July 2018 (anticipated) \\
\hline Contact information & $\begin{array}{l}\text { Jacqueline Close } \\
\text { Neuroscience Research Australia } \\
\text { Barker Street, Randwick NSW } 2031 \\
\text { PO Box } 1165 \\
\text { Australia }\end{array}$ \\
\hline Notes & Listed as "Recruiting" as of 6 July 2017 \\
\hline
\end{tabular}

Multifactorial and multiple component interventions for preventing falls in older people living in the community (Review) 
Trial name or title Reducing falls after hospital discharge: a protocol for a randomised controlled trial evaluating an individualised multimodal falls education programme for older adults

\begin{tabular}{|c|c|}
\hline Methods & $\begin{array}{l}\text { Study design: RCT } \\
\text { Number of study arms: } 2\end{array}$ \\
\hline Participants & $\begin{array}{l}\text { Setting: Australia } \\
\text { Target sample size: } 390 \\
\text { Inclusion criteria: Aged } 60 \text { years or older, Abbreviated Mental Test Score }>7 / 10,34 \text { admitted to participating } \\
\text { wards for this trial, discharged to the community, able to understand English sufficiently to take part in the } \\
\text { education and receive telephone calls } \\
\text { Exclusion criteria: Unstable medical problem, discharged to transitional or residential care, requiring palliative } \\
\text { care, short-stay admissions that preclude screening, enrolment and intervention during the admission (defined } \\
\text { as admission planned of }<5 \text { days) }\end{array}$ \\
\hline Interventions & $\begin{array}{l}\text { Type of intervention: Multiple } \\
\text { 1. Falls prevention programme incorporating a video, workbook and individualised follow-up from an expert } \\
\text { health professional to foster capability and motivation to engage in falls prevention strategies } \\
\text { 2. Usual care plus social visit }\end{array}$ \\
\hline Outcomes & $\begin{array}{l}\text { 1. Rate of falls } \\
\text { 2. Number of people sustaining } 1 \text { or more falls } \\
\text { 3. Health-related quality of life }\end{array}$ \\
\hline Starting date & July 2015 to September 2016 (anticipated) \\
\hline Contact information & $\begin{array}{l}\text { Anne-Marie Hill } \\
\text { School of Physiotherapy and Exercise Science } \\
\text { Curtin University Kent St, Bentley WA } \\
\text { Australia }\end{array}$ \\
\hline Notes & Listed as "Recruiting" as of 6 July 2017 \\
\hline
\end{tabular}

\section{ISRCTN21120199}

Trial name or title The effect of an assessment-based falls prevention programme in elderly people utilising day-care services

\begin{tabular}{ll}
\hline Methods & $\begin{array}{l}\text { Study design: RCT (cluster RCT) } \\
\text { Number of study arms: } 2\end{array}$ \\
\hline
\end{tabular}

$\begin{array}{ll}\text { Participants } & \text { Setting: Japan } \\ & \text { Target sample size: } 5000 \\ & \text { Inclusion criteria: Aged over 65; using day-care services } \\ & \text { Exclusion criteria: Acute health conditions }\end{array}$

Interventions Type of intervention: Multifactorial

1. Fall risk assessment and fall-prevention education for care providers and elderly participants

2. Control: usual care 
ISRCTN21120199 (Continued)

\begin{tabular}{|c|c|}
\hline Outcomes & $\begin{array}{l}\text { 1. Rate of falls } \\
\text { 2. Number of people sustaining } 1 \text { or more falls }\end{array}$ \\
\hline Starting date & September 2008 to December 2009 \\
\hline Contact information & $\begin{array}{l}\text { Tokyo Metropolitan Institute of Gerontology } \\
\text { 35-2 Sakae-cho } \\
\text { Itabashi } \\
\text { Tokyo } \\
\text { Japan }\end{array}$ \\
\hline Notes & Listed as "Completed" but results not yet published \\
\hline
\end{tabular}

Landi 2017

Trial name or title The "Sarcopenia and Physical fRailty IN older people: multi-componenT Treatment strategies" (SPRINTT) randomized controlled trial: design and methods

\begin{tabular}{|c|c|}
\hline Methods & $\begin{array}{l}\text { Study design: RCT } \\
\text { Number of study arms: } 2\end{array}$ \\
\hline Participants & $\begin{array}{l}\text { Setting: Europe } \\
\text { Target sample size: } 1500 \text { (estimated) } \\
\text { Inclusion criteria: Age } \geq 70 \text { years; Short Physical Performance Battery (SPPB) score between } 3 \text { and 9; ability } \\
\text { to complete the } 400 \text {-metre walk test within } 15 \text { minutes without sitting, the help with another person or the } \\
\text { use of a walker; presence of low muscle mass } \\
\text { Exclusion criteria: Residence in long-term care; other health conditions such as lung, heart and inflammatory } \\
\text { disease }\end{array}$ \\
\hline Interventions & $\begin{array}{l}\text { Type of intervention: Multiple component } \\
\text { 1. Structured physical activity, nutritional counselling/dietary intervention, and an information and commu- } \\
\text { nication technology intervention } \\
\text { 2. Healthy ageing lifestyle education programme }\end{array}$ \\
\hline Outcomes & Number of people sustaining 1 or more falls \\
\hline Starting date & Not specified \\
\hline Contact information & $\begin{array}{l}\text { Francesco Landi } \\
\text { Department of Geriatrics, Neurosciences and Orthopedics } \\
\text { Catholic University of the Sacred Heart School of Medicine } \\
\text { Rome, Italy }\end{array}$ \\
\hline Notes & Listed as "Recruiting" as of 6 July 2017 \\
\hline
\end{tabular}




\begin{tabular}{|c|c|}
\hline Trial name or title & Reducing falls with RENEW in older adults who have fallen \\
\hline Methods & $\begin{array}{l}\text { Study design: RCT } \\
\text { Number of study arms: } 2\end{array}$ \\
\hline Participants & $\begin{array}{l}\text { Setting: USA } \\
\text { Target sample size: } 100 \\
\text { Inclusion criteria: Men and women between } 65 \text { and } 95 \text { years; } 2 \text { or more self-reported comorbid conditions; } \\
\text { history of } \geq 1 \text { fall in last } 12 \text { months; ambulatory; community-dwelling; gait speed } 25 \text { metres/minute to } 80 \\
\text { metres/minute; with permission from physician to participate in a } 60 \text {-minute (with rests) exercise programme; } \\
\text { capable of performing RENEW on the ergometer } \\
\text { Exclusion criteria: Dementia; progressive neurologic disease or disease affecting muscle, e.g. Parkinson's, } \\
\text { muscular dystrophy: participated in a regular ( } 3 \text { a week) aerobic or resistance exercise programme in past } 12 \\
\text { months; any contraindication to having magnetic resonance imaging }\end{array}$ \\
\hline Interventions & $\begin{array}{l}\text { Type of intervention: Multifactorial } \\
\text { 1. High-intensity (lower body) Resistance Exercise via Negative, Eccentrically-induced Work (RENEW) } \\
\text { 2. Traditional lower-body resistance exercise }\end{array}$ \\
\hline Outcomes & 1. Rate of falls \\
\hline Starting date & April 2008 to February 2013 \\
\hline Contact information & $\begin{array}{l}\text { Sheldon B Smith } \\
\text { Department of Physical Therapy } \\
\text { University of Utah } \\
\text { Salt Lake City } \\
\text { Utah } \\
\text { USA } \\
\text { Email: sheldon.smith@hsc.utah.edu }\end{array}$ \\
\hline Notes & $\begin{array}{l}\text { Listed as "Recruitment status unknown". Completion date has passed and the status has not been verified in } \\
\text { more than } 2 \text { years }\end{array}$ \\
\hline
\end{tabular}

\section{NCT01552551}

Trial name or title Assessment and referral versus exercise in primary prevention of falls: PA Healthy Steps Program

Methods Study design: RCT

Number of study arms: 3

Participants Setting: USA

Target sample size: 189

Inclusion criteria: Aged 50 and above; scoring in the lowest tertile on at least 1 test in the Healthy Steps lower extremity performance battery

Exclusion criteria: hospice enrollee or life-threatening illness; active cancer treatment; neurologic disease linked to falls risk, such as Parkinson's; unable to walk indoors; high likelihood of moving in next 6 months 


\section{NCT01552551 (Continued)}

\begin{tabular}{ll}
\hline Interventions & $\begin{array}{l}\text { Type of intervention: Multifactorial } \\
\text { 1. Asssessment and care by physician and home-safety assessment } \\
\text { 2. Exercise programme - Healthy Steps a 4-week exercise programme } \\
\text { 3. Control: usual care }\end{array}$ \\
\hline Outcomes & 1. Number of people sustaining 1 or more falls \\
\hline Starting date & January 2013 to December 2015 \\
\hline Contact information & $\begin{array}{l}\text { Steven Albert } \\
\text { University of Pittsburgh } \\
\text { USA }\end{array}$ \\
\hline Notes & Listed as "Completed" but the results have not yet been published \\
\hline
\end{tabular}

\section{NCT01713543}

Trial name or title Community-based falls prevention program for the elderly

\begin{tabular}{|c|c|}
\hline Methods & $\begin{array}{l}\text { Study design: RCT } \\
\text { Number of study arms: } 2\end{array}$ \\
\hline Participants & $\begin{array}{l}\text { Setting: Singapore } \\
\text { Target sample size: } 354 \\
\text { Inclusion criteria: Aged } 65 \text { and above; seen in the Emergency Department for a fall or injury related to a fall; } \\
\text { able to follow 3-step commands; Singapore citizen or Permanent Resident; living at home upon discharge; if } \\
\text { admitted to the hospital, the illness or disability is one from which they are expected to recover basic ADLs } \\
\text { or weight bearing of the lower extremity within the next month } \\
\text { Exclusion criteria: Severe physical and/or mental impairments which preclude participation in a programme } \\
\text { of physical therapy; unable to walk even with assistance; community-dwelling prior to ED visit; total blindness }\end{array}$ \\
\hline Interventions & $\begin{array}{l}\text { Type of intervention: Multifactorial } \\
\text { 1. Individually-tailored intervention programme based on a number of risk factors, including exercise, poor } \\
\text { vision, medication review and home-hazard modification } \\
\text { 2. Control: usual care }\end{array}$ \\
\hline Outcomes & $\begin{array}{l}\text { 1. Number of people who sustained } 1 \text { or more falls } \\
\text { 2. Number of people who experience a fall who require medical attention }\end{array}$ \\
\hline Starting date & December 2012 to April 2015 \\
\hline Contact information & $\begin{array}{l}\text { David B Matchar } \\
\text { Duke-NUS Graduate Medical School } \\
\text { Singapore }\end{array}$ \\
\hline Notes & Listed as "Completed" but results have not yet been published \\
\hline
\end{tabular}


Trial name or title Falls prevention in older people receiving home-help services

\begin{tabular}{|c|c|}
\hline Methods & $\begin{array}{l}\text { Study design: RCT } \\
\text { Number of study arms: } 2\end{array}$ \\
\hline Participants & $\begin{array}{l}\text { Setting: Norway } \\
\text { Target sample size: } 155 \\
\text { Inclusion criteria: Aged } 67 \text { and above; has fallen at least once in the last } 12 \text { months; receives home-help } \\
\text { services; able to walk independently indoors with or without walking aid } \\
\text { Exclusion criteria: Medical contraindication to exercise; life expectancy < } 1 \text { year; scores under } 23 \text { points on } \\
\text { MMSE; participating in another falls-prevention programme }\end{array}$ \\
\hline Interventions & $\begin{array}{l}\text { Type of intervention: Multiple component } \\
\text { 1. Exercise and education, 12-week tailored exercise programme in accordance with Otago exercise pro- } \\
\text { gramme, education on motivation and importance of adherence to exercise } \\
\text { 2. Control: usual care }\end{array}$ \\
\hline Outcomes & $\begin{array}{l}\text { 1. Number of people sustaining } 1 \text { or more falls } \\
\text { 2. Health-related quality of life }\end{array}$ \\
\hline Starting date & February 2016 to January 2018 (anticipated) \\
\hline Contact information & $\begin{array}{l}\text { Astrid Bergland } \\
\text { Oslo University College of Applied Sciences } \\
\text { Norway }\end{array}$ \\
\hline Notes & Listed as "Ongoing but not recruiting" \\
\hline
\end{tabular}

\section{NCT02631330}

Trial name or title

Effect on falls reduction of a multimodal intervention in frail and pre-frail elderly community-dwelling people (FAREMAVA)

\begin{tabular}{ll} 
Methods & $\begin{array}{l}\text { Study design: RCT } \\
\text { Number of study arms: } 2\end{array}$ \\
\hline Participants & $\begin{array}{l}\text { Setting: Spain } \\
\text { Target sample size: } 466 \\
\text { Inclusion criteria: Aged } 70 \text { and above; independent ambulation; Linda Freid's criteria for pre-frailty } \\
\text { Exclusion criteria: Life expectancy of }<6 \text { months; institutionalised patients; severe hearing or visual deficits; } \\
\text { contraindication to physical exercise; serious psychiatric illness or moderate or severe cognitive impairment }\end{array}$ \\
\hline Interventions & $\begin{array}{l}\text { Type of intervention: Multifactorial } \\
\text { 1. Monthly talk on potential falls hazards, exercise component } 60 \text { minutes including balance, muscle and } \\
\text { strength training and medication review } \\
\text { 2. Control: no intervention }\end{array}$
\end{tabular}




\begin{tabular}{ll}
\hline Outcomes & $\begin{array}{l}\text { 1. Rate of falls } \\
\text { 2. Number of people who experience a fall that requires medical attention } \\
\text { 3. Number of people who experience a fall that requires hospital admission } \\
\text { 4. Health-related quality of life }\end{array}$ \\
\hline Starting date & December 2016 to December 2017 (anticipated) \\
\hline Contact information & $\begin{array}{l}\text { Francisco J Tarazona-Santabalbina } \\
\text { Hospital Universitario de la Ribera } \\
\text { Spain }\end{array}$ \\
\hline Notes & Listed as "Recruiting" as of 6 July 2017 \\
\hline
\end{tabular}

\section{Sherrington 2016}

Trial name or title RESTORE: Recovery exercises and Stepping On after fracture

\begin{tabular}{|c|c|}
\hline Methods & $\begin{array}{l}\text { Study design: RCT } \\
\text { Number of study arms: } 2\end{array}$ \\
\hline Participants & $\begin{array}{l}\text { Setting: Australia } \\
\text { Target sample size: } 350 \\
\text { Inclusion criteria: People with a fall-related lower limb or pelvic fracture who have completed active physio- } \\
\text { therapy or rehabilitation or both, and who are living at home or in a hostel } \\
\text { Exclusion criteria: Residing in nursing home; MMSE < 24; insufficient English language skills; inability to } \\
\text { walk } 10 \text { metres despite assistance from another person or walking aid; progressive neurological disease; a } \\
\text { medical condition precluding exercise }\end{array}$ \\
\hline Interventions & $\begin{array}{l}\text { Type of intervention: Multifactorial } \\
\text { 1. Home visits from a physiotherapist to prescribe an individualised exercise programme and use motivational } \\
\text { interviewing and goal-setting to encourage behaviour change about exercise, also offered the Stepping On } \\
\text { programme as implemented by the NSW Department of Health: weekly 2-hour group discussion sessions } \\
\text { for } 7 \text { weeks plus an additional booster session at } 3 \text { months } \\
\text { 2. Usual care control }\end{array}$ \\
\hline Outcomes & $\begin{array}{l}\text { 1. Rate of falls } \\
\text { 2. Number of people who experience a fall that requires medical attention } \\
\text { 3. Health-related quality of life }\end{array}$ \\
\hline Starting date & September 2010 \\
\hline Contact information & $\begin{array}{l}\text { C Sherrington } \\
\text { The George Institute for Global Health } \\
\text { PO Box M201 } \\
\text { Missenden Rd NSW } 2050 \\
\text { Australia }\end{array}$ \\
\hline
\end{tabular}


Sherrington 2016 (Continued)

Notes $\quad$ Listed as "Recruiting” as of 6 July 2017

Tan 2014

Trial name or title An individually-tailored multifactorial intervention program for older fallers in a middle-income developing country: Malaysian Falls Assessment and Intervention Trial (MyFAIT)

\begin{tabular}{|c|c|}
\hline Methods & $\begin{array}{l}\text { Study design: RCT } \\
\text { Number of study arms: } 2\end{array}$ \\
\hline Participants & $\begin{array}{l}\text { Setting: Malaysia } \\
\text { Target sample size: } 300 \\
\text { Inclusion criteria: Aged } 65 \text { and above; } 2 \text { or more falls or } 1 \text { injurious fall over the past } 12 \text { months } \\
\text { Exclusion criteria: Clinically-diagnosed dementia (ICD-10 definition); severe physical disabilities (i.e. unable } \\
\text { to walk with a walking aid); major psychiatric illnesses, psychosis (i.e. schizophrenia, paranoia) or brain } \\
\text { damage }\end{array}$ \\
\hline Interventions & $\begin{array}{l}\text { Type of intervention: Multifactorial } \\
\text { 1. Individually-tailored, multifaceted interventions involving modifiable risk factors for falls: cardiovascular } \\
\text { assessment and intervention; medication review; physiotherapy prescribed strength and balance exercise pro- } \\
\text { gramme; home-hazards Intervention; visual assessment and intervention; others as required } \\
\text { 2. Control: usual care }\end{array}$ \\
\hline Outcomes & $\begin{array}{l}\text { 1. Rate of falls } \\
\text { 2. Health-related quality of life }\end{array}$ \\
\hline Starting date & July 2012 to February 2016 \\
\hline Contact information & $\begin{array}{l}\text { Pey June Tan } \\
\text { Ageing and Age-Associated Disorders Research Group } \\
\text { Health and Translational Medicine Cluster } \\
\text { University of Malaya } \\
\text { Kuala Lumpur } \\
\text { Malaysia }\end{array}$ \\
\hline Notes & Listed as "Completed" but results not yet published (protocol published) \\
\hline
\end{tabular}

MMSE: Mini Mental State Examination; TUG: timed up and go 
DATA AND ANALYSES

Comparison 1. Multifactorial intervention vs usual care or attention control

\begin{tabular}{|c|c|c|c|c|}
\hline Outcome or subgroup title & $\begin{array}{l}\text { No. of } \\
\text { studies }\end{array}$ & $\begin{array}{c}\text { No. of } \\
\text { participants }\end{array}$ & Statistical method & Effect size \\
\hline $\begin{array}{l}1 \text { Rate of falls (falls per person } \\
\text { years) }\end{array}$ & 19 & 5853 & Rate Ratio (Random, 95\% CI) & $0.77[0.67,0.87]$ \\
\hline $\begin{array}{l}2 \text { Number of people sustaining } \\
\text { one or more falls }\end{array}$ & 29 & 9637 & Risk Ratio (Random, 95\% CI) & $0.96[0.90,1.03]$ \\
\hline $\begin{array}{l}3 \text { Number of people sustaining } \\
\text { recurrent falls (defined as two } \\
\text { or more falls in a specified time } \\
\text { period) }\end{array}$ & 12 & 3368 & Risk Ratio (Random, 95\% CI) & $0.87[0.74,1.03]$ \\
\hline $\begin{array}{l}4 \text { Number of people sustaining one } \\
\text { or more fall-related fractures }\end{array}$ & 9 & 2850 & Risk Ratio (Random, 95\% CI) & $0.73[0.53,1.01]$ \\
\hline $\begin{array}{l}5 \text { Number of people who } \\
\text { experience a fall that required } \\
\text { hospital admission }\end{array}$ & 15 & 5227 & Risk Ratio (Random, 95\% CI) & $1.00[0.92,1.07]$ \\
\hline $\begin{array}{l}6 \text { Number of people who } \\
\text { experience a fall that require } \\
\text { medical attention }\end{array}$ & 8 & 3078 & Risk Ratio (Random, 95\% CI) & $0.91[0.75,1.10]$ \\
\hline $\begin{array}{l}7 \text { Health-related quality of life: } \\
\text { endpoint score }\end{array}$ & 9 & 2373 & Std. Mean Difference (IV, Random, 95\% CI) & $0.19[0.03,0.35]$ \\
\hline $\begin{array}{l}8 \text { Health-related quality of life } \\
\text { (mental): endpoint score }\end{array}$ & 3 & 376 & Std. Mean Difference (IV, Random, 95\% CI) & $0.27[-0.03,0.56]$ \\
\hline $\begin{array}{l}9 \text { Health-related quality of life } \\
\text { (physical): endpoint score }\end{array}$ & 3 & 376 & Std. Mean Difference (IV, Random, 95\% CI) & $0.39[-0.00,0.79]$ \\
\hline
\end{tabular}

Comparison 2. Multifactorial intervention vs exercise

\begin{tabular}{|c|c|c|c|c|}
\hline Outcome or subgroup title & $\begin{array}{l}\text { No. of } \\
\text { studies }\end{array}$ & $\begin{array}{c}\text { No. of } \\
\text { participants }\end{array}$ & Statistical method & Effect size \\
\hline $\begin{array}{l}1 \text { Rate of falls (falls per person } \\
\text { years) }\end{array}$ & 1 & & Rate Ratio (Random, 95\% CI) & Subtotals only \\
\hline $\begin{array}{l}2 \text { Number of people sustaining } \\
\text { one or more falls }\end{array}$ & 1 & & Risk Ratio (Random, 95\% CI) & Subtotals only \\
\hline
\end{tabular}

Multifactorial and multiple component interventions for preventing falls in older people living in the community (Review) 


\begin{tabular}{|c|c|c|c|c|}
\hline Outcome or subgroup title & $\begin{array}{l}\text { No. of } \\
\text { studies }\end{array}$ & $\begin{array}{c}\text { No. of } \\
\text { participants }\end{array}$ & Statistical method & Effect size \\
\hline $\begin{array}{l}1 \text { Rate of falls (falls per person } \\
\text { years) }\end{array}$ & 6 & 1085 & Rate Ratio (Random, 95\% CI) & $0.74[0.60,0.91]$ \\
\hline $\begin{array}{l}1.1 \text { Exercise, home safety and } \\
\text { nutrition }\end{array}$ & 1 & 145 & Rate Ratio (Random, 95\% CI) & $0.70[0.53,0.95]$ \\
\hline 1.2 Exercise and nutrition & 2 & 335 & Rate Ratio (Random, 95\% CI) & $0.87[0.69,1.09]$ \\
\hline $\begin{array}{l}1.3 \text { Exercise, home safety and } \\
\text { vision }\end{array}$ & 1 & 310 & Rate Ratio (Random, 95\% CI) & $0.69[0.50,0.96]$ \\
\hline $\begin{array}{l}1.4 \text { Exercise and psychological } \\
\text { component }\end{array}$ & 1 & 116 & Rate Ratio (Random, 95\% CI) & $0.40[0.11,1.53]$ \\
\hline $\begin{array}{l}1.5 \text { Nutrition and } \\
\text { psychological component }\end{array}$ & 1 & 151 & Rate Ratio (Random, 95\% CI) & $0.39[0.22,0.68]$ \\
\hline 1.6 Exercise and home safety & 1 & 28 & Rate Ratio (Random, 95\% CI) & $1.20[0.59,2.42]$ \\
\hline $\begin{array}{l}2 \text { Number of people sustaining } \\
\text { one or more falls }\end{array}$ & 11 & 1980 & Risk Ratio (Random, 95\% CI) & $0.82[0.74,0.90]$ \\
\hline $\begin{array}{l}2.1 \text { Exercise, home safety and } \\
\text { nutrition }\end{array}$ & 1 & 145 & Risk Ratio (Random, 95\% CI) & $0.77[0.57,1.03]$ \\
\hline 2.2 Exercise and nutrition & 1 & 146 & Risk Ratio (Random, 95\% CI) & $0.78[0.58,1.04]$ \\
\hline $\begin{array}{l}2.3 \text { Exercise, home safety and } \\
\text { vision }\end{array}$ & 2 & 479 & Risk Ratio (Random, 95\% CI) & $0.84[0.71,1.00]$ \\
\hline 2.4 Exercise and vision & 1 & 170 & Risk Ratio (Random, 95\% CI) & $0.75[0.56,1.00]$ \\
\hline 2.5 Exercise and home safety & 3 & 219 & Risk Ratio (Random, 95\% CI) & $0.84[0.65,1.09]$ \\
\hline 2.6 Home safety and vision & 1 & 171 & Risk Ratio (Random, 95\% CI) & $0.88[0.65,1.18]$ \\
\hline $\begin{array}{l}\text { 2.7 Exercise and psychological } \\
\text { component }\end{array}$ & 2 & 149 & Risk Ratio (Random, 95\% CI) & $0.84[0.25,2.77]$ \\
\hline 2.8 Education and exercise & 2 & 192 & Risk Ratio (Random, 95\% CI) & $1.09[0.57,2.11]$ \\
\hline $\begin{array}{l}\text { 2.9 Nutrition and } \\
\text { psychological component }\end{array}$ & 1 & 210 & Risk Ratio (Random, 95\% CI) & $0.41[0.21,0.82]$ \\
\hline $\begin{array}{l}2.10 \text { Exercise, nutrition and } \\
\text { psychological component }\end{array}$ & 1 & 99 & Risk Ratio (Random, 95\% CI) & $0.41[0.08,1.99]$ \\
\hline $\begin{array}{l}3 \text { Number of people sustaining } \\
\text { recurrent falls (defined as two } \\
\text { or more falls in a specified time } \\
\text { period) }\end{array}$ & 4 & 662 & Risk Ratio (Random, 95\% CI) & $0.81[0.63,1.05]$ \\
\hline $\begin{array}{l}3.1 \text { Exercise, home safety and } \\
\text { nutrition }\end{array}$ & 1 & 146 & Risk Ratio (Random, 95\% CI) & $0.79[0.45,1.36]$ \\
\hline 3.2 Exercise and home safety & 2 & 173 & Risk Ratio (Random, 95\% CI) & $0.89[0.54,1.46]$ \\
\hline $\begin{array}{l}3.3 \text { Exercise, home safety and } \\
\text { vision }\end{array}$ & 1 & 310 & Risk Ratio (Random, 95\% CI) & $0.74[0.52,1.05]$ \\
\hline $\begin{array}{l}3.4 \text { Exercise and psychological } \\
\text { component }\end{array}$ & 1 & 33 & Risk Ratio (Random, 95\% CI) & $5.00[0.68,36.94]$ \\
\hline $\begin{array}{l}4 \text { Number of people sustaining one } \\
\text { or more fall-related fractures }\end{array}$ & 2 & 232 & Risk Ratio (Random, 95\% CI) & $0.50[0.05,5.32]$ \\
\hline $\begin{array}{l}\text { 4.1 Nutrition and } \\
\text { psychological component }\end{array}$ & 1 & 210 & Risk Ratio (Random, 95\% CI) & $0.50[0.02,14.89]$ \\
\hline 4.2 Exercise and home safety & 1 & 22 & Risk Ratio (Random, 95\% CI) & $0.50[0.02,13.50]$ \\
\hline
\end{tabular}

Multifactorial and multiple component interventions for preventing falls in older people living in the community (Review)

Copyright ( 2018 The Cochrane Collaboration. Published by John Wiley \& Sons, Ltd. 
5 Number of people who experience a fall that required hospital admission

5.1 Exercise, nutrition and psychological component

6 Number of people who experience a fall that required medical attention

6.1 Exercise, home safety and nutrition

6.2 Exercise and nutrition

7 Health-related quality of life: endpoint score

7.1 Exercise and nutrition

7.2 Exercise and psychological component

7.3 Exercise, nutrition and psychological component

8 Health-related quality of life (mental): endpoint score 8.1 Exercise and home safety 8.2 Exercise, nutrition and psychological component

9 Health-related quality of life (physical): endpoint score 9.1 Exercise and home safety 9.2 Exercise, nutrition and psychological component

1
Risk Ratio (Random, 95\% CI)

Totals not selected

Risk Ratio (Random, 95\% CI)

$0.0[0.0,0.0]$ 291

Risk Ratio (Random, 95\% CI)

$0.95[0.67,1.35]$

$0.91[0.56,1.49]$

$0.99[0.61,1.62]$

145 Risk Ratio (Random, 95\% CI)

$0.77[0.16,1.39]$

Std. Mean Difference (IV, Random, 95\% CI)

$0.07[-0.27,0.41]$

$1.23[0.92,1.54]$

Std. Mean Difference (IV, Random, 95\% CI)

Std. Mean Difference (IV, Random, 95\% CI)

Std. Mean Difference (IV, Random, 95\% CI)

$0.57[0.07,1.07]$

Std. Mean Difference (IV, Random, 95\% CI)

$0.69[0.26,1.11]$

Std. Mean Difference (IV, Random, 95\% CI)

Std. Mean Difference (IV, Random, 95\% CI)

$0.80[0.02,1.57]$

$0.64[0.14,1.14]$

$0.12[-0.53,0.77]$

Std. Mean Difference (IV, Random, 95\% CI)

Std. Mean Difference (IV, Random, 95\% CI)

$-0.27[-1.02,0.47]$

Std. Mean Difference (IV, Random, 95\% CI)

$0.40[-0.10,0.90]$

\section{Comparison 4. Multiple intervention vs exercise}

\begin{tabular}{|c|c|c|c|c|}
\hline Outcome or subgroup title & $\begin{array}{l}\text { No. of } \\
\text { studies }\end{array}$ & $\begin{array}{c}\text { No. of } \\
\text { participants }\end{array}$ & Statistical method & Effect size \\
\hline $\begin{array}{l}1 \text { Rate of falls (falls per person } \\
\text { years) }\end{array}$ & 1 & & Rate Ratio (Random, 95\% CI) & Totals not selected \\
\hline 1.1 Exercise and nutrition & 1 & & Rate Ratio (Random, 95\% CI) & $0.0[0.0,0.0]$ \\
\hline $\begin{array}{l}2 \text { Number of people sustaining } \\
\text { one or more falls }\end{array}$ & 3 & 863 & Risk Ratio (Random, 95\% CI) & $0.93[0.78,1.10]$ \\
\hline 2.1 Education and exercise & 1 & 87 & Risk Ratio (Random, 95\% CI) & $2.23[0.11,46.43]$ \\
\hline $\begin{array}{l}2.2 \text { Education, nutrition and } \\
\text { psychological component }\end{array}$ & 1 & 97 & Risk Ratio (Random, 95\% CI) & $0.65[0.11,3.72]$ \\
\hline 2.3 Exercise and vision & 1 & 170 & Risk Ratio (Random, 95\% CI) & $0.87[0.61,1.24]$ \\
\hline 2.4 Exercise and home safety & 1 & 169 & Risk Ratio (Random, 95\% CI) & $0.95[0.68,1.33]$ \\
\hline 2.5 Home safety and vision & 1 & 171 & Risk Ratio (Random, 95\% CI) & $1.02[0.73,1.42]$ \\
\hline $\begin{array}{l}2.6 \text { Exercise, home safety and } \\
\text { vision }\end{array}$ & 1 & 169 & Risk Ratio (Random, 95\% CI) & $0.86[0.60,1.22]$ \\
\hline
\end{tabular}

Multifactorial and multiple component interventions for preventing falls in older people living in the community (Review) 


\section{Comparison 5. Multifactorial intervention vs control: subgroup analysis by intensity of intervention}

\begin{tabular}{|c|c|c|c|c|}
\hline Outcome or subgroup title & $\begin{array}{l}\text { No. of } \\
\text { studies }\end{array}$ & $\begin{array}{c}\text { No. of } \\
\text { participants }\end{array}$ & Statistical method & Effect size \\
\hline $\begin{array}{l}1 \text { Rate of falls (falls per person } \\
\text { years) }\end{array}$ & 19 & 5853 & Rate Ratio (Random, 95\% CI) & $0.77[0.67,0.87]$ \\
\hline $\begin{array}{l}1.1 \text { Assessment and active } \\
\text { intervention }\end{array}$ & 11 & 2630 & Rate Ratio (Random, 95\% CI) & $0.74[0.58,0.95]$ \\
\hline $\begin{array}{l}1.2 \text { Assessment and referral or } \\
\text { provision of information }\end{array}$ & 8 & 3223 & Rate Ratio (Random, 95\% CI) & $0.78[0.69,0.88]$ \\
\hline $\begin{array}{l}2 \text { Number of people sustaining } \\
\text { one or more falls }\end{array}$ & 29 & 9637 & Risk Ratio (Random, 95\% CI) & $0.96[0.90,1.03]$ \\
\hline $\begin{array}{l}2.1 \text { Assessment and active } \\
\text { intervention }\end{array}$ & 13 & 3677 & Risk Ratio (Random, 95\% CI) & $0.93[0.86,1.01]$ \\
\hline $\begin{array}{l}2.2 \text { Assessment and referral or } \\
\text { provision of information }\end{array}$ & 16 & 5960 & Risk Ratio (Random, 95\% CI) & $1.00[0.89,1.13]$ \\
\hline $\begin{array}{l}3 \text { Number of people sustaining } \\
\text { recurrent falls (defined as two } \\
\text { or more falls in a specified time } \\
\text { period) }\end{array}$ & 12 & 3368 & Risk Ratio (Random, 95\% CI) & $0.87[0.74,1.03]$ \\
\hline $\begin{array}{l}3.1 \text { Assessment and active } \\
\text { intervention }\end{array}$ & 7 & 2191 & Risk Ratio (Random, 95\% CI) & $0.82[0.66,1.03]$ \\
\hline $\begin{array}{l}3.2 \text { Assessment and referral or } \\
\text { provision of information }\end{array}$ & 5 & 1177 & Risk Ratio (Random, 95\% CI) & $0.96[0.74,1.23]$ \\
\hline
\end{tabular}

Comparison 6. Multifactorial intervention vs control: subgroup analysis by falls risk at baseline

\begin{tabular}{lcclc} 
Outcome or subgroup title & $\begin{array}{c}\text { No. of } \\
\text { studies }\end{array}$ & $\begin{array}{c}\text { No. of } \\
\text { participants }\end{array}$ & Statistical method & Effect size \\
\hline $\begin{array}{l}\text { 1 Rate of falls (falls per person } \\
\text { years) }\end{array}$ & 19 & 5853 & Rate Ratio (Random, 95\% CI) & $0.77[0.67,0.87]$ \\
$\quad \begin{array}{l}1.1 \text { Selected for higher risk of } \\
\text { falling }\end{array}$ & 16 & 5112 & Rate Ratio (Random, 95\% CI) & $0.78[0.68,0.89]$ \\
$\quad \begin{array}{l}1.2 \text { Not selected for higher } \\
\text { risk of falling }\end{array}$ & 3 & 741 & Rate Ratio (Random, 95\% CI) & $0.67[0.36,1.25]$ \\
$\begin{array}{l}\text { Number of people sustaining } \\
\text { one or more falls }\end{array}$ & 29 & 9637 & Risk Ratio (Random, 95\% CI) & $0.96[0.90,1.03]$
\end{tabular}

Multifactorial and multiple component interventions for preventing falls in older people living in the community (Review) 


$\begin{array}{lcccc}\begin{array}{l}\text { 2.1 Selected for higher risk of } \\ \text { falling }\end{array} & 22 & 6975 & \text { Risk Ratio (Random, 95\% CI) } & 0.97[0.90,1.04] \\ \begin{array}{l}2.2 \text { Not selected for higher } \\ \text { risk of falling }\end{array} & 7 & 2662 & \text { Risk Ratio (Random, 95\% CI) } & 0.92[0.75,1.12] \\ \begin{array}{l}\text { Number of people sustaining } \\ \text { recurrent falls (defined as two } \\ \text { or more falls in a specified time } \\ \text { period) }\end{array} & 12 & 3368 & \text { Risk Ratio (Random, 95\% CI) } & 0.87[0.74,1.03] \\ \begin{array}{l}3.1 \text { Selected for higher risk of } \\ \text { falling }\end{array} & 10 & 2824 & \text { Risk Ratio (Random, 95\% CI) } & 0.91[0.76,1.10] \\ \begin{array}{l}3.2 \text { Not selected for higher } \\ \text { risk of falling }\end{array} & 2 & 544 & \text { Risk Ratio (Random, 95\% CI) } & 0.70[0.54,0.90]\end{array}$

\section{Comparison 7. Multiple intervention vs control: subgroup analysis by falls risk at baseline}

\begin{tabular}{|c|c|c|c|c|}
\hline Outcome or subgroup title & $\begin{array}{l}\text { No. of } \\
\text { studies }\end{array}$ & $\begin{array}{c}\text { No. of } \\
\text { participants }\end{array}$ & Statistical method & Effect size \\
\hline $\begin{array}{l}1 \text { Rate of falls (falls per person } \\
\text { years) }\end{array}$ & 6 & 1085 & Rate Ratio (Random, 95\% CI) & $0.74[0.60,0.91]$ \\
\hline $\begin{array}{l}\text { 1.1 Selected for higher risk of } \\
\text { falling }\end{array}$ & 4 & 818 & Rate Ratio (Random, 95\% CI) & $0.79[0.68,0.93]$ \\
\hline $\begin{array}{l}1.2 \text { Not selected for higher } \\
\text { risk of falling }\end{array}$ & 2 & 267 & Rate Ratio (Random, 95\% CI) & $0.39[0.23,0.66]$ \\
\hline $\begin{array}{l}2 \text { Number of people sustaining } \\
\text { one or more falls }\end{array}$ & 11 & 1980 & Risk Ratio (Random, 95\% CI) & $0.82[0.74,0.90]$ \\
\hline $\begin{array}{l}\text { 2.1 Selected for higher risk of } \\
\text { falling }\end{array}$ & 7 & 872 & Risk Ratio (Random, 95\% CI) & $0.86[0.75,0.98]$ \\
\hline $\begin{array}{l}2.2 \text { Not selected for higher } \\
\text { risk of falling }\end{array}$ & 4 & 1108 & Risk Ratio (Random, 95\% CI) & $0.77[0.67,0.89]$ \\
\hline $\begin{array}{l}3 \text { Number of people sustaining } \\
\text { recurrent falls (defined as two } \\
\text { or more falls in a specified time } \\
\text { period) }\end{array}$ & 4 & & Risk Ratio (Random, 95\% CI) & Subtotals only \\
\hline $\begin{array}{l}\text { 3.1 Selected for higher risk of } \\
\text { falling }\end{array}$ & 4 & 662 & Risk Ratio (Random, 95\% CI) & $0.81[0.63,1.05]$ \\
\hline $\begin{array}{l}3.2 \text { Not selected for higher } \\
\text { risk of falling }\end{array}$ & 0 & 0 & Risk Ratio (Random, 95\% CI) & $0.0[0.0,0.0]$ \\
\hline
\end{tabular}

Multifactorial and multiple component interventions for preventing falls in older people living in the community (Review) 


\begin{tabular}{lcccc} 
Outcome or subgroup title & $\begin{array}{c}\text { No. of } \\
\text { studies }\end{array}$ & $\begin{array}{c}\text { No. of } \\
\text { participants }\end{array}$ & Statistical method & Effect size \\
\hline $\begin{array}{l}1 \text { Rate of falls (falls per person } \\
\text { years) }\end{array}$ & 8 & 3516 & Rate Ratio (Random, 95\% CI) & $0.80[0.66,0.98]$ \\
$\begin{array}{l}2 \text { Number of people sustaining } \\
\text { one or more falls }\end{array}$ & 12 & 4692 & Risk Ratio (Random, 95\% CI) & $0.98[0.86,1.10]$ \\
$\begin{array}{l}3 \text { Number of people sustaining } \\
\text { recurrent falls (defined as two } \\
\text { or more falls in a specified time } \\
\text { period) }\end{array}$ & 6 & 1862 & Risk Ratio (Random, 95\% CI) & $0.85[0.62,1.15]$ \\
\hline
\end{tabular}

Comparison 9. Multifactorial intervention vs control: sensitivity analysis by low risk of detection bias

\begin{tabular}{lcccc} 
Outcome or subgroup title & $\begin{array}{c}\text { No. of } \\
\text { studies }\end{array}$ & $\begin{array}{c}\text { No. of } \\
\text { participants }\end{array}$ & Statistical method & Effect size \\
\hline $\begin{array}{l}1 \text { Rate of falls (falls per person } \\
\text { years) }\end{array}$ & 12 & 3718 & Rate Ratio (Random, 95\% CI) & $0.78[0.66,0.91]$ \\
$\begin{array}{l}2 \text { Number of people sustaining } \\
\text { one or more falls }\end{array}$ & 16 & 4380 & Risk Ratio (Random, 95\% CI) & $0.97[0.88,1.07]$ \\
$\begin{array}{l}3 \text { Number of people sustaining } \\
\text { recurrent falls (defined as two } \\
\text { or more falls in a specified time } \\
\text { period) }\end{array}$ & 10 & 3033 & Risk Ratio (Random, 95\% CI) & $0.89[0.73,1.08]$ \\
\hline
\end{tabular}

Comparison 10. Multifactorial intervention vs control: sensitivity analysis by low risk of attrition bias

\begin{tabular}{lcccc} 
Outcome or subgroup title & $\begin{array}{c}\text { No. of } \\
\text { studies }\end{array}$ & $\begin{array}{c}\text { No. of } \\
\text { participants }\end{array}$ & Statistical method & Effect size \\
\hline $\begin{array}{l}1 \text { Rate of falls (falls per person } \\
\text { years) }\end{array}$ & 11 & 4125 & Rate Ratio (Random, 95\% CI) & $0.77[0.66,0.89]$ \\
$\begin{array}{l}2 \text { Number of people sustaining } \\
\text { one or more falls }\end{array}$ & 13 & 4452 & Risk Ratio (Random, 95\% CI) & $0.95[0.88,1.02]$ \\
$\begin{array}{l}3 \text { Number of people sustaining } \\
\text { recurrent falls (defined as two } \\
\text { or more falls in a specified time } \\
\text { period) }\end{array}$ & 5 & 1402 & Risk Ratio (Random, 95\% CI) & $0.96[0.81,1.13]$ \\
\hline
\end{tabular}


Comparison 11. Multifactorial intervention vs control: sensitivity analysis by individual randomisation

\begin{tabular}{lcccc} 
Outcome or subgroup title & $\begin{array}{c}\text { No. of } \\
\text { studies }\end{array}$ & $\begin{array}{c}\text { No. of } \\
\text { participants }\end{array}$ & Statistical method & Effect size \\
\hline $\begin{array}{l}1 \text { Rate of falls (falls per person } \\
\text { years) }\end{array}$ & 18 & 5562 & Rate Ratio (Random, 95\% CI) & $0.78[0.68,0.89]$ \\
$\begin{array}{l}2 \text { Number of people sustaining } \\
\text { one or more falls }\end{array}$ & 26 & 8774 & Risk Ratio (Random, 95\% CI) & $0.97[0.89,1.04]$ \\
$\begin{array}{l}3 \text { Number of people sustaining } \\
\text { recurrent falls (defined as two } \\
\text { or more falls in a specified time }\end{array}$ & 12 & 3368 & Risk Ratio (Random, 95\% CI) & $0.87[0.74,1.03]$ \\
period) & & & & \\
\hline
\end{tabular}

Comparison 12. Multiple intervention vs control: sensitivity analysis by low risk of selection bias

\begin{tabular}{lcccc} 
Outcome or subgroup title & $\begin{array}{c}\text { No. of } \\
\text { studies }\end{array}$ & $\begin{array}{c}\text { No. of } \\
\text { participants }\end{array}$ & Statistical method & Effect size \\
\hline $\begin{array}{l}1 \text { Rate of falls (falls per person } \\
\text { years) }\end{array}$ & 4 & 584 & Rate Ratio (Random, 95\% CI) & $0.68[0.51,0.92]$ \\
$\begin{array}{l}2 \text { Number of people sustaining } \\
\text { one or more falls }\end{array}$ & 8 & 1478 & Risk Ratio (Random, 95\% CI) & $0.78[0.70,0.88]$ \\
$\begin{array}{l}3 \text { Number of people sustaining } \\
\text { recurrent falls (defined as two } \\
\text { or more falls in a specified time } \\
\text { period) }\end{array}$ & 3 & 352 & Risk Ratio (Random, 95\% CI) & $0.90[0.62,1.30]$ \\
\hline
\end{tabular}

Comparison 13. Multiple intervention vs control: sensitivity analysis by low risk of detection bias

\begin{tabular}{lcccc} 
Outcome or subgroup title & $\begin{array}{c}\text { No. of } \\
\text { studies }\end{array}$ & $\begin{array}{c}\text { No. of } \\
\text { participants }\end{array}$ & Statistical method & Effect size \\
\hline $\begin{array}{l}1 \text { Rate of falls (falls per person } \\
\text { years) }\end{array}$ & 5 & 969 & Rate Ratio (Random, 95\% CI) & $0.75[0.60,0.93]$ \\
$\begin{array}{l}2 \text { Number of people sustaining } \\
\text { one or more falls }\end{array}$ & 5 & 1518 & Risk Ratio (Random, 95\% CI) & $0.81[0.73,0.89]$ \\
$\begin{array}{l}3 \text { Number of people sustaining } \\
\text { recurrent falls (defined as two } \\
\text { or more falls in a specified time } \\
\text { period) }\end{array}$ & 3 & 629 & Risk Ratio (Random, 95\% CI) & $0.79[0.61,1.02]$ \\
\hline
\end{tabular}


Comparison 14. Multiple intervention vs control: sensitivity analysis by low risk of attrition bias

\begin{tabular}{lcccc} 
Outcome or subgroup title & $\begin{array}{c}\text { No. of } \\
\text { studies }\end{array}$ & $\begin{array}{c}\text { No. of } \\
\text { participants }\end{array}$ & Statistical method & Effect size \\
\hline $\begin{array}{l}1 \text { Rate of falls (falls per person } \\
\text { years) }\end{array}$ & 3 & 596 & Rate Ratio (Random, 95\% CI) & $0.79[0.66,0.96]$ \\
$\begin{array}{l}2 \text { Number of people sustaining } \\
\text { one or more falls }\end{array}$ & 3 & 506 & Risk Ratio (Random, 95\% CI) & $0.75[0.62,0.92]$ \\
$\begin{array}{l}3 \text { Number of people sustaining } \\
\text { recurrent falls (defined as two } \\
\text { or more falls in a specified time } \\
\text { period) }\end{array}$ & 1 & 291 & Risk Ratio (Random, 95\% CI) & $0.84[0.57,1.23]$ \\
\hline
\end{tabular}

Comparison 15. Multiple intervention vs control: sensitivity analysis by individual randomisation

\begin{tabular}{lcccc} 
Outcome or subgroup title & $\begin{array}{c}\text { No. of } \\
\text { studies }\end{array}$ & $\begin{array}{c}\text { No. of } \\
\text { participants }\end{array}$ & Statistical method & Effect size \\
\hline $\begin{array}{l}1 \text { Rate of falls (falls per person } \\
\text { years) }\end{array}$ & 6 & 1085 & Rate Ratio (Random, 95\% CI) & $0.74[0.60,0.91]$ \\
$\begin{array}{l}2 \text { Number of people sustaining } \\
\text { one or more falls }\end{array}$ & 10 & 1877 & Risk Ratio (Random, 95\% CI) & $0.81[0.74,0.90]$ \\
$\begin{array}{l}3 \text { Number of people sustaining } \\
\text { recurrent falls (defined as two } \\
\text { or more falls in a specified time } \\
\text { period) }\end{array}$ & 4 & 662 & Risk Ratio (Random, 95\% CI) & $0.81[0.63,1.05]$ \\
\hline
\end{tabular}


Analysis I.I. Comparison I Multifactorial intervention vs usual care or attention control, Outcome I Rate of falls (falls per person years).

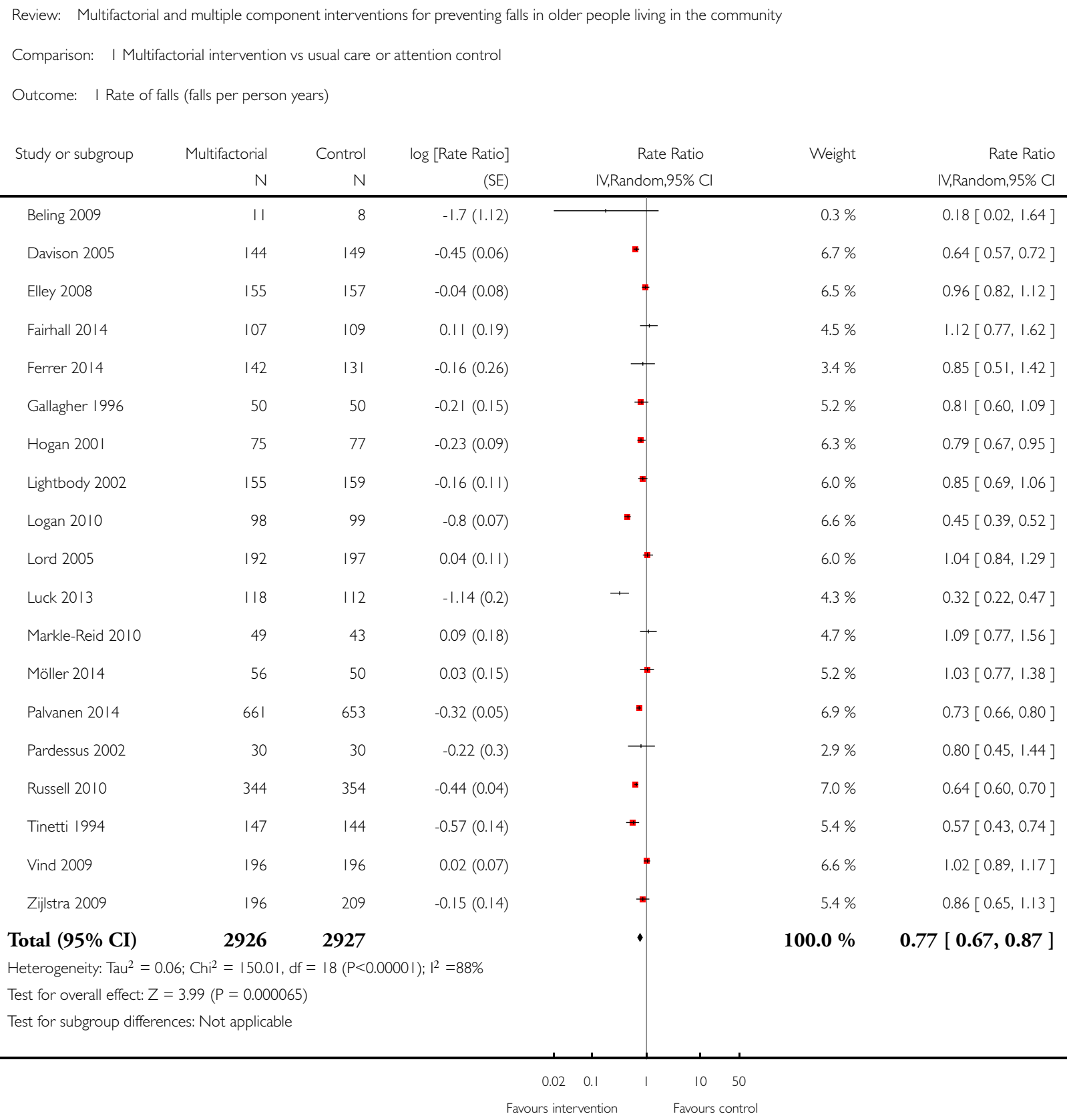




\section{Analysis I.2. Comparison I Multifactorial intervention vs usual care or attention control, Outcome 2}

Number of people sustaining one or more falls.

Review: Multifactorial and multiple component interventions for preventing falls in older people living in the community

Comparison: I Multifactorial intervention vs usual care or attention control

Outcome: 2 Number of people sustaining one or more falls

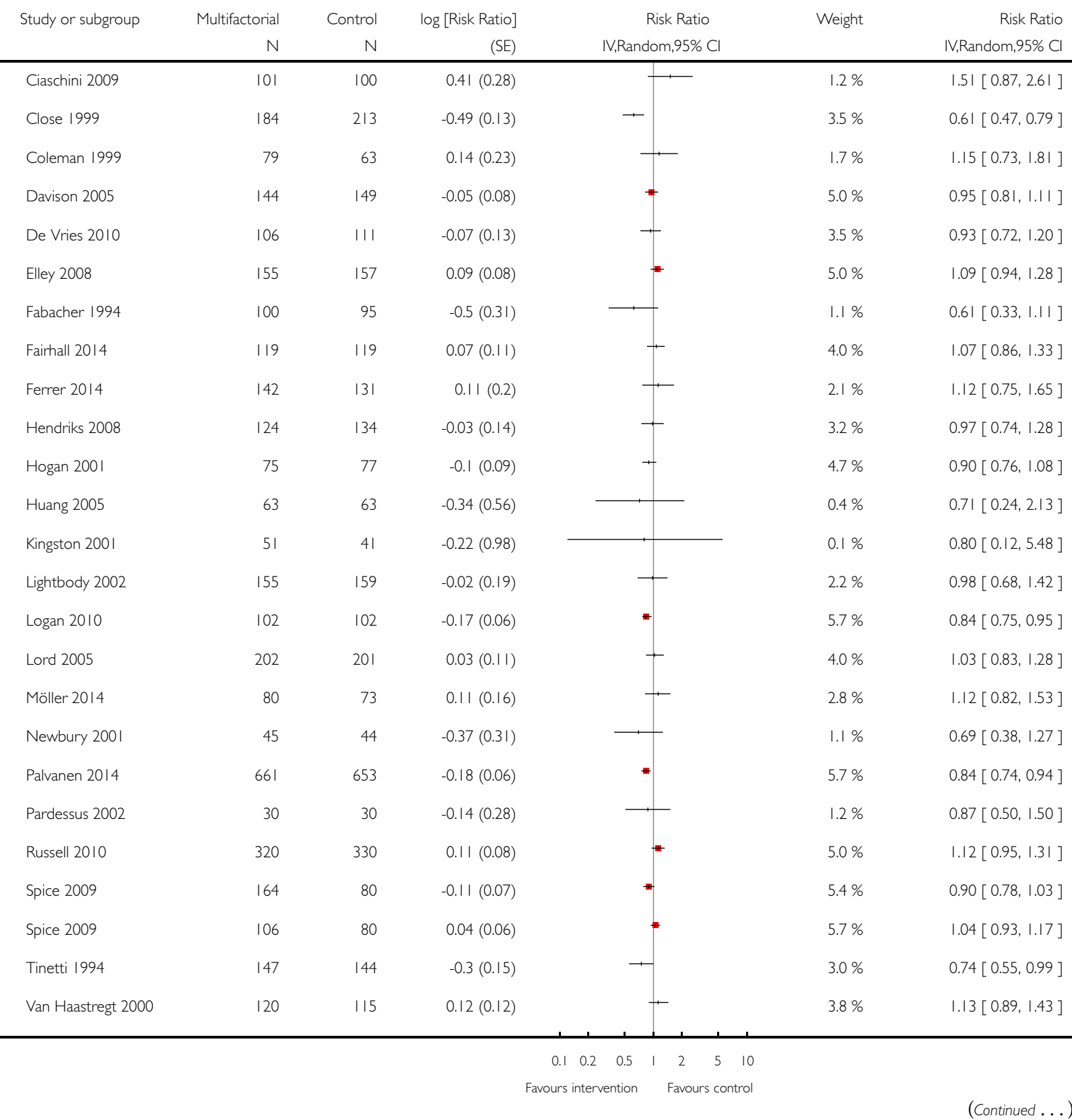




\begin{tabular}{|c|c|c|c|c|c|c|}
\hline \multirow[t]{2}{*}{ Study or subgroup } & Multifactorial & Control & log [Risk Ratio] & Risk Ratio & Weight & $\begin{array}{c}\text { (... Continued) } \\
\text { Risk Ratio }\end{array}$ \\
\hline & $\mathrm{N}$ & $\mathrm{N}$ & $(\mathrm{SE})$ & IV,Random,95\% Cl & & IV,Random,95\% Cl \\
\hline Vetter 1992 & 240 & 210 & $0.25(0.13)$ & + & $3.5 \%$ & $1.28[1.00,1.66]$ \\
\hline Vind 2009 & 196 & 196 & $0.09(0.09)$ & + & $4.7 \%$ & $1.09[0.92,1.31]$ \\
\hline Wagner 1994 (I) & 635 & 607 & $-0.29(0.08)$ & \# & $5.0 \%$ & $0.75[0.64,0.88]$ \\
\hline Whitehead 2003 & 58 & 65 & $0.74(0.26)$ & $\longrightarrow$ & $1.4 \%$ & $2.10[1.26,3.49]$ \\
\hline Zijlstra 2009 & 188 & 203 & $-0.17(0.1)$ & + & $4.4 \%$ & $0.84[0.69,1.03]$ \\
\hline Total $(95 \%$ CI) & 4892 & 4745 & & - & $100.0 \%$ & $0.96[0.90,1.03]$ \\
\hline \multicolumn{7}{|c|}{ Heterogeneity: $\mathrm{Tau}^{2}=0.02 ; \mathrm{Chi}^{2}=72.98, \mathrm{df}=29(\mathrm{P}=0.0000 \mathrm{I}) ; \mathrm{I}^{2}=60 \%$} \\
\hline \multicolumn{7}{|c|}{ Test for overall effect: $Z=1.16(P=0.24)$} \\
\hline \multicolumn{7}{|c|}{ Test for subgroup differences: Not applicable } \\
\hline
\end{tabular}

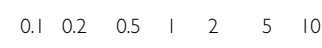

Favours intervention Favours control

(I) Multifactorial arm vs control 
Analysis I.3. Comparison I Multifactorial intervention vs usual care or attention control, Outcome 3 Number of people sustaining recurrent falls (defined as two or more falls in a specified time period).

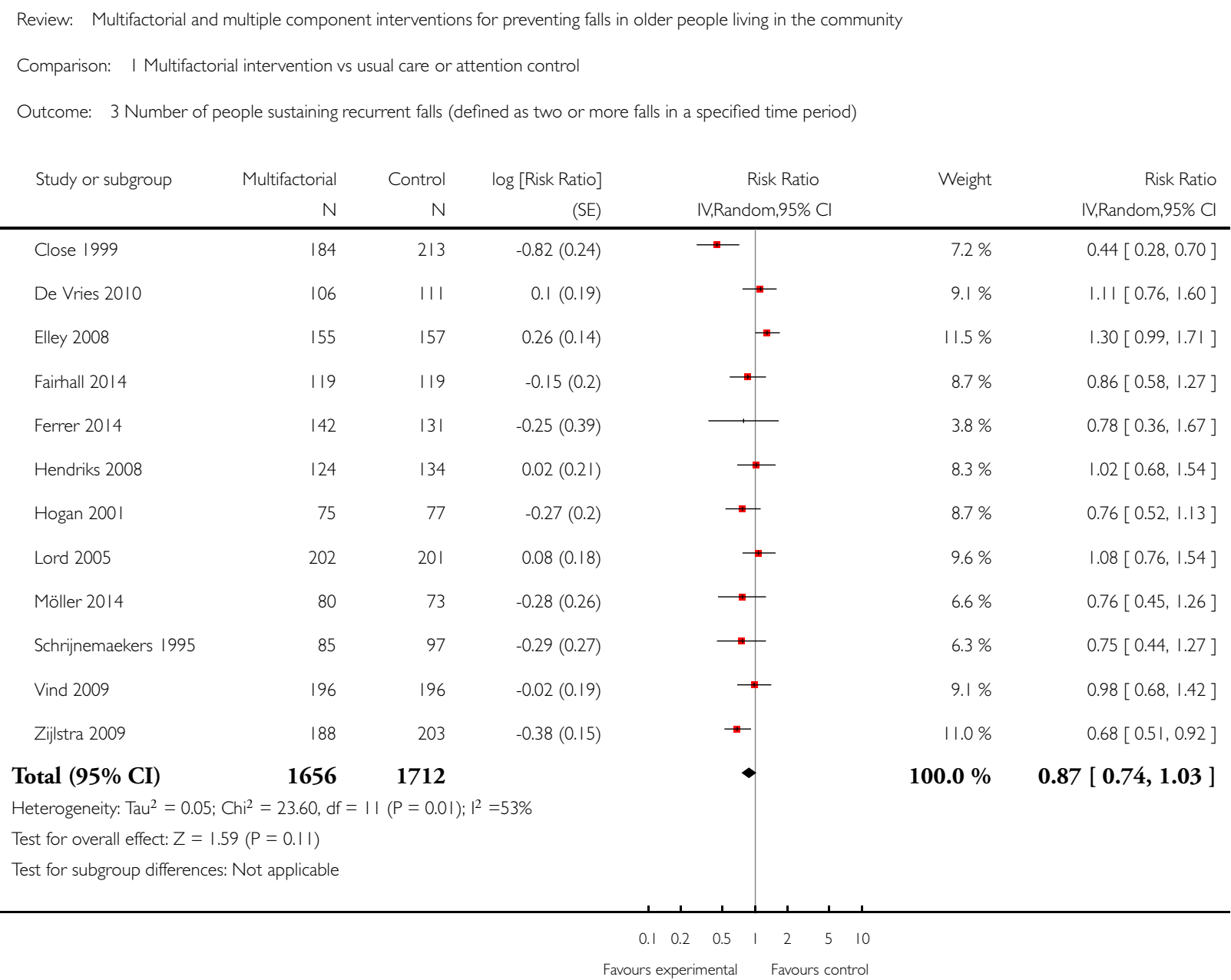


Analysis 1.4. Comparison I Multifactorial intervention vs usual care or attention control, Outcome 4 Number of people sustaining one or more fall-related fractures.

Review: Multifactorial and multiple component interventions for preventing falls in older people living in the community

Comparison: I Multifactorial intervention vs usual care or attention control

Outcome: 4 Number of people sustaining one or more fall-related fractures

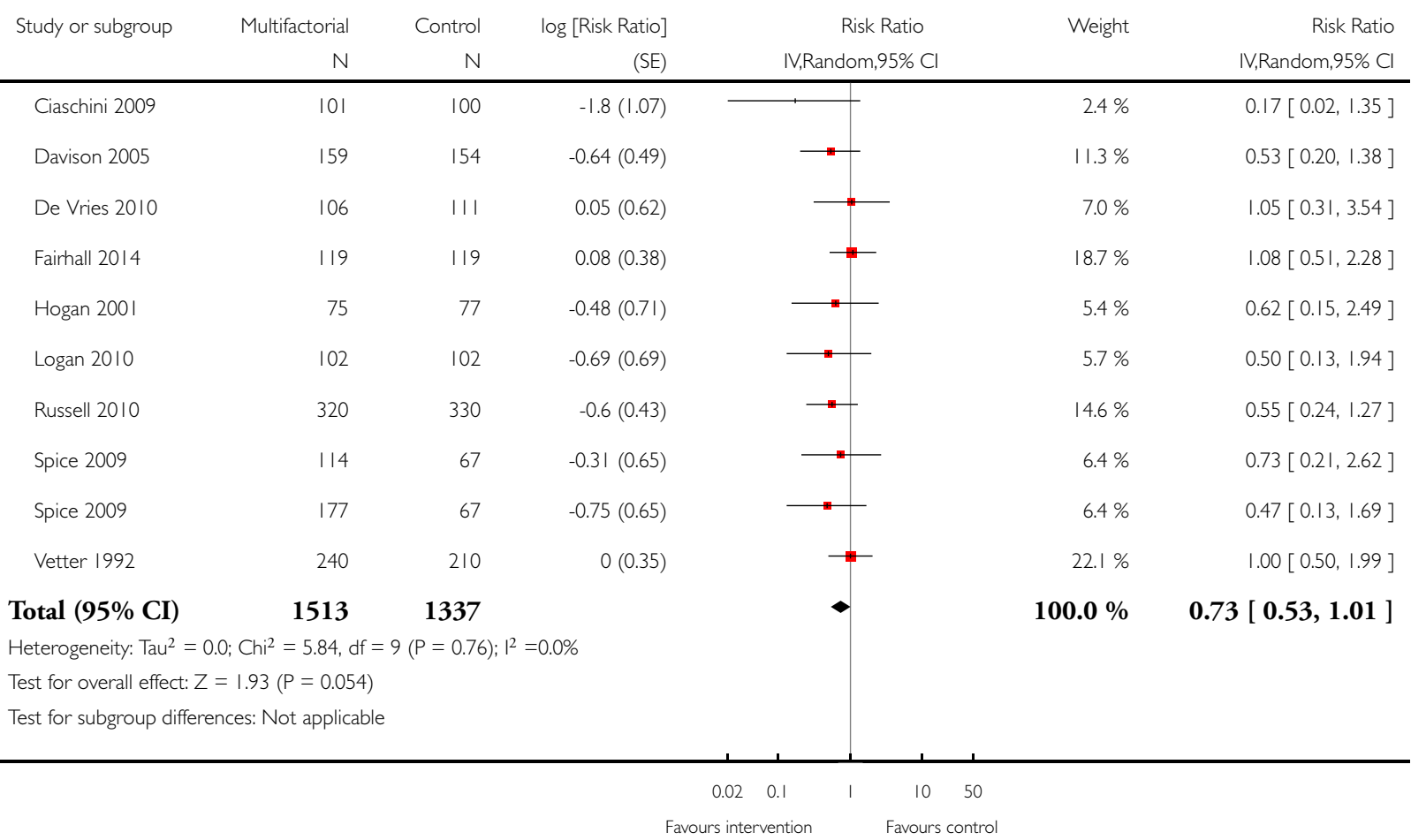



Analysis I.5. Comparison I Multifactorial intervention vs usual care or attention control, Outcome 5
Number of people who experience a fall that required hospital admission.

Review: Multifactorial and multiple component interventions for preventing falls in older people living in the community

Comparison: I Multifactorial intervention vs usual care or attention control

Outcome: 5 Number of people who experience a fall that required hospital admission

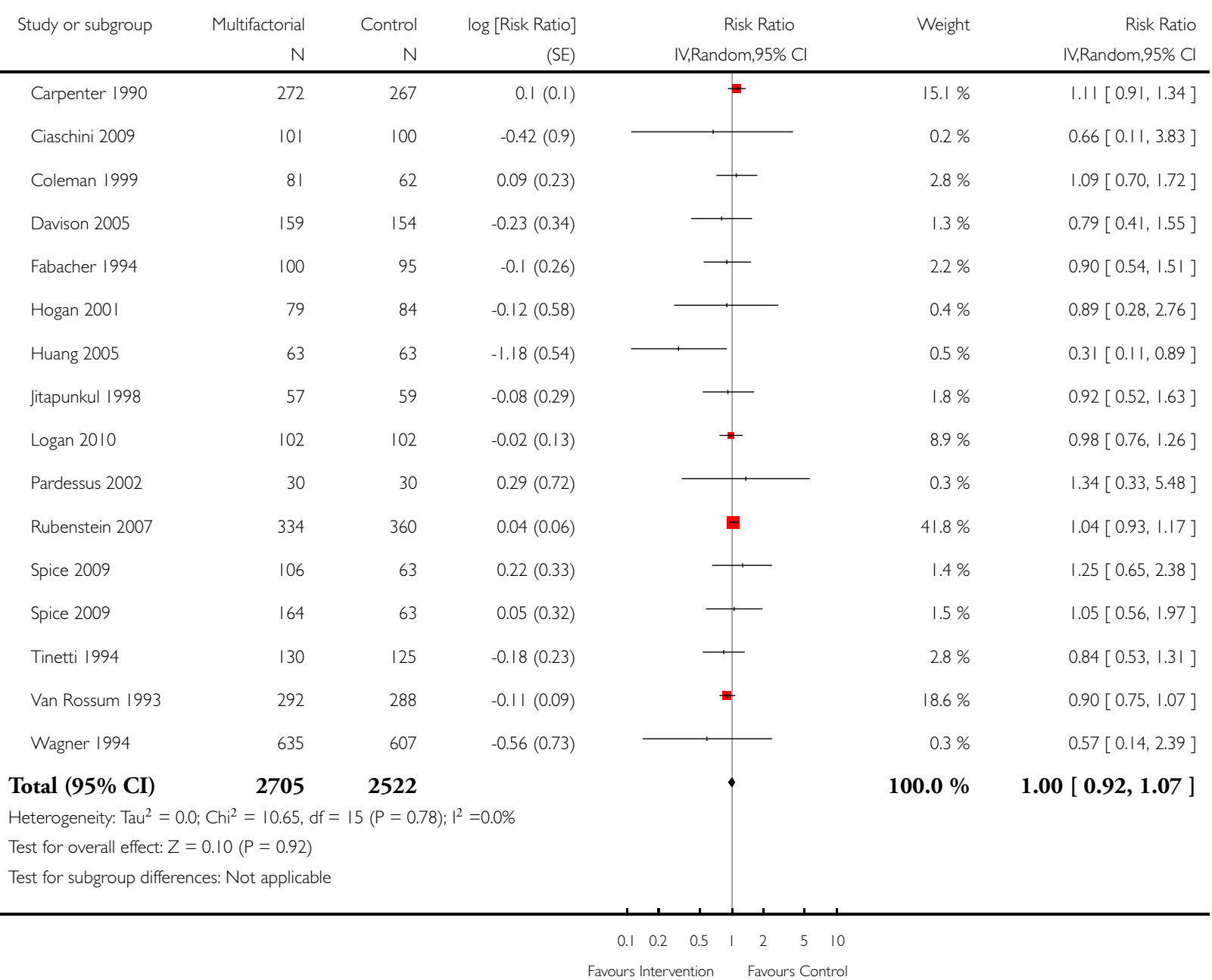


Analysis I.6. Comparison I Multifactorial intervention vs usual care or attention control, Outcome 6 Number of people who experience a fall that require medical attention.

Review: Multifactorial and multiple component interventions for preventing falls in older people living in the community

Comparison: I Multifactorial intervention vs usual care or attention control

Outcome: 6 Number of people who experience a fall that require medical attention

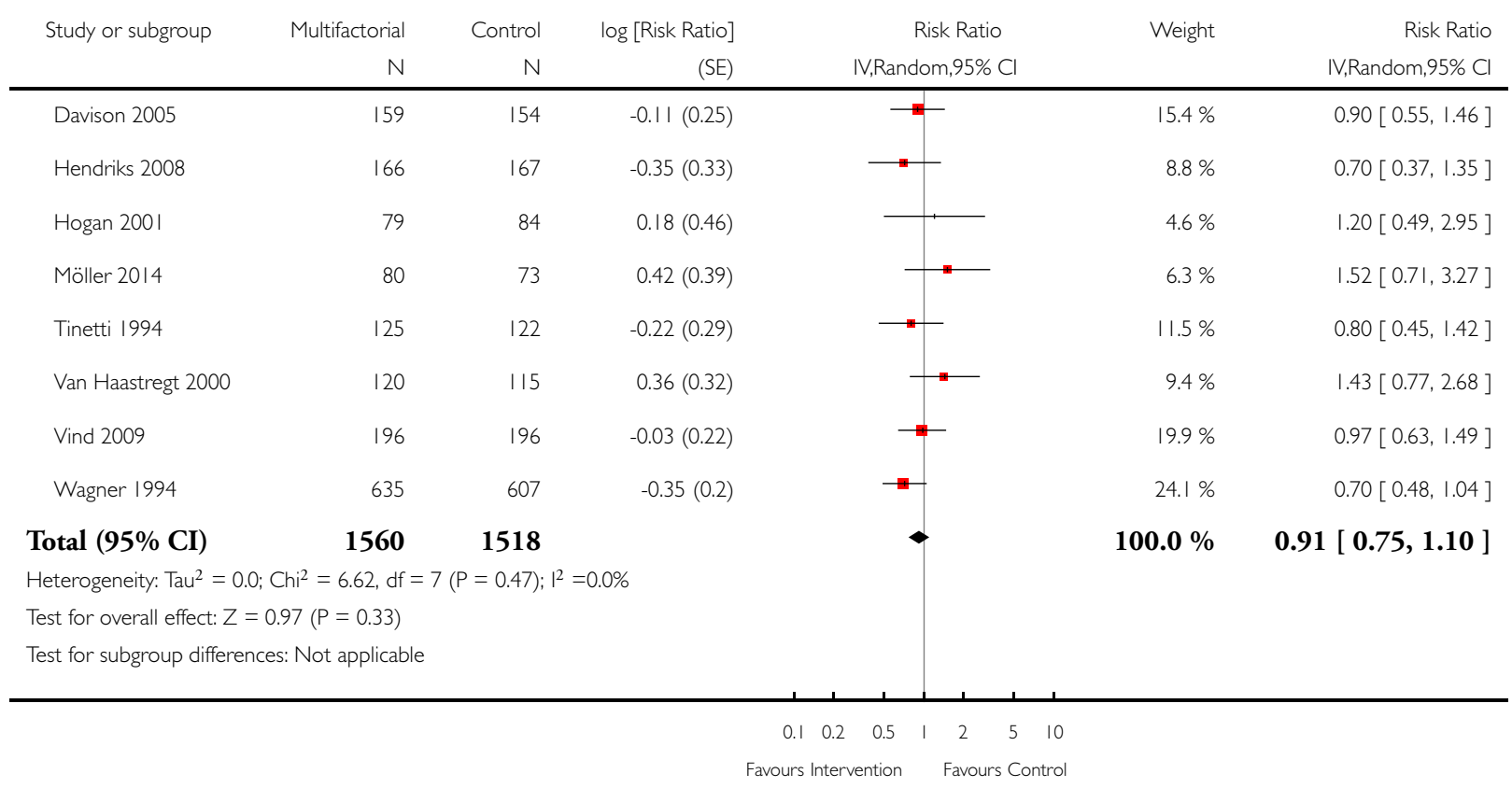


Analysis I.7. Comparison I Multifactorial intervention vs usual care or attention control, Outcome 7 Health-related quality of life: endpoint score.

Review: Multifactorial and multiple component interventions for preventing falls in older people living in the community

Comparison: I Multifactorial intervention vs usual care or attention control

Outcome: 7 Health-related quality of life: endpoint score

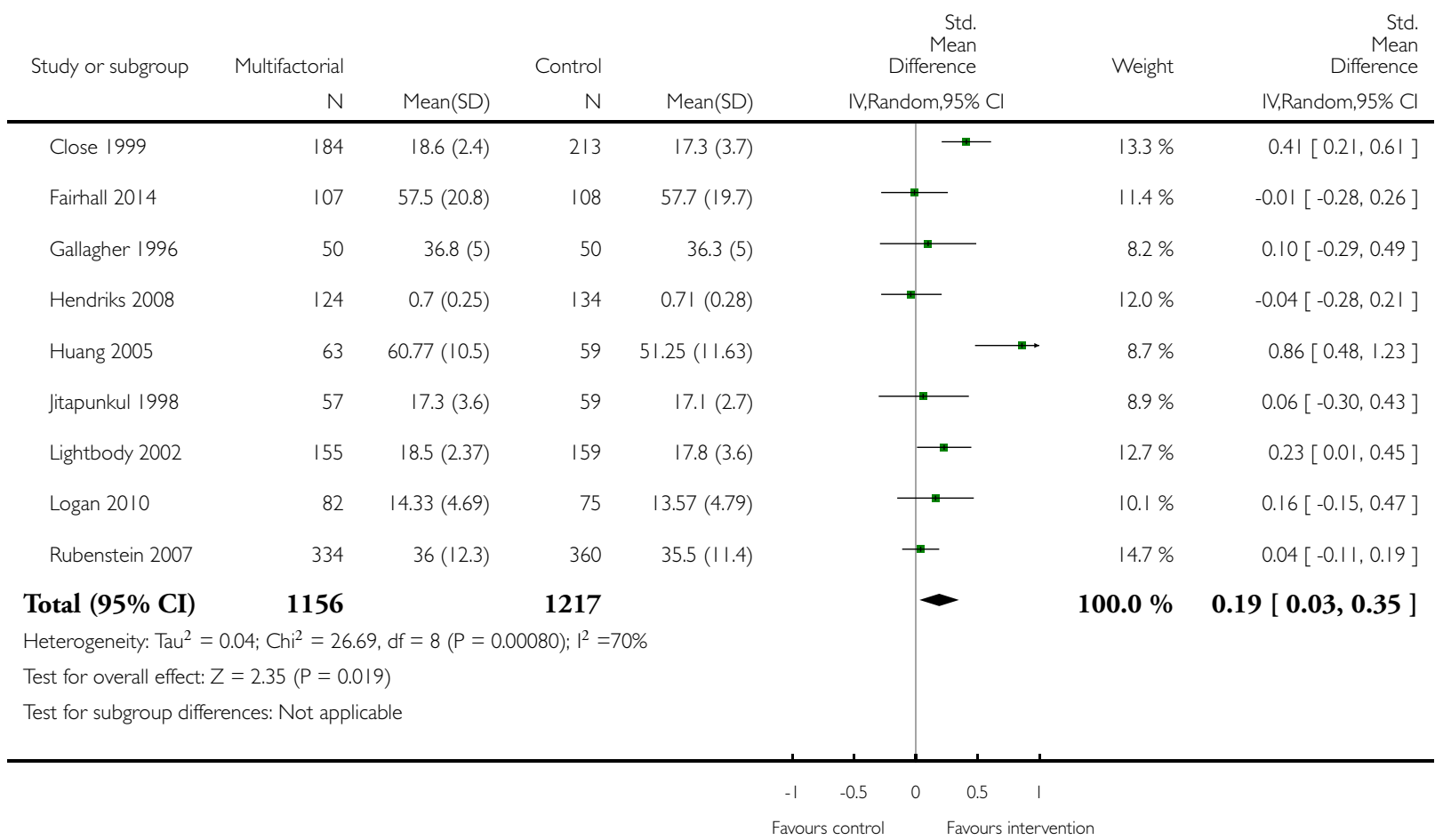


Analysis I.8. Comparison I Multifactorial intervention vs usual care or attention control, Outcome 8 Health-related quality of life (mental): endpoint score.

Review: Multifactorial and multiple component interventions for preventing falls in older people living in the community

Comparison: I Multifactorial intervention vs usual care or attention control

Outcome: 8 Health-related quality of life (mental): endpoint score

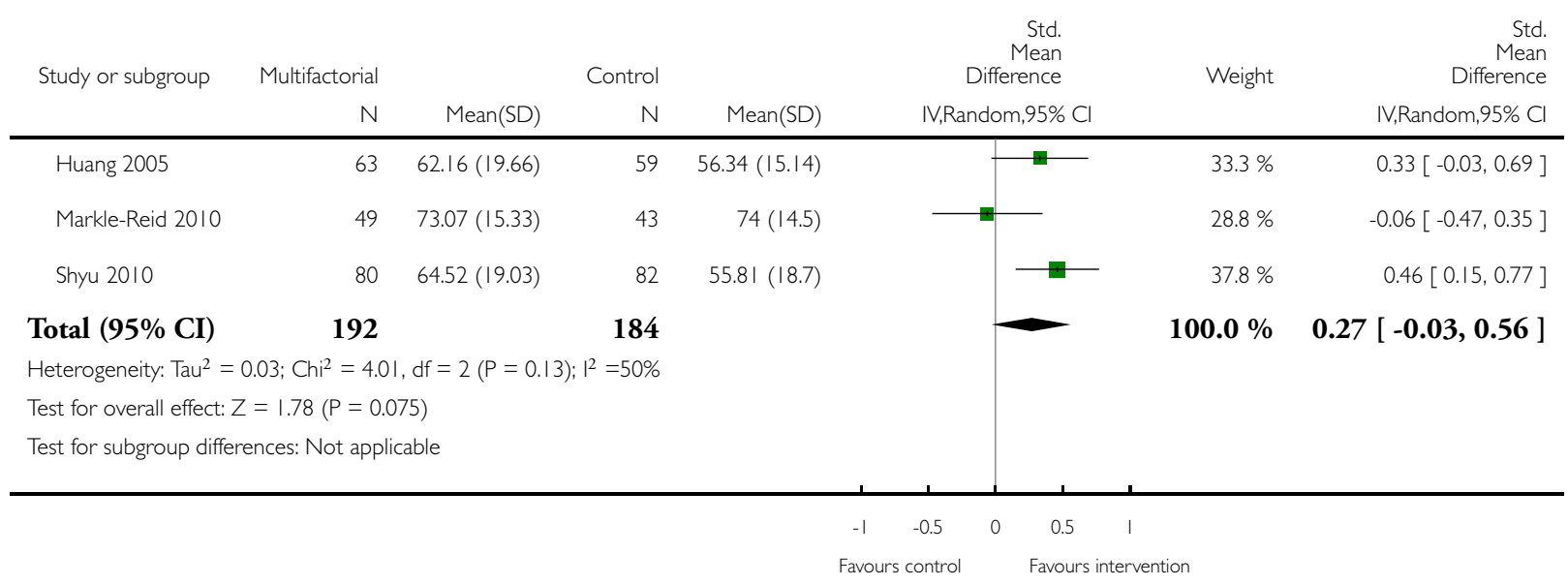




\section{Analysis I.9. Comparison I Multifactorial intervention vs usual care or attention control, Outcome 9}

Health-related quality of life (physical): endpoint score.

Review: Multifactorial and multiple component interventions for preventing falls in older people living in the community

Comparison: I Multifactorial intervention vs usual care or attention control

Outcome: 9 Health-related quality of life (physical): endpoint score

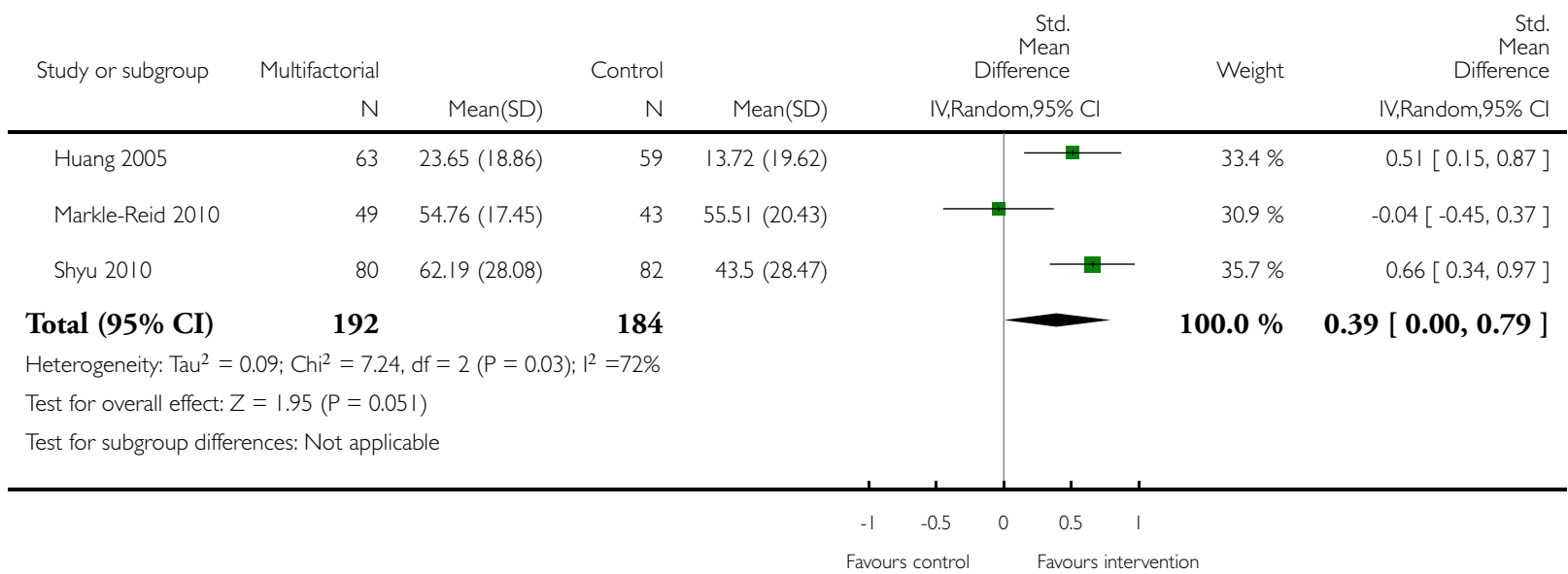

Analysis 2.I. Comparison 2 Multifactorial intervention vs exercise, Outcome I Rate of falls (falls per person years).

Review: Multifactorial and multiple component interventions for preventing falls in older people living in the community

Comparison: 2 Multifactorial intervention vs exercise

Outcome: I Rate of falls (falls per person years)

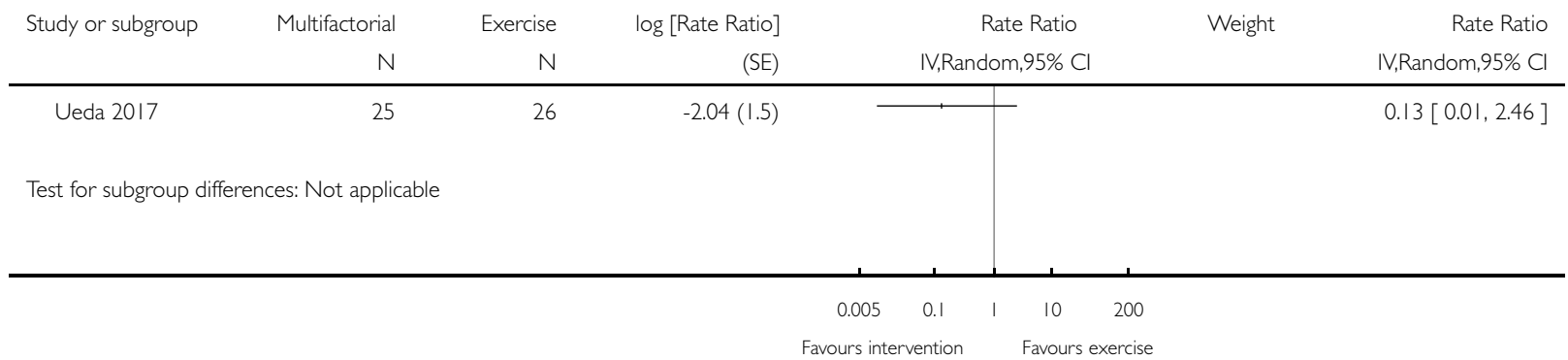




\section{Analysis 2.2. Comparison 2 Multifactorial intervention vs exercise, Outcome 2 Number of people} sustaining one or more falls.

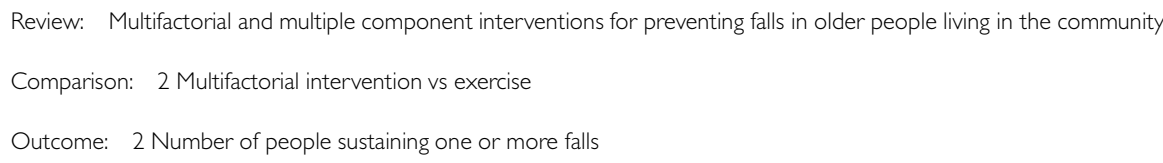

5)

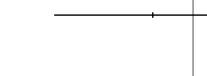

\section{Test for subgroup differences: Not applicable}

\begin{tabular}{|c|c|c|c|c|}
\hline 0.01 & 0.1 & 1 & 10 & 100 \\
\hline Favours int & entior & & Favour: & exercise \\
\hline
\end{tabular}

\section{Analysis 3.I. Comparison 3 Multiple intervention vs usual care or attention control, Outcome I Rate of falls (falls per person years).}

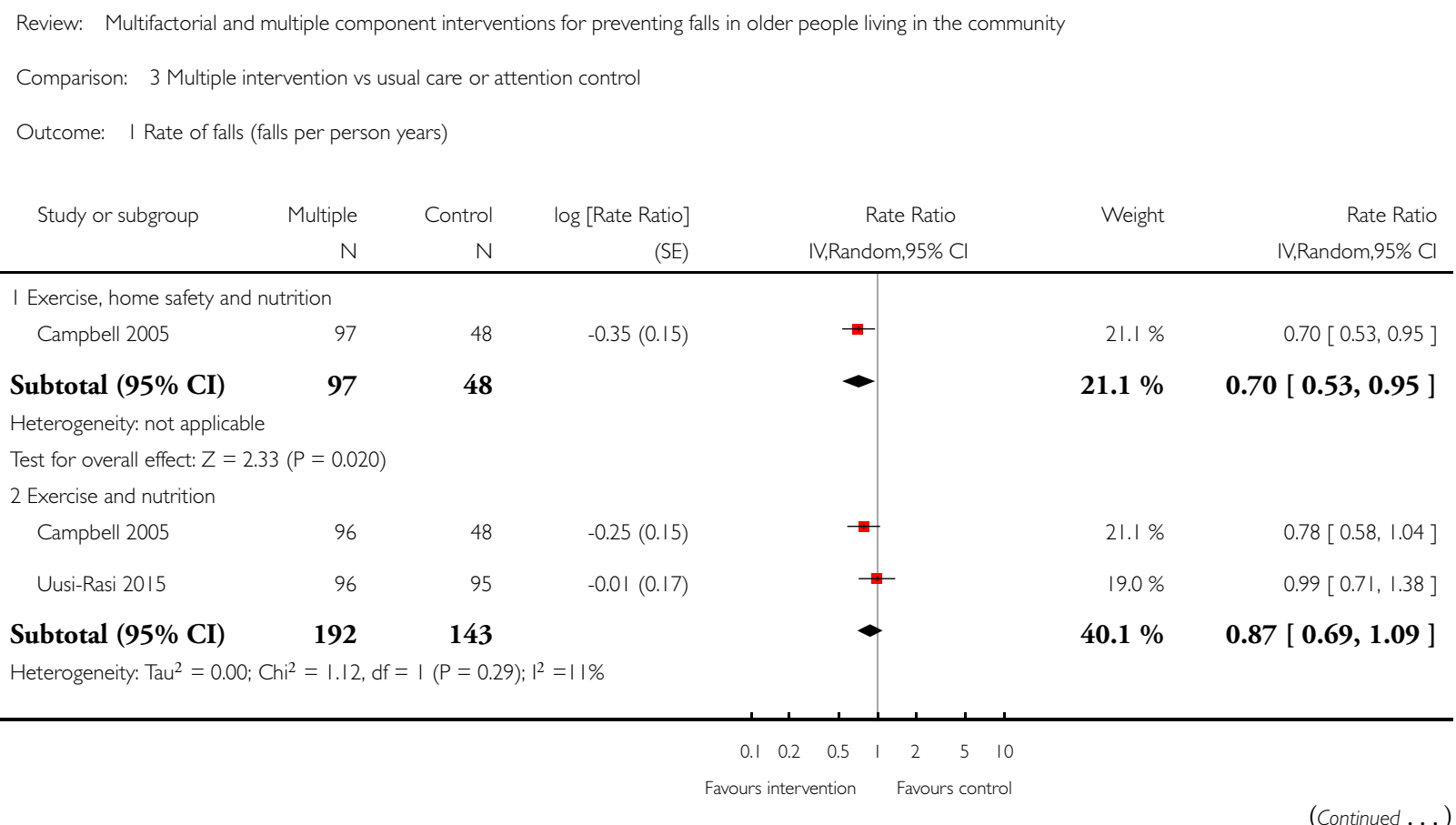




\begin{tabular}{|c|c|c|c|c|c|c|}
\hline \multirow[t]{2}{*}{ Study or subgroup } & Multiple & Control & log [Rate Ratio] & \multirow{2}{*}{$\begin{array}{c}\text { Rate Ratio } \\
\text { IV,Random,95\% Cl }\end{array}$} & \multirow{2}{*}{\multicolumn{2}{|c|}{$\begin{array}{r}(\ldots \text { Continued }) \\
\text { Rate Ratio } \\
\text { IV,Random, } 95 \% \mathrm{Cl} \\
\end{array}$}} \\
\hline & $\mathrm{N}$ & $\mathrm{N}$ & (SE) & & & \\
\hline \multicolumn{7}{|c|}{ Test for overall effect: $Z=1.20(P=0.23)$} \\
\hline \multicolumn{7}{|c|}{3 Exercise, home safety and vision } \\
\hline Clemson 2004 & 157 & 153 & $-0.37(0.17)$ & $\mp$ & $19.0 \%$ & $0.69[0.50,0.96]$ \\
\hline Subtotal $(95 \% \mathrm{CI})$ & 157 & 153 & & - & $19.0 \%$ & $0.69[0.50,0.96]$ \\
\hline \multicolumn{7}{|c|}{ Heterogeneity: not applicable } \\
\hline \multicolumn{7}{|c|}{ Test for overall effect: $Z=2.18(P=0.030)$} \\
\hline \multicolumn{7}{|c|}{4 Exercise and psychological component } \\
\hline Huang 201I & 56 & 60 & $-0.9 \mid(0.68)$ & & $2.4 \%$ & $0.40[0.11,1.53]$ \\
\hline Subtotal $(95 \% \mathrm{CI})$ & 56 & 60 & & & $2.4 \%$ & $0.40[0.11,1.53]$ \\
\hline \multicolumn{7}{|c|}{ Heterogeneity: not applicable } \\
\hline \multicolumn{7}{|c|}{ Test for overall effect: $Z=1.34(P=0.18)$} \\
\hline \multicolumn{7}{|c|}{5 Nutrition and psychological component } \\
\hline Neelemaat 2012 & 76 & 75 & $-0.95(0.29)$ & $\longrightarrow$ & $10.1 \%$ & $0.39[0.22,0.68]$ \\
\hline Subtotal $(95 \% \mathrm{CI})$ & 76 & 75 & & - & $10.1 \%$ & $0.39[0.22,0.68]$ \\
\hline \multicolumn{7}{|c|}{ Heterogeneity: not applicable } \\
\hline \multicolumn{7}{|c|}{ Test for overall effect: $Z=3.28(P=0.001 \mathrm{I})$} \\
\hline \multicolumn{7}{|l|}{6 Exercise and home safety } \\
\hline Waterman 2016 & 15 & 13 & $0.18(0.36)$ & 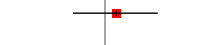 & $7.3 \%$ & $1.20[0.59,2.42]$ \\
\hline Subtotal $(95 \% \mathrm{CI})$ & 15 & 13 & & & $7.3 \%$ & $1.20[0.59,2.42]$ \\
\hline \multicolumn{7}{|c|}{ Heterogeneity: not applicable } \\
\hline \multicolumn{7}{|c|}{ Test for overall effect: $Z=0.50(P=0.62)$} \\
\hline Total $(95 \% \mathrm{CI})$ & 593 & 492 & & $\bullet$ & $100.0 \%$ & $0.74[0.60,0.91]$ \\
\hline \multicolumn{7}{|c|}{ Heterogeneity: $\mathrm{Tau}^{2}=0.03 ; \mathrm{Ch}^{2}=10.88, \mathrm{df}=6(\mathrm{P}=0.09) ; \mathrm{I}^{2}=45 \%$} \\
\hline \multicolumn{7}{|c|}{ Test for overall effect: $Z=2.78(P=0.0055)$} \\
\hline \multicolumn{7}{|c|}{ Test for subgroup differences: $\mathrm{Chi}^{2}=9.61, \mathrm{df}=5(\mathrm{P}=0.09), \mathrm{I}^{2}=48 \%$} \\
\hline
\end{tabular}




\section{Analysis 3.2. Comparison 3 Multiple intervention vs usual care or attention control, Outcome 2 Number of}

people sustaining one or more falls.

Review: Multifactorial and multiple component interventions for preventing falls in older people living in the community

Comparison: 3 Multiple intervention vs usual care or attention control

Outcome: 2 Number of people sustaining one or more falls

\begin{tabular}{|c|c|c|c|c|c|c|}
\hline \multirow[t]{2}{*}{ Study or subgroup } & Multiple & Control & log [Risk Ratio] & Risk Ratio & \multirow[t]{2}{*}{ Weight } & \multirow{2}{*}{$\begin{array}{r}\text { Risk Ratio } \\
\text { IV,Random,95\% Cl }\end{array}$} \\
\hline & N & N & (SE) & IV,Random,95\% Cl & & \\
\hline \multicolumn{7}{|c|}{ I Exercise, home safety and nutrition } \\
\hline Campbell 2005 & 97 & 48 & $-0.26(0.15)$ & $\rightarrow$ & $10.9 \%$ & $0.77[0.57,1.03]$ \\
\hline Subtotal (95\% CI) & 97 & 48 & & - & $10.9 \%$ & $0.77[0.57,1.03]$ \\
\hline \multicolumn{7}{|c|}{ Heterogeneity: not applicable } \\
\hline \multicolumn{7}{|c|}{ Test for overall effect: $Z=1.73(P=0.083)$} \\
\hline \multicolumn{7}{|l|}{2 Exercise and nutrition } \\
\hline Campbell 2005 & 98 & 48 & $-0.25(0.15)$ & $\rightarrow$ & $10.9 \%$ & $0.78[0.58,1.04]$ \\
\hline Subtotal (95\% CI) & 98 & 48 & & - & $10.9 \%$ & $0.78[0.58,1.04]$ \\
\hline \multicolumn{7}{|c|}{ Heterogeneity: not applicable } \\
\hline \multicolumn{7}{|c|}{ Test for overall effect: $Z=1.67(P=0.096)$} \\
\hline \multicolumn{7}{|c|}{3 Exercise, home safety and vision } \\
\hline Clemson 2004 & 157 & 153 & $-0.11(0.1)$ & + & $24.5 \%$ & $0.90[0.74,1.09]$ \\
\hline Day 2002 & 135 & 34 & $-0.3(0.15)$ & ¥ & $10.9 \%$ & $0.74[0.55,0.99]$ \\
\hline Subtotal (95\% CI) & 292 & 187 & & $\bullet$ & $35.5 \%$ & $0.84[0.71,1.00]$ \\
\hline \multicolumn{7}{|c|}{ Heterogeneity: Tau $^{2}=0.00 ; \mathrm{Chi}^{2}=1.1 \mathrm{l}, \mathrm{df}=1(P=0.29) ;\left.\right|^{2}=10 \%$} \\
\hline \multicolumn{7}{|c|}{ Test for overall effect: $Z=1.93(P=0.053)$} \\
\hline \multicolumn{7}{|l|}{4 Exercise and vision } \\
\hline Day 2002 & 136 & 34 & $-0.29(0.15)$ & $\rightarrow$ & $10.9 \%$ & $0.75[0.56,1.00]$ \\
\hline Subtotal $(95 \% \mathrm{CI})$ & 136 & 34 & & - & $10.9 \%$ & $0.75[0.56,1.00]$ \\
\hline \multicolumn{7}{|c|}{ Heterogeneity: not applicable } \\
\hline \multicolumn{7}{|c|}{ Test for overall effect: $Z=1.93(P=0.053)$} \\
\hline \multicolumn{7}{|l|}{5 Exercise and home safety } \\
\hline Day 2002 & 135 & 34 & $-0.19(0.15)$ & $\rightarrow$ & $10.9 \%$ & $0.83[0.62,1.11]$ \\
\hline Waterman 2016 & 15 & 13 & $-0.03(0.3)$ & — & $2.7 \%$ & $0.97[0.54,1.75]$ \\
\hline Wesson 2013 & 11 & 11 & $-0.69(0.75)$ & & $0.4 \%$ & $0.50[0.12,2.18]$ \\
\hline Subtotal (95\% CI) & 161 & 58 & & - & $14.1 \%$ & $0.84[0.65,1.09]$ \\
\hline \multicolumn{7}{|c|}{ Heterogeneity: $\mathrm{Tau}^{2}=0.0 ; \mathrm{Chi}^{2}=0.72, \mathrm{df}=2(\mathrm{P}=0.70) ; \mathrm{I}^{2}=0.0 \%$} \\
\hline \multicolumn{7}{|c|}{ Test for overall effect: $Z=1.32(P=0.19)$} \\
\hline \multicolumn{7}{|l|}{6 Home safety and vision } \\
\hline Day 2002 & 137 & 34 & $-0.13(0.15)$ & $\rightarrow$ & $10.9 \%$ & $0.88[0.65,1.18]$ \\
\hline Subtotal $(95 \% \mathrm{CI})$ & 137 & 34 & & - & $10.9 \%$ & $0.88[0.65,1.18]$ \\
\hline
\end{tabular}

(Continued...) 


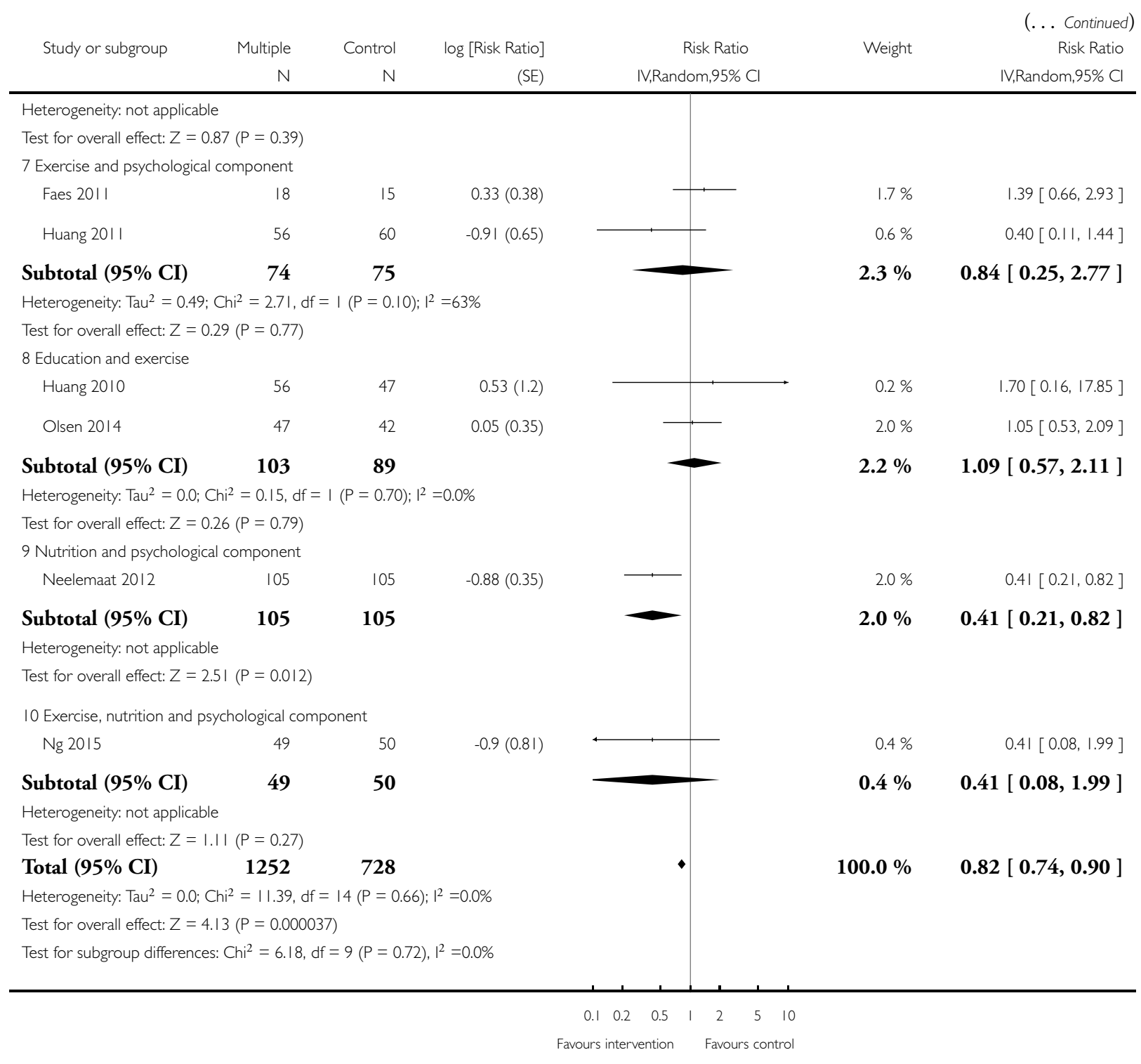


Analysis 3.3. Comparison 3 Multiple intervention vs usual care or attention control, Outcome 3 Number of people sustaining recurrent falls (defined as two or more falls in a specified time period).

Review: Multifactorial and multiple component interventions for preventing falls in older people living in the community

Comparison: 3 Multiple intervention vs usual care or attention control

Outcome: 3 Number of people sustaining recurrent falls (defined as two or more falls in a specified time period)

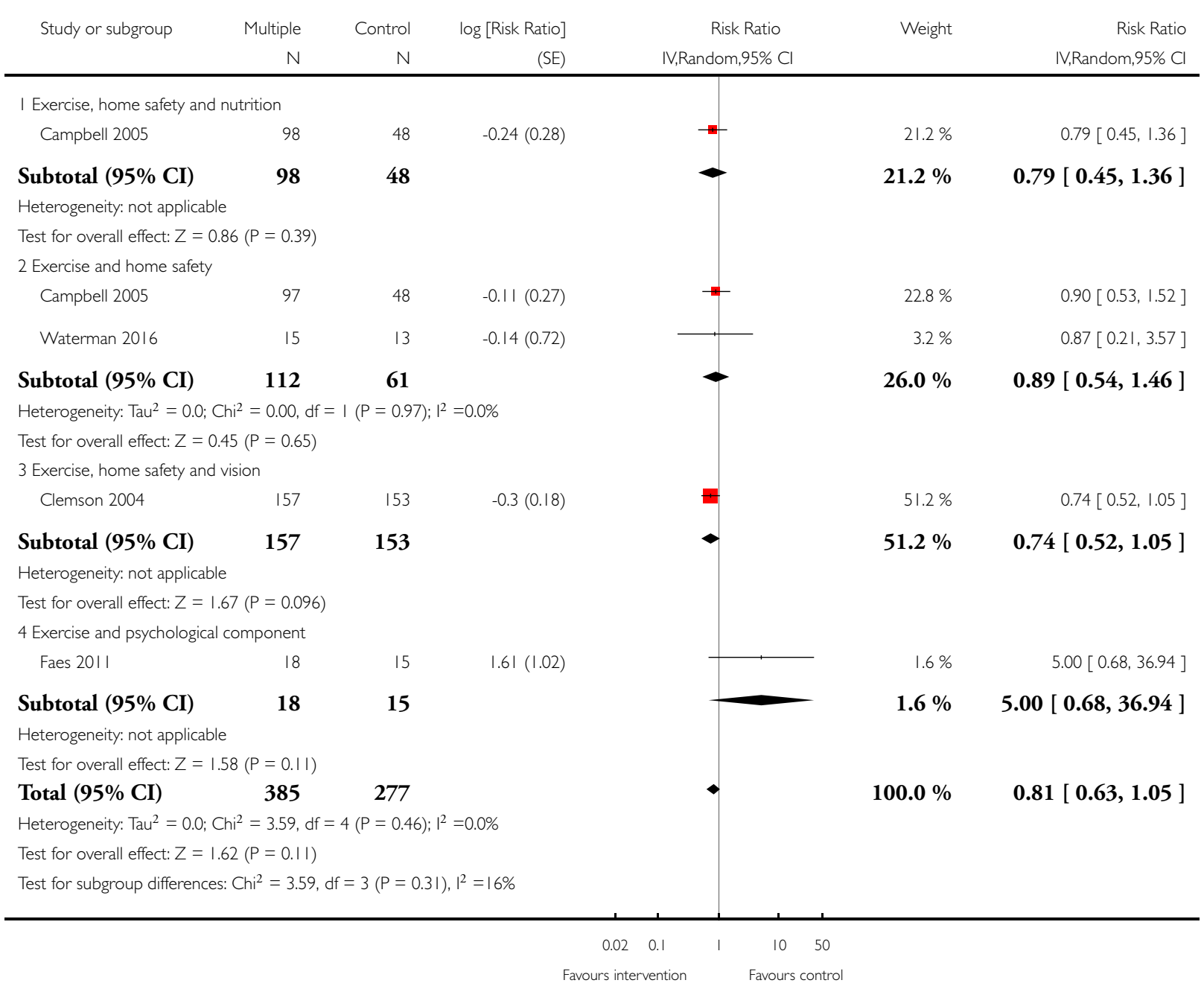


Analysis 3.4. Comparison 3 Multiple intervention vs usual care or attention control, Outcome 4 Number of people sustaining one or more fall-related fractures.

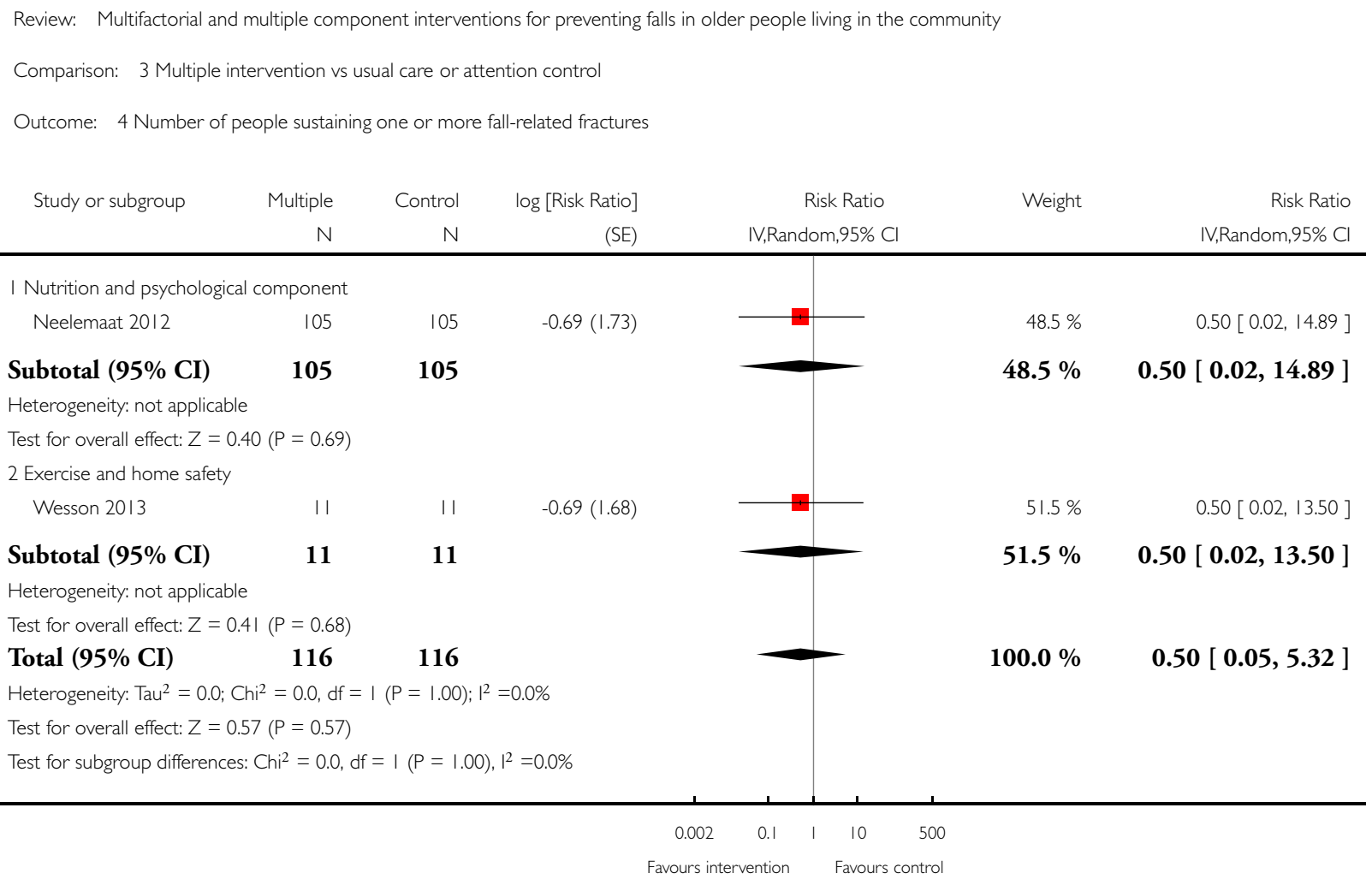


Analysis 3.5. Comparison 3 Multiple intervention vs usual care or attention control, Outcome 5 Number of people who experience a fall that required hospital admission.

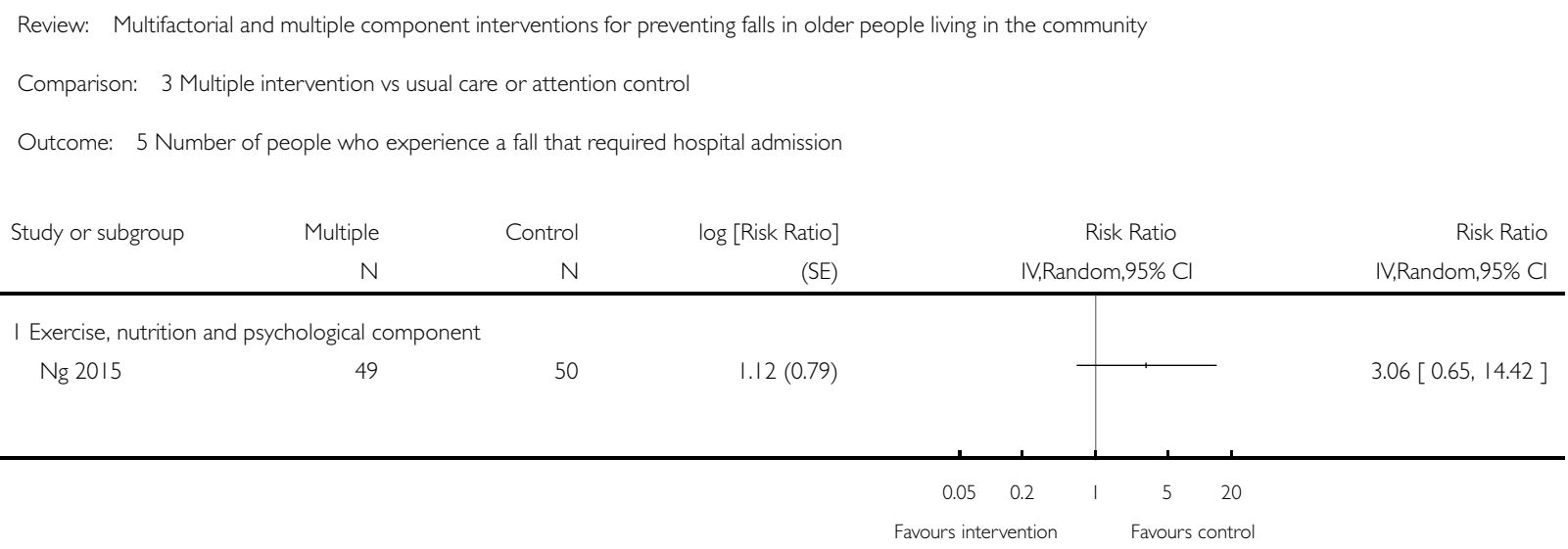

Analysis 3.6. Comparison 3 Multiple intervention vs usual care or attention control, Outcome 6 Number of people who experience a fall that required medical attention.

\begin{tabular}{|c|c|c|c|c|c|c|}
\hline \multicolumn{7}{|c|}{ Comparison: 3 Multiple intervention vs usual care or attention control } \\
\hline \multicolumn{7}{|c|}{ Outcome: 6 Number of people who experience a fall that required medical attention } \\
\hline \multirow[t]{2}{*}{ Study or subgroup } & Multiple & Control & log [Risk Ratio] & Risk Ratio & Weight & Risk Ratio \\
\hline & N & N & (SE) & \multicolumn{2}{|l|}{ IV,Random,95\% Cl } & IV,Random,95\% Cl \\
\hline \multicolumn{7}{|c|}{ I Exercise, home safety and nutrition } \\
\hline Campbell 2005 & 98 & 48 & $-0.09(0.25)$ & $\leftarrow$ & $50.0 \%$ & $0.91[0.56,1.49]$ \\
\hline Subtotal $(95 \% \mathrm{CI})$ & 98 & 48 & & & $50.0 \%$ & $0.91[0.56,1.49]$ \\
\hline \multicolumn{7}{|c|}{ Heterogeneity: not applicable } \\
\hline \multicolumn{7}{|c|}{ Test for overall effect: $Z=0.36(P=0.72)$} \\
\hline \multicolumn{7}{|l|}{2 Exercise and nutrition } \\
\hline Campbell 2005 & 97 & 48 & $-0.01(0.25)$ & & $50.0 \%$ & $0.99[0.61,1.62]$ \\
\hline Subtotal (95\% CI) & 97 & 48 & & - & $50.0 \%$ & $0.99[0.61,1.62]$ \\
\hline \multicolumn{7}{|c|}{ Heterogeneity: not applicable } \\
\hline \multicolumn{7}{|c|}{ Test for overall effect: $Z=0.04(P=0.97)$} \\
\hline Total $(95 \% \mathrm{CI})$ & 195 & 96 & & $<$ & $100.0 \%$ & $0.95[0.67,1.35]$ \\
\hline
\end{tabular}

(Continued...) 


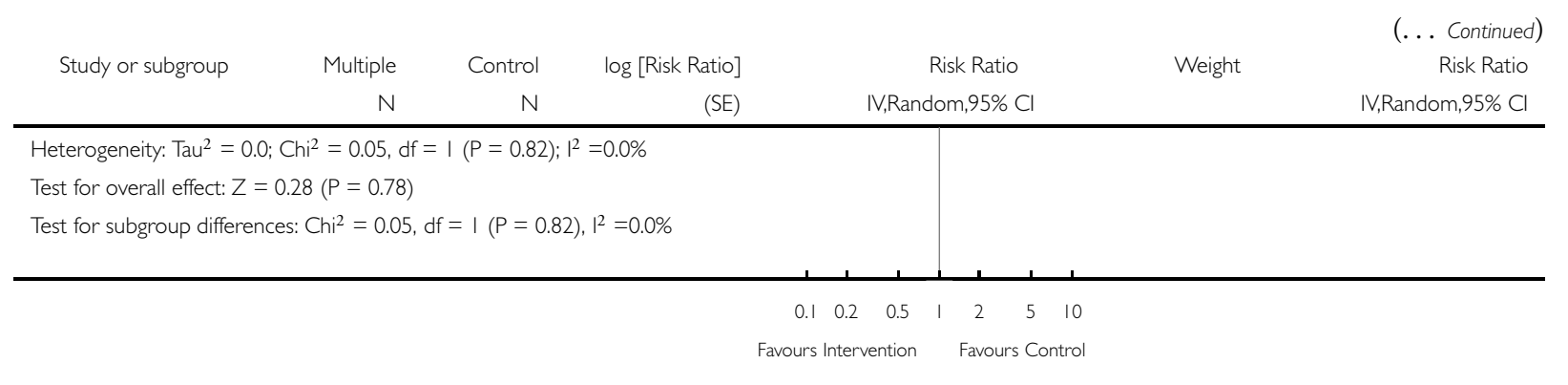

\section{Analysis 3.7. Comparison 3 Multiple intervention vs usual care or attention control, Outcome 7 Health- related quality of life: endpoint score.}

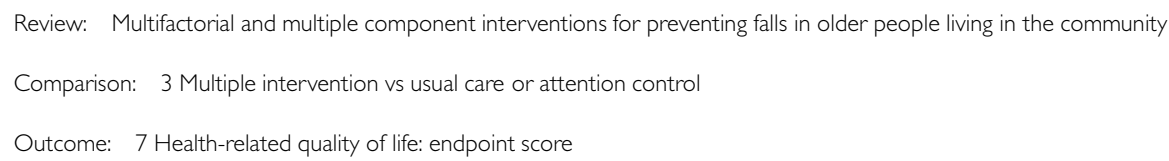

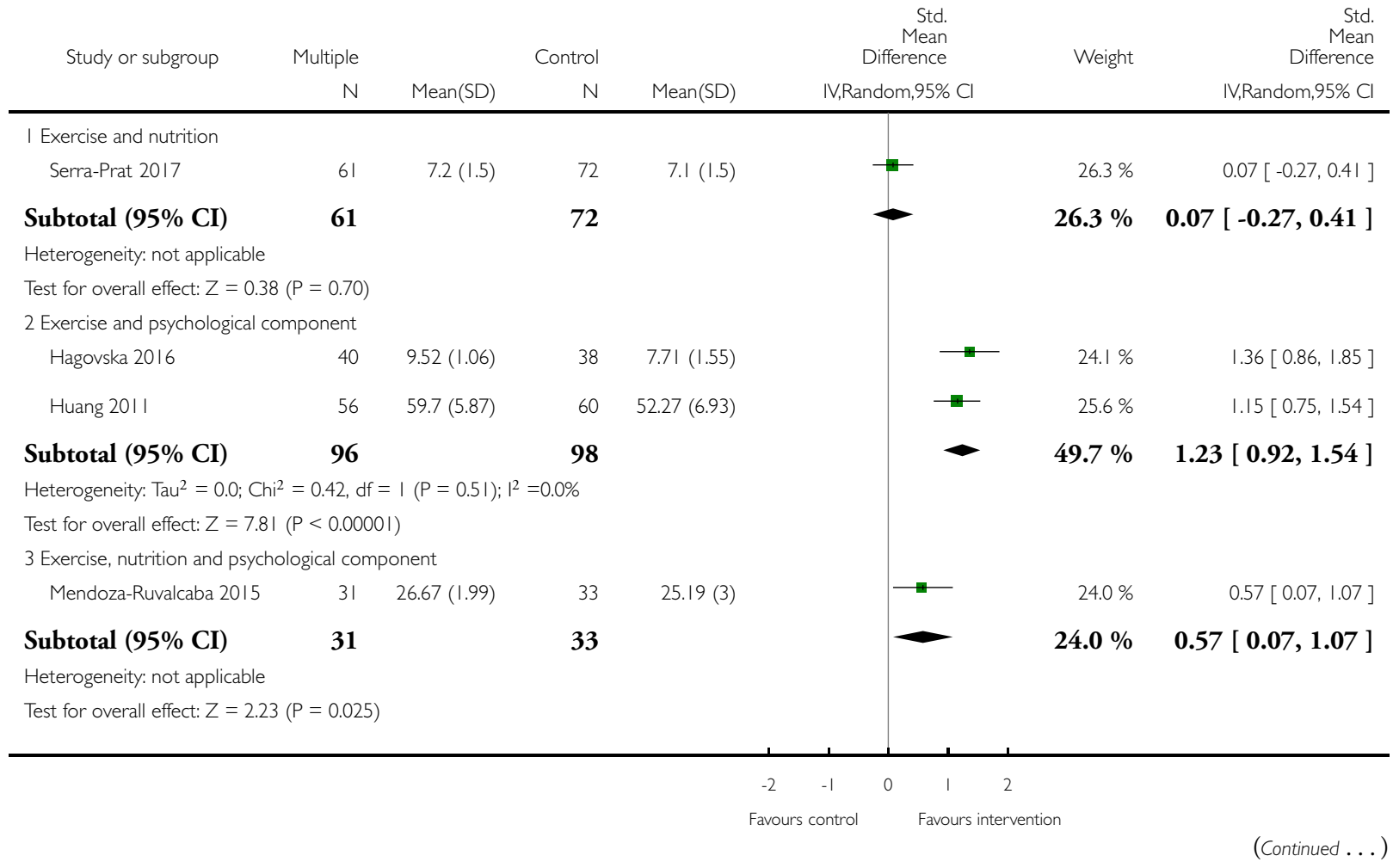




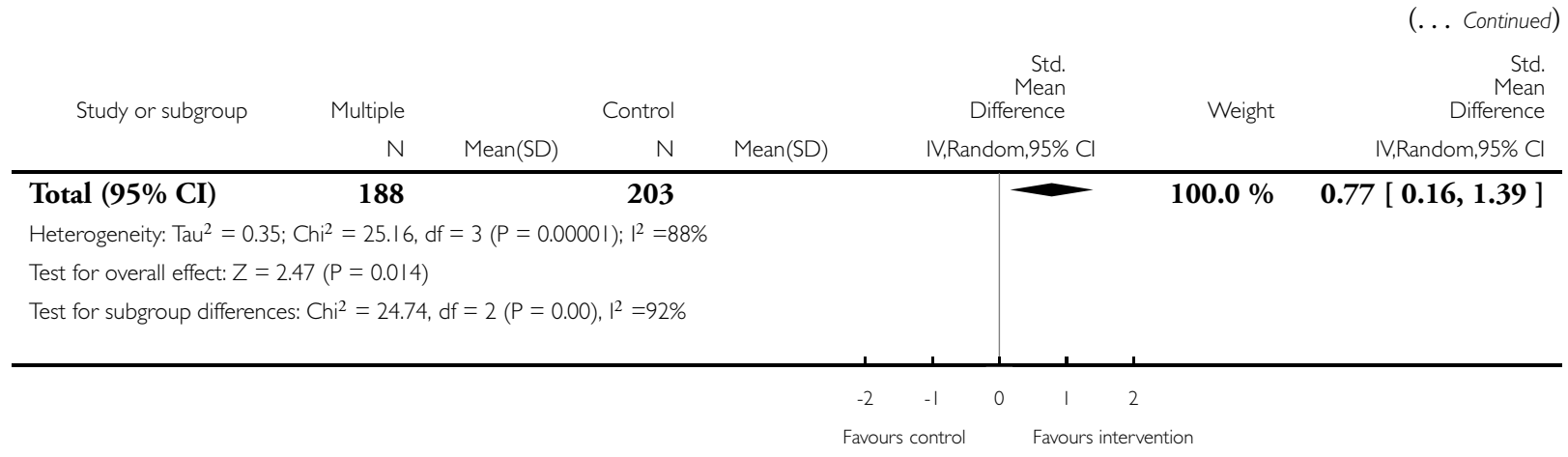

Analysis 3.8. Comparison 3 Multiple intervention vs usual care or attention control, Outcome 8 Healthrelated quality of life (mental): endpoint score.

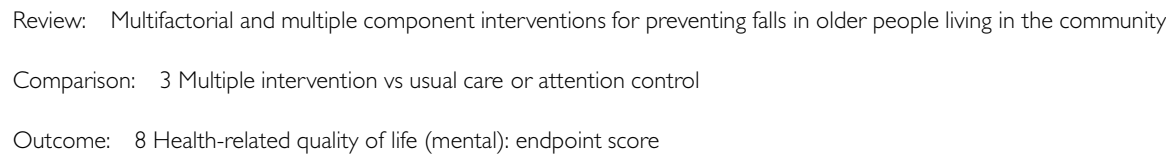

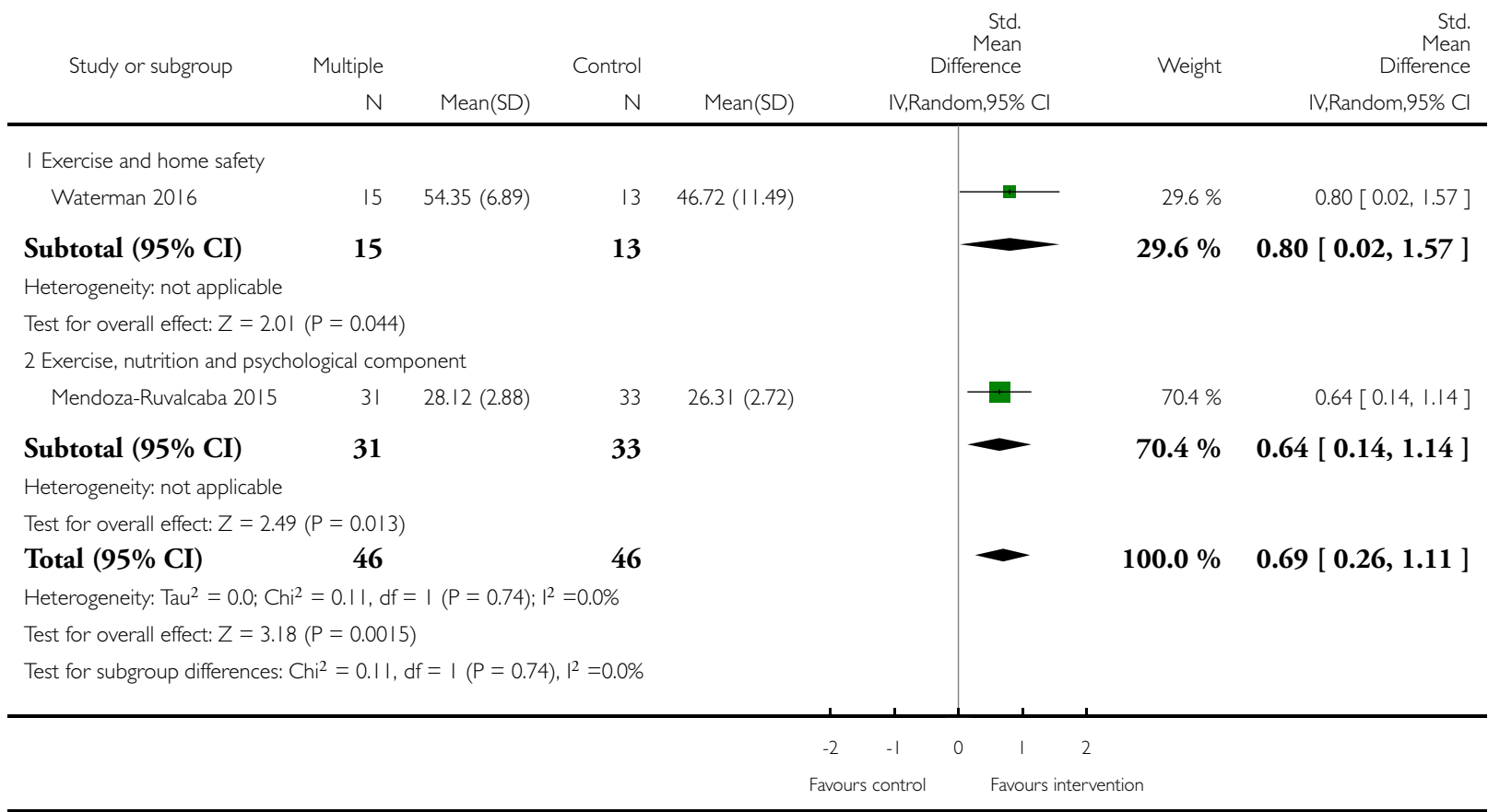


Analysis 3.9. Comparison 3 Multiple intervention vs usual care or attention control, Outcome 9 Healthrelated quality of life (physical): endpoint score.

Review: Multifactorial and multiple component interventions for preventing falls in older people living in the community

Comparison: 3 Multiple intervention vs usual care or attention control

Outcome: 9 Health-related quality of life (physical): endpoint score

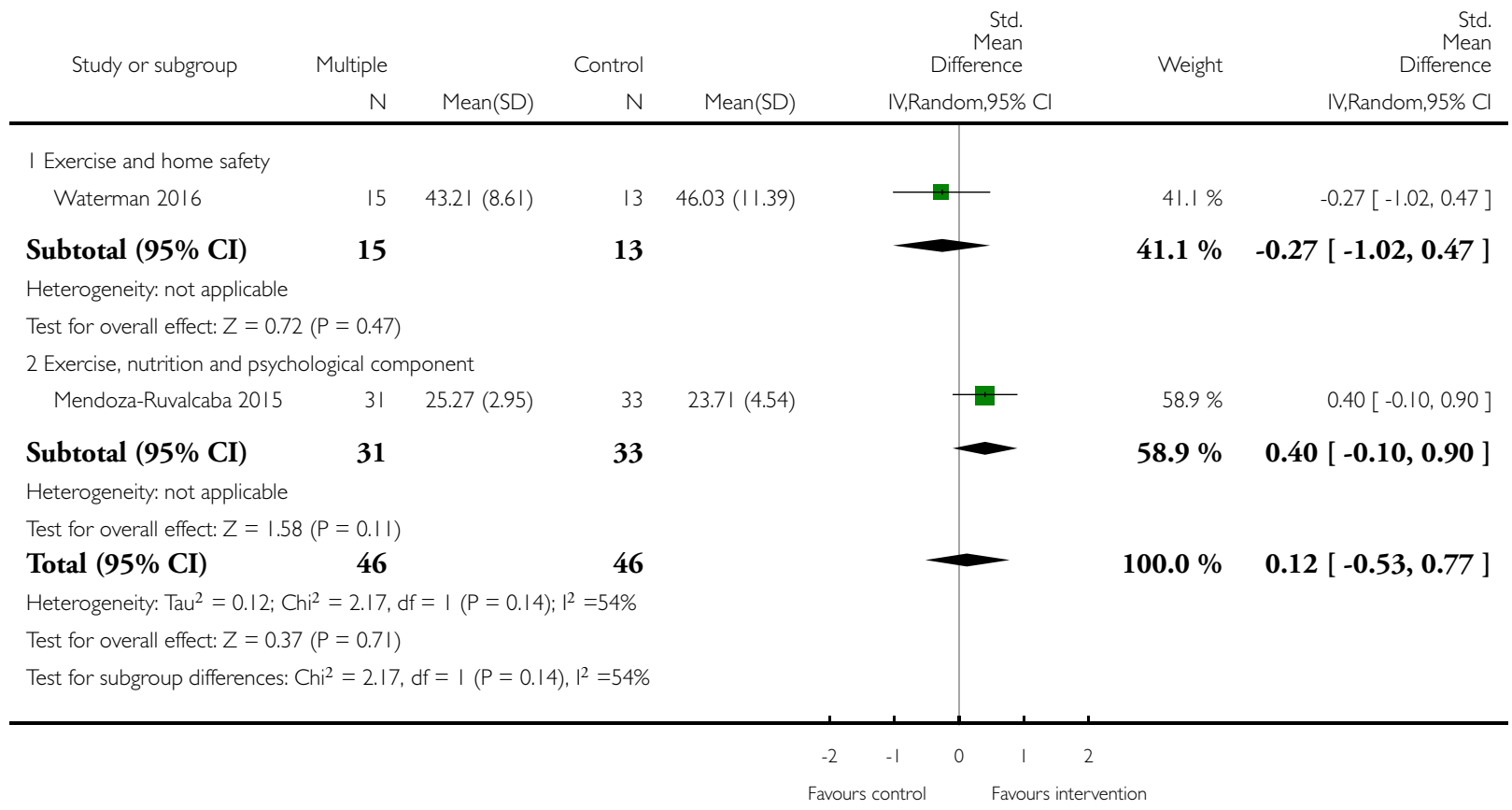


Analysis 4.I. Comparison 4 Multiple intervention vs exercise, Outcome I Rate of falls (falls per person years).

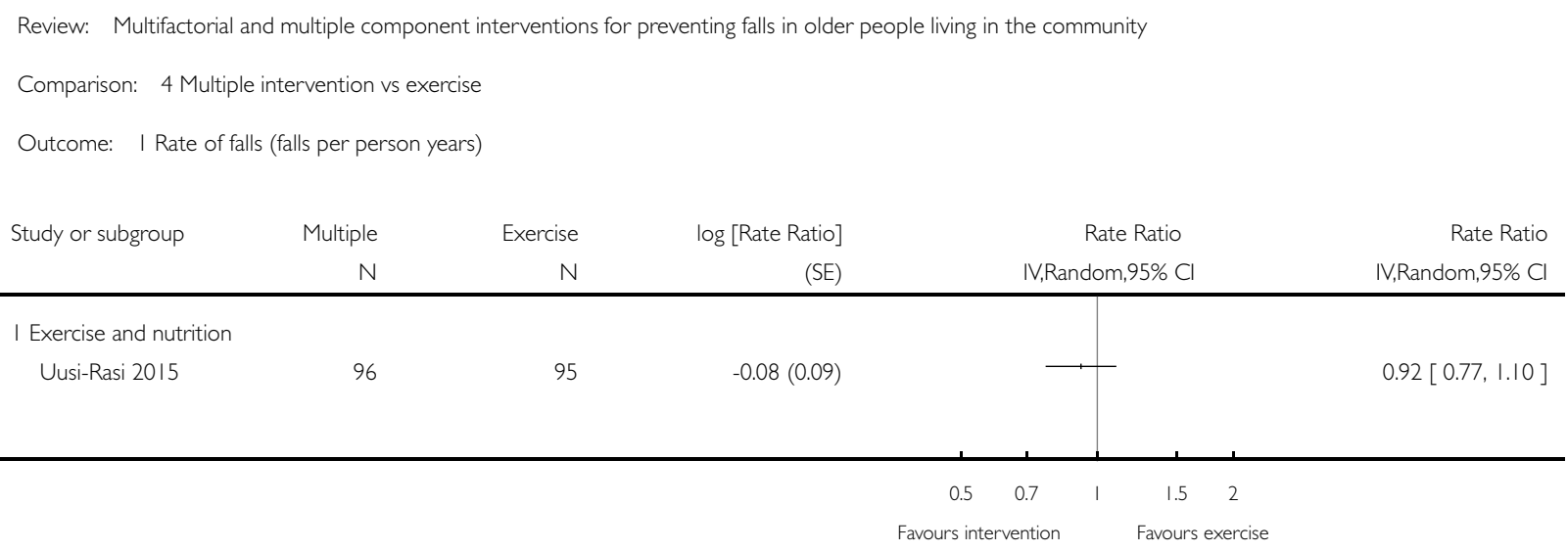

Analysis 4.2. Comparison 4 Multiple intervention vs exercise, Outcome 2 Number of people sustaining one or more falls.

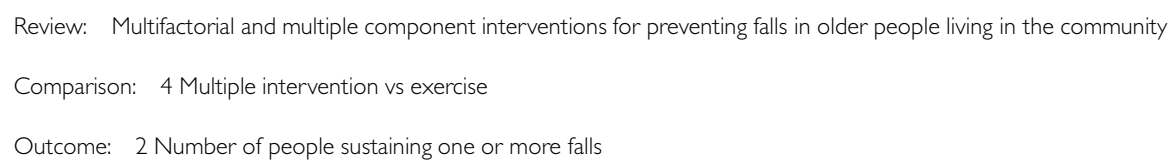

$\begin{array}{llll}\mathrm{N} & \mathrm{N} & (\mathrm{SE}) & \mathrm{IV}, \mathrm{Random}, 95 \% \mathrm{Cl}\end{array}$

I Education and exercise

$\begin{array}{llll}\text { Huang } 2010 & 56 & 31 & 0.8(1.55)\end{array}$

Subtotal (95\% CI)

$56 \quad 31$

31

Heterogeneity: not applicable

Test for overall effect: $Z=0.52(P=0.61)$

2 Education, nutrition and psychological component

$\begin{array}{llll}\mathrm{Ng} 2015 & 49 & 48 & -0.43(0.89)\end{array}$

Subtotal (95\% CI)

$49 \quad 48$

Heterogeneity: not applicable

Test for overall effect: $Z=0.48(P=0.63)$

3 Exercise and vision

\begin{tabular}{l|l} 
& \\
\hline & \\
\hline
\end{tabular}

$0.3 \%$

$2.23[0.11,46.43]$

$0.3 \%$

$2.23[0.11,46.43$ ]
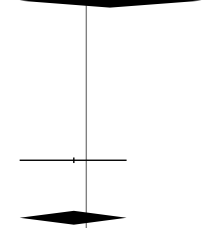

$1.0 \%$

$0.65[0.11,3.72]$

$1.0 \%$

$0.65[0.11,3.72]$ 


\begin{tabular}{|c|c|c|c|c|c|c|}
\hline \multirow[t]{2}{*}{ Study or subgroup } & Multiple & Exercise & log [Risk Ratio] & Risk Ratio & \multicolumn{2}{|r|}{$\begin{array}{c}\text { (... Continued) } \\
\text { Risk Ratio }\end{array}$} \\
\hline & N & $\mathrm{N}$ & (SE) & \multicolumn{2}{|l|}{ IV,Random,95\% Cl } & IV,Random, $95 \% \mathrm{Cl}$ \\
\hline Day 2002 & 136 & 34 & $-0.14(0.18)$ & + & $23.3 \%$ & $0.87[0.61,1.24]$ \\
\hline Subtotal $(95 \% \mathrm{CI})$ & 136 & 34 & & $\bullet$ & $23.3 \%$ & $0.87[0.61,1.24]$ \\
\hline \multicolumn{7}{|c|}{ Heterogeneity: not applicable } \\
\hline \multicolumn{7}{|c|}{ Test for overall effect: $Z=0.78(P=0.44)$} \\
\hline \multicolumn{7}{|l|}{4 Exercise and home safety } \\
\hline Day 2002 & 135 & 34 & $-0.05(0.17)$ & $\#$ & $26.1 \%$ & $0.95[0.68,1.33]$ \\
\hline Subtotal $(95 \% \mathrm{CI})$ & 135 & 34 & & $\bullet$ & $26.1 \%$ & $0.95[0.68,1.33]$ \\
\hline \multicolumn{7}{|c|}{ Heterogeneity: not applicable } \\
\hline \multicolumn{7}{|c|}{ Test for overall effect: $Z=0.29(P=0.77)$} \\
\hline \multicolumn{7}{|l|}{5 Home safety and vision } \\
\hline Day 2002 & 137 & 34 & $0.02(0.17)$ & \# & $26.1 \%$ & $1.02[0.73,1.42]$ \\
\hline Subtotal $(95 \% \mathrm{CI})$ & 137 & 34 & & $\longrightarrow$ & $26.1 \%$ & $1.02[0.73,1.42]$ \\
\hline \multicolumn{7}{|c|}{ Heterogeneity: not applicable } \\
\hline \multicolumn{7}{|c|}{ Test for overall effect: $Z=0.12(P=0.91)$} \\
\hline \multicolumn{7}{|c|}{6 Exercise, home safety and vision } \\
\hline Day 2002 & 135 & 34 & $-0.15(0.18)$ & \# & $23.3 \%$ & $0.86[0.60,1.22]$ \\
\hline Subtotal $(95 \% \mathrm{CI})$ & 135 & 34 & & $\bullet$ & $23.3 \%$ & $0.86[0.60,1.22]$ \\
\hline \multicolumn{7}{|c|}{ Heterogeneity: not applicable } \\
\hline \multicolumn{7}{|c|}{ Test for overall effect: $Z=0.83(P=0.40)$} \\
\hline Total $(95 \% \mathrm{CI})$ & 648 & 215 & & $\bullet$ & $100.0 \%$ & $0.93[0.78,1.10]$ \\
\hline \multicolumn{7}{|c|}{ Heterogeneity: $\mathrm{Tau}^{2}=0.0 ; \mathrm{Chi}^{2}=1.12, \mathrm{df}=5(\mathrm{P}=0.95) ; 1^{2}=0.0 \%$} \\
\hline \multicolumn{7}{|c|}{ Test for overall effect: $Z=0.89(P=0.38)$} \\
\hline \multicolumn{7}{|c|}{ Test for subgroup differences: $C h i^{2}=1.12, \mathrm{df}=5(P=0.95), 1^{2}=0.0 \%$} \\
\hline & & & & 10 & 100 & \\
\hline
\end{tabular}




\section{Analysis 4.3. Comparison 4 Multiple intervention vs exercise, Outcome 3 Number of people who} experience a fall that required hospital admission.

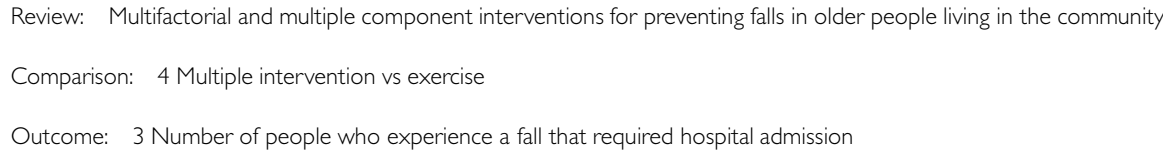

V.Random, $95 \%$ cl

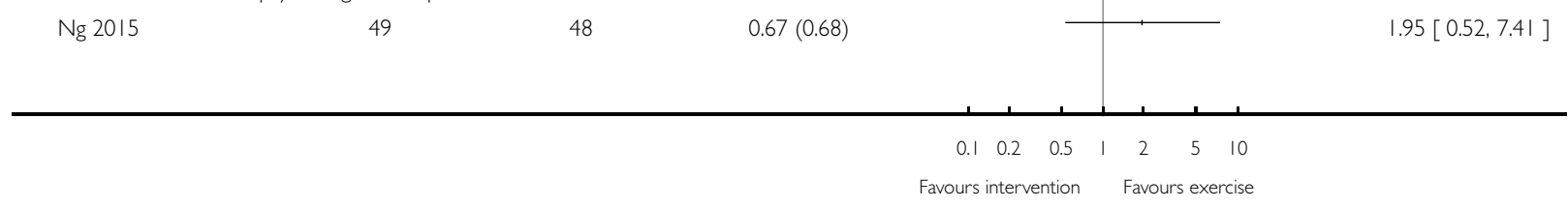

Analysis 5.I. Comparison 5 Multifactorial intervention vs control: subgroup analysis by intensity of intervention, Outcome I Rate of falls (falls per person years).

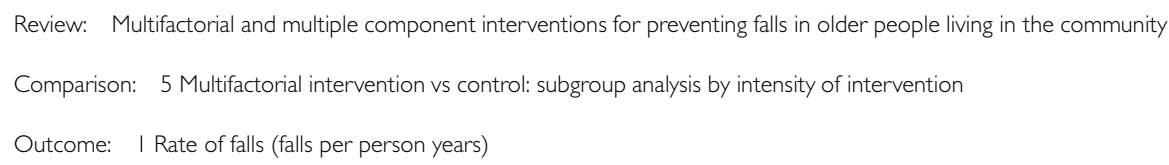
IV,Random,95\% Cl IV,Random,95\% Cl

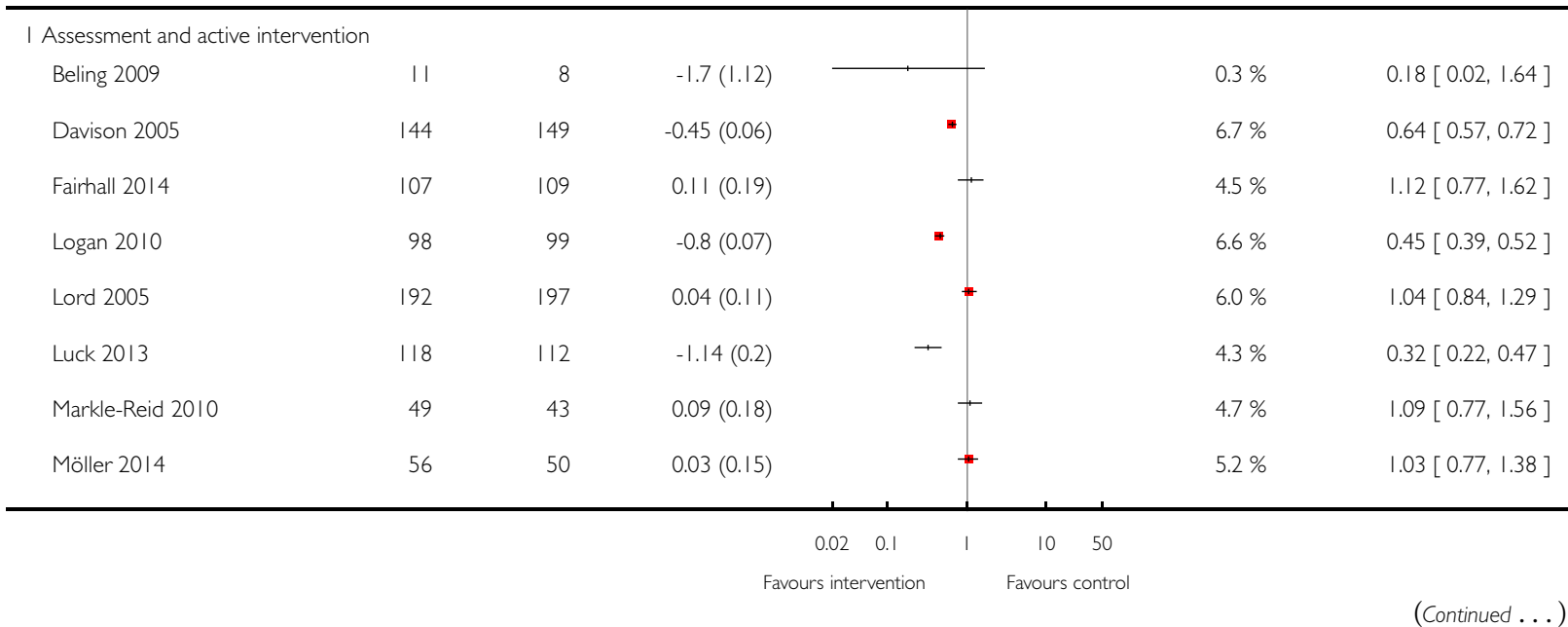




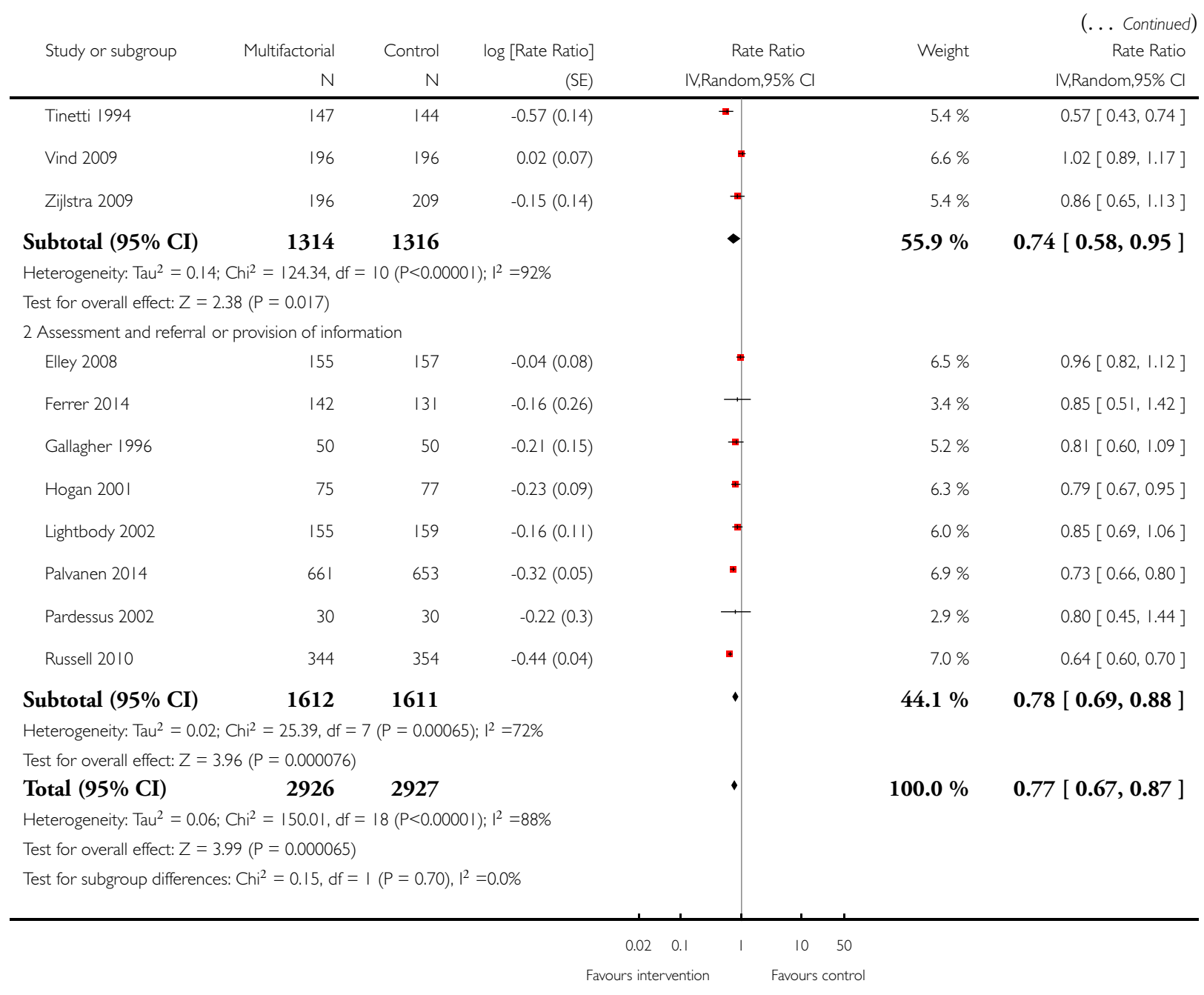


Analysis 5.2. Comparison 5 Multifactorial intervention vs control: subgroup analysis by intensity of intervention, Outcome 2 Number of people sustaining one or more falls.

Review: Multifactorial and multiple component interventions for preventing falls in older people living in the community

Comparison: 5 Multifactorial intervention vs control: subgroup analysis by intensity of intervention

Outcome: 2 Number of people sustaining one or more falls

Study or subgroup Multifactorial Control log [Risk Ratio] Risk Ratio Risk Ratio

$\mathrm{N} \quad \mathrm{N} \quad$ (SE) IV,Random,95\% Cl

V,Random,95\% Cl

I Assessment and active intervention

Close 1999

184

213

$-0.49(0.13)$

Coleman 1999

79

63

$0.14(0.23)$

Davison 2005

144

149

-0.05 (0.08)

De Vries 2010

106

| | |

$-0.07(0.13)$

Fairhall 2014

119

119

$0.07(0.11)$

Huang 2005

63

63

$-0.34(0.56)$

Logan 2010

$$
102
$$

102

$-0.17(0.06)$

Lord 2005

202

201

$0.03(0.11)$

Möller 2014

$$
80
$$

73

$0.11(0.16)$

Spice 2009

$$
164
$$

80

$-0.11(0.07)$

Spice 2009

106

80

$0.04(0.06)$

Tinetti 1994

Vind 2009

$$
147
$$

144

$-0.3(0.15)$

Zijlstra 2009

196

196

$0.09(0.09)$

$188 \quad 203$

$-0.17(0.1)$

$1880 \quad 1797$

Subtotal (95\% CI)

1880

1797

Heterogeneity: Tau $^{2}=0.01 ; \mathrm{Chi}^{2}=28.25, \mathrm{df}=\mathrm{I} 3(\mathrm{P}=0.0 \mathrm{I}) ; \mathrm{I}^{2}=54 \%$

Test for overall effect: $Z=1.74(P=0.082)$

2 Assessment and referral or provision of information

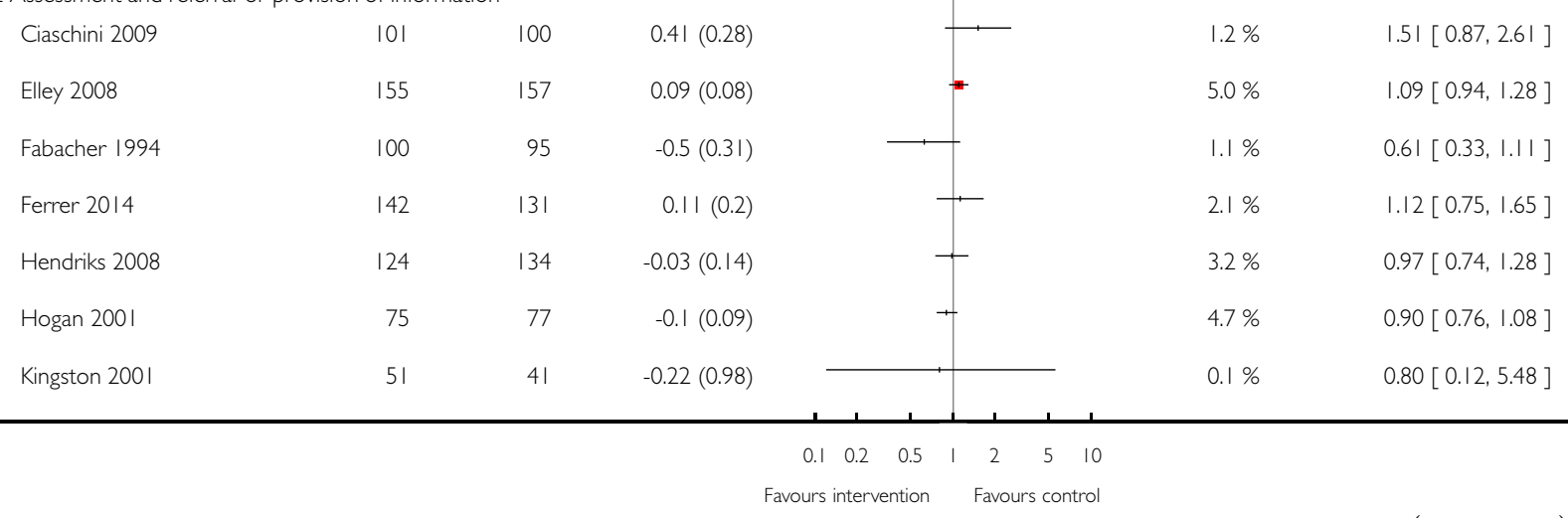

(Continued....) 


\begin{tabular}{|c|c|c|c|c|c|c|}
\hline Study or subgroup & Multifactorial & Control & log [Risk Ratio] & Risk Ratio & Weight & $\begin{array}{r}\text { (... Continued) } \\
\text { Risk Ratio }\end{array}$ \\
\hline Lightbody 2002 & 155 & 159 & $-0.02(0.19)$ & $\leftarrow$ & $2.2 \%$ & $0.98[0.68,1.42]$ \\
\hline Newbury 200I & 45 & 44 & $-0.37(0.31)$ & + & $1.1 \%$ & $0.69[0.38,1.27]$ \\
\hline Palvanen 2014 & 661 & 653 & $-0.18(0.06)$ & $=$ & $5.7 \%$ & $0.84[0.74,0.94]$ \\
\hline Pardessus 2002 & 30 & 30 & $-0.14(0.28)$ & $\longrightarrow$ & $1.2 \%$ & $0.87[0.50,1.50]$ \\
\hline Russell 2010 & 320 & 330 & $0.11(0.08)$ & 世 & $5.0 \%$ & $1.12[0.95,1.31]$ \\
\hline Van Haastregt 2000 & 120 & 115 & $0.12(0.12)$ & + & $3.8 \%$ & $1.13[0.89,1.43]$ \\
\hline Vetter 1992 & 240 & 210 & $0.25(0.13)$ & + & $3.5 \%$ & $1.28[1.00,1.66]$ \\
\hline Wagner 1994 (I) & 635 & 607 & $-0.29(0.08)$ & \# & $5.0 \%$ & $0.75[0.64,0.88]$ \\
\hline Whitehead 2003 & 58 & 65 & $0.74(0.26)$ & $\longrightarrow$ & $1.4 \%$ & $2.10[1.26,3.49]$ \\
\hline Subtotal $(95 \%$ CI $)$ & 3012 & 2948 & & $\bullet$ & $46.3 \%$ & $1.00[0.89,1.13]$ \\
\hline \multicolumn{7}{|c|}{ Heterogeneity: $\mathrm{Tau}^{2}=0.03 ; \mathrm{Chi}^{2}=44.22, \mathrm{df}=\mathrm{I} 5(\mathrm{P}=0.000 \mathrm{I} 0) ; \mathbf{1}^{2}=66 \%$} \\
\hline \multicolumn{7}{|c|}{ Test for overall effect: $Z=0.07(P=0.95)$} \\
\hline Total $(95 \% \mathrm{CI})$ & 4892 & 4745 & & - & $100.0 \%$ & $0.96[0.90,1.03]$ \\
\hline \multicolumn{7}{|c|}{ Heterogeneity: $\operatorname{Tau}^{2}=0.02 ; \mathrm{Chi}^{2}=72.98, \mathrm{df}=29(\mathrm{P}=0.0000 \mathrm{I}) ; \mathrm{I}^{2}=60 \%$} \\
\hline \multicolumn{7}{|c|}{ Test for overall effect: $Z=1.16(P=0.24)$} \\
\hline \multicolumn{7}{|c|}{ Test for subgroup differences: $\mathrm{Ch}^{2}=1.10, \mathrm{df}=1(P=0.29), \mathrm{I}^{2}=9 \%$} \\
\hline
\end{tabular}

(I) Multifactorial arm vs control 
Analysis 5.3. Comparison 5 Multifactorial intervention vs control: subgroup analysis by intensity of intervention, Outcome 3 Number of people sustaining recurrent falls (defined as two or more falls in a specified time period).

Review: Multifactorial and multiple component interventions for preventing falls in older people living in the community

Comparison: 5 Multifactorial intervention vs control: subgroup analysis by intensity of intervention

Outcome: 3 Number of people sustaining recurrent falls (defined as two or more falls in a specified time period)

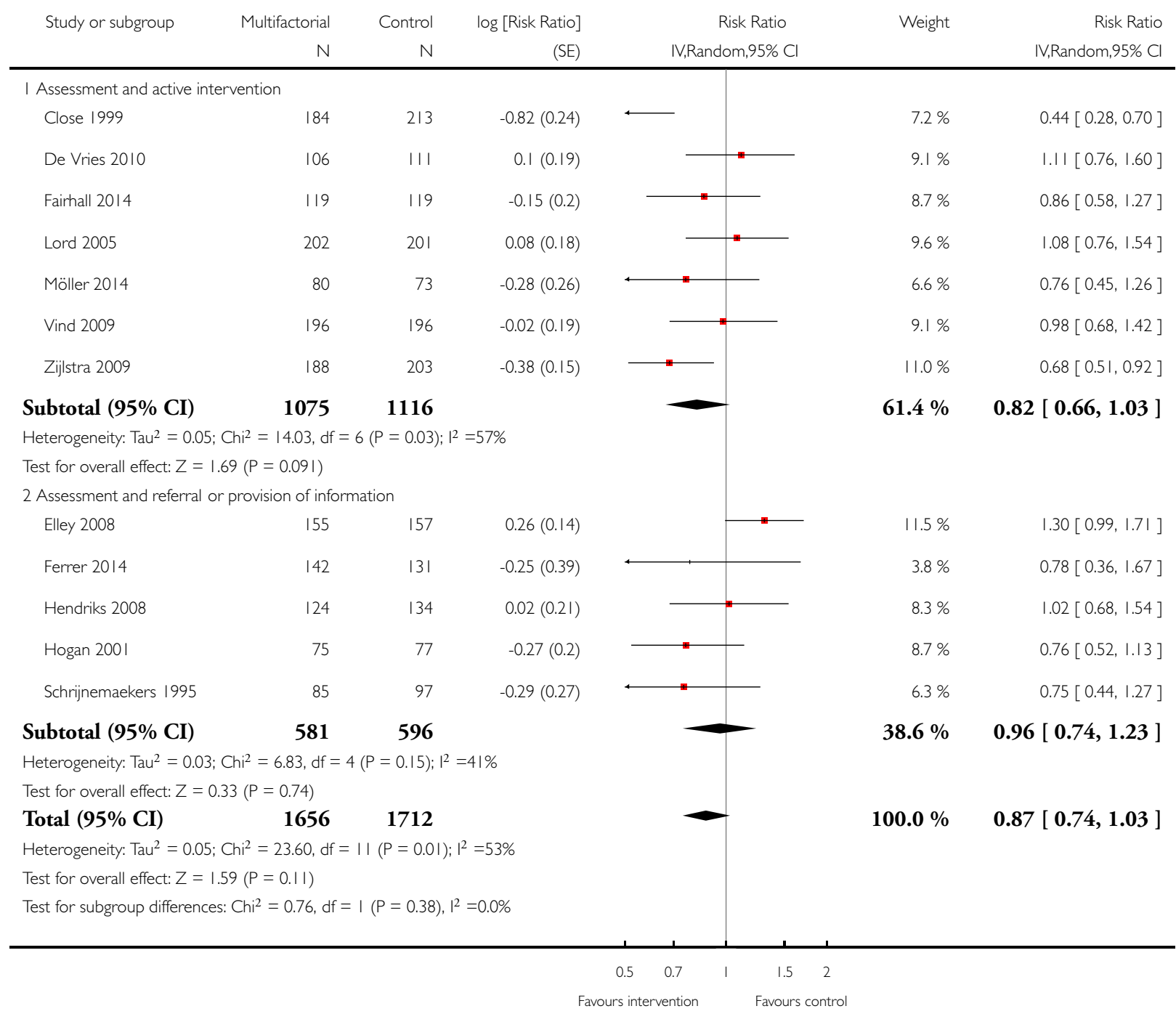


Analysis 6.I. Comparison 6 Multifactorial intervention vs control: subgroup analysis by falls risk at baseline, Outcome I Rate of falls (falls per person years).

Review: Multifactorial and multiple component interventions for preventing falls in older people living in the community

Comparison: 6 Multifactorial intervention vs control: subgroup analysis by falls risk at baseline

Outcome: I Rate of falls (falls per person years)

Study or subgroup Multifactorial Control log [Rate Ratio] Rate Ratio Ratio Ratio

N

N

(SE)

IV,Random,95\% Cl

IV,Random,95\% C

I Selected for higher risk of falling

Beling 2009

$\begin{array}{lll}11 & 8 & -1.7(1.12)\end{array}$

Davison 2005

$144 \quad 149$

$-0.45(0.06)$

Elley 2008

155

157

$-0.04(0.08)$

Fairhall 2014

107

109

$0.11(0.19)$

Ferrer 2014

131

$-0.16(0.26)$

Gallagher 1996

50

$$
50
$$

$-0.21(0.15)$

Hogan 2001

75

77

$-0.23(0.09)$

Lightbody 2002

$$
155
$$

159

$-0.16(0.11)$

Logan 2010

Lord 2005

98

99

$-0.8(0.07)$

Markle-Reid 2010

192

197

$0.04(0.11)$

Palvanen 2014

49

43

$0.09(0.18)$

Pardessus 2002

$$
661
$$

653

$-0.32(0.05)$

Russell 2010

30

30

$-0.22(0.3)$

Tinetti 1994

$$
344
$$

354

$-0.44(0.04)$

Vind 2009

$147 \quad 144$

$-0.57(0.14)$

196

144

$0.02(0.07)$

Subtotal (95\% CI)

2556

2556

\begin{tabular}{|c|c|c|c|}
\hline Luck 2013 & 118 & 112 & $-1.14(0.2$ \\
\hline Möller 20|4 & 56 & 50 & $0.03(0.15$ \\
\hline Zijlstra 2009 & 196 & 209 & $-0.15(0.14$ \\
\hline Subtotal (95\% CI) & 370 & 371 & \\
\hline
\end{tabular}

Heterogeneity: Tau $^{2}=0.06 ; \mathrm{Chi}^{2}=126.21, \mathrm{df}=15(\mathrm{P}<0.0000 \mathrm{I}) ; \mathrm{I}^{2}=88 \%$

Test for overall effect: $Z=3.58(P=0.00034)$

2 Not selected for higher risk of falling

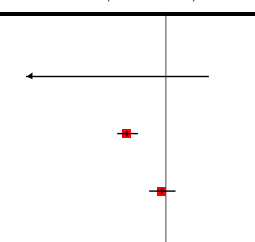

$0.3 \%$

$6.7 \%$

$6.5 \%$

$4.5 \%$

$3.4 \%$

$5.2 \%$

$6.3 \%$

$6.0 \%$

$6.6 \%$

$6.0 \%$

$4.7 \%$

$6.9 \%$

$2.9 \%$

$7.0 \%$

$5.4 \%$

$6.6 \%$

$85.0 \%$
$0.18[0.02,1.64]$

$0.64[0.57,0.72]$

$0.96[0.82,1.12]$

I.12 [0.77, I.62]

$0.85[0.51,1.42]$

$0.81[0.60,1.09]$

$0.79[0.67,0.95]$

$0.85[0.69,1.06]$

$0.45[0.39,0.52]$

$1.04[0.84,1.29]$

$1.09[0.77,1.56]$

$0.73[0.66,0.80]$

$0.80[0.45,1.44]$

$0.64[0.60,0.70]$

$0.57[0.43,0.74]$

$1.02[0.89,1.17]$

$0.78[0.68,0.89]$

$4.3 \%$

$0.32[0.22,0.47]$

$5.2 \%$

$1.03[0.77,1.38]$

$5.4 \%$

$0.86[0.65,1.13]$

$15.0 \%$

Test for overall effect: $Z=1.27(P=0.20)$

(Continued....)

Multifactorial and multiple component interventions for preventing falls in older people living in the community (Review) 


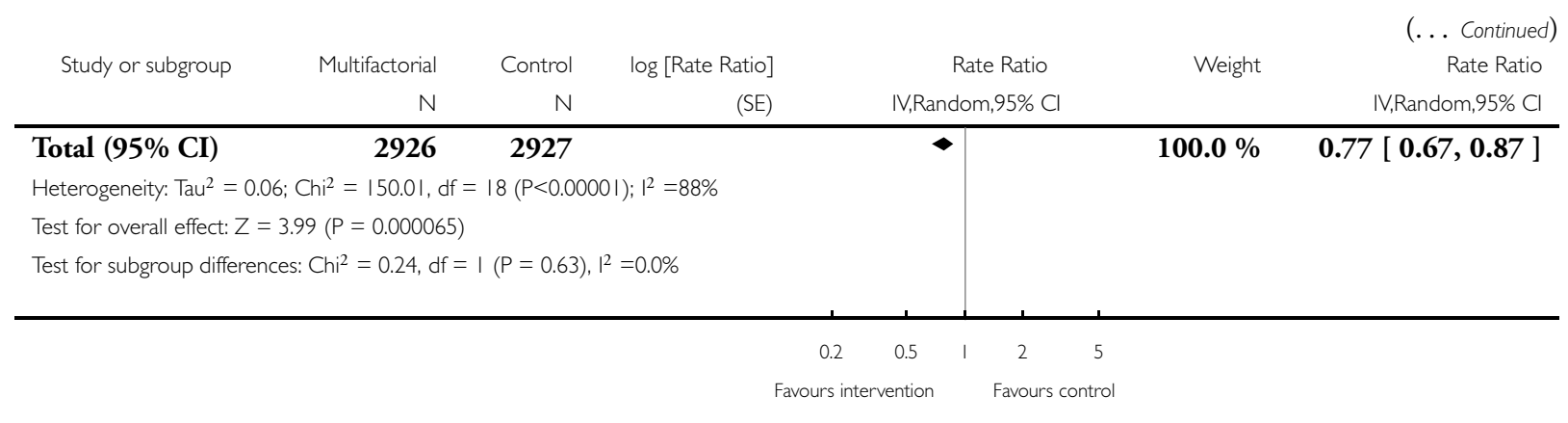

Analysis 6.2. Comparison 6 Multifactorial intervention vs control: subgroup analysis by falls risk at baseline, Outcome 2 Number of people sustaining one or more falls.

Review: Multifactorial and multiple component interventions for preventing falls in older people living in the community

Comparison: 6 Multifactorial intervention vs control: subgroup analysis by falls risk at baseline

Outcome: 2 Number of people sustaining one or more falls

$\begin{array}{lllll}\text { Study or subgroup } \quad \text { Multifactorial Control log [Risk Ratio] } & \text { Risk Ratio Ratio }\end{array}$

$\begin{array}{llll}\mathrm{N} & \mathrm{N} & \text { (SE) IV,Random,95\% Cl Random,95\% Cl }\end{array}$

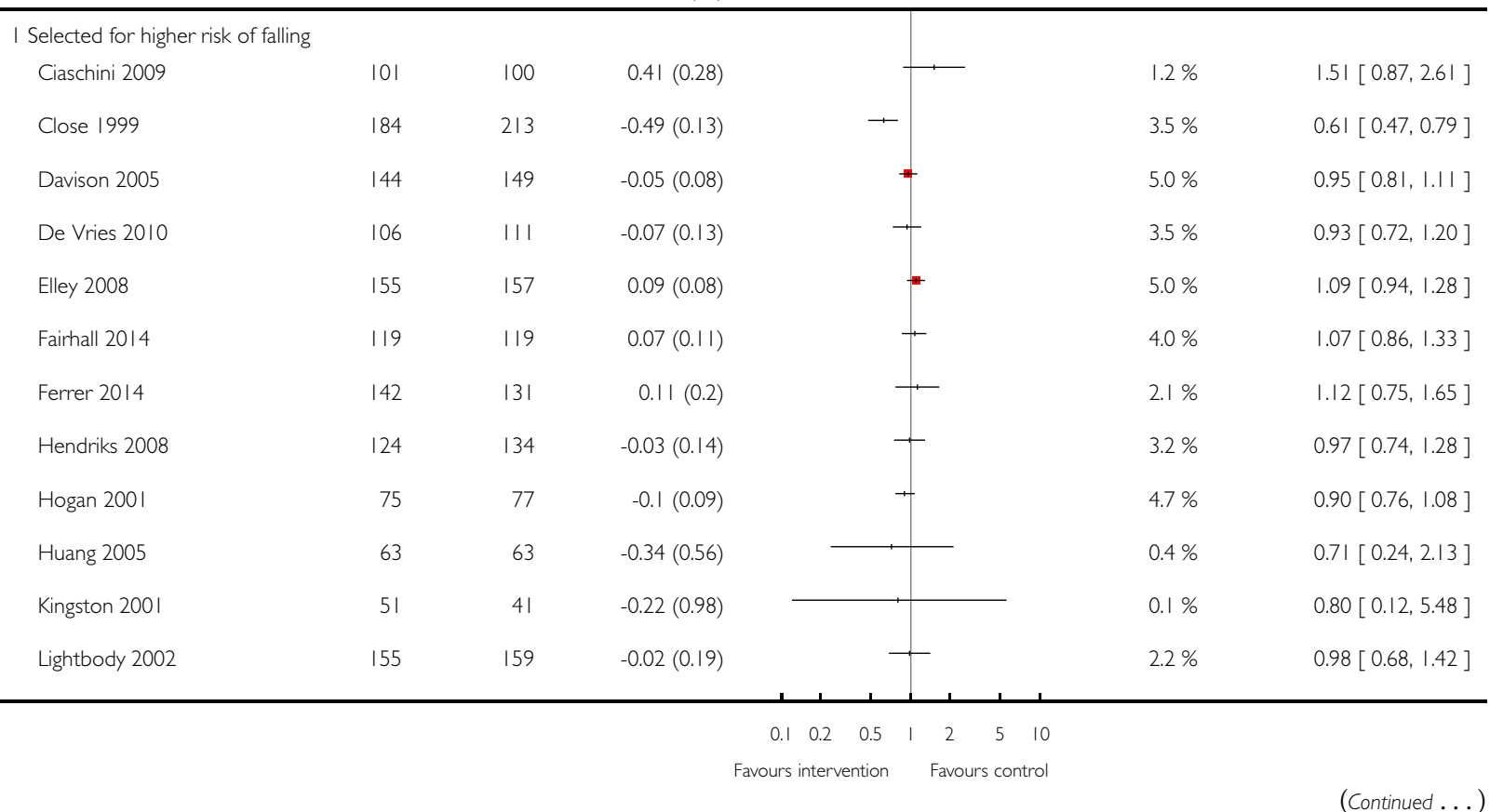




\begin{tabular}{|c|c|c|c|c|c|c|}
\hline \multirow[t]{2}{*}{ Study or subgroup } & Multifactorial & Control & log [Risk Ratio] & Risk Ratio & \multicolumn{2}{|r|}{ (... Continued) } \\
\hline & $N$ & $\mathrm{~N}$ & (SE) & IV,Random,95\% Cl & & IV,Random,95\% Cl \\
\hline Logan 2010 & 102 & 102 & $-0.17(0.06)$ & $\#$ & $5.7 \%$ & $0.84[0.75,0.95]$ \\
\hline Lord 2005 & 202 & 201 & $0.03(0.11)$ & + & $4.0 \%$ & $1.03[0.83,1.28]$ \\
\hline Palvanen 2014 & 661 & 653 & $-0.18(0.06)$ & $=$ & $5.7 \%$ & $0.84[0.74,0.94]$ \\
\hline Pardessus 2002 & 30 & 30 & $-0.14(0.28)$ & $\longrightarrow$ & $1.2 \%$ & $0.87[0.50,1.50]$ \\
\hline Russell 2010 & 320 & 330 & $0.11(0.08)$ & 世 & $5.0 \%$ & $1.12[0.95,1.31]$ \\
\hline Spice 2009 & 164 & 80 & $-0.11(0.07)$ & + & $5.4 \%$ & $0.90[0.78,1.03]$ \\
\hline Spice 2009 & 106 & 80 & $0.04(0.06)$ & $\#$ & $5.7 \%$ & $1.04[0.93,1.17]$ \\
\hline Tinetti 1994 & 147 & 144 & $-0.3(0.15)$ & $\rightarrow$ & $3.0 \%$ & $0.74[0.55,0.99]$ \\
\hline Van Haastregt 2000 & 120 & 115 & $0.12(0.12)$ & + & $3.8 \%$ & $1.13[0.89,1.43]$ \\
\hline Vind 2009 & 196 & 196 & $0.09(0.09)$ & + & $4.7 \%$ & $1.09[0.92,1.31]$ \\
\hline Whitehead 2003 & 58 & 65 & $0.74(0.26)$ & $\longrightarrow$ & $1.4 \%$ & $2.10[1.26,3.49]$ \\
\hline Subtotal $(95 \% \mathrm{CI})$ & 3525 & 3450 & & - & $80.6 \%$ & $0.97[0.90,1.04]$ \\
\hline \multicolumn{7}{|c|}{ Heterogeneity: $\mathrm{Tau}^{2}=0.01 ; \mathrm{Chi}^{2}=52.40, \mathrm{df}=22(\mathrm{P}=0.00027) ; \mathrm{I}^{2}=58 \%$} \\
\hline \multicolumn{7}{|c|}{ Test for overall effect: $Z=0.83(P=0.4 \mathrm{I})$} \\
\hline \multicolumn{7}{|c|}{2 Not selected for higher risk of falling } \\
\hline Coleman 1999 & 79 & 63 & $0.14(0.23)$ & 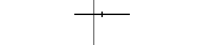 & $1.7 \%$ & $1.15[0.73,1.81]$ \\
\hline Fabacher 1994 & 100 & 95 & $-0.5(0.31)$ & 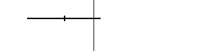 & $1.1 \%$ & $0.61[0.33,1.11]$ \\
\hline Möller 2014 & 80 & 73 & $0.11(0.16)$ & + & $2.8 \%$ & $1.12[0.82,1.53]$ \\
\hline Newbury 2001 & 45 & 44 & $-0.37(0.31)$ & - & $1.1 \%$ & $0.69[0.38,1.27]$ \\
\hline Vetter 1992 & 240 & 210 & $0.25(0.13)$ & + & $3.5 \%$ & $1.28[1.00,1.66]$ \\
\hline Wagner $1994(1)$ & 635 & 607 & $-0.29(0.08)$ & $\rightleftarrows$ & $5.0 \%$ & $0.75[0.64,0.88]$ \\
\hline Zijlstra 2009 & 188 & 203 & $-0.17(0.1)$ & $\rightarrow$ & $4.4 \%$ & $0.84[0.69,1.03]$ \\
\hline Subtotal (95\% CI) & 1367 & 1295 & & $\hookrightarrow$ & $19.4 \%$ & $0.92[0.75,1.12]$ \\
\hline \multicolumn{7}{|c|}{ Heterogeneity: $\mathrm{Tau}^{2}=0.04 ; \mathrm{Chi}^{2}=18.35, \mathrm{df}=6(P=0.01) ; \mathrm{I}^{2}=67 \%$} \\
\hline \multicolumn{7}{|c|}{ Test for overall effect: $Z=0.83(P=0.40)$} \\
\hline Total $(95 \% \mathrm{CI})$ & 4892 & 4745 & & $\cdot$ & $100.0 \%$ & $0.96[0.90,1.03]$ \\
\hline \multicolumn{7}{|c|}{ Heterogeneity: $\operatorname{Tau}^{2}=0.02 ; \mathrm{Chi}^{2}=72.98, \mathrm{df}=29(\mathrm{P}=0.0000 \mathrm{I}) ; \mathrm{I}^{2}=60 \%$} \\
\hline \multicolumn{7}{|c|}{ Test for overall effect: $Z=1.16(P=0.24)$} \\
\hline \multicolumn{7}{|c|}{ Test for subgroup differences: Chi $^{2}=0.26, \mathrm{df}=1(P=0.61), \mathrm{I}^{2}=0.0 \%$} \\
\hline
\end{tabular}

(I) Multifactorial arm vs control 
Analysis 6.3. Comparison 6 Multifactorial intervention vs control: subgroup analysis by falls risk at baseline, Outcome 3 Number of people sustaining recurrent falls (defined as two or more falls in a specified time period).

Review: Multifactorial and multiple component interventions for preventing falls in older people living in the community

Comparison: 6 Multifactorial intervention vs control: subgroup analysis by falls risk at baseline

Outcome: 3 Number of people sustaining recurrent falls (defined as two or more falls in a specified time period)

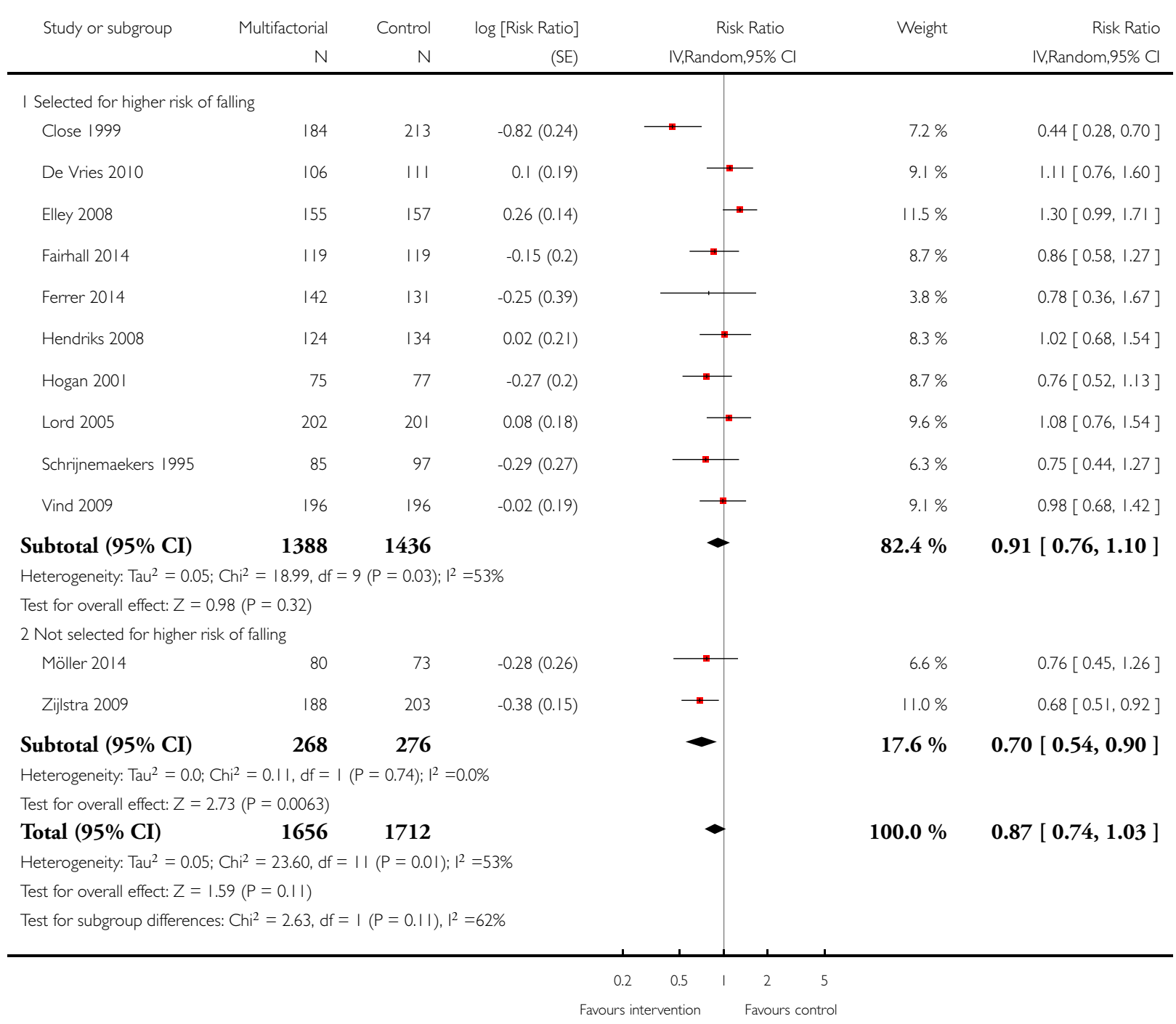


Analysis 7.I. Comparison 7 Multiple intervention vs control: subgroup analysis by falls risk at baseline, Outcome I Rate of falls (falls per person years).

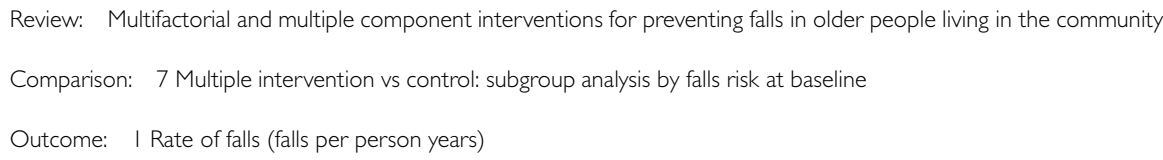

Subtotal (95\% CI)

$=0.00, d f=|(P=0.96) ;|^{2}=0.0 \%$

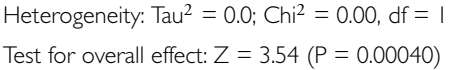

Total (95\% CI) $\mathbf{5 9 3} \quad \mathbf{4 9 2}$

492

Heterogeneity: $\mathrm{Tau}^{2}=0.03 ; \mathrm{Chi}^{2}=10.88, \mathrm{df}=6(\mathrm{P}=0.09) ; \mathrm{I}^{2}=45 \%$

Test for overall effect: $Z=2.78(P=0.0055)$

Test for subgroup differences: $C^{2} i^{2}=6.56, d f=I(P=0.01),\left.\right|^{2}=85 \%$

, Random,

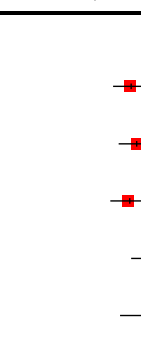
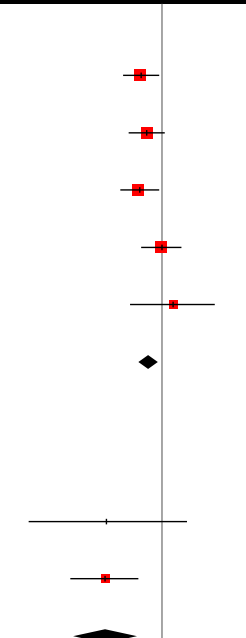

$\begin{array}{ll}21.1 \% & 0.70[0.53,0.95] \\ 21.1 \% & 0.78[0.58,1.04] \\ 19.0 \% & 0.69[0.50,0.96] \\ 19.0 \% & 0.99[0.71,1.38] \\ 7.3 \% & 1.20[0.59,2.42]\end{array}$

$87.5 \%$

$0.79[0.68,0.93$ ]

$2.4 \%$

$0.40[0.11,1.53]$

$10.1 \%$

$0.39[0.22,0.68]$

$12.5 \%$

$0.39[0.23,0.66$ ]

$100.0 \%$

$0.74[0.60,0.91]$ 
Analysis 7.2. Comparison 7 Multiple intervention vs control: subgroup analysis by falls risk at baseline, Outcome 2 Number of people sustaining one or more falls.

Review: Multifactorial and multiple component interventions for preventing falls in older people living in the community

Comparison: 7 Multiple intervention vs control: subgroup analysis by falls risk at baseline

Outcome: 2 Number of people sustaining one or more falls

Study or subgroup $\quad$ Multiple Control log [Risk Ratio] Risk Ratio Risk Ratio

N $\quad \mathrm{N}$

(SE)

IV,Random,95\% Cl

IV,Random,95\% Cl

I Selected for higher risk of falling

Campbell 2005

97

48

$-0.26(0.15)$

Campbell 2005

98

48

$-0.25(0.15)$

Clemson 2004

157

153

$-0.11(0.1)$

Faes 2011

18

15

$0.33(0.38)$

Ng 2015

49

50

$-0.9(0.81)$

Olsen 2014

47

42

$0.05(0.35)$

Waterman 2016

15

13

$-0.03(0.3)$

Wesson 2013

| I

| |

$-0.69(0.75)$

Subtotal (95\% CI)

492

380

Heterogeneity: $\mathrm{Tau}^{2}=0.0 ; \mathrm{Chi}^{2}=4.59, \mathrm{df}=7(\mathrm{P}=0.7 \mathrm{I}) ; \mathrm{I}^{2}=0.0 \%$

Test for overall effect: $Z=2.29(P=0.022)$

2 Not selected for higher risk of falling

$\begin{array}{lccc}\text { Day } 2002 & 135 & 34 & -0.19(0.15) \\ \text { Day 2002 } & 136 & 34 & -0.29(0.15) \\ \text { Day 2002 } & 137 & 34 & -0.13(0.15) \\ \text { Day 2002 } & 135 & 34 & -0.3(0.15) \\ \text { Huang 2010 } & 56 & 47 & 0.53(1.2) \\ \text { Huang 2011 } & 56 & 60 & -0.91(0.65) \\ \text { Neelemaat 2012 } & 105 & 105 & -0.88(0.35)\end{array}$

348

Subtotal (95\% CI)

760

Heterogeneity: Tau $^{2}=0.0 ; \mathrm{Chi}^{2}=5.65, \mathrm{df}=6(\mathrm{P}=0.46) ; \mathrm{I}^{2}=0.0 \%$

Test for overall effect: $Z=3.59(P=0.00033)$

Total (95\% CI) $\quad 1252 \quad 728$

Heterogeneity: $\mathrm{Tau}^{2}=0.0 ; \mathrm{Chi}^{2}=11.39, \mathrm{df}=14(\mathrm{P}=0.66) ; 1^{2}=0.0 \%$

Test for overall effect: $Z=4.13(P=0.000037)$

Test for subgroup differences: $\mathrm{Chi}^{2}=1.14 \mathrm{df}=\mathrm{I}(\mathrm{P}=0.29), \mathrm{I}^{2}=12 \%$

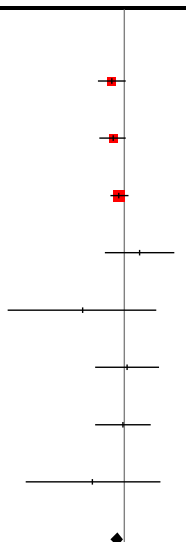

$10.9 \%$

$10.9 \%$

$24.5 \%$

$1.7 \%$

$0.4 \%$

$2.0 \%$

$2.7 \%$

$0.4 \%$

$53.6 \%$

$10.9 \%$

$10.9 \%$

$10.9 \%$

$10.9 \%$

$0.2 \%$

$0.6 \%$

$2.0 \%$

$46.4 \%$

-

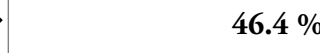

$100.0 \%$
$0.77[0.57,1.03]$

$0.78[0.58,1.04]$

$0.90[0.74,1.09]$

$1.39[0.66,2.93]$

$0.41[0.08,1.99]$

$1.05[0.53,2.09]$

$0.97[0.54,1.75]$

$0.50[0.12,2.18]$

0.86 [ $0.75,0.98$ ]

$0.83[0.62,1.11]$

$0.75[0.56,1.00]$

$0.88[0.65,1.18]$

$0.74[0.55,0.99]$

$1.70[0.16,17.85]$

$0.40[0.11,1.44]$

$0.41[0.21,0.82]$

$0.77[0.67,0.89]$

$0.82[0.74,0.90]$ 
Analysis 7.3. Comparison 7 Multiple intervention vs control: subgroup analysis by falls risk at baseline, Outcome 3 Number of people sustaining recurrent falls (defined as two or more falls in a specified time period).

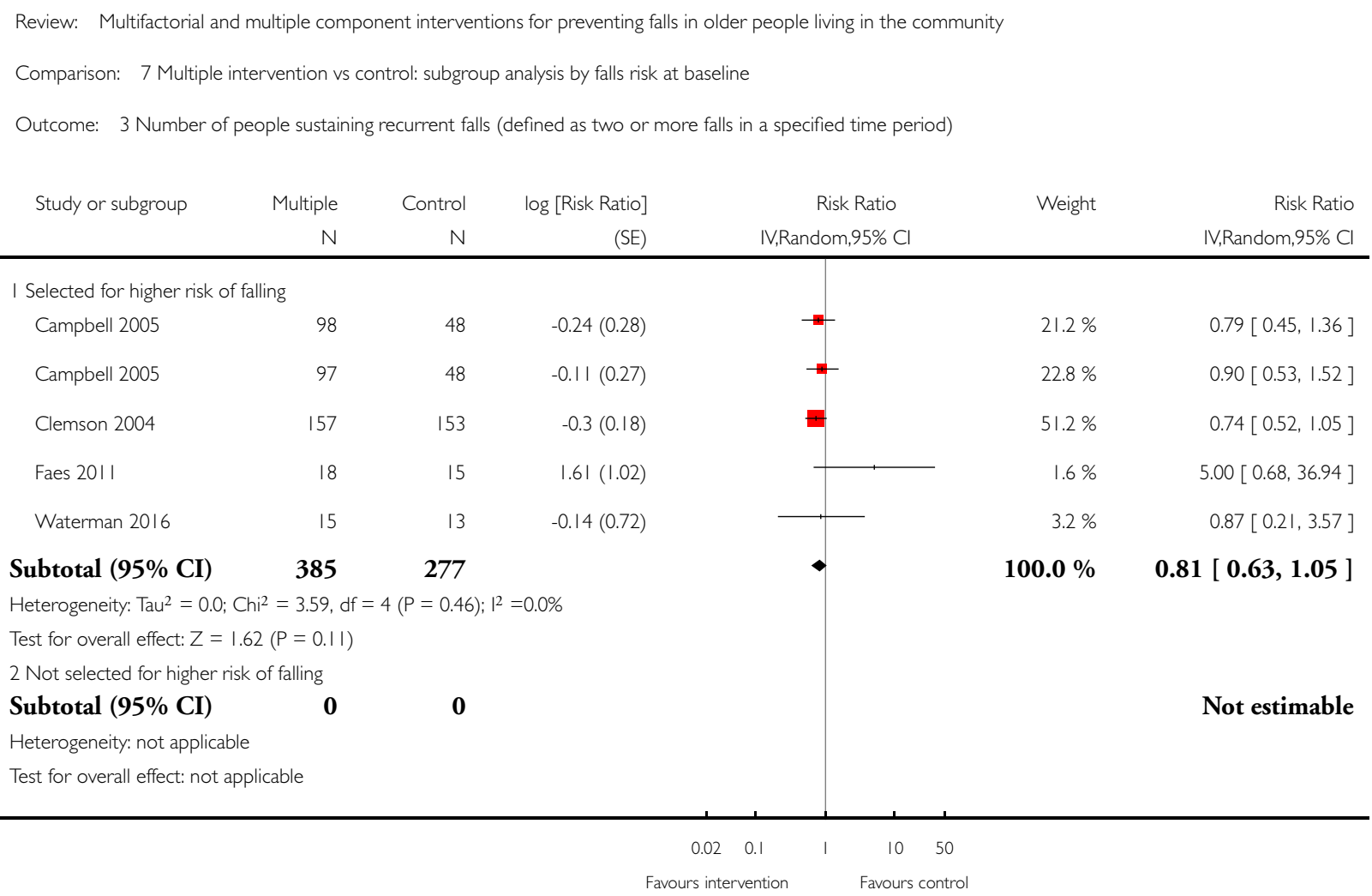


Analysis 8.1. Comparison 8 Multifactorial intervention vs control: sensitivity analysis by low risk of selection bias, Outcome I Rate of falls (falls per person years).

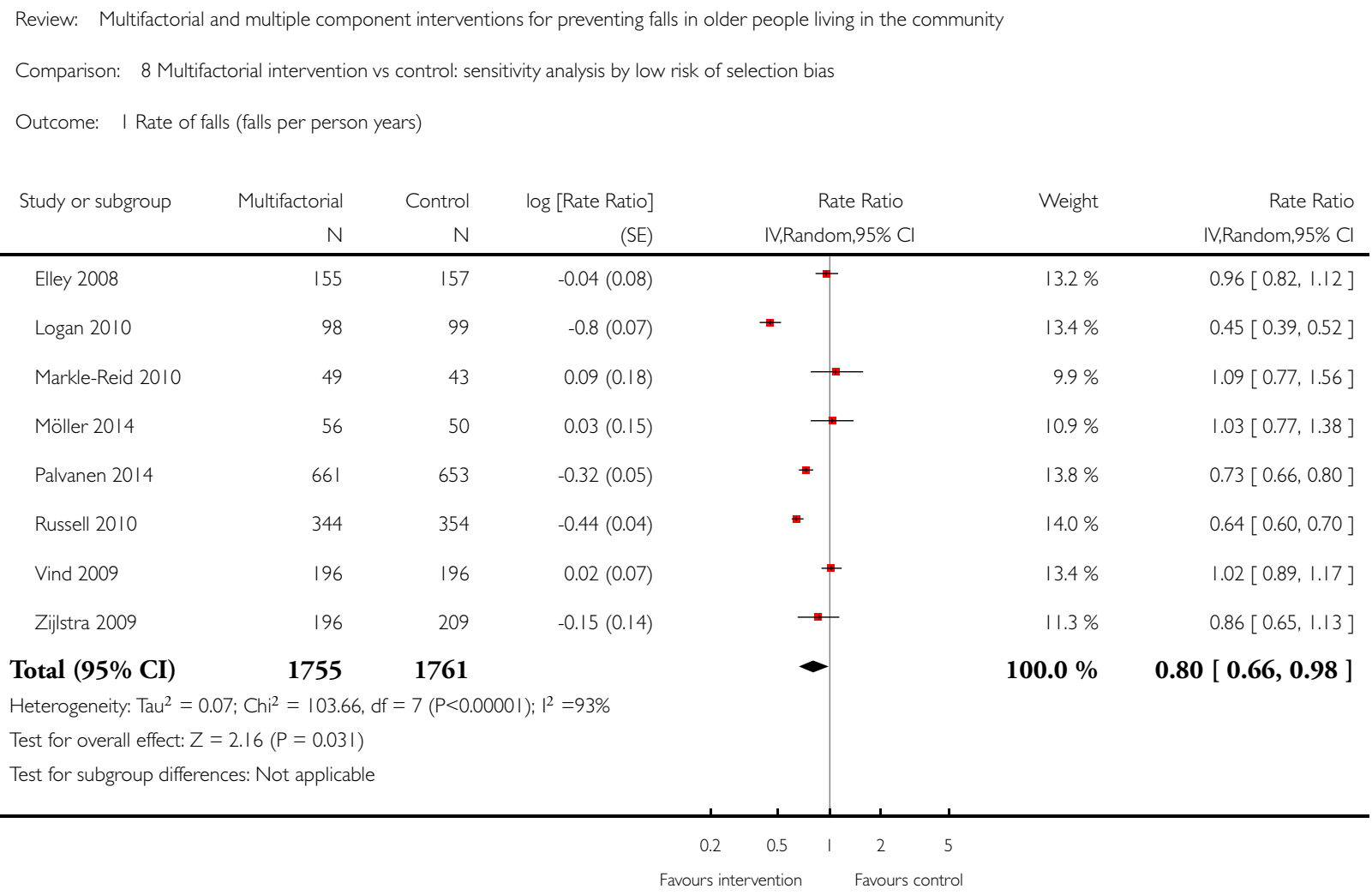


Analysis 8.2. Comparison 8 Multifactorial intervention vs control: sensitivity analysis by low risk of selection bias, Outcome 2 Number of people sustaining one or more falls.

\begin{tabular}{|c|c|c|c|c|c|c|}
\hline \multicolumn{7}{|c|}{ Comparison: 8 Multifactorial intervention vs control: sensitivity analysis by low risk of selection bias } \\
\hline \multicolumn{7}{|c|}{ Outcome: 2 Number of people sustaining one or more falls } \\
\hline \multirow[t]{2}{*}{ Study or subgroup } & Multifactorial & Control & $\log$ [Risk Ratio] & Risk Ratio & Weight & Risk Ratio \\
\hline & N & $N$ & $(\mathrm{SE})$ & \multicolumn{2}{|l|}{ IV,Random,95\% Cl } & IV,Random,95\% Cl \\
\hline Close 1999 & 184 & 213 & $-0.49(0.13)$ & 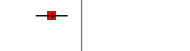 & $8.1 \%$ & $0.61[0.47,0.79]$ \\
\hline De Vries 2010 & 106 & 111 & $-0.07(0.13)$ & $\rightarrow$ & $8.1 \%$ & $0.93[0.72,1.20]$ \\
\hline Elley 2008 & 155 & 157 & $0.09(0.08)$ & ¥ & $10.3 \%$ & $1.09[0.94,1.28]$ \\
\hline Logan 2010 & 102 & 102 & $-0.17(0.06)$ & \pm & $11.1 \%$ & $0.84[0.75,0.95]$ \\
\hline Möller 2014 & 80 & 73 & $0.11(0.16)$ & $=$ & $6.8 \%$ & $1.12[0.82,1.53]$ \\
\hline Newbury 200I & 45 & 44 & $-0.37(0.31)$ & \multirow[b]{2}{*}{ \# } & $3.1 \%$ & $0.69[0.38,1.27]$ \\
\hline Palvanen 2014 & 661 & 653 & $-0.18(0.06)$ & & $11.1 \%$ & $0.84[0.74,0.94]$ \\
\hline Russell 2010 & 320 & 330 & $0.11(0.08)$ & + & $10.3 \%$ & $1.12[0.95,1.31]$ \\
\hline Vetter 1992 & 240 & 210 & $0.25(0.13)$ & 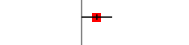 & $8.1 \%$ & $1.28[1.00,1.66]$ \\
\hline Vind 2009 & 196 & 196 & $0.09(0.09)$ & $\mp$ & $9.8 \%$ & $1.09[0.92,1.31]$ \\
\hline Whitehead 2003 & 58 & 65 & $0.74(0.26)$ & $\longrightarrow$ & $3.9 \%$ & $2.10[1.26,3.49]$ \\
\hline Zijlstra 2009 & 188 & 203 & $-0.17(0.1)$ & - & $9.4 \%$ & $0.84[0.69,1.03]$ \\
\hline Total (95\% CI) & 2335 & 2357 & & $\bullet$ & $100.0 \%$ & $0.98[0.86,1.10]$ \\
\hline \multicolumn{7}{|c|}{ Heterogeneity: $\mathrm{Tau}^{2}=0.03 ; \mathrm{Chi}^{2}=47.6 \mathrm{I}, \mathrm{df}=\mathrm{II}(\mathrm{P}<0.0000 \mathrm{I}) ; \mathrm{I}^{2}=77 \%$} \\
\hline \multicolumn{7}{|c|}{ Test for overall effect: $Z=0.40(P=0.69)$} \\
\hline \multicolumn{7}{|c|}{ Test for subgroup differences: Not applicable } \\
\hline
\end{tabular}


Analysis 8.3. Comparison 8 Multifactorial intervention vs control: sensitivity analysis by low risk of selection bias, Outcome 3 Number of people sustaining recurrent falls (defined as two or more falls in a specified time period).

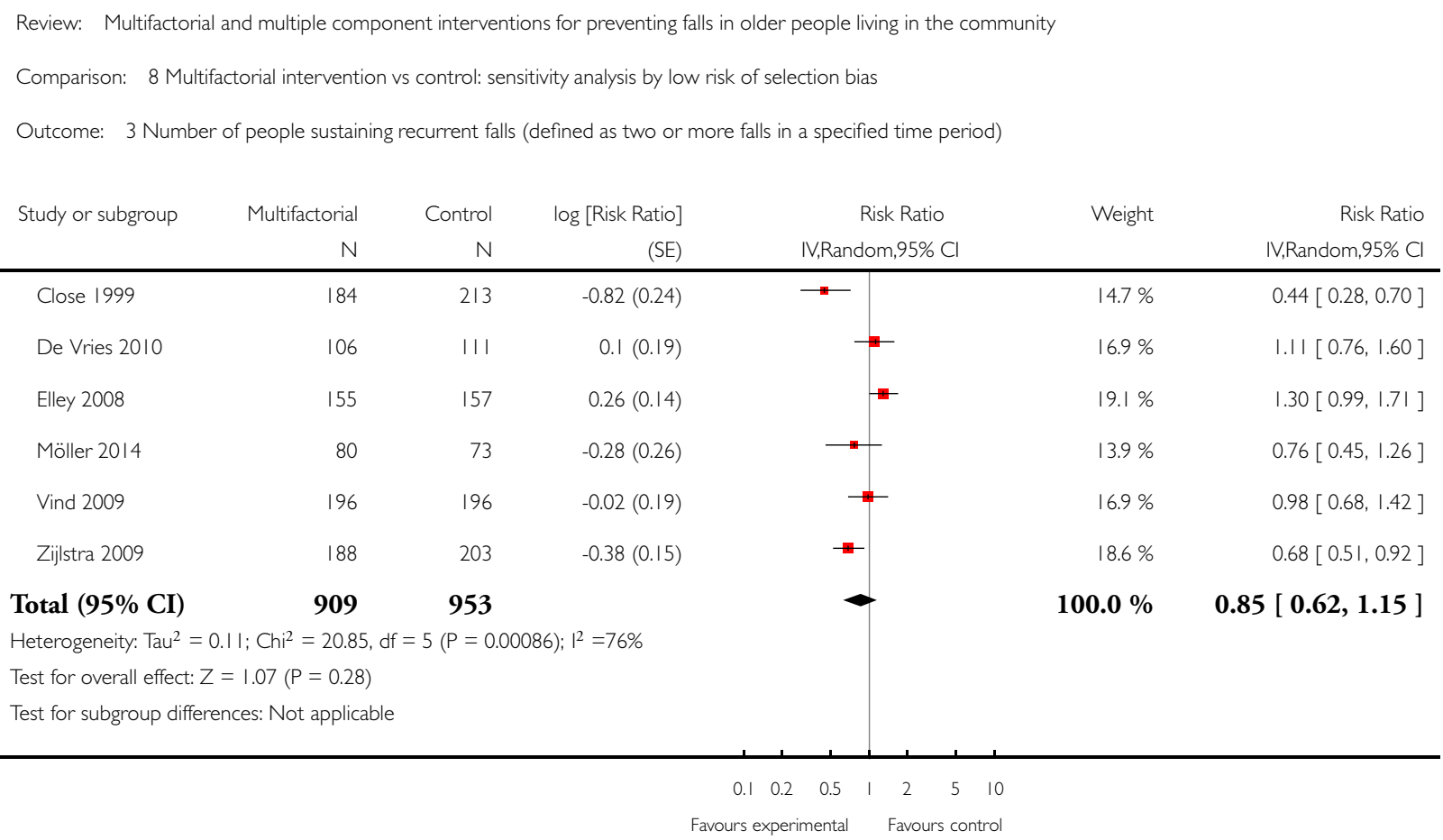




\section{Analysis 9.1. Comparison 9 Multifactorial intervention vs control: sensitivity analysis by low risk of}

detection bias, Outcome I Rate of falls (falls per person years).

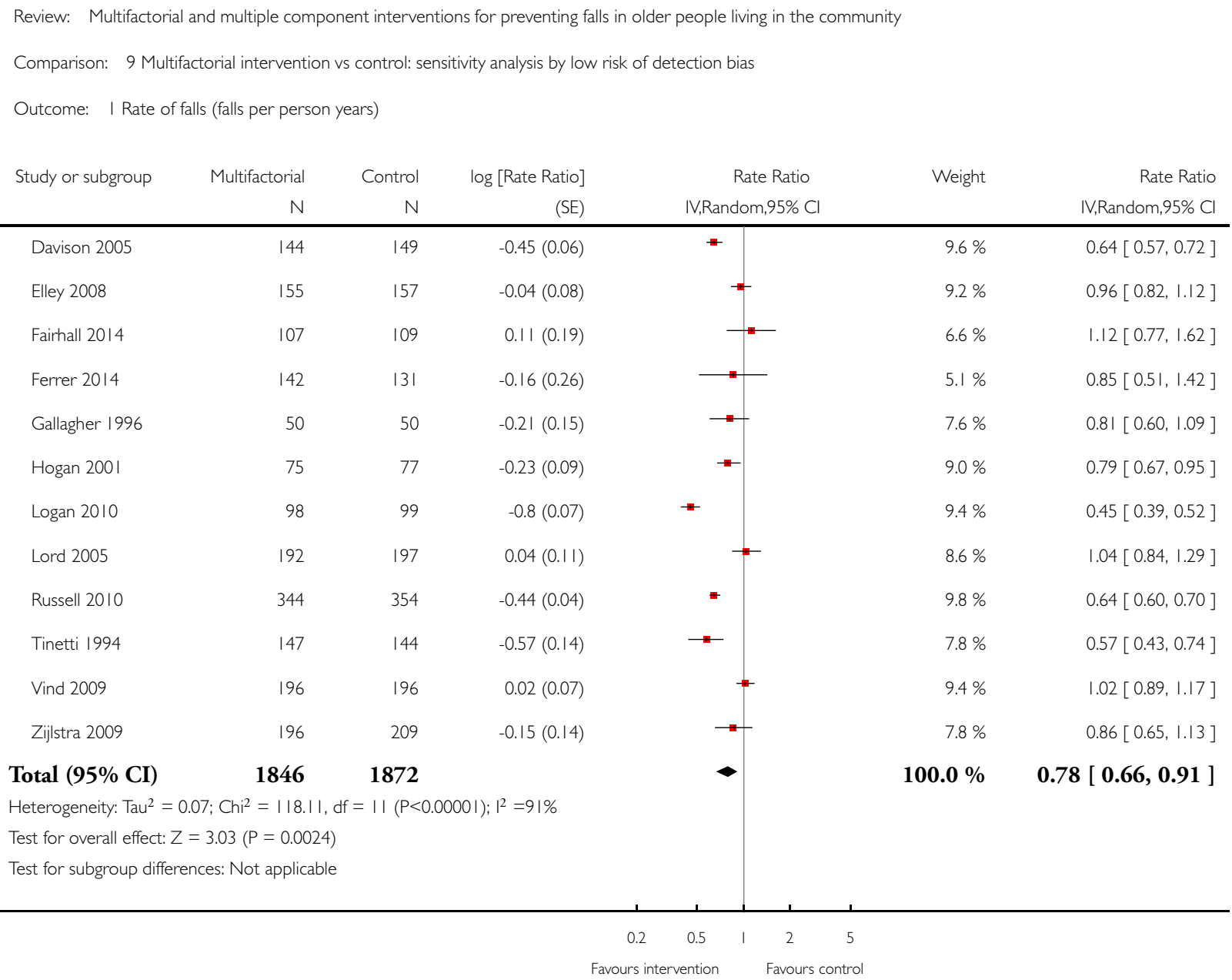


Analysis 9.2. Comparison 9 Multifactorial intervention vs control: sensitivity analysis by low risk of detection bias, Outcome 2 Number of people sustaining one or more falls.

Review: Multifactorial and multiple component interventions for preventing falls in older people living in the community

Comparison: 9 Multifactorial intervention vs control: sensitivity analysis by low risk of detection bias

Outcome: 2 Number of people sustaining one or more falls

\begin{tabular}{|c|c|c|c|c|c|c|}
\hline \multirow[t]{2}{*}{ Study or subgroup } & Multifactorial & Control & log [Risk Ratio] & \multirow{2}{*}{$\begin{array}{c}\text { Risk Ratio } \\
\text { IV,Random,95\% Cl }\end{array}$} & \multirow[t]{2}{*}{ Weight } & \multirow{2}{*}{$\begin{array}{r}\text { Risk Ratio } \\
\text { IV,Random,95\% Cl }\end{array}$} \\
\hline & $N$ & $N$ & (SE) & & & \\
\hline Ciaschini 2009 & 101 & 100 & $0.41(0.28)$ & \multirow[b]{2}{*}{$\rightarrow$} & $2.3 \%$ & $1.51[0.87,2.61]$ \\
\hline Close 1999 & 184 & 213 & $-0.49(0.13)$ & & $6.0 \%$ & $0.61[0.47,0.79]$ \\
\hline Davison 2005 & 144 & 149 & $-0.05(0.08)$ & 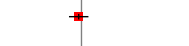 & $8.3 \%$ & $0.95[0.81,1.11]$ \\
\hline De Vries 2010 & 106 & 111 & $-0.07(0.13)$ & $\rightarrow$ & $6.0 \%$ & $0.93[0.72,1.20]$ \\
\hline Elley 2008 & 155 & 157 & $0.09(0.08)$ & $\Psi$ & $8.3 \%$ & $1.09[0.94,1.28]$ \\
\hline Fairhall 2014 & 119 & 119 & $0.07(0.11)$ & $\Psi$ & $6.8 \%$ & $1.07[0.86,1.33]$ \\
\hline Ferrer 2014 & 142 & $|3|$ & $0.11(0.2)$ & 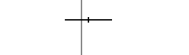 & $3.7 \%$ & $1.12[0.75,1.65]$ \\
\hline Hendriks 2008 & 124 & 134 & $-0.03(0.14)$ & $\rightarrow$ & $5.6 \%$ & $0.97[0.74,1.28]$ \\
\hline Hogan 200I & 75 & 77 & $-0.1(0.09)$ & $\boxplus$ & $7.8 \%$ & $0.90[0.76,1.08]$ \\
\hline Logan 2010 & 102 & 102 & $-0.17(0.06)$ & \pm & $9.2 \%$ & $0.84[0.75,0.95]$ \\
\hline Lord 2005 & 202 & 201 & $0.03(0.11)$ & + & $6.8 \%$ & $1.03[0.83,1.28]$ \\
\hline Tinetti 1994 & 147 & 144 & $-0.3(0.15)$ & $\rightarrow$ & $5.2 \%$ & $0.74[0.55,0.99]$ \\
\hline Van Haastregt 2000 & 120 & 115 & $0.12(0.12)$ & $\mp$ & $6.4 \%$ & $1.13[0.89,1.43]$ \\
\hline Vind 2009 & 196 & 196 & $0.09(0.09)$ & + & $7.8 \%$ & $1.09[0.92,1.31]$ \\
\hline Whitehead 2003 & 58 & 65 & $0.74(0.26)$ & $\longrightarrow$ & $2.5 \%$ & $2.10[1.26,3.49]$ \\
\hline Zijlstra 2009 & 188 & 203 & $-0.17(0.1)$ & $\Psi$ & $7.3 \%$ & $0.84[0.69,1.03]$ \\
\hline Total (95\% CI) & 2163 & 2217 & & $\bullet$ & $100.0 \%$ & $0.97[0.88,1.07]$ \\
\hline \multicolumn{7}{|c|}{ Heterogeneity: Tau $^{2}=0.02 ; \mathrm{Chi}^{2}=41.81, \mathrm{df}=15(\mathrm{P}=0.00024) ;\left.\right|^{2}=64 \%$} \\
\hline \multicolumn{7}{|c|}{ Test for overall effect: $Z=0.63(P=0.53)$} \\
\hline \multicolumn{4}{|c|}{ Test for subgroup differences: Not applicable } & & & \\
\hline
\end{tabular}

$\begin{array}{lllllll}0.1 & 0.2 & 0.5 & 1 & 2 & 5 & 10\end{array}$

Favours intervention Favours control 
Analysis 9.3. Comparison 9 Multifactorial intervention vs control: sensitivity analysis by low risk of detection bias, Outcome 3 Number of people sustaining recurrent falls (defined as two or more falls in a specified time period).

\begin{tabular}{|c|c|c|c|c|c|c|}
\hline \multicolumn{7}{|c|}{ Comparison: 9 Multifactorial intervention vs control: sensitivity analysis by low risk of detection bias } \\
\hline \multicolumn{7}{|c|}{ Outcome: 3 Number of people sustaining recurrent falls (defined as two or more falls in a specified time period) } \\
\hline \multirow[t]{2}{*}{ Study or subgroup } & Multifactorial & Control & $\log [$ Risk Ratio] & Risk Ratio & Weight & \multirow{2}{*}{$\begin{array}{r}\text { Risk Ratio } \\
\text { IV,Random,95\% Cl }\end{array}$} \\
\hline & N & N & (SE) & \multicolumn{2}{|l|}{ IV,Random,95\% Cl } & \\
\hline Close 1999 & 184 & 213 & $-0.82(0.24)$ & $\longrightarrow$ & $8.5 \%$ & $0.44[0.28,0.70]$ \\
\hline De Vries 2010 & 106 & 111 & $0.1(0.19)$ & $\longrightarrow$ & $10.5 \%$ & $1.11[0.76,1.60]$ \\
\hline Elley 2008 & 155 & 157 & $0.26(0.14)$ & 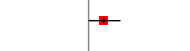 & $12.8 \%$ & $1.30[0.99,1.71]$ \\
\hline Fairhall 2014 & 119 & 119 & $-0.15(0.2)$ & $\rightarrow$ & $10.1 \%$ & $0.86[0.58,1.27]$ \\
\hline Ferrer 2014 & 142 & $|3|$ & $-0.25(0.39)$ & +1 & $4.6 \%$ & $0.78[0.36,1.67]$ \\
\hline Hendriks 2008 & 124 & 134 & $0.02(0.21)$ & $\rightarrow$ & $9.7 \%$ & $1.02[0.68,1.54]$ \\
\hline Hogan 2001 & 75 & 77 & $-0.27(0.2)$ & $\rightarrow$ & $10.1 \%$ & $0.76[0.52,1.13]$ \\
\hline Lord 2005 & 202 & 201 & $0.08(0.18)$ & $\rightarrow$ & $10.9 \%$ & $1.08[0.76,1.54]$ \\
\hline Vind 2009 & 196 & 196 & $-0.02(0.19)$ & $\rightarrow$ & $10.5 \%$ & $0.98[0.68,1.42]$ \\
\hline Zijlstra 2009 & 188 & 203 & $-0.38(0.15)$ & $\rightarrow$ & $12.3 \%$ & $0.68[0.51,0.92]$ \\
\hline Total $(95 \% \mathrm{CI})$ & 1491 & 1542 & & 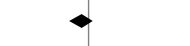 & $100.0 \%$ & $0.89[0.73,1.08]$ \\
\hline \multicolumn{7}{|c|}{ Heterogeneity: $\operatorname{Tau}^{2}=0.06 ; \mathrm{Chi}^{2}=22.61, \mathrm{df}=9(P=0.01) ; \mathrm{I}^{2}=60 \%$} \\
\hline \multicolumn{7}{|c|}{ Test for overall effect: $Z=1.20(P=0.23)$} \\
\hline \multicolumn{7}{|c|}{ Test for subgroup differences: Not applicable } \\
\hline
\end{tabular}


Analysis 10.I. Comparison 10 Multifactorial intervention vs control: sensitivity analysis by low risk of attrition bias, Outcome I Rate of falls (falls per person years).

Review: Multifactorial and multiple component interventions for preventing falls in older people living in the community

Comparison: 10 Multifactorial intervention vs control: sensitivity analysis by low risk of attrition bias

Outcome: I Rate of falls (falls per person years)

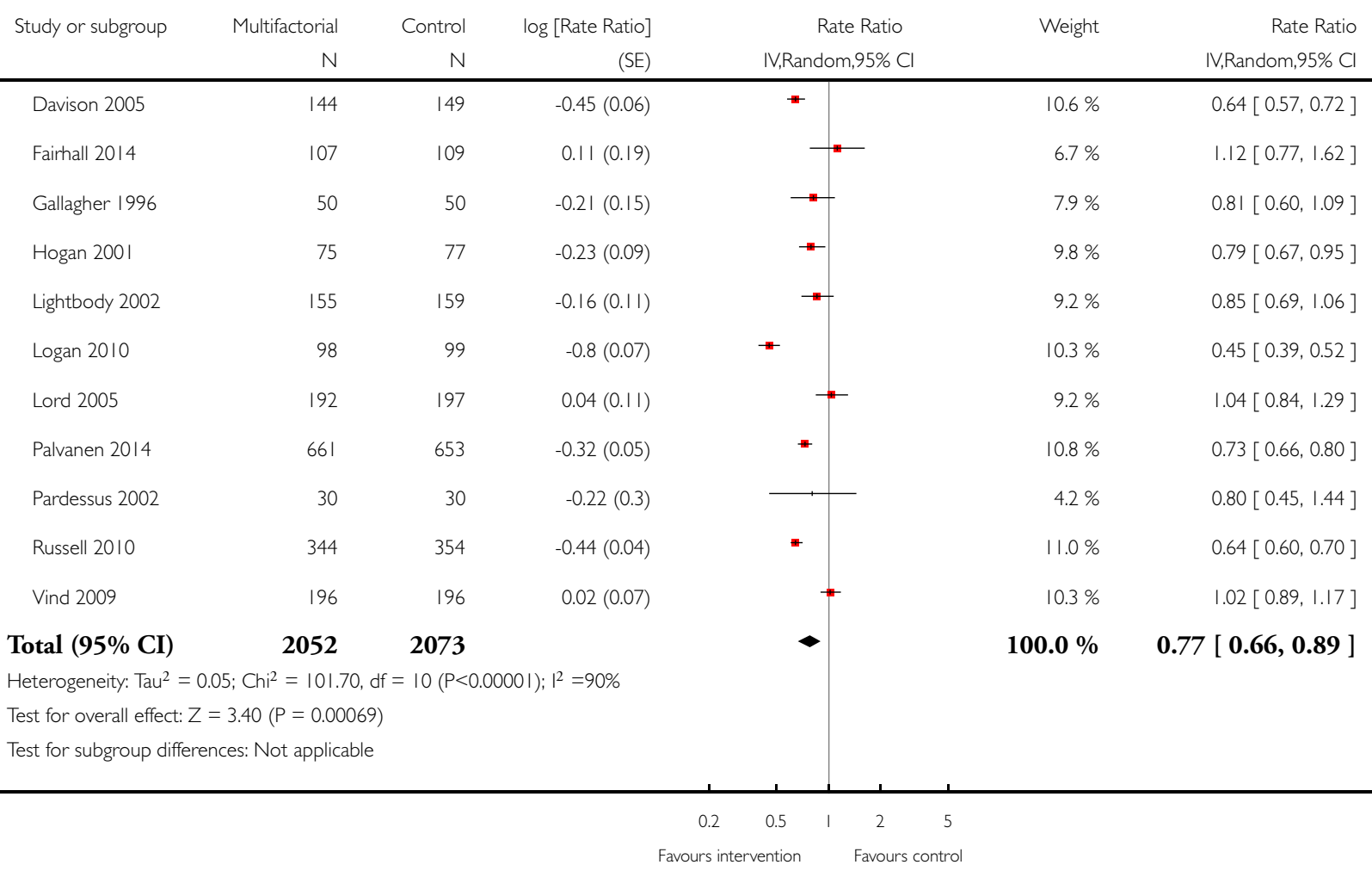


Analysis 10.2. Comparison 10 Multifactorial intervention vs control: sensitivity analysis by low risk of attrition bias, Outcome 2 Number of people sustaining one or more falls.

Review: Multifactorial and multiple component interventions for preventing falls in older people living in the community

Comparison: 10 Multifactorial intervention vs control: sensitivity analysis by low risk of attrition bias

Outcome: 2 Number of people sustaining one or more falls

\begin{tabular}{|c|c|c|c|c|c|c|}
\hline \multirow[t]{2}{*}{ Study or subgroup } & Multifactorial & Control & log [Risk Ratio] & \multirow{2}{*}{$\begin{array}{c}\text { Risk Ratio } \\
\text { IV,Random,95\% Cl }\end{array}$} & \multirow[t]{2}{*}{ Weight } & \multirow{2}{*}{$\begin{array}{r}\text { Risk Ratio } \\
\text { IV,Random,95\% Cl }\end{array}$} \\
\hline & $\mathrm{N}$ & $\mathrm{N}$ & (SE) & & & \\
\hline Davison 2005 & 144 & 149 & $-0.05(0.08)$ & + & $11.3 \%$ & $0.95[0.81,1.11]$ \\
\hline De Vries 2010 & 106 & 111 & $-0.07(0.13)$ & 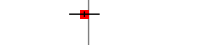 & $5.9 \%$ & $0.93[0.72,1.20]$ \\
\hline Fairhall 2014 & 119 & 119 & $0.07(0.11)$ & 世 & $7.6 \%$ & $1.07[0.86,1.33]$ \\
\hline Hogan 200I & 75 & 77 & $-0.1(0.09)$ & \# & $9.9 \%$ & $0.90[0.76,1.08]$ \\
\hline Huang 2005 & 63 & 63 & $-0.34(0.56)$ & 1 & $0.4 \%$ & $0.71[0.24,2.13]$ \\
\hline Lightbody 2002 & 155 & 159 & $-0.02(0.19)$ & 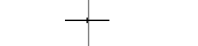 & $3.2 \%$ & $0.98[0.68,1.42]$ \\
\hline Logan 2010 & 102 & 102 & $-0.17(0.06)$ & $=$ & $15.0 \%$ & $0.84[0.75,0.95]$ \\
\hline Lord 2005 & 202 & 201 & $0.03(0.11)$ & + & $7.6 \%$ & $1.03[0.83,1.28]$ \\
\hline Newbury 200I & 45 & 44 & $-0.37(0.31)$ & + & $1.3 \%$ & $0.69[0.38,1.27]$ \\
\hline Palvanen 2014 & 661 & 653 & $-0.18(0.06)$ & \# & $15.0 \%$ & $0.84[0.74,0.94]$ \\
\hline Pardessus 2002 & 30 & 30 & $-0.14(0.28)$ & $\longrightarrow$ & $1.6 \%$ & $0.87[0.50,1.50]$ \\
\hline Russell 2010 & 320 & 330 & $0.11(0.08)$ & 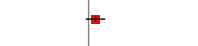 & $11.3 \%$ & $1.12[0.95,1.31]$ \\
\hline Vind 2009 & 196 & 196 & $0.09(0.09)$ & + & $9.9 \%$ & $1.09[0.92, \mid .31]$ \\
\hline Total (95\% CI) & 2218 & 2234 & & $\bullet$ & $100.0 \%$ & $0.95[0.88,1.02]$ \\
\hline \multicolumn{7}{|c|}{ Heterogeneity: $\mathrm{Tau}^{2}=0.01 ; \mathrm{Chi}^{2}=18.26, \mathrm{df}=12(\mathrm{P}=0.1 \mathrm{I}) ; \mathrm{I}^{2}=34 \%$} \\
\hline \multicolumn{7}{|c|}{ Test for overall effect: $Z=1.44(P=0.15)$} \\
\hline \multicolumn{7}{|c|}{ Test for subgroup differences: Not applicable } \\
\hline
\end{tabular}

$\begin{array}{lllllll}0.1 & 0.2 & 0.5 & 1 & 2 & 5 & 10\end{array}$

Favours intervention Favours control 
Analysis 10.3. Comparison 10 Multifactorial intervention vs control: sensitivity analysis by low risk of attrition bias, Outcome 3 Number of people sustaining recurrent falls (defined as two or more falls in a specified time period).

\begin{tabular}{|c|c|c|c|c|c|c|}
\hline \multicolumn{7}{|c|}{ Comparison: 10 Multifactorial intervention vs control: sensitivity analysis by low risk of attrition bias } \\
\hline \multicolumn{7}{|c|}{ Outcome: 3 Number of people sustaining recurrent falls (defined as two or more falls in a specified time period) } \\
\hline \multirow[t]{2}{*}{ Study or subgroup } & Multifactorial & Control & log [Risk Ratio] & Risk Ratio & Weight & Risk Ratio \\
\hline & N & $\mathrm{N}$ & (SE) & IV,Random,95\% Cl & & IV,Random, $95 \% \mathrm{Cl}$ \\
\hline De Vries 2010 & 106 & 111 & $0.1(0.19)$ & 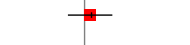 & $20.3 \%$ & $1.11[0.76,1.60]$ \\
\hline Fairhall 2014 & 119 & 119 & $-0.15(0.2)$ & $\rightarrow$ & $18.3 \%$ & $0.86[0.58,1.27]$ \\
\hline Hogan 2001 & 75 & 77 & $-0.27(0.2)$ & $\rightarrow$ & $18.3 \%$ & $0.76[0.52,1.13]$ \\
\hline Lord 2005 & 202 & 201 & $0.08(0.18)$ & $\rightarrow$ & $22.6 \%$ & $1.08[0.76,1.54]$ \\
\hline Vind 2009 & 196 & 196 & $-0.02(0.19)$ & $\rightarrow$ & $20.3 \%$ & $0.98[0.68,1.42]$ \\
\hline Total $(95 \% \mathrm{CI})$ & 698 & 704 & & $\bullet$ & $100.0 \%$ & $0.96[0.81,1.13]$ \\
\hline \multicolumn{7}{|c|}{ Heterogeneity: $\mathrm{Tau}^{2}=0.0 ; \mathrm{Chi}^{2}=2.62, \mathrm{df}=4(\mathrm{P}=0.62) ; \mathrm{I}^{2}=0.0 \%$} \\
\hline \multicolumn{7}{|c|}{ Test for overall effect: $Z=0.50(P=0.62)$} \\
\hline \multicolumn{7}{|c|}{ Test for subgroup differences: Not applicable } \\
\hline
\end{tabular}

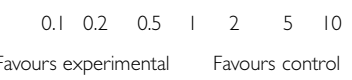




\section{Analysis I I.I. Comparison I I Multifactorial intervention vs control: sensitivity analysis by individual}

randomisation, Outcome I Rate of falls (falls per person years).

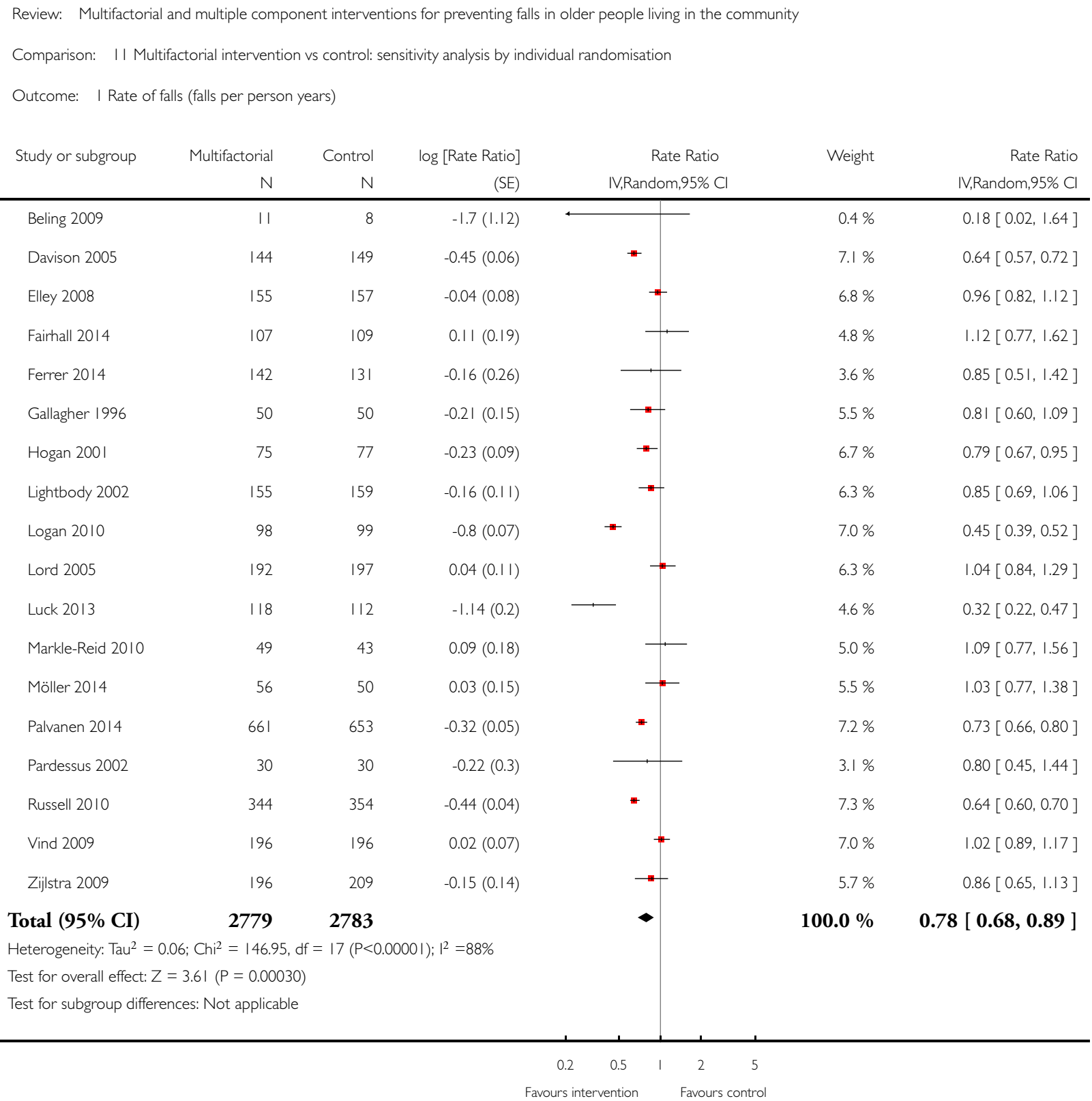


Analysis I I.2. Comparison I I Multifactorial intervention vs control: sensitivity analysis by individual randomisation, Outcome 2 Number of people sustaining one or more falls.

Review: Multifactorial and multiple component interventions for preventing falls in older people living in the community

Comparison: I I Multifactorial intervention vs control: sensitivity analysis by individual randomisation

Outcome: 2 Number of people sustaining one or more falls

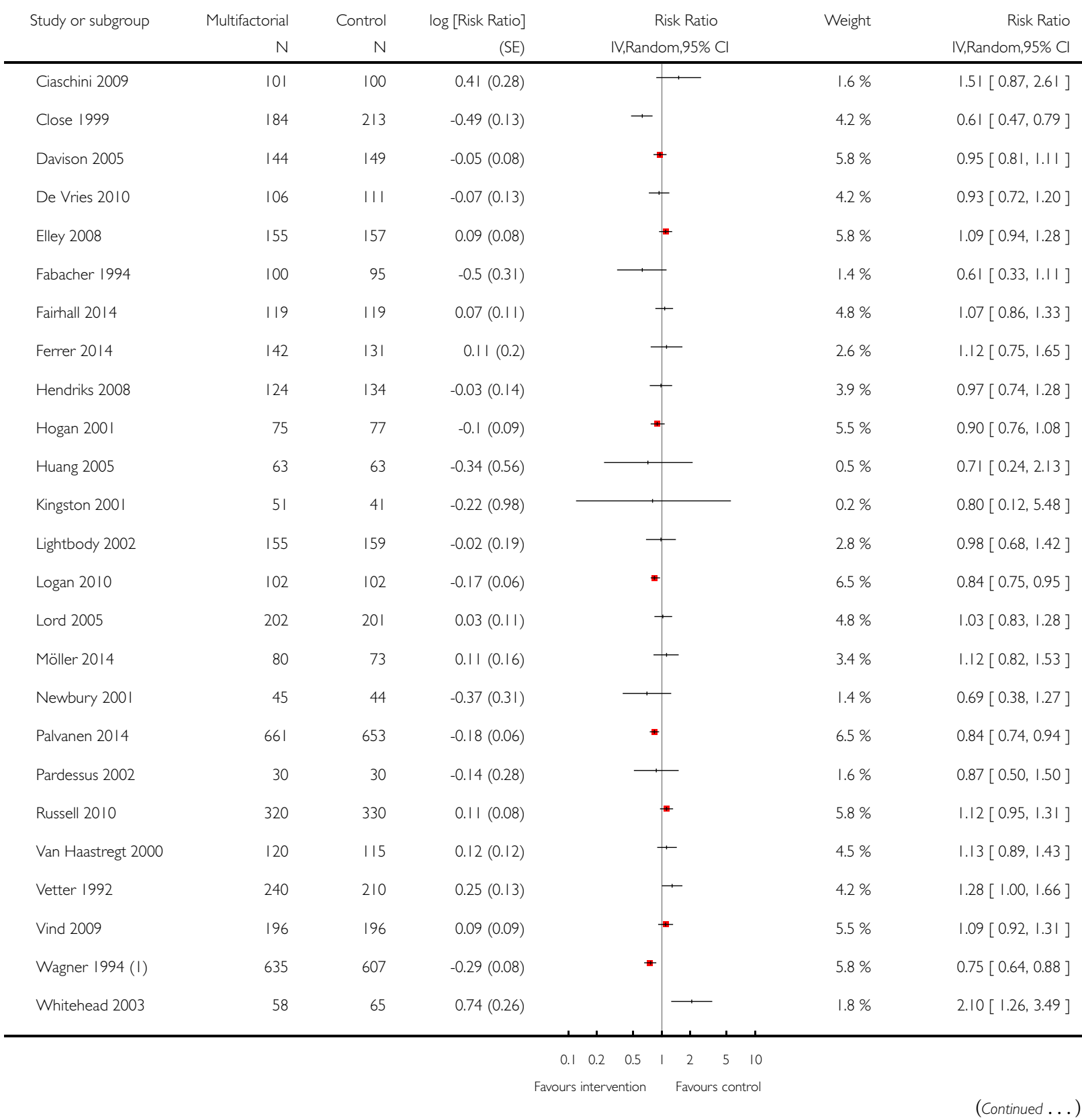




\begin{tabular}{|c|c|c|c|c|c|c|}
\hline \multirow[t]{2}{*}{ Study or subgroup } & Multifactorial & Control & $\log [$ Risk Ratio] & Risk Ratio & Weight & $\begin{array}{r}\text { (... Continued) } \\
\text { Risk Ratio }\end{array}$ \\
\hline & $\mathrm{N}$ & $\mathrm{N}$ & (SE) & IV,Random,95\% Cl & & IV,Random,95\% Cl \\
\hline Zijlstra 2009 & 188 & 203 & $-0.17(0.1)$ & \# & $5.1 \%$ & $0.84[0.69,1.03]$ \\
\hline Total $(95 \% \mathrm{CI})$ & 4396 & 4378 & & - & $100.0 \%$ & $0.97[0.89,1.04]$ \\
\hline \multicolumn{7}{|c|}{ Heterogeneity: $\mathrm{Tau}^{2}=0.02 ; \mathrm{Chi}^{2}=66.4 \mathrm{I}, \mathrm{df}=25(\mathrm{P}=0.0000 \mathrm{I}) ; \mathrm{I}^{2}=62 \%$} \\
\hline \multicolumn{7}{|c|}{ Test for overall effect: $Z=0.86(P=0.39)$} \\
\hline \multicolumn{7}{|c|}{ Test for subgroup differences: Not applicable } \\
\hline
\end{tabular}

(I) Multifactorial arm vs control

Analysis I I.3. Comparison I I Multifactorial intervention vs control: sensitivity analysis by individual randomisation, Outcome 3 Number of people sustaining recurrent falls (defined as two or more falls in a specified time period).

\footnotetext{
Review: Multifactorial and multiple component interventions for preventing falls in older people living in the community

Comparison: II Multifactorial intervention vs control: sensitivity analysis by individual randomisation

Outcome: 3 Number of people sustaining recurrent falls (defined as two or more falls in a specified time period)
}

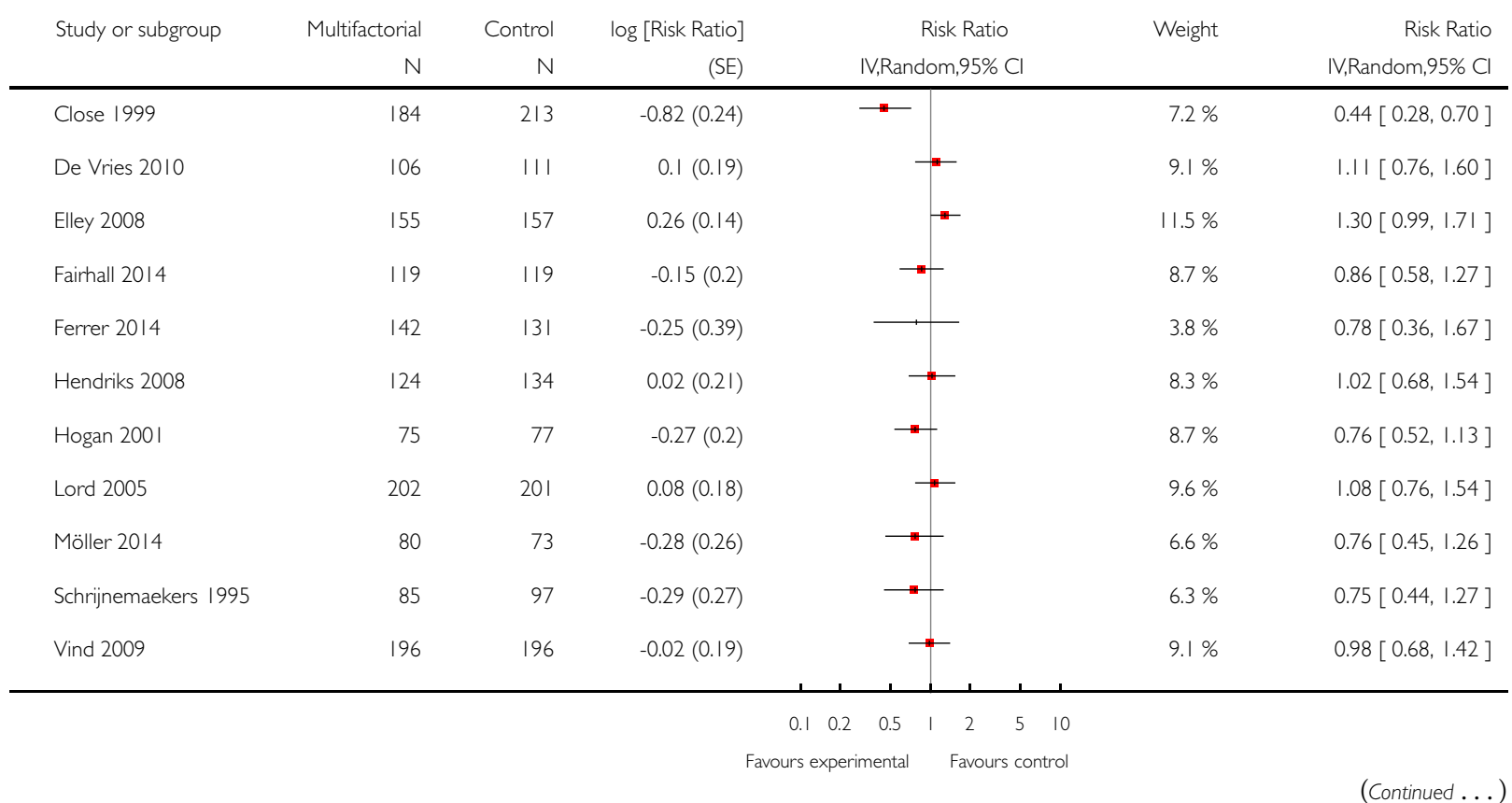




\begin{tabular}{|c|c|c|c|c|c|c|}
\hline \multirow[t]{2}{*}{ Study or subgroup } & Multifactorial & Control & log [Risk Ratio] & \multirow{2}{*}{$\begin{array}{c}\text { Risk Ratio } \\
\text { IV,Random,95\% Cl }\end{array}$} & \multirow[t]{2}{*}{ Weight } & $\begin{array}{r}\text { (... Continued) } \\
\text { Risk Ratio }\end{array}$ \\
\hline & N & N & (SE) & & & IV,Random,95\% Cl \\
\hline Zijlstra 2009 & 188 & 203 & $-0.38(0.15)$ & 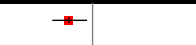 & $11.0 \%$ & $0.68[0.51,0.92]$ \\
\hline Total $(95 \% \mathrm{CI})$ & 1656 & 1712 & & $\bullet$ & $100.0 \%$ & $0.87[0.74,1.03]$ \\
\hline \multicolumn{7}{|c|}{ Heterogeneity: $\mathrm{Tau}^{2}=0.05 ; \mathrm{Ch}^{2}=23.60, \mathrm{df}=1 \mid(P=0.01) ; \mathrm{I}^{2}=53 \%$} \\
\hline \multicolumn{7}{|c|}{ Test for overall effect: $Z=1.59(P=0.11)$} \\
\hline \multicolumn{7}{|c|}{ Test for subgroup differences: Not applicable } \\
\hline
\end{tabular}

Analysis 12.I. Comparison I 2 Multiple intervention vs control: sensitivity analysis by low risk of selection bias, Outcome I Rate of falls (falls per person years).

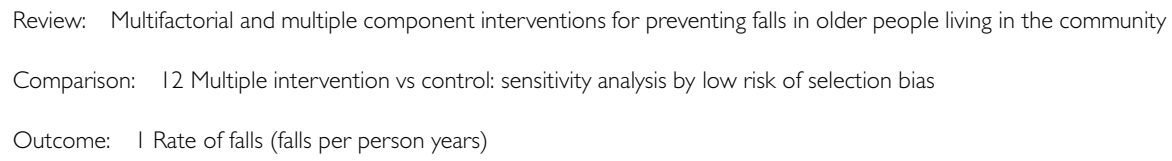

\begin{tabular}{|c|c|c|c|c|c|c|}
\hline \multirow[t]{2}{*}{ Study or subgroup } & Multiple & Control & $\log$ [Rate Ratio] & \multirow{2}{*}{$\begin{array}{c}\text { Rate Ratio } \\
\text { IV,Random,95\% Cl }\end{array}$} & \multirow[t]{2}{*}{ Weight } & \multirow{2}{*}{$\begin{array}{r}\text { Rate Ratio } \\
\text { IV,Random,95\% Cl }\end{array}$} \\
\hline & $N$ & $\mathrm{~N}$ & (SE) & & & \\
\hline Campbell 2005 & 96 & 48 & $-0.25(0.15)$ & $\rightarrow$ & $32.5 \%$ & $0.78[0.58,1.04]$ \\
\hline Campbell 2005 & 97 & 48 & $-0.35(0.15)$ & $\rightarrow$ & $32.5 \%$ & $0.70[0.53,0.95]$ \\
\hline Huang 201I & 56 & 60 & $-0.91(0.68)$ & - & $4.5 \%$ & $0.40[0.11,1.53]$ \\
\hline Neelemaat 2012 & 76 & 75 & $-0.95(0.29)$ & —- & $17.4 \%$ & $0.39[0.22,0.68]$ \\
\hline Waterman 2016 & 15 & 13 & $0.18(0.36)$ & 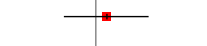 & $13.0 \%$ & $1.20[0.59,2.42]$ \\
\hline Total (95\% CI) & 340 & 244 & & - & $100.0 \%$ & $0.68[0.51,0.92]$ \\
\hline \multicolumn{7}{|c|}{ Heterogeneity: Tau $^{2}=0.05 ; \mathrm{Chi}^{2}=7.57, \mathrm{df}=4(\mathrm{P}=0.1 \mathrm{I}) ; \mathrm{I}^{2}=47 \%$} \\
\hline \multicolumn{7}{|c|}{ Test for overall effect: $Z=2.49(P=0.013)$} \\
\hline Test for subgroup diff & es: Not app & & & & & \\
\hline
\end{tabular}

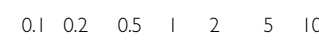

Favours intervention Favours control 
Analysis 12.2. Comparison 12 Multiple intervention vs control: sensitivity analysis by low risk of selection bias, Outcome 2 Number of people sustaining one or more falls.

Review: Multifactorial and multiple component interventions for preventing falls in older people living in the community

Comparison: 12 Multiple intervention vs control: sensitivity analysis by low risk of selection bias

Outcome: 2 Number of people sustaining one or more falls

\begin{tabular}{|c|c|c|c|c|c|c|}
\hline \multirow[t]{2}{*}{ Study or subgroup } & Multiple & Control & log [Risk Ratio] & Risk Ratio & Weight & Risk Ratio \\
\hline & N & $\mathrm{N}$ & $(\mathrm{SE})$ & IV,Random,95\% Cl & & IV,Random,95\% Cl \\
\hline Campbell 2005 & 97 & 48 & $-0.26(0.15)$ & 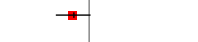 & $14.9 \%$ & $0.77[0.57,1.03]$ \\
\hline Campbell 2005 & 98 & 48 & $-0.25(0.15)$ & $\rightarrow$ & $14.9 \%$ & $0.78[0.58,1.04]$ \\
\hline Day 2002 & 137 & 34 & $-0.13(0.15)$ & $\rightarrow$ & $14.9 \%$ & $0.88[0.65,1.18]$ \\
\hline Day 2002 & 135 & 34 & $-0.19(0.15)$ & $\rightarrow$ & $14.9 \%$ & $0.83[0.62,1.11]$ \\
\hline Day 2002 & 135 & 34 & $-0.3(0.15)$ & $\rightarrow$ & $14.9 \%$ & $0.74[0.55,0.99]$ \\
\hline Day 2002 & 136 & 34 & $-0.29(0.15)$ & $\rightarrow$ & $14.9 \%$ & $0.75[0.56,1.00]$ \\
\hline Faes 201 I & 18 & 15 & $0.33(0.38)$ & - & $2.3 \%$ & $1.39[0.66,2.93]$ \\
\hline Huang 201I & 56 & 60 & $-0.9 \mid(0.65)$ & & $0.8 \%$ & $0.40[0.11,1.44]$ \\
\hline Neelemaat 2012 & 105 & 105 & $-0.88(0.35)$ & - & $2.7 \%$ & $0.41[0.21,0.82]$ \\
\hline Ng 2015 & 49 & 50 & $-0.9(0.81)$ & & $0.5 \%$ & $0.41[0.08,1.99]$ \\
\hline Waterman 2016 & 15 & 13 & $-0.03(0.3)$ & $\longrightarrow$ & $3.7 \%$ & $0.97[0.54,1.75]$ \\
\hline Wesson 2013 & 11 & 11 & $-0.69(0.75)$ & & $0.6 \%$ & $0.50[0.12,2.18]$ \\
\hline Total (95\% CI) & 992 & 486 & & $\bullet$ & $100.0 \%$ & $0.78[0.70,0.88]$ \\
\hline \multicolumn{7}{|c|}{ Heterogeneity: $\operatorname{Tau}^{2}=0.0 ; \mathrm{Chi}^{2}=9.1 \mathrm{I}, \mathrm{df}=\mathrm{II}(\mathrm{P}=0.6 \mathrm{I}) ; \mathrm{I}^{2}=0.0 \%$} \\
\hline \multicolumn{7}{|c|}{ Test for overall effect: $Z=4.23(P=0.000023)$} \\
\hline \multicolumn{7}{|c|}{ Test for subgroup differences: Not applicable } \\
\hline
\end{tabular}

$\begin{array}{lllllll}0.1 & 0.2 & 0.5 & 1 & 2 & 5 & 10\end{array}$

Favours intervention Favours control 
Analysis 12.3. Comparison I 2 Multiple intervention vs control: sensitivity analysis by low risk of selection bias, Outcome 3 Number of people sustaining recurrent falls (defined as two or more falls in a specified time period).

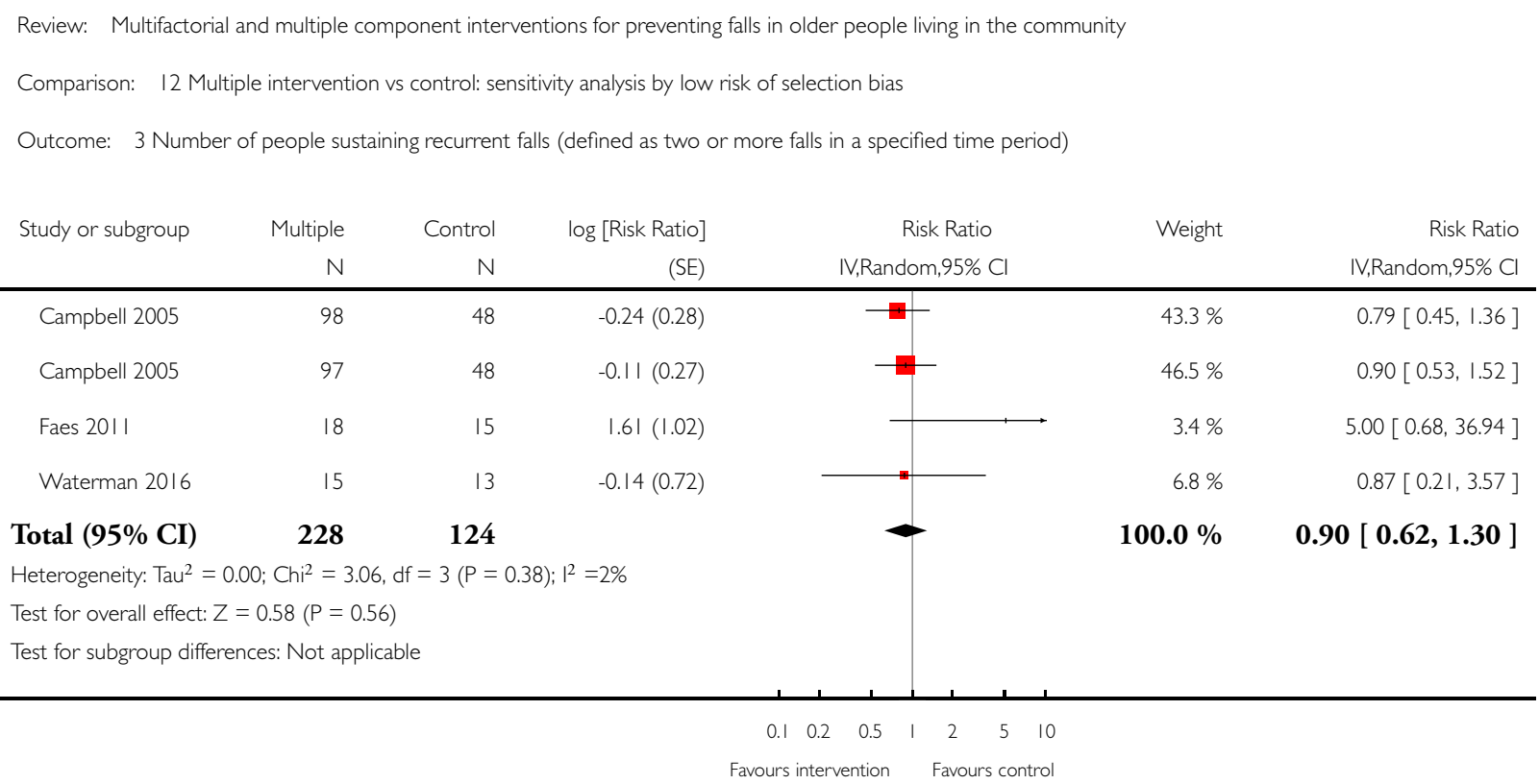


Analysis 13.I. Comparison 13 Multiple intervention vs control: sensitivity analysis by low risk of detection bias, Outcome I Rate of falls (falls per person years).

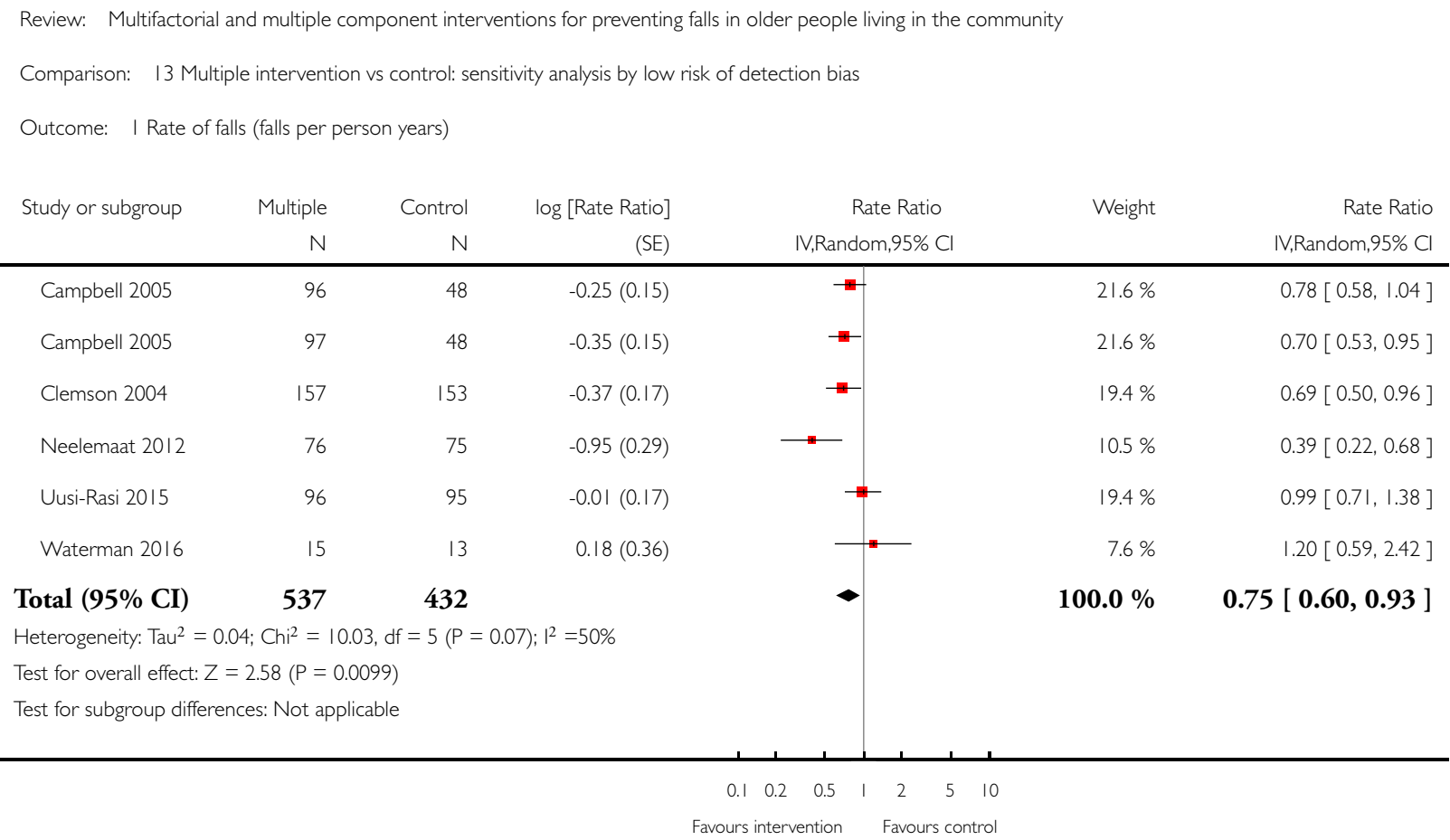


Analysis 13.2. Comparison 13 Multiple intervention vs control: sensitivity analysis by low risk of detection bias, Outcome 2 Number of people sustaining one or more falls.

Review: Multifactorial and multiple component interventions for preventing falls in older people living in the community

Comparison: 13 Multiple intervention vs control: sensitivity analysis by low risk of detection bias

Outcome: 2 Number of people sustaining one or more falls

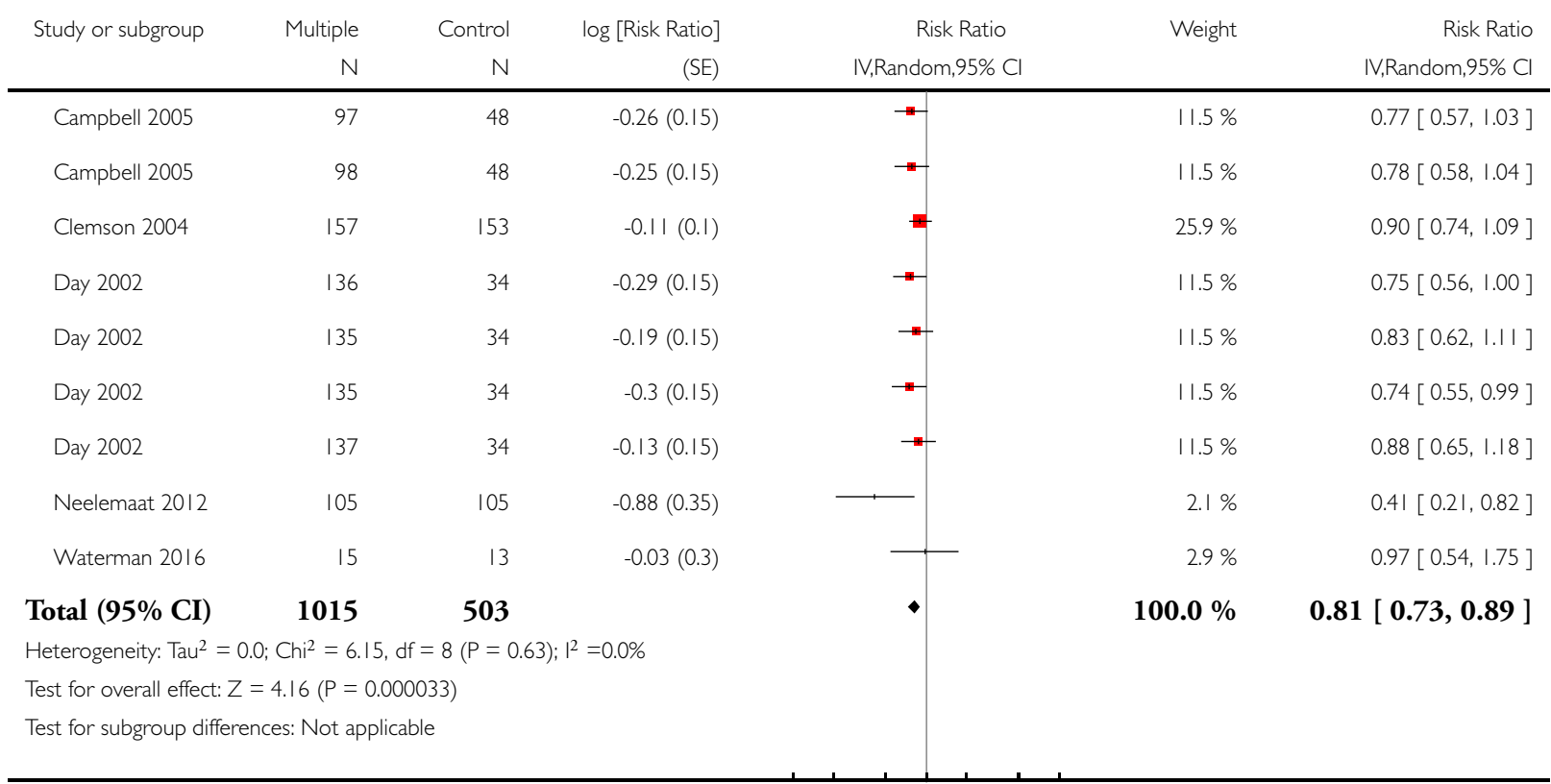

$\begin{array}{lllllll}0.1 & 0.2 & 0.5 & 1 & 2 & 5 & 10\end{array}$

Favours intervention Favours control 
Analysis 13.3. Comparison I3 Multiple intervention vs control: sensitivity analysis by low risk of detection bias, Outcome 3 Number of people sustaining recurrent falls (defined as two or more falls in a specified time period).

\begin{tabular}{|c|c|c|c|c|c|c|}
\hline \multicolumn{7}{|c|}{ Comparison: 13 Multiple intervention vs control: sensitivity analysis by low risk of detection bias } \\
\hline \multicolumn{7}{|c|}{ Outcome: 3 Number of people sustaining recurrent falls (defined as two or more falls in a specified time period) } \\
\hline \multirow[t]{2}{*}{ Study or subgroup } & Multiple & Control & log [Risk Ratio] & Risk Ratio & Weight & Risk Ratio \\
\hline & N & N & (SE) & \multicolumn{2}{|l|}{ IV,Random,95\% Cl } & IV,Random,95\% Cl \\
\hline Campbell 2005 & 97 & 48 & $-0.11(0.27)$ & $\longrightarrow$ & $23.1 \%$ & $0.90[0.53,1.52]$ \\
\hline Campbell 2005 & 98 & 48 & $-0.24(0.28)$ & $\rightarrow-$ & $21.5 \%$ & $0.79[0.45,1.36]$ \\
\hline Clemson 2004 & 157 & 153 & $-0.3(0.18)$ & $\rightarrow$ & $52.1 \%$ & $0.74[0.52,1.05]$ \\
\hline Waterman 2016 & 15 & 13 & $-0.14(0.72)$ & & $3.3 \%$ & $0.87[0.21,3.57]$ \\
\hline Total (95\% CI) & 367 & 262 & & $<$ & $100.0 \%$ & $0.79[0.61,1.02]$ \\
\hline \multicolumn{7}{|c|}{ Heterogeneity: $\mathrm{Tau}^{2}=0.0 ; \mathrm{Chi}^{2}=0.36, \mathrm{df}=3(\mathrm{P}=0.95) ;\left.\right|^{2}=0.0 \%$} \\
\hline \multicolumn{7}{|c|}{ Test for overall effect: $Z=1.83(P=0.067)$} \\
\hline \multicolumn{7}{|c|}{ Test for subgroup differences: Not applicable } \\
\hline
\end{tabular}


Analysis 14.1. Comparison 14 Multiple intervention vs control: sensitivity analysis by low risk of attrition bias, Outcome I Rate of falls (falls per person years).

Review: Multifactorial and multiple component interventions for preventing falls in older people living in the community

Comparison: 14 Multiple intervention vs control: sensitivity analysis by low risk of attrition bias

Outcome: I Rate of falls (falls per person years)

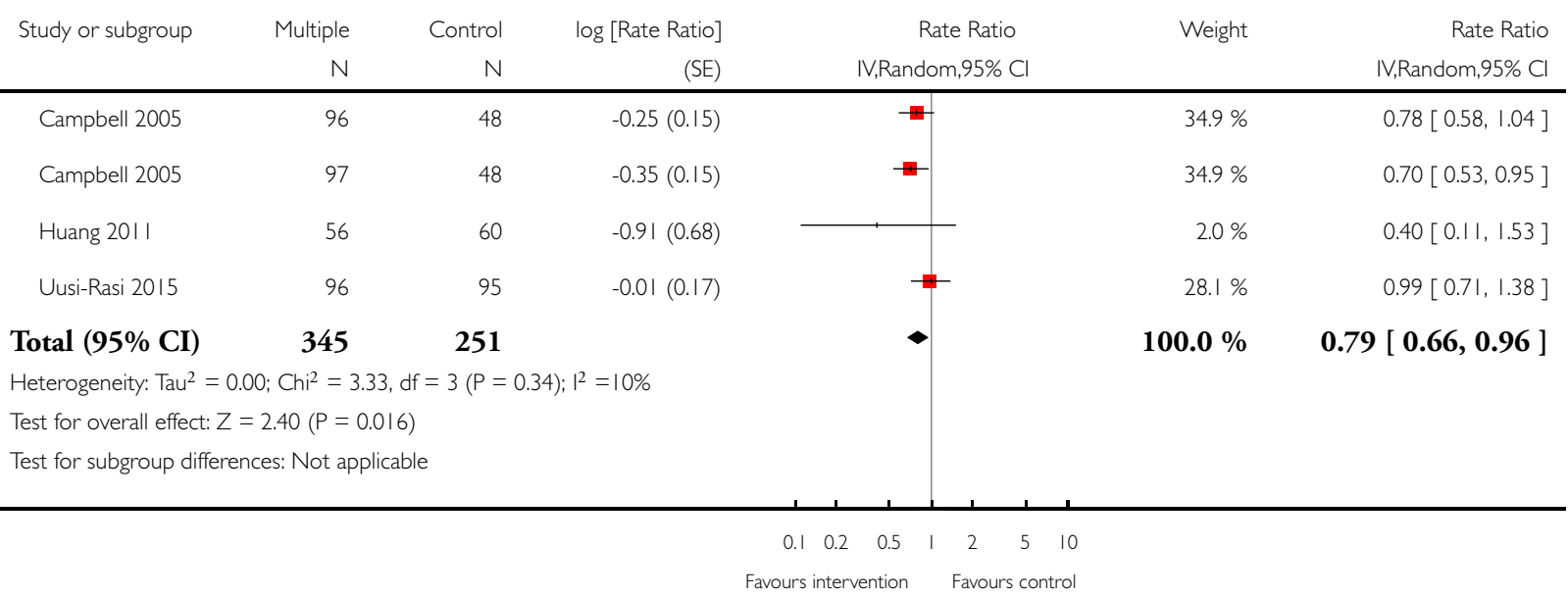




\section{Analysis 14.2. Comparison I4 Multiple intervention vs control: sensitivity analysis by low risk of attrition}

bias, Outcome 2 Number of people sustaining one or more falls.

Review: Multifactorial and multiple component interventions for preventing falls in older people living in the community

Comparison: 14 Multiple intervention vs control: sensitivity analysis by low risk of attrition bias

Outcome: 2 Number of people sustaining one or more falls

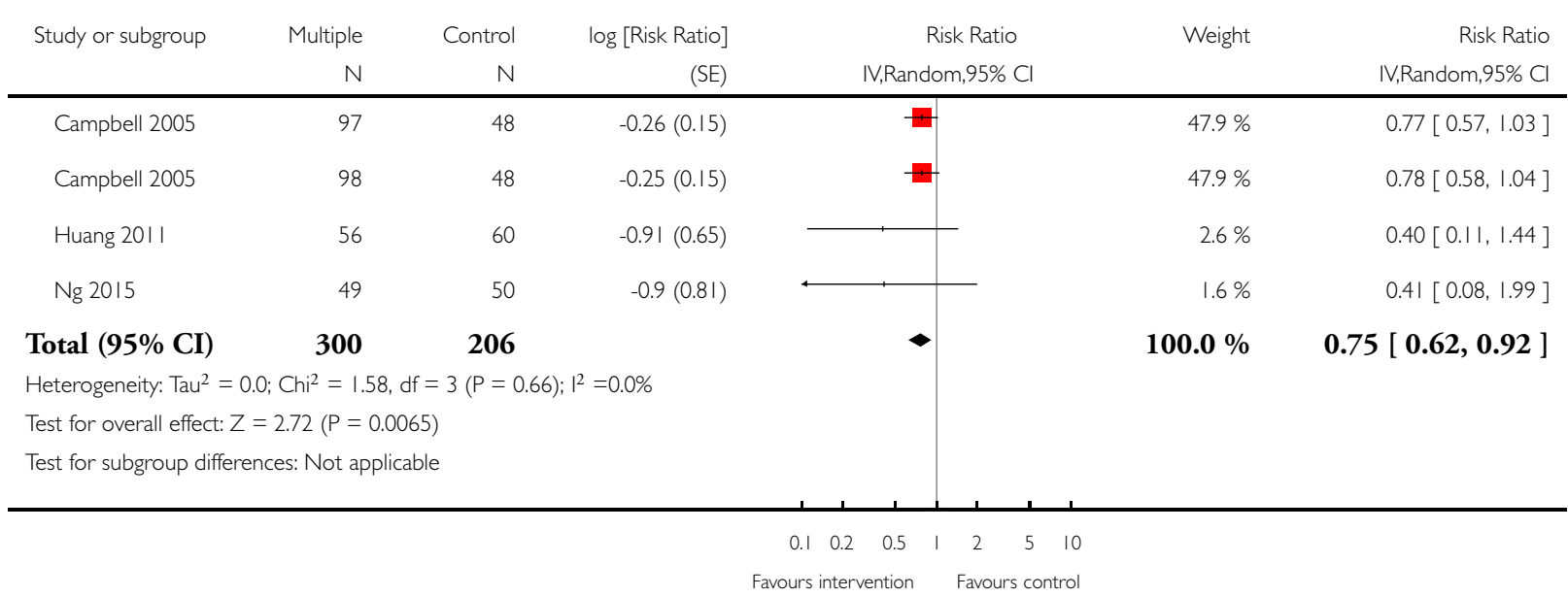


Analysis 14.3. Comparison 14 Multiple intervention vs control: sensitivity analysis by low risk of attrition bias, Outcome 3 Number of people sustaining recurrent falls (defined as two or more falls in a specified time period).

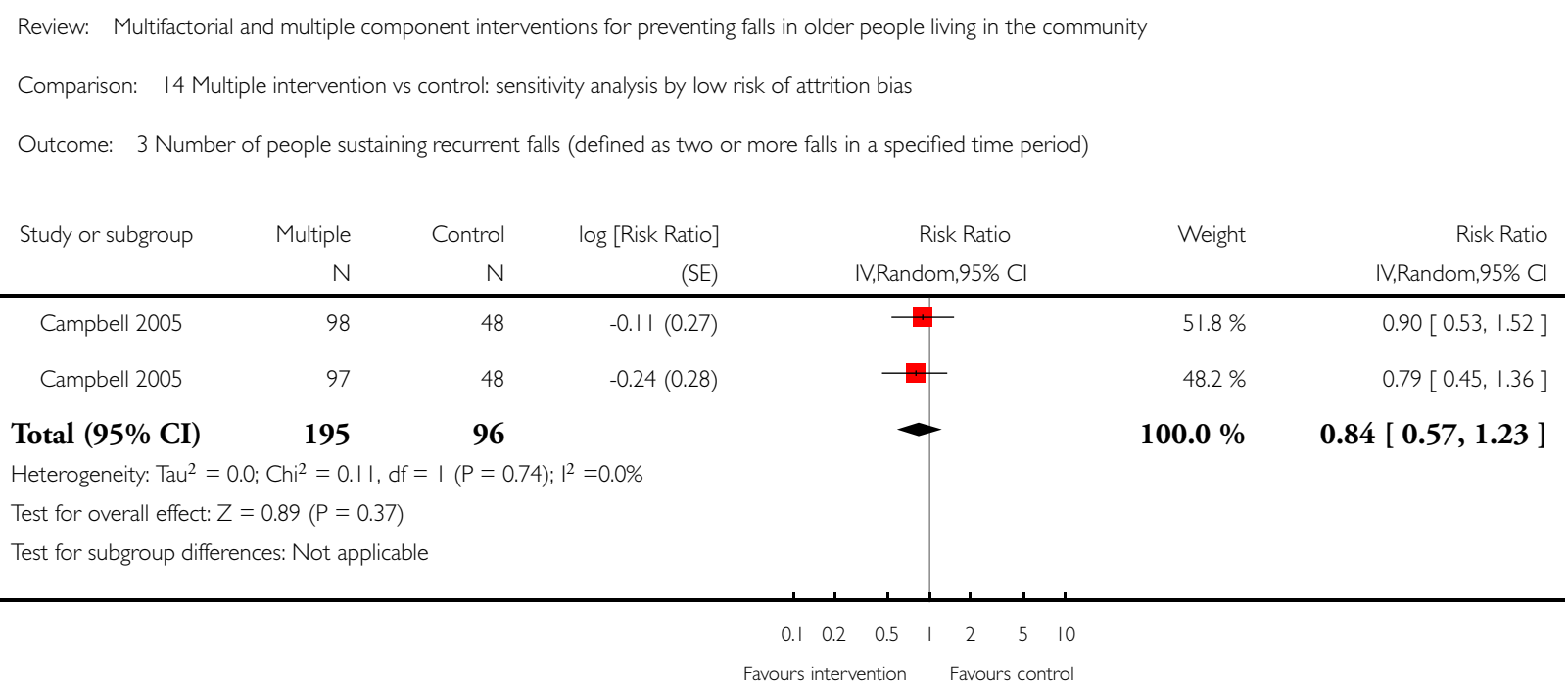


Analysis I5.I. Comparison I 5 Multiple intervention vs control: sensitivity analysis by individual randomisation, Outcome I Rate of falls (falls per person years).

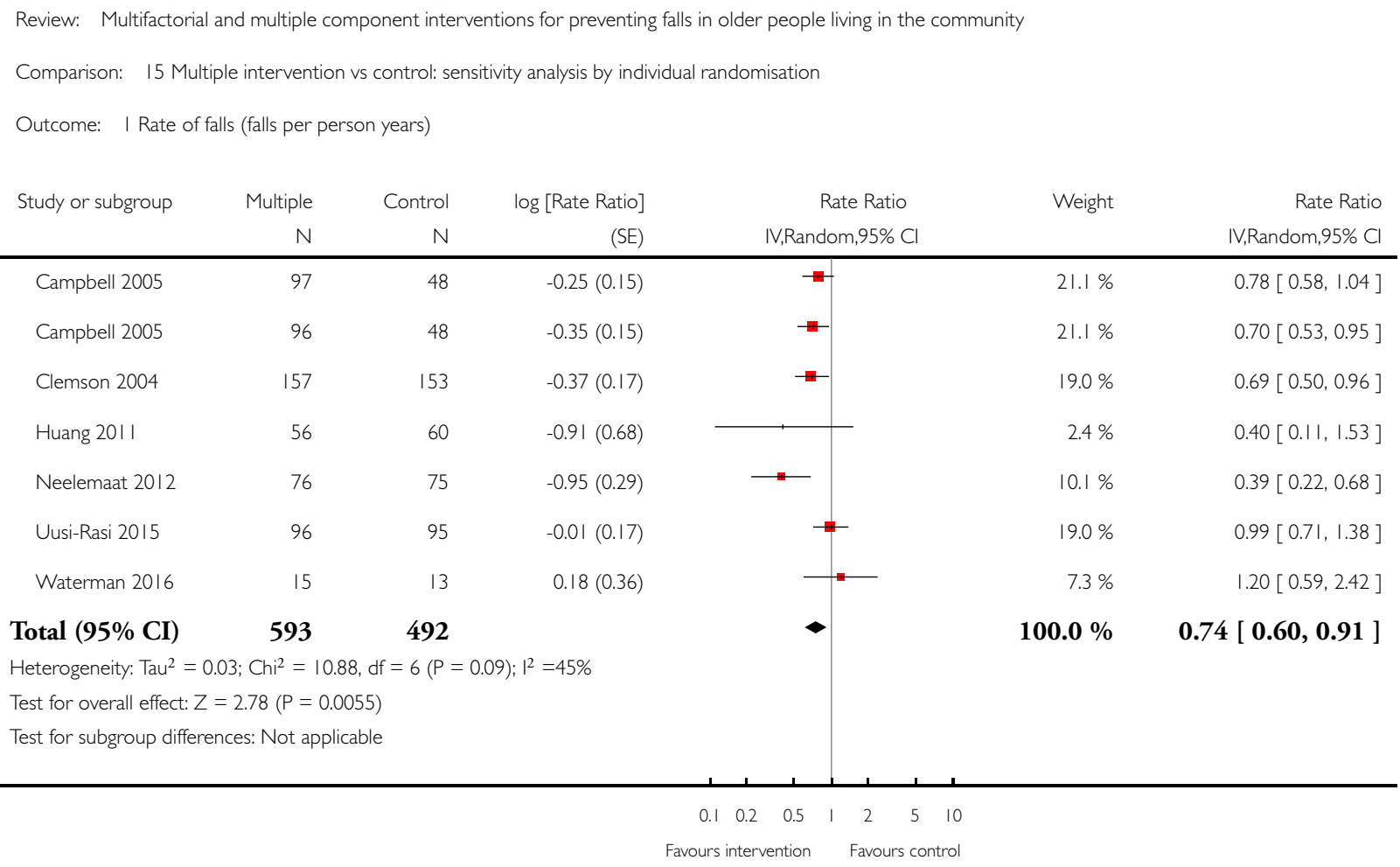


Analysis I5.2. Comparison I 5 Multiple intervention vs control: sensitivity analysis by individual randomisation, Outcome 2 Number of people sustaining one or more falls.

\begin{tabular}{|c|c|c|c|c|c|c|}
\hline \multicolumn{7}{|c|}{ Comparison: 15 Multiple intervention vs control: sensitivity analysis by individual randomisation } \\
\hline \multicolumn{7}{|c|}{ Outcome: 2 Number of people sustaining one or more falls } \\
\hline \multirow[t]{2}{*}{ Study or subgroup } & Multiple & Control & log [Risk Ratio] & Risk Ratio & Weight & \multirow{2}{*}{$\begin{array}{r}\text { Risk Ratio } \\
\text { IV,Random,95\% CI }\end{array}$} \\
\hline & $\mathrm{N}$ & $\mathrm{N}$ & (SE) & IV,Random,95\% Cl & & \\
\hline Campbell 2005 & 97 & 48 & $-0.26(0.15)$ & $\rightarrow$ & $10.9 \%$ & $0.77[0.57,1.03]$ \\
\hline Campbell 2005 & 98 & 48 & $-0.25(0.15)$ & $\rightarrow$ & $10.9 \%$ & $0.78[0.58,1.04]$ \\
\hline Clemson 2004 & 157 & 153 & $-0.11(0.1)$ & + & $24.6 \%$ & $0.90[0.74,1.09]$ \\
\hline Day 2002 & 137 & 34 & $-0.13(0.15)$ & $\rightarrow$ & $10.9 \%$ & $0.88[0.65,1.18]$ \\
\hline Day 2002 & 135 & 34 & $-0.3(0.15)$ & $\rightarrow$ & $10.9 \%$ & $0.74[0.55,0.99]$ \\
\hline Day 2002 & 135 & 34 & $-0.19(0.15)$ & $\rightarrow$ & $10.9 \%$ & $0.83[0.62,1.11]$ \\
\hline Day 2002 & 136 & 34 & $-0.29(0.15)$ & $\rightrightarrows$ & $10.9 \%$ & $0.75[0.56,1.00]$ \\
\hline Faes 2011 & 18 & 15 & $0.33(0.38)$ & +1 & $1.7 \%$ & $1.39[0.66,2.93]$ \\
\hline Huang 201 I & 56 & 60 & $-0.91(0.65)$ & \multirow{2}{*}{ 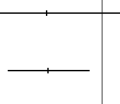 } & $0.6 \%$ & $0.40[0.11,1.44]$ \\
\hline Neelemaat 2012 & 105 & 105 & $-0.88(0.35)$ & & $2.0 \%$ & $0.41[0.21,0.82]$ \\
\hline Ng 2015 & 49 & 50 & $-0.9(0.81)$ & + & $0.4 \%$ & $0.41[0.08,1.99]$ \\
\hline Olsen 2014 & 47 & 42 & $0.05(0.35)$ & $\begin{array}{l}\square \\
\square\end{array}$ & $2.0 \%$ & $1.05[0.53,2.09]$ \\
\hline Waterman 2016 & 15 & 13 & $-0.03(0.3)$ & \begin{tabular}{l|l} 
\\
\end{tabular} & $2.7 \%$ & $0.97[0.54,1.75]$ \\
\hline Wesson 2013 & 11 & 11 & $-0.69(0.75)$ & \multirow[b]{2}{*}{ • } & $0.4 \%$ & $0.50[0.12,2.18]$ \\
\hline Total $(95 \% \mathrm{CI})$ & 1196 & 681 & & & $100.0 \%$ & $0.81[0.74,0.90]$ \\
\hline \multicolumn{7}{|c|}{ Heterogeneity: $\mathrm{Tau}^{2}=0.0 ; \mathrm{Chi}^{2}=11.01, \mathrm{df}=13(\mathrm{P}=0.61) ; \mathrm{I}^{2}=0.0 \%$} \\
\hline \multicolumn{7}{|c|}{ Test for overall effect: $Z=4.15(P=0.000033)$} \\
\hline \multicolumn{7}{|c|}{ Test for subgroup differences: Not applicable } \\
\hline
\end{tabular}


Analysis 15.3. Comparison I5 Multiple intervention vs control: sensitivity analysis by individual randomisation, Outcome 3 Number of people sustaining recurrent falls (defined as two or more falls in a specified time period).

\begin{tabular}{|c|c|c|c|c|c|c|}
\hline \multicolumn{7}{|c|}{ Comparison: 15 Multiple intervention vs control: sensitivity analysis by individual randomisation } \\
\hline \multicolumn{7}{|c|}{ Outcome: 3 Number of people sustaining recurrent falls (defined as two or more falls in a specified time period) } \\
\hline \multirow[t]{2}{*}{ Study or subgroup } & Multiple & Control & $\log$ [Risk Ratio] & Risk Ratio & Weight & Risk Ratio \\
\hline & $\mathrm{N}$ & $\mathrm{N}$ & (SE) & IV,Random,95\% Cl & & IV,Random,95\% Cl \\
\hline Campbell 2005 & 97 & 48 & $-0.24(0.28)$ & $\longrightarrow$ & $21.2 \%$ & $0.79[0.45,1.36]$ \\
\hline Campbell 2005 & 98 & 48 & $-0.11(0.27)$ & $\longrightarrow$ & $22.8 \%$ & $0.90[0.53,1.52]$ \\
\hline Clemson 2004 & 157 & 153 & $-0.3(0.18)$ & $\rightarrow$ & $51.2 \%$ & $0.74[0.52,1.05]$ \\
\hline Faes 2011 & 18 & 15 & $1.61(1.02)$ & - & $1.6 \%$ & $5.00[0.68,36.94]$ \\
\hline Waterman 2016 & 15 & 13 & $-0.14(0.72)$ & & $3.2 \%$ & $0.87[0.21,3.57]$ \\
\hline Total $(95 \% \mathrm{CI})$ & 385 & 277 & & 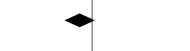 & $100.0 \%$ & $0.81[0.63,1.05]$ \\
\hline \multicolumn{7}{|c|}{ Heterogeneity: $\mathrm{Tau}^{2}=0.0 ; \mathrm{Chi}^{2}=3.59, \mathrm{df}=4(\mathrm{P}=0.46) ; \mathrm{I}^{2}=0.0 \%$} \\
\hline \multicolumn{7}{|c|}{ Test for overall effect: $Z=1.62(P=0.11)$} \\
\hline \multicolumn{7}{|c|}{ Test for subgroup differences: Not applicable } \\
\hline
\end{tabular}

\section{ADDITIONAL TABLES}

Table 1. Multifactorial interventions: study design, setting and trial size

\begin{tabular}{|c|c|c|c|c|c|c|c|c|}
\hline Study ID & $\begin{array}{l}\text { Study } \\
\text { design }\end{array}$ & No. arms & $\begin{array}{l}\text { Study cen- } \\
\text { tres }\end{array}$ & $\begin{array}{l}\text { Length of } \\
\text { follow-up }\end{array}$ & Setting & $\begin{array}{l}\text { No. } \\
\text { randomised }\end{array}$ & $\begin{array}{l}\text { No. } \\
\text { analysed }\end{array}$ & $\begin{array}{l}\% \text { lost to } \\
\text { follow-up }\end{array}$ \\
\hline $\begin{array}{l}\text { Beling } \\
2009\end{array}$ & Parallel & 2 & Single & 3 months & USA & 23 & 19 & $17 \%$ \\
\hline $\begin{array}{l}\text { Carpenter } \\
1990\end{array}$ & Parallel & 2 & Multiple & 36 months & $\begin{array}{l}\text { United } \\
\text { Kingdom }\end{array}$ & 539 & 367 & $32 \%$ \\
\hline $\begin{array}{l}\text { Carter } \\
1997\end{array}$ & Parallel & 3 & Unclear & 12 months & Australia & 657 & 457 & $30 \%$ \\
\hline $\begin{array}{l}\text { Ciaschini } \\
2009\end{array}$ & Parallel & 2 & Single & 12 months & Canada & 201 & 176 & $12 \%$ \\
\hline
\end{tabular}


Table 1. Multifactorial interventions: study design, setting and trial size (Continued)

\begin{tabular}{|c|c|c|c|c|c|c|c|c|}
\hline Close 1999 & Parallel & 2 & Unclear & 12 months & $\begin{array}{l}\text { United } \\
\text { Kingdom }\end{array}$ & 397 & 304 & $23 \%$ \\
\hline $\begin{array}{l}\text { Coleman } \\
1999\end{array}$ & Cluster & 2 & Multiple & 12 months & USA & 169 & 142 & $16 \%$ \\
\hline $\begin{array}{l}\text { Davison } \\
2005\end{array}$ & Parallel & 2 & Unclear & 12 months & $\begin{array}{l}\text { United } \\
\text { Kingdom }\end{array}$ & 313 & 282 & $10 \%$ \\
\hline $\begin{array}{l}\text { De Vries } \\
2010\end{array}$ & Parallel & 2 & Multiple & 12 months & $\begin{array}{l}\text { The Nether- } \\
\text { lands }\end{array}$ & 217 & 187 & $14 \%$ \\
\hline Elley 2008 & Parallel & 2 & Multiple & 12 months & $\begin{array}{l}\text { New } \\
\text { Zealand }\end{array}$ & 312 & 280 & $10 \%$ \\
\hline $\begin{array}{l}\text { Fabacher } \\
1994\end{array}$ & Parallel & 2 & Single & 12 months & USA & 254 & 195 & $23 \%$ \\
\hline $\begin{array}{l}\text { Fairhall } \\
2014\end{array}$ & Parallel & 2 & Single & 12 months & Australia & 241 & 216 & $10 \%$ \\
\hline Ferrer 2014 & Parallel & 2 & Single & 12 months & Spain & 328 & 273 & $17 \%$ \\
\hline $\begin{array}{l}\text { Gallagher } \\
1996\end{array}$ & Parallel & 2 & Unclear & 6 months & Canada & 100 & 100 & $0 \%$ \\
\hline $\begin{array}{l}\text { Hendriks } \\
2008\end{array}$ & Parallel & 2 & Single & 12 months & $\begin{array}{l}\text { The Nether- } \\
\text { lands }\end{array}$ & 333 & 258 & $23 \%$ \\
\hline $\begin{array}{l}\text { Hogan } \\
2001\end{array}$ & Parallel & 2 & Unclear & 24 months & Canada & 163 & 139 & $15 \%$ \\
\hline $\begin{array}{l}\text { Huang } \\
2005\end{array}$ & Parallel & 2 & Single & 3 months & Taiwan & 141 & 126 & $11 \%$ \\
\hline Imhof 2012 & Parallel & 2 & Single & 9 months & Switzerland & 461 & 413 & $10 \%$ \\
\hline $\begin{array}{l}\text { Jitapunkul } \\
1998\end{array}$ & Parallel & 2 & Unclear & 36 months & Thailand & 160 & 116 & $28 \%$ \\
\hline $\begin{array}{l}\text { Kingston } \\
2001\end{array}$ & Parallel & 2 & Single & 3 months & $\begin{array}{l}\text { United } \\
\text { Kingdom }\end{array}$ & 109 & 92 & $16 \%$ \\
\hline $\begin{array}{l}\text { Lightbody } \\
2002\end{array}$ & Parallel & 2 & Single & 6 months & $\begin{array}{l}\text { United } \\
\text { Kingdom }\end{array}$ & 348 & 314 & $10 \%$ \\
\hline Logan 2010 & Parallel & 2 & Unclear & 12 months & $\begin{array}{l}\text { United } \\
\text { Kingdom }\end{array}$ & 204 & 157 & $23 \%$ \\
\hline
\end{tabular}


Table 1. Multifactorial interventions: study design, setting and trial size (Continued)

\begin{tabular}{|c|c|c|c|c|c|c|c|c|}
\hline Lord 2005 & Parallel & 3 & Single & 12 months & Australia & 620 & 578 & $7 \%$ \\
\hline Luck 2013 & Parallel & 2 & Multiple & 18 months & Germany & 305 & 230 & $26 \%$ \\
\hline $\begin{array}{l}\text { Markle- } \\
\text { Reid } 2010\end{array}$ & Parallel & 4 & Multiple & 6 months & Canada & 109 & 92 & $16 \%$ \\
\hline $\begin{array}{l}\text { Metzelthin } \\
2013\end{array}$ & Cluster & 2 & Multiple & 24 months & $\begin{array}{l}\text { The Nether- } \\
\text { lands }\end{array}$ & 346 & 270 & $22 \%$ \\
\hline $\begin{array}{l}\text { Möller } \\
2014\end{array}$ & Parallel & 2 & Multiple & 12 months & Sweden & 153 & 106 & $31 \%$ \\
\hline $\begin{array}{l}\text { Newbury } \\
2001\end{array}$ & Parallel & 2 & Multiple & 12 months & Australia & 100 & 89 & $11 \%$ \\
\hline $\begin{array}{l}\text { Palvanen } \\
2014\end{array}$ & Parallel & 2 & Multiple & 12 months & Finland & 1314 & 1145 & $13 \%$ \\
\hline $\begin{array}{l}\text { Pardessus } \\
2002\end{array}$ & Parallel & 2 & Single & 12 months & France & 60 & 51 & $15 \%$ \\
\hline $\begin{array}{l}\text { Rubenstein } \\
2007\end{array}$ & Parallel & 2 & Single & 12 months & USA & 792 & 694 & $12 \%$ \\
\hline $\begin{array}{l}\text { Russell } \\
2010\end{array}$ & Parallel & 2 & Multiple & 12 months & Australia & 712 & 650 & $9 \%$ \\
\hline $\begin{array}{l}\text { Schrijne- } \\
\text { maekers } \\
1995\end{array}$ & Parallel & 2 & Single & 36 months & $\begin{array}{l}\text { The Nether- } \\
\text { lands }\end{array}$ & 222 & 182 & $18 \%$ \\
\hline $\begin{array}{l}\text { Sheffield } \\
2013\end{array}$ & Parallel & 2 & Single & 3 months & USA & 90 & 60 & $33 \%$ \\
\hline Shyu 2010 & Parallel & 2 & Single & 12 months & Taiwan & 162 & 122 & $25 \%$ \\
\hline Spice 2009 & Cluster & 3 & Multiple & 12 months & $\begin{array}{l}\text { United } \\
\text { Kingdom }\end{array}$ & 516 & 422 & $18 \%$ \\
\hline $\begin{array}{l}\text { Tinetti } \\
1994\end{array}$ & Cluster & 2 & Multiple & 12 months & USA & 301 & 291 & $3 \%$ \\
\hline Ueda $2017^{a}$ & Parallel & 2 & Single & 1 month & Japan & 60 & 51 & $15 \%$ \\
\hline $\begin{array}{l}\text { Van } \\
\text { Haastregt } \\
2000\end{array}$ & Parallel & 2 & Multiple & 18 months & $\begin{array}{l}\text { The Nether- } \\
\text { lands }\end{array}$ & 316 & 235 & $26 \%$ \\
\hline
\end{tabular}


Table 1. Multifactorial interventions: study design, setting and trial size (Continued)

\begin{tabular}{|c|c|c|c|c|c|c|c|c|}
\hline $\begin{array}{l}\text { Van } \\
\text { Rossum } \\
1993\end{array}$ & Parallel & 2 & Unclear & 36 months & $\begin{array}{l}\text { The Nether- } \\
\text { lands }\end{array}$ & 580 & 493 & $15 \%$ \\
\hline Vetter 1992 & Parallel & 2 & Single & 48 months & $\begin{array}{l}\text { United } \\
\text { Kingdom }\end{array}$ & 674 & 450 & $33 \%$ \\
\hline Vind 2009 & Parallel & 2 & Single & 12 months & Denmark & 392 & 364 & $7 \%$ \\
\hline $\begin{array}{l}\text { Wagner } \\
1994\end{array}$ & Parallel & 3 & Multiple & 24 months & USA & 1559 & Not reported & Not reported \\
\hline $\begin{array}{l}\text { Whitehead } \\
2003\end{array}$ & Parallel & 2 & Single & 6 months & Australia & 140 & 123 & $12 \%$ \\
\hline $\begin{array}{l}\text { Zijlstra } \\
2009\end{array}$ & Parallel & 2 & Multiple & 14 months & $\begin{array}{l}\text { The Nether- } \\
\text { lands }\end{array}$ & 540 & 405 & $25 \%$ \\
\hline
\end{tabular}

${ }^{a}$ Only trial with an active comparator (exercise)

Table 2. Multifactorial interventions: participants, intervention approach, comparator and compliance

\begin{tabular}{|c|c|c|c|c|c|c|}
\hline Study ID & Age (mean) & \% Women & High risk of falls & Active /referral & Comparator & $\begin{array}{l}\text { Compliance as- } \\
\text { sessed }\end{array}$ \\
\hline Beling 2009 & 80 & $42 \%$ & Yes & Active & Usual care & No \\
\hline Carpenter 1990 & $\geq 75$ years & $65 \%$ & No & Referral & Usual care & No \\
\hline Carter 1997 & $34 \%>80$ years & $66 \%$ & No & Referral & Usual care & Yes \\
\hline Ciaschini 2009 & 72 & $94 \%$ & Yes & Referral & Usual care & Yes \\
\hline Close 1999 & 78 & $68 \%$ & Yes & Active & Usual care & No \\
\hline Coleman 1999 & 77 & $49 \%$ & No & Active & Usual care & Yes \\
\hline Davison 2005 & 77 & $72 \%$ & Yes & Active & Usual care & Yes \\
\hline De Vries 2010 & 80 & $71 \%$ & Yes & Active & Usual care & Yes \\
\hline Elley 2008 & 81 & $69 \%$ & Yes & Referral & Attention control & Yes \\
\hline Fabacher 1994 & 73 & $2 \%$ & No & Referral & Usual care & Yes \\
\hline Fairhall 2014 & 83 & $68 \%$ & Yes & Active & Usual care & Yes \\
\hline
\end{tabular}


Table 2. Multifactorial interventions: participants, intervention approach, comparator and compliance

\begin{tabular}{|c|c|c|c|c|c|c|}
\hline Ferrer 2014 & 81 & $62 \%$ & Yes & Active & Usual care & Yes \\
\hline Gallagher 1996 & 75 & $80 \%$ & Yes & Referral & Usual care & Yes \\
\hline Hendriks 2008 & 75 & $68 \%$ & Yes & Referral & Usual care & Yes \\
\hline Hogan 2001 & 78 & $72 \%$ & Yes & Referral & Usual care & Yes \\
\hline Huang 2005 & 77 & $69 \%$ & Yes & Active & Usual care & No \\
\hline Imhof 2012 & 85 & $73 \%$ & Yes & Active & Usual care & No \\
\hline $\begin{array}{l}\text { Jitapunkul } \\
1998\end{array}$ & 76 & $65 \%$ & No & Referral & Usual care & No \\
\hline Kingston 2001 & 72 & $100 \%$ & Yes & Referral & Usual care & No \\
\hline $\begin{array}{l}\text { Lightbody } \\
2002\end{array}$ & $\begin{array}{l}\text { Median } 75 \text { (IQR } \\
70 \text { to } 81)\end{array}$ & $74 \%$ & Yes & Referral & Usual care & No \\
\hline Logan 2010 & $\begin{array}{l}\text { Median } 83 \text { (IQR } \\
77 \text { to } 86)\end{array}$ & $65 \%$ & Yes & Active & Usual care & No \\
\hline Lord 2005 & 80 & $66 \%$ & Yes & $\begin{array}{l}\text { Active and Refer- } \\
\text { ral }\end{array}$ & Usual care & Yes \\
\hline Luck 2013 & 85 & $69 \%$ & No & Active & Usual care & Yes \\
\hline $\begin{array}{l}\text { Markle-Reid } \\
2010\end{array}$ & Range 75 to 84 & $72 \%$ & Yes & Active & Usual care & Yes \\
\hline $\begin{array}{l}\text { Metzelthin } \\
2013\end{array}$ & 77 & $58 \%$ & Yes & Referral & Usual care & No \\
\hline Möller 2014 & 82 & $67 \%$ & No & Active & Usual care & No \\
\hline Newbury 2001 & Median 79 & $63 \%$ & No & Referral & Usual care & No \\
\hline Palvanen 2014 & 77 & $86 \%$ & Yes & Referral & Attention control & Yes \\
\hline Pardessus 2002 & 83 & $78 \%$ & Yes & Referral & Usual care & Yes \\
\hline $\begin{array}{l}\text { Rubenstein } \\
2007\end{array}$ & 75 & $3 \%$ & Yes & Referral & Usual care & Yes \\
\hline Russell 2010 & $\geq 75(51 \%)$ & $70 \%$ & Yes & Referral & Usual care & Yes \\
\hline
\end{tabular}


Table 2. Multifactorial interventions: participants, intervention approach, comparator and compliance

(Continued)

\begin{tabular}{|c|c|c|c|c|c|c|}
\hline $\begin{array}{l}\text { Schrijnemaek- } \\
\text { ers } \\
1995\end{array}$ & $>77$ & $70 \%$ & Yes & Referral & Usual care & Yes \\
\hline Sheffield 2013 & 82 & $80 \%$ & No & Active & Usual care & No \\
\hline Shyu 2010 & 78 & $69 \%$ & Yes & Active & Usual care & No \\
\hline Spice 2009 & 82 & Not reported & Yes & Active & Usual care & Yes \\
\hline Tinetti 1994 & 79 & $69 \%$ & Yes & Active & Usual care & Yes \\
\hline Ueda $2017^{a}$ & 76 & $69 \%$ & Yes & Active & Exercise & No \\
\hline $\begin{array}{l}\text { Van Haastregt } \\
2000\end{array}$ & 77 & $66 \%$ & Yes & Referral & Usual care & No \\
\hline $\begin{array}{l}\text { Van Rossum } \\
1993\end{array}$ & Range 75 to 84 & $58 \%$ & No & Referral & Usual care & No \\
\hline Vetter 1992 & $>70$ & Not reported & No & Referral & Usual care & Yes \\
\hline Vind 2009 & 74 & $74 \%$ & Yes & Active & Usual care & No \\
\hline Wagner 1994 & 72 & $59 \%$ & No & Referral & Usual care & Yes \\
\hline $\begin{array}{l}\text { Whitehead } \\
2003\end{array}$ & 78 & $71 \%$ & Yes & Referral & Usual care & Yes \\
\hline Zijlstra 2009 & 75 & $72 \%$ & No & Active & Usual care & Yes \\
\hline
\end{tabular}

$\bar{a}$ Only trial with an active comparator (exercise).

Table 3. Multifactorial interventions: key components of the interventions ${ }^{a}$

\begin{tabular}{|c|c|c|c|c|c|c|c|c|c|}
\hline Study ID & $\begin{array}{l}\text { Referral / } \\
\text { active }\end{array}$ & Exercise & $\begin{array}{l}\text { Medica- } \\
\text { tion (drug } \\
\text { target) }\end{array}$ & $\begin{array}{l}\text { Medi- } \\
\text { cation (re- } \\
\text { view) }\end{array}$ & Surgery & $\begin{array}{l}\text { Manage- } \\
\text { ment } \\
\text { of urinary } \\
\text { inconti- } \\
\text { nence }\end{array}$ & $\begin{array}{l}\text { Fluid or } \\
\text { nutrition } \\
\text { therapy }\end{array}$ & $\begin{array}{l}\text { Psycho- } \\
\text { logical in- } \\
\text { tervention }\end{array}$ & $\begin{array}{l}\text { Environ- } \\
\text { ment/ } \\
\text { assis- } \\
\text { tive tech- } \\
\text { nology } \\
\text { (external) }\end{array}$ \\
\hline $\begin{array}{l}\text { Beling } \\
2009\end{array}$ & Active & $\begin{array}{l}\text { Balance } \\
\text { training to } \\
\text { address } \\
\text { risk factors }\end{array}$ & - & $\begin{array}{l}\text { Medica- } \\
\text { tion review }\end{array}$ & - & - & - & - & $\begin{array}{l}\text { Home as- } \\
\text { sessment } \\
\text { for falls } \\
\text { risk } \\
\text { with writ- }\end{array}$ \\
\hline
\end{tabular}

Multifactorial and multiple component interventions for preventing falls in older people living in the community (Review) 
Table 3. Multifactorial interventions: key components of the interventions ${ }^{a} \quad$ (Continued)

\begin{tabular}{|c|c|c|c|c|c|c|c|}
\hline & & & & & & & $\begin{array}{l}\text { ten recom- } \\
\text { menda- } \\
\text { tions }\end{array}$ \\
\hline $\begin{array}{l}\text { Carpenter } \\
1990\end{array}$ & Referral & - & - & - & - & $\begin{array}{l}\text { Referral to } \\
\text { psy- } \\
\text { chogeri- } \\
\text { atric } \\
\text { day hospi- } \\
\text { tal or nurs- } \\
\text { ing services }\end{array}$ & $\begin{array}{l}\text { Refer- } \\
\text { ral for aids } \\
\text { to daily liv- } \\
\text { ing, e.g. } \\
\text { bath seat }\end{array}$ \\
\hline $\begin{array}{l}\text { Carter } \\
1997 \\
\text { (group 1) }\end{array}$ & Referral & - & - & - & - & - & $\begin{array}{l}\text { Home as- } \\
\text { sessment } \\
\text { for falls } \\
\text { risk with } \\
\text { written } \\
\text { summary } \\
\text { of hazards }\end{array}$ \\
\hline $\begin{array}{l}\text { Carter } \\
1997 \\
\text { (group 2) }\end{array}$ & Referral & - & $\begin{array}{l}\text { Medica- } \\
\text { tion review }\end{array}$ & - & - & - & $\begin{array}{l}\text { Home as- } \\
\text { sessment } \\
\text { for falls } \\
\text { risk with } \\
\text { written } \\
\text { summary } \\
\text { of hazards } \\
\text { and refer- } \\
\text { ral to local } \\
\text { services to } \\
\text { make } \\
\text { changes }\end{array}$ \\
\hline $\begin{array}{l}\text { Ciaschini } \\
2009\end{array}$ & Referral & $\begin{array}{l}\text { Refer- } \\
\text { ral to phys- } \\
\text { iotherapy } \\
\text { (strength- } \\
\text { ening, gait } \\
\text { and bal- } \\
\text { ance train- } \\
\text { ing, refer- } \\
\text { ral to activ- } \\
\text { ities such } \\
\text { as Tai Chi) }\end{array}$ & $\begin{array}{l}\text { Medica- } \\
\text { tion review }\end{array}$ & - & - & $\begin{array}{l}\text { Referral to } \\
\text { occupa- } \\
\text { tional ther- } \\
\text { apy (cogni- } \\
\text { tive assess- } \\
\text { ment) }\end{array}$ & $\begin{array}{l}\text { Referral to } \\
\text { occupa- } \\
\text { tional ther- } \\
\text { apy (home } \\
\text { environ- } \\
\text { mental as- } \\
\text { sessment) }\end{array}$ \\
\hline $\begin{array}{l}\text { Close } \\
1999\end{array}$ & Active & - & $\begin{array}{l}\text { Medica- } \\
\text { tion review }\end{array}$ & - & - & $\begin{array}{l}\text { Cog- } \\
\text { nition and } \\
\text { depression } \\
\text { assessment }\end{array}$ & $\begin{array}{l}\text { Occupa- } \\
\text { tional ther- } \\
\text { apy home } \\
\text { visit assess- }\end{array}$ \\
\hline
\end{tabular}

Multifactorial and multiple component interventions for preventing falls in older people living in the community (Review) 
Table 3. Multifactorial interventions: key components of the interventions ${ }^{a} \quad$ (Continued)

\begin{tabular}{|c|c|c|c|c|c|c|c|c|}
\hline & & & & & & & & $\begin{array}{l}\text { ing envi- } \\
\text { ronmen- } \\
\text { tal hazards } \\
\text { with home } \\
\text { modifica- } \\
\text { tions }\end{array}$ \\
\hline $\begin{array}{l}\text { Coleman } \\
1999\end{array}$ & Active & $\begin{array}{l}\text { Problem } \\
\text { solving } \\
\text { on physical } \\
\text { activity }\end{array}$ & - & $\begin{array}{l}\text { Session } \\
\text { with phar- } \\
\text { macist ad- } \\
\text { dressing } \\
\text { polyphar- } \\
\text { macy and } \\
\text { medica- } \\
\text { tions asso- } \\
\text { ciated with } \\
\text { functional } \\
\text { decline }\end{array}$ & - & $\begin{array}{l}\text { Problem- } \\
\text { solving on } \\
\text { nutrition }\end{array}$ & $\begin{array}{l}\text { Self- } \\
\text { manage- } \\
\text { ment skills } \\
\text { and group } \\
\text { problem- } \\
\text { solving }\end{array}$ & \\
\hline $\begin{array}{l}\text { Davison } \\
2005\end{array}$ & Active & $\begin{array}{l}\text { Physio- } \\
\text { therapist } \\
\text { assessment } \\
\text { of gait and } \\
\text { balance, } \\
\text { functional } \\
\text { training } \\
\text { pro- } \\
\text { gramme }\end{array}$ & $\begin{array}{l}\text { Medica- } \\
\text { tion } \\
\text { to achieve } \\
\text { target } \\
\text { blood pres- } \\
\text { sure }\end{array}$ & $\begin{array}{l}\text { Medica- } \\
\text { tion review }\end{array}$ & - & - & $\begin{array}{l}\text { Neurolog- } \\
\text { ical exami- } \\
\text { nation }\end{array}$ & $\begin{array}{l}\text { Occupa- } \\
\text { tional ther- } \\
\text { apy home } \\
\text { visit assess- } \\
\text { ing envi- } \\
\text { ronmen- } \\
\text { tal hazards } \\
\text { with home } \\
\text { modifica- } \\
\text { tions } \\
\text { and assis- } \\
\text { tive devices }\end{array}$ \\
\hline $\begin{array}{l}\text { De Vries } \\
2010\end{array}$ & Active & $\begin{array}{l}\text { Balance } \\
\text { and } \\
\text { strength } \\
\text { exercises }\end{array}$ & Vitamin D & $\begin{array}{l}\text { Medica- } \\
\text { tion review }\end{array}$ & - & - & - & $\begin{array}{l}\text { Home haz- } \\
\text { ard reduc- } \\
\text { tion }\end{array}$ \\
\hline Elley 2008 & Referral & $\begin{array}{l}\text { Strength } \\
\text { and } \\
\text { balance ex- } \\
\text { ercise pro- } \\
\text { gramme }\end{array}$ & $\begin{array}{l}\text { Vitamin D } \\
\text { and } \\
\text { calcium }\end{array}$ & $\begin{array}{l}\text { Medica- } \\
\text { tion review }\end{array}$ & - & - & - & $\begin{array}{l}\text { Home haz- } \\
\text { ard assess- } \\
\text { ment } \\
\text { with home } \\
\text { modi- } \\
\text { fications or } \\
\text { referral } \\
\text { to occupa- } \\
\text { tional ther- } \\
\text { apy service }\end{array}$ \\
\hline
\end{tabular}


Table 3. Multifactorial interventions: key components of the interventions ${ }^{a} \quad$ (Continued)

\begin{tabular}{|c|c|c|c|c|c|c|c|c|c|}
\hline $\begin{array}{l}\text { Fabacher } \\
1994\end{array}$ & Referral & $\begin{array}{l}\text { Gait and } \\
\text { balance as- } \\
\text { sessment }\end{array}$ & - & $\begin{array}{l}\text { Medica- } \\
\text { tion review }\end{array}$ & - & - & - & $\begin{array}{l}\text { Mental sta- } \\
\text { tus exami- } \\
\text { nation }\end{array}$ & $\begin{array}{l}\text { Home haz- } \\
\text { ard assess- } \\
\text { ment }\end{array}$ \\
\hline $\begin{array}{l}\text { Fairhall } \\
2014\end{array}$ & Active & $\begin{array}{l}\text { Physio- } \\
\text { ther- } \\
\text { apy visits, } \\
\text { strength } \\
\text { and bal- } \\
\text { ance train- } \\
\text { ing }\end{array}$ & - & - & - & $\begin{array}{l}\text { Referral to } \\
\text { urinary in- } \\
\text { continence } \\
\text { clinic }\end{array}$ & $\begin{array}{l}\text { Nutrition } \\
\text { assessment } \\
\text { and man- } \\
\text { agement }\end{array}$ & - & $\begin{array}{l}\text { Home haz- } \\
\text { ard assess- } \\
\text { ment } \\
\text { with home } \\
\text { modifica- } \\
\text { tions, mo- } \\
\text { bility aids } \\
\text { and safety } \\
\text { advice, re- } \\
\text { ferral } \\
\text { to occupa- } \\
\text { tional ther- } \\
\text { apist }\end{array}$ \\
\hline $\begin{array}{l}\text { Ferrer } \\
2014\end{array}$ & Referral & $\begin{array}{l}\text { Gait and } \\
\text { balance as- } \\
\text { sessment, } \\
\text { referral for } \\
\text { physical } \\
\text { therapy }\end{array}$ & - & $\begin{array}{l}\text { Med- } \\
\text { ication re- } \\
\text { view, rec- } \\
\text { ommen- } \\
\text { dations to } \\
\text { dis- } \\
\text { cuss medi- } \\
\text { cation with } \\
\text { physician }\end{array}$ & - & - & $\begin{array}{l}\text { Malnutri- } \\
\text { tion } \\
\text { screen- } \\
\text { ing, nutri- } \\
\text { tion or vi- } \\
\text { tamin sup- } \\
\text { plementa- } \\
\text { tion }\end{array}$ & $\begin{array}{l}\text { Cogni- } \\
\text { tive screen- } \\
\text { ing, educa- } \\
\text { tion, refer- } \\
\text { ral to } \\
\text { physician } \\
\text { for fur- } \\
\text { ther cogni- } \\
\text { tive testing }\end{array}$ & $\begin{array}{l}\text { Home haz- } \\
\text { ard assess- } \\
\text { ment } \\
\text { with home } \\
\text { modifica- } \\
\text { tions and } \\
\text { recom- } \\
\text { menda- } \\
\text { tions }\end{array}$ \\
\hline $\begin{array}{l}\text { Gallagher } \\
1996^{2}\end{array}$ & Referral & - & - & - & - & - & - & - & \\
\hline $\begin{array}{l}\text { Hendriks } \\
2008\end{array}$ & Referral & $\begin{array}{l}\text { Assess- } \\
\text { ment by re- } \\
\text { habilita- } \\
\text { tion physi- } \\
\text { cian }\end{array}$ & - & - & - & - & - & - & $\begin{array}{l}\text { Home haz- } \\
\text { ard assess- } \\
\text { ment } \\
\text { with home } \\
\text { modifica- } \\
\text { tions, mo- } \\
\text { bility aids } \\
\text { and safety } \\
\text { advice }\end{array}$ \\
\hline $\begin{array}{l}\text { Hogan } \\
2001\end{array}$ & Referral & $\begin{array}{l}\text { Balance } \\
\text { and gait as- } \\
\text { sessment, } \\
\text { referral } \\
\text { to exercise } \\
\text { class, rec- } \\
\text { ommen- } \\
\text { dations for }\end{array}$ & - & $\begin{array}{l}\text { Medica- } \\
\text { tion review }\end{array}$ & - & - & - & $\begin{array}{l}\text { Neuro- } \\
\text { logic } \\
\text { screening }\end{array}$ & $\begin{array}{l}\text { Home haz- } \\
\text { ard assess- } \\
\text { ment with } \\
\text { recom- } \\
\text { menda- } \\
\text { tions, ad- } \\
\text { vice on as- }\end{array}$ \\
\hline
\end{tabular}

Multifactorial and multiple component interventions for preventing falls in older people living in the community (Review) 
Table 3. Multifactorial interventions: key components of the interventions ${ }^{a} \quad$ (Continued)

\begin{tabular}{|c|c|c|c|c|c|c|c|c|c|}
\hline & & $\begin{array}{l}\text { home exer- } \\
\text { cise }\end{array}$ & & & & & & & $\begin{array}{l}\text { sistive de- } \\
\text { vices }\end{array}$ \\
\hline $\begin{array}{l}\text { Huang } \\
2005\end{array}$ & Active & $\begin{array}{l}\text { Assess- } \\
\text { ment of re- } \\
\text { habil- } \\
\text { itation fa- } \\
\text { cility needs }\end{array}$ & - & $\begin{array}{l}\text { Education } \\
\text { on medica- } \\
\text { tion }\end{array}$ & - & - & - & - & $\begin{array}{l}\text { Ed- } \\
\text { ucation on } \\
\text { environ- } \\
\text { mental sa- } \\
\text { fety, assis- } \\
\text { tance } \\
\text { devices }\end{array}$ \\
\hline $\begin{array}{l}\text { Imhof } \\
2012\end{array}$ & Active & $\begin{array}{l}\text { Mobility } \\
\text { assessment }\end{array}$ & $\begin{array}{l}\text { Pain assess- } \\
\text { ment }\end{array}$ & - & - & - & $\begin{array}{l}\mathrm{Nu}- \\
\text { trition and } \\
\text { bladder } \\
\text { control as- } \\
\text { sessments }\end{array}$ & $\begin{array}{l}\text { Cognitive } \\
\text { screening }\end{array}$ & \\
\hline $\begin{array}{l}\text { Jita- } \\
\text { punkul } \\
1998\end{array}$ & Referral & $\begin{array}{l}\text { Nurse- } \\
\text { provided } \\
\text { rehabili- } \\
\text { tation pro- } \\
\text { gramme }\end{array}$ & $\begin{array}{l}\text { Medi- } \\
\text { cation pre- } \\
\text { scription }\end{array}$ & - & - & - & - & - & $\begin{array}{l}\text { Assistive } \\
\text { aids }\end{array}$ \\
\hline $\begin{array}{l}\text { Kingston } \\
2001\end{array}$ & Referral & $\begin{array}{l}\text { Advice on } \\
\text { exercise to } \\
\text { strengthen } \\
\text { muscles } \\
\text { and joints }\end{array}$ & $\begin{array}{l}\text { Pain } \\
\text { control ad- } \\
\text { vice, medi- } \\
\text { cation }\end{array}$ & $\begin{array}{l}\text { Advice on } \\
\text { risk factors } \\
\text { related to } \\
\text { drugs }\end{array}$ & - & - & $\begin{array}{l}\text { Advice on } \\
\text { diet and vi- } \\
\text { tamin sup- } \\
\text { plementa- } \\
\text { tion }\end{array}$ & - & $\begin{array}{l}\text { Educa- } \\
\text { tion on en- } \\
\text { vironmen- } \\
\text { tal risks in } \\
\text { the home }\end{array}$ \\
\hline $\begin{array}{l}\text { Light- } \\
\text { body } \\
2002\end{array}$ & Referral & $\begin{array}{l}\text { Balance } \\
\text { and mobil- } \\
\text { ity assess- } \\
\text { ment, } \\
\text { refer- } \\
\text { ral to phys- } \\
\text { iotherapy, } \\
\text { advised on } \\
\text { simple ex- } \\
\text { ercises }\end{array}$ & - & $\begin{array}{l}\text { Medica- } \\
\text { tion review }\end{array}$ & - & - & - & $\begin{array}{l}\text { Cogni- } \\
\text { tive screen- } \\
\text { ing, refer- } \\
\text { ral to GP }\end{array}$ & $\begin{array}{l}\text { Home haz- } \\
\text { ard assess- } \\
\text { ment } \\
\text { with home } \\
\text { modifica- } \\
\text { tions and } \\
\text { recom- } \\
\text { menda- } \\
\text { tions }\end{array}$ \\
\hline $\begin{array}{l}\text { Logan } \\
2010\end{array}$ & Active & $\begin{array}{l}\text { Strength } \\
\text { and bal- } \\
\text { ance train- } \\
\text { ing }\end{array}$ & - & $\begin{array}{l}\text { Medica- } \\
\text { tion review }\end{array}$ & - & - & - & - & $\begin{array}{l}\text { Home haz- } \\
\text { ard assess- } \\
\text { ment } \\
\text { with home } \\
\text { modifica- } \\
\text { tions and } \\
\text { recom- } \\
\text { menda- } \\
\text { tions }\end{array}$ \\
\hline
\end{tabular}

Multifactorial and multiple component interventions for preventing falls in older people living in the community (Review) 
Table 3. Multifactorial interventions: key components of the interventions ${ }^{a} \quad$ (Continued)

\begin{tabular}{|c|c|c|c|c|c|c|c|c|c|}
\hline Lord 2005 & Active & $\begin{array}{l}\text { Strength } \\
\text { and } \\
\text { balance ex- } \\
\text { ercise pro- } \\
\text { gramme }\end{array}$ & - & - & $\begin{array}{l}\text { Referral } \\
\text { for cataract } \\
\text { surgery }\end{array}$ & - & - & - & $\begin{array}{l}\text { Ad- } \\
\text { vice on en- } \\
\text { vironmen- } \\
\text { tal risks }\end{array}$ \\
\hline $\begin{array}{l}\text { Luck } \\
2013\end{array}$ & Active & - & - & - & - & - & $\begin{array}{l}\text { Consulta- } \\
\text { tion } \\
\text { with nutri- } \\
\text { tionist }\end{array}$ & - & \\
\hline $\begin{array}{l}\text { Markle- } \\
\text { Reid } 2010\end{array}$ & Active & $\begin{array}{l}\text { Home sup- } \\
\text { port ex- } \\
\text { ercise pro- } \\
\text { gram }\end{array}$ & $\begin{array}{l}\text { Advice } \\
\text { to consider } \\
\text { vitamin } \\
\mathrm{D} \text { and cal- } \\
\text { cium sup- } \\
\text { plementa- } \\
\text { tion }\end{array}$ & $\begin{array}{l}\text { Medica- } \\
\text { tion review } \\
\text { and modi- } \\
\text { fication }\end{array}$ & - & $\begin{array}{l}\text { Incon- } \\
\text { tinence as- } \\
\text { sessment, } \\
\text { referral } \\
\text { to GP, ed- } \\
\text { ucation on } \\
\text { pelvic floor } \\
\text { exercises }\end{array}$ & $\begin{array}{l}\text { Nutri- } \\
\text { tion assess- } \\
\text { ment, re- } \\
\text { ferral to di- } \\
\text { etician }\end{array}$ & $\begin{array}{l}\text { Cogni- } \\
\text { tive assess- } \\
\text { ment, } \\
\text { referral to } \\
\text { physician } \\
\text { or commu- } \\
\text { nity men- } \\
\text { tal health } \\
\text { services }\end{array}$ & $\begin{array}{l}\text { Home haz- } \\
\text { ard assess- } \\
\text { ment } \\
\text { with home } \\
\text { modifica- } \\
\text { tions and } \\
\text { recom- } \\
\text { menda- } \\
\text { tions }\end{array}$ \\
\hline $\begin{array}{l}\text { Met- } \\
\text { zelthin } \\
2013\end{array}$ & Referral & $\begin{array}{l}\text { Assess- } \\
\text { ment } \\
\text { by physio- } \\
\text { therapist, } \\
\text { advice on } \\
\text { daily phys- } \\
\text { ical activity }\end{array}$ & - & - & - & - & - & - & $\begin{array}{l}\text { Assess- } \\
\text { ment } \\
\text { by occupa- } \\
\text { tional ther- } \\
\text { apist, rec- } \\
\text { ommen- } \\
\text { dations on } \\
\text { environ- } \\
\text { men- } \\
\text { tal adapta- } \\
\text { tions }\end{array}$ \\
\hline $\begin{array}{l}\text { Möller } \\
2014\end{array}$ & Active & $\begin{array}{l}\text { Tailored } \\
\text { exercise } \\
\text { program, } \\
\text { referral } \\
\text { to physical } \\
\text { therapist }\end{array}$ & - & - & - & - & - & - & $\begin{array}{l}\text { Home haz- } \\
\text { ard assess- } \\
\text { ment } \\
\text { with home } \\
\text { modifica- } \\
\text { tions and } \\
\text { recom- } \\
\text { men- } \\
\text { dations, re- } \\
\text { ferral } \\
\text { to occupa- } \\
\text { tional ther- } \\
\text { apist }\end{array}$ \\
\hline $\begin{array}{l}\text { Newbury } \\
2001^{b}\end{array}$ & Referral & - & - & - & - & & - & - & \\
\hline
\end{tabular}

Multifactorial and multiple component interventions for preventing falls in older people living in the community (Review) 
Table 3. Multifactorial interventions: key components of the interventions ${ }^{a} \quad$ (Continued)

\begin{tabular}{|c|c|c|c|c|c|c|c|c|c|}
\hline $\begin{array}{l}\text { Palvanen } \\
2014\end{array}$ & Referral & $\begin{array}{l}\text { Phys- } \\
\text { ical activity } \\
\text { prescrip- } \\
\text { tion, indi- } \\
\text { vidually } \\
\text { tailored or } \\
\text { group ex- } \\
\text { ercise }\end{array}$ & - & $\begin{array}{l}\text { Medica- } \\
\text { tion review }\end{array}$ & $\begin{array}{l}\text { Referral } \\
\text { for cataract } \\
\text { surgery }\end{array}$ & - & $\begin{array}{l}\text { Nutri- } \\
\text { tional ad- } \\
\text { vice }\end{array}$ & - & $\begin{array}{l}\text { Home haz- } \\
\text { ard assess- } \\
\text { ment } \\
\text { with home } \\
\text { modifica- } \\
\text { tions and } \\
\text { recom- } \\
\text { men- } \\
\text { dations, re- } \\
\text { ferral } \\
\text { to occupa- } \\
\text { tional ther- } \\
\text { apist }\end{array}$ \\
\hline $\begin{array}{l}\text { Pardessus } \\
2002\end{array}$ & Referral & $\begin{array}{l}\text { Physi- } \\
\text { cal therapy } \\
\text { (both } \\
\text { arms) }\end{array}$ & - & $\begin{array}{l}\text { Med- } \\
\text { ication re- } \\
\text { view (both } \\
\text { arms) }\end{array}$ & - & - & - & $\begin{array}{l}\text { Cognitive } \\
\text { assessment } \\
\text { (both } \\
\text { arms) }\end{array}$ & $\begin{array}{l}\text { Home haz- } \\
\text { ard assess- } \\
\text { ment } \\
\text { with home } \\
\text { modifica- } \\
\text { tions and } \\
\text { recom- } \\
\text { menda- } \\
\text { tions }\end{array}$ \\
\hline $\begin{array}{l}\text { Ruben- } \\
\text { stein } \\
2007\end{array}$ & Referral & $\begin{array}{l}\text { Physio- } \\
\text { therapy as- } \\
\text { sessment } \\
\text { of falls and } \\
\text { gait im- } \\
\text { pairment }\end{array}$ & - & - & - & $\begin{array}{l}\text { Urinary in- } \\
\text { con- } \\
\text { tinence as- } \\
\text { sessment, } \\
\text { treat- } \\
\text { ment over- } \\
\text { seen by ex- } \\
\text { pert geria- } \\
\text { trician }\end{array}$ & - & $\begin{array}{l}\text { Cogni- } \\
\text { tive assess- } \\
\text { ment, } \\
\text { referral for } \\
\text { mental } \\
\text { health sup- } \\
\text { port, refer- } \\
\text { ral to geri- } \\
\text { atric psy- } \\
\text { chiatrist }\end{array}$ & \\
\hline $\begin{array}{l}\text { Russell } \\
2010\end{array}$ & Referral & $\begin{array}{l}\text { Refer- } \\
\text { ral to phys- } \\
\text { iotherapy }\end{array}$ & - & $\begin{array}{l}\text { Med- } \\
\text { ication re- } \\
\text { view, refer- } \\
\text { ral to GP }\end{array}$ & - & - & $\begin{array}{l}\text { Referral to } \\
\text { dietetics }\end{array}$ & - & $\begin{array}{l}\text { Referral to } \\
\text { occupa- } \\
\text { tional ther- } \\
\text { apy, advice } \\
\text { on minor } \\
\text { home im- } \\
\text { prove- } \\
\text { ments }\end{array}$ \\
\hline $\begin{array}{l}\text { Schrijne- } \\
\text { maekers } \\
1995\end{array}$ & Referral & $\begin{array}{l}\text { Refer- } \\
\text { ral to phys- } \\
\text { iotherapy }\end{array}$ & $\begin{array}{l}\text { Advice } \\
\text { to stop / } \\
\text { start medi- } \\
\text { cation }\end{array}$ & $\begin{array}{l}\text { Medica- } \\
\text { tion review }\end{array}$ & - & - & $\begin{array}{l}\text { Advice on } \\
\text { diet }\end{array}$ & $\begin{array}{l}\text { Referral } \\
\text { to psychol- } \\
\text { ogist }\end{array}$ & \\
\hline
\end{tabular}

Multifactorial and multiple component interventions for preventing falls in older people living in the community (Review) 
Table 3. Multifactorial interventions: key components of the interventions ${ }^{a} \quad$ (Continued)

\begin{tabular}{|c|c|c|c|c|c|c|c|c|c|}
\hline $\begin{array}{l}\text { Sheffield } \\
2013\end{array}$ & Active & - & $\begin{array}{l}\text { Training in } \\
\text { medica- } \\
\text { tion man- } \\
\text { agement }\end{array}$ & - & - & - & - & - & $\begin{array}{l}\text { Home haz- } \\
\text { ard assess- } \\
\text { ment } \\
\text { with home } \\
\text { modifica- } \\
\text { tions and } \\
\text { recom- } \\
\text { menda- } \\
\text { tions, pro- } \\
\text { vision } \\
\text { of assistive } \\
\text { devices }\end{array}$ \\
\hline $\begin{array}{l}\text { Shyu } \\
2010\end{array}$ & Active & $\begin{array}{l}\text { Rehabili- } \\
\text { tation plan } \\
\text { including } \\
\text { exercise } \\
\text { to increase } \\
\text { physical } \\
\text { fitness and } \\
\text { home exer- } \\
\text { cise } \\
\text { sessions by } \\
\text { nurses }\end{array}$ & $\begin{array}{l}\text { Sug- } \\
\text { gestions on } \\
\text { antibiotics }\end{array}$ & $\begin{array}{l}\text { Medica- } \\
\text { tion review }\end{array}$ & $\begin{array}{l}\text { Sug- } \\
\text { gestions to } \\
\text { surgeon re- } \\
\text { garding } \\
\text { time of hip } \\
\text { fracture } \\
\text { surgery }\end{array}$ & $\begin{array}{l}\text { Sug- } \\
\text { gestions on } \\
\text { urinary } \\
\text { tract man- } \\
\text { agement }\end{array}$ & $\begin{array}{l}\text { Nutri- } \\
\text { tion assess- } \\
\text { ment, sug- } \\
\text { gestions on } \\
\text { nutri- } \\
\text { tion man- } \\
\text { agement }\end{array}$ & $\begin{array}{l}\text { Cogni- } \\
\text { tive assess- } \\
\text { ment, sug- } \\
\text { gestions on } \\
\text { delirium } \\
\text { manage- } \\
\text { ment and } \\
\text { prevention }\end{array}$ & \\
\hline $\begin{array}{l}\text { Spice } \\
2009 \text { (pri- } \\
\text { mary care } \\
\text { setting) }\end{array}$ & Active & $\begin{array}{l}\text { Mobil- } \\
\text { ity assess- } \\
\text { ment, } \\
\text { referral } \\
\text { to occupa- } \\
\text { tional ther- } \\
\text { apist } \\
\text { or physio- } \\
\text { therapist }\end{array}$ & $\begin{array}{l}\text { Medica- } \\
\text { tion } \\
\text { changes, e. } \\
\text { g. add cal- } \\
\text { cium and } \\
\text { vitamin D }\end{array}$ & $\begin{array}{l}\text { Med- } \\
\text { ication re- } \\
\text { view, refer- } \\
\text { ral to GP }\end{array}$ & - & - & - & - & $\begin{array}{l}\text { Environ- } \\
\text { mental } \\
\text { haz- } \\
\text { ard screen- } \\
\text { ing, refer- } \\
\text { ral to occu- } \\
\text { pational } \\
\text { therapist } \\
\text { or council- } \\
\text { run home } \\
\text { hazard as- } \\
\text { sessment } \\
\text { with home } \\
\text { modifica- } \\
\text { tions }\end{array}$ \\
\hline $\begin{array}{l}\text { Spice } \\
2009 \text { (sec- } \\
\text { ondary } \\
\text { care } \\
\text { setting) }\end{array}$ & Active & $\begin{array}{l}\text { Mobil- } \\
\text { ity assess- } \\
\text { ment, } \\
\text { referral } \\
\text { to occupa- } \\
\text { tional ther- } \\
\text { apist } \\
\text { or physio- }\end{array}$ & $\begin{array}{l}\text { Medica- } \\
\text { tion } \\
\text { changes, e. } \\
\text { g. add cal- } \\
\text { cium and } \\
\text { vitamin D }\end{array}$ & $\begin{array}{l}\text { Med- } \\
\text { ication re- } \\
\text { view, refer- } \\
\text { ral to GP }\end{array}$ & - & - & - & - & $\begin{array}{l}\text { Environ- } \\
\text { mental } \\
\text { haz- } \\
\text { ard screen- } \\
\text { ing, refer- } \\
\text { ral to occu- } \\
\text { pational }\end{array}$ \\
\hline
\end{tabular}

Multifactorial and multiple component interventions for preventing falls in older people living in the community (Review) 
Table 3. Multifactorial interventions: key components of the interventions ${ }^{a} \quad$ (Continued)

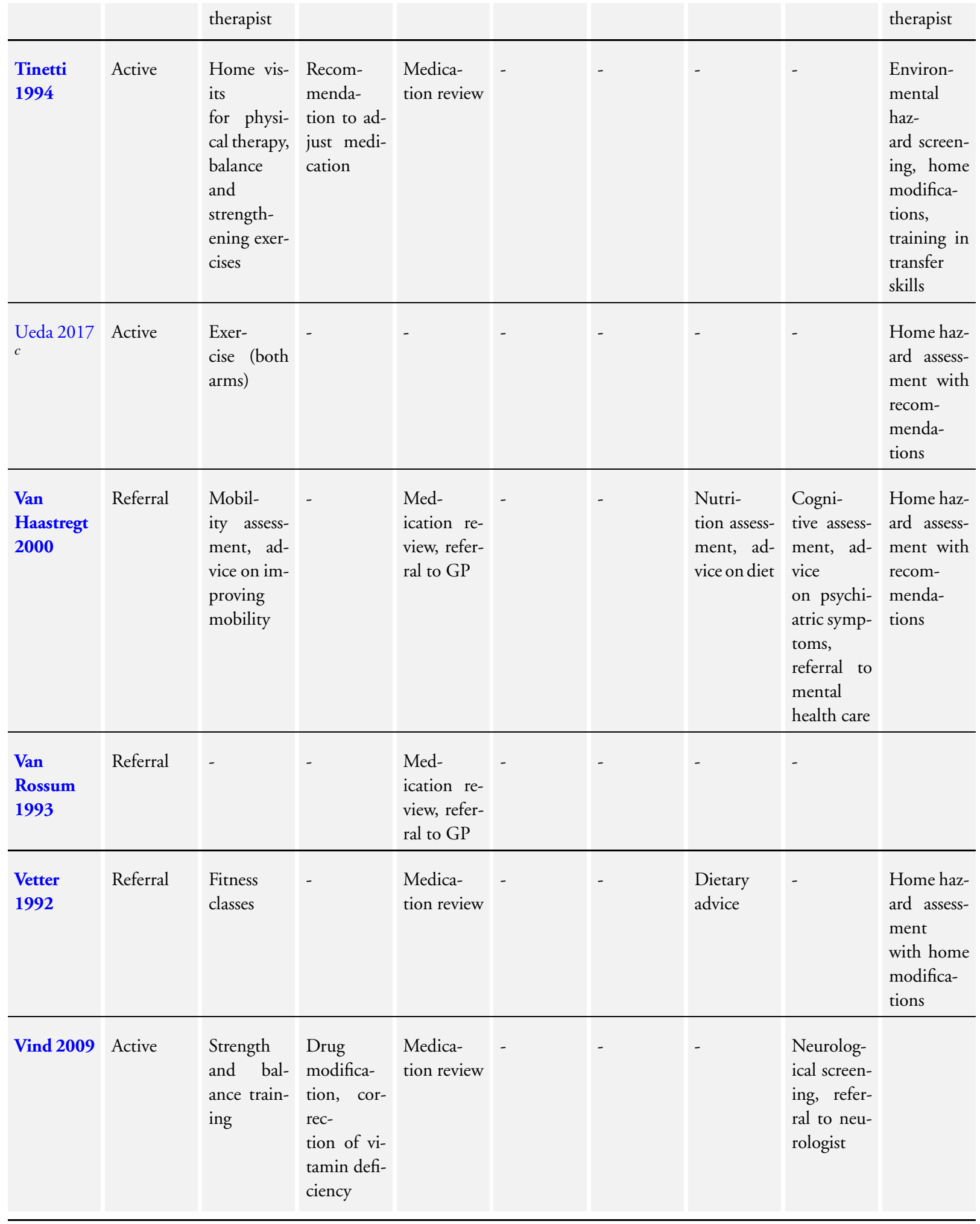

Multifactorial and multiple component interventions for preventing falls in older people living in the community (Review) 
Table 3. Multifactorial interventions: key components of the interventions ${ }^{a}$

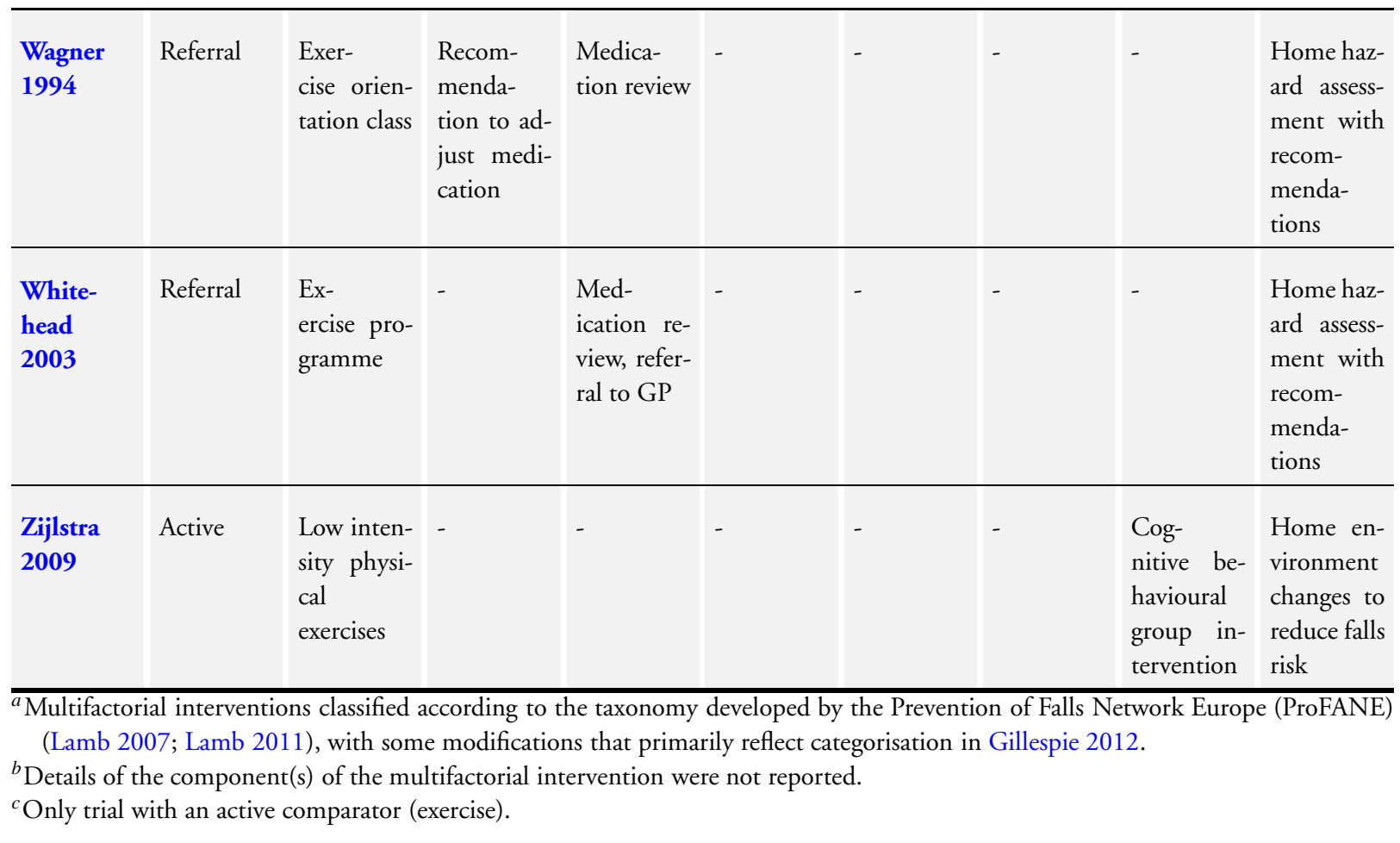

Table 4. Multifactorial interventions: outcomes

\begin{tabular}{|c|c|c|c|c|c|c|c|c|c|}
\hline Study ID & Rate falls & $\begin{array}{l}\text { Risk one } \\
\text { or more } \\
\text { falls }\end{array}$ & $\begin{array}{l}\text { Risk } \\
\text { recurrent } \\
\text { falls }\end{array}$ & $\begin{array}{l}\text { Risk } \\
\text { fall-re- } \\
\text { lated frac- } \\
\text { ture }\end{array}$ & $\begin{array}{l}\text { Risk fall- } \\
\text { related } \\
\text { hospital } \\
\text { admission }\end{array}$ & $\begin{array}{l}\text { Risk fall- } \\
\text { re- } \\
\text { lated med- } \\
\text { ical atten- } \\
\text { tion }\end{array}$ & $\begin{array}{l}\text { Health re- } \\
\text { lated qual- } \\
\text { ity of life }\end{array}$ & $\begin{array}{l}\text { Eco- } \\
\text { nomic in- } \\
\text { formation }\end{array}$ & $\begin{array}{l}\text { Adverse } \\
\text { events }^{a}\end{array}$ \\
\hline $\begin{array}{l}\text { Beling } \\
2009\end{array}$ & Yes & No & No & No & No & No & No & $\begin{array}{l}\text { Not } \\
\text { reported }\end{array}$ & $\begin{array}{l}\text { Not } \\
\text { reported }\end{array}$ \\
\hline $\begin{array}{l}\text { Carpenter } \\
1990\end{array}$ & Yes & No & No & No & Yes & No & No & $\begin{array}{l}\text { Not } \\
\text { reported }\end{array}$ & $\begin{array}{l}\text { Not } \\
\text { reported }\end{array}$ \\
\hline $\begin{array}{l}\text { Carter } \\
1997\end{array}$ & No & Yes & Yes & No & No & Yes & No & $\begin{array}{l}\text { Not } \\
\text { reported }\end{array}$ & $\begin{array}{l}\text { Not } \\
\text { reported }\end{array}$ \\
\hline $\begin{array}{l}\text { Ciaschini } \\
2009\end{array}$ & No & Yes & No & Yes & Yes & No & No & $\begin{array}{l}\text { Not } \\
\text { reported }\end{array}$ & $\begin{array}{l}\text { Not } \\
\text { reported }\end{array}$ \\
\hline $\begin{array}{l}\text { Close } \\
1999\end{array}$ & Yes & Yes & Yes & No & No & No & Yes & Yes & $\begin{array}{l}\text { Not } \\
\text { reported }\end{array}$ \\
\hline
\end{tabular}

Multifactorial and multiple component interventions for preventing falls in older people living in the community (Review) 
Table 4. Multifactorial interventions: outcomes

(Continued)

\begin{tabular}{|c|c|c|c|c|c|c|c|c|c|}
\hline $\begin{array}{l}\text { Coleman } \\
1999\end{array}$ & No & Yes & No & No & Yes & No & Yes & Yes & $\begin{array}{l}\text { Not } \\
\text { reported }\end{array}$ \\
\hline $\begin{array}{l}\text { Davison } \\
2005\end{array}$ & Yes & Yes & No & Yes & Yes & Yes & No & $\begin{array}{l}\text { Not } \\
\text { reported }\end{array}$ & $\begin{array}{l}\text { Not } \\
\text { reported }\end{array}$ \\
\hline $\begin{array}{l}\text { De Vries } \\
2010\end{array}$ & No & Yes & Yes & Yes & No & No & Yes & Yes & $\begin{array}{l}\text { Not } \\
\text { reported }\end{array}$ \\
\hline Elley 2008 & Yes & Yes & Yes & No & No & No & Yes & $\begin{array}{l}\text { Not } \\
\text { reported }\end{array}$ & $\begin{array}{l}\text { Not } \\
\text { reported }\end{array}$ \\
\hline $\begin{array}{l}\text { Fabacher } \\
1994\end{array}$ & No & Yes & No & No & Yes & No & No & $\begin{array}{l}\text { Not } \\
\text { reported }\end{array}$ & $\begin{array}{l}\text { Not } \\
\text { reported }\end{array}$ \\
\hline $\begin{array}{l}\text { Fairhall } \\
2014\end{array}$ & Yes & Yes & Yes & Yes & No & No & Yes & Yes & Yes \\
\hline $\begin{array}{l}\text { Ferrer } \\
2014\end{array}$ & Yes & Yes & Yes & No & No & No & No & $\begin{array}{l}\text { Not } \\
\text { reported }\end{array}$ & $\begin{array}{l}\text { Not } \\
\text { reported }\end{array}$ \\
\hline $\begin{array}{l}\text { Gallagher } \\
1996\end{array}$ & Yes & No & No & No & No & No & Yes & $\begin{array}{l}\text { Not } \\
\text { reported }\end{array}$ & $\begin{array}{l}\text { Not } \\
\text { reported }\end{array}$ \\
\hline $\begin{array}{l}\text { Hendriks } \\
2008\end{array}$ & No & Yes & Yes & No & No & Yes & Yes & Yes & $\begin{array}{l}\text { Not } \\
\text { reported }\end{array}$ \\
\hline $\begin{array}{l}\text { Hogan } \\
2001\end{array}$ & Yes & Yes & Yes & Yes & Yes & Yes & No & $\begin{array}{l}\text { Not } \\
\text { reported }\end{array}$ & $\begin{array}{l}\text { Not } \\
\text { reported }\end{array}$ \\
\hline $\begin{array}{l}\text { Huang } \\
2005\end{array}$ & No & Yes & No & No & Yes & No & Yes & $\begin{array}{l}\text { Not } \\
\text { reported }\end{array}$ & $\begin{array}{l}\text { Not } \\
\text { reported }\end{array}$ \\
\hline $\begin{array}{l}\text { Imhof } \\
2012\end{array}$ & No & Yes & No & No & Yes & No & Yes & Yes & $\begin{array}{l}\text { Not } \\
\text { reported }\end{array}$ \\
\hline $\begin{array}{l}\text { Jita- } \\
\text { punkul } \\
1998\end{array}$ & No & Yes & No & No & Yes & Yes & Yes & $\begin{array}{l}\text { Not } \\
\text { reported }\end{array}$ & $\begin{array}{l}\text { Not } \\
\text { reported }\end{array}$ \\
\hline $\begin{array}{l}\text { Kingston } \\
2001\end{array}$ & No & Yes & No & No & No & No & Yes & $\begin{array}{l}\text { Not } \\
\text { reported }\end{array}$ & $\begin{array}{l}\text { Not } \\
\text { reported }\end{array}$ \\
\hline $\begin{array}{l}\text { Light- } \\
\text { body } \\
2002\end{array}$ & Yes & Yes & No & No & No & No & Yes & Yes & $\begin{array}{l}\text { Not } \\
\text { reported }\end{array}$ \\
\hline
\end{tabular}


Table 4. Multifactorial interventions: outcomes

(Continued)

\begin{tabular}{|c|c|c|c|c|c|c|c|c|c|}
\hline $\begin{array}{l}\text { Logan } \\
2010\end{array}$ & Yes & Yes & No & Yes & Yes & No & Yes & Yes & $\begin{array}{l}\text { Not } \\
\text { reported }\end{array}$ \\
\hline Lord 2005 & Yes & Yes & Yes & No & No & No & No & $\begin{array}{l}\text { Not } \\
\text { reported }\end{array}$ & $\begin{array}{l}\text { Not } \\
\text { reported }\end{array}$ \\
\hline $\begin{array}{l}\text { Luck } \\
2013\end{array}$ & Yes & No & No & No & No & No & No & $\begin{array}{l}\text { Not } \\
\text { reported }\end{array}$ & $\begin{array}{l}\text { Not } \\
\text { reported }\end{array}$ \\
\hline $\begin{array}{l}\text { Markle- } \\
\text { Reid } 2010\end{array}$ & Yes & No & No & No & No & No & Yes & $\begin{array}{l}\text { Not } \\
\text { reported }\end{array}$ & $\begin{array}{l}\text { Not } \\
\text { reported }\end{array}$ \\
\hline $\begin{array}{l}\text { Met- } \\
\text { zelthin } \\
2013\end{array}$ & No & No & No & No & No & No & No & Yes & $\begin{array}{l}\text { Not } \\
\text { reported }\end{array}$ \\
\hline $\begin{array}{l}\text { Möller } \\
2014\end{array}$ & Yes & Yes & Yes & No & No & Yes & No & $\begin{array}{l}\text { Not } \\
\text { reported }\end{array}$ & $\begin{array}{l}\text { Not } \\
\text { reported }\end{array}$ \\
\hline $\begin{array}{l}\text { Newbury } \\
2001\end{array}$ & No & Yes & No & No & No & No & Yes & $\begin{array}{l}\text { Not } \\
\text { reported }\end{array}$ & $\begin{array}{l}\text { Not } \\
\text { reported }\end{array}$ \\
\hline $\begin{array}{l}\text { Palvanen } \\
2014\end{array}$ & Yes & Yes & No & No & No & No & No & $\begin{array}{l}\text { Not } \\
\text { reported }\end{array}$ & $\begin{array}{l}\text { Not } \\
\text { reported }\end{array}$ \\
\hline $\begin{array}{l}\text { Pardessus } \\
2002\end{array}$ & Yes & Yes & No & No & Yes & No & No & $\begin{array}{l}\text { Not } \\
\text { reported }\end{array}$ & $\begin{array}{l}\text { Not } \\
\text { reported }\end{array}$ \\
\hline $\begin{array}{l}\text { Ruben- } \\
\text { stein } \\
2007\end{array}$ & Yes & Yes & No & No & Yes & No & Yes & $\begin{array}{l}\text { Not } \\
\text { reported }\end{array}$ & $\begin{array}{l}\text { Not } \\
\text { reported }\end{array}$ \\
\hline $\begin{array}{l}\text { Russell } \\
2010\end{array}$ & Yes & Yes & No & Yes & No & No & No & $\begin{array}{l}\text { Not } \\
\text { reported }\end{array}$ & $\begin{array}{l}\text { Not } \\
\text { reported }\end{array}$ \\
\hline $\begin{array}{l}\text { Schrijne- } \\
\text { maekers } \\
1995\end{array}$ & No & No & Yes & No & No & No & No & $\begin{array}{l}\text { Not } \\
\text { reported }\end{array}$ & $\begin{array}{l}\text { Not } \\
\text { reported }\end{array}$ \\
\hline $\begin{array}{l}\text { Sheffield } \\
2013\end{array}$ & No & No & No & No & No & No & Yes & Yes & $\begin{array}{l}\text { Not } \\
\text { reported }\end{array}$ \\
\hline $\begin{array}{l}\text { Shyu } \\
2010\end{array}$ & No & Yes & No & No & Yes & Yes & Yes & Yes & $\begin{array}{l}\text { Not } \\
\text { reported }\end{array}$ \\
\hline $\begin{array}{l}\text { Spice } \\
2009\end{array}$ & No & Yes & No & Yes & Yes & No & Yes & $\begin{array}{l}\text { Not } \\
\text { reported }\end{array}$ & $\begin{array}{l}\text { Not } \\
\text { reported }\end{array}$ \\
\hline
\end{tabular}


Table 4. Multifactorial interventions: outcomes

(Continued)

\begin{tabular}{|c|c|c|c|c|c|c|c|c|c|}
\hline $\begin{array}{l}\text { Tinetti } \\
1994\end{array}$ & Yes & Yes & No & No & Yes & Yes & No & Yes & Yes \\
\hline$\underset{b}{\text { Ueda }} 2017$ & Yes & Yes & No & No & No & No & No & $\begin{array}{l}\text { Not } \\
\text { reported }\end{array}$ & $\begin{array}{l}\text { Not } \\
\text { reported }\end{array}$ \\
\hline $\begin{array}{l}\text { Van } \\
\text { Haastregt } \\
2000\end{array}$ & No & Yes & No & No & No & Yes & No & $\begin{array}{l}\text { Not } \\
\text { reported }\end{array}$ & $\begin{array}{l}\text { Not } \\
\text { reported }\end{array}$ \\
\hline $\begin{array}{l}\text { Van } \\
\text { Rossum } \\
1993\end{array}$ & No & No & No & No & Yes & No & No & Yes & $\begin{array}{l}\text { Not } \\
\text { reported }\end{array}$ \\
\hline $\begin{array}{l}\text { Vetter } \\
1992\end{array}$ & No & Yes & No & Yes & No & No & No & $\begin{array}{l}\text { Not } \\
\text { reported }\end{array}$ & $\begin{array}{l}\text { Not } \\
\text { reported }\end{array}$ \\
\hline Vind 2009 & Yes & Yes & Yes & No & No & Yes & No & $\begin{array}{l}\text { Not } \\
\text { reported }\end{array}$ & $\begin{array}{l}\text { Not } \\
\text { reported }\end{array}$ \\
\hline $\begin{array}{l}\text { Wagner } \\
1994\end{array}$ & No & Yes & No & No & Yes & Yes & No & $\begin{array}{l}\text { Not } \\
\text { reported }\end{array}$ & $\begin{array}{l}\text { Not } \\
\text { reported }\end{array}$ \\
\hline $\begin{array}{l}\text { White- } \\
\text { head } \\
2003\end{array}$ & No & Yes & No & No & No & No & No & $\begin{array}{l}\text { Not } \\
\text { reported }\end{array}$ & $\begin{array}{l}\text { Not } \\
\text { reported }\end{array}$ \\
\hline $\begin{array}{l}\text { Zijlstra } \\
2009\end{array}$ & Yes & Yes & Yes & No & No & No & No & $\begin{array}{l}\text { Not } \\
\text { reported }\end{array}$ & Yes \\
\hline
\end{tabular}

${ }^{a}$ Reported information on adverse events which may have been as a result of the intervention.

${ }^{b}$ Only trial with an active comparator (exercise).

Table 5. Multifactorial and multiple interventions: health economic information

\begin{tabular}{l|l|l|l|l}
\hline Study ID & Country & $\begin{array}{l}\text { Recruitment } \\
\text { period }\end{array}$ & $\begin{array}{l}\text { Cost information } \\
\text { reported }\end{array}$ & $\begin{array}{l}\text { Cost per fall pre- Cost per QALY } \\
\text { vented }\end{array}$ \\
\hline
\end{tabular}

\section{Multifactorial interventions}

\begin{tabular}{|c|c|c|c|c|c|}
\hline $\begin{array}{l}\text { Close } 1999 \text { (cost } \\
\text { analysis reported in } \\
\text { Close 2000) }\end{array}$ & UK & $\begin{array}{l}\text { December } 1995 \text { to } \\
\text { June } 1996\end{array}$ & $\begin{array}{l}\text { No significant differ- } \\
\text { ence between the } 2 \\
\text { groups for health ser- } \\
\text { vice costs. Costs re- } \\
\text { ported as GBP } 1953 \\
\text { in the interven- } \\
\text { tion group and GBP }\end{array}$ & Not reported & Not reported \\
\hline
\end{tabular}




\begin{tabular}{|c|c|c|c|c|c|}
\hline & & & $\begin{array}{l}2549 \text { in the control } \\
\text { group }\end{array}$ & & \\
\hline Coleman 1999 & USA & Not reported & $\begin{array}{l}\text { No significant differ- } \\
\text { ence between the } 2 \\
\text { groups for pharmacy } \\
\text { costs or total health } \\
\text { service costs. Cost re- } \\
\text { ported as USD } 9535 \\
\text { in the intervention } \\
\text { group and USD } 10 \text {, } \\
116 \text { in the control } \\
\text { group per year }\end{array}$ & Not reported & Not reported \\
\hline De Vries 2010 & The Netherlands & $\begin{array}{l}\text { April } 2005 \text { to July } \\
2008\end{array}$ & $\begin{array}{l}\text { No significant differ- } \\
\text { ence be- } \\
\text { tween groups. Mean } \\
\text { total healthcare costs } \\
\text { reported as EUR } \\
7740 \text { in the interven- } \\
\text { tion group and EUR } \\
6838 \text { in the control } \\
\text { group }\end{array}$ & $\begin{array}{l}\text { EUR } 226 \text { per per- } \\
\text { centage reduction in } \\
\text { fallers }\end{array}$ & $\begin{array}{l}\text { If EUR 300, } \\
000 \text { invested, proba- } \\
\text { bility that the inter- } \\
\text { vention would im- } \\
\text { prove quality of life } \\
\text { (utility) by } 1 \text { point } \\
\text { was } 0.30 \text { (incremen- } \\
\text { tal cost per QALY } \\
\text { gained not reported) }\end{array}$ \\
\hline Fairhall 2014 & Australia & 2011 & $\begin{array}{l}\text { No significant be- } \\
\text { tween-group differ- } \\
\text { ence in EQ- } 5 \text { D util- } \\
\text { ity scores. The cost } \\
\text { for } 1 \text { extra person } \\
\text { to transition out of } \\
\text { frailty was AUD } 15 \text {, } \\
955 \text { (at } 2011 \text { prices) }\end{array}$ & Not reported & Not reported \\
\hline
\end{tabular}

\begin{tabular}{|c|c|c|c|c|c|}
\hline Hendriks 2008 & The Netherlands & $\begin{array}{l}\text { January } 2003 \text { to } \\
\text { March } 2004\end{array}$ & $\begin{array}{l}\text { No significant differ- } \\
\text { ence be- } \\
\text { tween groups. Mean } \\
\text { total healthcare costs } \\
\text { reported as EUR } \\
4857 \text { in the interven- } \\
\text { tion group and EUR } \\
4991 \text { in the control } \\
\text { group }\end{array}$ & $\begin{array}{l}\text { Incremental ra- } \\
\text { tios not calculated as } \\
\text { intervention did not } \\
\text { reduce falls or result } \\
\text { in QALY gains }\end{array}$ & Not reported \\
\hline Imhof 2012 & Switerland & 2008 to 2011 & $\begin{array}{l}\text { The inter- } \\
\text { vention cost approx- } \\
\text { imately USD } 1250 \\
\text { per participant; costs } \\
\text { for the control group } \\
\text { not reported }\end{array}$ & Not reported & Not reported \\
\hline
\end{tabular}




\begin{tabular}{|c|c|c|c|c|c|}
\hline Lightbody 2002 & UK & $\begin{array}{l}\text { July } 1997 \text { to Decem- } \\
\text { ber } 1997\end{array}$ & $\begin{array}{l}\text { Total costs not re- } \\
\text { ported. There was a } \\
\text { cost saving in the } \\
\text { number of fall-re- } \\
\text { lated hospital bed } \\
\text { days reported (total } \\
\text { costs of bed days } \\
\text { GBP } 11,719 \text { in in- } \\
\text { tervention group } \\
\text { and GBP } 37,951 \text { in } \\
\text { control group) }\end{array}$ & Not reported & Not reported \\
\hline $\begin{array}{l}\text { Logan } 2010 \text { (cost } \\
\text { analysis reported } \\
\text { in Sach 2012) }\end{array}$ & UK & $\begin{array}{l}\text { September } 2005 \text { to } \\
\text { January } 2007\end{array}$ & $\begin{array}{l}\text { Mean total health- } \\
\text { care costs reported as } \\
\text { GBP } 15,266 \text { in the } \\
\text { intervention group } \\
\text { and GBP } 16,818 \text { in } \\
\text { the control group per } \\
\text { participant }\end{array}$ & Not reported & $\begin{array}{l}\text { Mean QALY per pa- } \\
\text { tient was }-0.059 \\
\text { (SD: } 0.269) \text { in the } \\
\text { intervention group } \\
\text { and }-0.129 \text { (SD } 0 \text {. } \\
238) \text { in the control } \\
\text { group. Mean differ- } \\
\text { ence of } 0.070(95 \% \\
\mathrm{CI}-0.010 \text { to } 0.150)\end{array}$ \\
\hline $\begin{array}{l}\text { Metzelthin } 2013 \\
\text { (costs reported in } \\
\text { Metzelthin 2015) }\end{array}$ & The Netherlands & $\begin{array}{l}\text { December } \\
2009 \text { (end date not } \\
\text { reported) }\end{array}$ & $\begin{array}{l}\text { Mean total health- } \\
\text { care costs were GBP } \\
26,503 \text { in the inter- } \\
\text { vention group and } \\
\text { GBP } 20,550 \text { in the } \\
\text { control group per } \\
\text { participant }\end{array}$ & Not reported & Not reported \\
\hline Sheffield 2013 & USA & Not reported & $\begin{array}{l}\text { Mean cost of the in- } \\
\text { tervention was USD } \\
1145 \text { per participant }\end{array}$ & Not reported & Not reported \\
\hline Shyu 2010 & Taiwan & $\begin{array}{l}\text { September } 2001 \text { to } \\
\text { November } 2003\end{array}$ & $\begin{array}{l}\text { Esti- } \\
\text { mated cost added by } \\
\text { the intervention pro- } \\
\text { gramme to the cur- } \\
\text { rent routine care was } \\
\text { USD } 438\end{array}$ & Not reported & Not reported \\
\hline $\begin{array}{l}\text { Tinetti } 1994 \\
\text { (costs reported in } \\
\text { Rizzo 1996) }\end{array}$ & USA & $\begin{array}{l}\text { October } 1990 \text { to } \\
\text { April } 1992\end{array}$ & $\begin{array}{l}\text { Mean total health- } \\
\text { care costs reported as } \\
\text { USD } 8310 \text { in the } \\
\text { intervention group } \\
\text { and USD } 10,439 \text { in } \\
\text { the control group }\end{array}$ & $\begin{array}{l}\text { USD } 1772 \text { per fall } \\
\text { prevented (interven- } \\
\text { tion costs only) }\end{array}$ & Not reported \\
\hline
\end{tabular}


Table 5. Multifactorial and multiple interventions: health economic information

\begin{tabular}{|l|l|l|l|}
\hline Van Rossum 1993 & The Netherlands & Not reported & $\begin{array}{l}\text { Mean total health- Not reported } \\
\text { care costs reported as } \\
\text { NLG 20,080 for the } \\
\text { intervention group } \\
\text { and NLG 19,321 in } \\
\text { the control group per } \\
\text { person }\end{array}$ \\
\hline
\end{tabular}

Multiple component interventions

\begin{tabular}{|c|c|c|c|c|c|}
\hline Campbell 2005 & New Zealand & $\begin{array}{l}\text { October } 2002 \text { to Oc- } \\
\text { tober } 2003\end{array}$ & $\begin{array}{l}\text { Home safety pro- } \\
\text { gramme cost NZD } \\
64,337 \text { to deliver } \\
\text { to the } 198 \text { partici- } \\
\text { pants in } 2 \text { centres, or } \\
\text { NZD } 325 \text { per per- } \\
\text { son (other compo- } \\
\text { nents not reported) }\end{array}$ & $\begin{array}{l}\text { NZD } 650 \text { per fall } \\
\text { prevented (home sa- } \\
\text { fety programme im- } \\
\text { plementation costs } \\
\text { only) }\end{array}$ & Not reported \\
\hline $\begin{array}{l}\text { Uusi-Rasi } \\
2015 \text { (cost analy- } \\
\text { sis reported in Patil } \\
2016 \text { ) }\end{array}$ & Finland & $\begin{array}{l}\text { April } 2010 \text { to March } \\
2013\end{array}$ & $\begin{array}{l}\text { Mean healthcare re- } \\
\text { ported as costs re- } \\
\text { ported as EUR } 188 \\
\text { for in the exercise } \\
\text { and vitamin D group } \\
\text { and EUR } 73.4 \text { in the } \\
\text { exercise-only group } \\
\text { per participant per } \\
\text { year }\end{array}$ & $\begin{array}{l}\text { Cost per fall pre- } \\
\text { vented is EUR } 3920 \\
\text { for the exercise and } \\
\text { vitamin D group }\end{array}$ & Not reported \\
\hline Waterman 2016 & UK & $\begin{array}{l}\text { March } 2012 \text { to Oc- } \\
\text { tober } 2012\end{array}$ & $\begin{array}{l}\text { Cost of the home sa- } \\
\text { fety and exercise pro- } \\
\text { gramme was GBP } \\
674 \text { per participant }\end{array}$ & $\begin{array}{l}\text { No difference } \\
\text { in number of falls be- } \\
\text { tween groups and so } \\
\text { cost per fall was not } \\
\text { calculated }\end{array}$ & Not reported \\
\hline
\end{tabular}

GBP: United Kingdom pound sterling

EUR: Euro

NLG: Dutch guilder

NZD: New Zealand dollar

USD: US dollar 
Table 6. Multiple interventions: study design, setting and trial size

\begin{tabular}{|c|c|c|c|c|c|c|c|c|}
\hline Study ID & $\begin{array}{l}\text { Study } \\
\text { design }\end{array}$ & $\begin{array}{l}\text { No. study } \\
\text { arms }\end{array}$ & $\begin{array}{l}\text { Study cen- } \\
\text { tres }\end{array}$ & $\begin{array}{l}\text { Length of } \\
\text { follow-up }\end{array}$ & Setting & $\begin{array}{l}\text { No. } \\
\text { randomised }\end{array}$ & $\begin{array}{l}\text { No. } \\
\text { analysed }\end{array}$ & $\begin{array}{l}\% \text { lost to } \\
\text { follow-up }\end{array}$ \\
\hline $\begin{array}{l}\text { Campbell } \\
2005\end{array}$ & Factorial & 4 & Multiple & 12 months & $\begin{array}{l}\text { New } \\
\text { Zealand }\end{array}$ & 391 & 360 & $8 \%$ \\
\hline $\begin{array}{l}\text { Clemson } \\
2004\end{array}$ & Parallel & 2 & Multiple & 14 months & Australia & 310 & 285 & $8 \%$ \\
\hline Day $2002^{a}$ & Factorial & 8 & Multiple & 18 months & Australia & 1107 & 1090 & $2 \%$ \\
\hline Faes 2011 & Parallel & 2 & Multiple & 6 months & $\begin{array}{l}\text { The Nether- } \\
\text { lands }\end{array}$ & 320 & $\begin{array}{l}\text { Not } \\
\text { reported }\end{array}$ & $\begin{array}{l}\text { Not } \\
\text { reported }\end{array}$ \\
\hline $\begin{array}{l}\text { Freiberger } \\
2012\end{array}$ & Parallel & 4 & Single & 24 months & Germany & 280 & 201 & $28 \%$ \\
\hline $\begin{array}{l}\text { Hagovska } \\
2016\end{array}$ & Parallel & 2 & Single & 3 months & Slovakia & 80 & 78 & $3 \%$ \\
\hline$\underset{a}{\text { Huang }} 2010$ & Cluster & 4 & Multiple & 18 months & Taiwan & 261 & 163 & $38 \%$ \\
\hline $\begin{array}{l}\text { Huang } \\
2011\end{array}$ & Parallel & 3 & Unclear & 5 months & Taiwan & 186 & 176 & $5 \%$ \\
\hline $\begin{array}{l}\text { Mendoza- } \\
\text { Ruvalcaba } \\
2015\end{array}$ & Parallel & 2 & Multiple & 6 months & Mexico & 72 & 64 & $11 \%$ \\
\hline $\begin{array}{l}\text { Neelemaat } \\
2012\end{array}$ & Parallel & 2 & Multiple & 3 months & $\begin{array}{l}\text { The Nether- } \\
\text { lands }\end{array}$ & 210 & 150 & $29 \%$ \\
\hline $\operatorname{Ng} 2015^{a}$ & Parallel & 5 (3 eligible) & Single & 12 months & Singapore & 147 & 138 & $6 \%$ \\
\hline Olsen 2014 & Parallel & 2 & Single & 12 months & Norway & 89 & 70 & $21 \%$ \\
\hline $\begin{array}{l}\text { Serra-Prat } \\
2017\end{array}$ & Parallel & 2 & Multiple & 12 months & Spain & 172 & 133 & $23 \%$ \\
\hline $\begin{array}{l}\text { Sosnoff } \\
2015^{a}\end{array}$ & Factorial & 4 & Single & 6 months & Canada & 37 & 34 & $8 \%$ \\
\hline $\begin{array}{l}\text { Uusi-Rasi } \\
2015^{b}\end{array}$ & Factorial & 4 & Multiple & 24 months & Finland & 409 & 370 & $10 \%$ \\
\hline $\begin{array}{l}\text { Waterman } \\
2016\end{array}$ & Parallel & 3 & Unclear & 6 months & $\begin{array}{l}\text { United } \\
\text { Kingdom }\end{array}$ & 49 & 43 & $12 \%$ \\
\hline
\end{tabular}


Table 6. Multiple interventions: study design, setting and trial size (Continued)

\begin{tabular}{|c|c|c|c|c|c|c|c|c|}
\hline $\begin{array}{l}\text { Wesson } \\
2013\end{array}$ & Parallel & 2 & Single & 3 months & Australia & 22 & 22 & $0 \%$ \\
\hline $\begin{array}{l}\text { Wilder } \\
2001\end{array}$ & Parallel & 3 & Unclear & 9 months & USA & 60 & $\begin{array}{l}\text { Not } \\
\text { reported }\end{array}$ & $\begin{array}{l}\text { Not } \\
\text { reported }\end{array}$ \\
\hline
\end{tabular}

${ }^{a}$ Trials also compared with an active comparator (exercise).

${ }^{b}$ Trial only compared with an active comparator (exercise).

Table 7. Multiple interventions: participants, intervention, comparator and compliance

\begin{tabular}{|c|c|c|c|c|c|c|}
\hline Study ID & Age (mean) & $\%$ Women & High risk of falls & $\begin{array}{l}\text { Multiple } \\
\text { intervention }\end{array}$ & Comparator & $\begin{array}{l}\text { Compliance } \\
\text { assessed }\end{array}$ \\
\hline Campbell 2005 & 84 & $68 \%$ & Yes & $\begin{array}{l}\text { Exercise, home sa- } \\
\text { fety and nutrition }\end{array}$ & Attention control & Yes \\
\hline Clemson 2004 & 78 & $74 \%$ & Yes & $\begin{array}{l}\text { Exercise, home sa- } \\
\text { fety and vision }\end{array}$ & Attention control & Yes \\
\hline Day $2002^{a}$ & 76 & $60 \%$ & No & $\begin{array}{l}\text { Exercise, home sa- } \\
\text { fety and vision }\end{array}$ & $\begin{array}{l}\text { Usual care and Ex- } \\
\text { ercise }\end{array}$ & No \\
\hline Faes 2011 & 79 & $70 \%$ & Yes & $\begin{array}{l}\text { Exercise and psy- } \\
\text { chological }\end{array}$ & Usual care & No \\
\hline Freiberger 2012 & 76 & $44 \%$ & Yes & $\begin{array}{l}\text { Exercise and edu- } \\
\text { cation }\end{array}$ & Usual care & Yes \\
\hline Hagovska 2016 & 67 & $49 \%$ & No & $\begin{array}{l}\text { Exercise and psy- } \\
\text { chological }\end{array}$ & Usual care & No \\
\hline Huang $2010^{a}$ & 71 & $48 \%$ & No & $\begin{array}{l}\text { Exercise and edu- } \\
\text { cation }\end{array}$ & $\begin{array}{l}\text { Usual care and Ex- } \\
\text { ercise }\end{array}$ & No \\
\hline Huang 2011 & Not reported & $59 \%$ & No & $\begin{array}{l}\text { Exercise and psy- } \\
\text { chological }\end{array}$ & Usual care & No \\
\hline $\begin{array}{l}\text { Mendoza- } \\
\text { Ruvalcaba } 2015\end{array}$ & 71 & $90 \%$ & No & $\begin{array}{l}\text { Exercise, nutrition } \\
\text { and psychological }\end{array}$ & Usual care & No \\
\hline $\begin{array}{l}\text { Neelemaat } \\
2012\end{array}$ & 75 & Not reported & No & $\begin{array}{l}\text { Nutrition and psy- } \\
\text { chological }\end{array}$ & Usual care & Yes \\
\hline $\operatorname{Ng} 2015^{a}$ & 70 & $61 \%$ & Yes & $\begin{array}{l}\text { Exercise, nutrition } \\
\text { and psychological }\end{array}$ & $\begin{array}{l}\text { Usual care and Ex- } \\
\text { ercise }\end{array}$ & Yes \\
\hline
\end{tabular}


Table 7. Multiple interventions: participants, intervention, comparator and compliance

\begin{tabular}{|c|c|c|c|c|c|c|}
\hline Olsen 2014 & 71 & $100 \%$ & Yes & $\begin{array}{l}\text { Exercise and edu- } \\
\text { cation }\end{array}$ & Usual care & Yes \\
\hline Serra-Prat 2017 & 78 & $57 \%$ & Yes & $\begin{array}{l}\text { Exercise and nu- } \\
\text { trition }\end{array}$ & Usual care & Yes \\
\hline Sosnoff $2015^{a}$ & 62 & $65 \%$ & Yes & $\begin{array}{l}\text { Exercise and edu- } \\
\text { cation }\end{array}$ & $\begin{array}{l}\text { Usual care and Ex- } \\
\text { ercise }\end{array}$ & Yes \\
\hline Uusi-Rasi $2015^{b}$ & 74 & $100 \%$ & Yes & $\begin{array}{l}\text { Exercise and nu- } \\
\text { trition }\end{array}$ & Exercise & Yes \\
\hline Waterman 2016 & 81 & $61 \%$ & Yes & $\begin{array}{l}\text { Exercise and home } \\
\text { safety }\end{array}$ & Usual care & Yes \\
\hline Wesson 2013 & 76 & $41 \%$ & Yes & $\begin{array}{l}\text { Exercise and home } \\
\text { safety }\end{array}$ & Usual care & Yes \\
\hline Wilder 2001 & Not reported & Not reported & No & $\begin{array}{l}\text { Exercise and home } \\
\text { safety }\end{array}$ & Usual care & No \\
\hline
\end{tabular}

${ }^{a}$ Trials also compared with an active comparator (exercise).

${ }^{b}$ Trial only compared with an active comparator (exercise).

Table 8. Multiple interventions: outcomes

\begin{tabular}{|c|c|c|c|c|c|c|c|c|c|}
\hline Study ID & Rate falls & $\begin{array}{l}\text { Risk one } \\
\text { or more } \\
\text { falls }\end{array}$ & $\begin{array}{l}\text { Risk } \\
\text { recurrent } \\
\text { falls }\end{array}$ & $\begin{array}{l}\text { Risk fall- } \\
\text { related } \\
\text { fracture }\end{array}$ & $\begin{array}{l}\text { Risk fall- } \\
\text { related } \\
\text { hospital } \\
\text { admission }\end{array}$ & $\begin{array}{l}\text { Risk fall- } \\
\text { re- } \\
\text { lated med- } \\
\text { ical atten- } \\
\text { tion }\end{array}$ & $\begin{array}{l}\text { Health-re- } \\
\text { lated qual- } \\
\text { ity of life }\end{array}$ & $\begin{array}{l}\text { Eco- } \\
\text { nomic in- } \\
\text { formation }\end{array}$ & $\begin{array}{l}\text { Adverse } \\
\text { events }^{a}\end{array}$ \\
\hline $\begin{array}{l}\text { Campbell } \\
2005\end{array}$ & Yes & Yes & Yes & No & No & Yes & No & Yes & Yes \\
\hline $\begin{array}{l}\text { Clemson } \\
2004\end{array}$ & Yes & Yes & Yes & No & No & No & Yes & $\begin{array}{l}\text { Not } \\
\text { reported }\end{array}$ & $\begin{array}{l}\text { Not } \\
\text { reported }\end{array}$ \\
\hline Day $2002^{b}$ & Yes & Yes & No & No & No & No & No & $\begin{array}{l}\text { Not } \\
\text { reported }\end{array}$ & $\begin{array}{l}\text { Not } \\
\text { reported }\end{array}$ \\
\hline Faes 2011 & No & Yes & Yes & No & No & No & Yes & $\begin{array}{l}\text { Not } \\
\text { reported }\end{array}$ & $\begin{array}{l}\text { Not } \\
\text { reported }\end{array}$ \\
\hline $\begin{array}{l}\text { Freiberger } \\
2012\end{array}$ & Yes & No & No & No & No & No & No & $\begin{array}{l}\text { Not } \\
\text { reported }\end{array}$ & Yes \\
\hline
\end{tabular}


Table 8. Multiple interventions: outcomes

(Continued)

\begin{tabular}{|c|c|c|c|c|c|c|c|c|c|}
\hline $\begin{array}{l}\text { Hagovska } \\
2016\end{array}$ & No & No & No & No & No & No & Yes & $\begin{array}{l}\text { Not } \\
\text { reported }\end{array}$ & $\begin{array}{l}\text { Not } \\
\text { reported }\end{array}$ \\
\hline $\begin{array}{l}\text { Huang } \\
2010^{b}\end{array}$ & No & Yes & No & No & No & No & No & $\begin{array}{l}\text { Not } \\
\text { reported }\end{array}$ & $\begin{array}{l}\text { Not } \\
\text { reported }\end{array}$ \\
\hline $\begin{array}{l}\text { Huang } \\
2011\end{array}$ & Yes & Yes & No & No & No & No & Yes & $\begin{array}{l}\text { Not } \\
\text { reported }\end{array}$ & $\begin{array}{l}\text { Not } \\
\text { reported }\end{array}$ \\
\hline $\begin{array}{l}\text { Mendoza- } \\
\text { Ruvalcaba } \\
2015\end{array}$ & No & No & No & No & No & No & Yes & $\begin{array}{l}\text { Not } \\
\text { reported }\end{array}$ & $\begin{array}{l}\text { Not } \\
\text { reported }\end{array}$ \\
\hline $\begin{array}{l}\text { Neele- } \\
\text { maat } \\
2012\end{array}$ & Yes & Yes & No & Yes & No & No & No & $\begin{array}{l}\text { Not } \\
\text { reported }\end{array}$ & $\begin{array}{l}\text { Not } \\
\text { reported }\end{array}$ \\
\hline $\operatorname{Ng} 2015^{b}$ & No & Yes & No & No & Yes & No & No & $\begin{array}{l}\text { Not } \\
\text { reported }\end{array}$ & Yes \\
\hline $\begin{array}{l}\text { Olsen } \\
2014\end{array}$ & No & Yes & No & No & No & No & No & $\begin{array}{l}\text { Not } \\
\text { reported }\end{array}$ & Yes \\
\hline $\begin{array}{l}\text { Serra-Prat } \\
2017\end{array}$ & No & Yes & No & No & No & No & Yes & $\begin{array}{l}\text { Not } \\
\text { reported }\end{array}$ & Yes \\
\hline $\begin{array}{l}\text { Sosnoff } \\
2015^{b}\end{array}$ & No & Yes & No & No & No & No & No & $\begin{array}{l}\text { Not } \\
\text { reported }\end{array}$ & $\begin{array}{l}\text { Not } \\
\text { reported }\end{array}$ \\
\hline $\begin{array}{l}\text { Uusi-Rasi } \\
2015^{c}\end{array}$ & Yes & No & No & No & No & No & No & Yes & Yes \\
\hline $\begin{array}{l}\text { Waterman } \\
2016\end{array}$ & Yes & Yes & Yes & No & No & No & Yes & Yes & Yes \\
\hline $\begin{array}{l}\text { Wesson } \\
2013\end{array}$ & No & Yes & No & Yes & No & No & No & $\begin{array}{l}\text { Not } \\
\text { reported }\end{array}$ & Yes \\
\hline $\begin{array}{l}\text { Wilder } \\
2001\end{array}$ & No & Yes & No & No & No & No & No & $\begin{array}{l}\text { Not } \\
\text { reported }\end{array}$ & $\begin{array}{l}\text { Not } \\
\text { reported }\end{array}$ \\
\hline
\end{tabular}

${ }^{a}$ Reported information on adverse events which may have been as a result of the intervention.

${ }^{b}$ Trials also compared with an active comparator (exercise).

${ }^{c}$ Trial only compared with an active comparator (exercise). 
Table 9. Multifactorial interventions versus control: sensitivity analyses

\begin{tabular}{|c|c|c|c|c|c|}
\hline Outcome & $\begin{array}{l}\text { Selection bias (low } \\
\text { risk) }\end{array}$ & $\begin{array}{l}\text { Detection } \\
\text { (low risk) }\end{array}$ & $\begin{array}{l}\text { Attrition bias (low } \\
\text { risk) }\end{array}$ & $\begin{array}{l}\text { Individ- } \\
\text { ually randomised } \\
\text { (excluding cluster) }\end{array}$ & $\begin{array}{l}\text { Overall treatment } \\
\text { effect }\end{array}$ \\
\hline Rate of falls & $\begin{array}{l}\text { RaR } 0.80(95 \% \text { CI } \\
0.66 \text { to } 0.98) ; 8 \\
\text { trials; } 3516 \text { partici- } \\
\text { pants; } I^{2}=93 \%\end{array}$ & $\begin{array}{l}\mathrm{RaR} 0.78(95 \% \mathrm{CI} \\
0.66 \text { to } 0.91) ; 12 \\
\text { trials; } 3718 \text { partici- } \\
\text { pants; } \mathrm{I}^{2}=91 \%\end{array}$ & $\begin{array}{l}\mathrm{RaR} 0.77(95 \% \mathrm{CI} \\
0.66 \text { to } 0.89) ; 11 \\
\text { trials; } 4125 \text { partici- } \\
\text { pants; } \mathrm{I}^{2}=90 \%\end{array}$ & $\begin{array}{l}\text { RaR } 0.78(95 \% \mathrm{CI} \\
0.68 \text { to } 0.89) ; 18 \\
\text { trials; } 5562 \text { partici- } \\
\text { pants; } \mathrm{I}^{2}=88 \%\end{array}$ & $\begin{array}{l}\text { RaR } 0.77(95 \% \mathrm{CI} \\
0.67 \text { to } 0.87) ; 19 \\
\text { trials; } 5853 \text { partici- } \\
\text { pants; } \mathrm{I}^{2}=88 \%\end{array}$ \\
\hline $\begin{array}{l}\text { Risk of sustaining } \\
\text { one or more falls }\end{array}$ & $\begin{array}{l}\text { RR } 0.98(95 \% \text { CI } \\
0.86 \text { to } 1.10) ; 12 \\
\text { trials; } 4692 \text { partici- } \\
\text { pants; } I^{2}=77 \%\end{array}$ & $\begin{array}{l}\text { RR } 0.97(95 \% \text { CI } \\
0.88 \text { to } 1.07) ; 16 \\
\text { trials; } 4380 \text { partici- } \\
\text { pants; } I^{2}=64 \%\end{array}$ & $\begin{array}{l}\text { RR } 0.95(95 \% \text { CI } \\
0.88 \text { to } 1.02) ; 13 \\
\text { trials; } 4452 \text { partici- } \\
\text { pants; } I^{2}=34 \%\end{array}$ & $\begin{array}{l}\text { RR } 0.97(95 \% \text { CI } \\
0.89 \text { to } 1.04) ; 26 \\
\text { trials; } 8774 \text { partici- } \\
\text { pants; } I^{2}=62 \%\end{array}$ & $\begin{array}{l}\text { RR } 0.96(95 \% \text { CI } \\
0.90 \text { to } 1.03) ; 29 \\
\text { trials; } 9637 \text { partici- } \\
\text { pants; } I^{2}=60 \%\end{array}$ \\
\hline $\begin{array}{l}\text { Risk of recurrent } \\
\text { falls }\end{array}$ & $\begin{array}{l}\text { RR } 0.85 \text { (95\% CI } 0 . \\
62 \text { to } 1.15) ; 6 \text { trials; } \\
1862 \text { participants; I } \\
2=76 \%\end{array}$ & $\begin{array}{l}\text { RR } 0.89(95 \% \text { CI } \\
0.73 \text { to } 1.08) ; 10 \\
\text { trials; } 3033 \text { partici- } \\
\text { pants; } I^{2}=60 \%\end{array}$ & $\begin{array}{l}\text { RR } 0.96 \text { (95\% CI } 0 . \\
81 \text { to } 1.13) ; 5 \text { trials; } \\
1402 \text { participants; I } \\
2=0 \%\end{array}$ & $\begin{array}{l}\text { RR } 0.87(95 \% \text { CI } \\
0.74 \text { to } 1.03) ; 12 \\
\text { trials; } 3368 \text { partici- } \\
\text { pants; } I^{2}=53 \%\end{array}$ & $\begin{array}{l}\text { RR } 0.87(95 \% \text { CI } \\
0.74 \text { to } 1.03) ; 12 \\
\text { trials; } 3368 \text { partici- } \\
\text { pants; } I^{2}=53 \%\end{array}$ \\
\hline $\begin{array}{l}\text { Risk of fall-related } \\
\text { fractures }\end{array}$ & $\begin{array}{l}\text { RR } 0.78 \text { (95\% CI } 0 . \\
49 \text { to } 1.23) ; 4 \text { trials; } \\
1521 \text { participants; I } \\
2=0 \%\end{array}$ & $\begin{array}{l}\text { RR } 0.47 \text { (95\% CI } 0 . \\
24 \text { to } 0.93) ; 3 \text { trials; } \\
1055 \text { participants; I } \\
2=0 \%\end{array}$ & $\begin{array}{l}\text { RR } 0.72(95 \% \text { CI } 0 . \\
48 \text { to } 1.08) ; 6 \text { trials; } \\
1774 \text { participants; I } \\
2=0 \%\end{array}$ & $\begin{array}{l}\text { RR } 0.75 \text { ( } 95 \% \text { CI } 0 \text {. } \\
53 \text { to } 1.06) ; 8 \text { trials; } \\
2425 \text { participants; I } \\
2=0 \%\end{array}$ & $\begin{array}{l}\text { RR } 0.73 \text { (95\% CI } 0 . \\
53 \text { to } 1.01) \text {; } 9 \text { trials; } \\
2850 \text { participants; I } \\
2=0 \%\end{array}$ \\
\hline $\begin{array}{l}\text { Risk of experienc- } \\
\text { ing a fall that re- } \\
\text { quired hospital ad- } \\
\text { mission }\end{array}$ & $\begin{array}{l}\text { RR } 0.98 \text { (95\% CI } 0 . \\
76 \text { to } 1.26) ; 1 \text { trial; } \\
204 \text { participants }\end{array}$ & $\begin{array}{l}\text { RR } 0.94 \text { (95\% CI } 0 . \\
74 \text { to } 1.18) ; 4 \text { trials; } \\
1960 \text { participants; I } \\
2=0 \%\end{array}$ & $\begin{array}{l}\text { RR } 1.03 \text { (95\% CI } 0 . \\
92 \text { to } 1.14) ; 7 \text { trials; } \\
2099 \text { participants; I } \\
2=7 \%\end{array}$ & $\begin{array}{l}\text { RR } 0.99(95 \% \text { CI } \\
0.92 \text { to } 1.08) ; 12 \\
\text { trials; } 4433 \text { partici- } \\
\text { pants; } I^{2}=0 \%\end{array}$ & $\begin{array}{l}\text { RR } 1.00(95 \% \mathrm{CI} \\
0.92 \text { to } 1.07) ; 15 \\
\text { trials; } 5227 \text { partici- } \\
\text { pants; } \mathrm{I}^{2}=0 \%\end{array}$ \\
\hline $\begin{array}{l}\text { Risk of experienc- } \\
\text { ing a fall that re- } \\
\text { quired medical at- } \\
\text { tention }\end{array}$ & $\begin{array}{l}\text { RR } 1.08 \text { (95\% CI } 0 . \\
74 \text { to } 1.58) ; 2 \text { trials; } \\
545 \text { participants; } I^{2} \\
=1 \%\end{array}$ & $\begin{array}{l}\text { RR } 0.83 \text { (95\% CI } 0 . \\
65 \text { to } 1.07) ; 3 \text { trials; } \\
1947 \text { participants; I } \\
2=0 \%\end{array}$ & $\begin{array}{l}\text { RR } 0.96(95 \% \text { CI } 0 . \\
71 \text { to } 1.31) ; 3 \text { trials; } \\
868 \text { participants; } I^{2} \\
=0 \%\end{array}$ & $\begin{array}{l}\text { RR } 0.93 \text { (95\% CI } 0 . \\
75 \text { to } 1.15) ; 7 \text { trials; } \\
2831 \text { participants; I } \\
2=6 \%\end{array}$ & $\begin{array}{l}\text { RR } 0.91 \text { (95\% CI } 0 . \\
75 \text { to } 1.10) \text {; } 8 \text { trials; } \\
3078 \text { participants; I } \\
2=0 \%\end{array}$ \\
\hline $\begin{array}{l}\text { Health re- } \\
\text { lated quality of life } \\
\text { (endpoint scores) }\end{array}$ & $\begin{array}{l}\text { SMD } 0.32(95 \% \mathrm{CI} \\
0.08 \text { to } 0.55) ; 2 \\
\text { trials; } 554 \text { partici- } \\
\text { pants; } \mathrm{I}^{2}=43 \%\end{array}$ & No trials remain & $\begin{array}{l}\text { SMD } 0.20(95 \% \text { CI } \\
0.00 \text { to } 0.41) ; \quad 6 \\
\text { trials; } 1602 \text { partici- } \\
\text { pants; } I^{2}=72 \%\end{array}$ & $\begin{array}{l}\text { SMD } 0.19(95 \% \mathrm{CI} \\
0.03 \text { to } 0.35) ; \quad 9 \\
\text { trials; } 2373 \text { partici- } \\
\text { pants; } \mathrm{I}^{2}=70 \%\end{array}$ & $\begin{array}{l}\text { SMD } 0.19(95 \% \mathrm{CI} \\
0.03 \text { to } 0.35) ; 9 \\
\text { trials; } 2373 \text { partici- } \\
\text { pants; } \mathrm{I}^{2}=70 \%\end{array}$ \\
\hline
\end{tabular}

Individual results for prespecified sensitivity analyses for primary and secondary outcomes. 
Table 10. Multiple interventions versus control: sensitivity analyses

\begin{tabular}{|c|c|c|c|c|c|}
\hline Outcome & $\begin{array}{l}\text { Selection bias (low } \\
\text { risk) }\end{array}$ & $\begin{array}{l}\text { Detection bias } \\
\text { (low risk) }\end{array}$ & $\begin{array}{l}\text { Attrition bias (low } \\
\text { risk) }\end{array}$ & $\begin{array}{l}\text { Individ- } \\
\text { ually randomised } \\
\text { (excluding cluster) }\end{array}$ & $\begin{array}{l}\text { Overall treatment } \\
\text { effect }\end{array}$ \\
\hline Rate of falls & $\begin{array}{l}\text { RaR } 0.68(95 \% \text { CI } \\
0.51 \text { to } 0.92) ; \quad 4 \\
\text { trials; } 584 \text { partici- } \\
\text { pants; } I^{2}=47 \%\end{array}$ & $\begin{array}{l}\mathrm{RaR} 0.75(95 \% \mathrm{CI} \\
0.60 \text { to } 0.93) ; \quad 5 \\
\text { trials; } 969 \text { partici- } \\
\text { pants; } \mathrm{I}^{2}=50 \%\end{array}$ & $\begin{array}{l}\mathrm{RaR} 0.79(95 \% \mathrm{CI} \\
0.66 \text { to } 0.96) ; \quad 3 \\
\text { trials; } 596 \text { partici- } \\
\text { pants; } \mathrm{I}^{2}=10 \%\end{array}$ & $\begin{array}{l}\text { RaR } 0.74(95 \% \text { CI } \\
0.60 \text { to } 0.91) ; 6 \\
\text { trials; } 1085 \text { partici- } \\
\text { pants; } I^{2}=45 \%\end{array}$ & $\begin{array}{l}\text { RaR } 0.74(95 \% \mathrm{CI} \\
0.60 \text { to } 0.91) ; 6 \\
\text { trials; } 1085 \text { partici- } \\
\text { pants; } \mathrm{I}^{2}=45 \%\end{array}$ \\
\hline $\begin{array}{l}\text { Risk of sustaining } \\
\text { one or more falls }\end{array}$ & $\begin{array}{l}\text { RR } 0.78 \text { (95\% CI } 0 . \\
70 \text { to } 0.88) ; 8 \text { trials; } \\
1478 \text { participants; I } \\
2=0 \%\end{array}$ & $\begin{array}{l}\text { RR } 0.81 \text { (95\% CI } 0 . \\
73 \text { to } 0.89) ; 5 \text { trials; } \\
1518 \text { participants; I } \\
2=0 \%\end{array}$ & $\begin{array}{l}\text { RR } 0.75(95 \% \text { CI } 0 . \\
62 \text { to } 0.92) ; 3 \text { trials; } \\
506 \text { participants; } I^{2} \\
=0 \%\end{array}$ & $\begin{array}{l}\text { (RR } 0.81(95 \% \mathrm{CI} \\
0.74 \text { to } 0.90) ; 10 \\
\text { trials; } 1877 \text { partici- } \\
\text { pants; } \mathrm{I}^{2}=0 \%\end{array}$ & $\begin{array}{l}\text { RR } 0.82(95 \% \text { CI } \\
0.74 \text { to } 0.90) ; 11 \\
\text { trials; } 1980 \text { partici- } \\
\text { pants; } I^{2}=0 \%\end{array}$ \\
\hline $\begin{array}{l}\text { Risk of recurrent } \\
\text { falls }\end{array}$ & $\begin{array}{l}\text { RR } 0.90(95 \% \text { CI } 0 . \\
62 \text { to } 1.30) ; 3 \text { trials; } \\
352 \text { participants; } I^{2} \\
=1 \%\end{array}$ & $\begin{array}{l}\text { RR } 0.79(95 \% \text { CI } 0 . \\
61 \text { to } 1.02) ; 3 \text { trials; } \\
629 \text { participants; } I^{2} \\
=0 \%\end{array}$ & $\begin{array}{l}\text { RR } 0.84(95 \% \text { CI } 0 \text {. } \\
57 \text { to } 1.23) ; 1 \text { trial; } \\
291 \text { participants; } I^{2} \\
=0 \%\end{array}$ & $\begin{array}{l}\text { RR } 0.81 \text { (95\% CI } 0 . \\
63 \text { to } 1.05) ; 4 \text { trials; } \\
662 \text { participants; } \mathrm{I}^{2} \\
=0 \%\end{array}$ & $\begin{array}{l}\text { RR } 0.81 \text { (95\% CI } 0 . \\
63 \text { to } 1.05) ; 4 \text { trials; } \\
662 \text { participants; } I^{2} \\
=0 \%\end{array}$ \\
\hline $\begin{array}{l}\text { Risk of fall-related } \\
\text { fractures }\end{array}$ & $\begin{array}{l}\text { RR } 0.50(95 \% \text { CI } 0 . \\
05 \text { to } 5.32) ; 2 \text { trials; } \\
232 \text { participants; } I^{2} \\
=0 \%\end{array}$ & $\begin{array}{l}\text { RR } 0.50 \text { ( } 95 \% \text { CI } 0 . \\
02 \text { to } 1.73) ; 1 \text { trial; } \\
210 \text { participants }\end{array}$ & $\begin{array}{l}\text { Both trials were at } \\
\text { unclear/high risk of } \\
\text { attrition bias }\end{array}$ & $\begin{array}{l}\text { RR } 0.50 \text { (95\% CI } 0 . \\
50 \text { to } 5.32) ; 2 \text { trials; } \\
232 \text { participants; } I^{2} \\
=0 \%\end{array}$ & $\begin{array}{l}\text { RR } 0.50(95 \% \text { CI } 0 . \\
05 \text { to } 5.32) ; 2 \text { trials; } \\
232 \text { participants; } I^{2} \\
=0 \%\end{array}$ \\
\hline $\begin{array}{l}\text { Risk of experienc- } \\
\text { ing a fall that re- } \\
\text { quired hospital ad- } \\
\text { mission }\end{array}$ & No trials remain & No trials remain & No trials remain & No trials remain & $\begin{array}{l}\text { RR } 3.06 \text { (95\% CI } 0 . \\
65 \text { to } 14.42) \text {; } 1 \text { trial; } \\
99 \text { participants }\end{array}$ \\
\hline $\begin{array}{l}\text { Risk of experienc- } \\
\text { ing a fall that re- } \\
\text { quired medical at- } \\
\text { tention }\end{array}$ & No trials remain & No trials remain & No trials remain & No trials remain & $\begin{array}{l}\text { RR } 0.95 \text { (95\% CI } 0 . \\
67 \text { to } 1.35) ; 1 \text { trial; } \\
291 \text { participants }\end{array}$ \\
\hline $\begin{array}{l}\text { Health-re- } \\
\text { lated quality of life } \\
\text { (endpoint scores) }\end{array}$ & $\begin{array}{l}\text { SMD } 0.84(95 \% \text { CI } \\
0.02 \text { to } 1.67) ; \quad 3 \\
\text { trials; } 327 \text { partici- } \\
\text { pants; } I^{2}=92 \%\end{array}$ & No trials remain & $\begin{array}{l}\text { SMD } 1.15 \text { (95\% } \\
\text { CI } 0.75 \text { to } 1.54) \text {; } \\
1 \text { trial; } 116 \text { partici- } \\
\text { pants }\end{array}$ & $\begin{array}{l}\text { SMD } 0.77(95 \% \text { CI } \\
0.16 \text { to } 1.39) ; \quad 4 \\
\text { trials; } 391 \text { partici- } \\
\text { pants; } I^{2}=88 \%\end{array}$ & $\begin{array}{l}\text { SMD } 0.77(95 \% \text { CI } \\
0.16 \text { to } 1.39) ; \quad 4 \\
\text { trials; } 391 \text { partici- } \\
\text { pants; } I^{2}=92 \%\end{array}$ \\
\hline
\end{tabular}

Individual results for prespecified sensitivity analyses for primary and secondary outcomes 


\section{A P PENDICES}

\section{Appendix I. Search strategies}

We carried out the MEDLINE, Embase and CINAHL searches in two stages: the first search was run from 2012 to June 2016 and a top-up search was run from June 2016 to June 2017.

\section{CENTRAL (CRS Online)}

\section{March 2012 to June 2017}

\#1 MESH DESCRIPTOR Accidental Falls EXPLODE ALL TREES (1167)

\#2 (falls or faller*):TI,AB,KY (3872)

\#3 \#1 or \#2 (3872)

\#4 MESH DESCRIPTOR Aged EXPLODE ALL TREES (1098)

\#5 (senior* or elder* or old* or aged or ag?ing or postmenopausal or community dwelling):TI,AB,KY (426265)

\#6 \#4 or \#5 (426265)

$\# 7 \# 3$ and \#6 (2947)

\#8 14/03/2012 TO 30/06/2017:DL (400529)

\#9 \#7 AND \#8 (1483)

\section{MEDLINE (Ovid Interface)}

2012 to June 2016

1 Accidental Falls/ (18084)

2 (falls or faller*1).tw. (35089)

3 or/1-2 (43653)

4 exp Aged/ (2586317)

5 (senior* 1 or elder* ${ }^{*}$ or old* or aged or ag?ing or postmenopausal or community dwelling).tw. (1743445)

6 or/4-5 (3816139)

73 and 6 (21745)

8 Randomized controlled trial.pt. (420633)

9 Controlled clinical trial.pt. (90997)

10 randomized.ab. (358562)

11 placebo.ab. (173730)

12 Clinical trials as topic.sh. (177470)

13 randomly.ab. (256508)

14 trial.ti. (156075)

158 or 9 or 10 or 11 or 12 or 13 or 14 (1041329)

16 exp Animals/ not Humans/ (4260951)

1715 not 16 (960106)

187 and 17 (2485)

$19\left(2012^{*}\right.$ or $2013^{*}$ or $2014^{*}$ or $2015^{*}$ or $\left.2016^{*}\right)$.ed,dc. $(5462305)$

2018 and 19 (949)

Top up search June 2016 to June 2017: (394)

\section{Embase (Ovid Interface)}

1 Falling/ (29668)

2 (falls or fallers).tw. (43232)

3 or/1-2 (59763)

4 exp Aged/ (2415181)

5 (senior* 1 or elder* or old* or aged or ag?ing or postmenopausal or community dwelling).tw. (2188057)

6 or/4-5 (4022662)

Multifactorial and multiple component interventions for preventing falls in older people living in the community (Review) 
73 and 6 (29465)

8 exp Randomized Controlled Trial/ or exp Single Blind Procedure/ or exp Double Blind Procedure/ or Crossover Procedure/ (456331)

9 (random* or RCT or placebo or allocat* or crossover* or 'cross over' or trial or (doubl* adj1 blind*) or (singl* adj1 blind*)).ti,ab.

(1513727)

108 or $9(1593780)$

11 (exp Animal/ or animal.hw. or Nonhuman/) not (exp Human/ or Human cell/ or (human or humans).ti.) (5531185)

1210 not $11(1408433)$

137 and 12 (4198)

$14\left(2012^{*}\right.$ or $2013^{*}$ or $2014^{*}$ or $2015^{*}$ or $\left.2016^{*}\right) . e m, d d .(7072579)$

1513 and 14 (1917)

Top-up search June 2016 to June 2017: (253)

\section{CINAHL (Ebsco)}

S1 (MH “Accidental Falls") $(14,885)$

S2 TI ( falls or faller*) OR AB ( falls or faller*) $(19,097)$

S3 S1 OR S2 $(26,576)$

S4 (MH “Aged+") $(561,909)$

S5 TI ( senior* or elder* or old* or aged or ag?ing or postmenopausal or community dwelling ) OR AB ( senior* or elder* or old* or aged or ag?ing or postmenopausal or community dwelling ) $(313,241)$

S6 S4 OR S5 $(738,634)$

S7 S3 AND S6 $(13,989)$

S8 PT Clinical Trial $(79,704)$

S9 (MH “Clinical Trials+”) $(198,945)$

S10 TI clinical trial* OR AB clinical trial* $(53,785)$

S11 TI ( (single blind* or double blind*) ) OR AB ( (single blind* or double blind*) ) $(24,624)$

S12 TI random* OR AB random* $(174,084)$

S13 S8 OR S9 OR S10 OR S11 OR S12 $(312,167)$

S14 S7 AND S13 $(1,850)$

S15 EM 2012 OR EM 2013 OR EM 2014 OR EM 2015 OR EM $2016(1,539,278)$

S16 S14 AND S15 (602)

Top-up search 2016 to June 2017: (175)

\section{WHO ICTRP}

1. FALLS and ELDERLY in title

2. FALLS and ELDERLY in title + MULTIPLE and/ or MULTIFACTORIAL in intervention

3. PREVENTION and FALLS in title

4. ELDERLY in condition AND PREVENTION and FALLS in intervention

5. INJURIOUS and FALLS in title, and ELDERLY in condition

(each of the search strings were run separately and then the records combined and duplicates removed)

\section{ClinicalTrials.gov}




\section{Appendix 2. 'Risk of bias' assessment tool}

\section{Domain}

Random sequence generation relating to selection bias (biased allocation to interventions) due to inadequate generation of a randomised sequence

\section{Criteria for judging risk of bias}

- Judgement of 'low risk' if the trial authors described a random component in the sequence generation, e.g. referring to a random number table; using a computer random number generator; coin tossing; shuffling cards or envelopes; throwing dice; drawing of lots; minimisation.

- Judgement of 'high risk' if the trial used a systematic nonrandom method, e.g. date of admission; odd or even date of birth; case record number; clinician judgement; participant preference; patient risk factor score or test results; availability of intervention.

- Judgement of 'unclear' if there is insufficient information about the sequence generation process to permit judgement of 'low risk' or 'high risk'.

Allocation concealment relating to selection bias (biased allocation to interventions) due to inadequate concealment of allocations prior to assignment
- Judgement of 'low risk' in studies using: $\circ$ individual randomisation if the trial described allocation concealment as by central allocation (telephone, internet-based or pharmacy-controlled randomisation); sequentially-numbered identical drug containers; sequentiallynumbered, opaque, sealed envelopes;

o cluster randomisation if allocation of all cluster units performed at the start of the study and individual participant recruitment was completed prior to assignment of the cluster, and the same participants were followed up over time or individual participants were recruited after cluster assignment, but recruitment carried out by a person unaware of group allocation and participant characteristics (e.g. fall history) or individual participants in intervention and control arms were invited by mail questionnaire with identical information.

- Judgement of 'high risk' in studies using: $\circ$ individual randomisation if investigators enrolling participants could possibly foresee assignments and thus introduce selection bias, e.g. using an open random allocation schedule (e.g. a list of random numbers); assignment envelopes unsealed, non-opaque, or not sequentially numbered; alternation or rotation; date of birth; case record number; or any other explicitly unconcealed procedure;

$\circ$ cluster-randomisation if individual participant recruitment was undertaken after group allocation by a person who was unblinded and may have had knowledge of participant characteristics.

- Judgement of 'unclear' if insufficient information to permit judgement of 'low risk' or 'high risk'. This is usually the case if the method of concealment is not described or not described in sufficient detail to allow a definite judgement, e.g. if the use of 
assignment envelopes is described, but it remains unclear whether envelopes were sequentially numbered, opaque and sealed.

Blinding of participants and personnel relating to performance bias due to knowledge of the allocated interventions by participants and personnel carrying out the interventions
- Judgement of 'low risk' if blinding of participants and personnel implementing the interventions was ensured, and unlikely that the blinding could have been broken (e.g. control group received matching placebo medication prepared by a pharmacist) OR no blinding or incomplete blinding, but the review authors judge that the outcomes (falls and fractures) are unlikely to be influenced by lack of blinding.

- Judgement of 'high risk' if participants or intervention delivery personnel, or both, were not blinded to group allocation (e.g. exercise intervention), and the outcomes (falls and fractures) are likely to be influenced by lack of blinding.

- Judgement of 'unclear' if there is insufficient information to make a judgement of 'low risk' or 'high risk'.

Blinding of outcome assessment relating to detection bias due to knowledge of the allocated interventions by outcome assessors

- Falls and fallers:

○ judgement of 'low risk' if falls were recorded/ confirmed in all allocated groups using the same method and the personnel recording/confirming falls were blind to group allocation;

○ judgement of 'high risk' if falls were not recorded/ confirmed in all allocated groups using the same method or the personnel recording/confirming falls were NOT blind to group allocation;

$\circ$ judgement of 'unclear' if there is insufficient information to make a judgement of 'low risk' or 'high risk'.

- Fractures:

$\circ$ judgement of 'low risk' if fractures were recorded/ confirmed in all allocated groups using the same method and fractures were confirmed by the results of radiological examination or from primary care case records and the personnel recording/confirming fractures were blind to group allocation;

$\circ$ judgement of 'High risk' if fractures were not recorded/confirmed in all allocated groups using the same method or the only evidence for fractures was from self reports from participants or carers;

$\circ$ judgement of 'Unclear' if there is insufficient information to make a judgement of 'low risk' or 'high risk'.

- Hospital admission and medical attention:

$\circ$ judgement of 'low risk' if requiring hospital admission/medical attention as a result of a fall was recorded/ confirmed in all allocated groups using the same method (e.g. from primary care records);

○ judgement of 'high risk' if requiring hospital admission/medical attention as a result of a fall was not recorded/confirmed in all allocated groups using the same 
method (e.g. from primary care records) or the only evidence for requiring medical attention was from self reports from participants or carers;

$\circ$ judgement of 'unclear' if there is insufficient information to make a judgement of 'low risk' or 'high risk'.

Incomplete outcome data relating to attrition bias due to amount, nature or handling of incomplete outcome data
- Judgement of 'low risk' if there are no missing outcome data, or less than $20 \%$ of missing outcome data are missing and losses are balanced in numbers across intervention groups with similar reasons for missing data across groups or missing data have been imputed using appropriate methods.

- Judgement of 'high risk' if greater than $20 \%$ of missing outcome data, or reason for missing outcome data likely to be related to true outcome, with either imbalance in numbers or reasons for missing data across intervention groups, or 'astreated' analysis done with substantial departure of the intervention received from that assigned at randomisation or potentially inappropriate application of simple imputation.

- Judgement of 'unclear' if there is insufficient information to make a judgement of 'low risk' or 'high risk'.
Selective outcome reporting relating to bias due to the selective reporting or non reporting of findings
- Judgement of 'low risk' if the study protocol is available and all prespecified study outcomes are reported in the prespecified way or the study protocol is unavailable but it is clear the published repot includes all expected outcomes.

- Judgement of 'high risk' if not all prespecified study outcomes are reported, or one or more primary outcomes are reported in ways which were not prespecified, or one or more outcomes are reported incompletely or the study fails to include results for a key outcome that would be expected to be reported.

- Judgement of 'unclear' if there is insufficient information to make a judgement of 'low risk' or 'high risk'.

Method of ascertaining falls relating to bias in the recall of falls due to unreliable methods of ascertainment

- Judgement of 'low risk' if the study used some form of concurrent collection of data about falling, e.g. participants given postcards to fill in daily and mail back monthly, calendar to mark etc, with monthly, or more frequent, follow-up by the researchers.

- Judgement of 'high risk' if ascertainment relied on participant recall at longer intervals than 1 month during the study or at its conclusion.

- Judgement of 'unclear' if there was retrospective recall over a short period only, or if the trial authors did not describe details of ascertainment, i.e. insufficient information was provided to allow a judgement of 'low risk' or 'high risk'.

Cluster randomised trials bias relating to i) recruitment bias, ii) baseline imbalance, iii) loss of clusters, iv) incorrect analysis and v) comparability with individually-randomised trials,
Specfically for cluster randomised trials bias relating to: i) recruitment bias - judged at 'high risk' if individuals were recruited to the trial after the clusters had been randomised 
ii) baseline imbalance - judged at 'high risk' if there was baseline imbalance between randomised groups, in terms of either clusters or individuals, statistical adjustment for balance line imbalance not performed

iii) loss of clusters - judged at 'high risk' if complete clusters were lost from the trial and omitted from the analysis

iv) incorrect analysis - judged at 'high risk' if clustering not taken into account in the analysis

v) comparability with individually-randomised trials - judged at 'high risk' if differences between individually randomised and cluster randomised trials in a meta-analysis

- Judgement of 'low risk' if the study is judged to be at 'low risk' of bias across all of the five biases related to cluster randomised trials.

- Judgment of 'high risk' if the study is judged to be a 'high risk' of bias across one or more of the five biases related to cluster randomised trials.

- Judgement of 'unclear risk' if there is insufficient information to make a judgment of 'low risk or 'high risk' across one or more of the five biases related to cluster randomised trials.

We adapted this from Table 8.5.a 'The Cochrane Collaboration's tool for assessing risk of bias' and Table 8.5.d 'Criteria for judging risk of bias in the 'Risk of bias' assessment tool' (Higgins 2011a).

\section{Appendix 3. Supplementary data table: raw data for rate of falls}

\begin{tabular}{|c|c|c|c|c|c|c|c|}
\hline Study ID & $\begin{array}{l}\text { Intervention } \\
\text { arm: } \\
\text { Number of } \\
\text { falls }\end{array}$ & $\begin{array}{l}\text { Control arm: } \\
\text { Number of } \\
\text { falls }\end{array}$ & $\begin{array}{l}\text { Intervention } \\
\text { arm: } \\
\text { Num- } \\
\text { ber of person } \\
\text { months }\end{array}$ & $\begin{array}{l}\text { Control arm: } \\
\text { Num- } \\
\text { ber of person } \\
\text { months }\end{array}$ & $\begin{array}{l}\text { Intervention } \\
\text { arm: } \\
\text { Number of } \\
\text { person years }\end{array}$ & $\begin{array}{l}\text { Control arm: } \\
\text { Number of } \\
\text { person years }\end{array}$ & $\begin{array}{l}\text { Details if } 2 \\
\text { or more com- } \\
\text { parisons }\end{array}$ \\
\hline Beling 2009 & 1 & 4 & 33 & 24 & 3 & 2 & - \\
\hline $\begin{array}{l}\text { Campbell } \\
2005 \\
\text { comparison a }\end{array}$ & 108 & 76 & 1107 & 548 & 92 & 46 & $\begin{array}{l}\text { Interven- } \\
\text { tion 1: Exer- } \\
\text { cise, home sa- } \\
\text { fety } \\
\text { programme } \\
\text { and vitamin D } \\
\text { Control: } \\
\text { Usual care }\end{array}$ \\
\hline $\begin{array}{l}\text { Campbell } \\
2005 \\
\text { comparison b }\end{array}$ & 120 & 76 & 1112 & 548 & 93 & 46 & $\begin{array}{l}\text { Inter- } \\
\text { vention 2: Ex- } \\
\text { ercise and vita- } \\
\text { min D }\end{array}$ \\
\hline
\end{tabular}

Multifactorial and multiple component interventions for preventing falls in older people living in the community (Review) 


\begin{tabular}{|c|c|c|c|c|c|c|c|}
\hline $\begin{array}{l}\text { Carpenter } \\
1990\end{array}$ & .- & .- & \multicolumn{4}{|c|}{$\begin{array}{l}\text { Data not included since the number of falls was only recorded for } \\
\text { a small interval of the total follow-up period ( } 1 \text { month prior to } \\
\text { final interview at } 3 \text { years) }\end{array}$} & - \\
\hline $\begin{array}{l}\text { Clemson } \\
2004\end{array}$ & - & - & \multicolumn{4}{|c|}{ Reported Rate Ratio 0.69 (95\% CI 0.50 to 0.96 ) } & - \\
\hline Close $1999^{a}$ & 183 & 510 & \multicolumn{4}{|c|}{$\begin{array}{l}\text { Data not included since the number of person months could not } \\
\text { be accurately calculated due to high attrition }\end{array}$} & - \\
\hline $\begin{array}{l}\text { Davison } \\
2005\end{array}$ & 387 & 617 & 1656 & 1676 & 138 & 140 & - \\
\hline Day 2002 & & & \multicolumn{4}{|c|}{ Data not included since the Hazard Ratio was reported } & - \\
\hline Elley 2008 & 285 & 299 & 1860 & 1884 & 155 & 157 & - \\
\hline Fairhall $2014^{a}$ & 183 & 178 & \multicolumn{4}{|c|}{ Reported Incidence Rate Ratio 1.12 (95\% CI 0.78 to 1.63 ) } & - \\
\hline Ferrer $2014^{a}$ & 57 & 62 & \multicolumn{4}{|c|}{ Reported Incidence Rate Ratio 0.85 (95\% CI 0.51 to 1.40 ) } & - \\
\hline $\begin{array}{l}\text { Freiberger } \\
2012\end{array}$ & - & - & \multicolumn{4}{|c|}{$\begin{array}{l}\text { Data not included since the number of falls only reported during } \\
\text { interval period ( } 12 \text { to } 24 \text { months) }\end{array}$} & - \\
\hline $\begin{array}{l}\text { Gallagher } \\
1996\end{array}$ & 85 & 105 & 300 & 300 & 25 & 25 & - \\
\hline Hogan 2001 & 241 & 311 & 1800 & 1848 & 150 & 154 & - \\
\hline Huang 2011 & 3 & 8 & 168 & 180 & 14 & 15 & - \\
\hline $\begin{array}{l}\text { Lightbody } \\
2002\end{array}$ & 141 & 171 & 1026 & 1062 & 86 & 89 & - \\
\hline Logan 2010 & 307 & 649 & 1063 & 1014 & 89 & 85 & - \\
\hline Lord 2005 & 183 & 175 & 2424 & 2412 & 202 & 201 & - \\
\hline Luck $2013^{a}$ & 260 & 414 & $\begin{array}{l}\text { Repo } \\
\text { on ch }\end{array}$ & $\begin{array}{l}\text { ence } \mathrm{Ra} \\
\text { baseli }\end{array}$ & $.32(9$ & 2 to 0.49 ) (based & - \\
\hline $\begin{array}{l}\text { Markle-Reid } \\
2010\end{array}$ & 71 & 57 & 294 & 258 & 25 & 22 & - \\
\hline Möller 2014 & 96 & 85 & 960 & 876 & 80 & 73 & . \\
\hline
\end{tabular}


(Continued)

\begin{tabular}{|c|c|c|c|c|c|c|c|}
\hline $\begin{array}{l}\text { Neelemaat } \\
2012\end{array}$ & 16 & 41 & 228 & 225 & 19 & 19 & - \\
\hline $\begin{array}{l}\text { Palvanen } \\
2014\end{array}$ & 608 & 825 & 7932 & 7836 & 661 & 653 & - \\
\hline $\begin{array}{l}\text { Pardessus } \\
2002\end{array}$ & 20 & 25 & 360 & 360 & 30 & 30 & - \\
\hline $\begin{array}{l}\text { Rubenstein } \\
2007\end{array}$ & $\cdot$ & . & \multicolumn{4}{|c|}{$\begin{array}{l}\text { Data not included since the number of falls was only recorded for } \\
\text { a partial interval of the total follow-up period ( } 12 \text { to } 24 \text { months) }\end{array}$} & - \\
\hline Russell 2010 & 908 & 1449 & 4128 & 4248 & 344 & 354 & - \\
\hline Tinetti $1994^{b}$ & 80 & 139 & 1495 & 1464 & 125 & 122 & - \\
\hline Ueda 2017 & $0.5^{c}$ & 4 & 25 & 26 & 2 & 2 & - \\
\hline $\begin{array}{l}\text { Uusi-Rasi } \\
2015 \\
\text { comparison a }\end{array}$ & 230 & 241 & 2336 & 2265 & 195 & 189 & $\begin{array}{l}\text { Inter- } \\
\text { vention: Vita- } \\
\text { min D and ex- } \\
\text { ercise } \\
\text { Control 1: Ex- } \\
\text { ercise }\end{array}$ \\
\hline $\begin{array}{l}\text { Uusi-Rasi } \\
2015 \\
\text { comparison b } \\
a\end{array}$ &.- &.- & \multicolumn{4}{|c|}{ Reported Incidence Rate Ratio 0.99 (95\% CI 0.72 to 1.39) } & $\begin{array}{l}\text { Inter- } \\
\text { vention: Vita- } \\
\text { min D and ex- } \\
\text { ercise } \\
\text { Control } 2 \text { : } \\
\text { Usual care }\end{array}$ \\
\hline Vind 2009 & 422 & 398 & 2289 & 2213 & 191 & 184 & - \\
\hline $\begin{array}{l}\text { Waterman } \\
2016\end{array}$ & 18 & 13 & 90 & 78 & 8 & 7 & - \\
\hline Zijlstra $2009^{a}$ & 302 & 381 & \multicolumn{4}{|c|}{ Reported Incidence Rate Ratio 0.86 (95\% CI 0.65 to 1.14 ) } & - \\
\hline $\begin{array}{l}{ }^{a} \text { Could not acc } \\
{ }^{b} \text { We performed } \\
{ }^{c} 0.5 \text { used for th }\end{array}$ & $\begin{array}{l}\text { urately } \\
\text { adjus }\end{array}$ & $\begin{array}{l}\text { e nun } \\
\text { usterir }\end{array}$ & $\begin{array}{l}\text { son m } \\
\text { ed in } \\
\text { ats }\end{array}$ & ds. Th & data & & \\
\hline
\end{tabular}


Appendix 4. Supplementary data table: raw data for number of fallers

\begin{tabular}{|c|c|c|c|c|c|}
\hline Study ID & $\begin{array}{l}\text { Intervention } \\
\text { arm: } \\
\text { Number of fall- } \\
\text { ers }\end{array}$ & $\begin{array}{l}\text { Control arm: } \\
\text { Number of fall- } \\
\text { ers }\end{array}$ & $\begin{array}{l}\text { Intervention } \\
\text { arm: } \\
\text { Number of par- } \\
\text { ticipants }\end{array}$ & $\begin{array}{l}\text { Control arm: } \\
\text { Number of par- } \\
\text { ticipants }\end{array}$ & $\begin{array}{l}\text { Details if } \\
2 \text { or more com- } \\
\text { parisons }\end{array}$ \\
\hline $\begin{array}{l}\text { Campbell } 2005 \\
\text { comparison a }\end{array}$ & 47 & 30 & 98 & 48 & $\begin{array}{l}\text { Interven- } \\
\text { tion 1: Exercise, } \\
\text { home safety pro- } \\
\text { gramme and vi- } \\
\text { tamin D } \\
\text { Control: Usual } \\
\text { care }\end{array}$ \\
\hline $\begin{array}{l}\text { Campbell } 2005 \\
\text { comparison b }\end{array}$ & 47 & 30 & 97 & 48 & $\begin{array}{l}\text { Intervention 2: } \\
\text { Exercise and vi- } \\
\text { tamin D } \\
\text { Control: Usual } \\
\text { care }\end{array}$ \\
\hline Ciaschini 2009 & 26 & 17 & 101 & 100 & - \\
\hline Clemson $2004^{a}$ & . & . & 157 & 153 & - \\
\hline Close 1999 & 59 & 111 & 184 & 213 & - \\
\hline Coleman $1999^{b}$ & 29 & 20 & 67 & 53 & - \\
\hline Davison 2005 & 94 & 102 & 144 & 149 & - \\
\hline $\begin{array}{l}\text { Day } 2002 \text { com- } \\
\text { parison a }\end{array}$ & 66 & 22 & 136 & 34 & $\begin{array}{l}\text { In- } \\
\text { tervention 1: Ex- } \\
\text { ercise and vision } \\
\text { improvement } \\
\text { Control 1: Usual } \\
\text { care }\end{array}$ \\
\hline $\begin{array}{l}\text { Day } 2002 \text { com- } \\
\text { parison b }\end{array}$ & 72 & 22 & 135 & 34 & $\begin{array}{l}\text { In- } \\
\text { tervention 2: Ex- } \\
\text { ercise and home } \\
\text { assessment } \\
\text { Control 1: Usual } \\
\text { care }\end{array}$ \\
\hline $\begin{array}{l}\text { Day } 2002 \text { com- } \\
\text { parison c }\end{array}$ & 78 & 22 & 137 & 34 & $\begin{array}{l}\text { Intervention } \\
\text { 3: Home assess- } \\
\text { ment and vision } \\
\text { improvement } \\
\text { Control 1: Usual } \\
\text { care }\end{array}$ \\
\hline
\end{tabular}

Multifactorial and multiple component interventions for preventing falls in older people living in the community (Review) 


\begin{tabular}{|c|c|c|c|c|c|}
\hline $\begin{array}{l}\text { Day } 2002 \text { com- } \\
\text { parison d }\end{array}$ & 65 & 22 & 135 & 34 & $\begin{array}{l}\text { Interven- } \\
\text { tion 4: Exercise, } \\
\text { home assessment } \\
\text { and vision im- } \\
\text { provement } \\
\text { Control 1: Usual } \\
\text { care }\end{array}$ \\
\hline $\begin{array}{l}\text { Day } 2002 \text { com- } \\
\text { parison e }\end{array}$ & 66 & 19 & 136 & 34 & $\begin{array}{l}\text { In- } \\
\text { tervention 1: Ex- } \\
\text { ercise and vision } \\
\text { improvement } \\
\text { Control 2: Exer- } \\
\text { cise }\end{array}$ \\
\hline $\begin{array}{l}\text { Day } 2002 \mathrm{com}- \\
\text { parison } f\end{array}$ & 72 & 19 & 135 & 34 & $\begin{array}{l}\text { In- } \\
\text { tervention 2: Ex- } \\
\text { ercise and home } \\
\text { assessment } \\
\text { Control 2: Exer- } \\
\text { cise }\end{array}$ \\
\hline $\begin{array}{l}\text { Day } 2002 \text { com- } \\
\text { parison } \mathrm{g}\end{array}$ & 78 & 19 & 137 & 34 & $\begin{array}{l}\text { Intervention } \\
\text { 3: Home assess- } \\
\text { ment and vision } \\
\text { improvement } \\
\text { Control 2: Exer- } \\
\text { cise }\end{array}$ \\
\hline $\begin{array}{l}\text { Day } 2002 \text { com- } \\
\text { parison h }\end{array}$ & 65 & 19 & 135 & 34 & $\begin{array}{l}\text { Interven- } \\
\text { tion 4: Exercise, } \\
\text { home assessment } \\
\text { and vision im- } \\
\text { provement } \\
\text { Control 2: Exer- } \\
\text { cise }\end{array}$ \\
\hline De Vries 2010 & 55 & 62 & 106 & 111 & \\
\hline Elley 2008 & 106 & 98 & 155 & 157 & \\
\hline Fabacher 1994 & 14 & 22 & 100 & 95 & - \\
\hline Faes 2011 & 10 & 6 & 18 & 15 & - \\
\hline Fairhall 2014 & 72 & 67 & 119 & 119 & - \\
\hline Ferrer 2014 & 40 & 33 & 142 & 131 & - \\
\hline
\end{tabular}


(Continued)

\begin{tabular}{|c|c|c|c|c|c|}
\hline Hendriks 2008 & 55 & 61 & 124 & 134 & - \\
\hline Hogan 2001 & 54 & 61 & 75 & 77 & - \\
\hline Huang 2005 & 5 & 7 & 63 & 63 & - \\
\hline $\begin{array}{l}\text { Huang } 2010 \\
\text { comparison }^{b}\end{array}$ & 2 & 1 & 34 & 29 & $\begin{array}{l}\text { Inter- } \\
\text { vention: Educa- } \\
\text { tion and Tai Chi } \\
\text { Control 1: Usual } \\
\text { care }\end{array}$ \\
\hline $\begin{array}{l}\text { Huang } 2010 \\
\text { comparison } \mathbf{b}^{b}\end{array}$ & 2 & 0.5 & 34 & 19 & $\begin{array}{l}\text { Inter- } \\
\text { vention: Educa- } \\
\text { tion and Tai Chi } \\
\text { Control 2: Exer- } \\
\text { cise }\end{array}$ \\
\hline Huang 2011 & 3 & 8 & 56 & 60 & - \\
\hline Kingston $2001^{c}$ & 2 & 2 & 51 & 41 & - \\
\hline $\begin{array}{l}\text { Lightbody } \\
2002\end{array}$ & 39 & 41 & 155 & 159 & - \\
\hline Logan 2010 & 81 & 96 & 102 & 102 & - \\
\hline Lord 2005 & 93 & 90 & 202 & 201 & - \\
\hline Möller 2014 & 43 & 35 & 80 & 73 & - \\
\hline $\begin{array}{l}\text { Neelemaat } \\
2012\end{array}$ & 10 & 24 & 105 & 105 & - \\
\hline Newbury 2001 & 12 & 17 & 45 & 44 & - \\
\hline $\operatorname{Ng} 2015$ a & 2 & 3 & 49 & 48 & $\begin{array}{l}\text { Interven- } \\
\text { tion: Physical ac- } \\
\text { tivity, nutritional } \\
\text { supplements and } \\
\text { cognitive train- } \\
\text { ing } \\
\text { Control 1: Exer- } \\
\text { cise }\end{array}$ \\
\hline Ng 2015 b & 2 & 5 & 49 & 50 & $\begin{array}{l}\text { Interven- } \\
\text { tion: Physical ac- } \\
\text { tivity, nutritional } \\
\text { supplements and }\end{array}$ \\
\hline
\end{tabular}

Multifactorial and multiple component interventions for preventing falls in older people living in the community (Review) 
(Continued)

cognitive train-

ing

Control 2: Usual

care

\begin{tabular}{llllll}
\hline Olsen 2014 & 13 & 11 & 47 & 42 & - \\
\hline Palvanen 2014 & 296 & 349 & 661 & 653 & - \\
\hline Pardessus 2002 & 13 & 15 & 30 & 30 & - \\
\hline
\end{tabular}

Rubenstein Data not included since the number of falls was only recorded for 3 month 2007 intervals during the follow up period

\begin{tabular}{|c|c|c|c|c|c|}
\hline Russell 2010 & 163 & 151 & 320 & 330 & - \\
\hline $\begin{array}{l}\text { Spice } 2009 \text { com- } \\
\text { parison } \mathbf{a}^{b, d}\end{array}$ & 92 & 52 & 106 & 62 & $\begin{array}{l}\text { Intervention } \\
\text { 1: Primary care } \\
\text { multifactorial in- } \\
\text { tervention } \\
\text { Control: Usual } \\
\text { care }\end{array}$ \\
\hline $\begin{array}{l}\text { Spice } 2009 \text { com- } \\
\text { parison } \mathbf{b}^{b, d}\end{array}$ & 123 & 52 & 164 & 62 & $\begin{array}{l}\text { Inter- } \\
\text { vention 2: Sec- } \\
\text { ondary care mul- } \\
\text { tifactorial inter- } \\
\text { vention } \\
\text { Control: Usual } \\
\text { care }\end{array}$ \\
\hline Tinetti $1994^{b}$ & 44 & 58 & 125 & 122 & - \\
\hline Ueda 2017 & $0.5^{e}$ & 2 & 25 & 26 & - \\
\hline $\begin{array}{l}\text { Van Haastregt } \\
2000\end{array}$ & 68 & 58 & 120 & 115 & - \\
\hline Vetter 1992 & 95 & 65 & 240 & 210 & - \\
\hline Vind 2009 & 110 & 101 & 196 & 196 & - \\
\hline Wagner 1994 & 175 & 223 & 635 & 607 & - \\
\hline Waterman 2016 & 9 & 8 & 15 & 13 & - \\
\hline Wesson 2013 & 2 & 4 & 11 & 11 & - \\
\hline $\begin{array}{l}\text { Whitehead } \\
2003\end{array}$ & 28 & 15 & 58 & 65 & - \\
\hline
\end{tabular}




\begin{tabular}{|c|c|c|c|c|c|}
\hline Zijlstra 2009 & 91 & 117 & 188 & 203 & - \\
\hline
\end{tabular}

${ }^{a}$ For Clemson 2004, we used the reported risk ratio $(0.90,95 \%$ CI 0.73 to 1.10$)$

${ }^{b}$ We performed adjustment for clustering as specified in the Methods. The adjusted data are presented

${ }^{c}$ Study article states the proportion of fallers were $4 \%$ and $5 \%$ and thus we used 2 as the number of fallers in each group in the analysis. However, a second point in the article refers to 9 fallers, raising concern on the accuracy of this data

${ }^{d}$ We used the conservative analysis for Spice 2009 presented in the main trial report in the meta-analysis. This assumed those who were lost to follow-up had a fall during the follow-up period. In meta-analysis, the control arm was incorrectly not adjusted for clustering.

${ }^{e} 0.5$ used for the purposes of the analysis: zero events.

\section{Appendix 5. Supplementary data table: raw data for number of recurrent fallers}

\begin{tabular}{|c|c|c|c|c|c|}
\hline Study ID & $\begin{array}{l}\text { Intervention } \\
\text { arm: } \\
\text { Number of re- } \\
\text { current fallers }\end{array}$ & $\begin{array}{l}\text { Control arm: } \\
\text { Number of re- } \\
\text { current fallers }\end{array}$ & $\begin{array}{l}\text { Intervention } \\
\text { arm: } \\
\text { Number of par- } \\
\text { ticipants }\end{array}$ & $\begin{array}{l}\text { Control arm: } \\
\text { Number of par- } \\
\text { ticipants }\end{array}$ & $\begin{array}{l}\text { Details if } \\
2 \text { or more com- } \\
\text { parisons }\end{array}$ \\
\hline $\begin{array}{l}\text { Campbell } 2005 \\
\text { comparison a }\end{array}$ & 24 & 15 & 98 & 48 & $\begin{array}{l}\text { Interven- } \\
\text { tion 1: Exercise, } \\
\text { home safety pro- } \\
\text { gramme and vi- } \\
\text { tamin D } \\
\text { Control: Usual } \\
\text { care }\end{array}$ \\
\hline $\begin{array}{l}\text { Campbell } 2005 \\
\text { comparison b }\end{array}$ & 27 & 15 & 97 & 48 & $\begin{array}{l}\text { Intervention 2: } \\
\text { Exercise and vi- } \\
\text { tamin D } \\
\text { Control: Usual } \\
\text { care }\end{array}$ \\
\hline Clemson $2004^{a}$ & - & - & 157 & 153 & - \\
\hline Close 1999 & 21 & 55 & 184 & 213 & - \\
\hline De Vries 2010 & 37 & 35 & 106 & 111 & - \\
\hline Elley 2008 & 69 & 54 & 155 & 157 & - \\
\hline Faes 2011 & 6 & 1 & 18 & 15 & - \\
\hline Fairhall 2014 & 32 & 37 & 119 & 119 & - \\
\hline
\end{tabular}


(Continued)

\begin{tabular}{|c|c|c|c|c|c|}
\hline Ferrer 2014 & 11 & 13 & 142 & 131 & - \\
\hline Hendriks 2008 & 32 & 34 & 124 & 134 & - \\
\hline Hogan 2001 & 26 & 35 & 75 & 77 & - \\
\hline Lord 2005 & 49 & 45 & 202 & 201 & - \\
\hline Möller 2014 & 19 & 23 & 80 & 73 & - \\
\hline $\begin{array}{l}\text { Schrijnemaek- } \\
\text { ers } \\
1995\end{array}$ & 17 & 26 & 85 & 97 & - \\
\hline Vind 2009 & 43 & 44 & 196 & 196 & - \\
\hline Waterman 2016 & 3 & 3 & 15 & 13 & - \\
\hline Zijlstra 2009 & 48 & 76 & 188 & 203 & - \\
\hline
\end{tabular}

\section{Appendix 6. Supplementary data table: raw data for number of people sustaining a fracture}

\begin{tabular}{|c|c|c|c|c|c|}
\hline Study ID & $\begin{array}{l}\text { Intervention arm: } \\
\text { Number of people } \\
\text { sustaining a frac- } \\
\text { ture }\end{array}$ & $\begin{array}{l}\text { Control arm: } \\
\text { Number of people } \\
\text { sustaining a frac- } \\
\text { ture }\end{array}$ & $\begin{array}{l}\text { Intervention arm: } \\
\text { Number of partici- } \\
\text { pants }\end{array}$ & $\begin{array}{l}\text { Control arm: } \\
\text { Number of partici- } \\
\text { pants }\end{array}$ & $\begin{array}{l}\text { Details given if } 2 \\
\text { or more compar- } \\
\text { isons and outcome } \\
\text { reported }\end{array}$ \\
\hline Ciaschini 2009 & 1 & 6 & 101 & 100 & - \\
\hline Davison 2005 & 6 & 11 & 159 & 154 & - \\
\hline De Vries 2010 & 5 & 5 & 106 & 111 & - \\
\hline Fairhall $2014^{a}$ & 13 & 12 & 119 & 119 & - \\
\hline Hogan 2001 & 3 & 5 & 75 & 77 & - \\
\hline Logan 2010 & 3 & 6 & 102 & 102 & - \\
\hline Neelemaat 2012 & $0.5^{b}$ & 1 & 105 & 105 & - \\
\hline Russell 2010 & 8 & 15 & 320 & 330 & - \\
\hline
\end{tabular}


(Continued)

\begin{tabular}{|c|c|c|c|c|c|}
\hline $\begin{array}{l}\text { Spice } 2009 \text { com- } \\
\text { parison } \mathbf{a}^{c}\end{array}$ & 5 & 4 & 89 & 52 & $\begin{array}{l}\text { Intervention } 1 \text { : Pri- } \\
\text { mary care multifac- } \\
\text { torial intervention } \\
\text { Control: Usual care }\end{array}$ \\
\hline $\begin{array}{l}\text { Spice } 2009 \text { com- } \\
\text { parison } \mathbf{b}^{c}\end{array}$ & 5 & 4 & 138 & 52 & $\begin{array}{l}\text { Intervention } 2 \text { : Sec- } \\
\text { ondary care mul- } \\
\text { tifactorial interven- } \\
\text { tion } \\
\text { Control: Usual care }\end{array}$ \\
\hline Vetter 1992 & 16 & 14 & 240 & 210 & - \\
\hline Wesson 2013 & $0.5^{2}$ & 1 & 11 & 11 & - \\
\hline \multicolumn{6}{|c|}{$\begin{array}{l}{ }^{a} \text { Outcome data from Fairhall } 2014 \text { measures the numb } \\
\text { number of people with fractures } \\
{ }^{b} 0.5 \text { used for the purposes of the analysis: zero events. } \\
{ }^{c} \text { We performed adjustment for clustering as specified in }\end{array}$} \\
\hline
\end{tabular}

\section{Appendix 7. Supplementary data table: raw data for number of people sustaining a fall requiring hospital admission}

\begin{tabular}{|c|c|c|c|c|c|}
\hline Study ID & $\begin{array}{l}\text { Intervention arm: } \\
\text { Number of people } \\
\text { who experience a } \\
\text { fall requiring hos- } \\
\text { pital admission }^{a}\end{array}$ & $\begin{array}{l}\text { Control arm: } \\
\text { Number of people } \\
\text { who experience a } \\
\text { fall requiring hos- } \\
\text { pital admission }^{a}\end{array}$ & $\begin{array}{l}\text { Intervention arm: } \\
\text { Number of partici- } \\
\text { pants }\end{array}$ & $\begin{array}{l}\text { Control arm: } \\
\text { Number of partici- } \\
\text { pants }\end{array}$ & $\begin{array}{l}\text { Details given if } 2 \\
\text { or more compar- } \\
\text { isons and outcome } \\
\text { reported }\end{array}$ \\
\hline Carpenter 1990 & 121 & 107 & 272 & 267 & - \\
\hline Ciaschini 2009 & 2 & 3 & 101 & 100 & - \\
\hline Coleman $1999^{b}$ & 30 & 21 & 81 & 62 & - \\
\hline Davison 2005 & 14 & 17 & 159 & 154 & - \\
\hline Fabacher 1994 & 22 & 23 & 100 & 95 & - \\
\hline Hogan 2001 & 5 & 6 & 79 & 84 & - \\
\hline Huang 2005 & 4 & 13 & 63 & 63 & - \\
\hline Jitapunkul 1998 & 16 & 18 & 57 & 59 & - \\
\hline
\end{tabular}




\begin{tabular}{|c|c|c|c|c|c|}
\hline Logan 2010 & 53 & 54 & 102 & 102 & - \\
\hline $\begin{array}{l}\text { Ng } 2015 \text { compari- } \\
\text { son a }\end{array}$ & 6 & 3 & 49 & 48 & $\begin{array}{l}\text { Intervention: Phys- } \\
\text { ical activity, nutri- } \\
\text { tional supplements } \\
\text { and cognitive train- } \\
\text { ing } \\
\text { Control 1: Exercise }\end{array}$ \\
\hline $\begin{array}{l}\text { Ng } 2015 \text { compari- } \\
\text { son b }\end{array}$ & 6 & 2 & 49 & 50 & $\begin{array}{l}\text { Intervention: Phys- } \\
\text { ical activity, nutri- } \\
\text { tional supplements } \\
\text { and cognitive train- } \\
\text { ing } \\
\text { Control 2: Usual } \\
\text { care }\end{array}$ \\
\hline Pardessus 2002 & 4 & 3 & 30 & 30 & - \\
\hline Rubenstein 2007 & 210 & 217 & 334 & 360 & - \\
\hline $\begin{array}{l}\text { Spice } 2009 \text { com- } \\
\text { parison a } \mathbf{a}^{b, c}\end{array}$ & 23 & 11 & 106 & 63 & $\begin{array}{l}\text { Intervention 1: Pri- } \\
\text { mary care multifac- } \\
\text { torial intervention } \\
\text { Control: Usual care }\end{array}$ \\
\hline $\begin{array}{l}\text { Spice } 2009 \text { com- } \\
\text { parison } \mathbf{b}^{b, c}\end{array}$ & 30 & 11 & 164 & 63 & $\begin{array}{l}\text { Intervention } 2 \text { : Sec- } \\
\text { ondary care mul- } \\
\text { tifactorial interven- } \\
\text { tion } \\
\text { Control: Usual care }\end{array}$ \\
\hline Tinetti $1994^{b}$ & 27 & 31 & 130 & 125 & - \\
\hline Van Rossum 1993 & 121 & 133 & 292 & 288 & - \\
\hline Wagner 1994 & 3 & 5 & 635 & 607 & - \\
\hline
\end{tabular}

${ }^{a}$ Due to poor reporting, it was sometimes unclear how many hospital admissions were falls-related. Therefore, we also included outcome data on hospital admissions in general.

${ }^{b}$ We performed adjustment for clustering as specified in the Methods. The adjusted data are presented

${ }^{c}$ We used the conservative analysis for Spice 2009 presented in the main trial report in the meta-analysis. This assumed those who were lost to follow-up had a hospital admission during the follow-up period 
Appendix 8. Supplementary data table: raw data for number of people sustaining a fall requiring medical attention

\begin{tabular}{|c|c|c|c|c|c|}
\hline Study ID & $\begin{array}{l}\text { Intervention arm: } \\
\text { Number of people } \\
\text { who experience a } \\
\text { fall requiring med- } \\
\text { ical attention }\end{array}$ & $\begin{array}{l}\text { Control arm: } \\
\text { Number of people } \\
\text { who experience a } \\
\text { fall requiring med- } \\
\text { ical attention }\end{array}$ & $\begin{array}{l}\text { Intervention arm: } \\
\text { Number of partici- } \\
\text { pants }\end{array}$ & $\begin{array}{l}\text { Control arm: } \\
\text { Number of partici- } \\
\text { pants }\end{array}$ & $\begin{array}{l}\text { Details given if } 2 \\
\text { or more compar- } \\
\text { isons and outcome } \\
\text { reported }\end{array}$ \\
\hline $\begin{array}{l}\text { Campbell } 2005 \\
\text { comparison a }\end{array}$ & 30 & 16 & 98 & 48 & $\begin{array}{l}\text { Intervention 1: Ex- } \\
\text { ercise, home safety } \\
\text { programme and vi- } \\
\text { tamin D } \\
\text { Control: Usual care }\end{array}$ \\
\hline $\begin{array}{l}\text { Campbell } 2005 \\
\text { comparison b }\end{array}$ & 32 & 16 & 97 & 48 & $\begin{array}{l}\text { Intervention 2: Ex- } \\
\text { ercise and vitamin D } \\
\text { Control: Usual care }\end{array}$ \\
\hline Davison 2005 & 25 & 27 & 159 & 154 & - \\
\hline Hendriks 2008 & 14 & 20 & 166 & 167 & - \\
\hline Hogan 2001 & 9 & 8 & 79 & 84 & - \\
\hline Möller 2014 & 15 & 9 & 80 & 73 & - \\
\hline Tinetti $1994^{a}$ & 18 & 22 & 125 & 122 & - \\
\hline $\begin{array}{l}\text { Van } \quad \text { Haastregt } \\
2000\end{array}$ & 21 & 14 & 120 & 115 & - \\
\hline Vind 2009 & 34 & 35 & 196 & 196 & - \\
\hline Wagner 1994 & 42 & 57 & 635 & 607 & - \\
\hline
\end{tabular}

Appendix 9. Supplementary data table: raw data for health-related quality of life (multifactorial interventions)

\begin{tabular}{|c|c|c|c|c|c|c|c|c|}
\hline Study ID & $\begin{array}{l}\text { Included in } \\
\text { HRQoL } \\
\text { meta- } \\
\text { analysis }\end{array}$ & $\begin{array}{l}\text { Outcome } \\
\text { measure } \\
\text { Range and } \\
\text { direction }\end{array}$ & $\begin{array}{l}\text { Mean } \\
(S D)\end{array}$ & No. pts & $\begin{array}{c}\text { Mean } \\
(\mathrm{SD})\end{array}$ & No. Pts & Effect measure & Summary data \\
\hline
\end{tabular}


(Continued)

\begin{tabular}{|c|c|c|c|c|c|c|c|c|}
\hline Close 1999 & Yes & $\begin{array}{l}\text { Barthel in- } \\
\text { dex } \\
\text { Range: } 0 \quad- \\
\text { 20; higher is } \\
\text { better }\end{array}$ & $\begin{array}{l}18.6 \\
(2.4)\end{array}$ & 184 & $\begin{array}{l}17.3 \\
(3.7)\end{array}$ & 213 & $\mathrm{MD}$ & $\begin{array}{l}1.30(0.69 \text { to } 1 . \\
91)\end{array}$ \\
\hline $\begin{array}{l}\text { Coleman } \\
1999\end{array}$ & $\begin{array}{l}\text { Data only } \\
\text { presented } \\
\text { separately } \\
\text { for physical } \\
\text { health- } \\
\text { related qual- } \\
\text { ity of life }\end{array}$ & $\begin{array}{l}\text { SF-36 phys- } \\
\text { ical } \\
\text { Range: } 0- \\
100 \text {; higher } \\
\text { is better }\end{array}$ & $\begin{array}{l}37.5 \\
(-)\end{array}$ & 78 & $\begin{array}{l}37.5 \\
(-)\end{array}$ & 49 & - & $\begin{array}{l}\text { (no overall dif- } \\
\text { ference } \\
\text { observed) }\end{array}$ \\
\hline $\begin{array}{l}\text { De Vries } \\
2010\end{array}$ & $\begin{array}{l}\text { Not pooled - } \\
\text { change score }\end{array}$ & $\begin{array}{l}\text { EQ-5D } \\
\text { Range: } 0 \text { - } \\
1 \text {; higher is } \\
\text { better }\end{array}$ & $\begin{array}{l}0.01 \\
(0.16)\end{array}$ & 106 & $\begin{array}{l}0.07 \\
(0.16)\end{array}$ & 106 & $\begin{array}{l}\text { MD of change } \\
\text { score }\end{array}$ & $\begin{array}{l}-0.06 \quad(-0.10 \\
\text { to }-0.02)\end{array}$ \\
\hline \multirow[t]{2}{*}{ Elley 2008} & \multirow[t]{2}{*}{$\begin{array}{l}\text { Data only } \\
\text { presented } \\
\text { separately } \\
\text { for physical } \\
\text { health- } \\
\text { related qual- } \\
\text { ity of life }\end{array}$} & $\begin{array}{l}\text { SF-36 phys- } \\
\text { ical } \\
\text { Range: } 0 \text { - } \\
100 ; \\
\text { higher is } \\
\text { better }\end{array}$ & $\begin{array}{l}\text { Median } 39 . \\
4(\text { IQR } 29.9 \\
-46.0)\end{array}$ & - & $\begin{array}{l}\text { Median } 37.2 \\
\text { (IQR } 29.0 \text { - } \\
45.4)\end{array}$ & - & - & $\begin{array}{l}\text { Only } \\
\text { median and in- } \\
\text { terquartile range } \\
\text { reported }\end{array}$ \\
\hline & & $\begin{array}{l}\text { SF-36 men- } \\
\text { tal } \\
\text { Range: } 0 \text { - } \\
100 \text {; higher } \\
\text { is better }\end{array}$ & $\begin{array}{l}\text { Median } 56 . \\
7 \text { (IQR } 48.8 \\
-61.3)\end{array}$ & - & $\begin{array}{l}\text { Median } 57.7 \\
\text { (IQR } 49.4 \text { - } \\
61.9 \text { ) }\end{array}$ & . & . & $\begin{array}{l}\text { Only } \\
\text { median and in- } \\
\text { terquartile range } \\
\text { reported }\end{array}$ \\
\hline $\begin{array}{l}\text { Fairhall } \\
2014\end{array}$ & Yes & $\begin{array}{l}\text { ED-5D VAS } \\
\text { Range: } 0 \text { - } \\
100 ; \\
\text { higher is } \\
\text { better }\end{array}$ & $\begin{array}{l}57.5 \\
(20.8)\end{array}$ & 107 & $\begin{array}{l}57.7 \\
(19.7)\end{array}$ & 108 & MD & $\begin{array}{l}-0.20 \quad(-5.62 \\
\text { to } 5.22)\end{array}$ \\
\hline $\begin{array}{l}\text { Gallagher } \\
1996\end{array}$ & Yes & $\begin{array}{l}\text { SF-36 } \\
\text { Range: } 0 \quad- \\
100 ; \\
\text { higher is } \\
\text { better, }\end{array}$ & $\begin{array}{l}36.8 \\
(5)\end{array}$ & 50 & $\begin{array}{l}36.3 \\
(5)\end{array}$ & 50 & MD & $\begin{array}{l}0.50(-1.46 \text { to } \\
2.46)\end{array}$ \\
\hline $\begin{array}{l}\text { Hendriks } \\
2008\end{array}$ & Yes & $\begin{array}{l}\text { EuroQoL } \\
\text { Range: } 0-1 \text {; } \\
\text { higher is } \\
\text { better, }\end{array}$ & $\begin{array}{l}0.7 \\
(0.25)\end{array}$ & 124 & $\begin{array}{l}0.71 \\
(0.28)\end{array}$ & 134 & MD & $\begin{array}{l}-0.01 \quad(-0.07 \\
\text { to } 0.05)\end{array}$ \\
\hline
\end{tabular}




\begin{tabular}{|c|c|c|c|c|c|c|c|c|}
\hline $\begin{array}{l}\text { Huang } \\
2005\end{array}$ & Yes & $\begin{array}{l}\text { SF-36 } \\
\text { Range: } 0 \\
\text { 100; higher } \\
\text { is better }\end{array}$ & $\begin{array}{l}60.77 \\
(10.5)\end{array}$ & 63 & $\begin{array}{l}51.25 \\
(11.63)\end{array}$ & 59 & $\mathrm{MD}$ & $\begin{array}{l}9.52 \quad(5.58 \text { to } \\
13.46)\end{array}$ \\
\hline Imhof 2012 & $\begin{array}{l}\text { Data not us- } \\
\text { able }\end{array}$ & $\begin{array}{l}\text { WHO- } \\
\text { QOL-BREF } \\
\text { (German) } \\
\text { Range: 0- } \\
\text { 100; higher } \\
\text { is better }\end{array}$ & . & . & . & . & . & $\begin{array}{l}\text { Only } \\
\text { reports ICC and } \\
\text { p-value } \\
\text { as part of mul- } \\
\text { tivariate analysis } \\
\text { (no overall dif- } \\
\text { ference } \\
\text { observed) }\end{array}$ \\
\hline $\begin{array}{l}\text { Jitapunkul } \\
1998\end{array}$ & Yes & $\begin{array}{l}\text { Barthel in- } \\
\text { dex } \\
\text { Range: } 0 \text { - } \\
\text { 20; higher is } \\
\text { better }\end{array}$ & $\begin{array}{l}17.3 \\
(3.6)\end{array}$ & 57 & $\begin{array}{l}17.1 \\
(2.7)\end{array}$ & 59 & $\mathrm{MD}$ & $\begin{array}{l}0.20(-0.96 \text { to } \\
1.36)\end{array}$ \\
\hline $\begin{array}{l}\text { Kingston } \\
2001\end{array}$ & $\begin{array}{l}\text { Data not us- } \\
\text { able }\end{array}$ & $\begin{array}{l}\text { SF-36 } \\
\text { Range: } 0 \quad- \\
100 ; \\
\text { higher is } \\
\text { better, }\end{array}$ & - & - & - & - & - & $\begin{array}{l}\text { Only re- } \\
\text { ports mean and } \\
\text { P value for each } \\
\text { domain sep- } \\
\text { arately (no over- } \\
\text { all difference } \\
\text { observed) }\end{array}$ \\
\hline $\begin{array}{l}\text { Lightbody } \\
2002\end{array}$ & Yes & $\begin{array}{l}\text { Barthel in- } \\
\text { dex } \\
\text { Range: } 0 \text { - } \\
\text { 20; higher is } \\
\text { better }\end{array}$ & $\begin{array}{l}18.5 \\
(2.37)\end{array}$ & 155 & $\begin{array}{l}17.8 \\
(3.6)\end{array}$ & 159 & MD & $\begin{array}{l}0.70(0.03 \text { to } 1 . \\
37)\end{array}$ \\
\hline Logan 2010 & Yes & $\begin{array}{l}\text { Barthel in- } \\
\text { dex } \\
\text { Range: } 0 \text { - } \\
20 \text {; higher is } \\
\text { better }\end{array}$ & $\begin{array}{l}14.33 \\
(4.69)\end{array}$ & 82 & $\begin{array}{l}13.57 \\
(4.79)\end{array}$ & 75 & MD & $\begin{array}{l}0.76(-0.73 \text { to } \\
2.25)\end{array}$ \\
\hline $\begin{array}{l}\text { Markle- } \\
\text { Reid } 2010\end{array}$ & $\begin{array}{l}\text { Data only } \\
\text { presented } \\
\text { separately } \\
\text { for men- } \\
\text { tal and phys- } \\
\text { ical health- } \\
\text { related qual- } \\
\text { ity of life }\end{array}$ & $\begin{array}{l}\text { SF-36 phys- } \\
\text { ical } \\
\text { Range: } 0 \text { - } \\
100 ; \\
\text { higher is } \\
\text { better }\end{array}$ & $\begin{array}{l}54.76 \\
(17.45)\end{array}$ & 49 & $\begin{array}{l}55.51 \\
(20.43)\end{array}$ & 43 & MD & $\begin{array}{l}-0.75 \quad(-8.57 \\
\text { to } 7.07)\end{array}$ \\
\hline
\end{tabular}




\begin{tabular}{|c|c|c|c|c|c|c|c|c|}
\hline & & $\begin{array}{l}\text { SF-36 men- } \\
\text { tal } \\
\text { Range: } 0- \\
100 \text {; higher } \\
\text { is better }\end{array}$ & $\begin{array}{l}73.07 \\
(15.33)\end{array}$ & 49 & $\begin{array}{l}74 \\
(14.5)\end{array}$ & 43 & $\mathrm{MD}$ & $\begin{array}{l}-0.93(-7.03 \\
\text { to } 5.17)\end{array}$ \\
\hline $\begin{array}{l}\text { Newbury } \\
2001\end{array}$ & $\begin{array}{l}\text { Data not us- } \\
\text { able }\end{array}$ & $\begin{array}{l}\text { Barthel in- } \\
\text { dex } \\
\text { Range: } 0- \\
\text { 20; higher is } \\
\text { better }\end{array}$ & - & - & - & - & - & $\begin{array}{l}\text { Only } \\
\text { reported physi- } \\
\text { cal function (no } \\
\text { overall differ- } \\
\text { ence observed) }\end{array}$ \\
\hline $\begin{array}{l}\text { Rubenstein } \\
2007\end{array}$ & Yes & $\begin{array}{l}\text { SF-36 } \\
\text { Range: } 0 \quad- \\
100 ; \\
\text { higher is } \\
\text { better }\end{array}$ & $\begin{array}{l}36 \\
(12.3)\end{array}$ & 334 & $\begin{array}{l}35.5 \\
(11.4)\end{array}$ & 360 & MD & $\begin{array}{l}0.50(-1.27 \text { to } \\
2.27)\end{array}$ \\
\hline $\begin{array}{l}\text { Sheffield } \\
2013\end{array}$ & $\begin{array}{l}\text { Data not us- } \\
\text { able }\end{array}$ & $\begin{array}{l}\text { EuroQoL } \\
\text { Range: } 0-1 \text {; } \\
\text { higher is } \\
\text { better }\end{array}$ & - & - & - & - & $\begin{array}{l}\text { Coef- } \\
\text { ficient in regres- } \\
\text { sion model }\end{array}$ & $\begin{array}{l}0.08 \text { (SE } 0 . \\
04) \text { representing } \\
\text { an } 8 \% \text { improve- } \\
\text { ment relative to } \\
\text { control }\end{array}$ \\
\hline \multirow[t]{2}{*}{ Shyu 2010} & \multirow{2}{*}{$\begin{array}{l}\text { Data only } \\
\text { presented } \\
\text { separately } \\
\text { for men- } \\
\text { tal and phys- } \\
\text { ical health- } \\
\text { related qual- } \\
\text { ity of life }\end{array}$} & $\begin{array}{l}\text { SF-36 phys- } \\
\text { ical } \\
\text { Range: } 0 \text { - } \\
100 ; \\
\text { higher is } \\
\text { better }\end{array}$ & $\begin{array}{l}64.52 \\
(19.03)\end{array}$ & 80 & $\begin{array}{l}55.81 \\
(18.7)\end{array}$ & 82 & MD & $\begin{array}{l}18.69 \\
27.40)\end{array}$ \\
\hline & & $\begin{array}{l}\text { SF-36 men- } \\
\text { tal } \\
\text { Range: } 0 \text { - } \\
100 ; \\
\text { higher is } \\
\text { better }\end{array}$ & $\begin{array}{l}62.19 \\
(28.08)\end{array}$ & 80 & $\begin{array}{l}43.5 \\
(28.47)\end{array}$ & 82 & $\mathrm{MD}$ & $\begin{array}{l}8.71 \quad(2.90 \text { to } \\
14.52)\end{array}$ \\
\hline Spice 2009 & $\begin{array}{l}\text { Data not us- } \\
\text { able }\end{array}$ & $\begin{array}{l}\text { Mod- } \\
\text { ified Barthel } \\
\text { index } \\
\text { Range: } 0 \text { - } \\
\text { 20; higher is } \\
\text { better }\end{array}$ & - & - & - & - & MD & $\begin{array}{l}\text { For primary care } \\
\text { intervention: } 0 . \\
07 \text { ( }-0.54 \text { to } 0 . \\
67) \\
\text { For sec- } \\
\text { ondary care in- } \\
\text { tervention: } 0.63 \\
(0.10 \text { to } 1.16)\end{array}$ \\
\hline
\end{tabular}




\section{Appendix 10. Supplementary data table: raw data for health-related quality of life (multiple interventions)}

\begin{tabular}{|c|c|c|c|c|c|c|c|c|}
\hline Study ID & $\begin{array}{l}\text { Included in } \\
\text { HRQoL } \\
\text { meta- } \\
\text { analysis }\end{array}$ & $\begin{array}{l}\text { Outcome } \\
\text { measure } \\
\text { Range and } \\
\text { direction }\end{array}$ & $\begin{array}{l}\text { Mean } \\
(S D)\end{array}$ & No. pts & $\begin{array}{l}\text { Mean } \\
(\mathrm{SD})\end{array}$ & No. pts & Effect measure & Summary data \\
\hline \multirow[t]{2}{*}{$\begin{array}{l}\text { Clemson } \\
2004\end{array}$} & \multirow{2}{*}{$\begin{array}{l}\text { Not pooled- } \\
\text { change score } \\
\text { and data } \\
\text { only pre- } \\
\text { sented sepa- } \\
\text { rately for } \\
\text { men- } \\
\text { tal and phys- } \\
\text { ical health- } \\
\text { related qual- } \\
\text { ity of life }\end{array}$} & $\begin{array}{l}\text { SF-36 phys- } \\
\text { ical } \\
\text { Range: } 0- \\
100 \text {; higher } \\
\text { is better }\end{array}$ & $\begin{array}{l}-0.52 \\
(10)\end{array}$ & 125 & $\begin{array}{l}0.01 \\
(9.65)\end{array}$ & 133 & $\begin{array}{l}\text { MD of change } \\
\text { score }\end{array}$ & $\begin{array}{l}0.53(-2.95 \text { to } \\
1.88)\end{array}$ \\
\hline & & $\begin{array}{l}\text { SF-36 men- } \\
\text { tal } \\
\text { Range: } 0 \text { - } \\
100 \text {; higher } \\
\text { is better }\end{array}$ & $\begin{array}{l}0.68 \\
(9.04)\end{array}$ & 125 & $\begin{array}{l}-0.02 \\
(8.34)\end{array}$ & 133 & $\begin{array}{l}\text { MD of change } \\
\text { score }\end{array}$ & $\begin{array}{l}0.70(-2.94 \text { to } \\
1.88)\end{array}$ \\
\hline Faes 2011 & $\begin{array}{l}\text { Not pooled - } \\
\text { change score }\end{array}$ & $\begin{array}{l}\text { EQ-5D VAS } \\
\text { Range: } \\
0 \text { - 100; pos- } \\
\text { itive change } \\
\text { is better }\end{array}$ & $\begin{array}{l}-10.54 \\
(17.19)\end{array}$ & 18 & $\begin{array}{l}9.19 \\
(15.64)\end{array}$ & 15 & $\begin{array}{l}\text { MD of change } \\
\text { score }\end{array}$ & $\begin{array}{l}-12.86 \quad(-28 . \\
30 \text { to } 2.58)\end{array}$ \\
\hline $\begin{array}{l}\text { Hagovska } \\
2016\end{array}$ & Yes & $\begin{array}{l}\text { QL-index } \\
\text { Range } 0 \quad- \\
10 ; \\
\text { higher is } \\
\text { better }\end{array}$ & $\begin{array}{l}9.52 \\
(1.06)\end{array}$ & 40 & $\begin{array}{l}7.71 \\
(1.55)\end{array}$ & 38 & $\mathrm{MD}$ & $\begin{array}{l}1.81 \text { (1.22 to } 2 . \\
40)\end{array}$ \\
\hline $\begin{array}{l}\text { Huang } \\
2011\end{array}$ & Yes & $\begin{array}{l}\text { WHO- } \\
\text { QOL-BREF } \\
\text { (Taiwanese) } \\
\text { Range: } 16 \text { - } \\
80 \text {; higher is } \\
\text { better }\end{array}$ & $\begin{array}{l}59.7 \\
(5.87)\end{array}$ & 56 & $\begin{array}{l}52.27 \\
(6.93)\end{array}$ & 60 & $\mathrm{MD}$ & $\begin{array}{l}7.43(5.10 \text { to } 9 . \\
76)\end{array}$ \\
\hline $\begin{array}{l}\text { Mendoza- } \\
\text { Ruvalcaba } \\
2015\end{array}$ & Yes & $\begin{array}{l}\text { Spanish ver- } \\
\text { sion } \\
\text { of Quality of } \\
\text { Life Index } \\
\text { Range: } 0 \text { - } \\
30 \text {; } \\
\text { higher is } \\
\text { better }\end{array}$ & $\begin{array}{l}26.67 \\
(1.99)\end{array}$ & 31 & $\begin{array}{l}25.19 \\
(3)\end{array}$ & 33 & MD & $\begin{array}{l}1.48(0.24 \text { to } 2 . \\
72)\end{array}$ \\
\hline $\begin{array}{l}\text { Serra-Prat } \\
2017\end{array}$ & Yes & $\begin{array}{l}\text { QoL } 0-10 \\
\text { VAS } \\
\text { Range: } 0-\end{array}$ & $\begin{array}{l}7.2 \\
(1.5)\end{array}$ & 61 & $\begin{array}{l}7.1 \\
(1.5)\end{array}$ & 72 & MD & $\begin{array}{l}0.10(-0.41 \text { to } \\
0.61)\end{array}$ \\
\hline
\end{tabular}




\begin{tabular}{|c|c|c|c|c|c|c|c|c|}
\hline & & $\begin{array}{l}10 \text {; higher is } \\
\text { better }\end{array}$ & & & & & & \\
\hline \multirow[t]{2}{*}{$\begin{array}{l}\text { Waterman } \\
2016\end{array}$} & \multirow{2}{*}{$\begin{array}{l}\text { Yes, data } \\
\text { only pre- } \\
\text { sented sepa- } \\
\text { rately for } \\
\text { men- } \\
\text { tal and phys- } \\
\text { ical health- } \\
\text { related qual- } \\
\text { ity of life }\end{array}$} & $\begin{array}{l}\text { SF-12 phys- } \\
\text { ical } \\
\text { Range: } 0 \text { - } \\
100 \text {; higher } \\
\text { is better }\end{array}$ & $\begin{array}{l}43.21 \\
(8.61)\end{array}$ & 15 & $\begin{array}{l}46.03 \\
(11.39)\end{array}$ & 13 & MD & $\begin{array}{l}-2.82(-10.39 \\
\text { to } 4.75)\end{array}$ \\
\hline & & $\begin{array}{l}\text { SF-12 men- } \\
\text { tal } \\
\text { Range: } 0- \\
100 \text {; higher } \\
\text { is better }\end{array}$ & $\begin{array}{l}54.35 \\
(6.89)\end{array}$ & 15 & $\begin{array}{l}46.72 \\
(11.49)\end{array}$ & 13 & MD & $\begin{array}{l}7.63 \quad(0.48 \text { to } \\
14.78)\end{array}$ \\
\hline
\end{tabular}

\section{CONTRIBUTIONS OFAUTHORS}

SH was involved in screening, data extraction, data analysis, led writing of the review and acted as guarantor of the review.

$\mathrm{OA}$ and $\mathrm{BC}$ were involved in screening, data extraction, data analysis and contributed to writing the review.

GB was involved in screening, data extraction, and commented on the draft.

SL, CS, LC and JC contributed to writing of the review and commented on the draft.

\section{DECLARATIONSOF INTEREST}

$\mathrm{SH}$ has no known conflicts of interest.

OA is funded on a NIHR Research Methods Programme Systematic Review Fellowship funded by the NIHR (NIHR-RMFI-201506-63). The views expressed in this publication are those of the protocol authors and not necessarily those of the NHS, the NIHR or the Department of Health.

BC has no known conflicts of interest.

GB has no known conflicts of interest.

CS is an author of several trials considered in this review, including an included trial (Fairhall 2014).

LC is an author of several trials considered in this review, including an included trial (Clemson 2004).

JC is an author of several trials considered in this review, including an included trial (Close 1999).

SL is lead author of the ProFaNE consensus for falls guidance and is an author of one of the trials considered in this review.

No review author was involved in study selection or processing of any trials of which they were or are involved.

Multifactorial and multiple component interventions for preventing falls in older people living in the community (Review) 


\section{SOURCES OF SUPPORT}

\section{Internal sources}

- No sources of support supplied

\section{External sources}

- National Institute for Health Research, UK.

This research is supported by the National Institute for Health Research (NIHR) Collaboration for Leadership in Applied Health Research and Care Oxford at Oxford Health NHS Foundation Trust, and the NIHR Oxford Musculoskeletal Biomedical Research Unit. OA is funded on a NIHR Research Methods Programme Systematic Review Fellowship funded by the NIHR (NIHR-RMFI2015-06-63). The views expressed in this publication are those of the authors and not necessarily those of the NHS, the NIHR or the Department of Health.

\section{DIFFERENCES BETWEEN PROTOCOLANDREVIEW}

The following changes between the protocol and review are described in the Methods section:

\section{Outcomes}

We noted when trials had performed an economic evaluation and summarised the key findings in a table.

\section{Search methods for identification of studies}

We did not search ClinicalTrials.gov. As ClinicalTrials.gov is included as one of the registers within the WHO ICTRP portal we considered a search of the latter sufficient.

\section{Risk of bias assessment}

We have added an assessment of risk of bias specifically for cluster-randomised trials. We assessed the risk of additional bias relating to recruitment, baseline imbalance, loss of clusters, incorrect analysis and comparability with individually-randomised trials, as described in Chapter 16 of the Cochrane Handbook for Systematic Reviews of Interventions ( Higgins 2011).

\section{Data synthesis}

We planned to make assessments at short-term (less than 12 months) and long-term (12 months or longer) follow-up. However, because of the limited number of studies for some outcomes, we combined both short- and long-term follow-up and reported duration of follow-up for each study in the Characteristics of included studies.

We planned to group multiple component interventions by the combination of interventions (i.e. where the same combination of single categories of intervention are delivered to all participants) and analyse each combination separately. Exercise was a key component in all but one of the 18 multiple component interventions and statistical heterogeneity $\left(\mathrm{I}^{2}\right)$ was $0 \%$. We therefore decided to present the results for the pooled analyses, in addition to subgroup totals for the different combinations.

\section{Subgroup analysis}

We planned to perform a subgroup analysis for multiple interventions which included a vitamin D component, comparing trials that recruited participants with lower baseline vitamin D levels versus those that did not. However, only four (Campbell 2005; Neelemaat 2012; Ng 2015; Uusi-Rasi 2015) of the 15 trials of multiple interventions included a vitamin D component and none specified the participants' baseline vitamin D level.

We restricted subgroup analyses to primary outcomes and where there were sufficient data.

Multifactorial and multiple component interventions for preventing falls in older people living in the community (Review) 


\section{Sensitivity analysis}

We planned to perform sensitivity analyses based on the choice of statistical model for pooling (fixed-effect versus random-effects). However, due to the heterogeneity in the type of interventions and participants identified, we decided to use only a random-effect model.

We planned to perform sensitivity analyses based on the effect of time on the impact of the intervention (i.e. comparing differences in treatment effect over time: earlier trials versus later trials). However, we did not set a cut-off year beforehand. Moreover, when we ordered studies by year of publication in RevMan, there was no obvious pattern over time and we therefore decided there was insufficient justification for arbitrarily choosing a cut-off year to select a subgroup of more recent trials.

\section{NOTES}

This review provides updated evidence for two of the intervention categories (multifactorial and multiple intervention) covered in the Cochrane Review Interventions for preventing falls in older people living in the community (Gillespie 2012). We took some of the wording in several sections of the review protocol, such as Background/Description of the condition, from Gillespie 2012. This reflected shared authorship of the two publications but also attempted to maintain continuity with the Gillespie 2012 review, and links between our review and other reviews that will cover other intervention categories, such as exercise (Sherrington 2016a).

Multifactorial and multiple component interventions for preventing falls in older people living in the community (Review) 